\title{
High-Speed PLIF Imaging of Hypersonic Transition over Discrete Cylindrical Roughness
}

\author{
P. M. Danehy ${ }^{*}$, C. B. Ivey ${ }^{\dagger}$, J. A. Inman ${ }^{\star}$, B. F. Bathel ${ }^{\S}$, S. B. Jones ${ }^{* *}$, A. C. McCrea ${ }^{\dagger \dagger}$ \\ NASA Langley Research Center, Hampton VA, 23681-2199 \\ N. Jiang ${ }^{\sharp}$, M. Webster ${ }^{\S}$, W. Lempert ${ }^{* * *}$ \\ The Ohio State University, Columbus $\mathrm{OH}$ \\ J. Miller, ${ }^{\dagger \dagger}$ T. Meyer ${ }^{\text {tt }}$ \\ The Iowa State University, Ames IA
}

\begin{abstract}
Transitional flow over cylindrical protuberances attached to a flat plate has been studied in a hypersonic wind tunnel. The tests were performed in the 31-Inch Mach 10 Air Tunnel at NASA Langley Research Center. Cylindrical protuberances with 4-mm diameter and heights of 1,2 , and $4 \mathrm{~mm}$, were attached to the flat plate. The plate angle of attack and tunnel stagnation pressure were varied to achieve desired combinations of boundary layer edge Mach number and the boundary layer thickness. Several different types of flow visualization based on nitric oxide planar laser-induced fluorescence (NO PLIF) were used. A 10-Hz NO PLIF system was used to produce a thin laser sheet. This laser sheet was oriented perpendicular to the flat plate on some runs and parallel to the flat plate on others. A thick laser sheet was also used in some runs to provide visualization of entire flow structures inside the boundary layer. Viewing the PLIF intensity generated with the thick laser sheet using two cameras allowed stereoscopic visualization of the flow. In post processing, these images were combined to produce anaglyphs. Viewing these anaglyphs through blue and red 3D-glasses allows the three-dimensional flow structures to be observed. Another measurement system was a MHz-rate NO PLIF imaging system. Both PLIF measurement systems have better than one microsecond time resolution, which is fast enough to freeze the flow. The MHz system allows subsequent images to be obtained at one or two microsecond time intervals, resulting in movies of the flow. The data reported in this paper show the size, shape, and speed of flow structures in transitional hypersonic boundary layer flows and will provide valuable comparison data for computations of these flows.
\end{abstract}

\section{Introduction}

Cylindrical-shaped protuberances will be present on the heatshield of NASA's Orion Crew Exploration Vehicle (CEV). ${ }^{1}$ Tension ties that hold the capsule to the launch vehicle will be cylindrical. The length of these ties considerably exceeds their diameter. Remnants of these ties will protrude from the heatshield during re-entry, probably burning off during descent. The compression pads that buffer the heatshield from the launch vehicle will also be

\footnotetext{
${ }^{*}$ Research Scientist, Advanced Sensing and Optical Measurement Branch, MS 493, AIAA Associate Fellow.

${ }^{\dagger}$ NASA LARSS Student, Advanced Sensing and Optical Measurements Branch, MS 493, and undergraduate student at Johns Hopkins University,

* Research Scientist, Advanced Sensing and Optical Measurement Branch, MS 493, AIAA Member.

${ }^{\S}$ PhD Student, University of Virginia, Charlottesville VA and graduate student at the National Institute of Aerospace, Hampton VA, AIAA Student Member.

** Technician, Advanced Sensing and Optical Measurement Branch, MS 493.

† ATK Space Systems, Hampton, VA.

* Post-Doctoral Researcher, AIAA Member.

$\S \S$ Graduate Research Assistant, AIAA Student Member.

*** Professor, AIAA Associate Fellow.

t† Graduate Research Assistant, AIAA Student Member.

\# Assistant Professor, AIAA Associate Fellow.
} 
cylindrical. Since these will be fabricated from a different material than the rest of the heat shield, they may end up being cylindrical protrusions if differential ablation occurs. These compression-pad protrusions will have a length much smaller than their diameter. Both of these types of discrete cylindrical roughness can cause the flow to transition from laminar to turbulent, significantly increasing the heat transfer to, and ablation of, the thermal protection material surrounding and downstream of the protrusions. Presently, the CEV is being designed conservatively - assuming a fully turbulent flow over the heatshield. However, a more detailed understanding of the physics of such transitional flows could reduce the uncertainty margin — and thus the associated weight — of heat shields for the CEV and other future aerospace vehicles.

Transition from laminar to turbulent flow caused by discrete protuberances is also relevant in many other hypersonic flight applications including flow-development for scramjet engine inlets ${ }^{2}$ and Space Shuttle Orbiter reentry. ${ }^{3}$ Tripped hypersonic flows have been studied for decades, as recently reviewed in Ref. 4. Various protuberance shapes have been investigated over the years, including spheres, rectangular fences, triangles, rectangles, pin-heads, and cylinders. Cylindrical protuberances have been studied by other researchers, owing to the relevance to the Orion CEV $\operatorname{program}^{1}$ as mentioned above, the simplicity of shape $\mathrm{e}^{5,6}$ and to easy availability of micrometer heads which allow precise and variable protuberance height. ${ }^{7}$

In this paper, we describe two separate wind tunnel entries in which advanced laser-based instrumentation has been developed and applied to visualize the hypersonic flow over cylindrical protrusions (or trips) on a flat plate. Upstream of these trips, trace quantities of nitric oxide (NO) were seeded into the boundary layer. The protuberances were sized to produce substantial perturbations of the incoming boundary layer. In the first test, a $10 \mathrm{~Hz}$ NO planar laser-induced fluorescence (NO PLIF) flow visualization system was used to provide wide-field-of-view, high-resolution images of the flowfield. The images had sub-microsecond time resolution, effectively freezing the flow during each camera exposure. These images, obtained with a time separation of 0.1 seconds, were uncorrelated with each other. Fluorescent oil-flow visualizations were also obtained during this test. In the second experiment, a laser and camera system capable of acquiring NO PLIF measurements at 1 million frames per second (1 MHz) was used. This system had lower spatial resolution and a smaller field of view, but the images were time correlated so that the temporal development of individual flow structures could be observed.

PLIF visualization allowed observation of both laminar and turbulent boundary layer flow downstream of the trips, depending on the flow conditions and experimental configuration. Parameters varied included trip height (from 1 to 4 $\mathrm{mm}$, while the trip diameter was held fixed at $4 \mathrm{~mm}$ ), model angle of attack and tunnel Reynolds number. By varying the angle of attack, the Mach number at the edge of the boundary layer was varied between approximately 3 and 8 , according to analytical oblique-shock calculations. The boundary layer thickness at the roughness location varied from 0.5 to 1.8 over the range of unit Reynolds numbers employed during the experiment, based on scaling of CFD computations described below. Experiments were performed in the NASA Langley Research Center 31-Inch Mach 10 Air Tunnel, which is an electrically-heated, blowdown facility.

In previous related work, flat plate flows over a triangular trip and a rectangular fence ${ }^{8}$ as well as a hemispherical roughness element ${ }^{9}$ were reported. In these papers, different methods of seeding NO were studied and analyzed to determine which method had the minimal impact on the flowfield. These minimally perturbing flow configurations were used in the present experiments. A companion paper details the operation and performance of the MHz-rate NO PLIF system. ${ }^{10}$ A second companion paper uses the same $10-\mathrm{Hz}$ and $\mathrm{MHz}$ instrumentation to study transitional flow over a protuberance shape similar to the Shuttle Orbiter Boundary Layer Transition flight experiment. ${ }^{11}$

To summarize the experimental setup, the model, shown in Fig. 1(a), was a sharp-leading-edge, wedge/flat-plate configuration with the cylindrical protuberance attached in the middle of the plate. Several trip inserts were used,

(a)

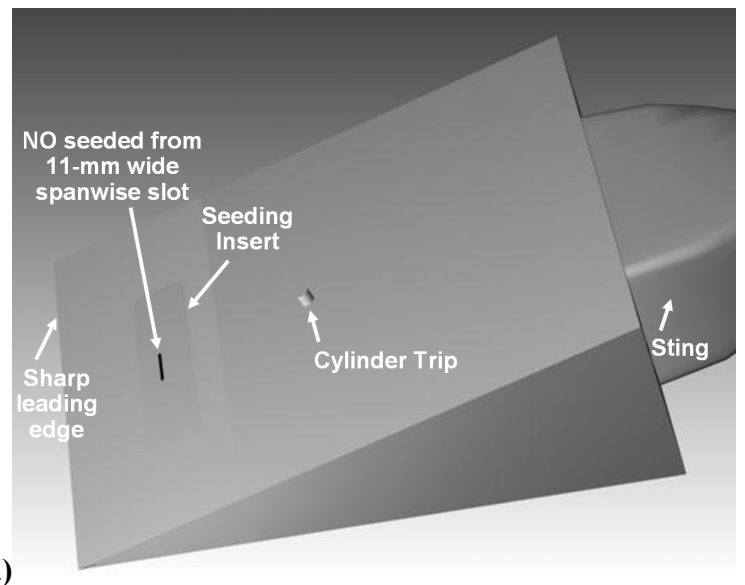

(b)

(c)

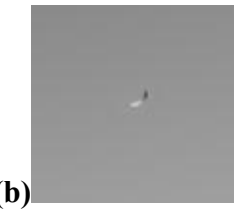

(d)

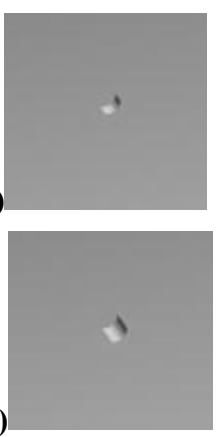

Figure 1. Test article used in the experiment, showing the assembled wedge model and trips. The model, including sting, flat plate, leading-edge seeding slot, and the 4-mm tall by 4-mm diameter cylindrical protuberance are shown in (a). Details of the three cylindrical trip configurations are shown in (b)-(d), 4-mm diameter trips with heights of 1-, 2-, and 4-mm respectively. A no-trip case was also studied. 
as shown in Fig. 1(b)-(d). NO was seeded into the flow through the slot indicated in Fig. 1(a) and interrogated by a UV laser sheet and captured by a camera to provide images of the flow. Three different unit Reynolds numbers, produced by varying the stagnation pressure of the wind tunnel, were tested with other test parameters remaining constant. In addition to varying the angle of attack and unit Reynolds number, different flow visualization techniques were used. Thin laser sheets were used to interrogate different planes in the flow, thick laser sheets were used to observe global flow structures as well as provide a stereoscopic imaging capability, and a high-framing rate laser system and camera were used to time-resolve the unsteady flow behavior.

\section{Experiment and Analysis Description}

The experiments were performed in the 31-Inch Mach 10 Air Tunnel at NASA Langley Research Center. The test apparatus consisted of five main components: the test article, the wind tunnel facility, the $10 \mathrm{~Hz}$ PLIF system, the MHz PLIF system and the oil flow visualization system. The analysis includes image processing and subsequent rendering in a three-dimensional software environment. These procedures are detailed in other papers and will only be briefly described herein, with references provided.

The test article was a $20^{\circ}$ full-angle wedge with a sharp leading edge. Figure 1 shows computer renderings of the trips and the model. The top surface of the wedge is a planar surface, herein referred to as a flat plate. The flat plate was $127.0-\mathrm{mm}$ (5-in.) wide and $162.5-\mathrm{mm}$ (6.4-in.) long. This flat plate was typically oriented at an angle of $20^{\circ}$ with respect to the oncoming flow (hereafter referred to the plate angle) during the experiments. In two of the runs reported herein, the plate angle was varied between $25^{\circ}$ and $5^{\circ}$ to alter the boundary layer edge conditions during a run. The edge Mach number $\left(\mathrm{M}_{\mathrm{e}}\right)$ and boundary layer thickness $(\delta)$ both decrease as the plate angle increases. The model was also rolled $1.5^{\circ}$ counterclockwise (as viewed from downstream) so the laser reflection from the bottom window of the tunnel would not illuminate the flow field; rather, the reflection hit the bottom of the model. This slight roll angle should have a negligible effect on the fluid mechanics of the experiment.

Three different protuberance shapes were attached to this surface to trip the flow from laminar to turbulent. The three cylindrical protuberances used had the following dimensions: (1) $1.05 \pm 0.03-\mathrm{mm}$ tall with a diameter of $4.08 \pm 0.03$ $\mathrm{mm}$, (2) $2.05 \pm 0.03-\mathrm{mm}$ tall with a diameter of $4.08 \pm 0.03 \mathrm{~mm}$, and (3) $4.04 \pm 0.03-\mathrm{mm}$ tall with a diameter of $4.11 \pm 0.03$ $\mathrm{mm}$ before being painted matte black. The edges of the trip were sharp. The center of each cylindrical trip was mounted 75.4 $\pm 0.2-\mathrm{mm}$ (2.97-in) downstream of the sharp leading edge of the model. Reference 9 and 11 describe the $11.0 \pm 0.1$ $\mathrm{mm}$ wide seeding slot and various issues regarding the potential perturbations to the flow caused by the seeding system used to inject NO into the laminar boundary layer upstream of the trip. Table 1 shows the NO flowrates used in each run. The model used in these experiments contained some imperfections. For the runs in Test 443 the imperfections are described in Ref. 9. For Test 463, the imperfections were the same as for Test 462 and are described in Ref. 11. These surface imperfections were typically an order of magnitude smaller than the 1- to 4-mm tall trips used to perturb the flow in this experiment. To first order, the model imperfections described should be negligible, but could have an effect on higher fidelity computations of the flow.

Table 1 summarizes the test conditions and configurations for the data presented in this paper. For the most part, three tunnel stagnation pressures, 2.4, 5.0 and 9.3 $\mathrm{MPa}$, were used to vary the unit Reynolds number. Hereafter these are referred to as low-, middle-, and high-Reynolds number conditions. In Test 463, two additional intermediate stagnation pressures were used. Further details of the flow properties at these conditions can be found in Hollis et al. ${ }^{12}$ or can be requested from the authors. 


\begin{tabular}{|c|c|c|c|c|c|c|c|c|c|c|c|c|}
\hline Test & Run & $\begin{array}{c}\text { Primary } \\
\text { Measurement }\end{array}$ & View & $\begin{array}{l}\text { Framing } \\
\text { Rate }\end{array}$ & Trip Type & $\begin{array}{c}\text { Trip } \\
\text { Height }\end{array}$ & $\begin{array}{c}\text { Trip } \\
\text { Width }\end{array}$ & $\begin{array}{c}\text { Stagnation } \\
\text { Pressure }\end{array}$ & $\begin{array}{l}\text { Stagnation } \\
\text { Temperature }\end{array}$ & $\begin{array}{c}\text { Unit } \\
\text { Reynolds } \\
\text { Number }\end{array}$ & Plate Angle & NO flow rate \\
\hline & & & & & & $(\mathrm{mm})$ & $(\mathrm{mm})$ & (MPa) & $(\mathrm{K})$ & $(1 / \mathrm{m})$ & (degrees) & $(\mathrm{sccm})$ \\
\hline 443 & 12 & PLIF Flow Vis & side & $10 \mathrm{~Hz}$ & cylinder & 1 & 4 & 9.34 & 1011 & 6.02 & $25 \rightarrow+5 \rightarrow 20$ & 300 \\
\hline 443 & 15 & PLIF Flow Vis & side & $10 \mathrm{~Hz}$ & cylinder & 2 & 4 & 9.30 & 1009 & 6.02 & $25 \rightarrow+5 \rightarrow 20$ & 300 \\
\hline 443 & 16 & PLIF Flow Vis & plan & $10 \mathrm{~Hz}$ & cylinder & 4 & 4 & 9.31 & 1012 & 6.00 & 20 & 300 \\
\hline 443 & 18 & PLIF Flow Vis & plan & $10 \mathrm{~Hz}$ & cylinder & 1 & 4 & 9.32 & 1011 & 6.01 & 20 & 300 \\
\hline 443 & 19 & PLIF Flow Vis & plan & $10 \mathrm{~Hz}$ & cylinder & 1 & 4 & 4.97 & 1016 & 3.27 & 20 & 300 \\
\hline 443 & 20 & PLIF Flow Vis & plan & $10 \mathrm{~Hz}$ & cylinder & 1 & 4 & 2.41 & 1006 & 1.66 & 20 & 300 \\
\hline 443 & 23 & PLIF Flow Vis & plan & $10 \mathrm{~Hz}$ & no trip & -- & -- & 9.35 & 999 & 6.16 & 20 & $300 ; 1000$ \\
\hline 443 & 24 & PLIF Flow Vis & plan & $10 \mathrm{~Hz}$ & no trip & -- & -- & 9.34 & 1000 & 6.14 & 20 & $100 ; 30$ \\
\hline 443 & 25 & PLIF Flow Vis & plan & $10 \mathrm{~Hz}$ & no trip & -- & -- & 4.98 & 1014 & 3.28 & 20 & $30 ; 300$ \\
\hline 443 & 31 & PLIF Flow Vis & plan & $10 \mathrm{~Hz}$ & cylinder & 2 & 4 & 9.33 & 1002 & 6.11 & 20 & 300 \\
\hline 443 & 47 & Stereo PLIF & plan & $10 \mathrm{~Hz}$ & cylinder & 1 & 4 & 4.97 & 1010 & 3.30 & 20 & $300 ; 300+5 S L P M ~ N 2$ \\
\hline 443 & 48 & Stereo PLIF & plan & $10 \mathrm{~Hz}$ & cylinder & 1 & 4 & 9.35 & 1001 & 6.14 & 20 & $600 ; 600+5$ SLPM N2 \\
\hline 443 & 49 & Stereo PLIF & plan & $10 \mathrm{~Hz}$ & cylinder & 4 & 4 & 9.35 & 995 & 6.17 & 20 & $600 ; 600+5$ SLPM N2 \\
\hline 443 & 53 & Oil Flow & plan & -- & no trip & -- & -- & 9.33 & 1000 & 6.13 & 20 & -- \\
\hline 443 & 54 & Oil Flow & plan & -- & cylinder & 1 & 4 & 4.97 & 1017 & 3.27 & 20 & -- \\
\hline 443 & 55 & Oil Flow & plan & -- & cylinder & 1 & 4 & 9.33 & 1000 & 6.13 & 20 & -- \\
\hline 443 & 56 & Oil Flow & plan & -- & cylinder & 4 & 4 & 9.31 & 1004 & 6.08 & 20 & -- \\
\hline 463 & 6 & PLIF Flow Vis & plan & $500 \mathrm{kHz}$ & cylinder & 2 & 4 & 4.97 & 995 & 3.39 & 20 & 300 \\
\hline 463 & 7 & PLIF Flow Vis & plan & $500 \mathrm{kHz}$ & cylinder & 2 & 4 & 2.40 & 979 & 1.74 & 20 & 150 \\
\hline 463 & 8 & PLIF Flow Vis & plan & $500 \mathrm{kHz}$ & cylinder & 2 & 4 & 9.34 & 994 & 6.20 & 20 & 300 \\
\hline 463 & 9 & PLIF Flow Vis & plan & $500 \mathrm{kHz}$ & cylinder & 2 & 4 & 3.45 & 987 & 2.42 & 20 & 230 \\
\hline 463 & 10 & PLIF Flow Vis & plan & $500 \mathrm{kHz}$ & cylinder & 1 & 4 & 6.91 & 992 & 4.67 & 20 & 300 \\
\hline 463 & 14 & PLIF Flow Vis & plan & $500 \mathrm{kHz}$ & no trip & -- & -- & 9.32 & 997 & 6.15 & 20 & 300 \\
\hline 463 & 17 & PLIF Flow Vis & plan & $1 \mathrm{MHz}$ & cylinder & 2 & 4 & 4.98 & 1003 & 3.35 & 20 & 300 \\
\hline
\end{tabular}

Table 1. Experimental test matrix.

As mentioned in the introduction, two different NO PLIF systems were used to obtain the data described in this paper. The $10 \mathrm{~Hz}$ system is detailed in Ref. 9, where use of the system to obtain thin-laser-sheet images is described. This system produced a laser sheet about $0.2 \mathrm{~mm}$ thick. Additionally, a $10-\mathrm{mm}$-thick laser sheet was used to obtain stereoscopic visualizations of the flow, as described in Ref. 13. The MHz NO PLIF system (detailed in Ref. 10 with additional results described in Ref. 11) provided movie sequence flow visualization using a thin laser sheet. Figure 2 shows experimental setups for these three different imaging configurations. Figure 2(a) shows both $10 \mathrm{~Hz}$ and $\mathrm{MHz}$ laser systems. Figure 2(b) shows a detailed view of the laser sheet entering the test section, passing over the model, and the pair of cameras used by the $10 \mathrm{~Hz}$ system to image the flow. The cameras were identical but had different lenses (focal lengths of $105-\mathrm{mm}$ and $45-\mathrm{mm}$ ) resulting in different fields of view and spatial resolutions. All images shown in this paper were obtained with the shorter focal length lens corresponding to the larger field of view. Figure 2(c) shows the stereoscopic imaging configuration used with the $10 \mathrm{~Hz}$ system. In this case, the two cameras were oriented in a vertical plane and used identical $105 \mathrm{~mm}$ focal length lenses. Figure 2(d) shows the cameras used when the MHz laser system (see Ref. 10 for details of this imaging system). Subsequent image processing, enhancement, and rendering of the PLIF images with a CAD (computer aided drafting) model of the test article provides a more intuitive visualization of the data. This rendering was performed using the Virtual Diagnostics Interface (ViDI), which is detailed in Refs. 11 and 14. A sheet position bar is shown on many of the images, indicating the height of the laser sheet above the surface of the flat plate. Due to vibrations of the model and laser sheet, as well as steady movement of the model during the run, this sheet position indicator is typically accurate to $\pm 0.3 \mathrm{~mm}$, as determined by identifying model features from the PLIF images. Some of this image-based evidence shows that the measured and reported sheet position can err by as much as $0.5 \mathrm{~mm}$ or more. Such outlier images were not selected for inclusion in this paper.

Oil flow measurements were conducted for several cases to show surface flow patterns. For each oil flow run, the upper surface of the wedge model was painted with a thin coat of high-viscosity oil. A dusting of green fluorescent powder was then applied to the oil-coated surface. Once wind tunnel flow was established, the model was injected for a few seconds-long enough for the surface flow pattern to appear without excessive blurring. The model was then retracted, and after the run, ultraviolet lighting was used to illuminate the fluorescent powder. Images were acquired with a digital camera. These images were dewarped using a dotcard image-registration process (described in Ref. 14), and imported into the ViDI environment.

Viscous CFD computations described in Ref. 8 were available for $0^{\circ}, 10^{\circ}$ and $20^{\circ}$ plate angles, but only for the middle unit Reynolds number condition. The boundary layer thickness and edge conditions (e.g. edge Mach number, $M_{\mathrm{e}}$ ) were computed for these three angles. At other tunnel operating conditions, the boundary layer thickness was estimated by scaling by $\mathrm{P}_{0}^{-1 / 2}$. At other plate angles, the boundary layer thickness was estimated by interpolation. The ratio of trip height to boundary layer thickness $(k / \delta)$ was similarly computed. For the 1-mm trip height and $20^{\circ}$ plate angle, the roughness Reynolds number, based on the trip height and the conditions at the trip height $\left(R e_{k}\right)$ was computed to be about 3000 . 


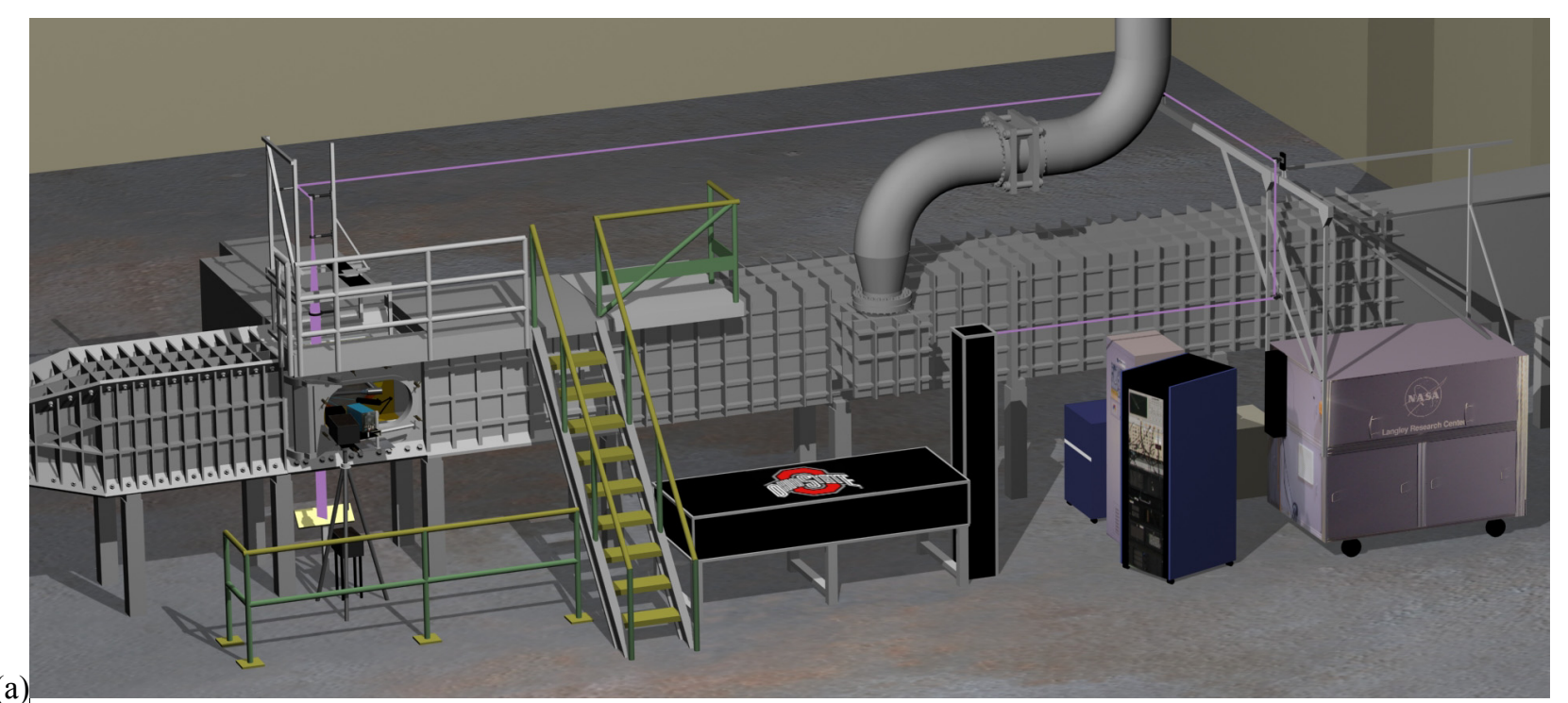

(a)

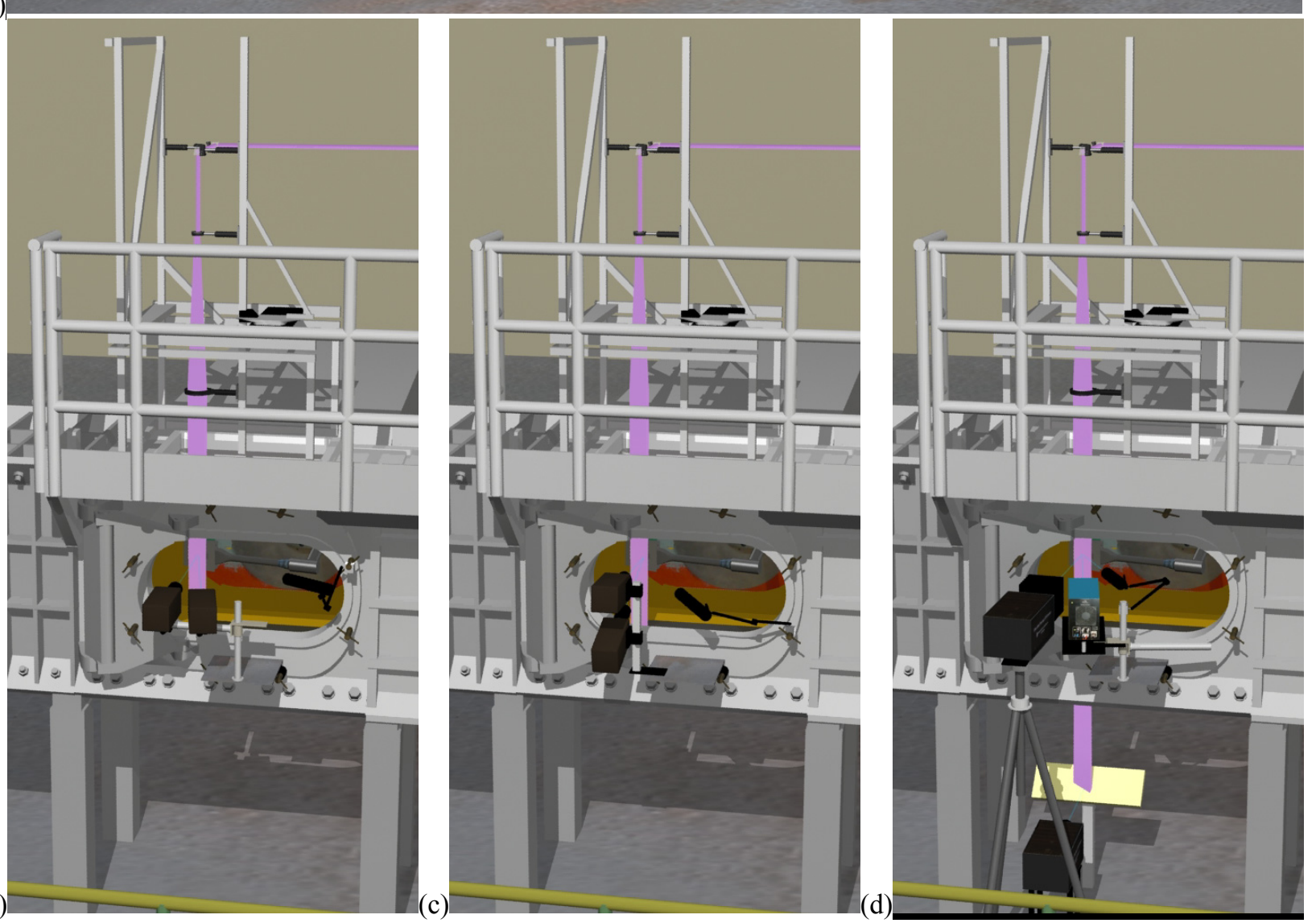

Figure 2. Schematic of the NO PLIF systems installed adjacent to the 31-Inch Mach 10 Air Tunnel for Tests 443 (10 Hz System) and 462 (MHz System). The NASA $10 \mathrm{~Hz}$ NO PLIF system is shown on the right side of (a) while the Ohio State University MHz NO PLIF system is shown to the right of the stairs. A close-up of the pair of cameras mounted near the tunnel window for Test 443 is shown in (b) while (c) shows the stereoscopic imaging configuration used in Test 443. The camera setup used for the MHz experiments in Test 462 is shown in (d). A second MHz rate camera below the tunnel is the sheet profiling camera that imaged the laser sheet which passes over the model, through the bottom window of the test section and onto a yellow painted plate. 


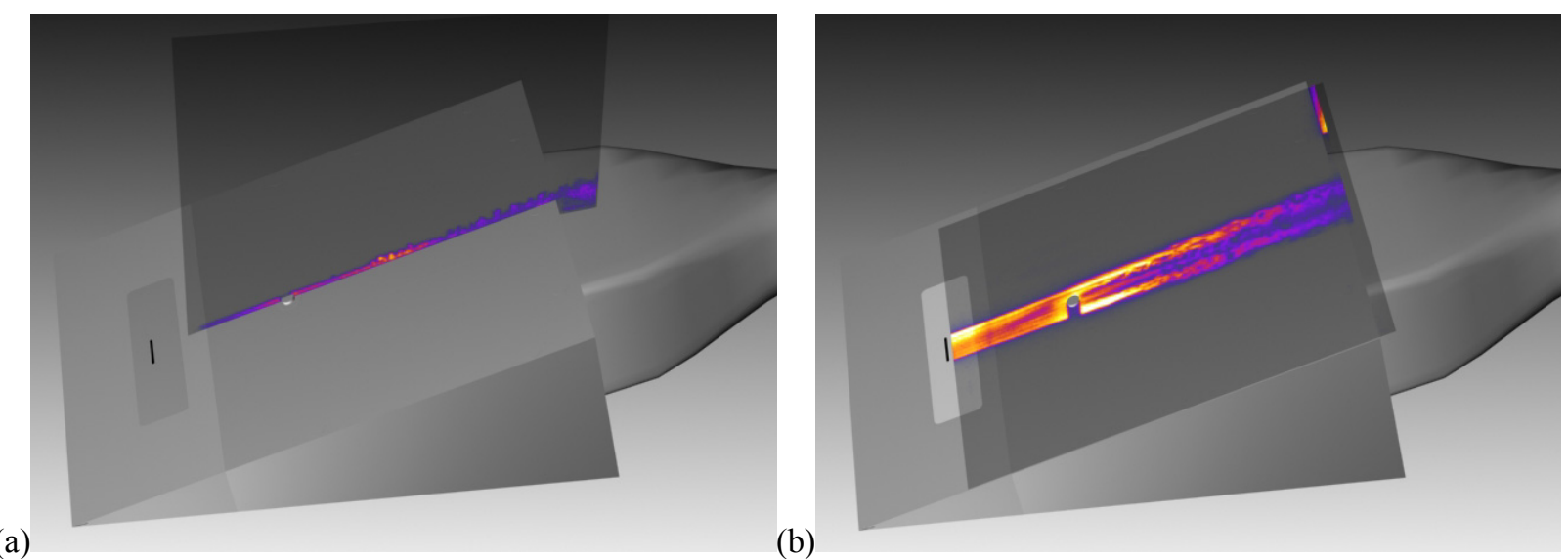

igure 3. Two different PLIF imaging configurations used in the experiment: (a) side view and (b) plan view. Both figures were obtained for a $1-\mathrm{mm}$ tall by $4-\mathrm{mm}$ diameter cylindrical protuberance with $\mathrm{P}_{0}=9.3 \mathrm{MPa}$ and a plate angle equal to $20^{\circ}$. Image (a) was obtained in Run 12 and image (b) was obtained in Run 18.

\section{Results}

This paper summarizes more than 20 wind tunnel runs, each having unique combinations of trip heights, tunnel operating conditions and/or imaging methods and configurations. Furthermore, each run typically consists of $>600$ images with different laser sheet positions and/or model angles of attack. For the sake of brevity, only a small subset of the data obtained in the test is described in this Results section. A few illustrative examples are selected from many of the runs to show some of the most important flow features and trends, and to illustrate the features of the different imaging methods. The appendix presents a much more thorough, though still incomplete, subset of the data obtained in the experiment.

\section{A. $10 \mathrm{~Hz}$ NO PLIF Flow visualization}

\section{Interpretation of NO PLIF visualizations}

Figure 3 shows ViDI renderings of typical $10 \mathrm{~Hz}$ NO PLIF flow visualization images obtained in Test 443 . Flow is from bottom left to top right over the model. In Fig. 3(a) the laser sheet was oriented perpendicular to the model surface and the laser entered from the top of the image. This imaging configuration is known as a side view. Fig. 3(b) shows a plan view imaging configuration where the laser was oriented parallel to the surface of the model and passed spanwise across the model (towards the viewer in this image). Consequently, a shadow forms under the trip as seen in Fig. 3(b).

Figure 4 shows a different view of an image obtained from Run 18. This view is used for most plan views in this paper because the flow is oriented to be nearly left-to-right, allowing many such images to be cropped and viewed on a single page. The legend on the left indicates the height of the laser sheet above the flat plate in both millimeters and inches. Between the slot and the trip, the flow is mostly smooth and laminar. Though some faint streaks are observed, no irregular flow structures are seen upstream of the trip. The origin of these streaks has not yet been identified, and their effect of flow interaction with the roughness element remains unknown.

In this run, the trip height is about $40 \%$ larger than the boundary layer thickness $(k / \delta=0.7)$. Consequently, the trip deflects part of the boundary layer flow towards the top of the image and the remaining flow towards the bottom of the image. Wake flow behind the trip shows substantially stronger streaks than the faint streaks in the upstream flow noted previously. These streaks are evidence of perturbations induced by the trip and may be associated with streamwise vorticity. Analogous streaks have been noted in recent computations modeling select flow conditions from the present experiment. ${ }^{15,16}$ High amplitude stationary streaks of this type can have strong temperature gradients (see for example, Fig. 11 of Ref. 15) and, furthermore, can transport unseeded fluid towards the wall, adjacent to the seeded fluid. Both of these effects (temperature and NO density gradients) contribute to the strong contrast in the fluorescence intensity in the image. The streaks within the roughness wake soon become unstable, oscillating with periodic structures and then breaking down into much more irregular structures. In the diagram, this region of the flow is labeled "Transition to turbulence," even though the onset of transition has not been formally established using conventional instrumentation.

While NO density and temperature gradients are primarily responsible for the intensity variations in the images, another factor is the laser sheet's width and intensity distribution. In many of the images, for example Fig. 5(a), the edge of the laser sheet is within the field of view of the camera, resulting in an abrupt drop in signal at the left edge of the image. A white arrow marks the left edge of the laser sheet in Fig. 5(a). NO exists upstream of this arrow but the laser does not illuminate it. Also, the laser's spatial intensity distribution varied laser-shot to laser-shot during the experiment. This resulted in left-to-right variations in the fluorescence even when the NO density is uniform (e.g. Fig 
5(a)). For the MHz PLIF experiment (Test 463) we measured the laser's intensity distribution on a shot-by-shot basis and corrected for the spatial variation in intensity, but for the earlier performed $10 \mathrm{~Hz}$ experiments (Test 443) described herein, we did not. Thus, for images acquired in Test 443, variations of PLIF signal parallel to the direction of the laser propagation (normal to the wall in the side-view images) have more fluid mechanical significance than gradual left-toright variations in intensity, which are often an artifact of the experiment.

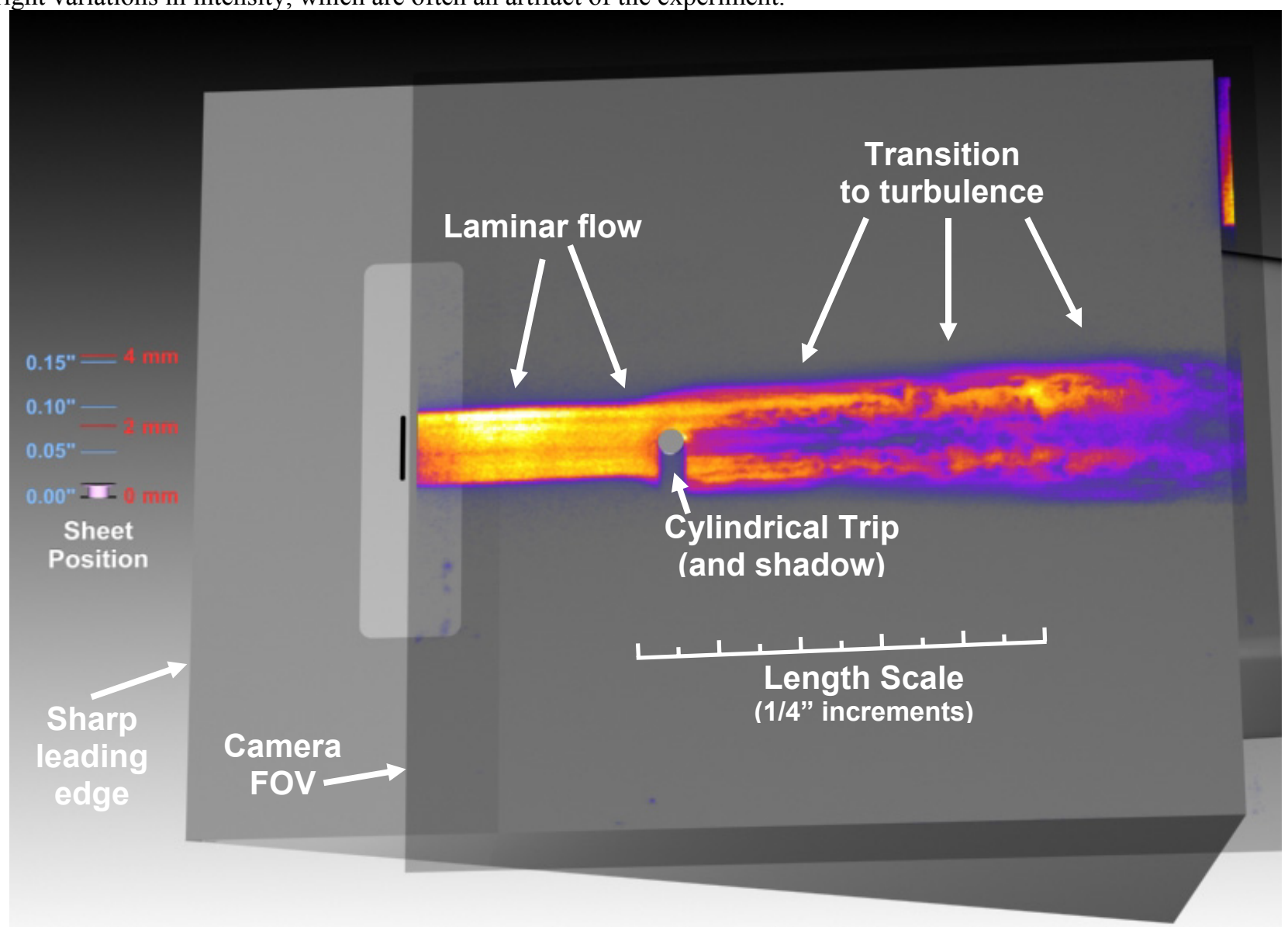

Figure 4. ViDI rendering of a typical $10 \mathrm{~Hz}$ NO PLIF image from Run 18, Test 443.

\section{Side View $10 \mathrm{~Hz}$ PLIF Images: Centerline Images with Angle of Attack Variation}

The laser sheet was oriented perpendicular to the model for the first series of measurements (Test 443, Runs 1-15). During these runs, the angle of attack of the model was swept so that the plate angle varied from $5^{\circ}$ to $25^{\circ}$. The oblique shock originating from the leading edge of the model varied in strength as the angle of attack was changed, thereby varying the edge Mach number and the boundary layer thickness, as indicated in Table 2. Using the highest tunnel stagnation pressure, two different trip heights were studied: 1 and $2 \mathrm{~mm}$ heights, both having a 4-mm diameter.

Figure 5 shows PLIF images resulting from an angle of attack sweep during Run 12, which used the 1-mm tall trip. During postprocessing of these data, a virtual camera was fixed relative to the model (rather than relative to the tunnel) in the ViDI rendering program. Consequently, the model appears stationary in the sequence of images in Fig. 5 even though in experiment the model angle of attack was varying.

\begin{tabular}{|c|c|c|c|}
\hline $\begin{array}{c}\text { Plate Angle } \\
\text { (degrees) }\end{array}$ & $\begin{array}{c}\text { M2 } \\
--\end{array}$ & $\begin{array}{c}\text { Me } \\
--\end{array}$ & $\begin{array}{c}\text { delta } \\
(\mathrm{mm})\end{array}$ \\
\hline 5 & 8.2 & & \\
7.5 & 7.3 & & \\
10 & 6.6 & 5.3 & 1.39 \\
12.5 & 5.9 & & \\
15 & 5.2 & & \\
17.5 & 4.7 & & \\
20 & 4.2 & 3.8 & 0.95 \\
22.5 & 3.7 & & \\
25 & 3.4 & & \\
\hline
\end{tabular}

Table 2. Computed post-oblique-shock $\left(\mathrm{M}_{2}\right)$ and edge $\left(\mathrm{M}_{\mathrm{e}}\right)$ Mach numbers and boundary layer thickness for the middle- $\operatorname{Re}\left(\mathrm{P}_{0}=5.0\right.$ $\mathrm{MPa}$ ) tunnel operating condition. $\mathrm{M}_{2}$ is computed from oblique shock theory while $\mathrm{M}_{\mathrm{e}}$ and $\delta$ were determined from CFD. ${ }^{8}$ 
(a)

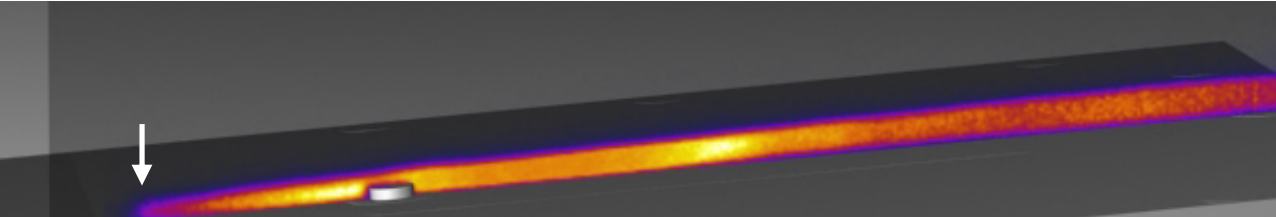

(b)

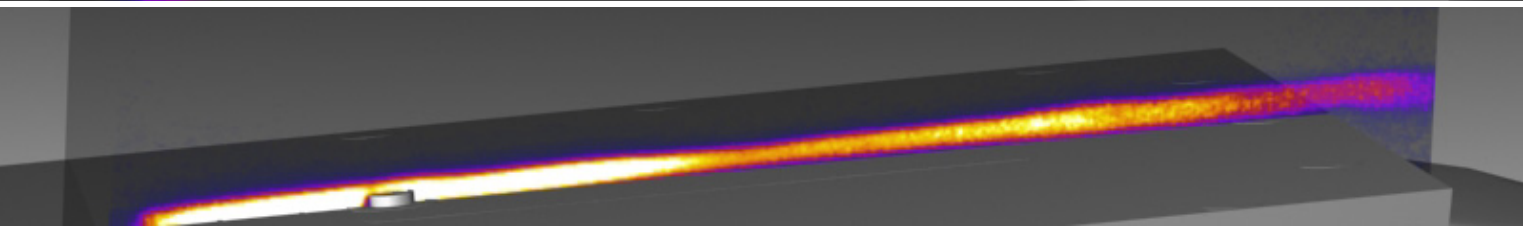

(c)

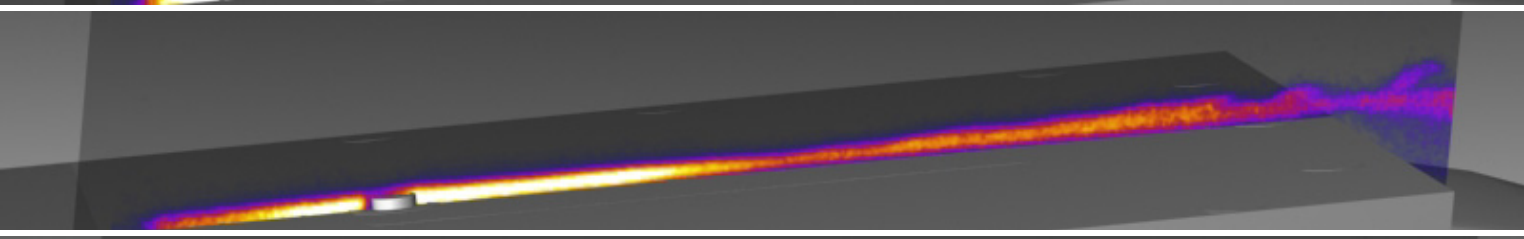

(d)

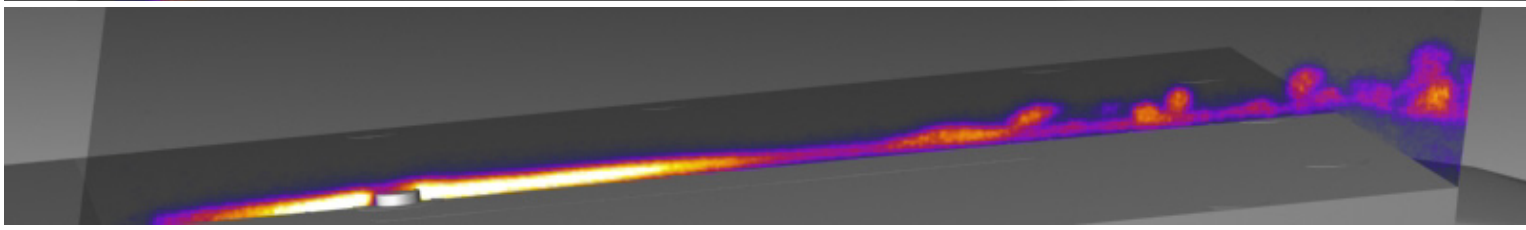

(e)

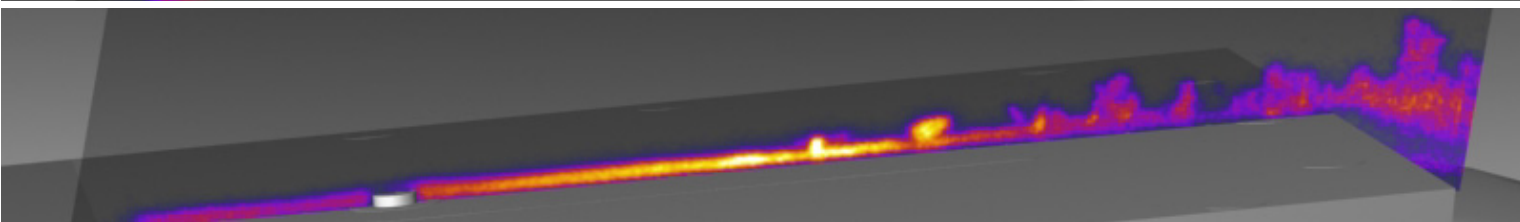

(f)

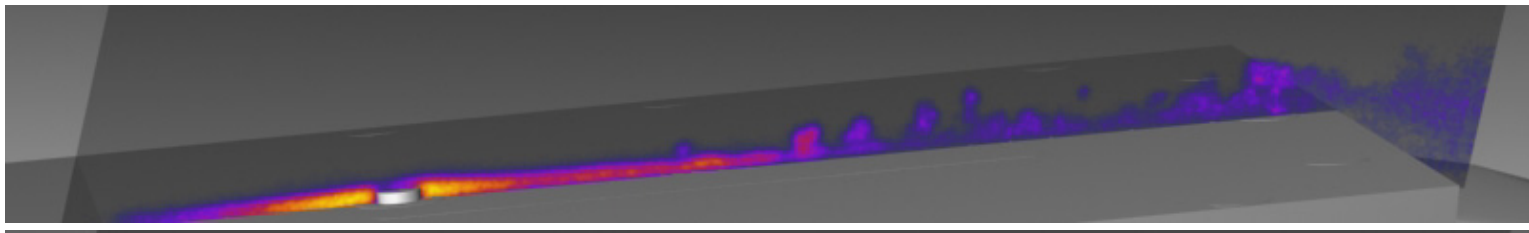

(g)

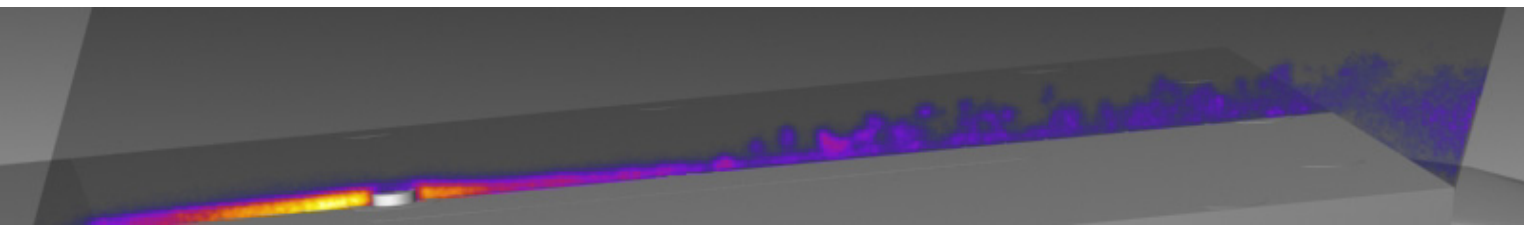

(h)

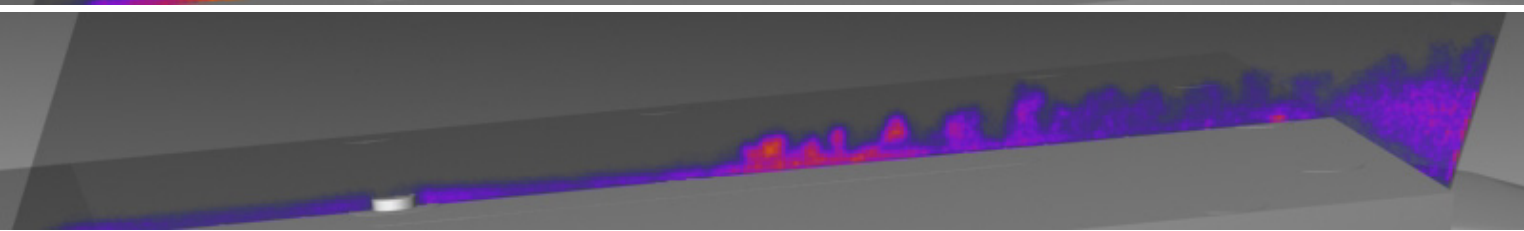

(i)

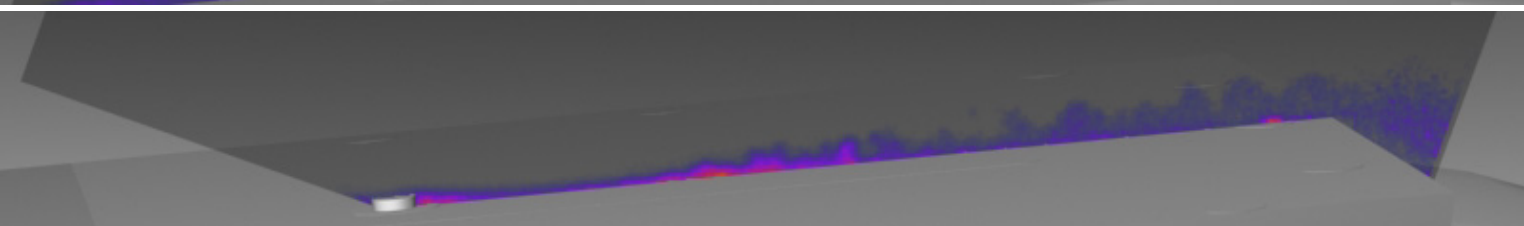

Figure 5. Angle of Attack Sweep. Test 443, Run 12, 1-mm tall by 4-mm wide cylinder, plate angle varies from 5 to $25^{\circ}$ in $2.5^{\circ}$ increments in (a)-(i), $11-\mathrm{mm}$ wide slot seeding, $\dot{m}=300 \mathrm{sccm}, \mathrm{P}_{0}=9.3 \mathrm{MPa}$, sheet position $=1 \mathrm{~mm}$ from centerline, framing rate $=10 \mathrm{~Hz}$. The white arrow in (a) indicates the left edge of the laser sheet. 
(a)

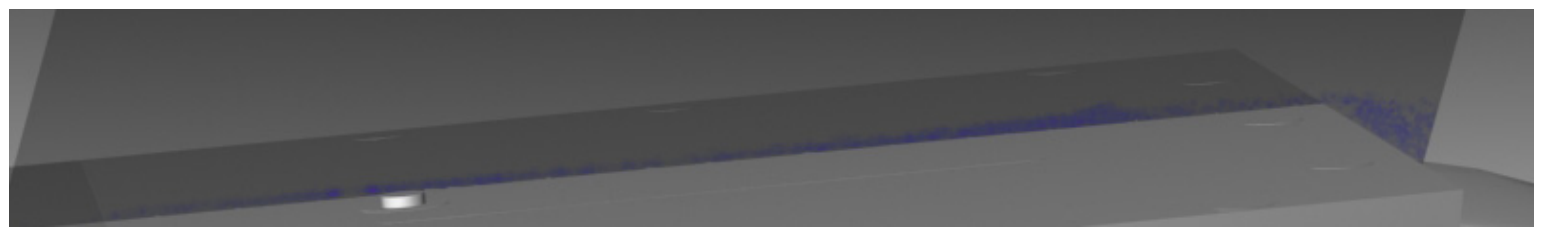

(b)

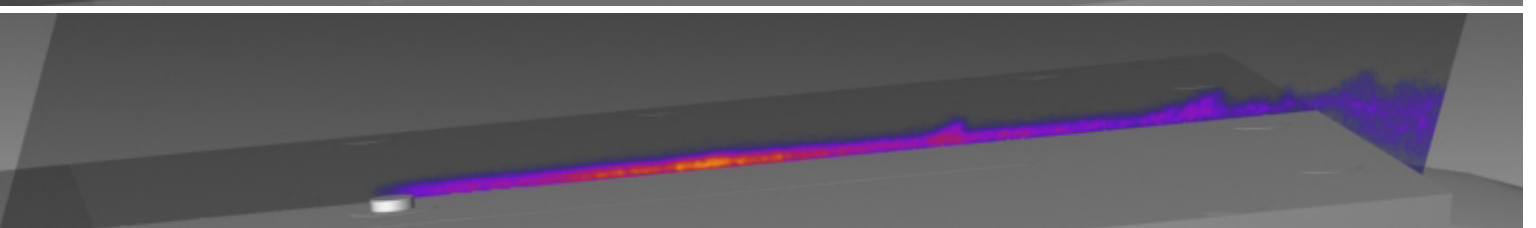

(c)

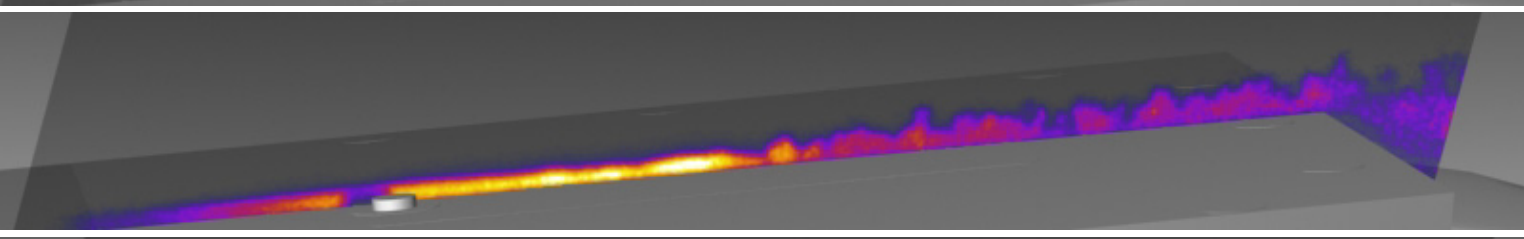

(d)

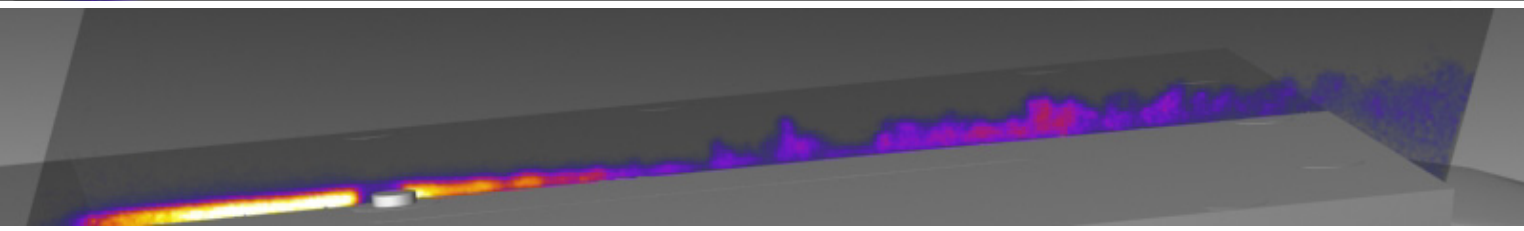

(e)

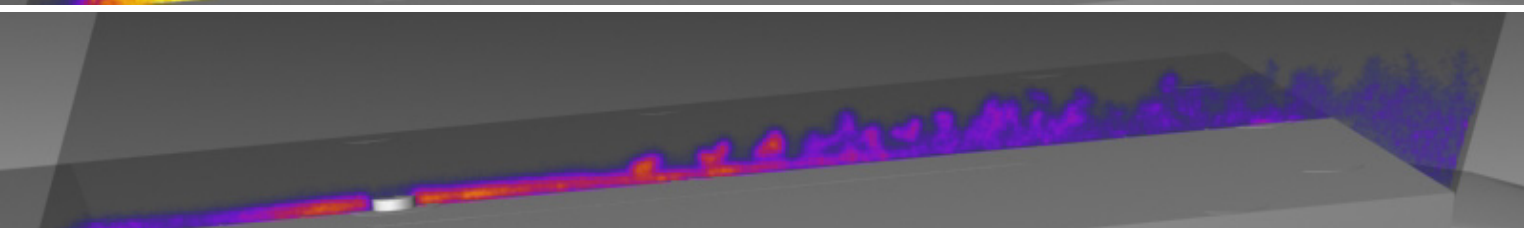

(f)

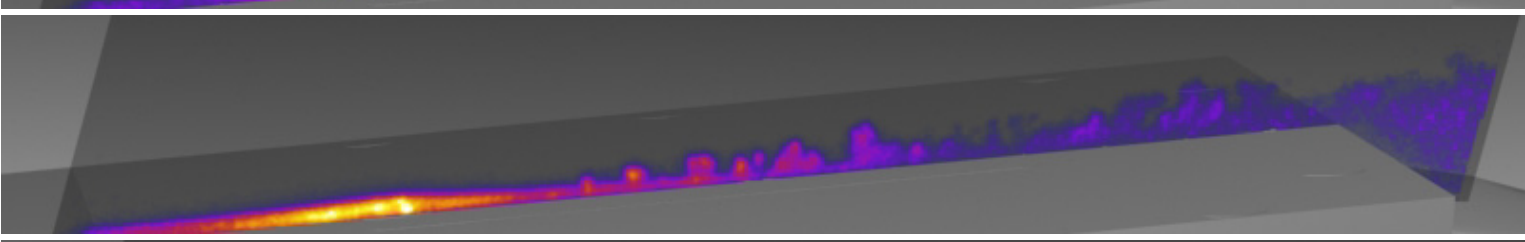

(g)

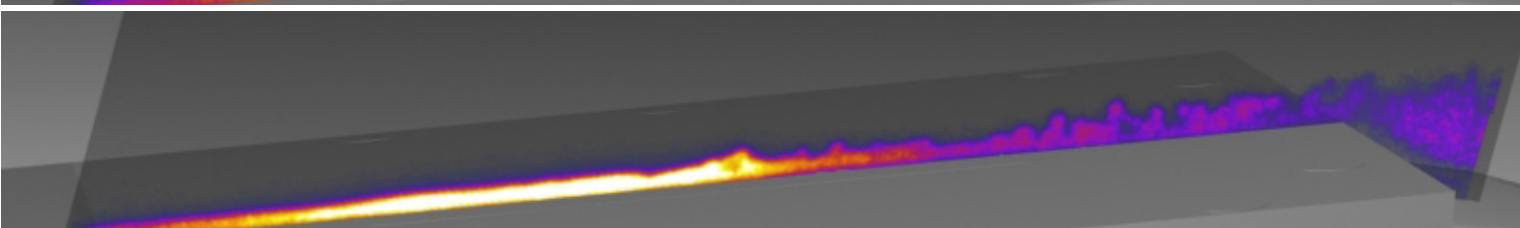

(h)

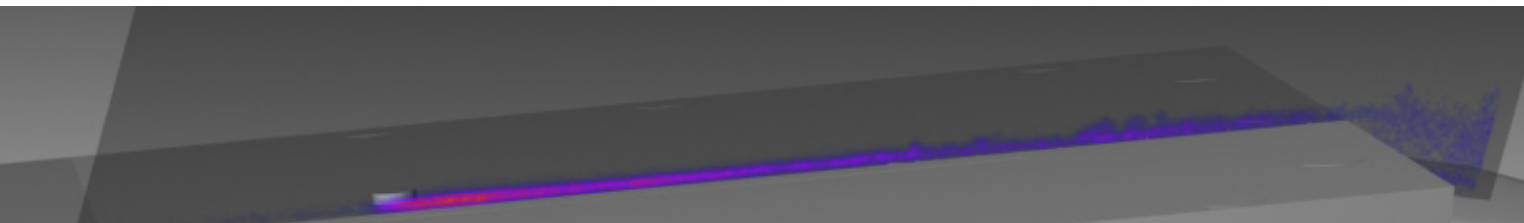

(i)

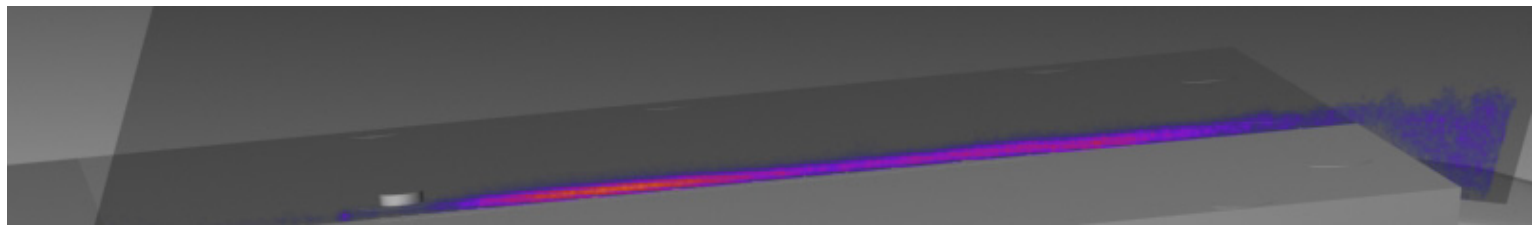

Figure 6. Spanwise Scan of Laser Sheet. Test 443, Run 12, 4-mm diameter cylinder with 1-mm height, plate angle $=20^{\circ}, 11-\mathrm{mm}$ wide slot seeding, $\dot{m}=300 \mathrm{sccm}, \mathrm{P}_{0}=9.3 \mathrm{MPa}$, sheet position varies from 10 to $-10 \mathrm{~mm}$ from centerline in $2.5 \mathrm{~mm}$ increments in (a)-(i), framing rate $=10 \mathrm{~Hz}$. 
The images show laminar flow at the $5^{\circ}$ plate angle in Fig. 5(a). As the angle of the model is increased to $7.5^{\circ}$ and then to $10^{\circ}$, some instabilities are observed in the boundary layer, particularly at the right-hand side of the images. Further increasing the plate angle results in large structures that transport away from surface of the plate. These structures are prevalent in the downstream region of the images. By the time the angle of the plate becomes greater than $15^{\circ}$, many structures are observed, and these structures originate closer to the trip as the angle of the plate increases to $25^{\circ}$. In some cases the structures are periodic near their onset-for example, Fig. 5(f)-but in other cases they are more irregular. There is a tendency for the structures to become smaller at higher angles of attack (i.e., higher $\mathrm{Re}_{\mathrm{k}}$ ).

Figure 6 shows a spanwise sweep of the laser sheet position for the case of the $20^{\circ}$ plate angle shown in Fig. $5(\mathrm{~g})$. We estimate that the laminar boundary layer thickness at the position of the trip for these conditions is approximately $0.7-\mathrm{mm}$, which is less than 1-mm trip height. The total spanwise travel of the laser sheet in Fig. 6 is $20-\mathrm{mm}$. On the far side of the trip (Fig 6(a)) there is no significant visible fluorescence. Slightly closer to the trip (Fig. 6 (b)), the flow looks laminar through most of the image and does not show signal forward of the trip. The next five images, (c) through $(\mathrm{g})$, show irregular transitional flow structures behind the trip, which clearly indicate the influence from the trip. As the laser sheet continues to scan towards the camera, the flow becomes more laminar again with less florescence forward at the trip as seen in Fig. 6(h) and (i). The signal forward of the trip in Fig. 6(c) through (g) is consistent with the NO seeding from the 11-mm wide slot which expands slightly in the spanwise direction as it travels downstream. Once it hits the trip however, the spanwise extent of the fluorescence is larger, consistent with the plan view images shown below.

In Run 15, a 2-mm tall trip was tested at the same conditions as the 1-mm trip previously discussed. These results are shown in Fig. 7 for an angle-of-attack sweep. As in Fig. 5, note that the boundary layer thickness decreases as the angle of attack increases. The biggest difference between Figs. 5 and 7 is that the larger trip in Fig. 7 blocks much of the seeded boundary layer flow resulting in very low PLIF signals downstream of the trip, particularly at the steeper plate angles. The boundary layer gas gets deflected around the sides of the trip by this 2-mm protuberance leaving a socalled "dead air region" immediately downstream of the trip. A small amount of NO is sometimes transported to this region allowing some flow visualization there. At the lowest plate angles (both $5^{\circ}$ and $7.5^{\circ}$ ), the boundary layer thickness becomes larger than the trip and the flow passes over the trip, resulting in good signal-to-noise flow visualizations immediately downstream of the trip. For the $5^{\circ}$ case, the flow is mostly laminar, as it was with the $1-\mathrm{mm}$ tall trip. At plate angles greater than $5^{\circ}$, however, instabilities are observed. These instabilities grow in frequency as the plate angle increases. The typical size of the flow structures resulting from these instabilities gets smaller as the plate angle increases from top to bottom in Fig. 7.

Figure 8 shows a spanwise laser sheet scan obtained for the $20^{\circ}$ plate angle corresponding to Fig. $7(\mathrm{~g})$. When the laser sheet is scanned away from the trip, much larger signal intensities are seen in the downstream portion of the images compared to the centerline. Similar to Fig. 6, the flow is observed to be mostly laminar at the two locations farthest from the trip in Fig. 8. As the laser sheet approaches the trip from either side, the images show stronger transitional behavior. Finally, as the laser reaches the center of the trip, the dead air region directly downstream of the trip is observed. The appendices show two additional spanwise laser sheet scans (at plate angles of $5^{\circ}$ and $25^{\circ}$ ) for both Runs 12 and 15. 
(a)

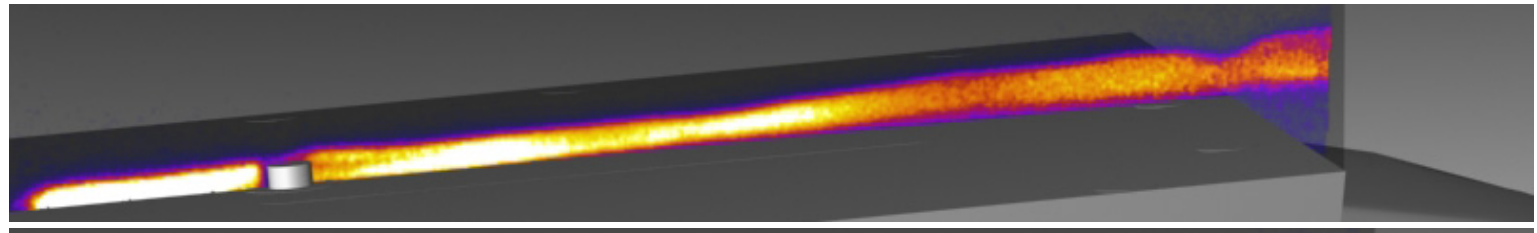

(b)

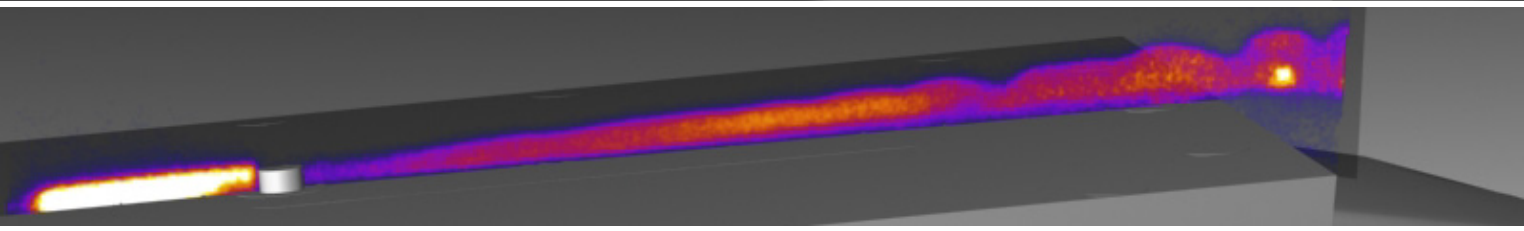

(c)

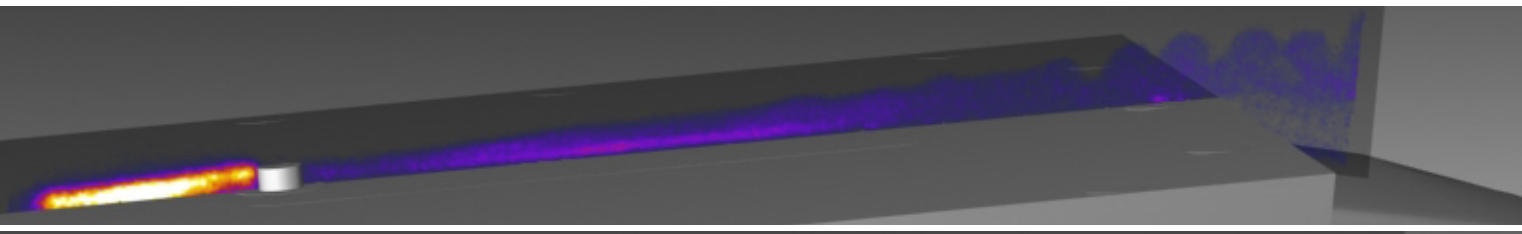

(d)

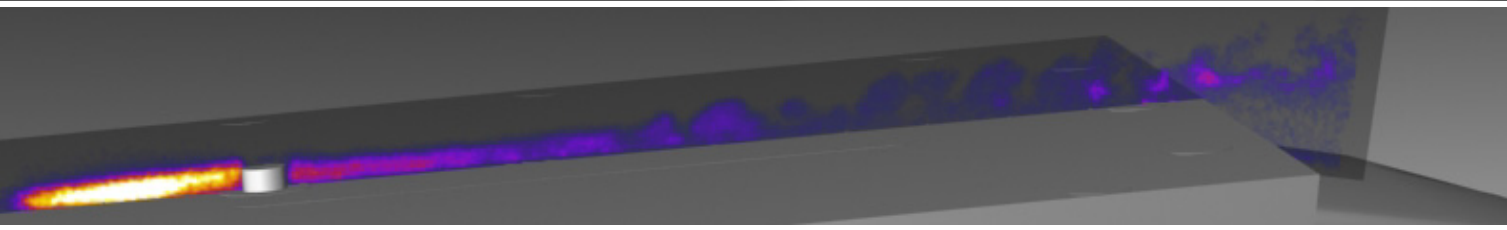

(e)

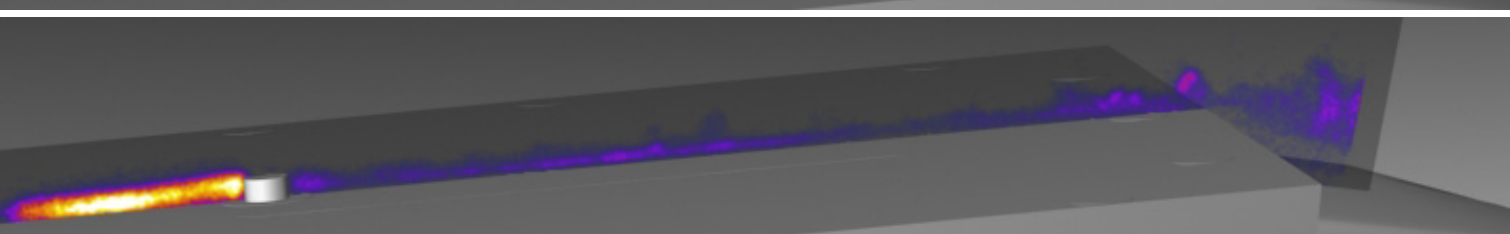

(f)

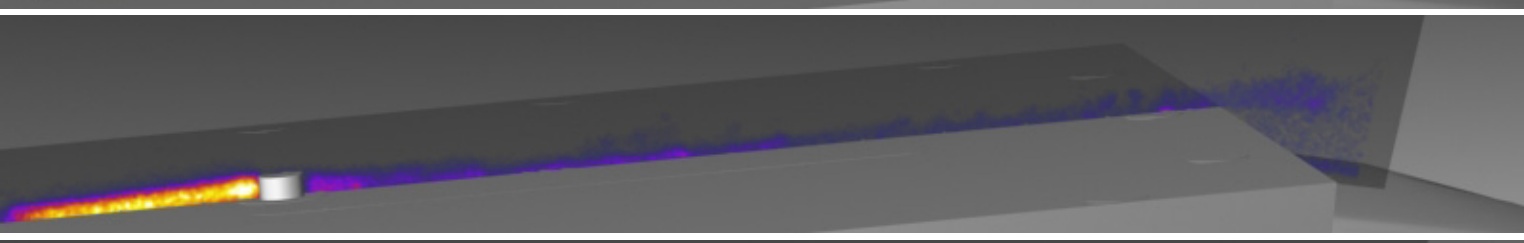

(g)

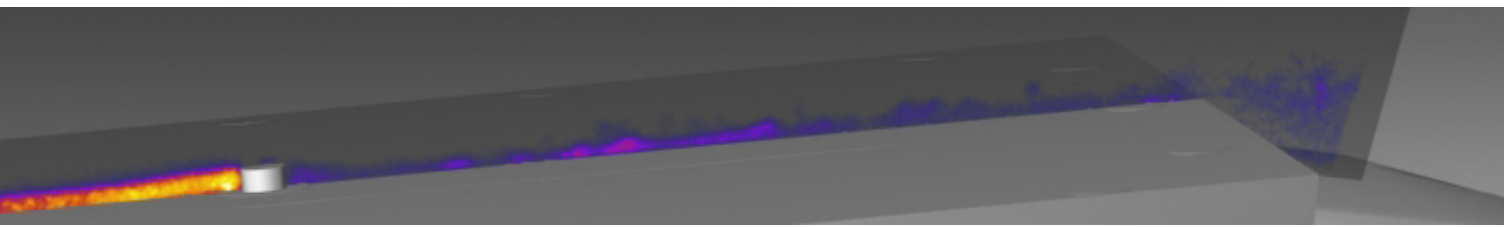

(h)

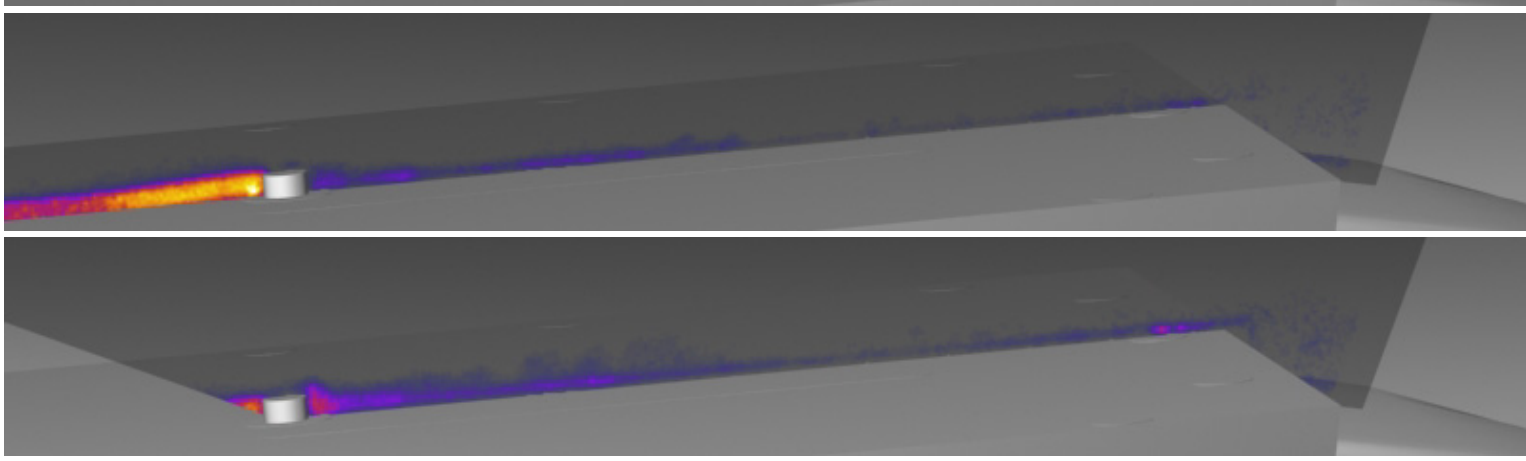

(i)

Figure 7. Angle of Attack Sweep. Test 443, Run 15, 2-mm tall by 4-mm wide cylinder, plate angle varies from 5 to $25^{\circ}$ in $2.5^{\circ}$ increments in (a)-(i), $11-\mathrm{mm}$ wide slot seeding, $\dot{m}=300 \mathrm{sccm}, \mathrm{P}_{0}=9.3 \mathrm{MPa}$, sheet position $=2 \mathrm{~mm}$ from centerline, framing rate $=10 \mathrm{~Hz}$. 
(a)

(b)

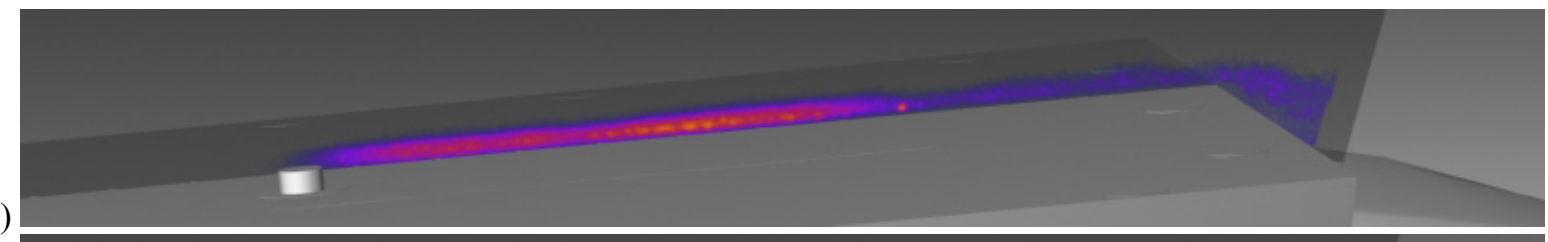

(c)

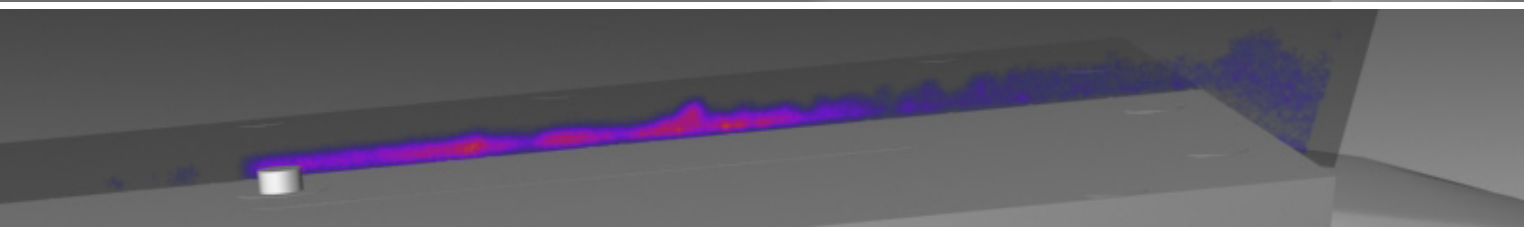

(d)

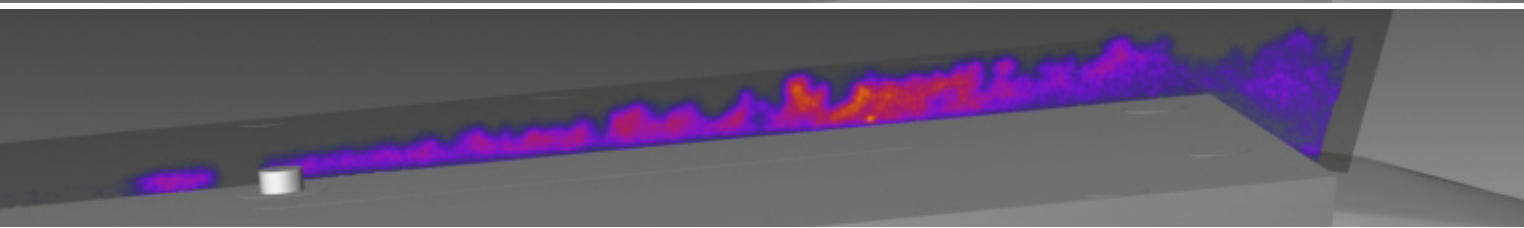

e)

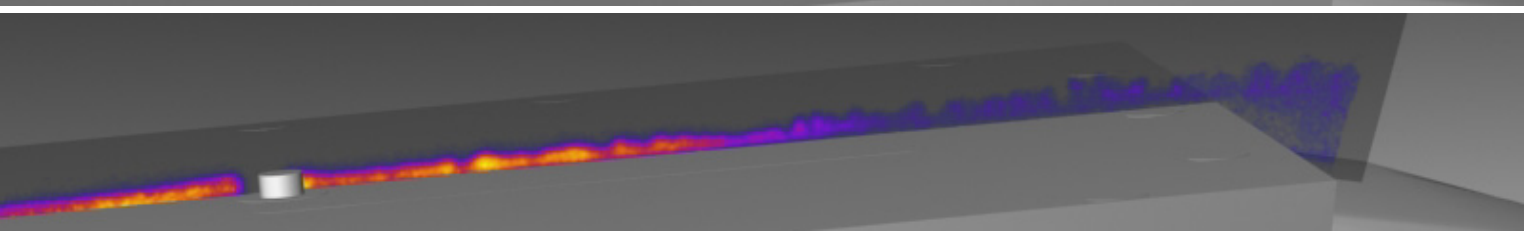

(f)

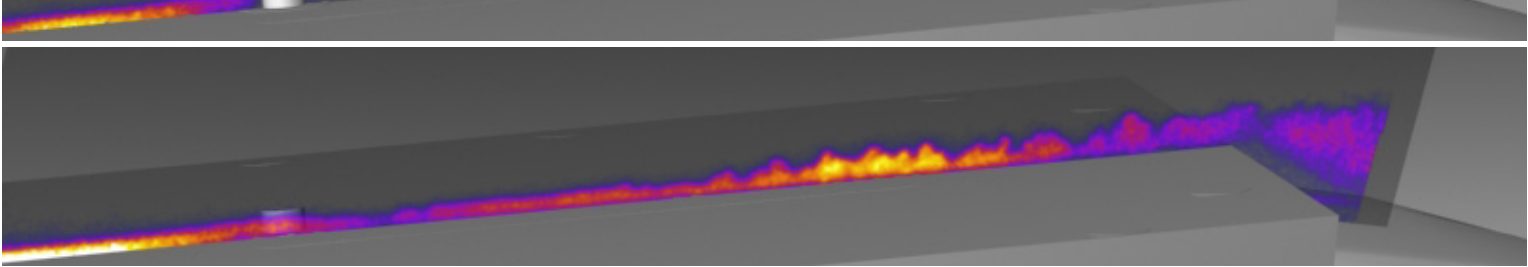

(g)

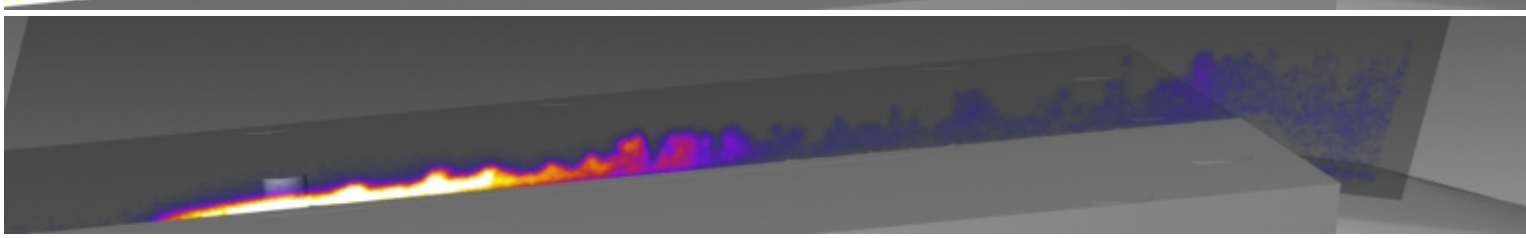

(h)

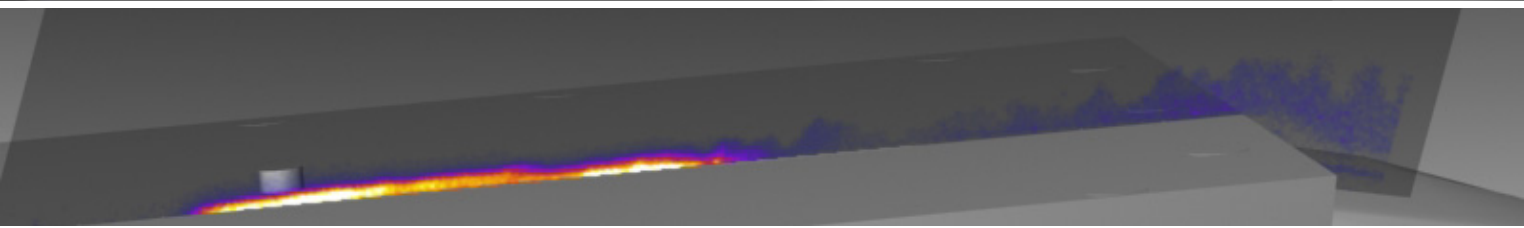

(i)

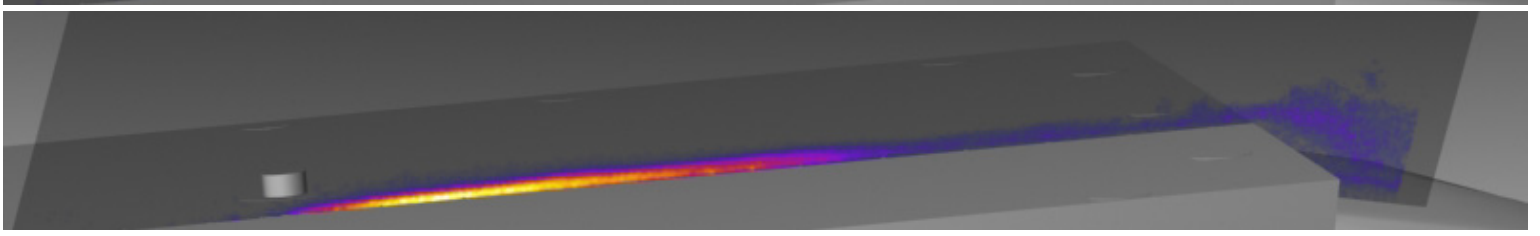

Figure 8. Spanwise Scan of Laser Sheet. Test 443, Run 15, 2-mm tall by 4-mm wide cylinder, plate angle $=20^{\circ}$, $11-\mathrm{mm}$ wide slot seeding, $\dot{m}=300 \mathrm{sccm}, \mathrm{P}_{0}=9.3 \mathrm{MPa}$, sheet position varies from 16 to $-16 \mathrm{~mm}$ from centerline in 4-mm increments in (a)-(i), framing rate $=10 \mathrm{~Hz}$. 


\section{Plan View10 Hz PLIF Images: Variation of Sheet Position and Reynolds Number}

In this section, plan views are used to investigate the variation in the flow structures with height above the surface of the plate. The variation of the flow structures with the unit Reynolds number is also investigated. Figure 9 shows the flow over a 1-mm tall cylindrical trip for the lowest Reynolds number tested. Flow is uniform and laminar as it passes from the seeding insert to the trip. Immediately downstream of the trip, the flow is also laminar. Halfway down the plate, it becomes unsteady in some of the images. The flow in images obtained closest to the surface of the plate, e.g. Fig. 9(a), appears to be more laminar whereas further away from the plate, e.g. Fig. 9(c)-(e), the flow exhibits more unstable behavior on the right half of the images. This is consistent with the conventional wisdom in supersonic and hypersonic boundary layers, in which transition to turbulence initiates closer to the edge of the boundary layer than the wall. ${ }^{17}$ In Fig. 9(d), periodic oscillatory waves are seen along the centerline of the flow. In Fig. 9(e), the seeded NO flow is deflected far off the surface ( $>2 \mathrm{~mm}$ compared to about a $1 \mathrm{~mm}$ laminar boundary layer thickness in the absence of the trip). All of these images show strong streamwise streaks generated downstream of the trip. These streaks are probably streamwise vortices. These images show many more unsteady and transitional flow features compared to Fig. 4 in Ref. 11 which was obtained at the same operating conditions. The trip studied in Fig. 4, Ref. 11 was the same height $(1 \mathrm{~mm})$ but was actually significantly wider $(11 \mathrm{~mm}$ versus $4 \mathrm{~mm})$. However, the trip in Fig. 4, Ref. 11 had a smooth and streamlined profile whereas the cylinder trip in the current work had a sharp edge around the top. This sharpness appears to have a significant effect promoting the near field development of the flow.

(a)

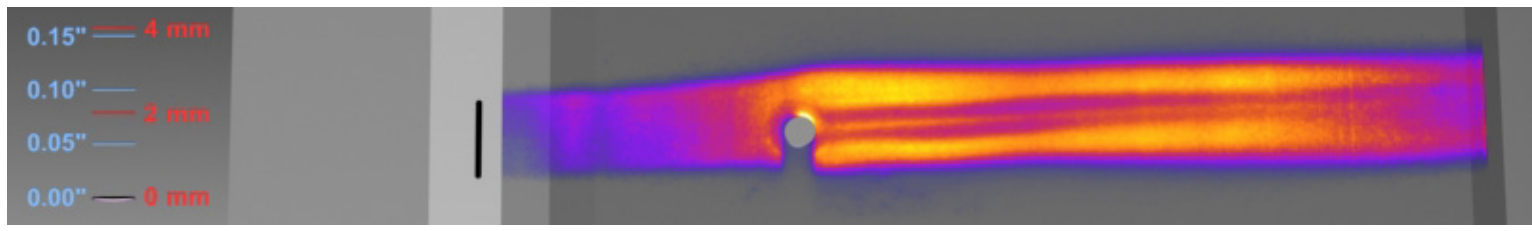

(b)

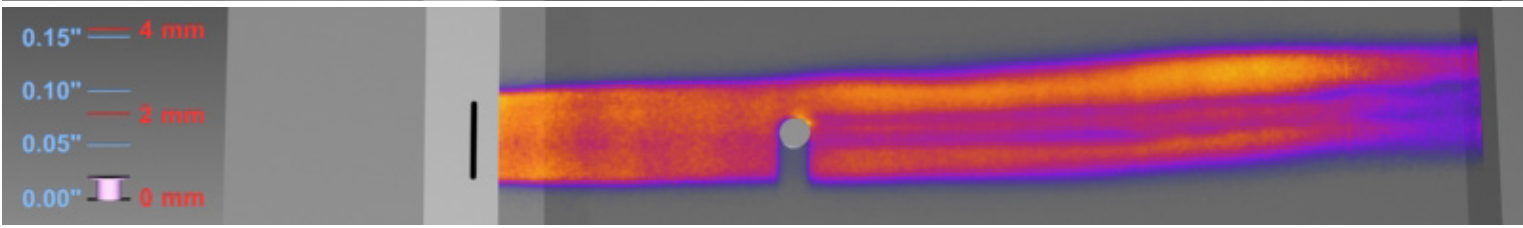

(c)

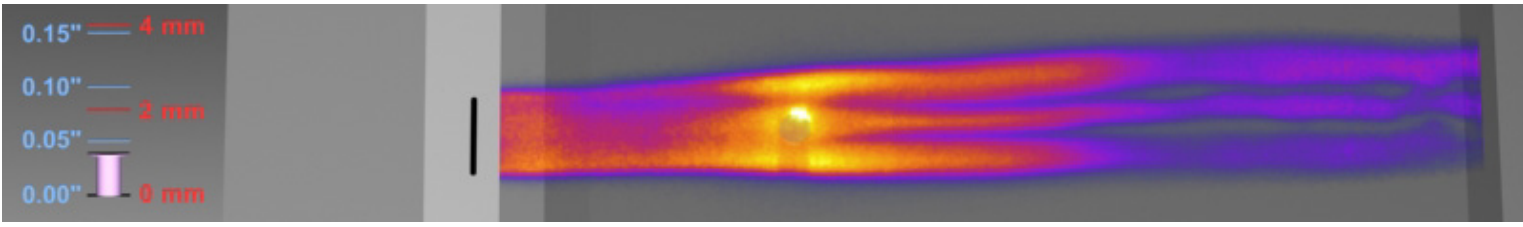

(d)

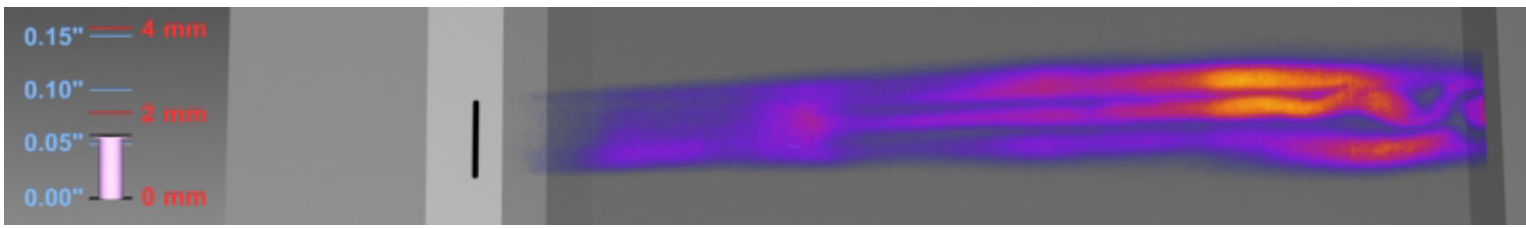

(e)

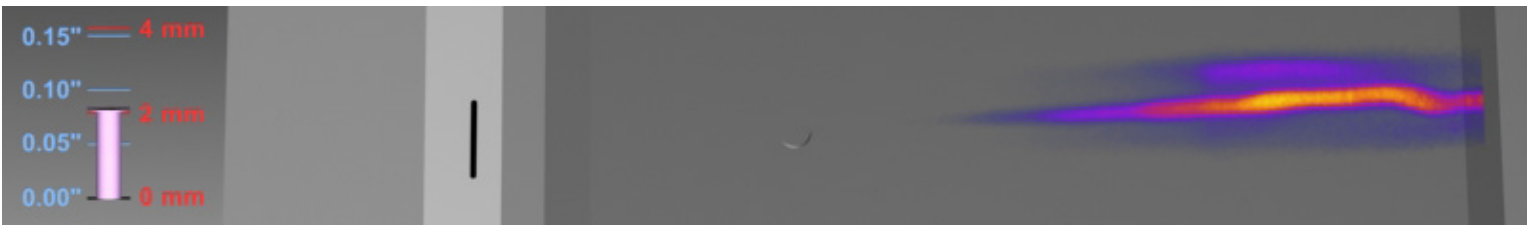

Figure 9. Test 443 , Run $20,1-\mathrm{mm}$ tall by $4-\mathrm{mm}$ wide cylinder, plate angle $=20^{\circ}, 11-\mathrm{mm}$ wide slot seeding, $\dot{m}=300 \mathrm{sccm}, \mathrm{P}_{0}=2.41 \mathrm{MPa}$, sheet position varies from 0.6 to $1.0 \mathrm{~mm}$ above the plate in $0.2 \mathrm{~mm}$ increments in (a)-(c), framing rate $=10 \mathrm{~Hz}$. 
Figure 10 shows the same imaging configuration as Fig. 9, but with the wind tunnel running at a higher stagnation pressure corresponding to the middle Reynolds number tested. In these images, laminar streaks are observed just downstream of the trip, for example in Figs. 10(a) and (b). However, these structures become oscillatory and then irregular over a shorter distance in this higher Reynolds number flow, as expected. Throughout Fig. 10, considering that flow is moving left to right, laminar structures on the left side of the image lead to periodic or irregular structures in the middle, which then lead to much smaller scale random structures on the right hand side of the image.

(a)

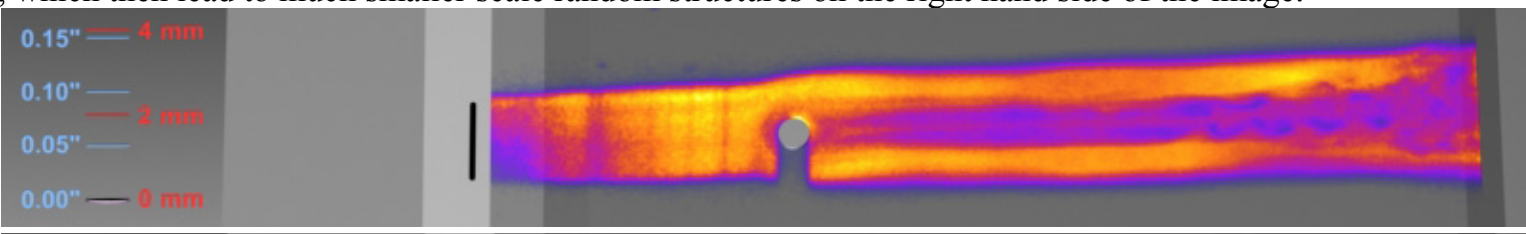

(b)

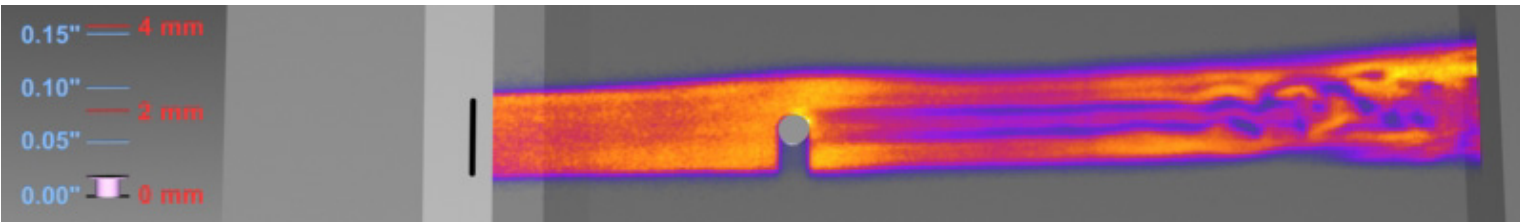

(c)

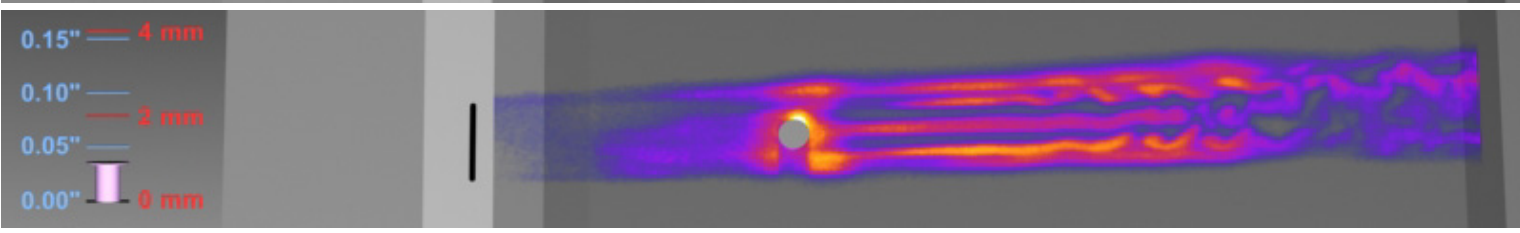

(d)

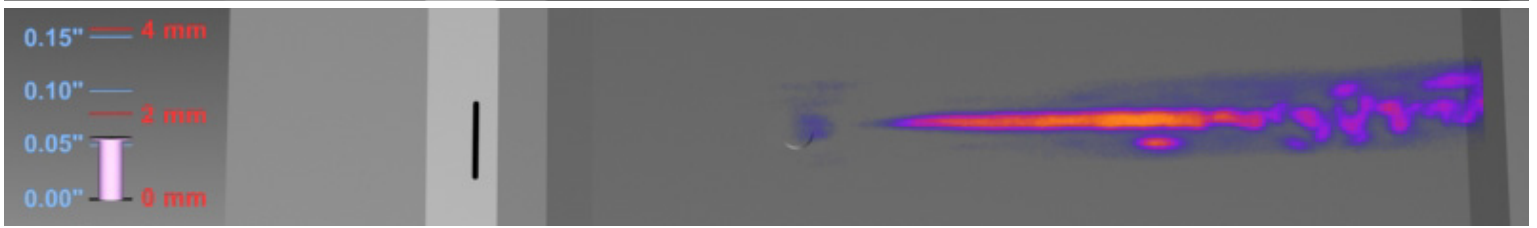

(e)

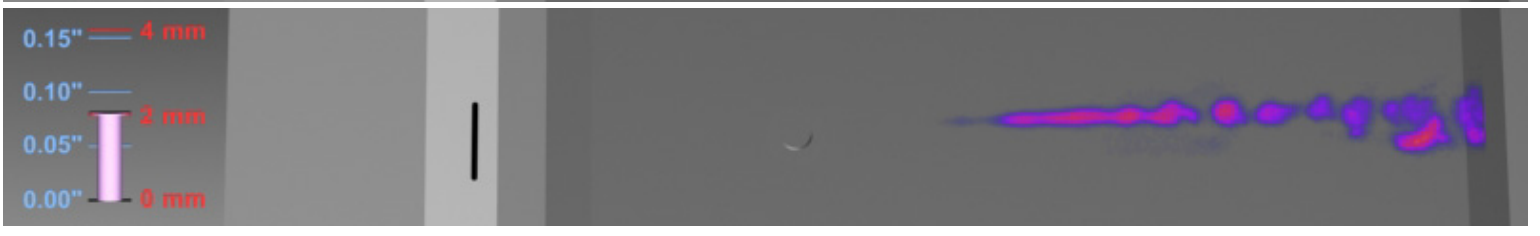

Figure 10. Test 443, Run 19, 1-mm tall by 4-mm wide cylinder, plate angle $=20^{\circ}$, 11-mm wide slot seeding, $\dot{m}=300 \mathrm{sccm}, \mathrm{P}_{0}=4.97 \mathrm{MPa}$, sheet position varies from 0.0 to $2.0 \mathrm{~mm}$ above the plate in $0.5 \mathrm{~mm}$ increments in (a)-(e), framing rate $=10 \mathrm{~Hz}$. 
Figure 11 shows the 1-mm tall trip tested at the highest Reynolds number condition. The transition from laminar to turbulent flow in the images moves closer to the trip, continuing the trend observed between Figs. 9 and 10. The flow structures observed in this case are much smaller than observed at the lower Reynolds number cases, particularly towards the right side of the images. Flow structures are also observed further off the surface than in the lower Reynolds number cases.

(a)

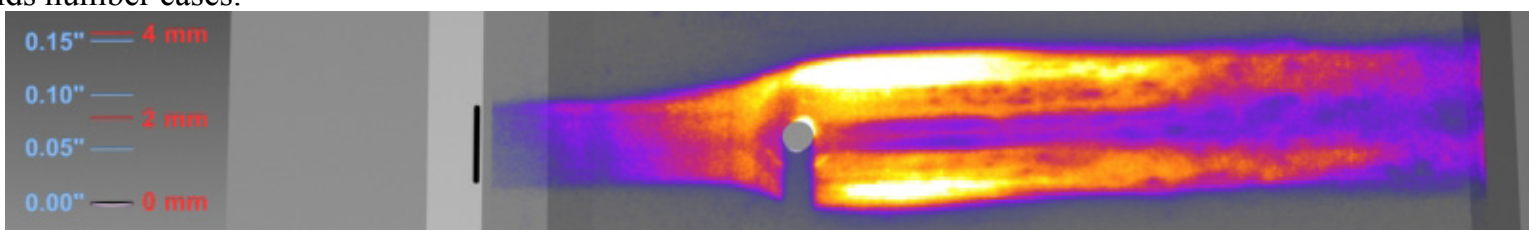

(b)

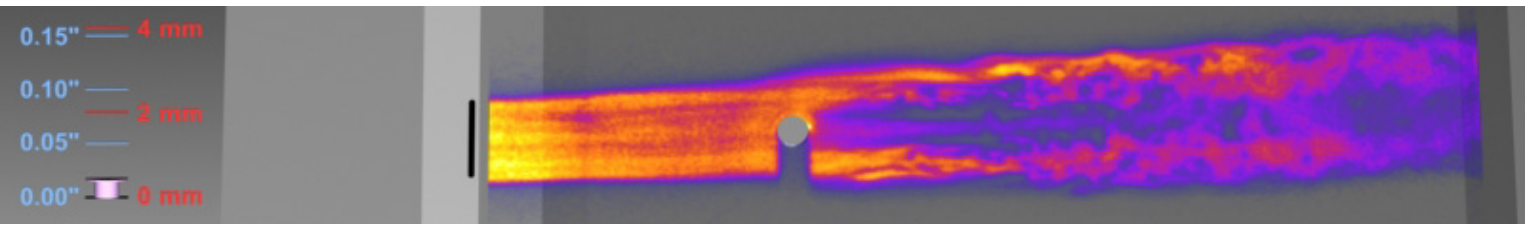

(c)

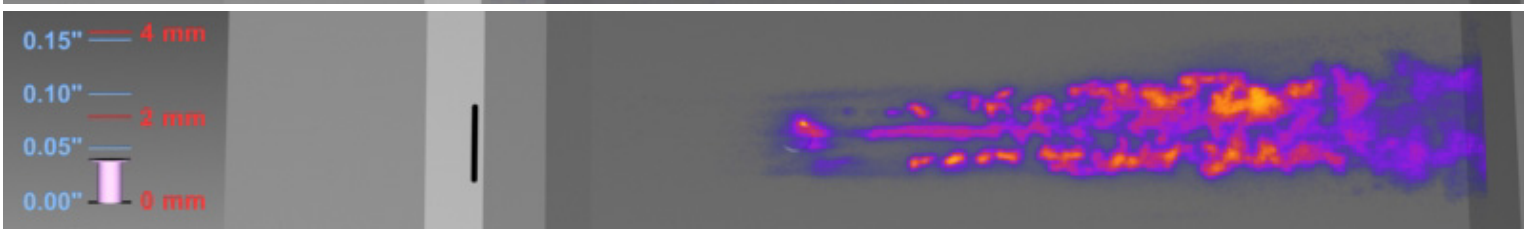

(d)

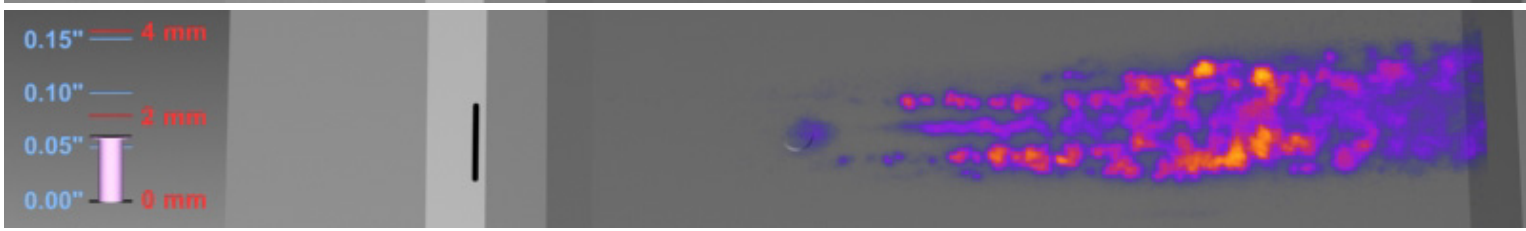

(e)

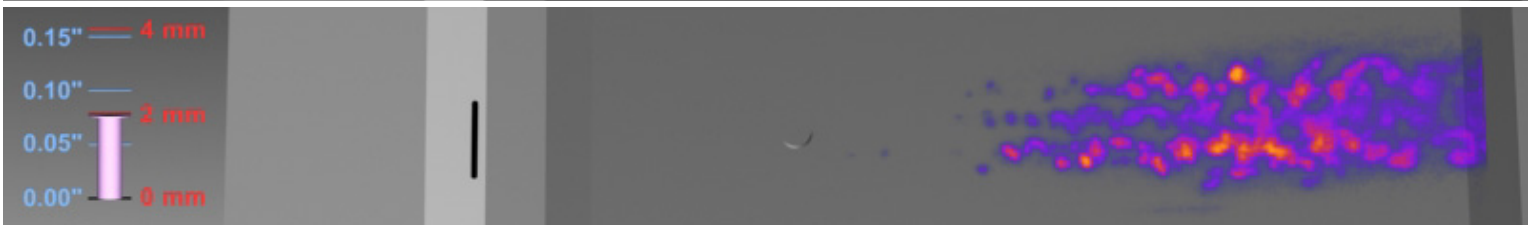

Figure 11. Test 443 , Run $18,1-\mathrm{mm}$ tall by $4-\mathrm{mm}$ wide cylinder, plate angle $=20^{\circ}, 11-\mathrm{mm}$ wide slot seeding, $\dot{m}=300 \mathrm{sccm}, \mathrm{P}_{0}=9.32 \mathrm{MPa}$, sheet position varies from 0.0 to $2.0 \mathrm{~mm}$ above the plate in $0.5 \mathrm{~mm}$ increments in (a)-(e), framing rate $=10 \mathrm{~Hz}$. 
In Fig. 12, the highest tunnel Reynolds number is again used but a larger 2-mm tall trip is placed on the flat plate. This 2-mm tall trip deflected the flow spanwise to a much larger extent than the 1-mm trip. In addition, a much larger dead air region is observed directly downstream of this $2 \mathrm{~mm}$ trip in agreement with the results observed in Fig(s). 7 and 8 for this same 2-mm tall trip and same Reynolds number condition. Based on the shapes of the flow structures in the image, transition appears to be starting immediately at or downstream of the location of the trip.

(a)

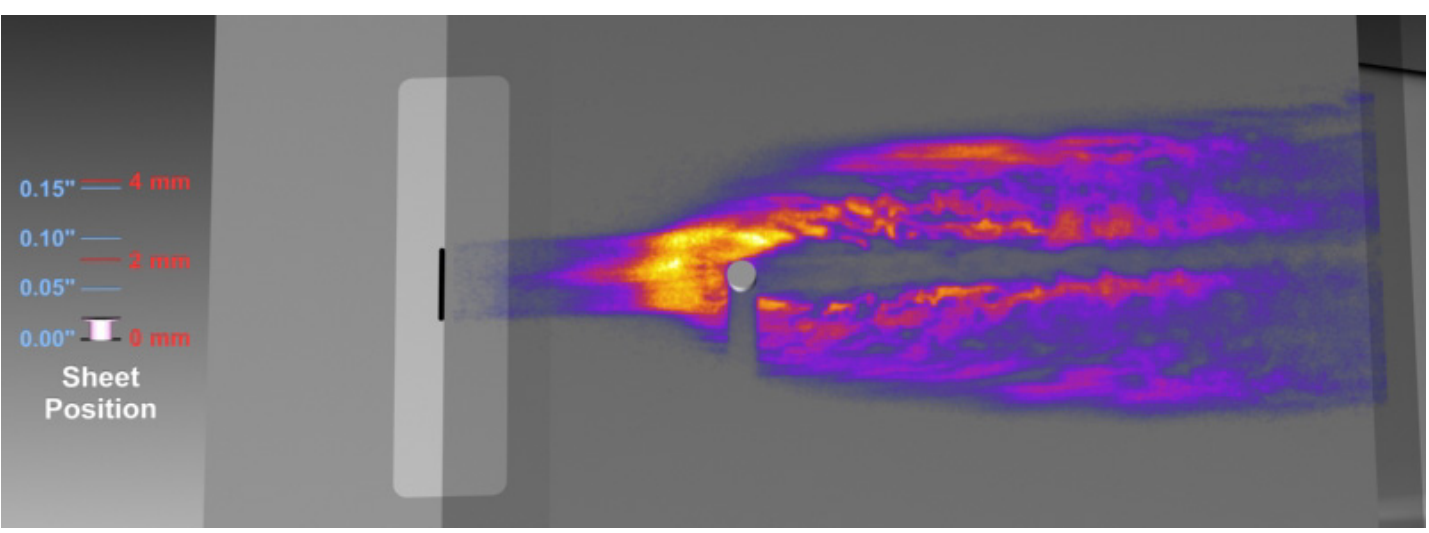

(b)
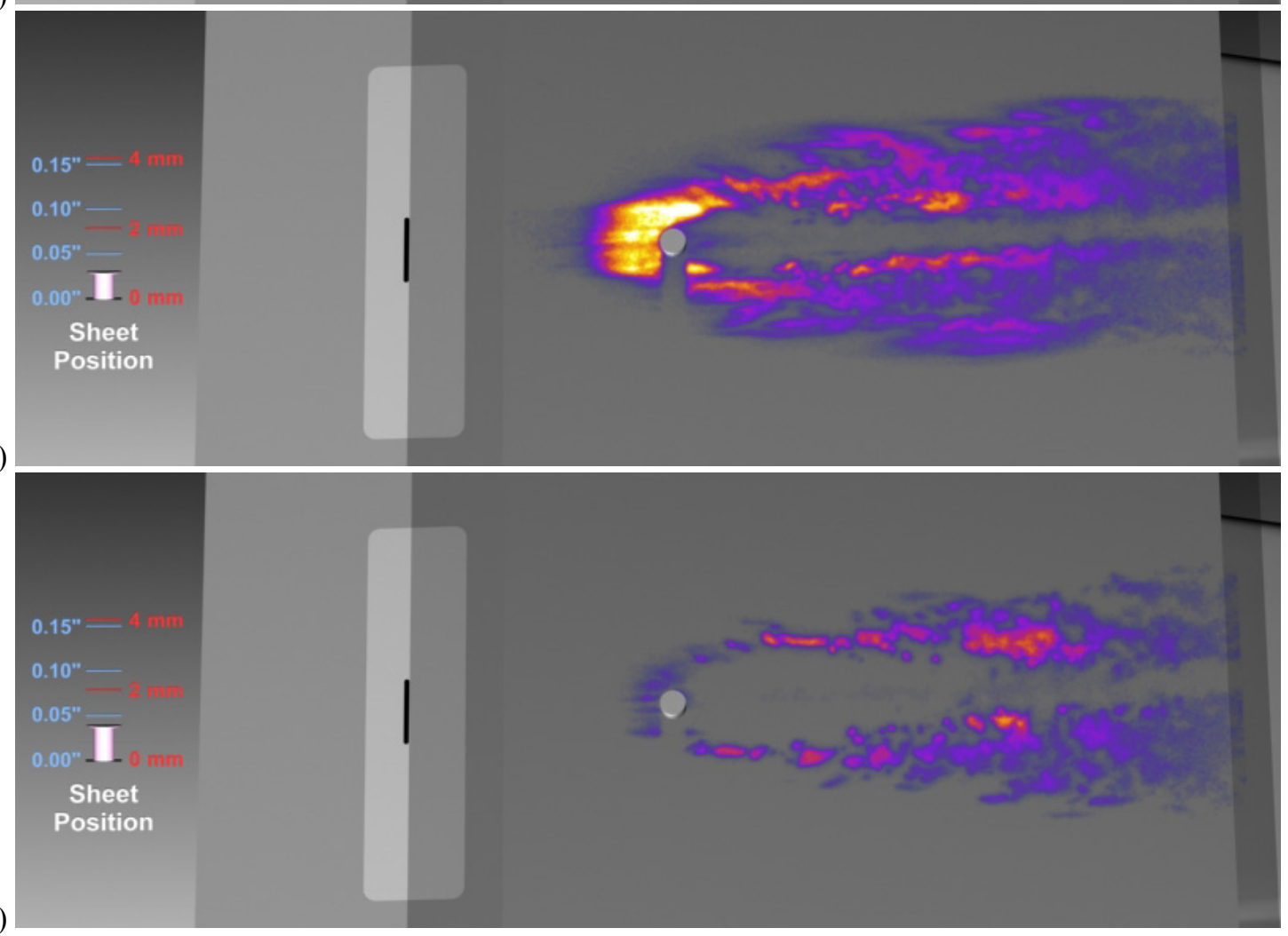

Figure 12. Test 443, Run 31, 2-mm tall by 4-mm wide cylinder, plate angle $=20^{\circ}$, 11-mm wide slot seeding, $\dot{m}=300 \mathrm{sccm}, \mathrm{P}_{0}=9.33 \mathrm{MPa}$, sheet position varies from 0.0 to $2.0 \mathrm{~mm}$ above the plate in $0.25 \mathrm{~mm}$ increments in (a)-(i), framing rate $=10 \mathrm{~Hz}$. 
When the 4-mm tall cylindrical trip is placed on the flat plate, the flow is even further perturbed, as shown in Fig. 13. The gas is significantly deflected to both sides of the trip: the full width of the deflection is about 20 trip diameters for images near the surface, in Fig. 13(a). This compares to a 12 trip-diameter deflection for the 2-mm tall trip in Fig. 12(a) and a 5 trip-diameter deflection for the 1-mm tall trip in Fig. 11(a). Also observed in Fig. 13(a) are various streamwise streaks and $45^{\circ}$ oriented flow structures downstream of the trip. Again, flow past this trip shows a very large dead air region immediately downstream of the trip.

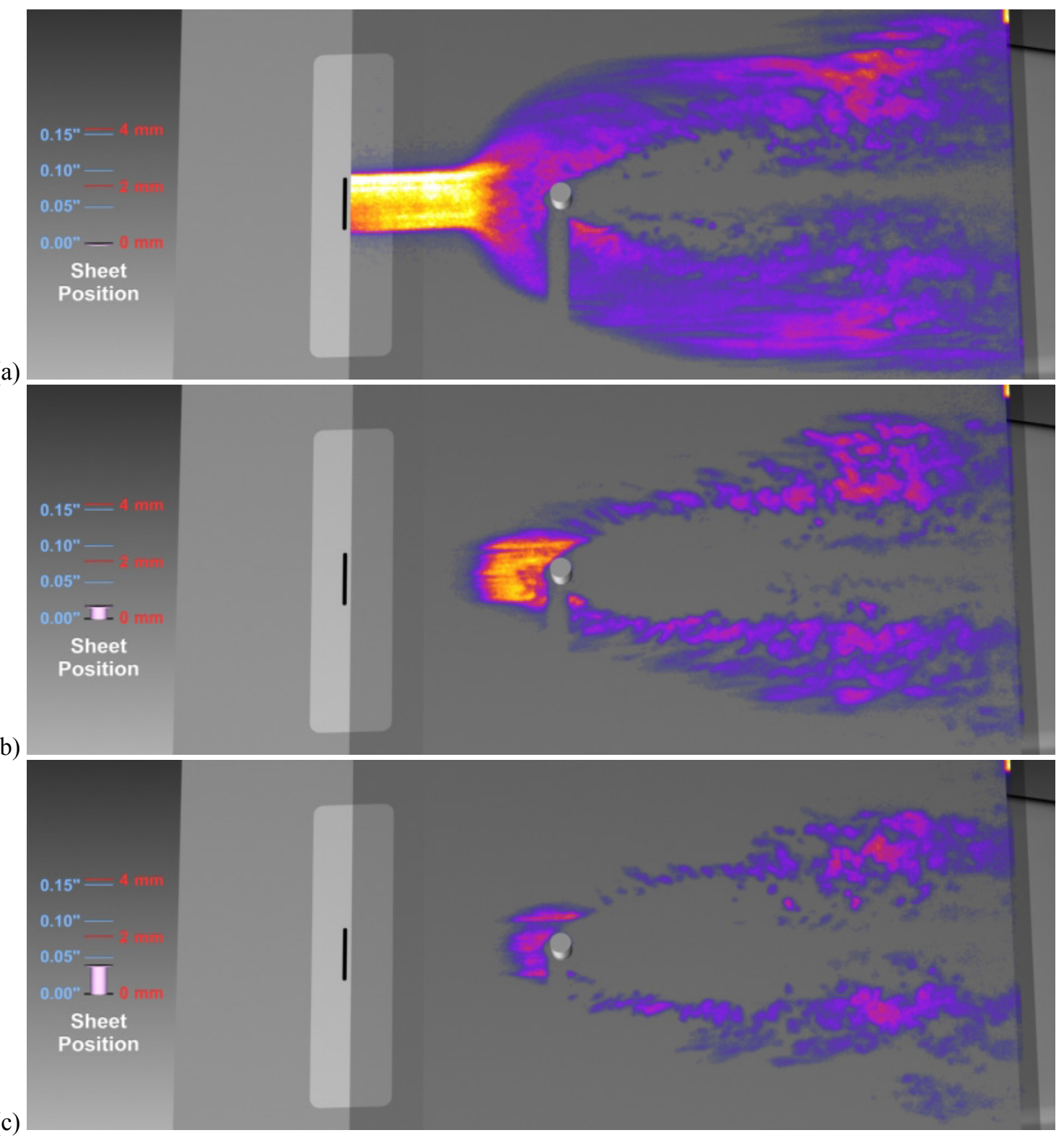

Figure 13. Test 443, Run 16, 4-mm tall by 4-mm wide cylinder, plate angle $=20^{\circ}$, 11-mm wide slot seeding, $\dot{m}=300 \mathrm{sccm}, \mathrm{P}_{0}=9.3 \mathrm{MPa}$, sheet position varies from 0.0 to $1.0 \mathrm{~mm}$ above the plate in $0.5-\mathrm{mm}$ increments in (a)(c), framing rate $=10 \mathrm{~Hz}$. 


\section{B. Oil Flow Visualizations and Comparison with Plan View 10 Hz PLIF Images}

Surface oil flow measurements were obtained in separate tunnel runs as indicated in Table 1. Oil flow visualizations from three separate runs are presented in Fig. 14. Figure 14(a) shows the center part of the model for a run without a protuberance. The flow is from left to right and appears to be uniform and parallel in the streamwise direction along the centerline. When the 1-mm cylindrical trip is in placed on the flat plate in Fig.14(d), the flow is observed to separate in front of the trip, probably with a horseshoe-shaped vortex rolling up at the base of the trip. The streamlines in the flow just upstream of this vortex are deflected away from the model centerline. The streamlines show that much of the boundary layer flow is deflected spanwise around the trip. Downstream of the trip, the flow along the surface is mainly parallel to the streamwise direction.

The oil flow map from the 4-mm tall trip is shown in Fig. 14(c). Far upstream of the trip, the flow is parallel and uniform, aligned in the streamwise direction. A very large flow separation is observed forward of and adjacent to the trip. Significant evidence of reversed flow is seen in the region just upstream of the trip. A large quantity of oil is transported to the interface between the oncoming boundary layer flow and the separated flow from the trip, resulting in the parabolic shaped structure in the image. Downstream of the trip, a complicated pattern emerges. Alternating bands of parallel lines indicate that multiple counterrotating vortices are present in the wake flow. Far downstream of the trip, the flow mostly becomes parallel as it exits the plate.

Also in Fig. 14, PLIF images have been superimposed onto the oil flow maps. Fig. 14(d) shows a uniform PLIF image for the case of no protuberance. The PLIF image has minimal spatial structure (other than a few streamwise streaks), indicating laminar flow. The edges of the fluorescing seeded gas - themselves providing streakline visualization - are observed to be parallel to the oil flow streamlines. Figure 14(e) and (f) show the superposition of the PLIF and oil flow images for the 1-mm and 4-mm tall trips, respectively. In both cases, the edges of the fluorescence pattern are observed to track the most deflected streamlines to the spanwise sides of the trip. The irregular structures observed in the PLIF images, which are suggestive of transition to turbulence, are not visible in the oil flow maps. The oil flow data, which is time averaged over the duration of the wind tunnel run, does not indicate if and where transition to turbulence may have occurred. 


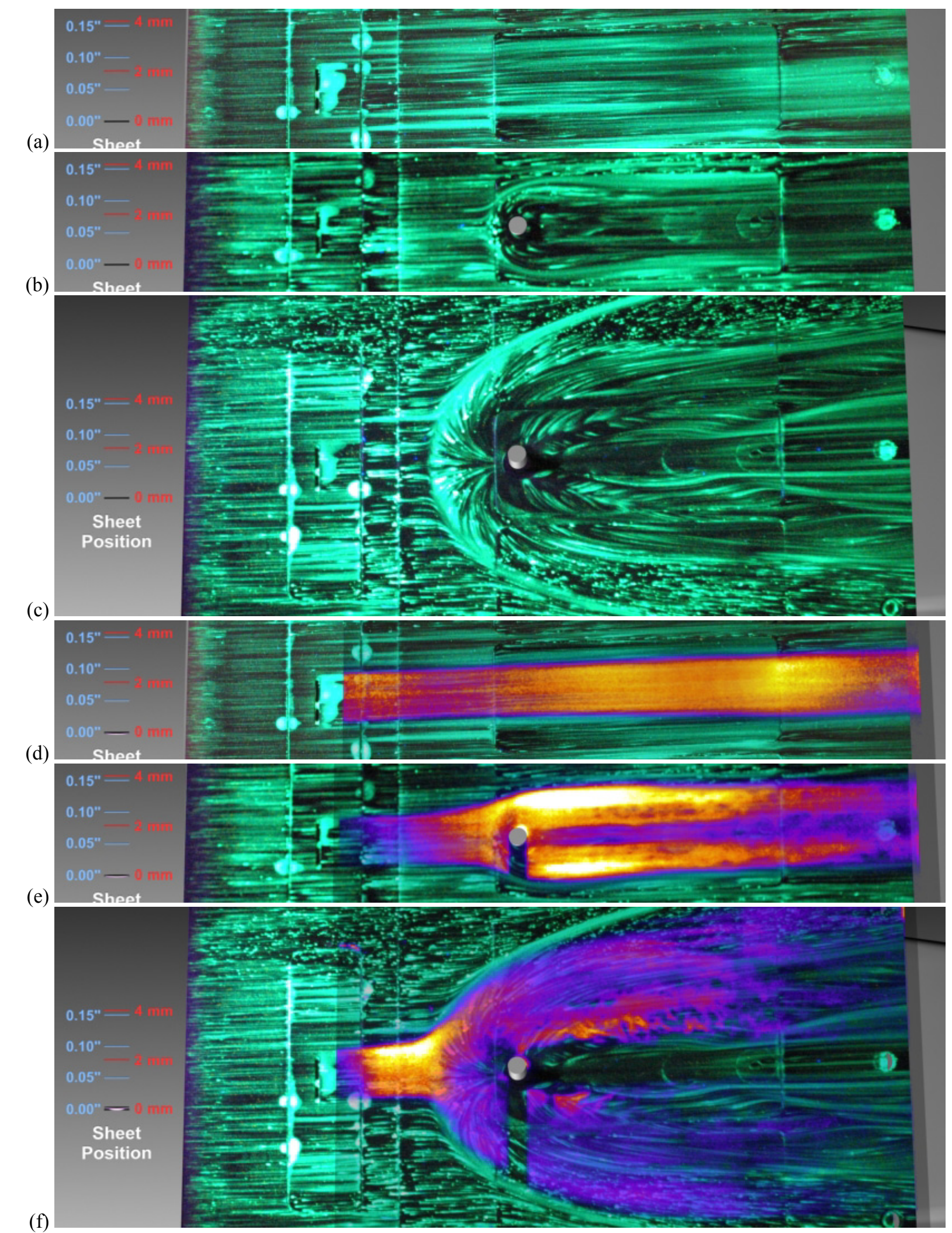

Figure 14. Oil flow visualizations from Test 443, (a) Run 53, no trip, (b) Run 55, 1-mm tall by 4-mm wide cylinder, and (c) Run 56, 4-mm tall by 4-mm wide cylinder. These same oil flow images are superimposed with PLIF images obtained at the respective runs: (d) Run 23, (e) Run 18, and (f) Run 16. For all images, plate angle = $20^{\circ}$, 11-mm wide slot seeding, $\dot{m}=300 \mathrm{sccm}, \mathrm{P}_{0}=9.3 \mathrm{MPa}$, sheet position $=0.0 \mathrm{~mm}$ above the plate. 
(a)
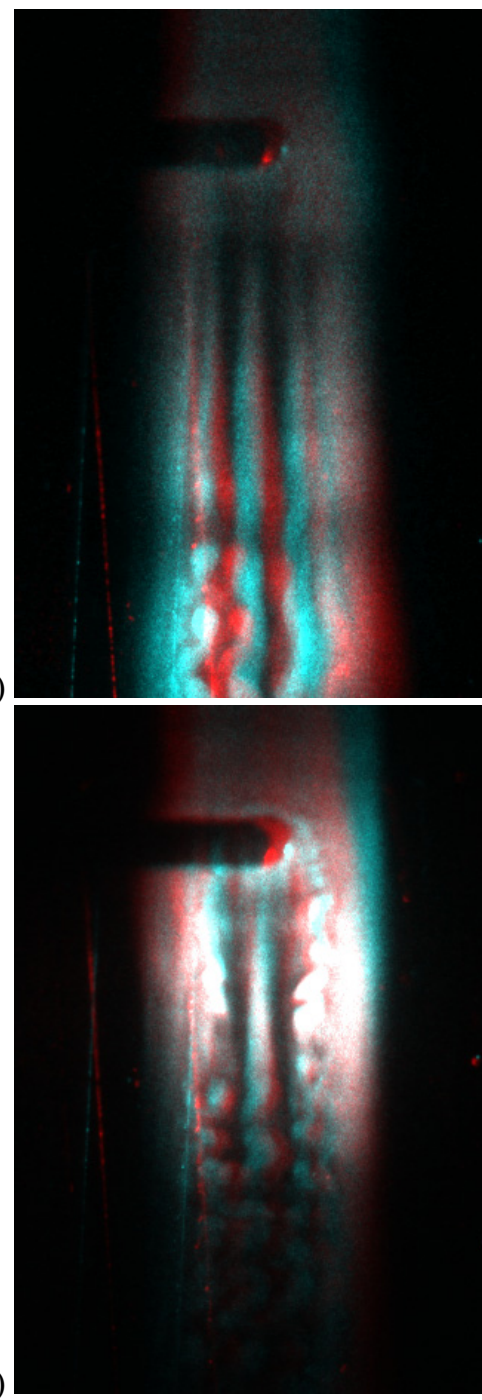

(d)

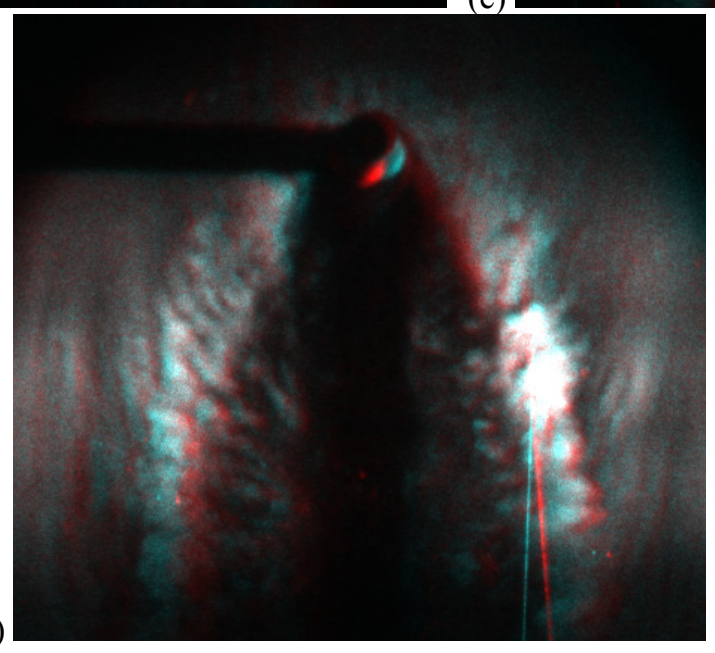

(b)

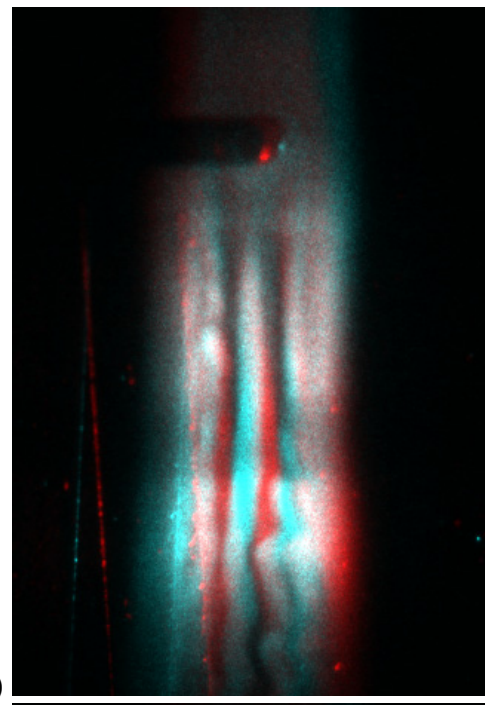

(c)

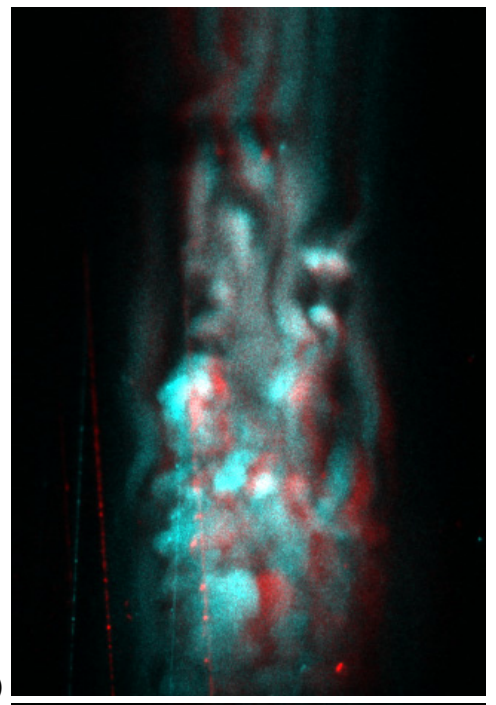

(f)

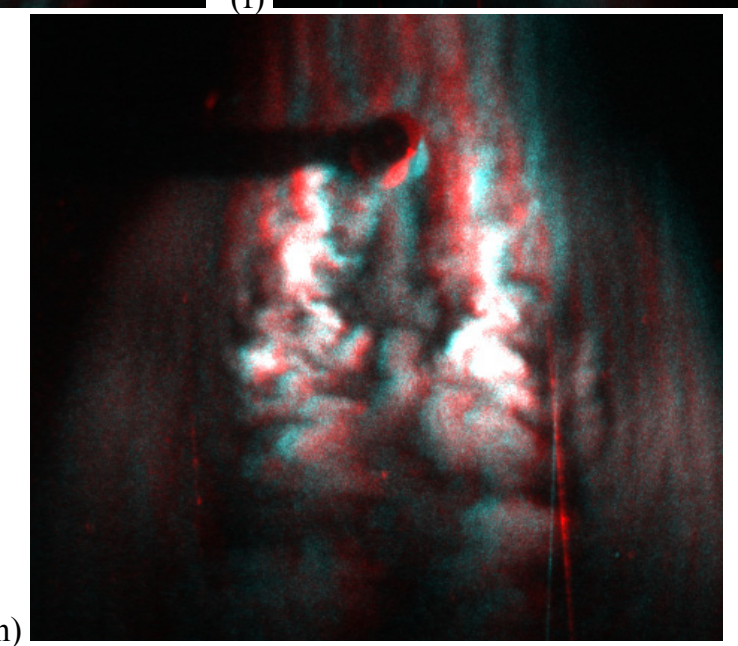

(h)

Figure 15. Stereoscopic PLIF visualization for Test 443, (a)-(c) are from Run 47: 1-mm tall trip, $\mathrm{P}_{0}=4.97 \mathrm{MPa}$, $\dot{m}=310 \mathrm{sccm}$ in (a) and (b) and $4300 \mathrm{sccm}$ in (c); (d)-(f) are from Run 48: 1-mm tall trip, $\mathrm{P}_{0}=9.35 \mathrm{MPa}, \dot{m}=620$ sccm in (d) and (e) and $4200 \mathrm{sccm}$ in (f); (g) and (h) are from Run 49: 4-mm tall trip, $\mathrm{P}_{0}=9.35 \mathrm{MPa}, \dot{m}=620 \mathrm{sccm}$ in $(\mathrm{g})$ and $4400 \mathrm{sccm}$ in (h), For call cases, cylindrical trip width $=4 \mathrm{~mm}$, plate angle $=20^{\circ}$, slot seeding $=11 \mathrm{~mm}$ wide, framing rate $=10 \mathrm{~Hz}$. Flow is from top to bottom in the images. The laser sheet is about $10 \mathrm{~mm}$ thick. 


\section{Thick Laser Sheet and Stereoscopic PLIF Visualization}

The drawback of probing the flow with a thin laser sheet is that only thin slices of structures are visualized, potentially limiting a holistic understanding of the flow features. By using a thick laser sheet, the entire boundary layer flow can be illuminated and imaged instantaneously, providing a more global view of the flow patterns. The excited florescence is viewed by a pair of cameras having identical lenses. While individual images provide useful visualization of the flow structures, the pair of images can be combined to provide qualitative three-dimensional information about the flow structures, as described in Ref. 13. Figure 15 shows several such images for operating conditions similar to those used in the prior sections of this paper. Special glasses containing a red lens over the left eye and a blue lens over the right eye should be worn while viewing this figure. Corresponding thick-laser-sheet single-camera images are shown in the appendix, if stereoscopic viewing glasses are not available.

Figures 15(a)-(c) show images from a run using the 1-mm tall trip with the wind tunnel operating at the middle Reynolds number. Figures 15(a) and (b) show two independent visualizations using the same NO mass flow rate. The cameras were oriented slightly downstream of the model so that the gas flow is from top to bottom, with the surface of flat plate appearing closer to the viewer at the bottom of the images and further away at the top, when viewed with 3-D glasses. Laminar flow enters from the top of the image and passes by the trip. Streaks are generated in the flow downstream of the trip. In both Fig. 15(a) and (b) a weak spiral or corkscrew-shaped vortex can be observed just left of the centerline. The flow immediately downstream of the trip is also unstable, perhaps breaking up in a varicose (sausage-like) mode. ${ }^{15}$ When the flow rate of $\mathrm{NO}$ is increased by over a factor of 10, in Fig. 15(c), the flow changes dramatically. The flow is no longer laminar upstream of the trip. Rather, well-defined streaks appear there. The interaction between the protuberance and this perturbed boundary layer flow causes the flow to transition to much smaller, random structures in the same field of view, compared to the case with a lower flow rate. Also the flow structures appear to penetrate further into the flow above the plate in this higher flowrate case. One streak even passes over the top of the trip in Fig. 15(c).

When the tunnel stagnation pressure is increased to operate at the highest Reynolds number case, the flow again shows similar trends to those observed in Figs. 10 and 11; irregular flow structures are observed much closer to the trip for this higher Reynolds number case when probing the 1-mm tall trip in Figs. 15(d) and (e). Furthermore, more vigorous corkscrew-shaped vortices are seen to the sides of the trip and slightly above the laminar layer directly adjacent to the wall. These vortices appear to meet in front of the trip in a classic horseshoe vortex pattern. Again consistent with Figs. 10 and 11, the observed flow structures downstream of the trip are smaller in the higher Reynolds number case. When the flow rate is increased, almost by a factor of 10 in Fig. 15(f), the flow becomes even more irregular and three-dimensional. Images acquired using the 4-mm tall trip at the highest Reynolds number condition are shown in Fig. 15(g) and (h). The lower flow rate image in 15(g) shows a buildup of seeded boundary layer fluid above the surface in an arc shape around the trip as well as a dead air region directly downstream of the trip, consistent with Fig. 13. When the flow rate is increased, the wake closes in a manner analogous to the flow separation behind a dimpled golf ball.

\section{Plan View MHz NO PLIF Flow Visualization}

The appendix shows 28 different MHz-rate flow visualization image sequences obtained at various measurement heights, using different trip heights and different tunnel operating conditions, as indicated in Table 1. As representative samples of this data set, selected images from four of these image sequences are shown in Figs. 16-19. Because the images are separated in time by a mere 1 or 2 microseconds (for the $1 \mathrm{MHz}$ and $500 \mathrm{kHz}$ framing rates, respectively), the evolution of individual flow structures can be observed from one image to the next. For Figs. 16-19, a 2-mm tall by 4-mm diameter cylindrical trip was used and approximately a 1-mm height above the flat plate was probed. Four different unit Reynolds number conditions were investigated: the same low, middle, and high stagnation pressure conditions used previously, plus one additional condition between the low and middle $\left(\mathrm{P}_{0}=3.45 \mathrm{MPa}\right)$, hereafter referred to as the lower-middle condition. 
(a)

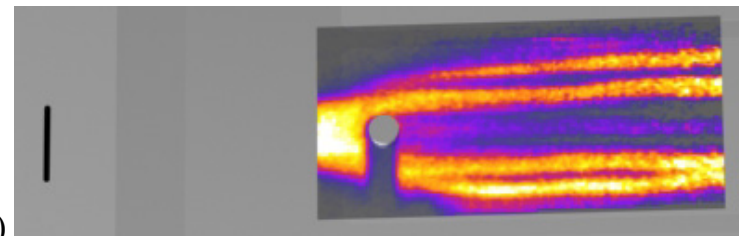

(c)
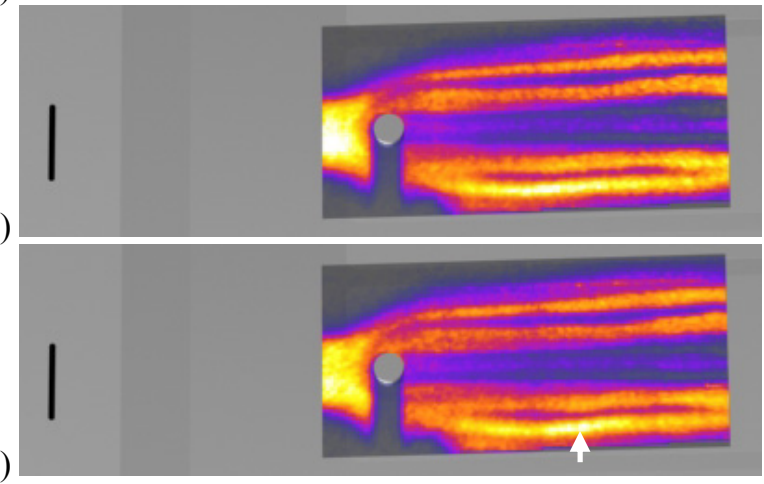

(b)

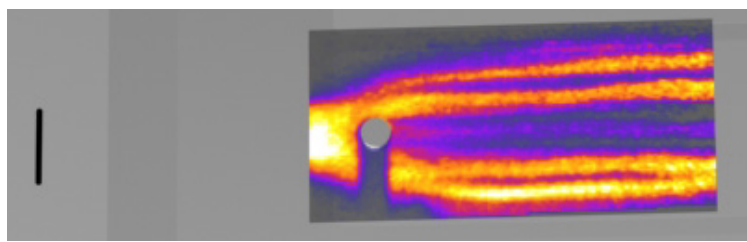

(d)

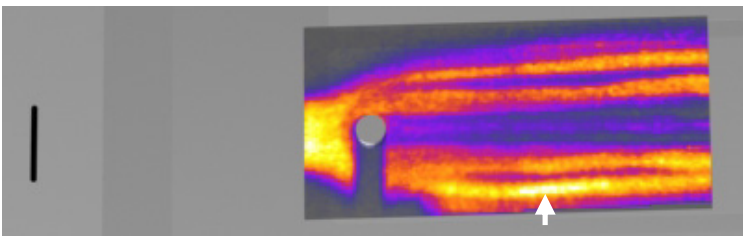

(f)

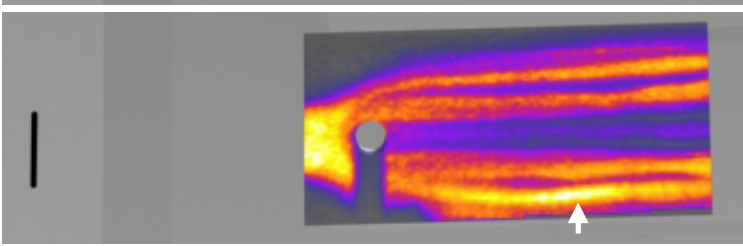

Figure 16. Test 463 , Run 7, 2-mm tall by $4 \mathrm{~mm}$ wide cylinder, plate angle $=20^{\circ}, 11$-mm wide slot seeding, $\dot{m}=300$ $\mathrm{sccm}, \mathrm{P}_{0}=2.4 \mathrm{MPa}$, sheet position $=1 \mathrm{~mm}$ above surface, framing rate $=500 \mathrm{kHz}$. The black, vertical segment near the left boundary of each image denotes the relative location and spanwise extent of the slot used for seeding NO into the boundary layer.

Figure 16 shows the lowest Reynolds number run. The imaged region is approximately 50 -mm (2-inches) long by $25-\mathrm{mm}$ (1 inch) tall. The trip height is approximately twice as large as the boundary layer thickness in this run. Consequently, the trip deflects the flow in the spanwise direction and a partial dead air region develops immediately downstream of the trip. A horseshoe vortex originating from the front of the trip appears to be laminar but with a long wavelength and low amplitude unsteadiness, which propagates downstream as the images progress. For example the arrows in Figs. 16(d)-(f) highlight a weak disturbance that moves downstream. Five distinct streamwise-oriented streaks are observed downstream of the trip.

When the Reynolds number is increased to the lower-middle condition in Fig. 17, the horseshoe vortex is again observed wrapping around the trip, but it develops into a highly periodic, larger amplitude and shorter wavelength corkscrew-shaped vortex on one side of trip and a longer wavelength disturbance on the other side.

(a)

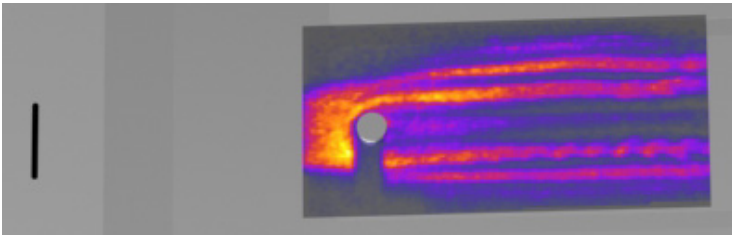

(c)
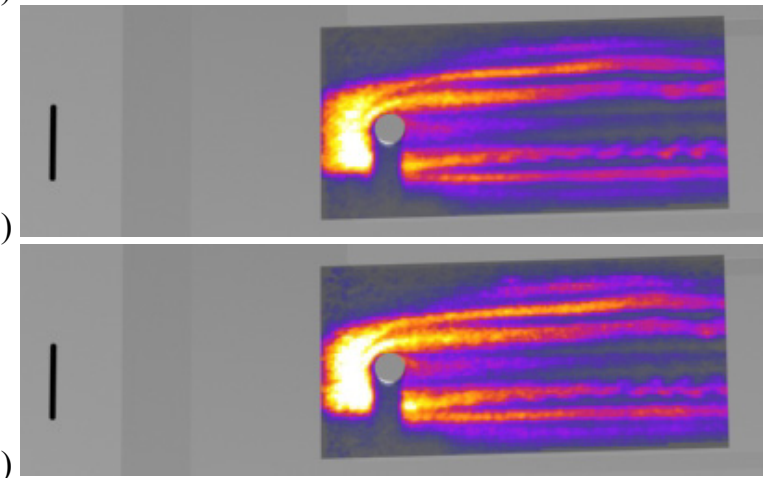

(b)

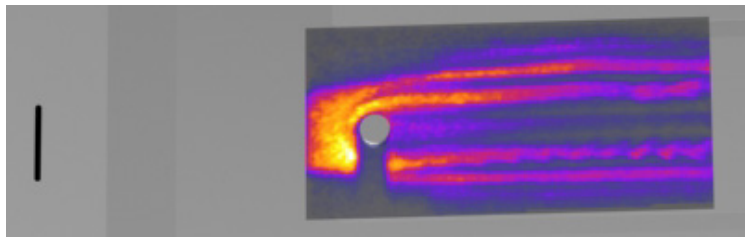

(d)

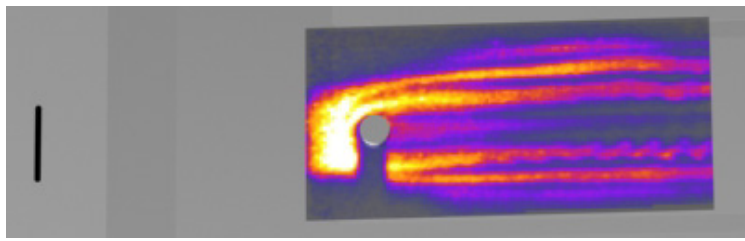

(f)

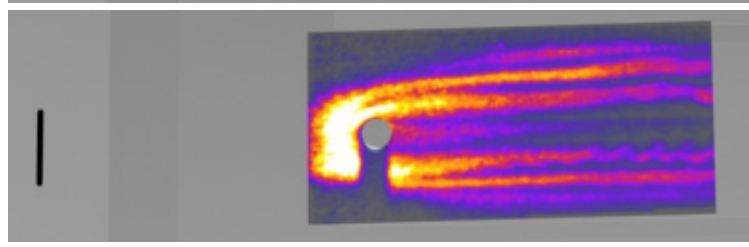

Figure 17. Test 463 , Run 9,2 -mm tall by $4 \mathrm{~mm}$ wide cylinder, plate angle $=20^{\circ}, 11$-mm wide slot seeding, $\dot{m}=300$ $\mathrm{sccm}, \mathrm{P}_{0}=3.45 \mathrm{MPa}$, sheet position $=1 \mathrm{~mm}$ above surface, framing rate $=500 \mathrm{kHz}$. 
At an even higher Reynolds number (the middle stagnation pressure tested) in Fig. 18, energetic, unsteady flow structures appear even closer to the trip. (Note that the framing rate of Fig. 18 is higher by a factor of two compared to Figs 16, 17, and 19.) The development of these vortical structures can be observed in the image sequences. The flow structures in this middle Reynolds number case are much more irregular than in the lower Reynolds number runs.

(a)

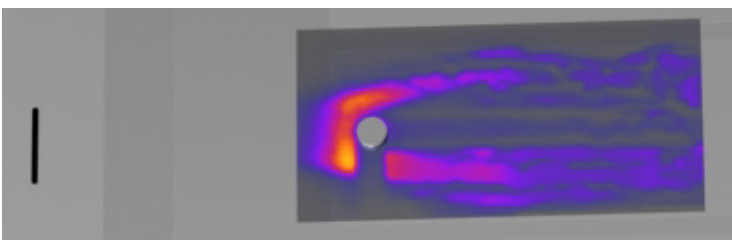

(c)

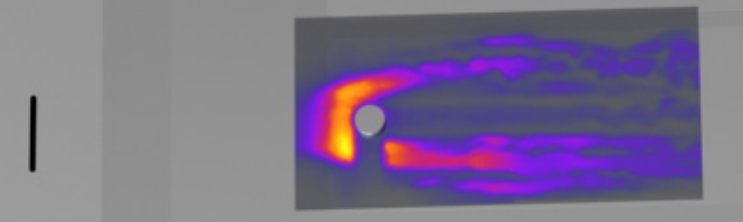

(e)

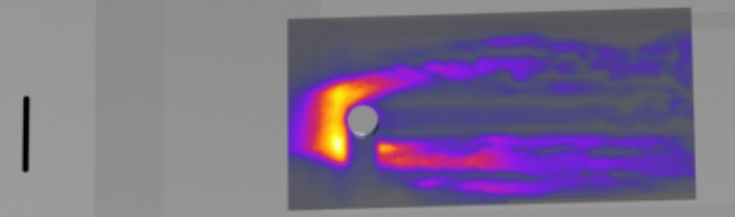

(b)

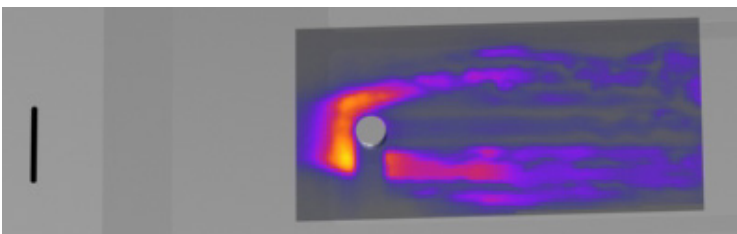

(d)

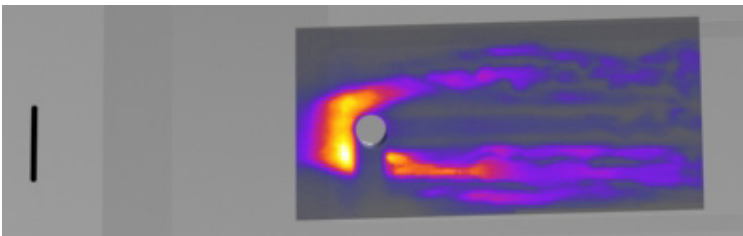

(f)

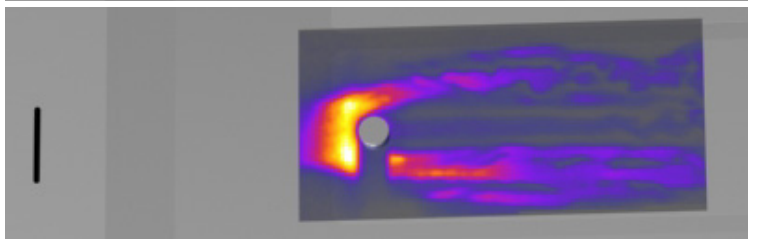

Figure 18. Test 463, Run 17, 2-mm tall x 4-mm wide cylinder on a flat plate, plate angle $=20^{\circ}, 11-\mathrm{mm}$ wide slot seeding, $\dot{m}=300 \mathrm{sccm}, \mathrm{P}_{0}=4.97 \mathrm{MPa}$, sheet position $=1.1 \mathrm{~mm}$ above surface, framing rate $=1 \mathrm{MHz}$.

The highest Reynolds number run, shown in Fig. 19, exhibits tightly wound corkscrew shaped vortices downstream on both sides of the trip. As they propagate downstream, they lose their organized structure - see for example the structure highlighted with the white oval in Fig. 19. Some structures transition into structures having smaller length scales - for example the two faint c-shaped structures that can be seen on the right side of the highlighted oval in Fig. 19(f).

(a)

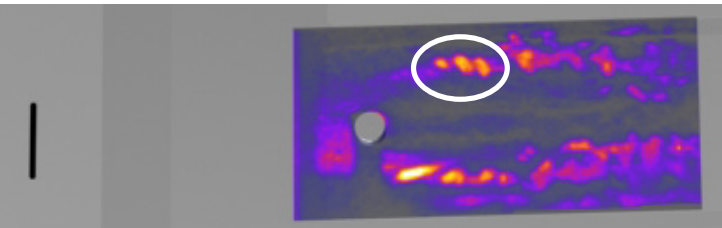

(c)
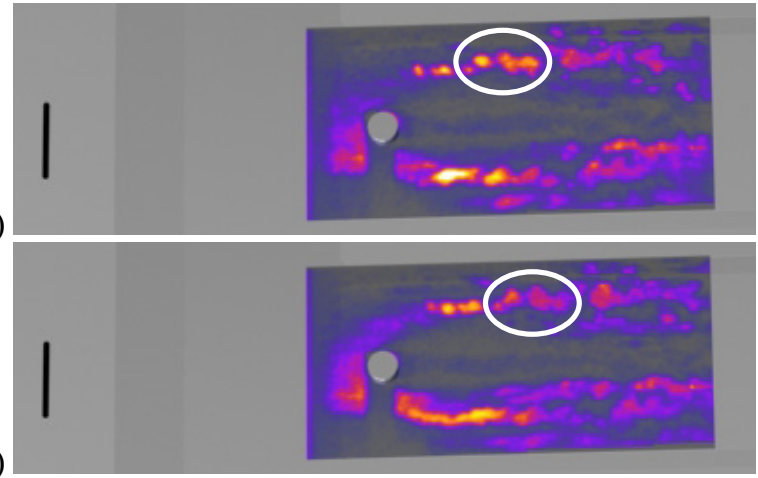

(b)

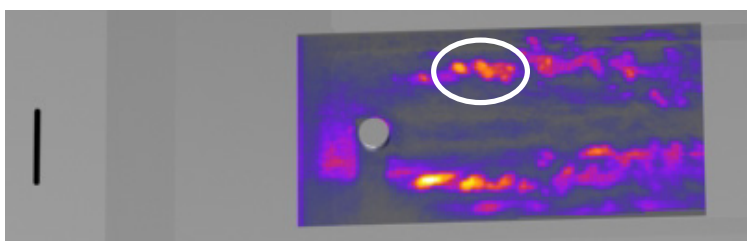

(d)

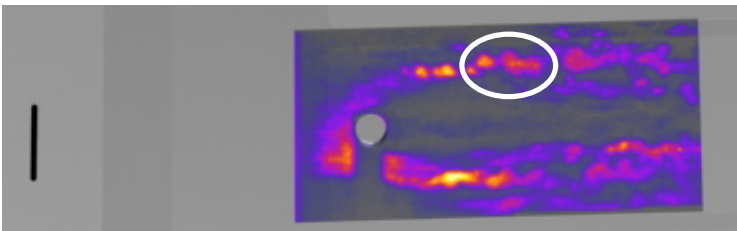

(f)

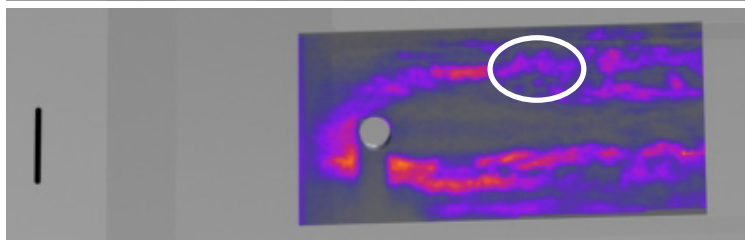

Figure 19. Test 463 , Run $8,2-\mathrm{mm}$ tall by $4-\mathrm{mm}$ wide cylinder, plate angle $=20^{\circ}, 11-\mathrm{mm}$ wide slot seeding, $\dot{m}=300 \mathrm{sccm}, \mathrm{P}_{0}=9.34 \mathrm{MPa}$, sheet position $=1.2 \mathrm{~mm}$ above surface, framing rate $=500 \mathrm{kHz}$. 


\section{Conclusion}

Several different imaging methods based on NO PLIF have been used to study the near-field of transitional flow over cylindrical protuberances on a flat plate. Side view imaging provided information about boundary layer thickness, and flow features normal to the wall. Plan view imaging shows the spanwise extent of the influence of the trip. For example, the $4 \mathrm{~mm}$ tall cylindrical trip deflected the seeded gas about 20 trip diameters whereas the $1 \mathrm{~mm}$ tall trip only deflected the seeded gas 5 trip diameters, for the same operating conditions. The plan views also helped identify the dominant flow structures within the strongly perturbed, unsteady wake behind the protuberance, such as the corkscrewshaped vortical structures originating from horse-shoe shaped vortices wrapping around the front of the trip. Such structures would not have been observed with NO PLIF centerline-only measurements or with surface measurement techniques alone. Complementary oil flow measurements indicated flow patterns that were consistent with the off-body NO PLIF images. Stereoscopic imaging has been employed to instantaneously image full flowfield structures instead of just obtaining individual slices. These images show the three-dimensionality of the flow. The MHz rate NO PLIF images show the evolution and development of the individual structures present in the flows and can potentially be processed in the future to provide information about the convective velocity of the dominant flow structures. These images further reveal how energy is transported from large scale to small scale structures. Finally, a detailed appendix provides a much more extensive data set that will prove useful for comparison with computational simulations of the flow. Such comparisons can make use of both qualitative visualizations and some quantitative data, such as spacing and number of streaks that develop downstream of the protuberances.

\section{Acknowledgments}

We wish to acknowledge the contribution to this project from the NASA Langley Research Center 31-Inch Mach 10 Air Tunnel technicians and engineers, including Kevin Hollingsworth, Sheila Wright, Paul Tucker, Tony Robbins, Henry Fitzgerald and Johnny Ellis. We appreciate the assistance of David Alderfer who contributed to the setup and acquisition of some of the data described herein and of Meelan Choudhari who made numerous improvements to the manuscript. This work was supported by the NASA Fundamental Aeronautics Hypersonics Program with support from both the Experimental Capabilities and Aerodynamics-Aerothermodynamics-Plasma Disciplines. Support for operation of the facility was also obtained from the Aeronautics Testing Program (ATP) and is appreciated. Thanks also to Rich Schwartz from ATK Space Systems, Hampton Virginia, for assisting with the computer visualizations of the data, to Mark Kulick, also of ATK, for building some of the test hardware. The authors from OSU and ISU also wish to acknowledge the Air Force Office of Scientific Research (Julian Tishkoff and John Schmisseur) and the Air Force Research Laboratory (James Gord) for their contributions to the development of the $\mathrm{MHz}$ laser and camera technologies.

\section{References}

${ }^{1}$ Liechty, D.S., "Aerothermodynamic Testing of Protuberances and Penetrations on the NASA Crew Exploration Vehicle Heat Shield," AIAA Paper 2008-1240, 46th AIAA Aerospace Sciences Meeting and Exhibit, Reno, NV, Jan. 710 (2008).

${ }^{2}$ S. A. Berry, A. H. Auslender, A. D. Dilley, J. F. Calleja, "Hypersonic Boundary-Layer Trip Development For HyperX,” Journal of Spacecraft and Rockets Vol. 38, No. 6, p. 853-864, Nov.-Dec, 2001

${ }^{3}$ S. Berry, T. Horvath, "Discrete-Roughness Transition for Hypersonic Flight Vehicles," Journal of Spacecraft and Rockets, vol.45 no. 2 p.216-227, 2008

${ }^{4}$ S. Schneider, "Effects of Roughness on Hypersonic Boundary-Layer Transition " Journal of Spacecraft and Rockets, v.45 n.2 p.193-209, 2008

${ }^{5}$ A. H. Whitehead. "Flow Field and Drag Characteristics of Several Boundary-Layer Tripping Elements in Hypersonic Flow” Technical Report TN D-5454, NASA Langley Research Center, October 1969

${ }^{6}$ A. S. Collier, J. F. Lafferty, S. S. Swinford, D, W. Witte, Aerodynamic heat transfer testing in hypersonic wind tunnels using an infrared imaging system AIAA-1990-189, Aerospace Sciences Meeting, 28th, Reno, NV, Jan 8-11, 1990.

${ }^{7}$ B. M. Wheaton and S. P. Schneider "Roughness- Induced Instability in a Laminar Boundary Layer at Mach 6" AIAA2010-1574, 48 ${ }^{\text {th }}$ AIAA Aerospace Sciences Meeting, Orlando, FL, January, 2010.

${ }^{8}$ P.M. Danehy, A.P. Garcia, S. Borg, A.A. Dyakonov, S.A. Berry, J.A. Wilkes Inman, D.W. Alderfer, "Fluorescence visualization of hypersonic flow past triangular and rectangular boundary-layer trips", AIAA-2007-0536, 45th AIAA Aerospace Sciences Meeting, Reno Nevada, January 8-11 (2007).

${ }^{9}$ P. M. Danehy, B. Bathel, C. Ivey, J. A. Inman, S. B. Jones, "NO PLIF study of hypersonic transition over a discrete hemispherical roughness element", AIAA-2009-0394, 47th AIAA Aerospace Sciences Meeting, Orlando, FL, January, 2009. 


\footnotetext{
${ }^{10}$ N. Jiang, M. Webster, W. R. Lempert, J. D. Miller and T. R. Meyer, P. M. Danehy, "MHz-Rate NO PLIF Imaging in a Mach 10 Hypersonic Wind Tunnel," Paper AIAA-2010-1407, the 48th AIAA Aerospace Sciences Meeting, AMT TC, Orlando Florida, January 2010.

${ }^{11}$ P.M. Danehy, C. B. Ivey, B. F. Bathel, J.A. Inman, S.B. Jones, A. N. Watkins, K. Goodman, A. C. McCrea, B, D. Leighty and W. K. Lipford, N. Jiang, M. Webster, W. Lempert, J. Miller, T. Meyer, "Orbiter BLT flight experiment wind tunnel simulations: nearfield flowfield imaging and surface thermography," Paper AIAA-2010-1571, the 48th AIAA Aerospace Sciences Meeting, Orlando Florida, January 2010.

${ }^{12}$ Hollis, B.R. "Real-gas flow properties for NASA Langley Research Center Aerothermodynamics Facilities Complex Wind Tunnels," NASA Contractor Report 4755, Sept. 1996.

${ }^{13}$ P. M. Danehy, J. A. Inman, B. Bathel, D. W. Alderfer, S. B. Jones, "Stereoscopic Imaging in Hypersonics Flows using Planar Laser Induced Fluorescence", Paper Number 2008-4267, 26th AIAA Aerodynamic Measurement Technology and Ground Testing Conference, Seattle, WA June 23-26, 2008

${ }^{14}$ P.M. Danehy, D.W. Alderfer , J.A. Inman, K.T. Berger, G.M. Buck and R. J. Schwartz "Fluorescence Imaging and Streamline Visualization of Hypersonic Flow over Rapid Prototype Wind-Tunnel Models," Proc. IMechE, Part G: J. Aerospace Engineering, 222(G5), 637-651 (2008).

${ }^{15}$ C. Chang, M. Choudhari, and F. Li, "Numerical Computations of Hypersonic Boundary-Layer over Surface Irregularities," AIAA Paper 2010-1572, 2010.

${ }^{16}$ M. Choudhari, F. Li, and J. A. Edwards, "Stability Analysis of Roughness Array Wake in a High-Speed Boundary Layer," AIAA Paper 2009-0170, 2009.

${ }^{17}$ M. C. Fischer, "Spreading of a Turbulent Disturbance," AIAA Journal, vol.10 no.7 p. 957-959, 1972.
} 


\section{Appendix}

This appendix contains selected ViDI-processed images from Tests 443 and 463 in the 31 Inch Mach 10 Wind Tunnel at NASA Langley Research Center. The table below summarizes the data contained in this appendix. Furthermore, oil flow visualizations from Runs 54, 55, and 56 of Test 443 are shown with PLIF images obtained at the same conditions in Figures A32, A26, and A20, respectively (see Table 1 in the paper for further information about these oil flow runs).

\begin{tabular}{|c|c|c|c|c|c|c|c|c|c|c|c|c|}
\hline Figures & Test & Run & $\begin{array}{c}\text { Primary } \\
\text { Measurement }\end{array}$ & View & $\begin{array}{l}\text { Framing } \\
\text { Rate }\end{array}$ & Trip Type & $\begin{array}{c}\text { Trip } \\
\text { Height }\end{array}$ & $\begin{array}{c}\text { Trip } \\
\text { Width }\end{array}$ & $\begin{array}{c}\text { Stagnation } \\
\text { Pressure }\end{array}$ & \begin{tabular}{|c|} 
Unit \\
Reynolds \\
Number \\
\end{tabular} & Plate Angle & NO flow rate \\
\hline & & & & & & & $(\mathrm{mm})$ & $(\mathrm{mm})$ & (MPa) & $(1 / \mathrm{m})$ & (degrees) & $(\mathrm{sccm})$ \\
\hline A1-A7 & 443 & 12 & PLIF Flow Vis & side & $10 \mathrm{~Hz}$ & cylinder & 1 & 4 & 9.34 & 6.02 & $25 \rightarrow+5 \rightarrow 20$ & 300 \\
\hline A8-A14 & 443 & 15 & PLIF Flow Vis & side & $10 \mathrm{~Hz}$ & cylinder & 2 & 4 & 9.30 & 6.02 & $25 \rightarrow+5 \rightarrow 20$ & 300 \\
\hline A15-A20 & 443 & 16 & PLIF Flow Vis & plan & $10 \mathrm{~Hz}$ & cylinder & 4 & 4 & 9.31 & 6.00 & 20 & 300 \\
\hline A21-A26 & 443 & 18 & PLIF Flow Vis & plan & $10 \mathrm{~Hz}$ & cylinder & 1 & 4 & 9.32 & 6.01 & 20 & 300 \\
\hline A27-A32 & 443 & 19 & PLIF Flow Vis & plan & $10 \mathrm{~Hz}$ & cylinder & 1 & 4 & 4.97 & 3.27 & 20 & 300 \\
\hline A33-A37 & 443 & 20 & PLIF Flow Vis & plan & $10 \mathrm{~Hz}$ & cylinder & 1 & 4 & 2.41 & 1.66 & 20 & 300 \\
\hline A38-A40 & 443 & 23 & PLIF Flow Vis & plan & $10 \mathrm{~Hz}$ & no trip & -- & -- & 9.35 & 6.16 & 20 & $300 ; 1000$ \\
\hline A41-A45 & 443 & 25 & PLIF Flow Vis & plan & $10 \mathrm{~Hz}$ & no trip & -- & -- & 4.98 & 3.28 & 20 & $30 ; 300$ \\
\hline A46-A49 & 443 & 31 & PLIF Flow Vis & plan & $10 \mathrm{~Hz}$ & cylinder & 2 & 4 & 9.33 & 6.11 & 20 & 300 \\
\hline A50-A53 & 443 & 47 & Stereo PLIF & plan & $10 \mathrm{~Hz}$ & cylinder & 1 & 4 & 4.97 & 3.30 & 20 & $300 ; 300+5 S L P M ~ N 2$ \\
\hline A54-A57 & 443 & 48 & Stereo PLIF & plan & $10 \mathrm{~Hz}$ & cylinder & 1 & 4 & 9.35 & 6.14 & 20 & $600 ; 600+5 S L P M ~ N 2$ \\
\hline A58-A61 & 443 & 49 & Stereo PLIF & plan & $10 \mathrm{~Hz}$ & cylinder & 4 & 4 & 9.35 & 6.17 & 20 & $600 ; 600+5$ SLPM N2 \\
\hline A62-A64 & 463 & 6 & PLIF Flow Vis & plan & $500 \mathrm{kHz}$ & cylinder & 2 & 4 & 4.97 & 3.39 & 20 & 300 \\
\hline A65-A68 & 463 & 7 & PLIF Flow Vis & plan & $500 \mathrm{kHz}$ & cylinder & 2 & 4 & 2.40 & 1.74 & 20 & 150 \\
\hline A69-A72 & 463 & 8 & PLIF Flow Vis & plan & $500 \mathrm{kHz}$ & cylinder & 2 & 4 & 9.34 & 6.20 & 20 & 300 \\
\hline A73-A77 & 463 & 9 & PLIF Flow Vis & plan & $500 \mathrm{kHz}$ & cylinder & 2 & 4 & 3.45 & 2.42 & 20 & 230 \\
\hline A78-A81 & 463 & 10 & PLIF Flow Vis & plan & $500 \mathrm{kHz}$ & cylinder & 1 & 4 & 6.91 & 4.67 & 20 & 300 \\
\hline A82 & 463 & 14 & PLIF Flow Vis & plan & $500 \mathrm{kHz}$ & no trip & -- & -- & 9.32 & 6.15 & 20 & 300 \\
\hline A83-A90 & 463 & 17 & PLIF Flow Vis & plan & $1 \mathrm{MHz}$ & cylinder & 2 & 4 & 4.98 & 3.35 & 20 & 300 \\
\hline
\end{tabular}

Table A1: Test matrix and figure summary. 
(a)

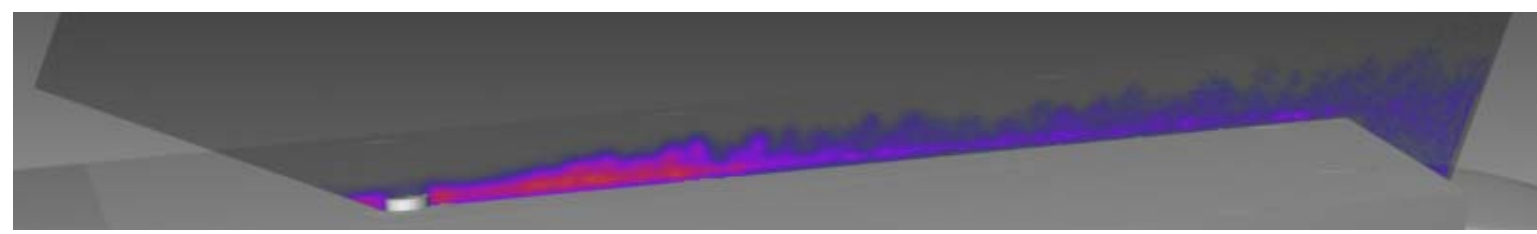

(b)

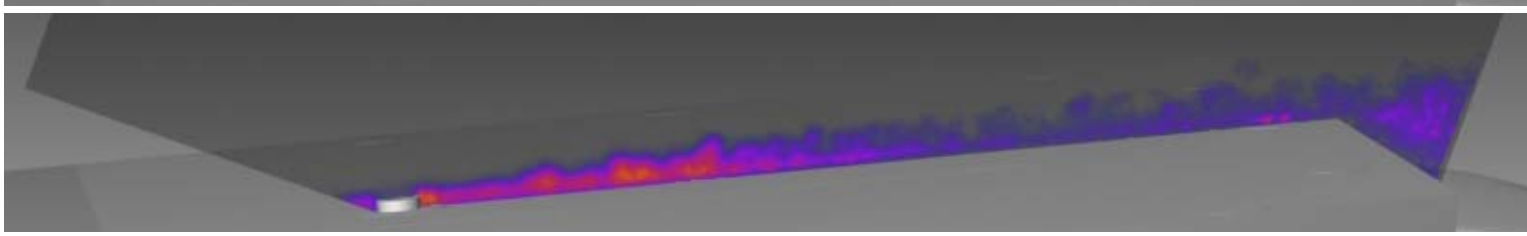

(c)

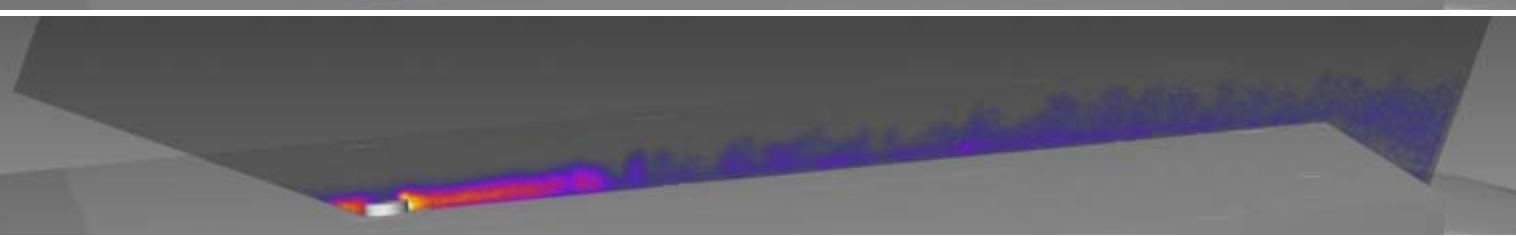

(d)

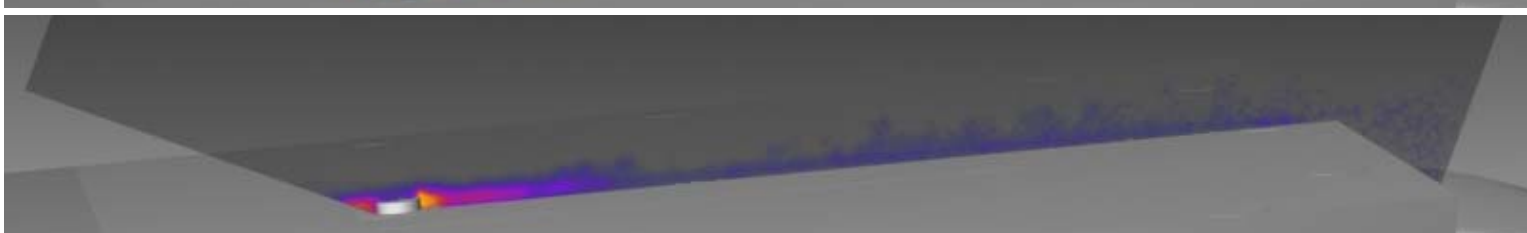

(e)

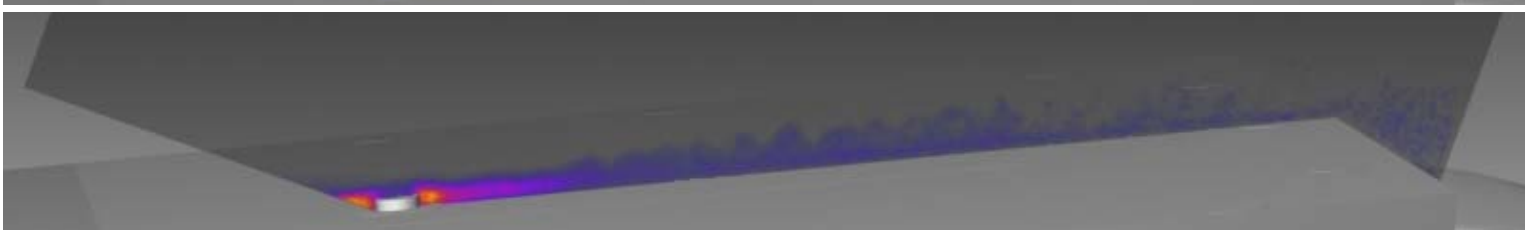

(f)

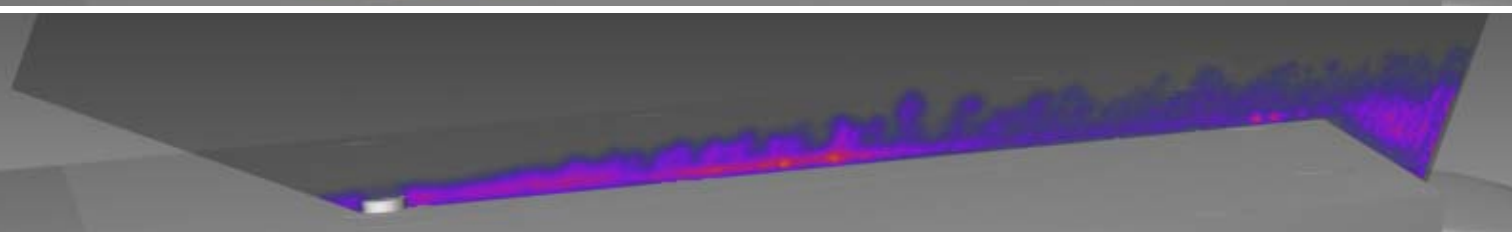

(g)

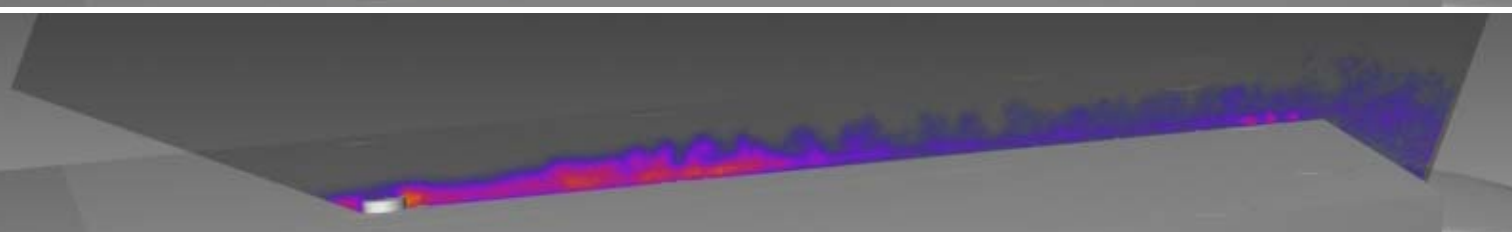

(h)

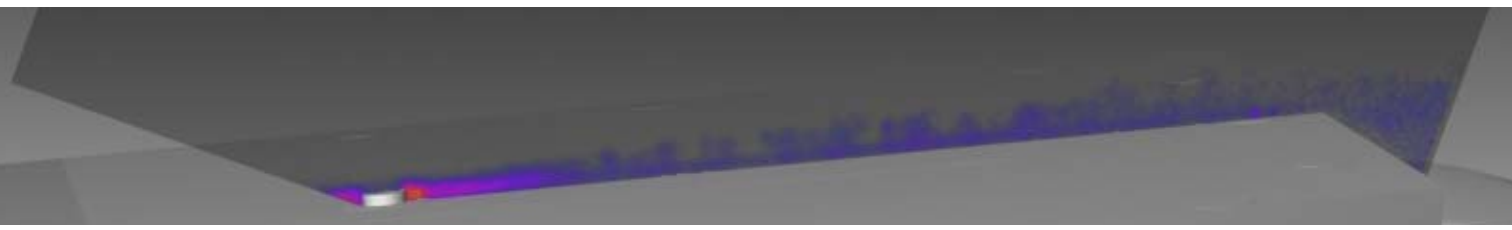

(i)

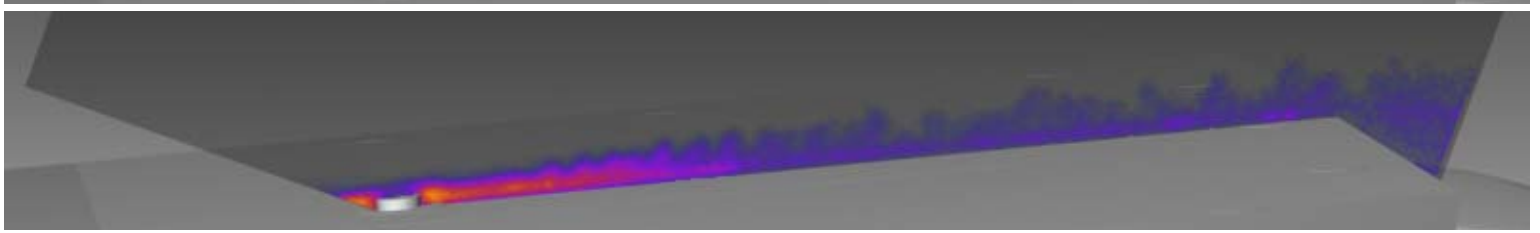

Figure A1: Test 443, Run 12, 1-mm tall by 4-mm wide cylinder, plate angle $=25^{\circ}, 11$-mm wide slot seeding, $\dot{m}=$ $300 \mathrm{sccm}, \mathrm{P}_{0}=9.3 \mathrm{MPa}$, sheet position $=1 \mathrm{~mm}$ from centerline, framing rate $=10 \mathrm{~Hz}$. 
(a)

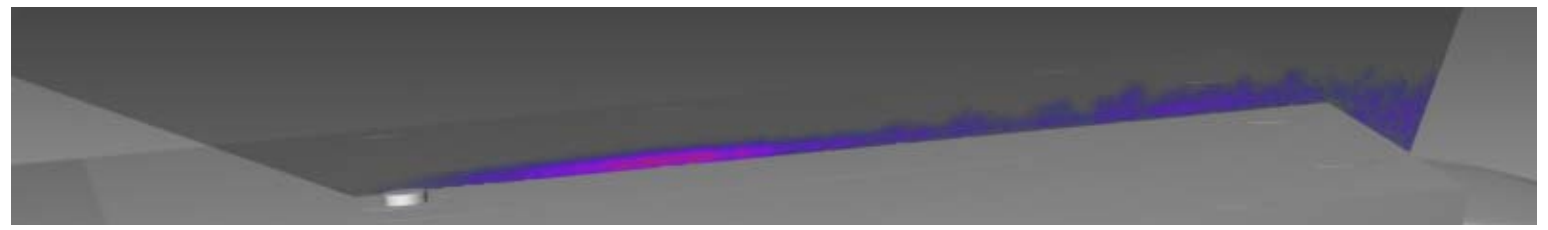

(b)

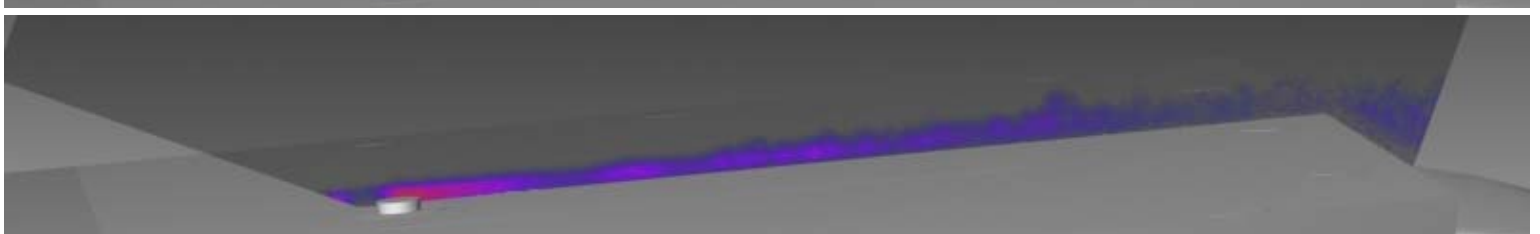

(c)

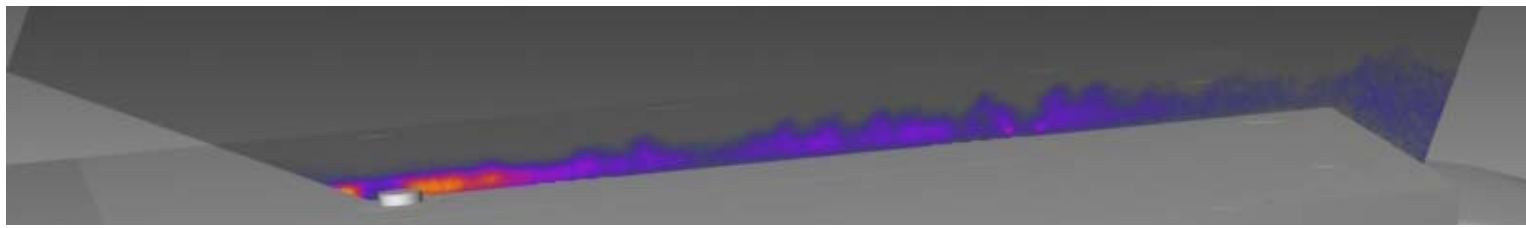

(d)

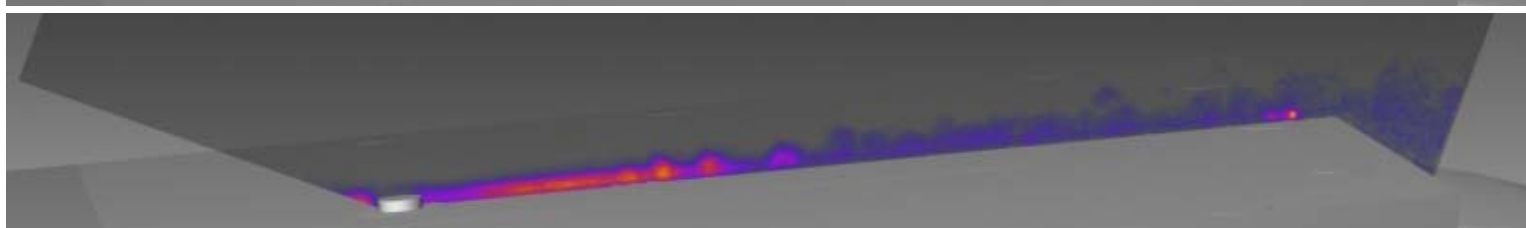

(e)

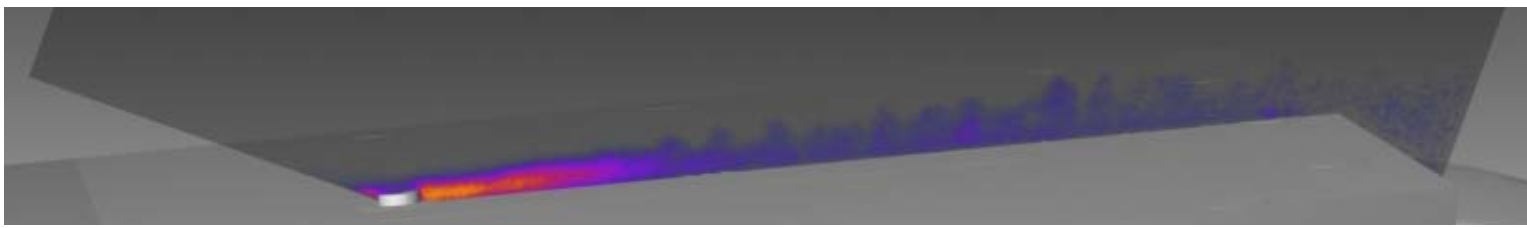

(f)

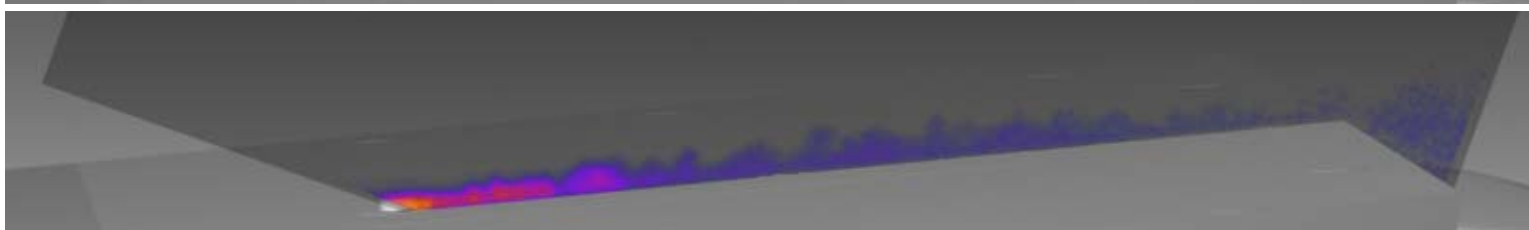

(g)

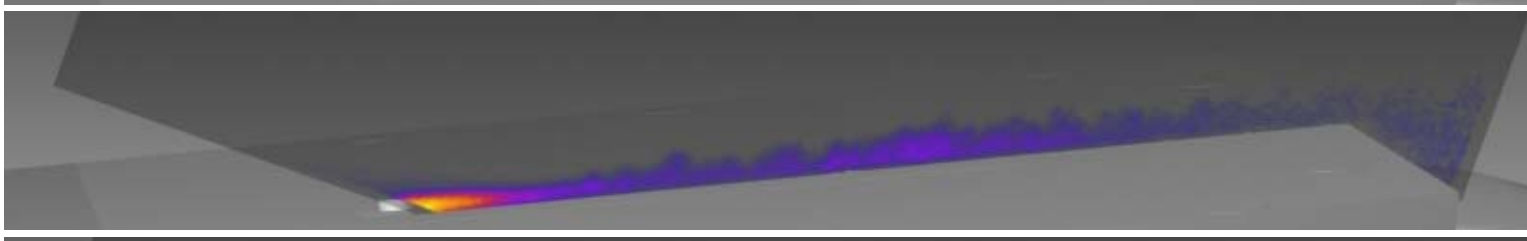

(h)

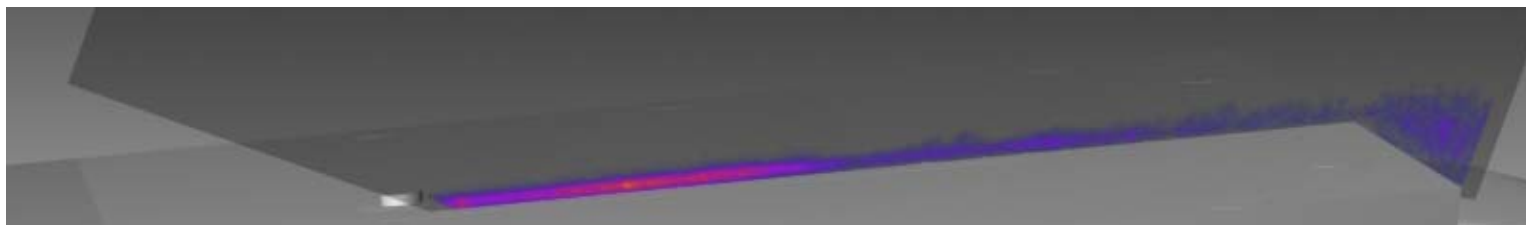

(i)

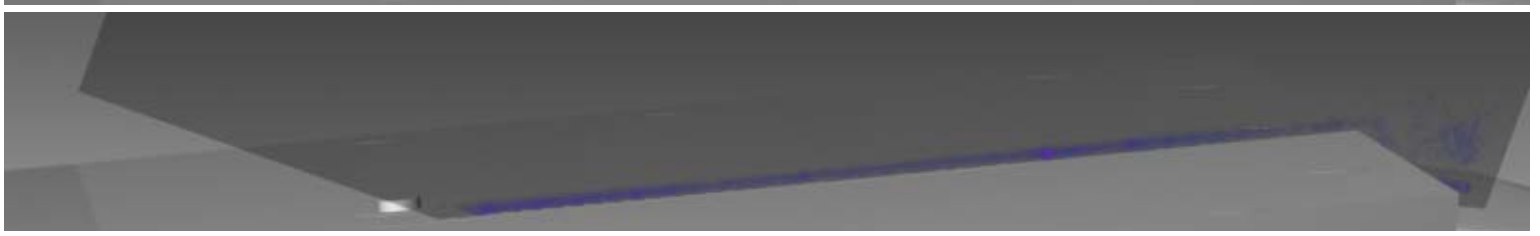

Figure A2: Test 443, Run 12, 1-mm tall by 4-mm wide cylinder, plate angle $=25^{\circ}, 11$-mm wide slot seeding, $\dot{m}=$ $300 \mathrm{sccm}, \mathrm{P}_{0}=9.3 \mathrm{MPa}$, sheet position varies from 10 to $-10 \mathrm{~mm}$ from centerline in $2.5 \mathrm{~mm}$ increments in (a)-(i), framing rate $=10 \mathrm{~Hz}$. 
(a)

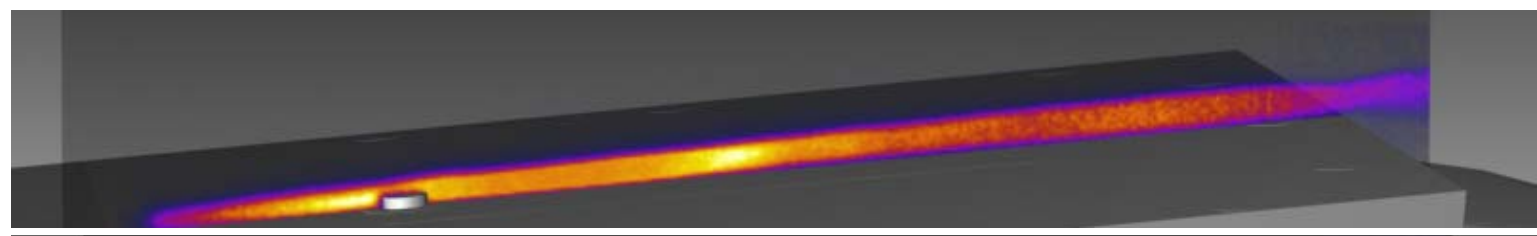

(b)

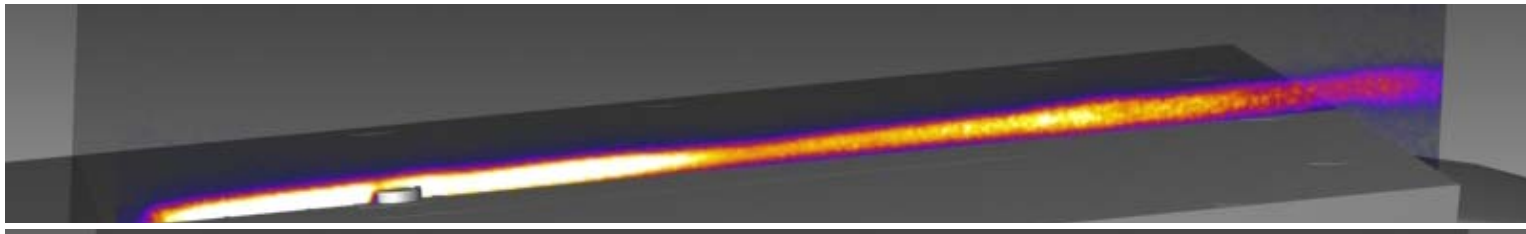

(c)

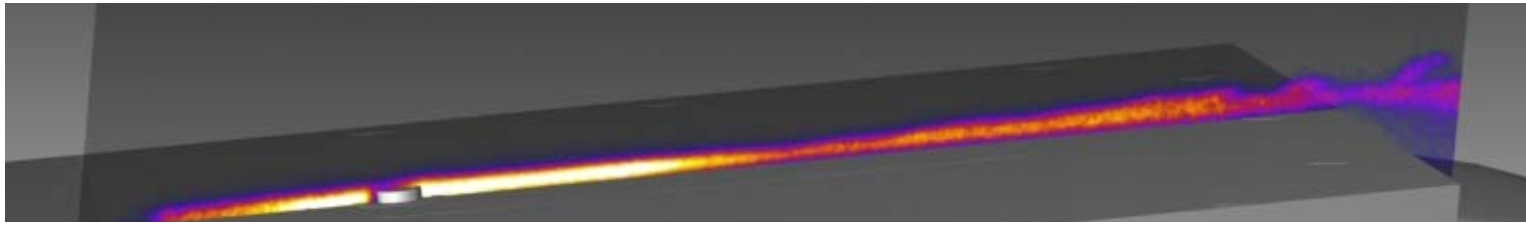

(d)

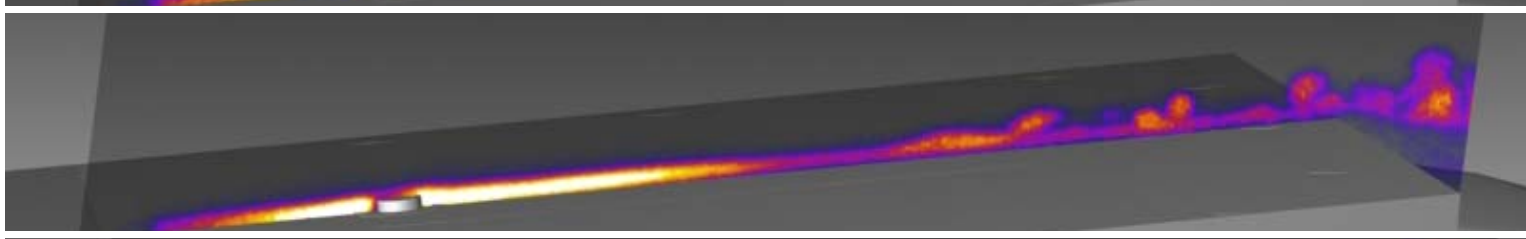

(e)

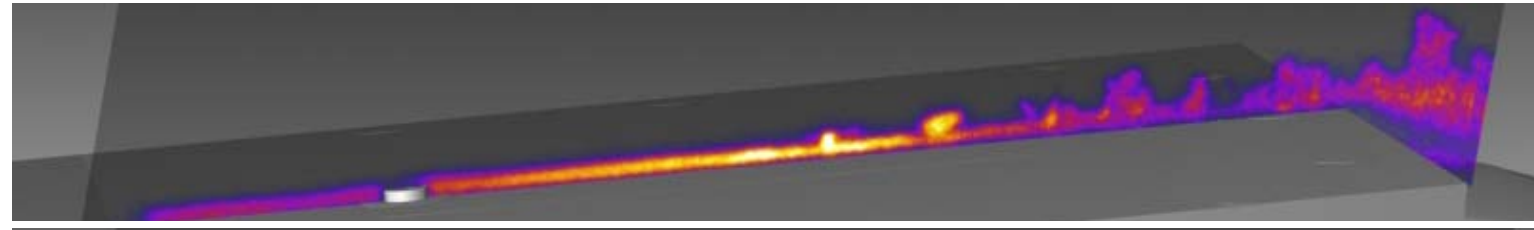

(f)

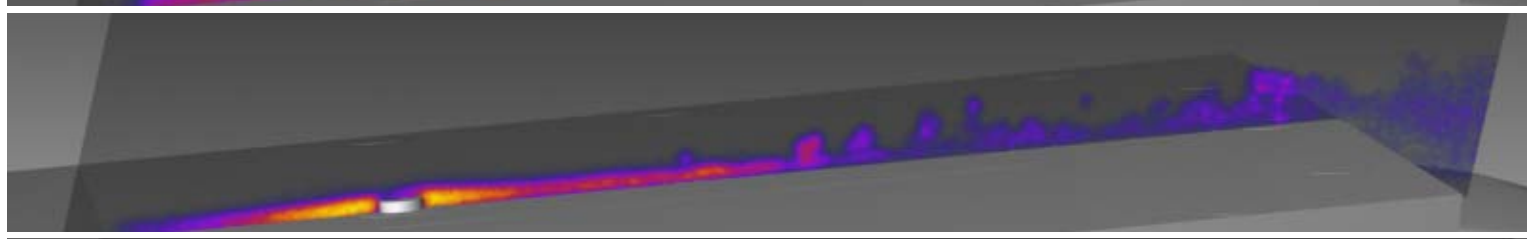

(g)

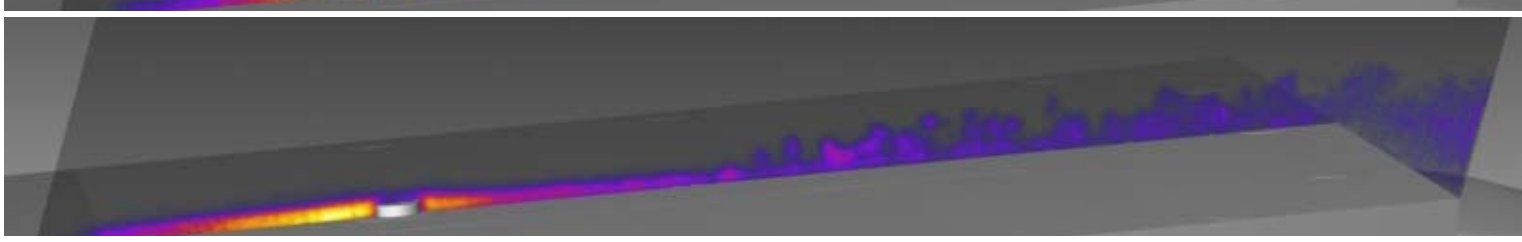

(h)

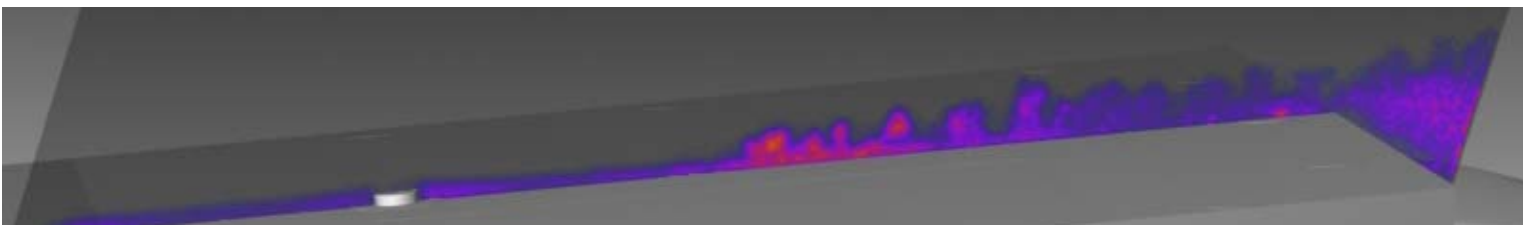

(i)

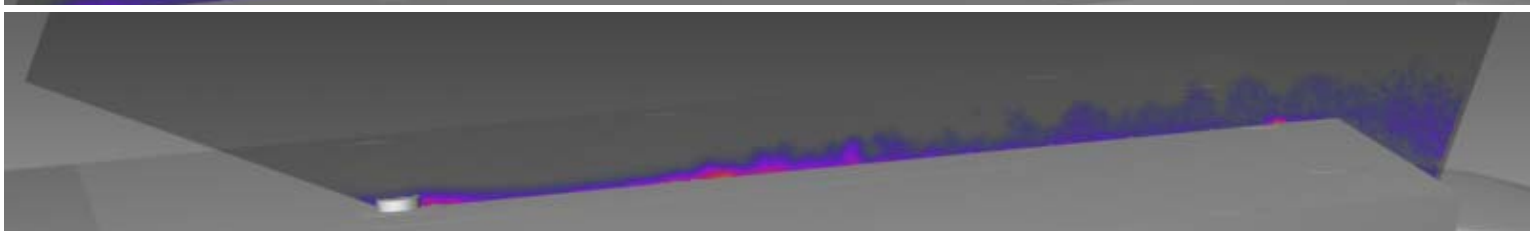

Figure A3: Test 443, Run 12, 1-mm tall by 4-mm wide cylinder, plate angle varies from 5 to $25^{\circ}$ in $2.5^{\circ}$ increments in (a)-(i), 11 -mm wide slot seeding, $\dot{m}=300 \mathrm{sccm}, \mathrm{P}_{0}=9.3 \mathrm{MPa}$, sheet position $=1 \mathrm{~mm}$ from centerline, framing rate $=10 \mathrm{~Hz}$. 
(a)

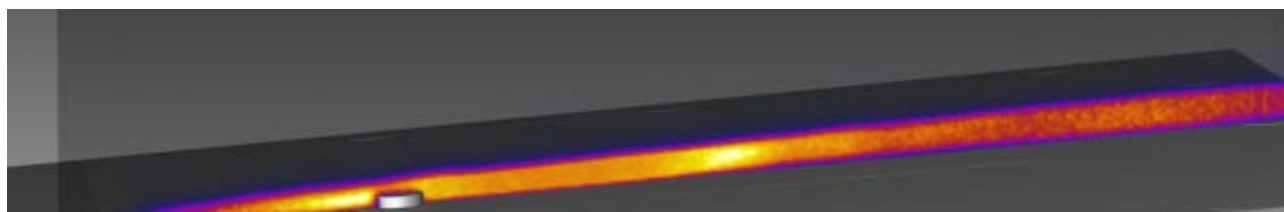

(b)

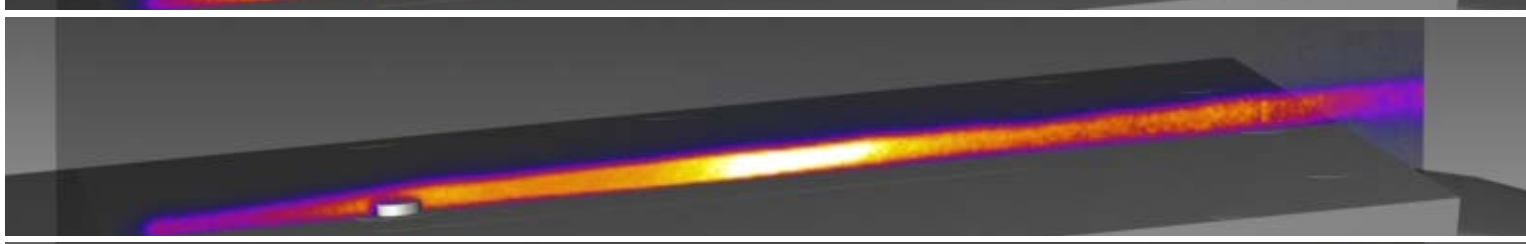

(c)

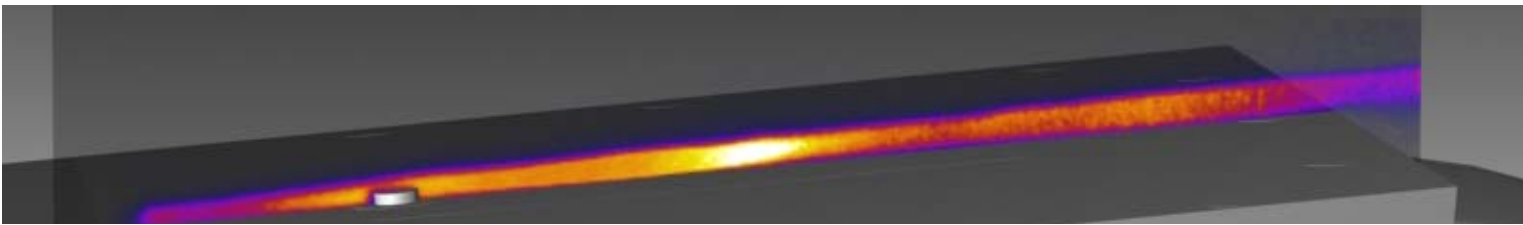

(d)

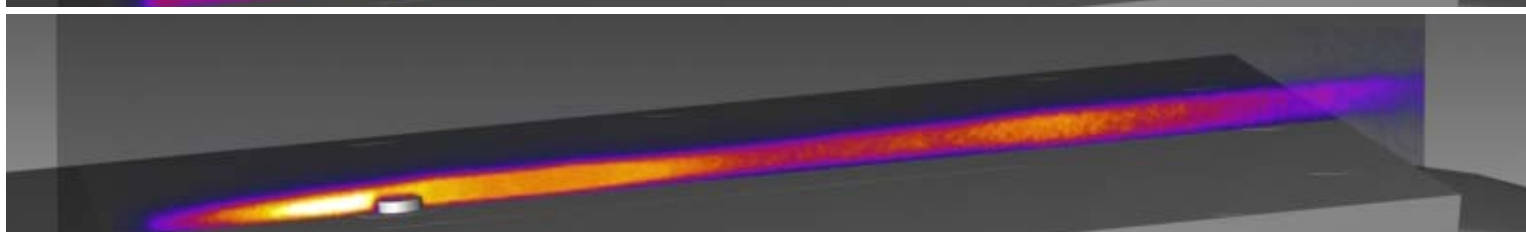

(e)

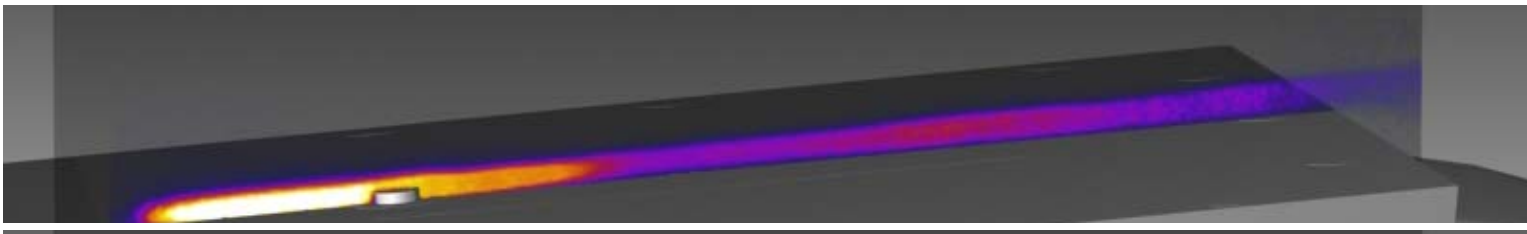

(f)

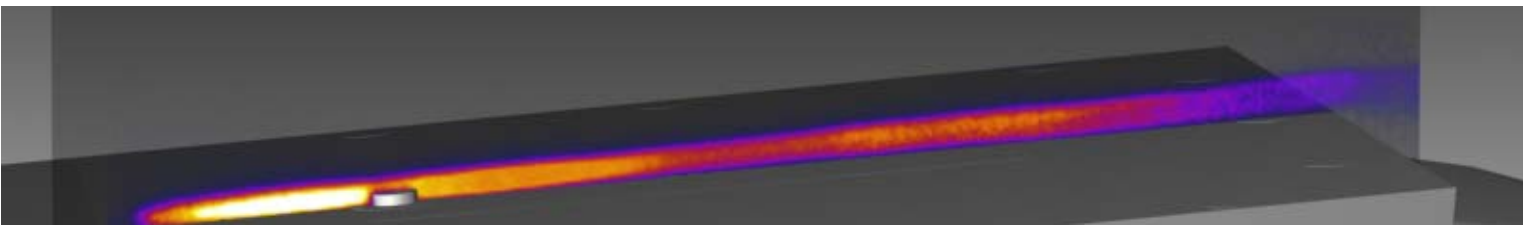

(g)

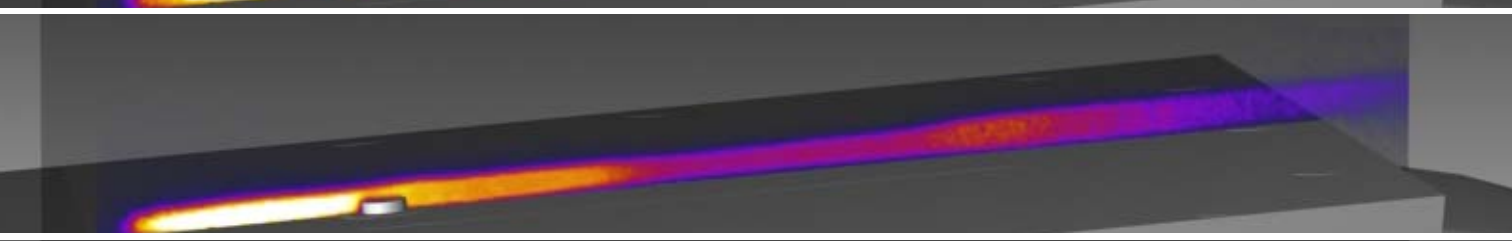

(h)

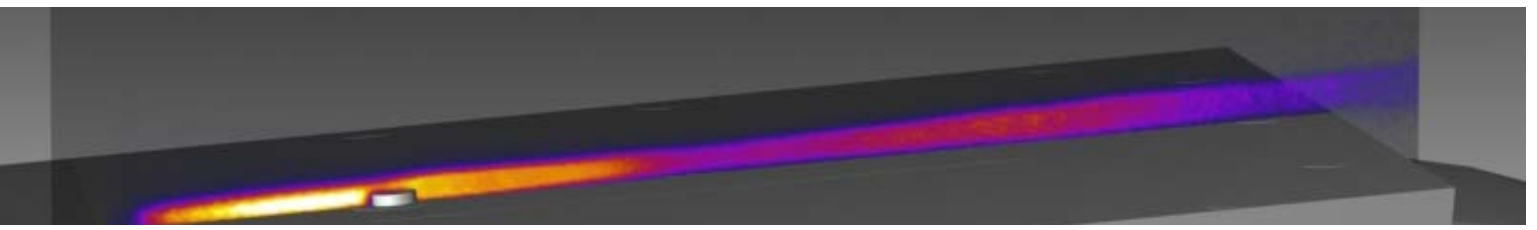

(i)

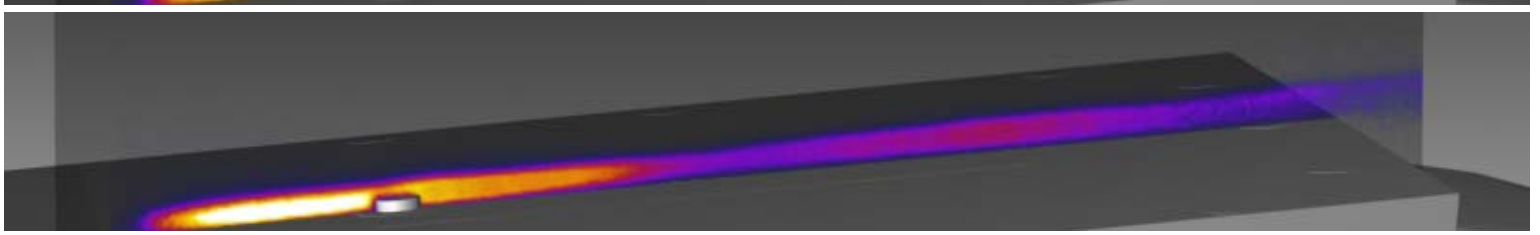

Figure A4: Test 443, Run 12, 1-mm tall by 4-mm wide cylinder, plate angle $=5^{\circ}, 11$-mm wide slot seeding, $\dot{m}=300$ sccm, $\mathrm{P}_{0}=9.3 \mathrm{MPa}$, sheet position $=1 \mathrm{~mm}$ from centerline, framing rate $=10 \mathrm{~Hz}$. 
(a)

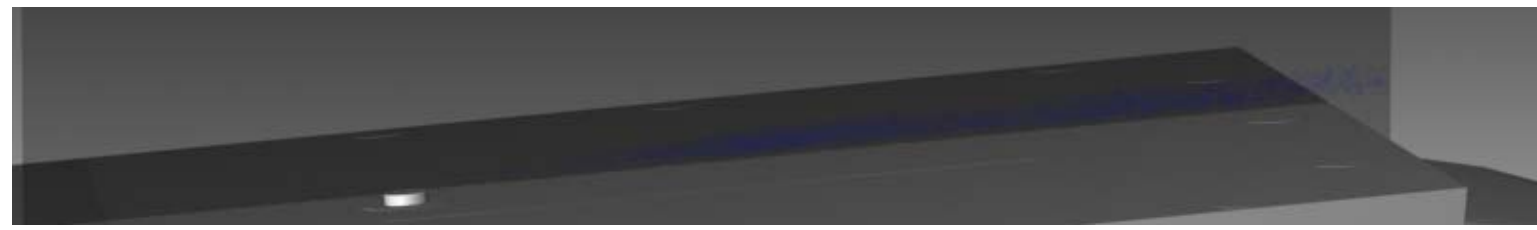

(b)

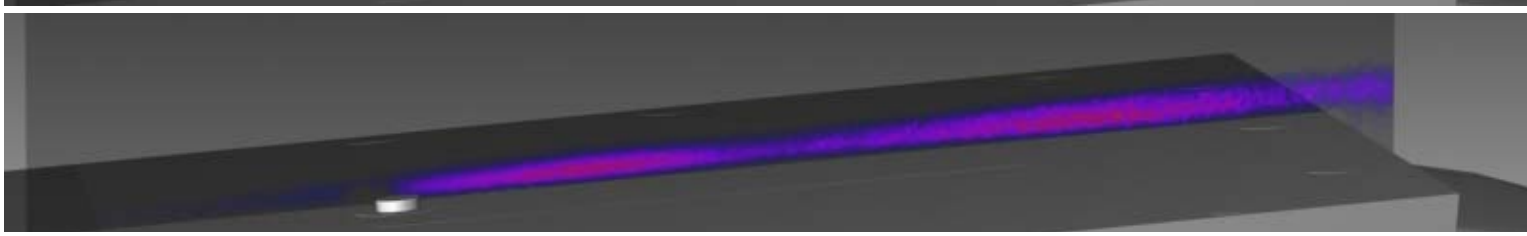

(c)

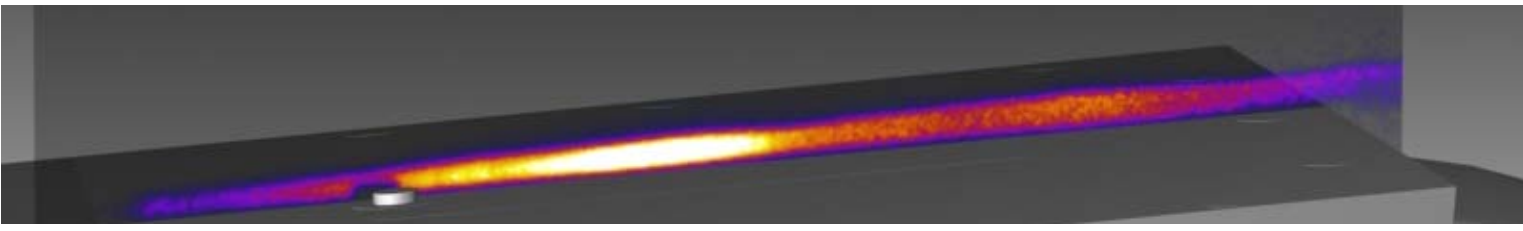

(d)

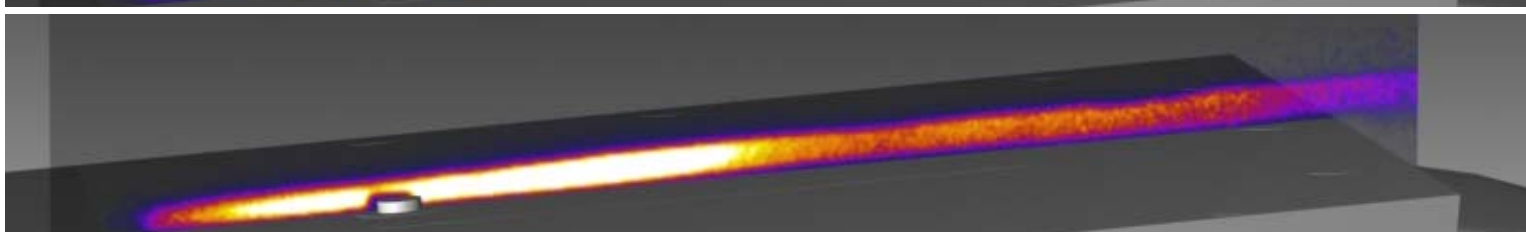

(e)

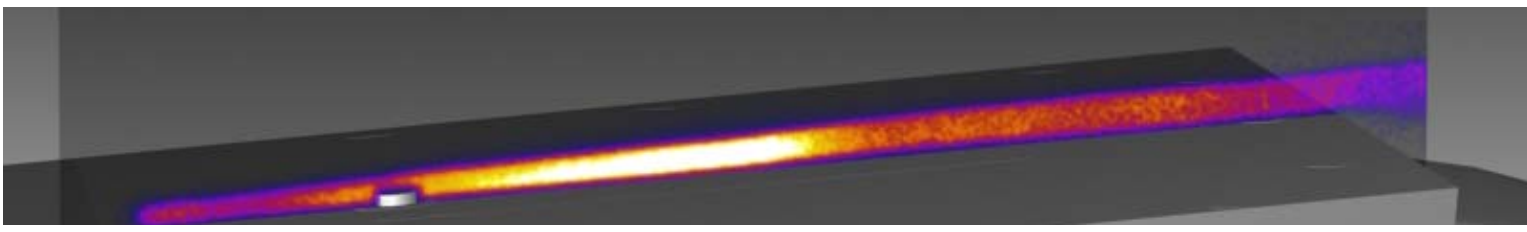

(f)

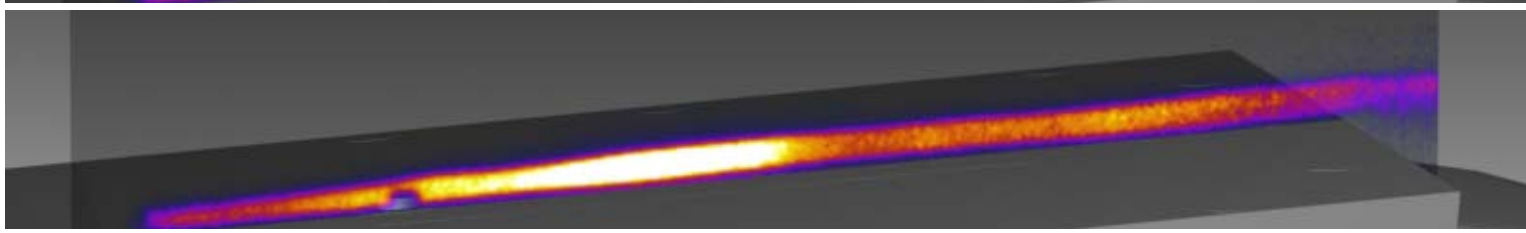

(g)

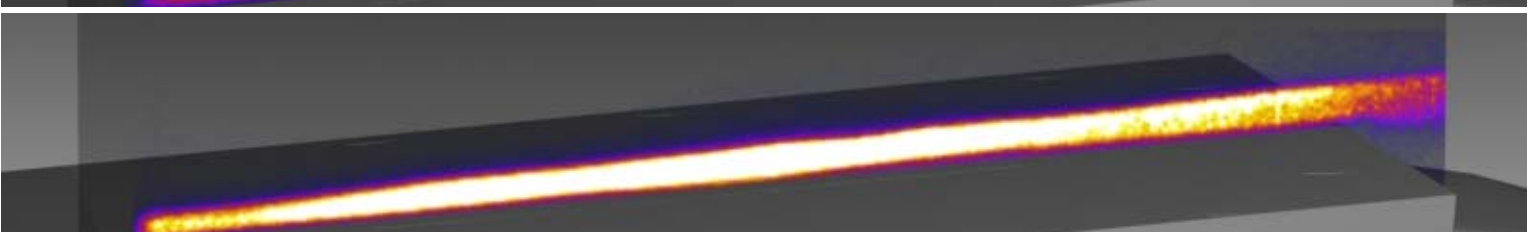

(h)

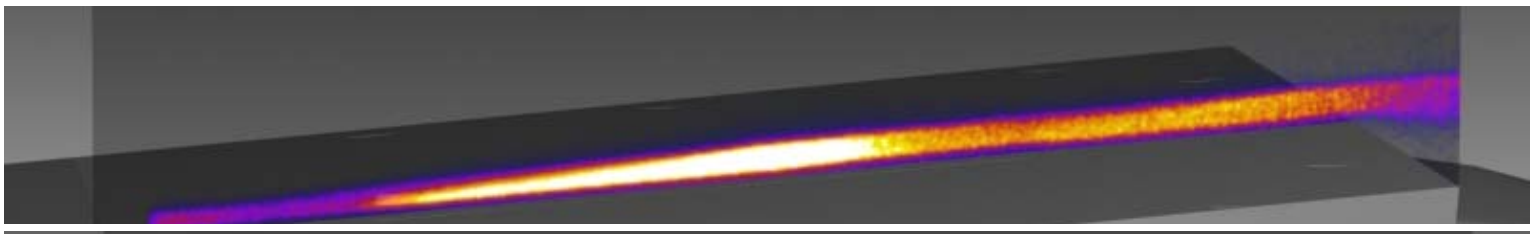

(i)

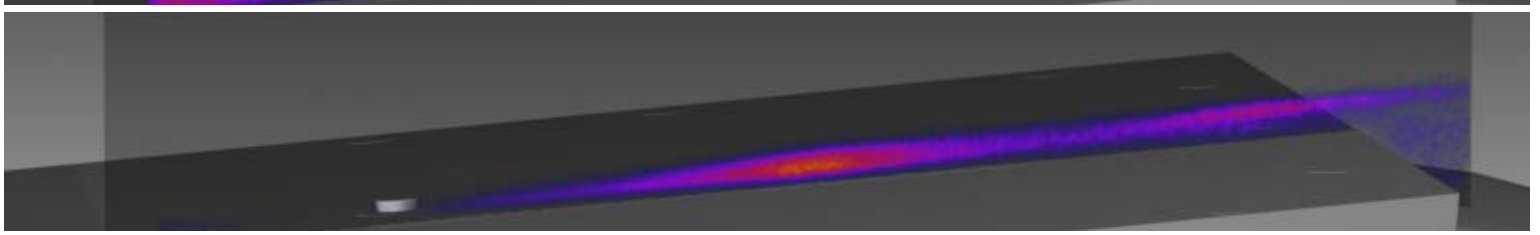

Figure A5: Test 443, Run 12, 1-mm tall by 4-mm wide cylinder, plate angle $=5^{\circ}, 11$-mm wide slot seeding, $\dot{m}=300$ sccm, $\mathrm{P}_{0}=9.3 \mathrm{MPa}$, sheet position varies from 10 to $-10 \mathrm{~mm}$ from centerline in $2.5 \mathrm{~mm}$ increments in (a)-(i), framing rate $=10 \mathrm{~Hz}$. 
(a)

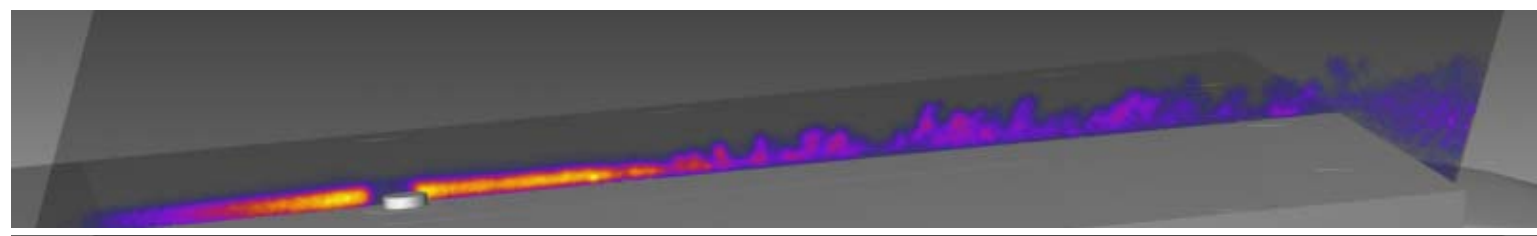

(b)

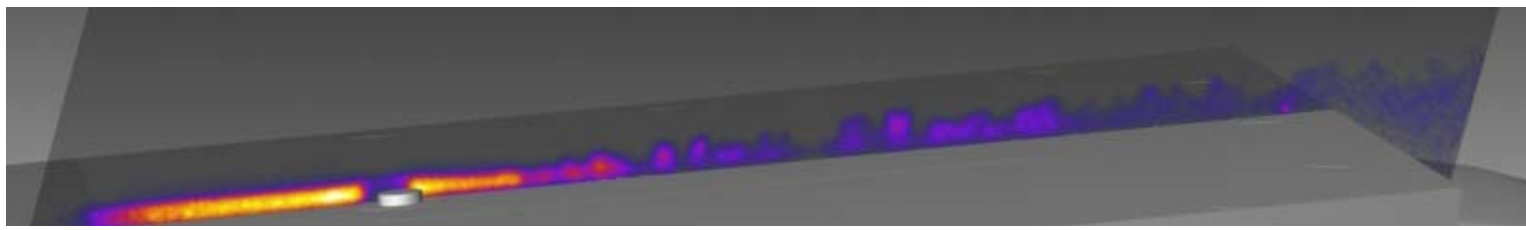

(c)

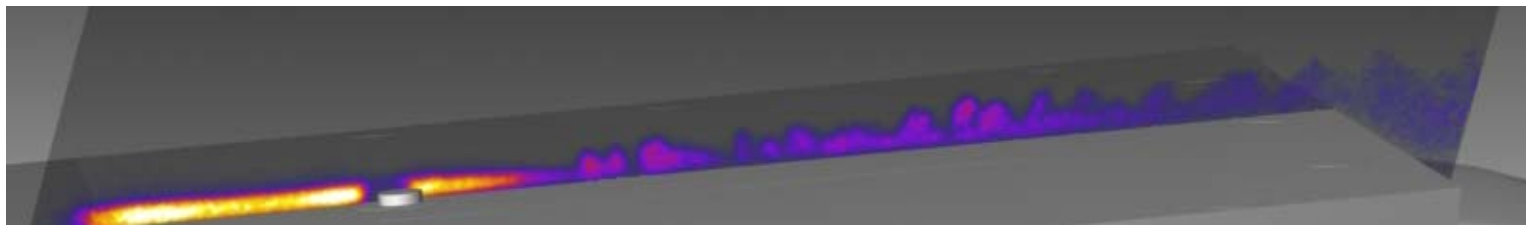

(d)

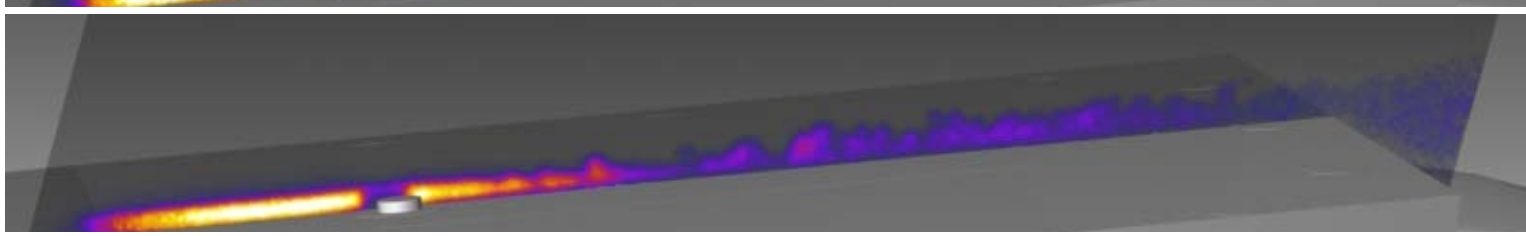

(e)

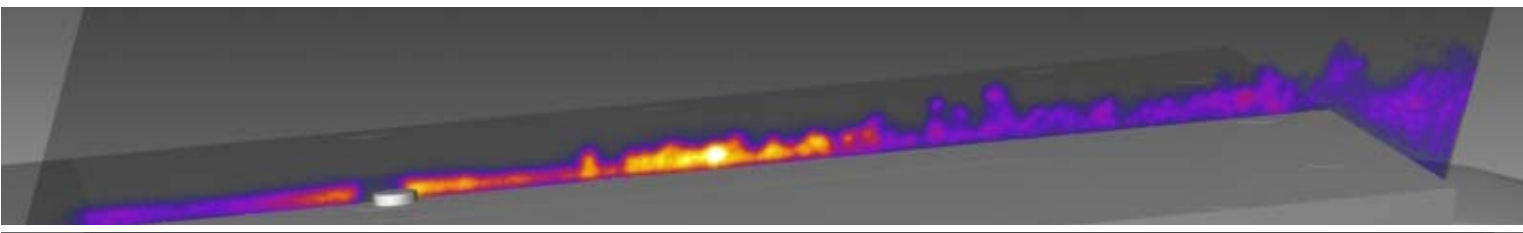

(f)
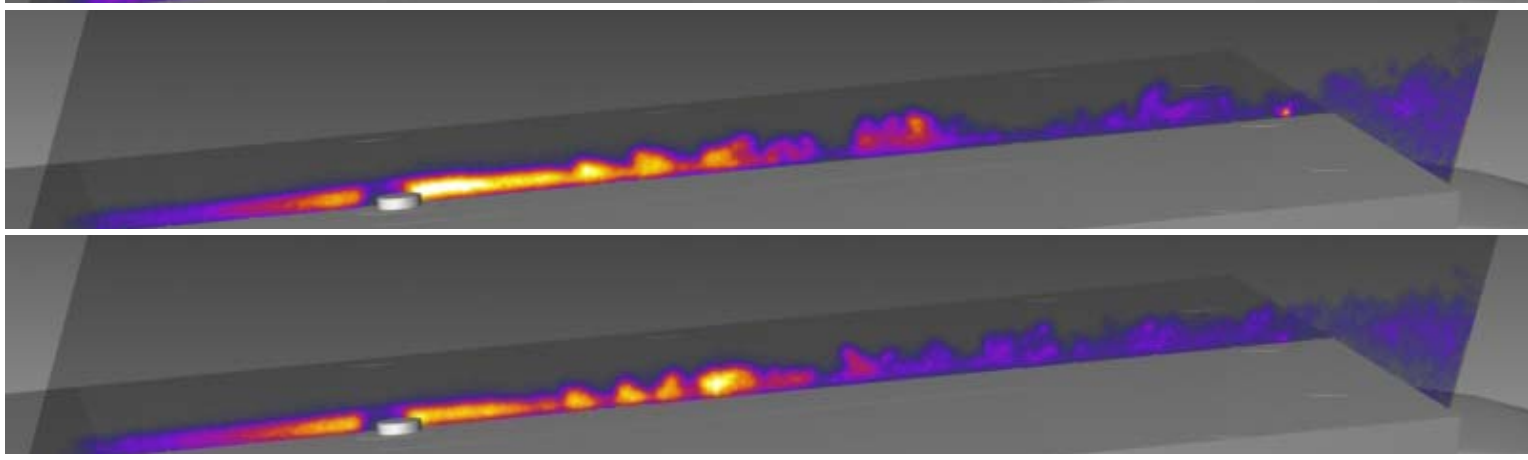

(h)

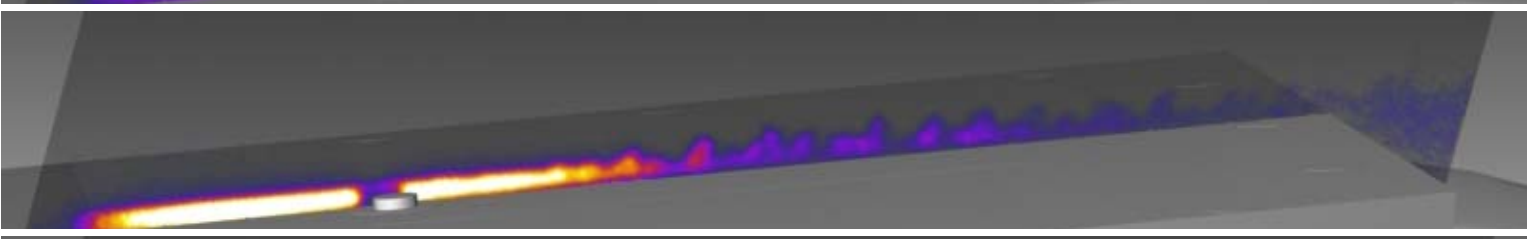

(i)

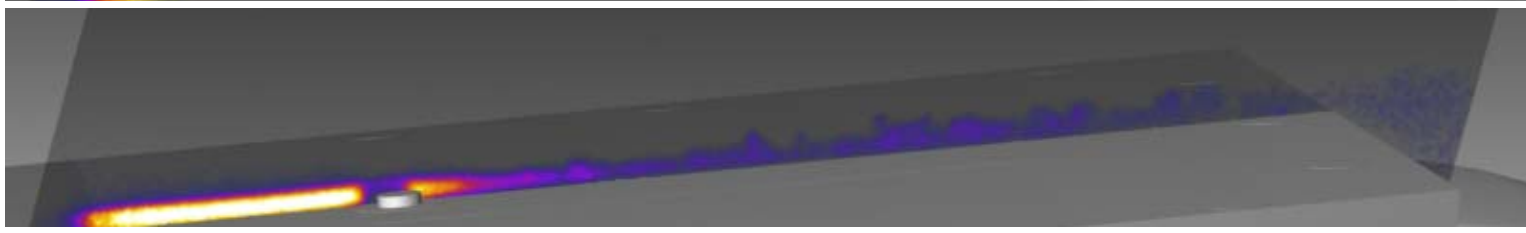

Figure A6: Test 443, Run 12, 1-mm tall by 4-mm wide cylinder, plate angle $=20^{\circ}$, 11-mm wide slot seeding, $\dot{m}=$ $300 \mathrm{sccm}, \mathrm{P}_{0}=9.3 \mathrm{MPa}$, sheet position $=3 \mathrm{~mm}$ from centerline, framing rate $=10 \mathrm{~Hz}$. 
(a)

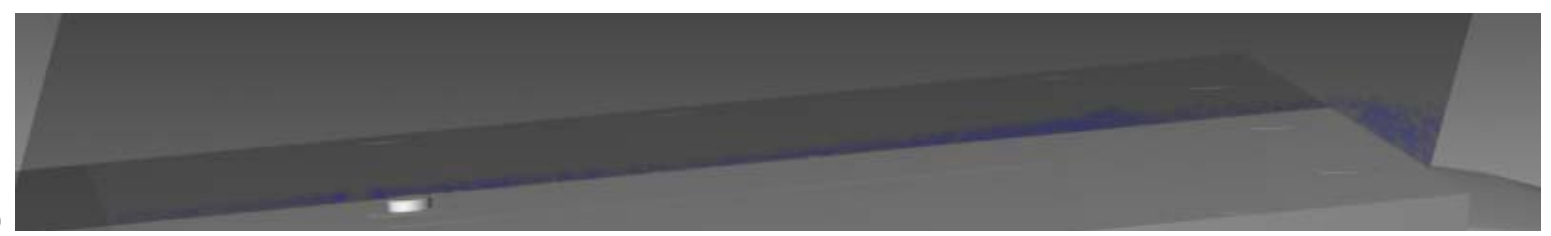

(b)

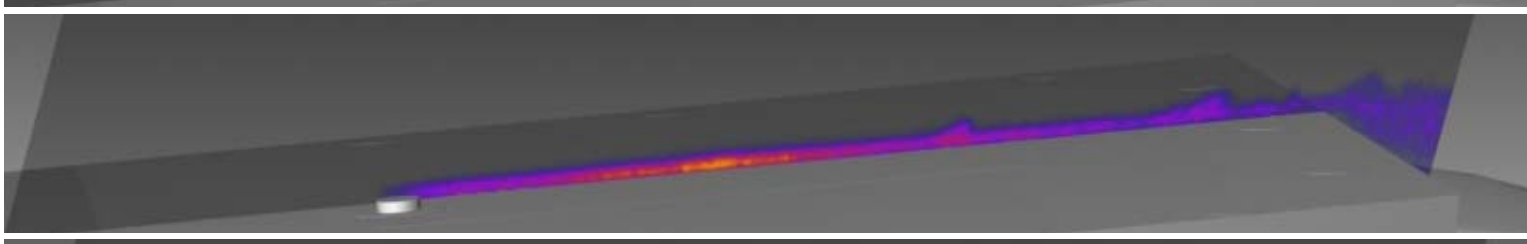

(c)

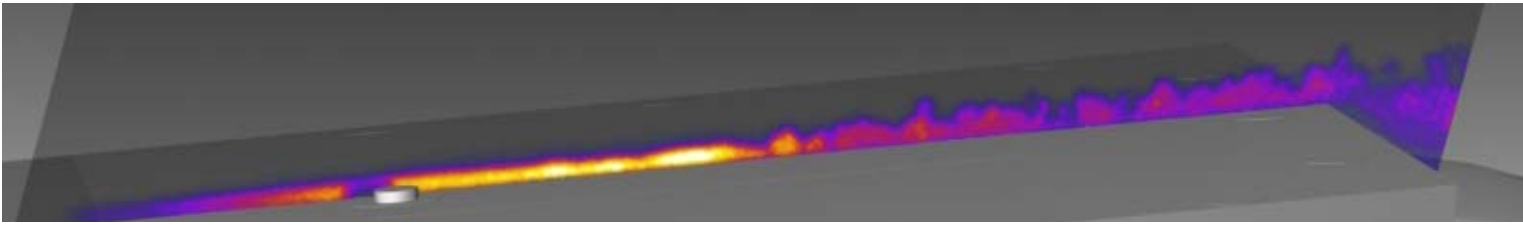

(d)

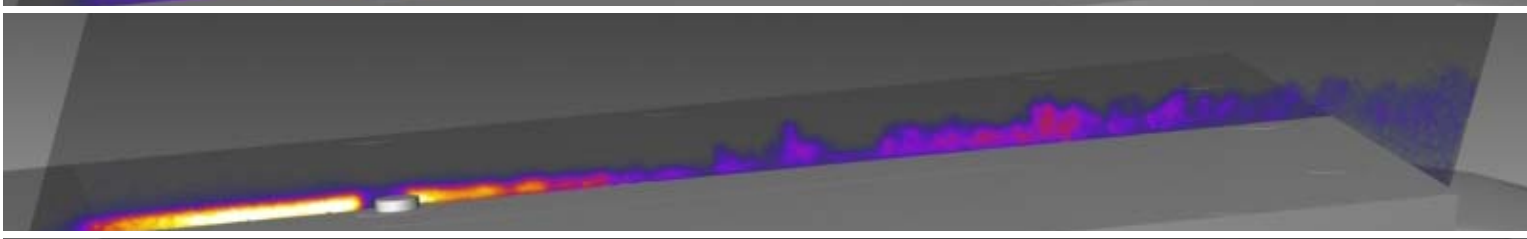

(e)

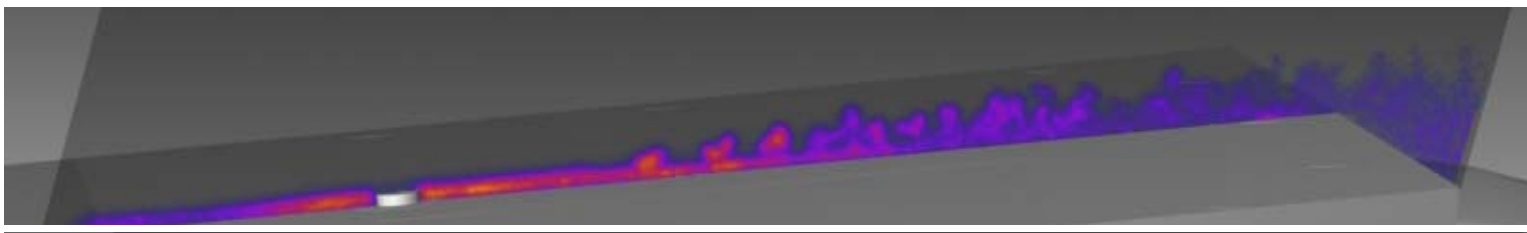

(f)

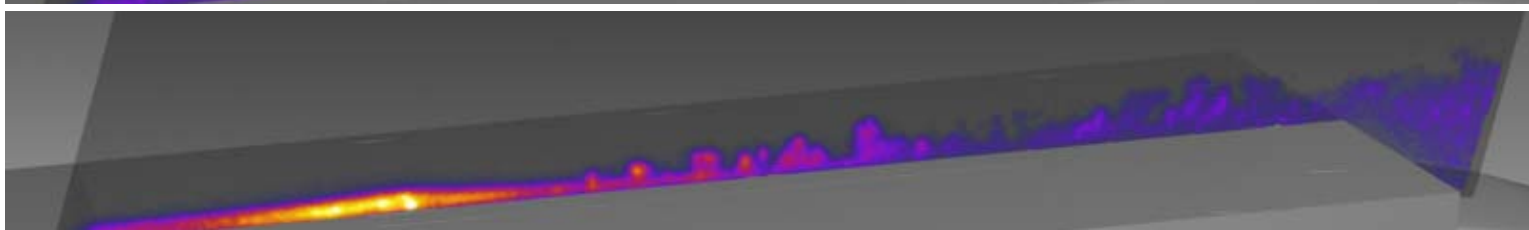

(g)

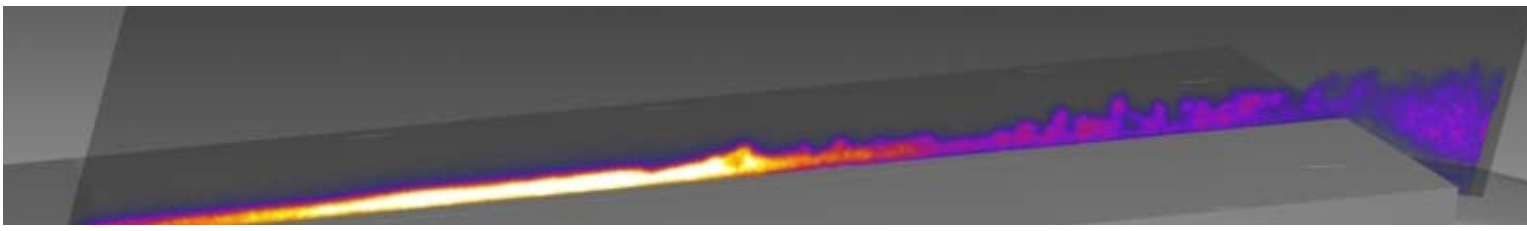

(h)

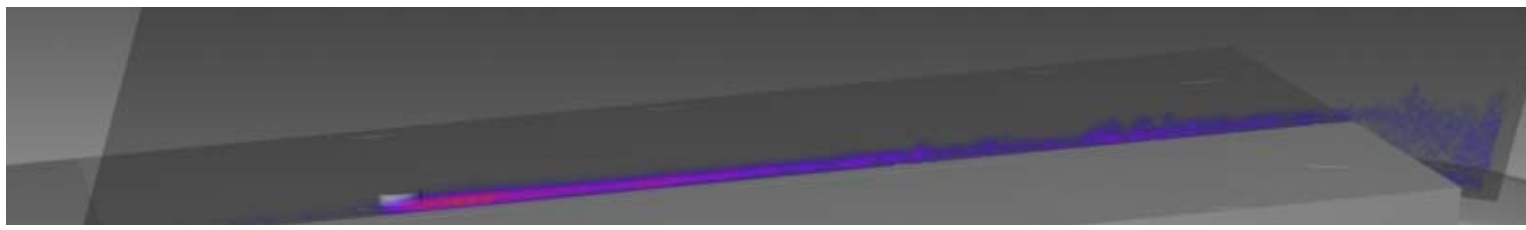

(i)

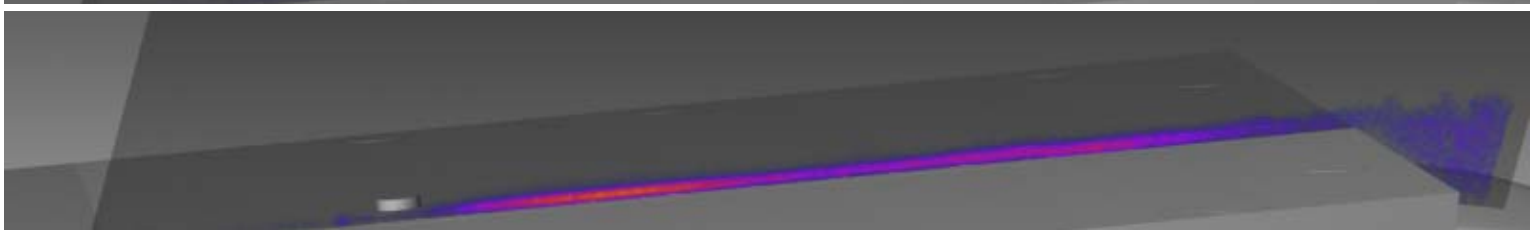

Figure A7: Test 443, Run 12, 1-mm tall by 4-mm wide cylinder, plate angle $=20^{\circ}, 11$-mm wide slot seeding, $\dot{m}=$ $300 \mathrm{sccm}, \mathrm{P}_{0}=9.3 \mathrm{MPa}$, sheet position varies from 10 to $-10 \mathrm{~mm}$ from centerline in $2.5 \mathrm{~mm}$ increments in (a)-(i), framing rate $=10 \mathrm{~Hz}$. 
(a)

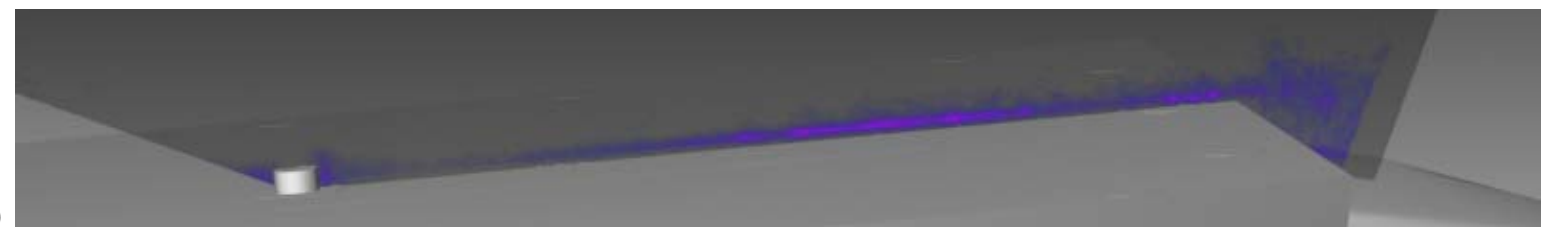

(b)

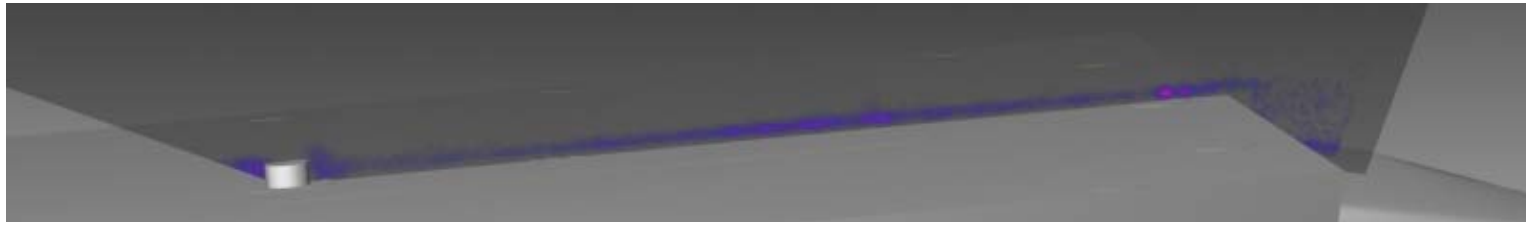

(c)

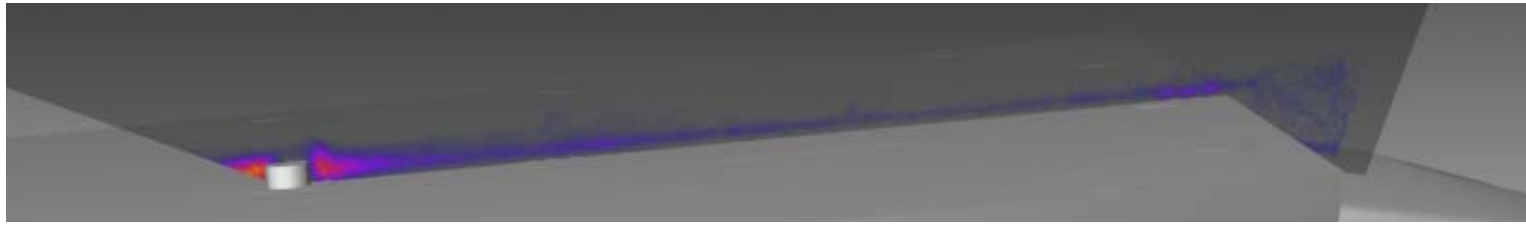

(d)

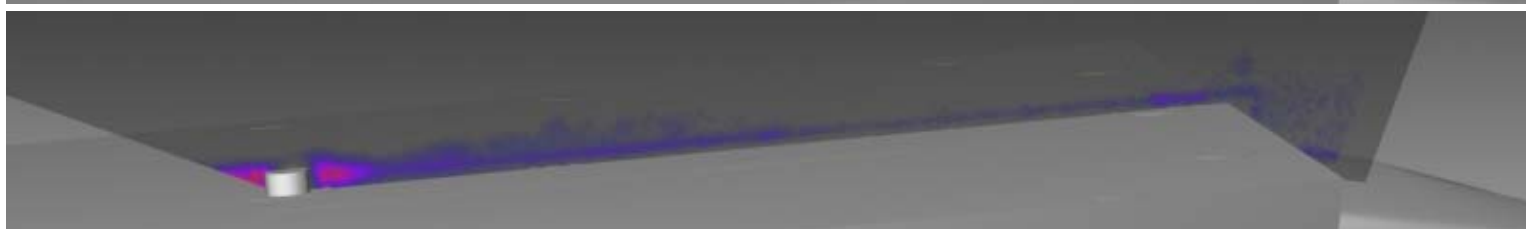

(e)

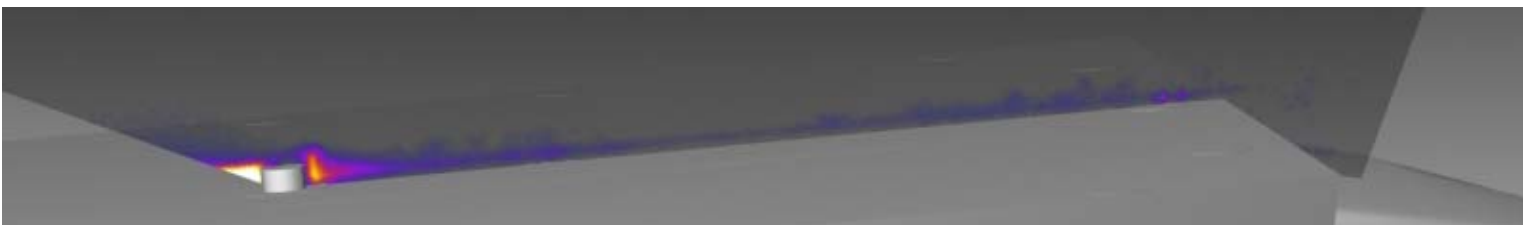

(f)

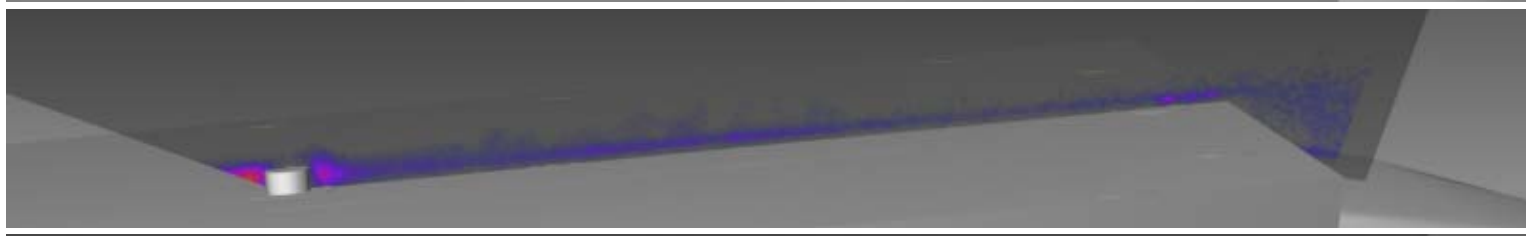

(g)

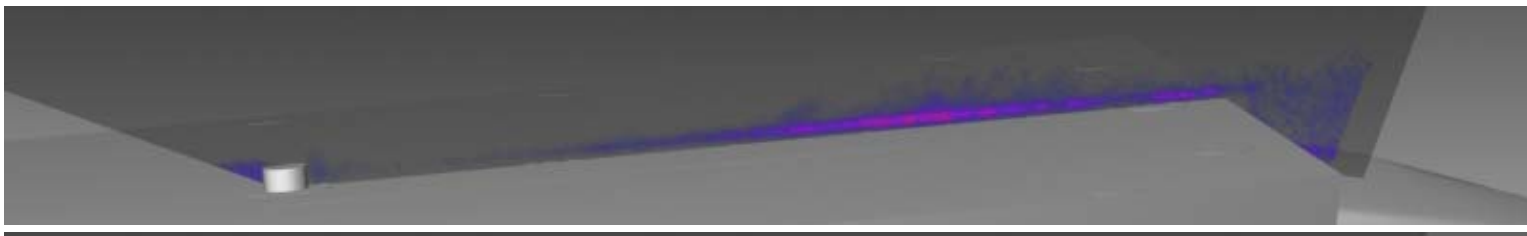

(h)

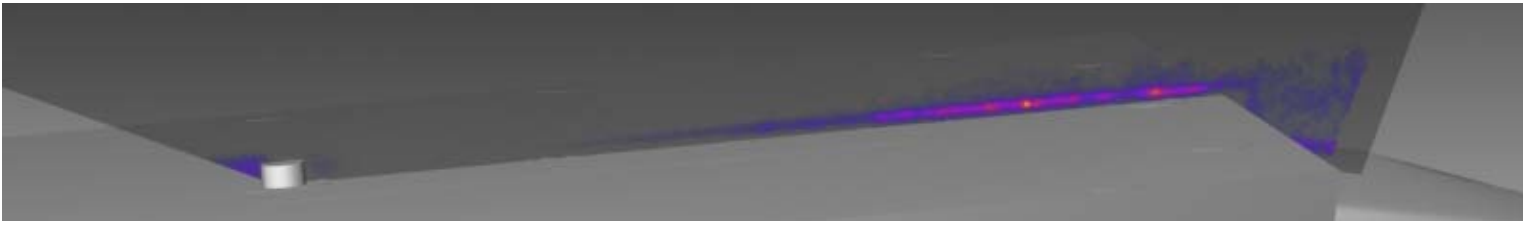

(i)

Figure A8: Test 443, Run 15, 2-mm tall by 4-mm wide cylinder, plate angle $=25^{\circ}, 11$-mm wide slot seeding, $\dot{m}=$ $300 \mathrm{sccm}, \mathrm{P}_{0}=9.3 \mathrm{MPa}$, sheet position $=2 \mathrm{~mm}$ from centerline, framing rate $=10 \mathrm{~Hz}$. 
(a)

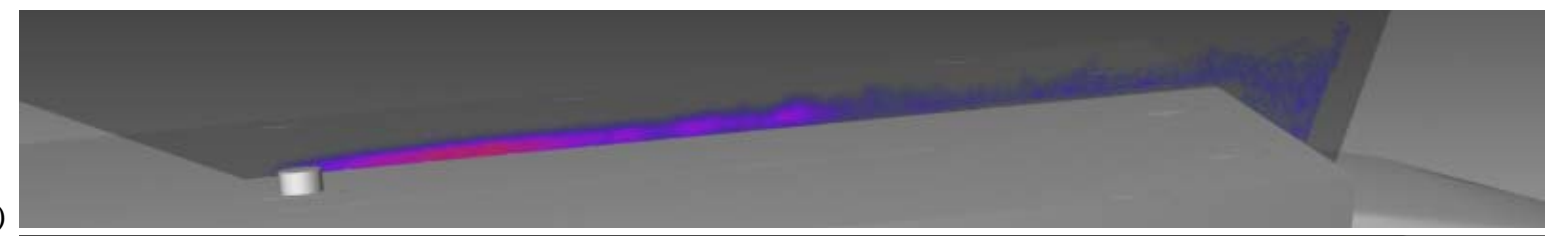

(b)

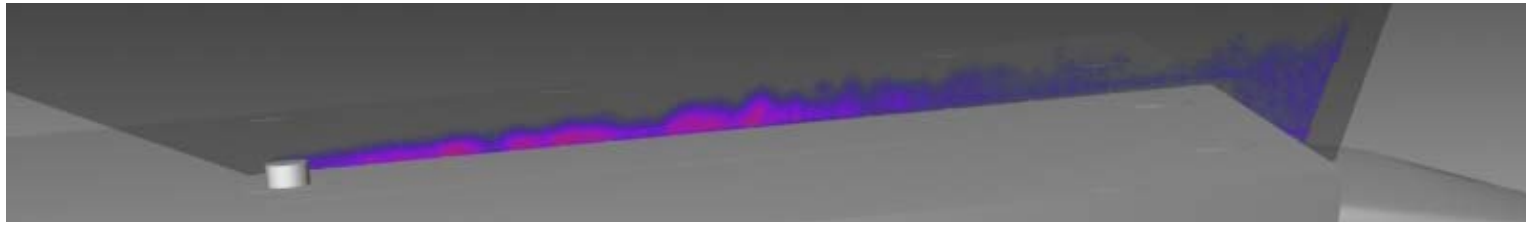

(c)

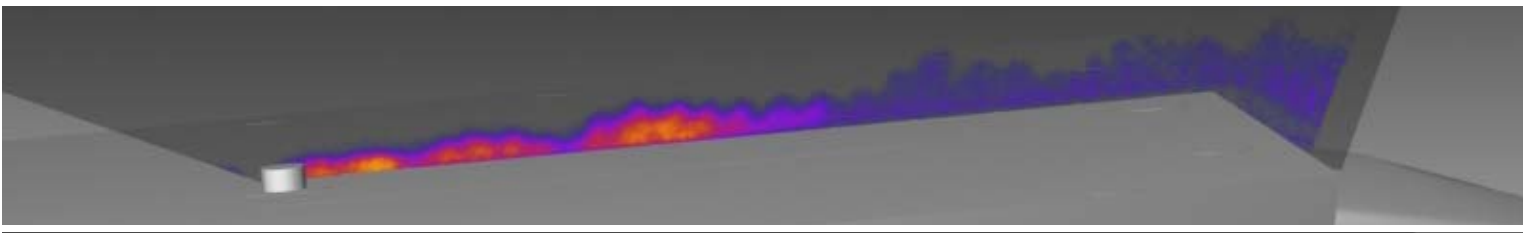

(d)

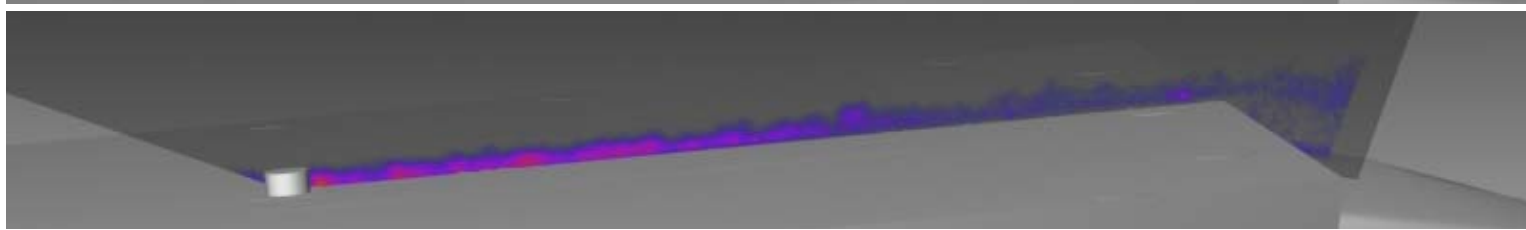

(e)

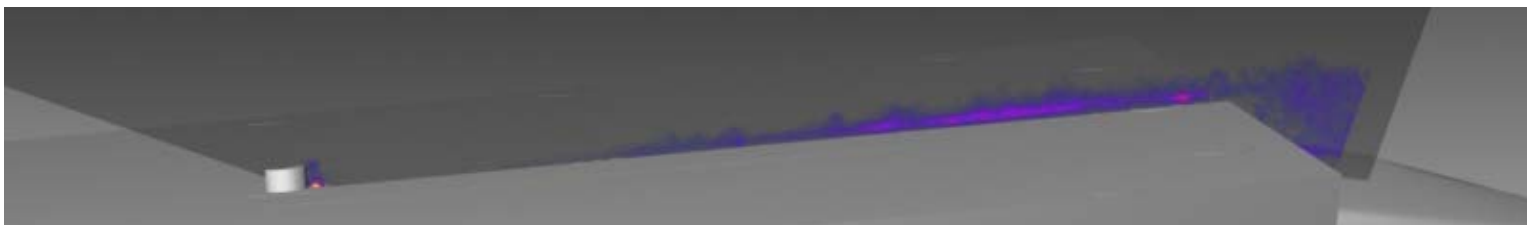

(f)

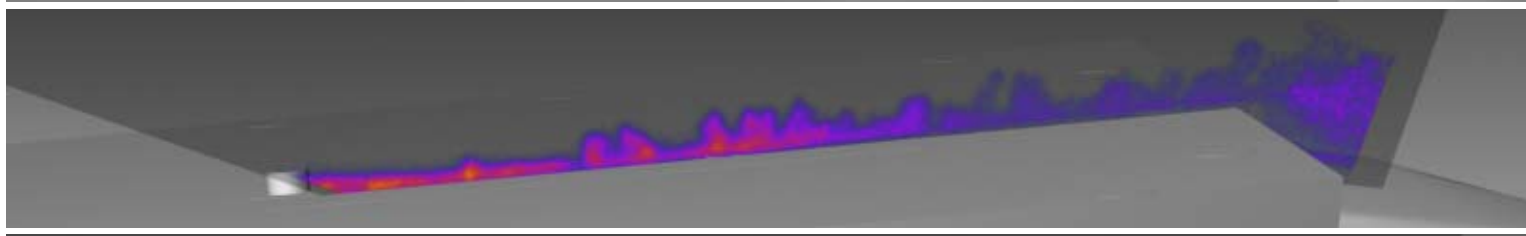

(g)

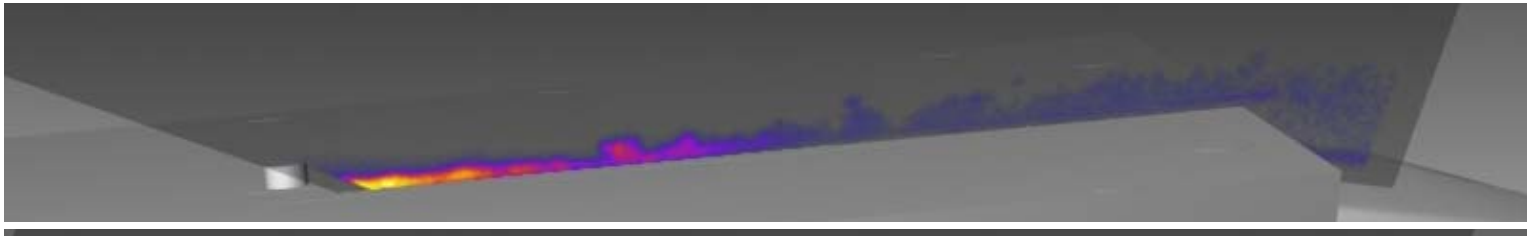

(h)

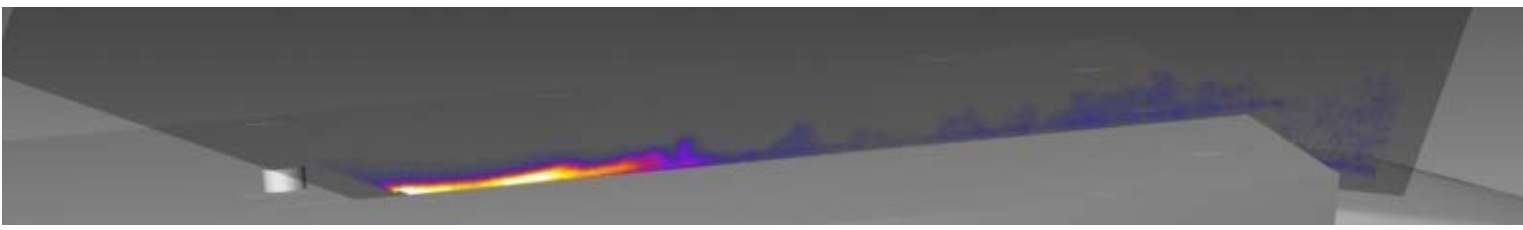

(i)

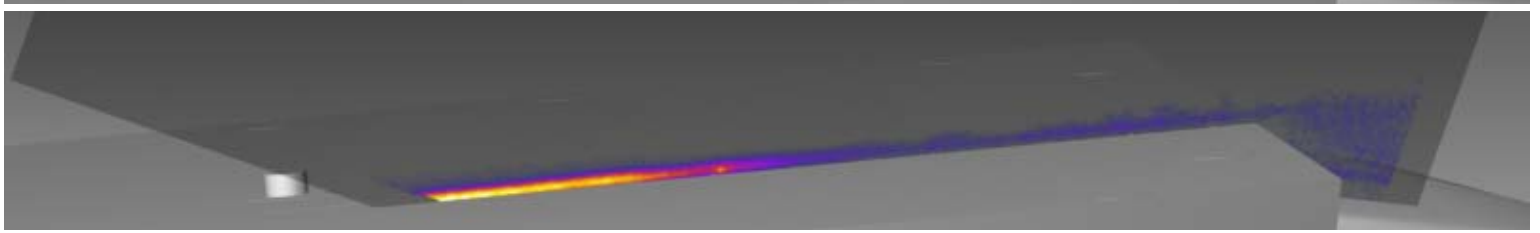

Figure A9: Test 443, Run 15, 2-mm tall by 4-mm wide cylinder, plate angle $=25^{\circ}, 11$-mm wide slot seeding, $\dot{m}=$ $300 \mathrm{sccm}, \mathrm{P}_{0}=9.3 \mathrm{MPa}$, sheet position varies from 16 to $-16 \mathrm{~mm}$ from centerline in $4 \mathrm{~mm}$ increments in (a)-(i), framing rate $=10 \mathrm{~Hz}$. 
(a)

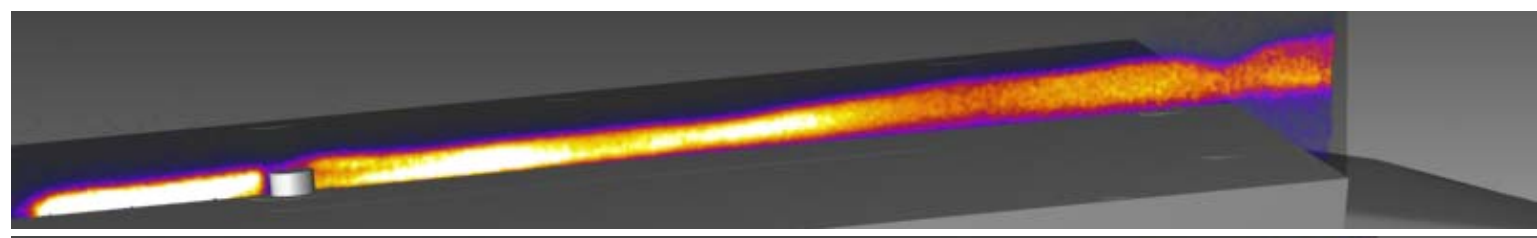

(b)

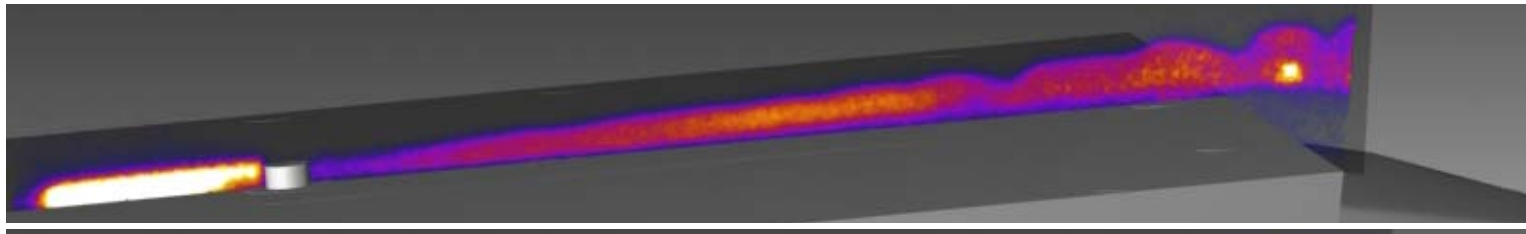

(c)

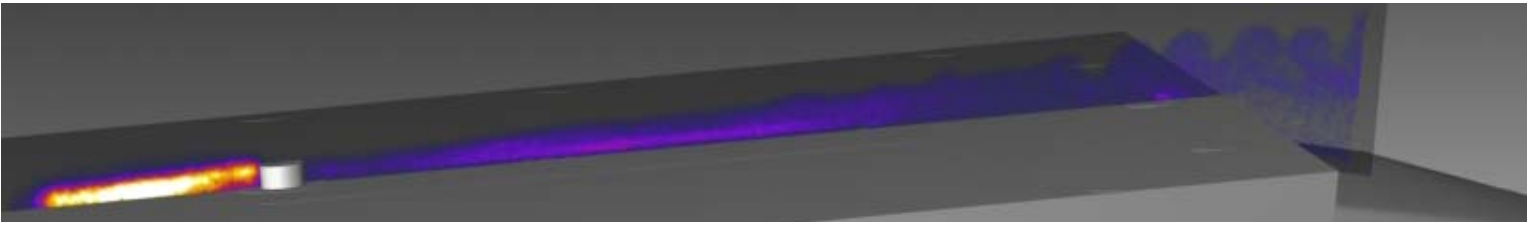

(d)

(e)

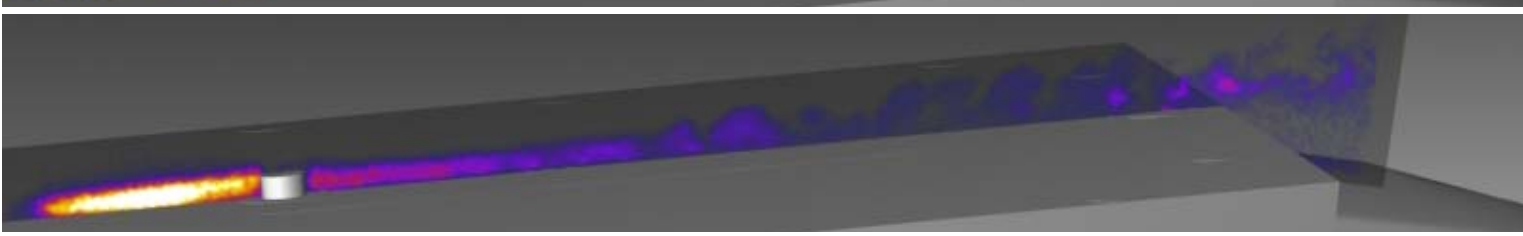

(f)

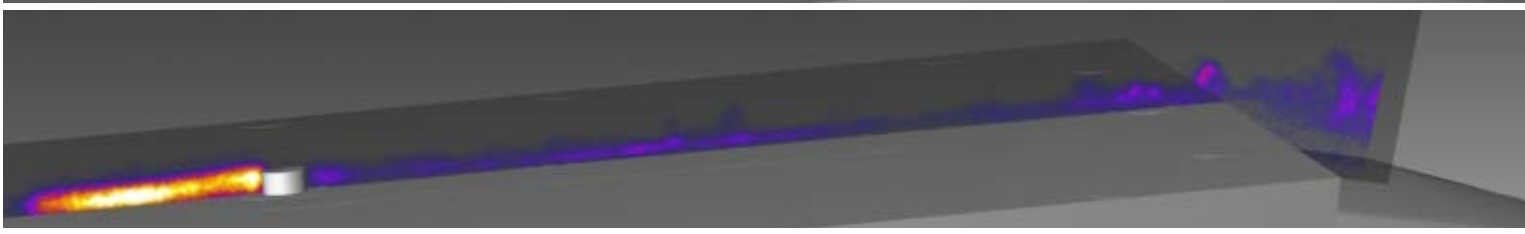

(g)

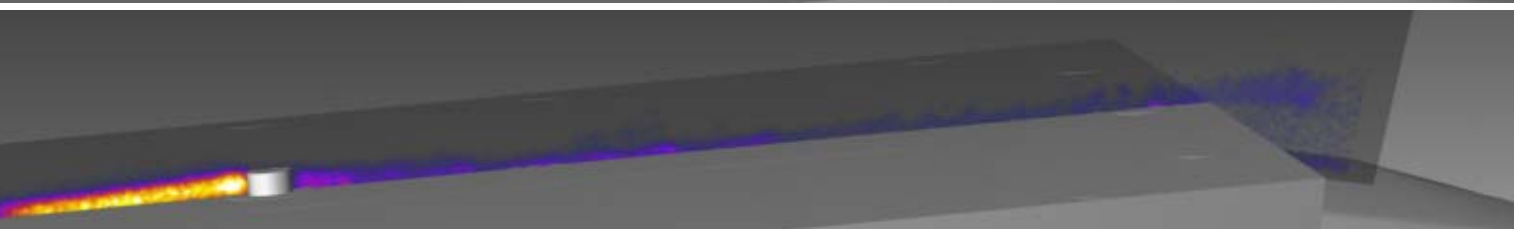

(h)

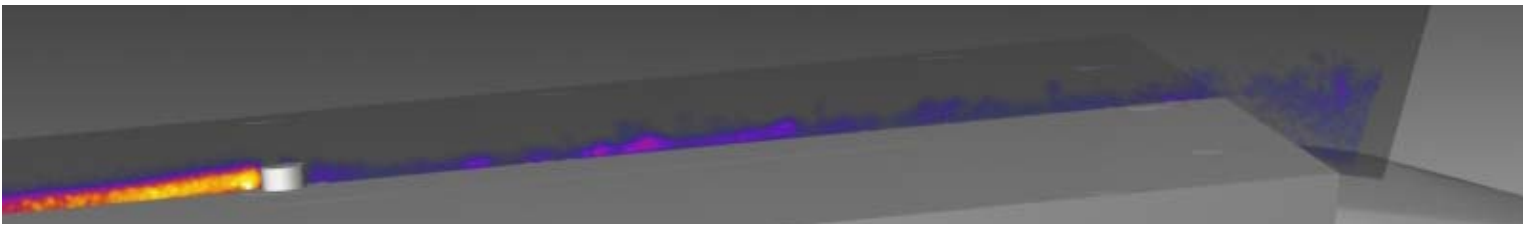

(i)

Figure A10: Test 443, Run 15, 2-mm tall by 4-mm wide cylinder, plate angle varies from 5 to $25^{\circ}$ in $2.5^{\circ}$ increments in (a)-(i), 11 -mm wide slot seeding, $\dot{m}=300 \mathrm{sccm}, \mathrm{P}_{0}=9.3 \mathrm{MPa}$, sheet position $=2 \mathrm{~mm}$ from centerline, framing rate $=10 \mathrm{~Hz}$. 
(a)

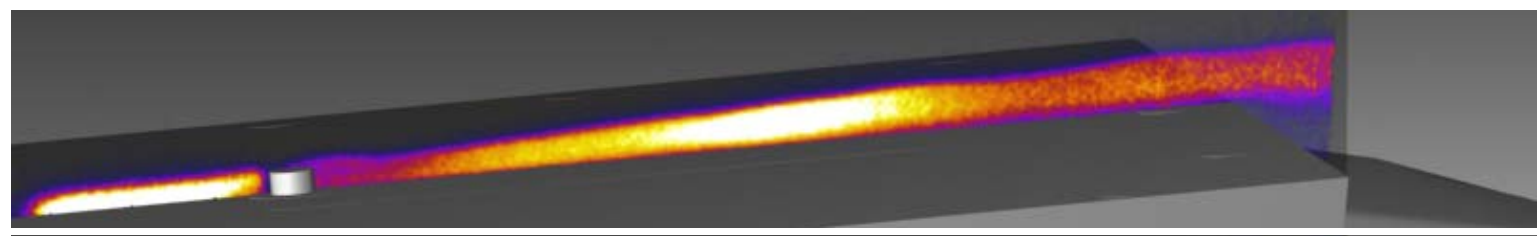

(b)

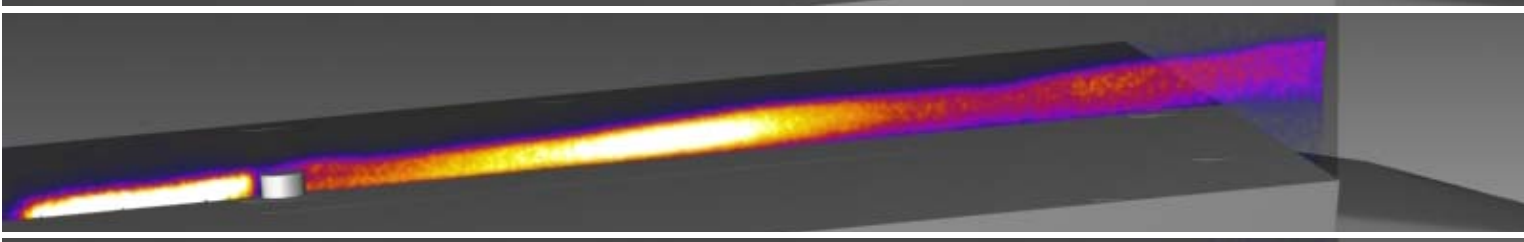

(c)

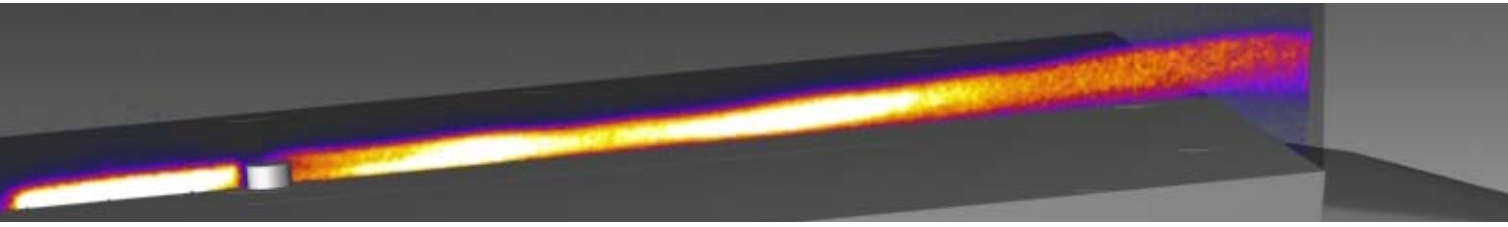

(d)

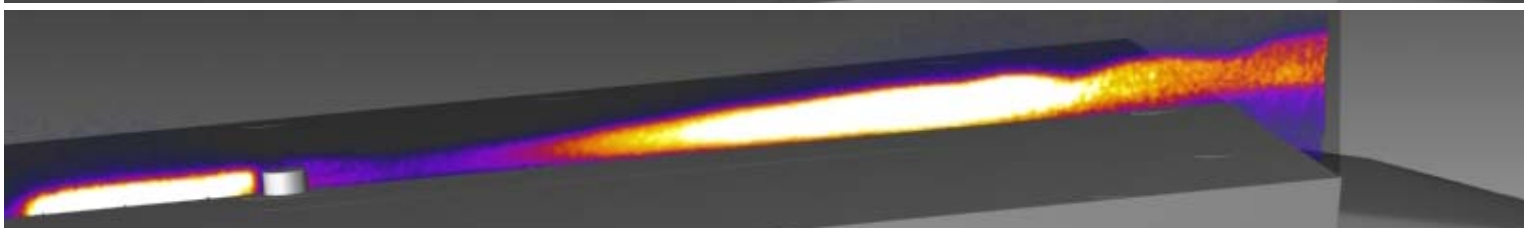

(e)

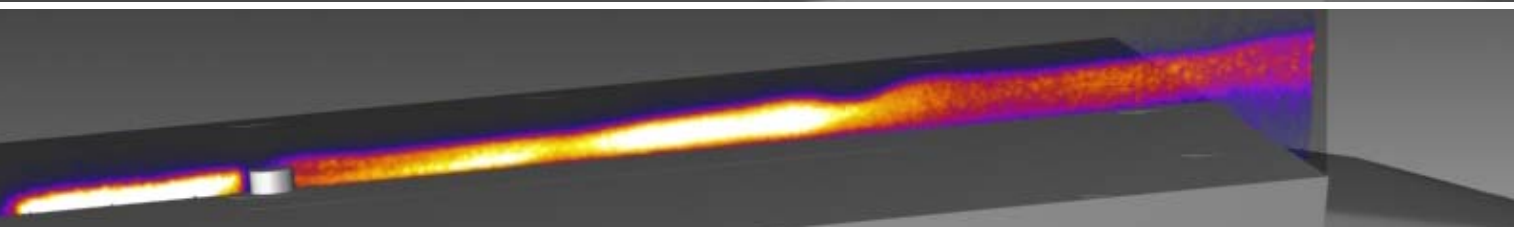

(f)

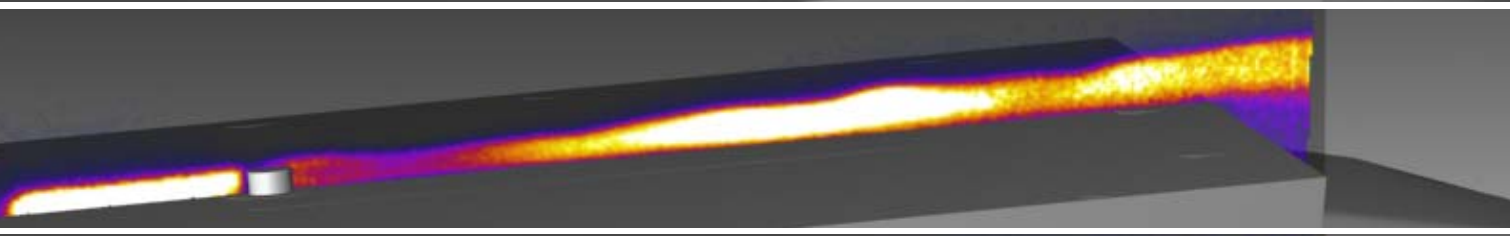

(g)

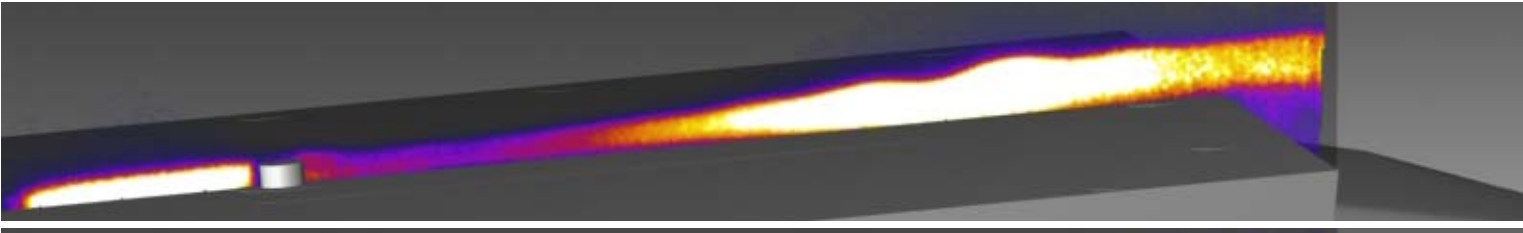

(h)

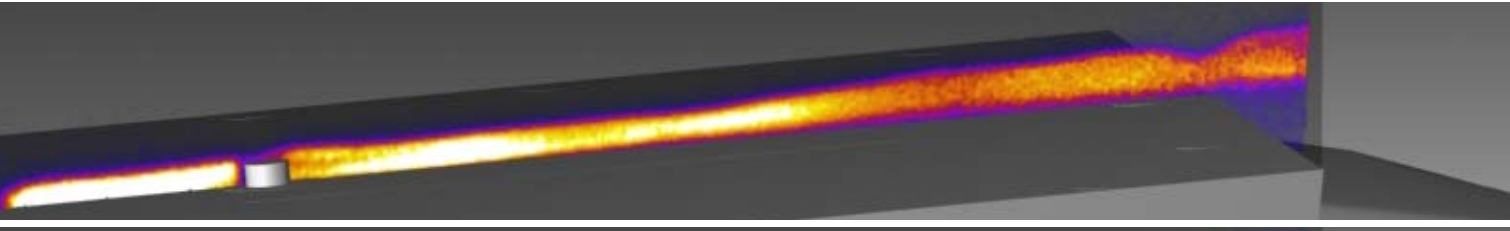

(i)

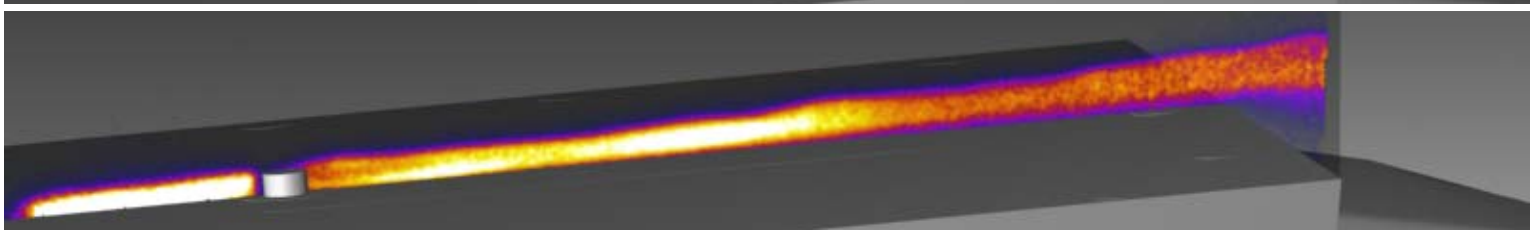

Figure A11: Test 443, Run 15, 2-mm tall by 4-mm wide cylinder, plate angle $=5^{\circ}$, 11 -mm wide slot seeding, $\dot{m}=$ $300 \mathrm{sccm}, \mathrm{P}_{0}=9.3 \mathrm{MPa}$, sheet position $=2 \mathrm{~mm}$ from centerline, framing rate $=10 \mathrm{~Hz}$. 
(a)

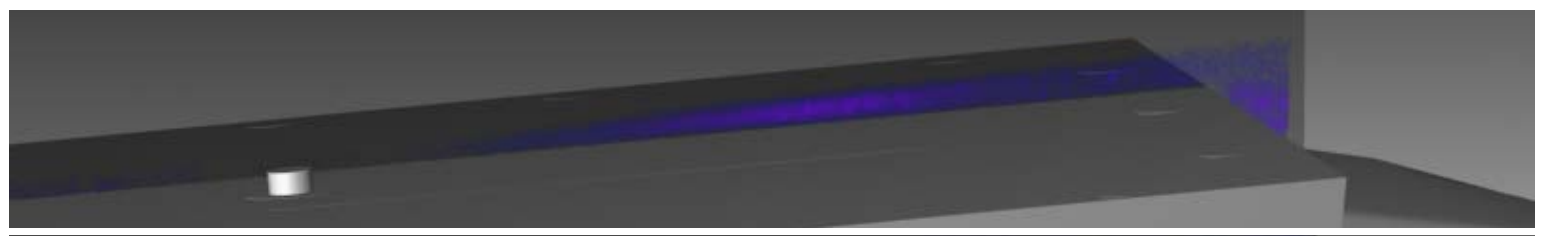

(b)

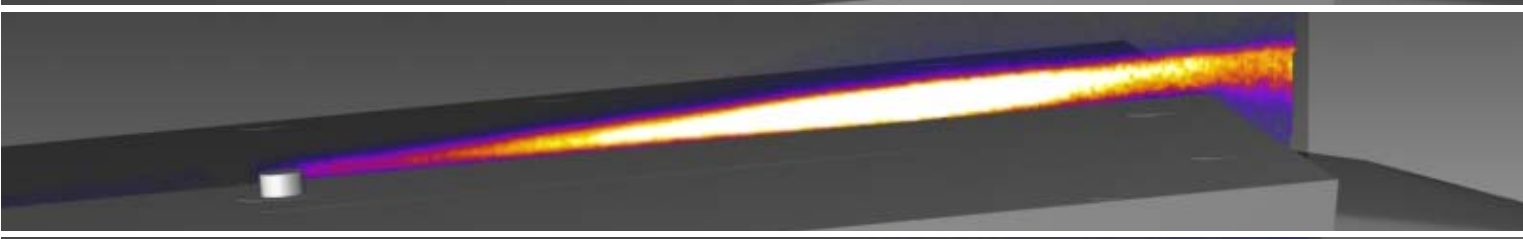

(c)

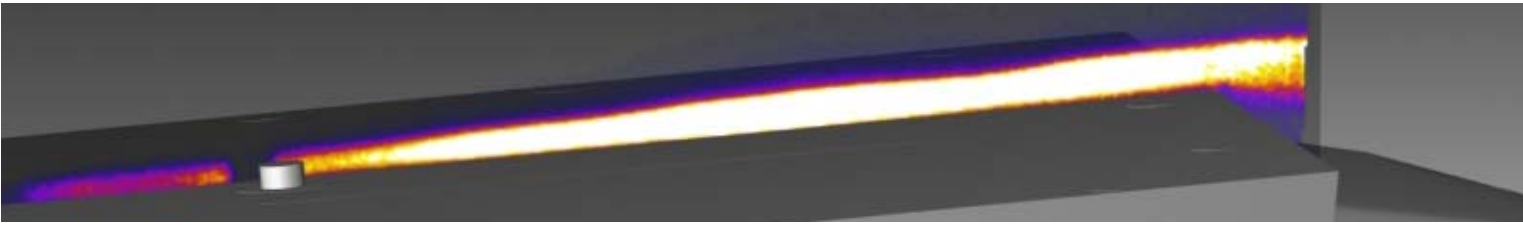

(d)

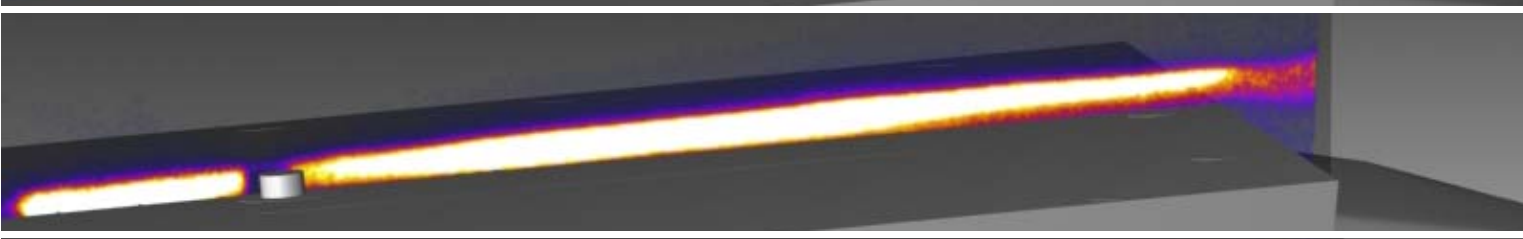

(e)

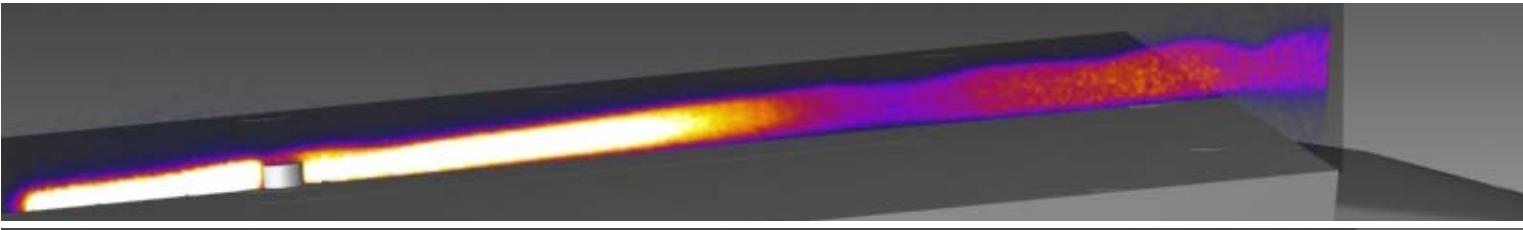

(f)

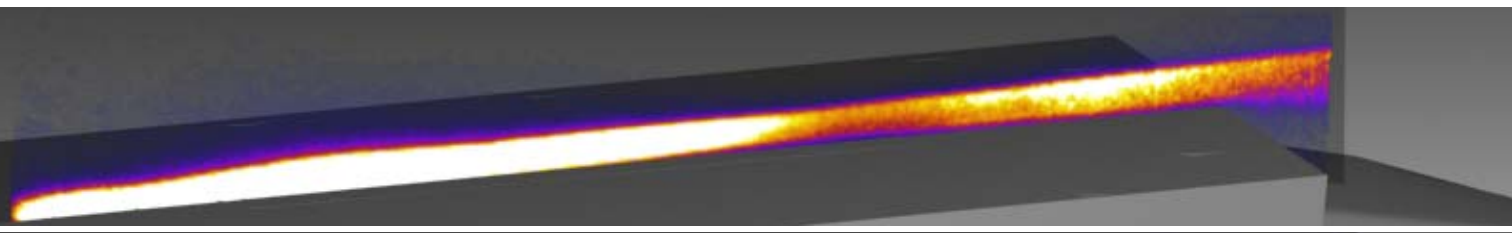

(g)

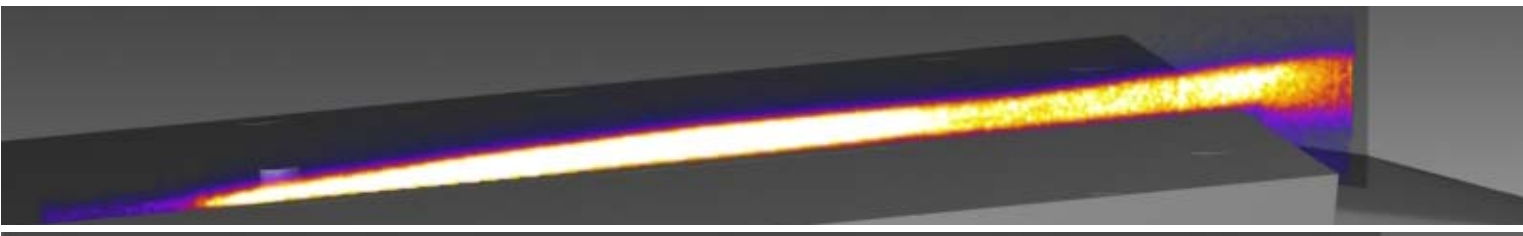

(h)

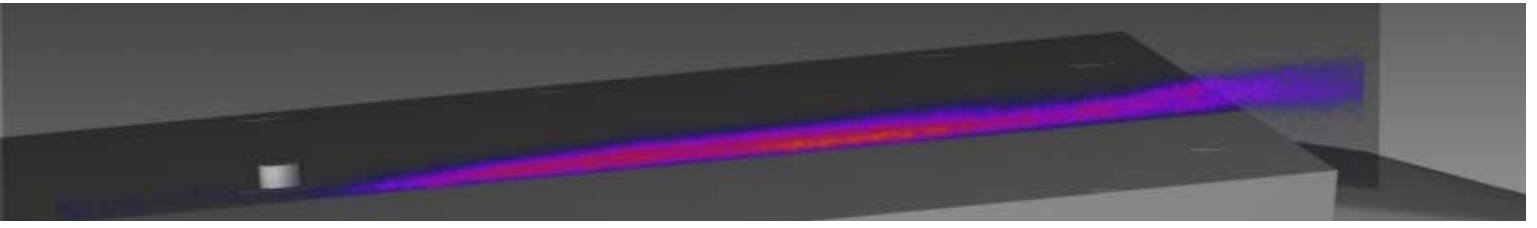

(i)

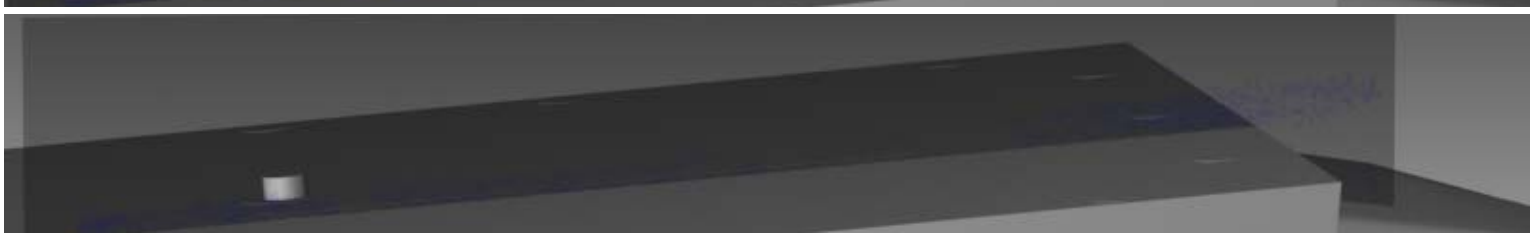

Figure A12: Test 443, Run 15, 2-mm tall by 4-mm wide cylinder, plate angle $=5^{\circ}, 11$-mm wide slot seeding, $\dot{m}=$ $300 \mathrm{sccm}, \mathrm{P}_{0}=9.3 \mathrm{MPa}$, sheet position varies from 16 to $-16 \mathrm{~mm}$ from centerline in $4 \mathrm{~mm}$ increments in (a)-(i), framing rate $=10 \mathrm{~Hz}$. 
(a)

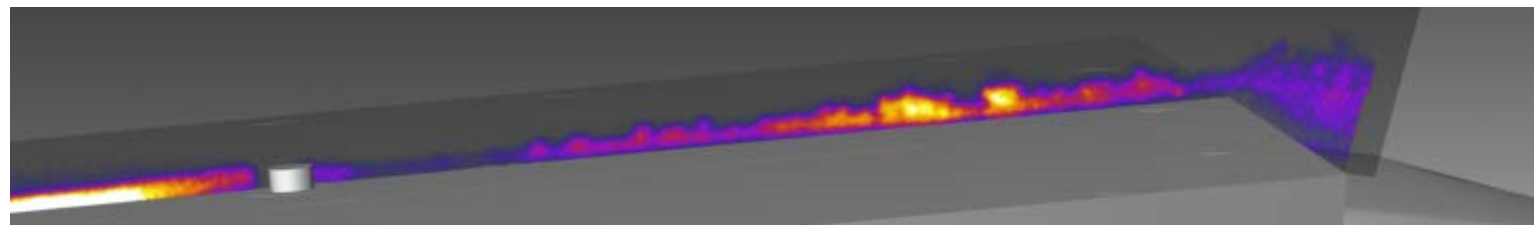

(b)

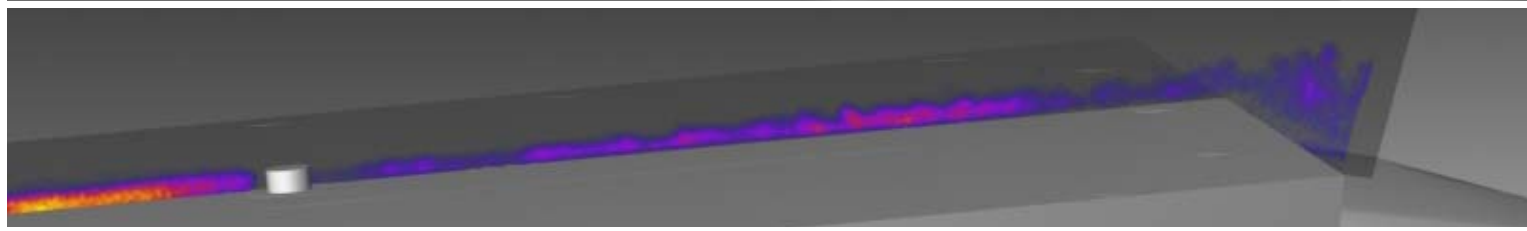

(c)

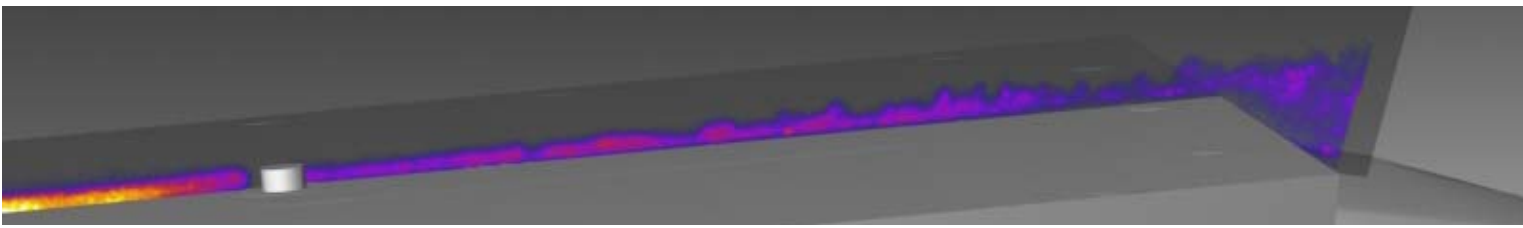

(d)

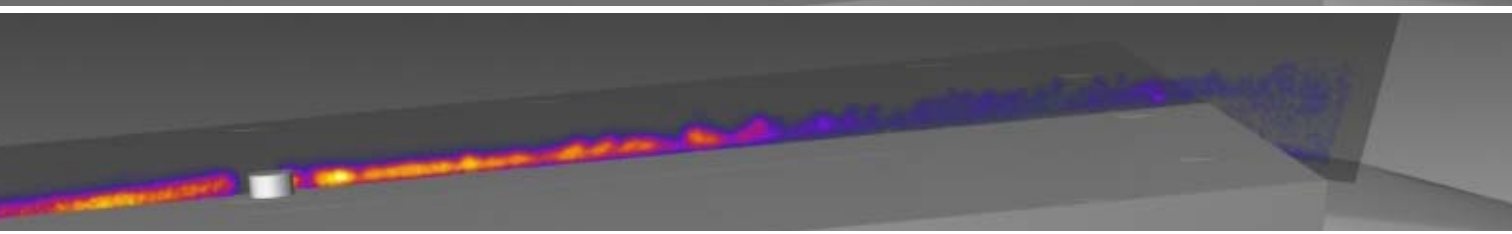

(e)

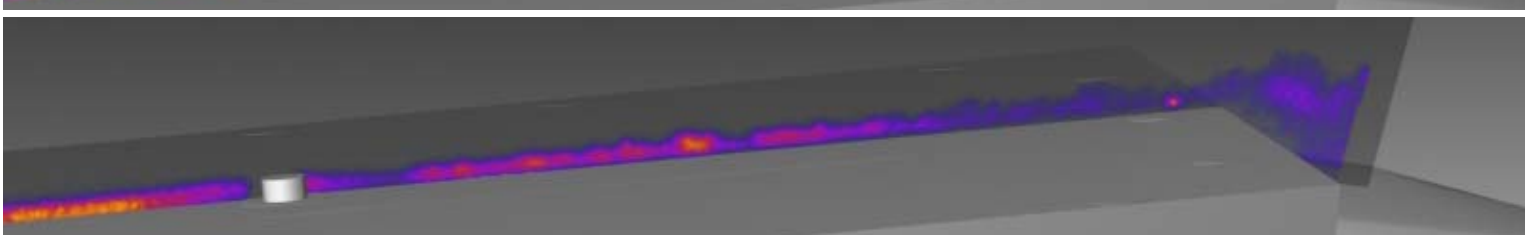

(f)

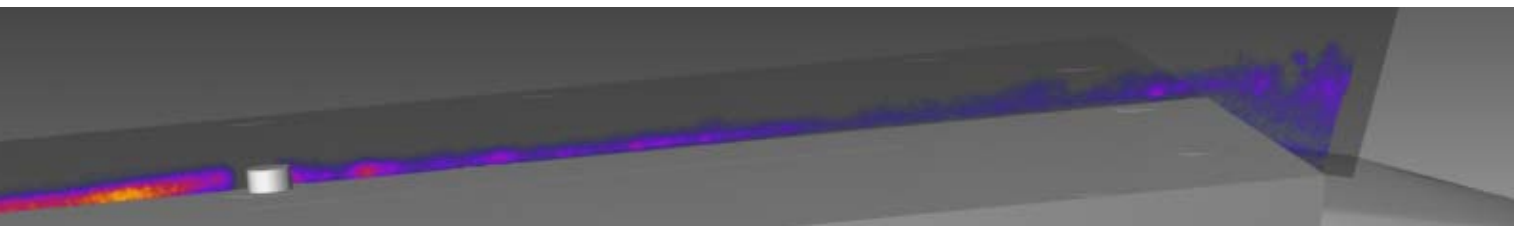

(g)

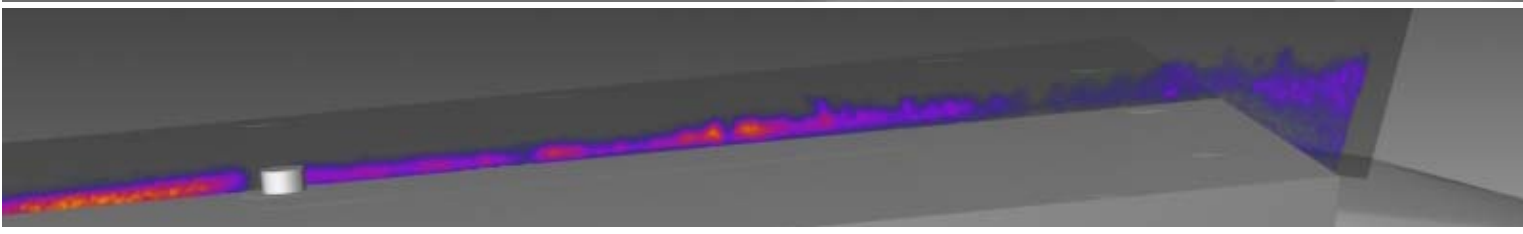

(h)

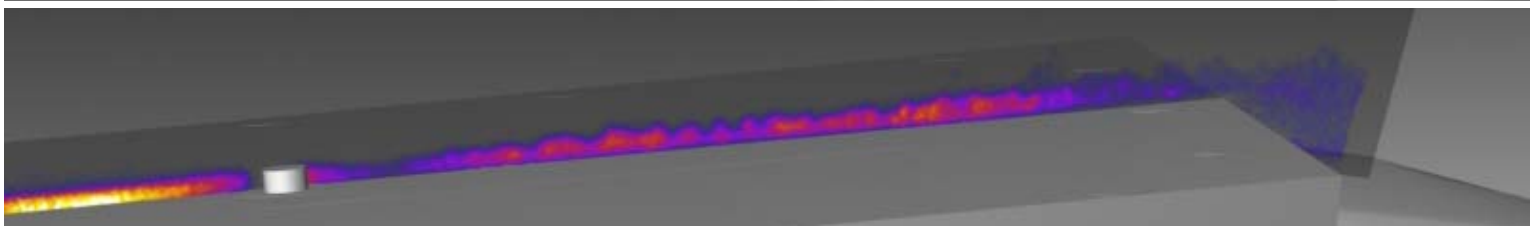

(i)

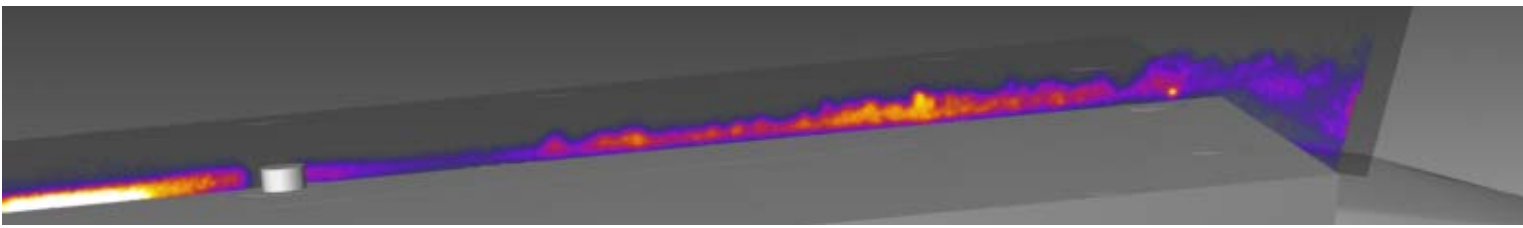

Figure A13: Test 443, Run 15, 2-mm tall by 4-mm wide cylinder, plate angle $=20^{\circ}, 11$-mm wide slot seeding, $\dot{m}=$ $300 \mathrm{sccm}, \mathrm{P}_{0}=9.3 \mathrm{MPa}$, sheet position $=3 \mathrm{~mm}$ from centerline, framing rate $=10 \mathrm{~Hz}$. 
(a)

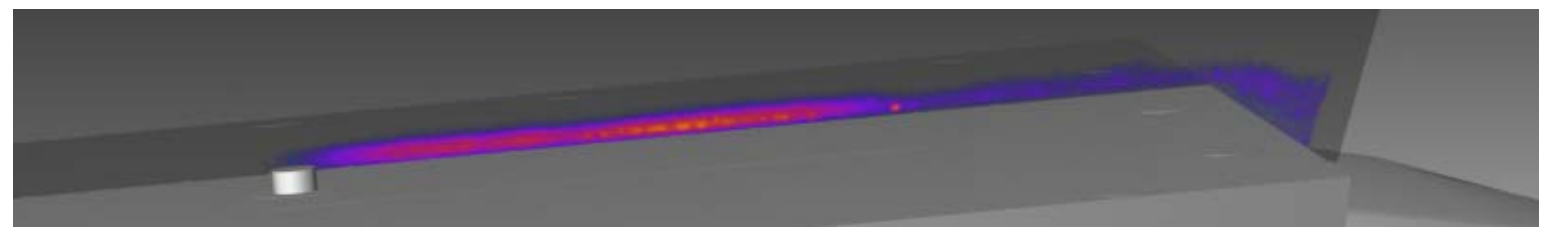

(b)

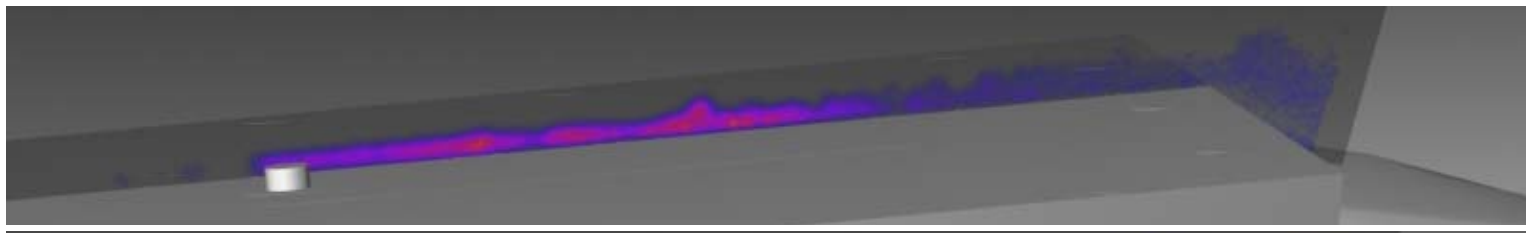

(c)

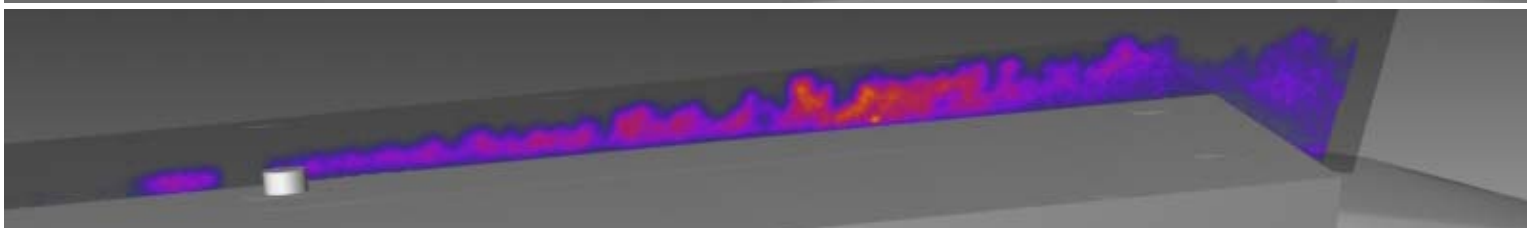

(d)

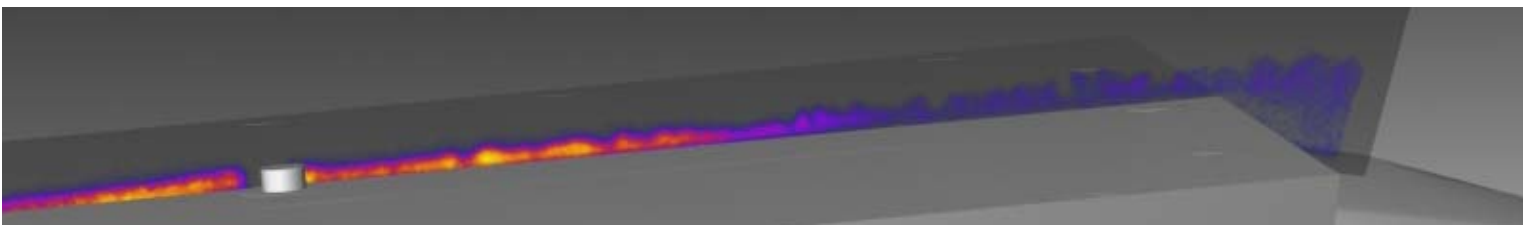

(e)

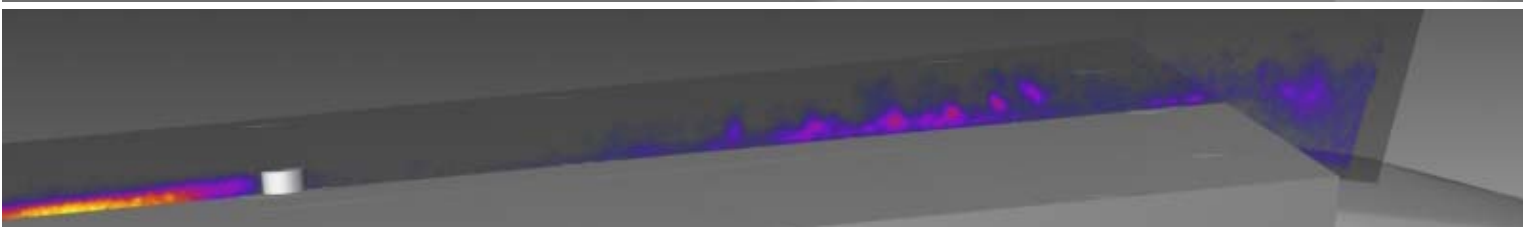

(f)

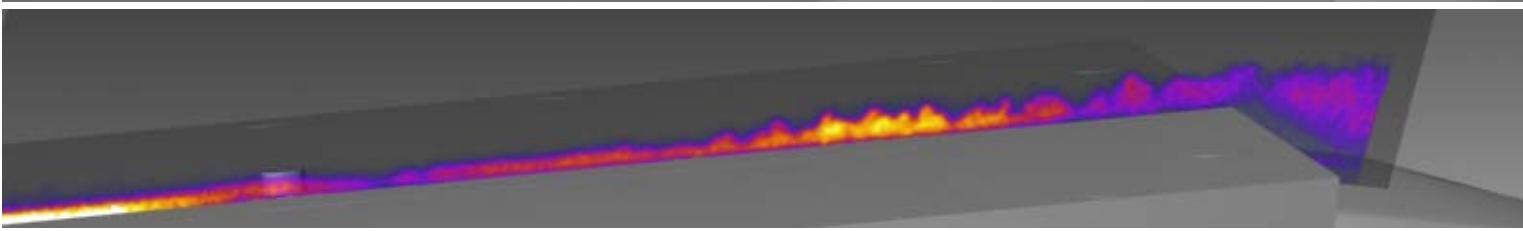

g)

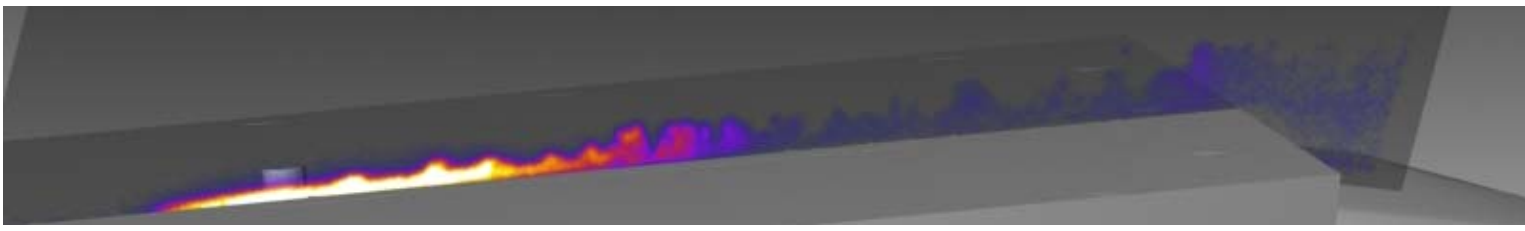

(h)

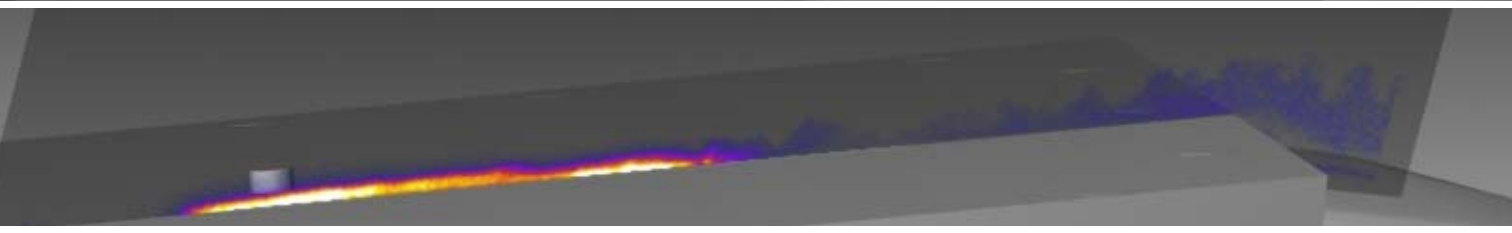

(i)

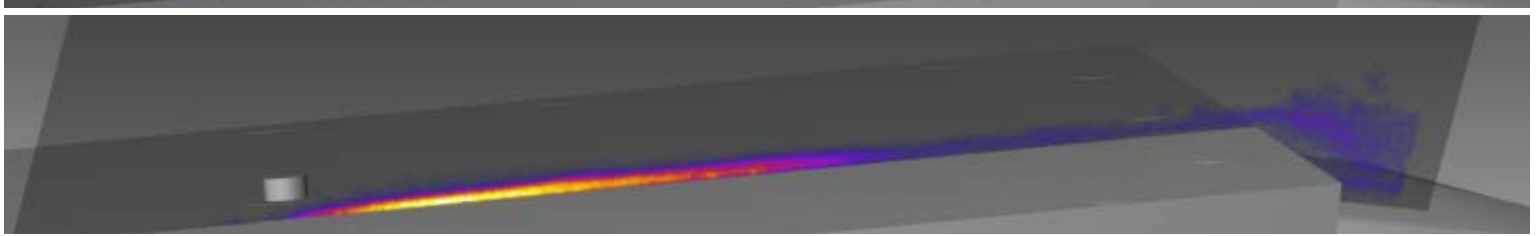

Figure A14: Test 443, Run 15, 2-mm tall by 4-mm wide cylinder, plate angle $=20^{\circ}, 11$-mm wide slot seeding, $\dot{m}=$ $300 \mathrm{sccm}, \mathrm{P}_{0}=9.3 \mathrm{MPa}$, sheet position varies from 16 to $-16 \mathrm{~mm}$ from centerline in $4 \mathrm{~mm}$ increments in (a)-(i), framing rate $=10 \mathrm{~Hz}$. 


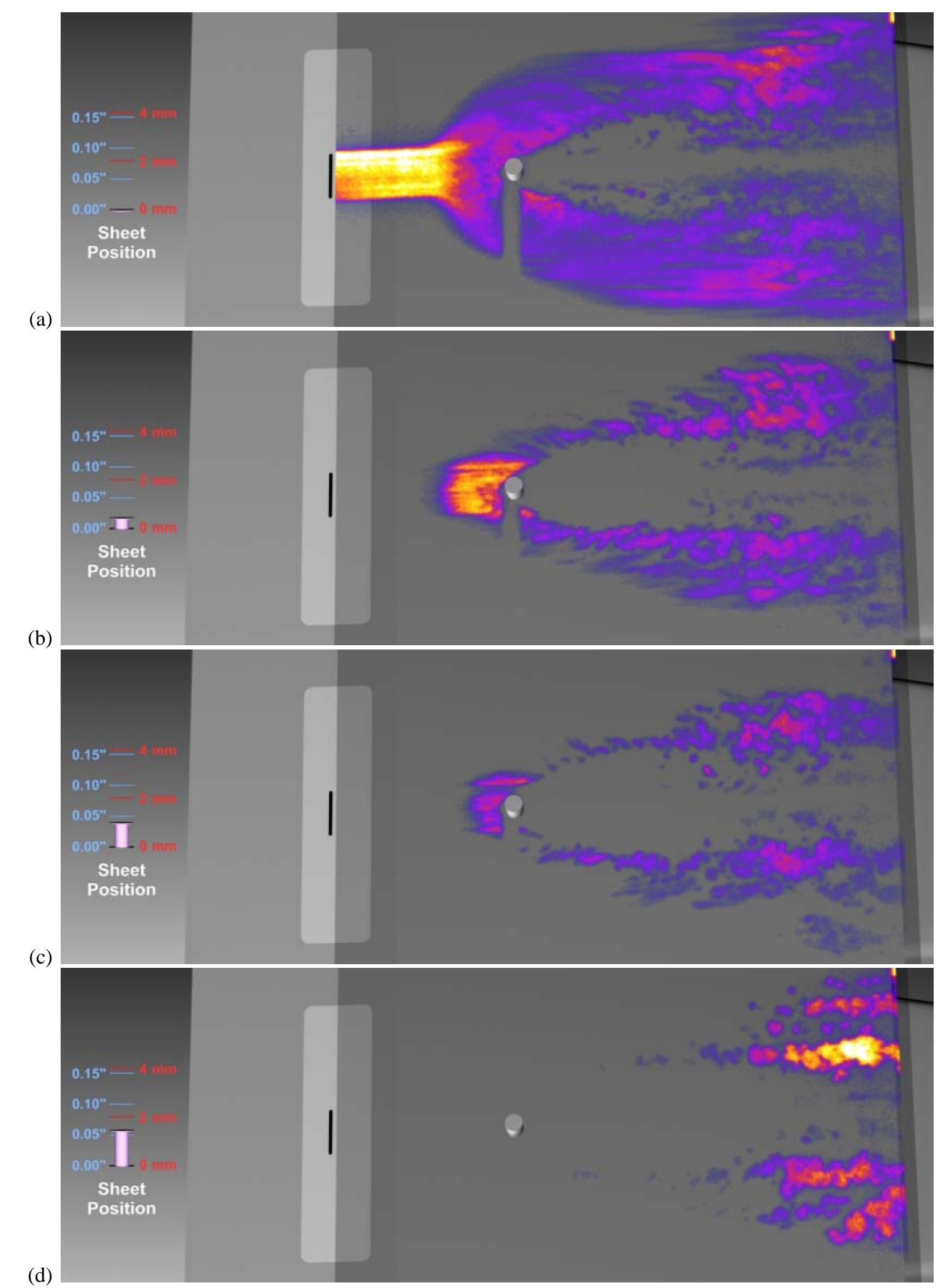

Figure A15: Test 443, Run 16, 4-mm tall by 4-mm wide cylinder, plate angle $=20^{\circ}$, 11 -mm wide slot seeding, $\dot{m}=$ $300 \mathrm{sccm}, \mathrm{P}_{0}=9.3 \mathrm{MPa}$, sheet position varies from 0.0 to $1.5 \mathrm{~mm}$ above the plate in $0.5 \mathrm{~mm}$ increments in (a)-(d), framing rate $=10 \mathrm{~Hz}$. 


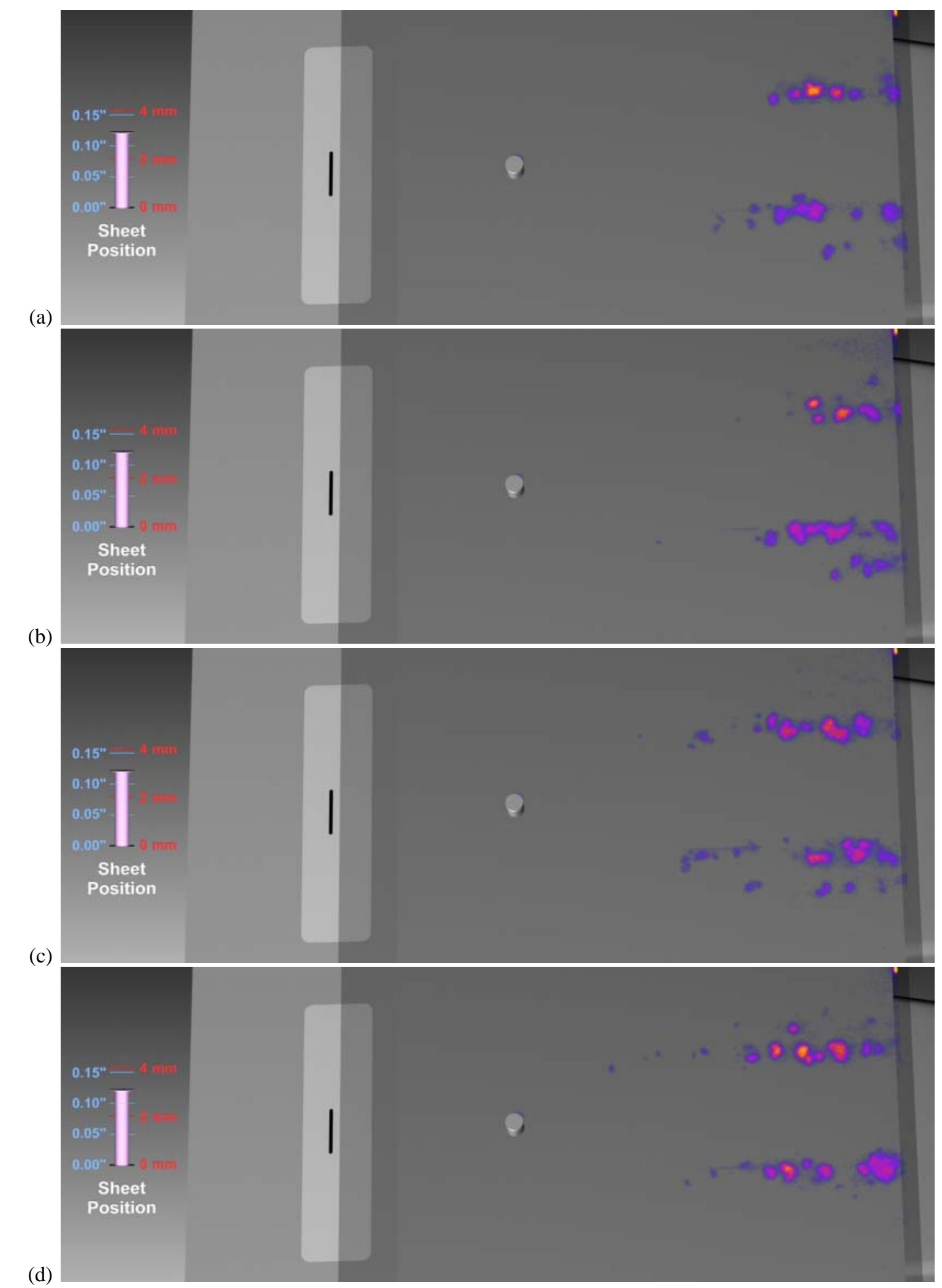

Figure A16: Test 443, Run 16, 4-mm tall by 4-mm wide cylinder, plate angle $=20^{\circ}$, 11-mm wide slot seeding, $\dot{m}=$ $300 \mathrm{sccm}, \mathrm{P}_{0}=9.3 \mathrm{MPa}$, sheet position $=3.1 \mathrm{~mm}$ above the plate, framing rate $=10 \mathrm{~Hz}$. 


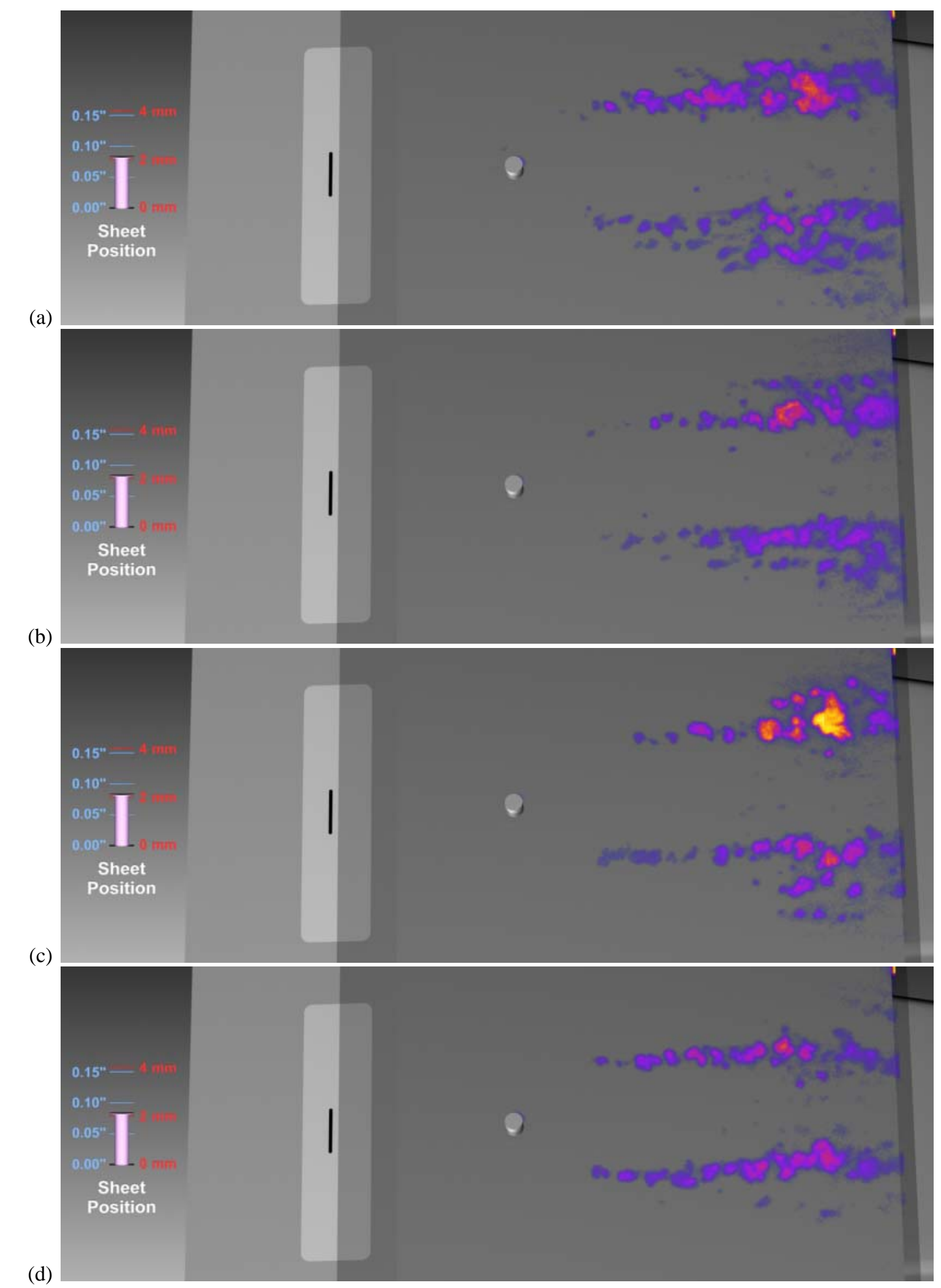

Figure A17: Test 443, Run 16, 4-mm tall by 4-mm wide cylinder, plate angle $=20^{\circ}$, 11-mm wide slot seeding, $\dot{m}=$ $300 \mathrm{sccm}, \mathrm{P}_{0}=9.3 \mathrm{MPa}$, sheet position $=2.1 \mathrm{~mm}$ above the plate, framing rate $=10 \mathrm{~Hz}$. 


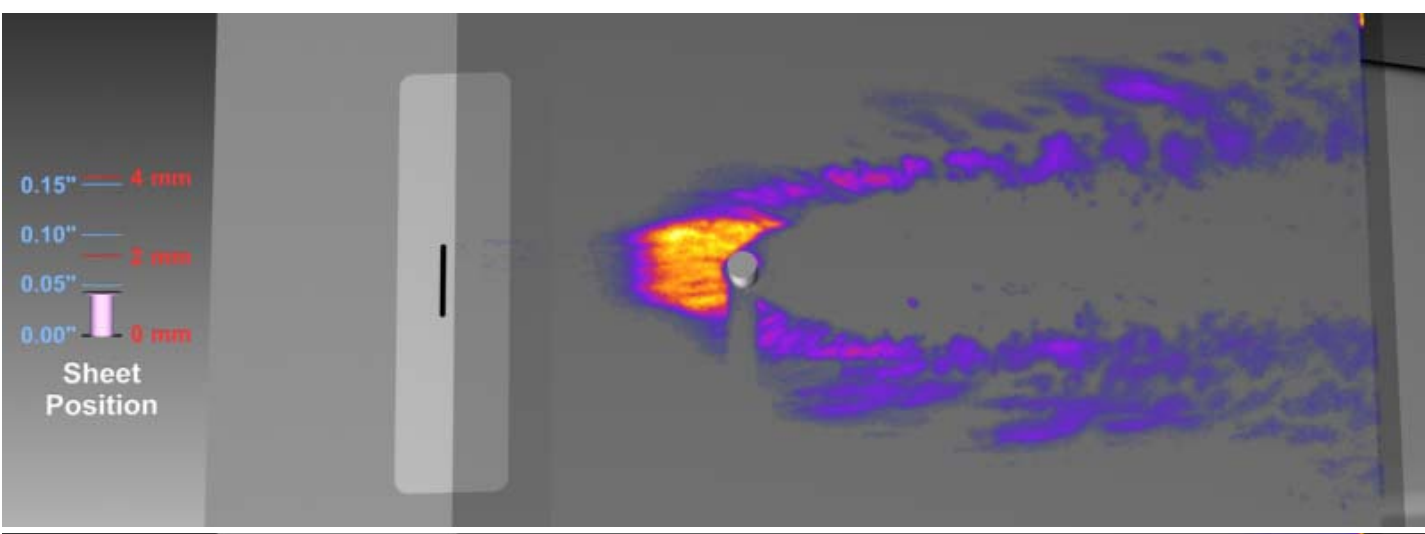

(a)

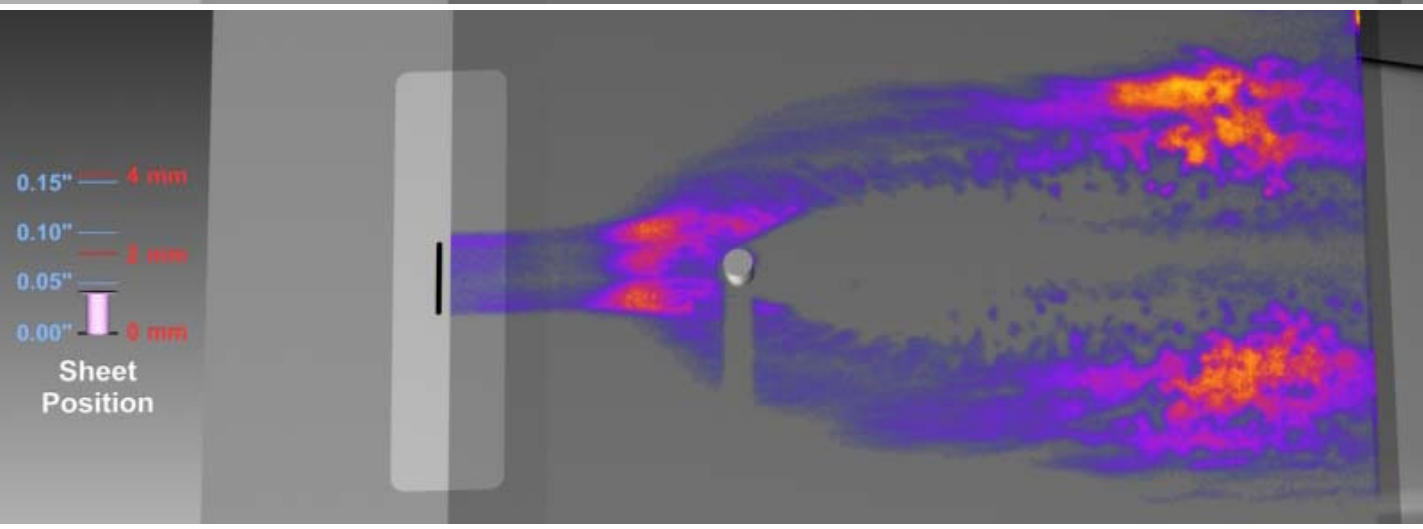

(b)

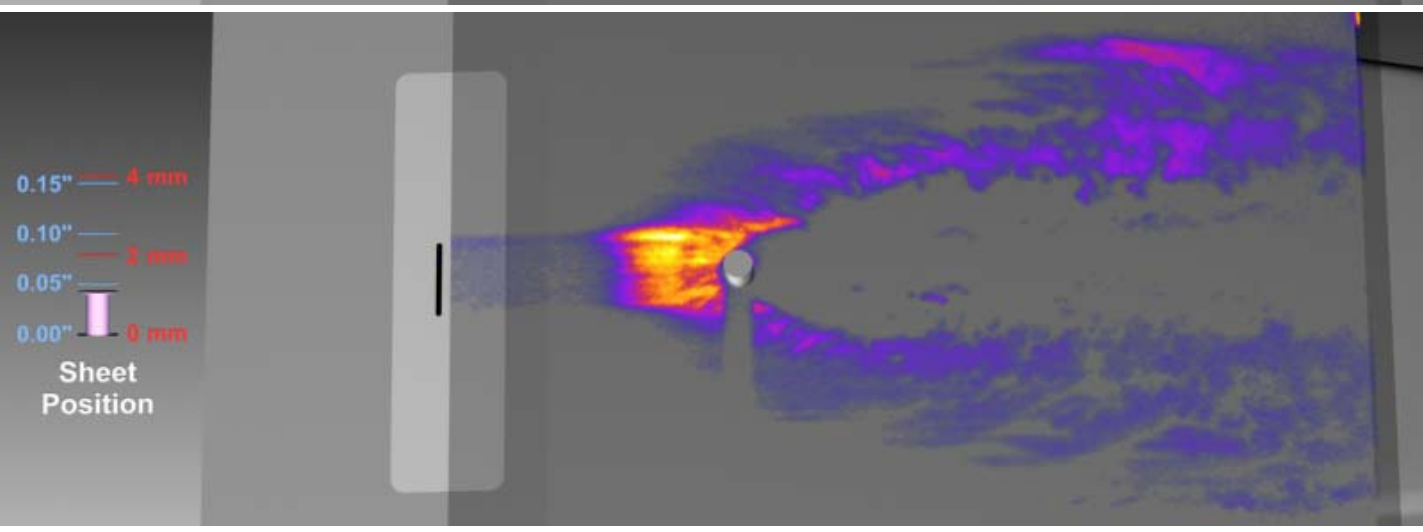

(c)

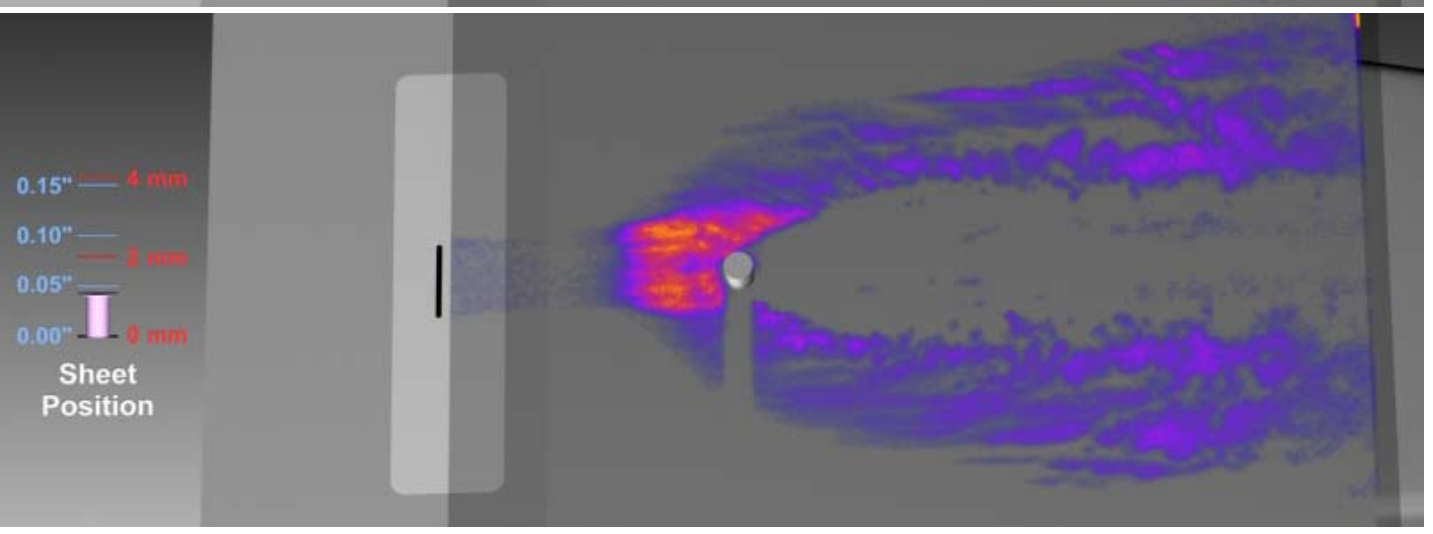

Figure A18: Test 443, Run 16, 4-mm tall by 4-mm wide cylinder, plate angle $=20^{\circ}$, 11-mm wide slot seeding, $\dot{m}=$ $300 \mathrm{sccm}, \mathrm{P}_{0}=9.3 \mathrm{MPa}$, sheet position $=1.1 \mathrm{~mm}$ above the plate, framing rate $=10 \mathrm{~Hz}$. 


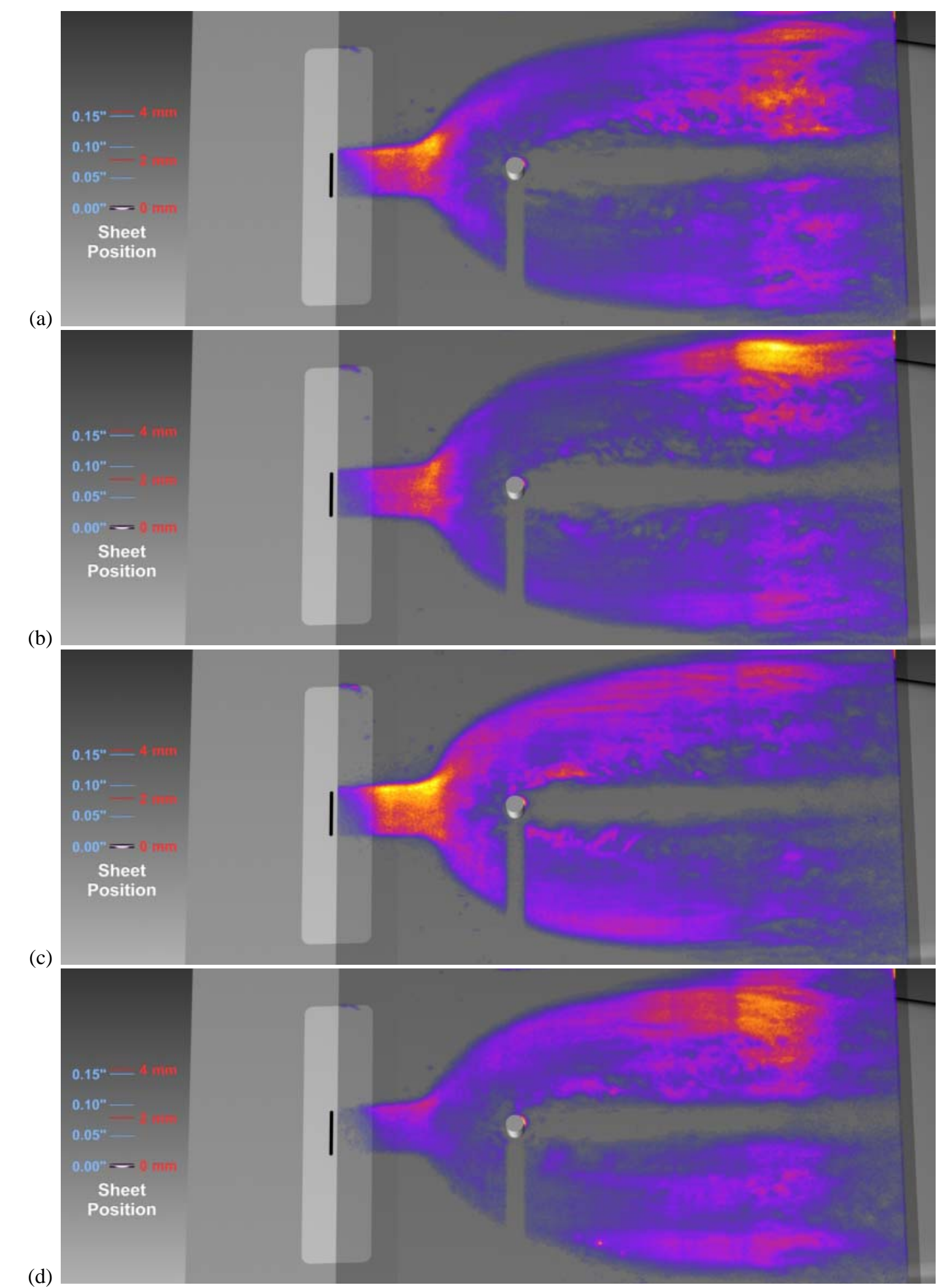

Figure A19: Test 443, Run 16, 4-mm tall by 4-mm wide cylinder, plate angle $=20^{\circ}, 11$-mm wide slot seeding, $\dot{m}=$ $300 \mathrm{sccm}, \mathrm{P}_{0}=9.3 \mathrm{MPa}$, sheet position $=0.1 \mathrm{~mm}$ above the plate, framing rate $=10 \mathrm{~Hz}$. 


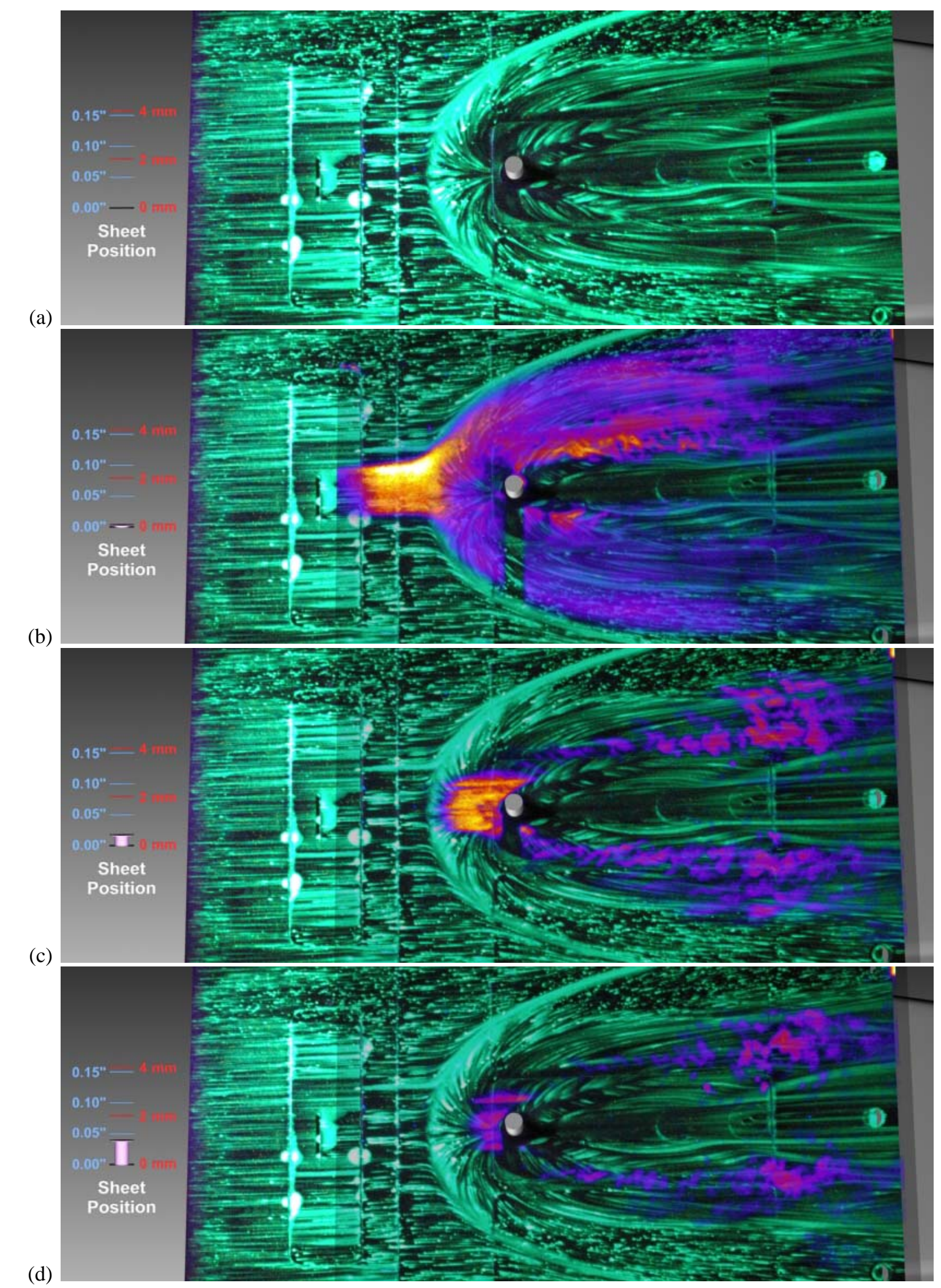

Figure A20: Test 443, Run 16, 4-mm tall by 4-mm wide cylinder with oil flow, plate angle $=20^{\circ}, 11$-mm wide slot seeding, $\dot{m}=300 \mathrm{sccm}, \mathrm{P}_{0}=9.3 \mathrm{MPa}$, sheet position varies: $0.0 \mathrm{~mm}$ in (a) and (b), $1.0 \mathrm{~mm}$ in (c) and $1.5 \mathrm{~mm}$ above the plate in (d), framing rate $=10 \mathrm{~Hz}$. 
(a)

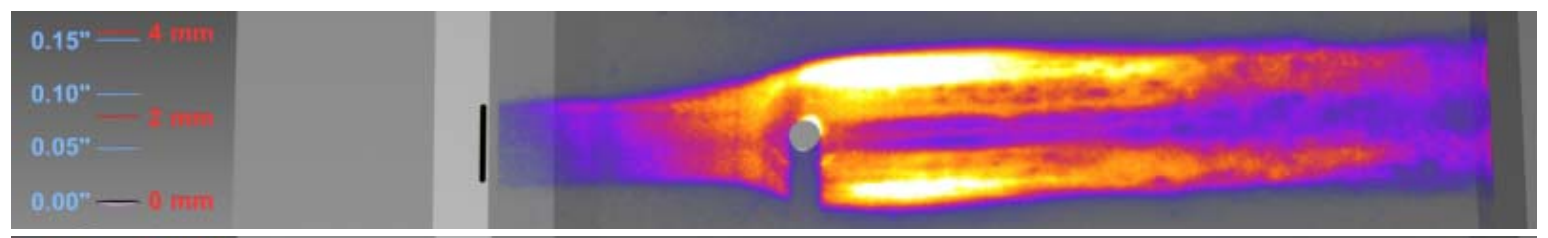

(b)

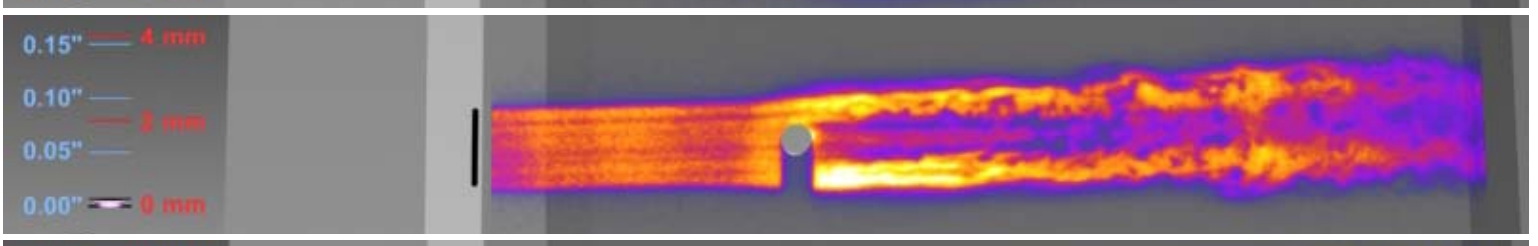

(c)

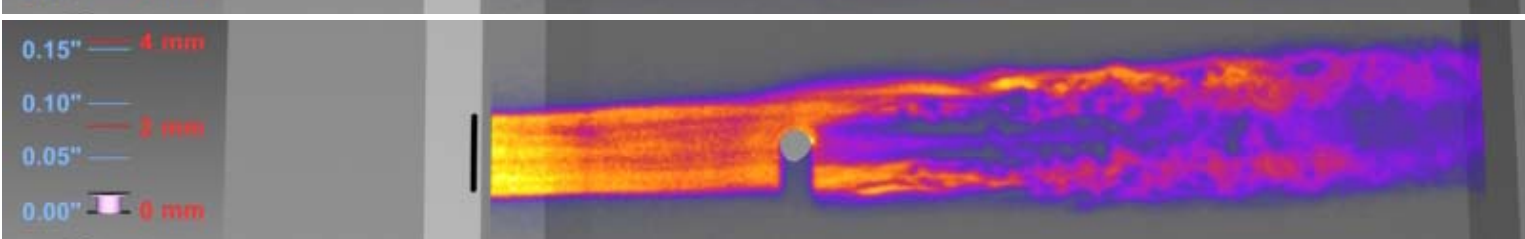

(d)

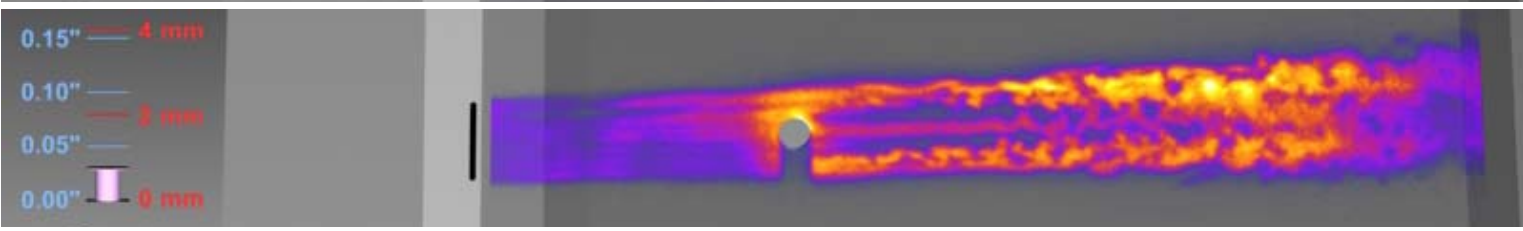

(e)

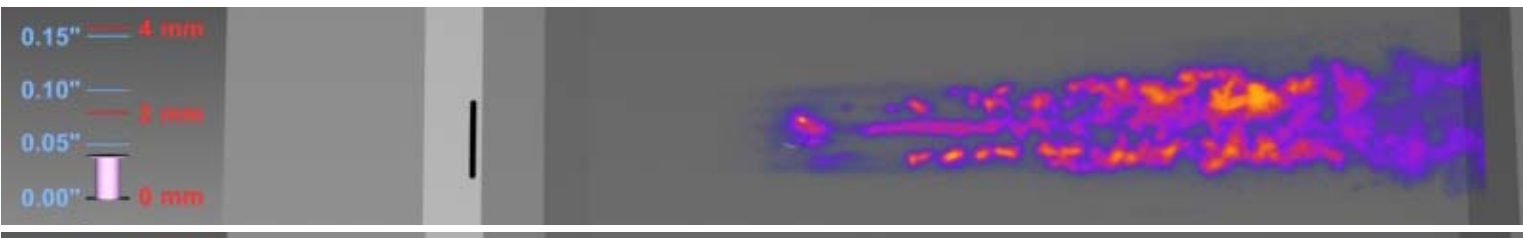

(f)

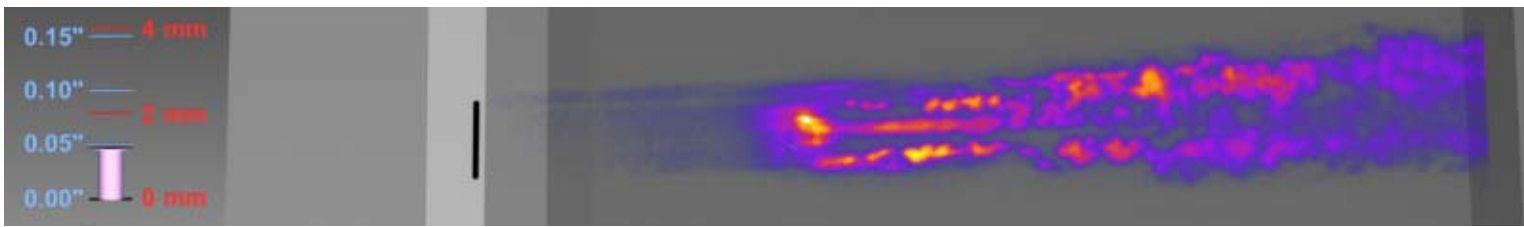

(g)

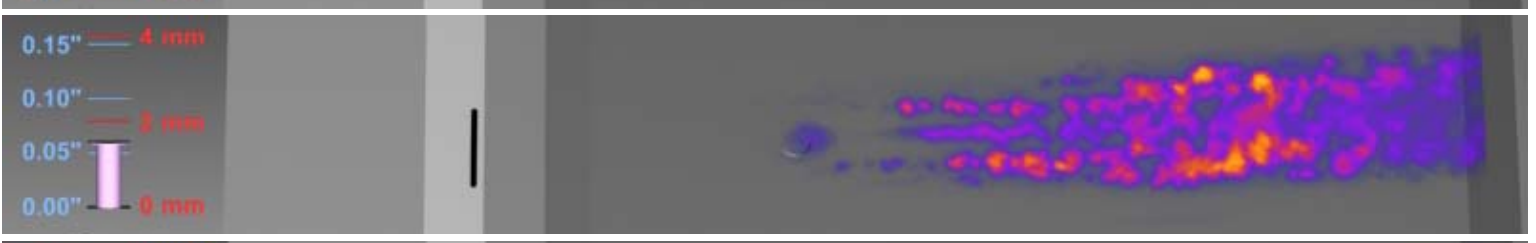

(h)

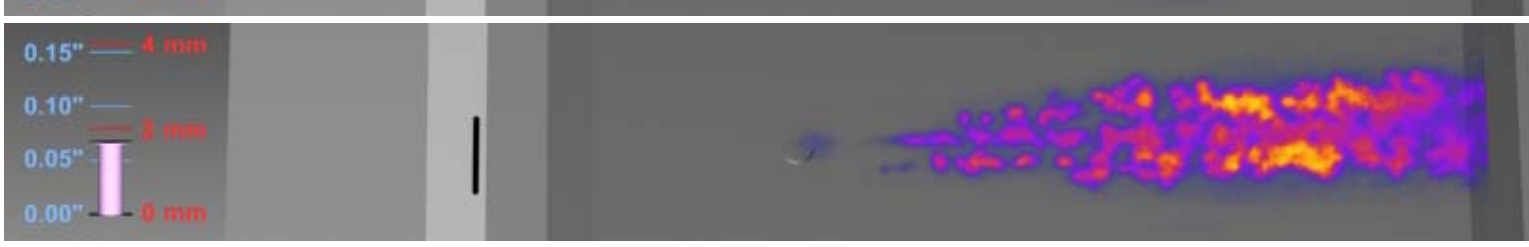

(i)

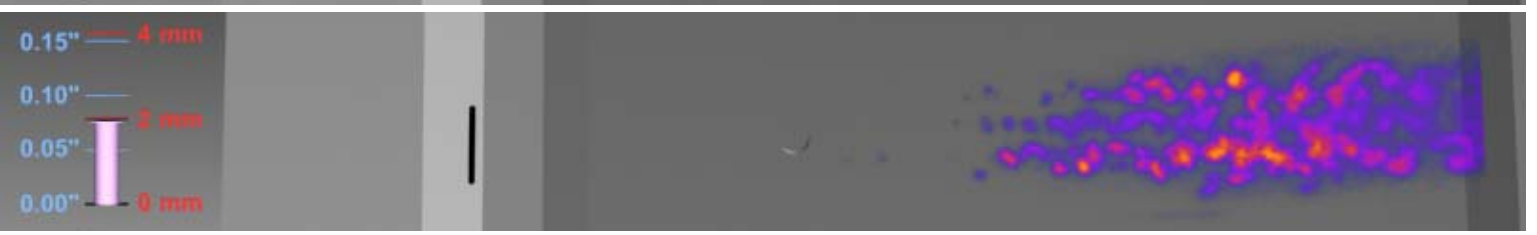

Figure A21: Test 443, Run 18, 1-mm tall by 4-mm wide cylinder, plate angle $=20^{\circ}, 11-\mathrm{mm}$ wide slot seeding, $\dot{m}=$ $300 \mathrm{sccm}, \mathrm{P}_{0}=9.32 \mathrm{MPa}$, sheet position varies from 0.0 to $2.0 \mathrm{~mm}$ above the plate in $0.25 \mathrm{~mm}$ increments in (a)-(i), framing rate $=10 \mathrm{~Hz}$. 
(a)

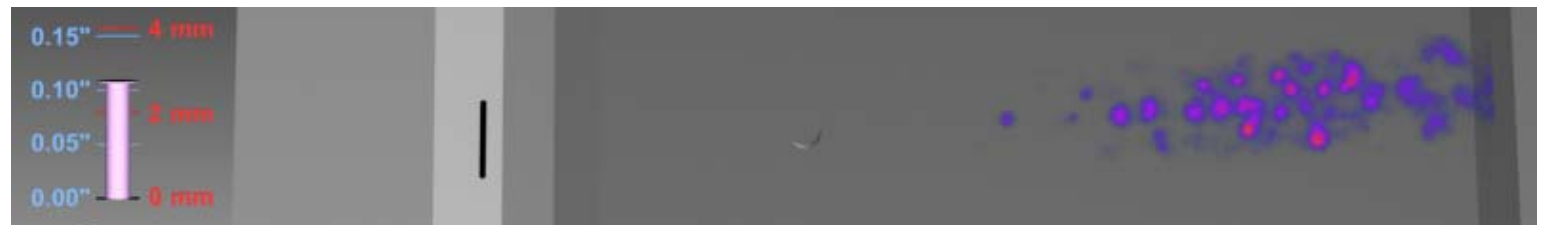

(b)

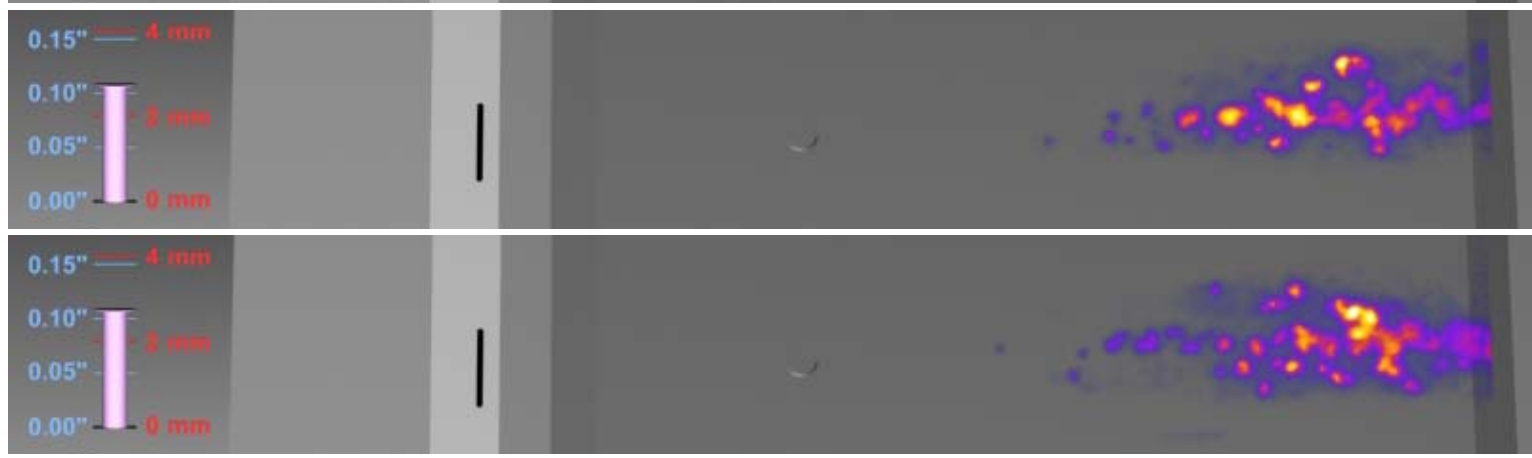

(c)

(d)

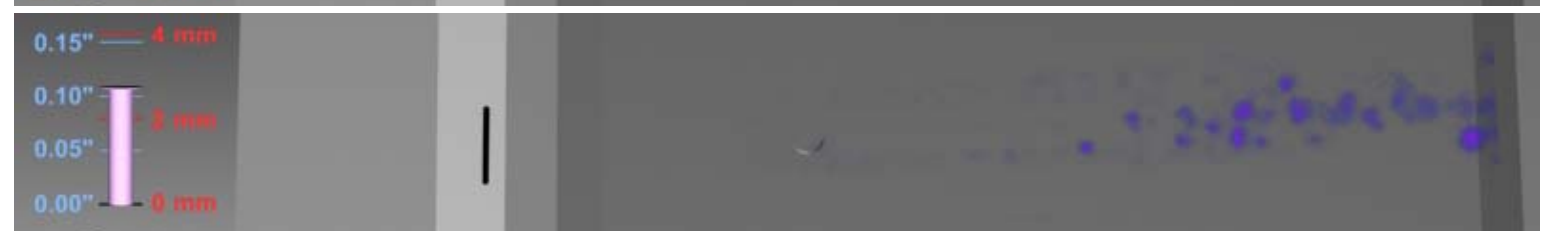

(e)

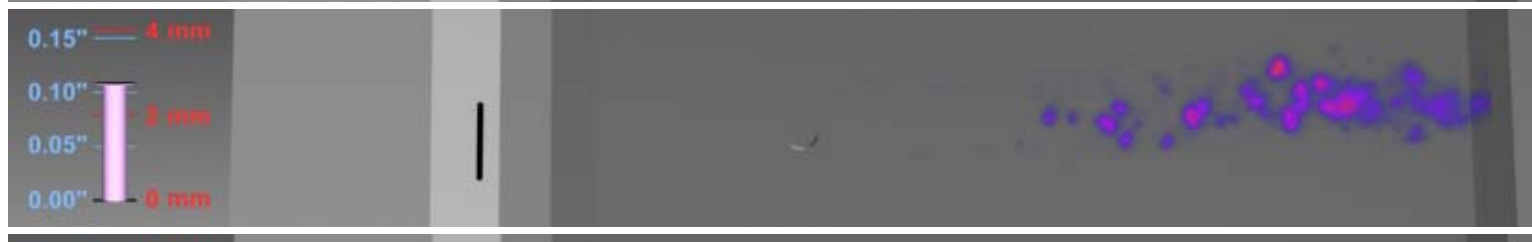

(f)

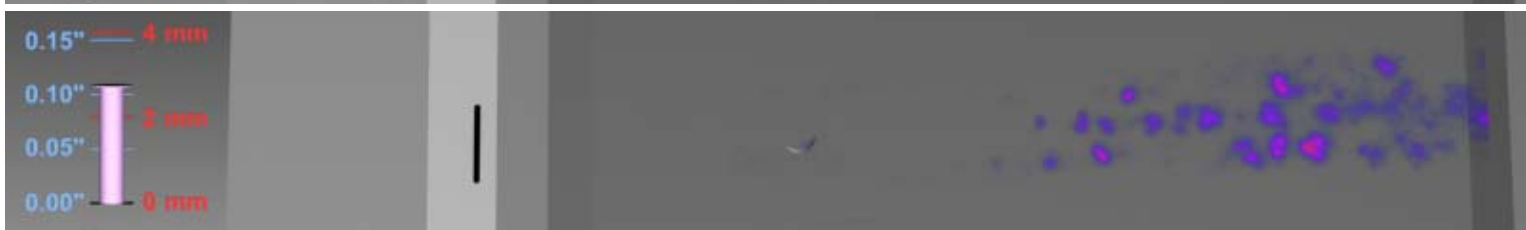

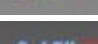

(g)

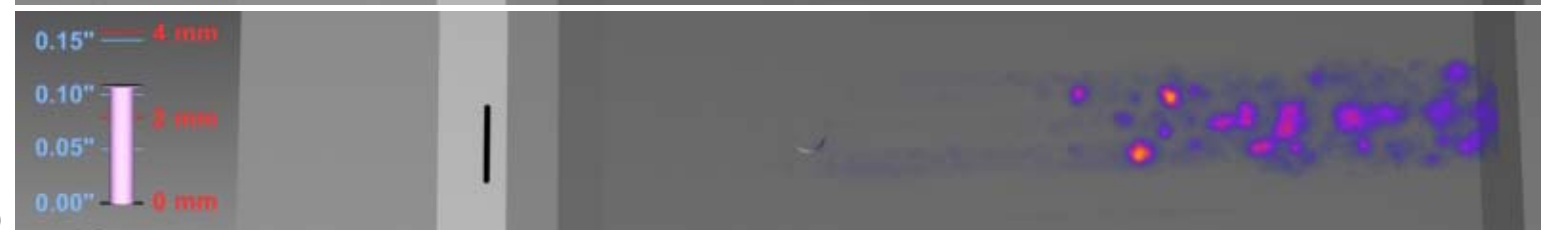

(h)

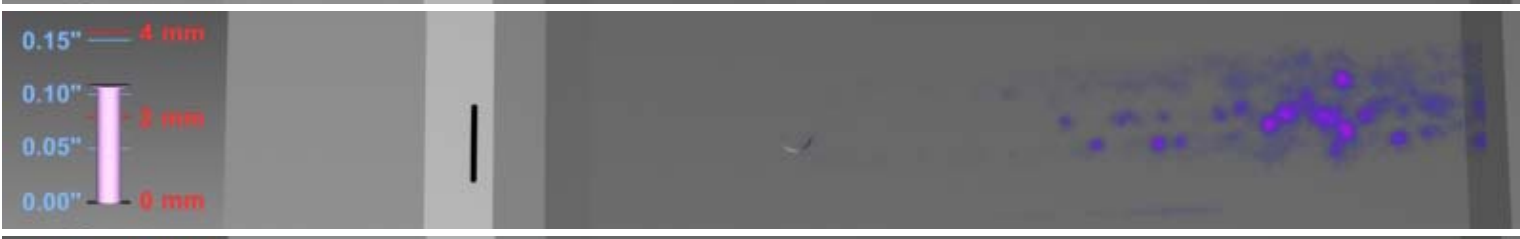

(i)

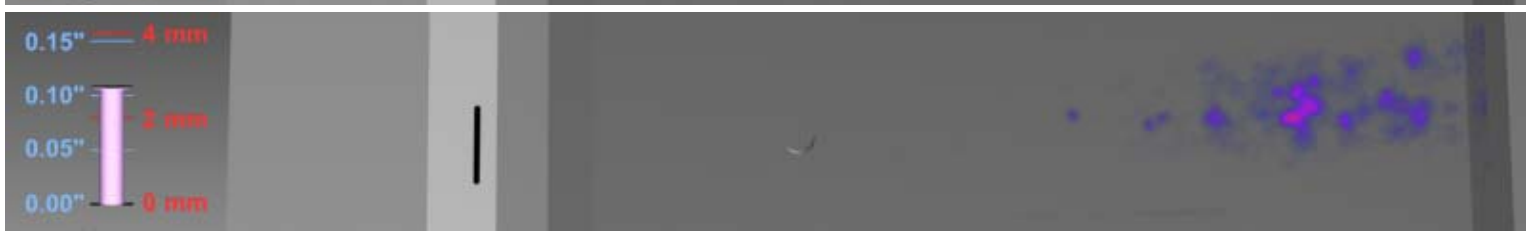

Figure A22: Test 443, Run 18, 1-mm tall by 4-mm wide cylinder, plate angle $=20^{\circ}, 11-\mathrm{mm}$ wide slot seeding, $\dot{m}=$ $300 \mathrm{sccm}, \mathrm{P}_{0}=9.32 \mathrm{MPa}$, sheet position $=2.8 \mathrm{~mm}$ above the plate, framing rate $=10 \mathrm{~Hz}$. 
(a)

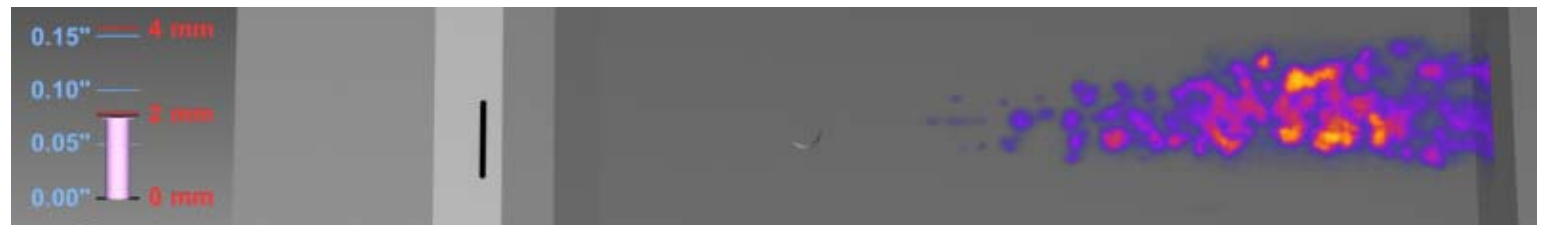

(b)

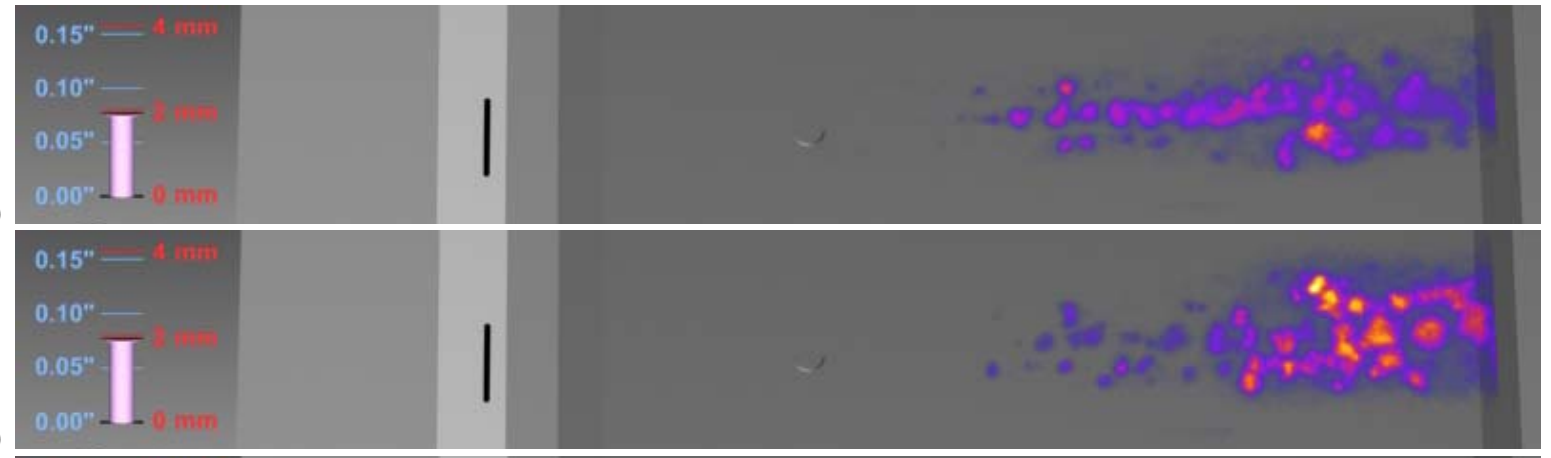

(d)

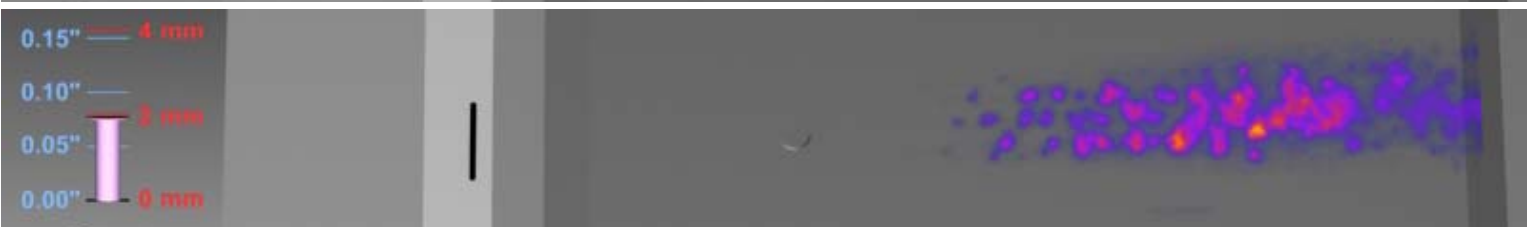

(e)

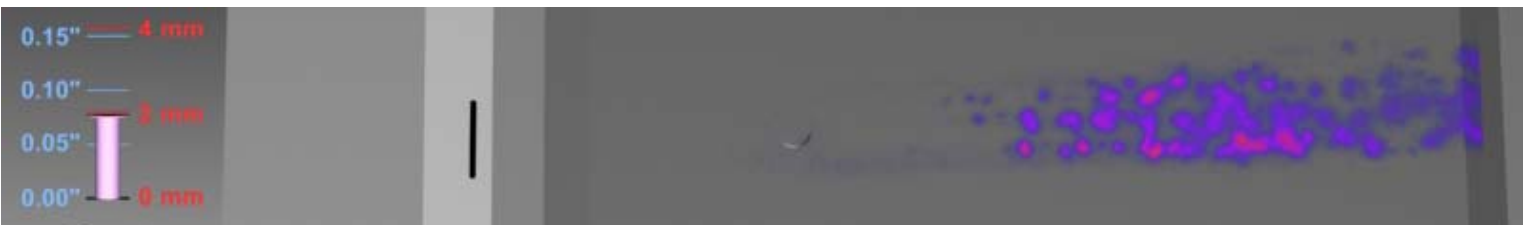

(f)

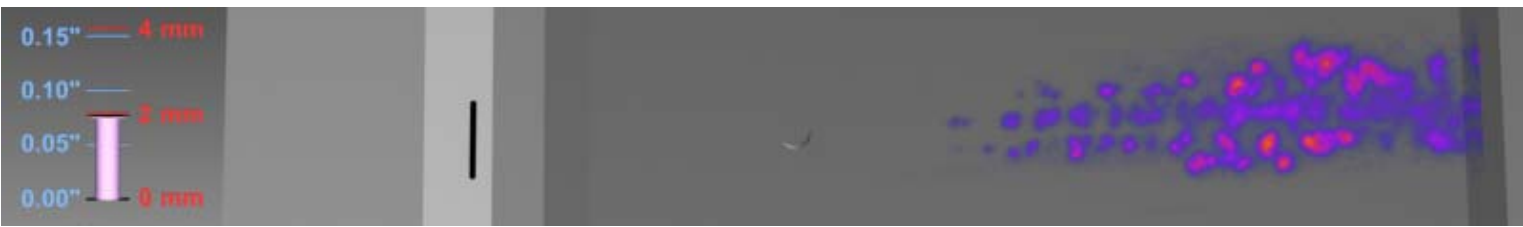

\section{$0.15 n$}

(g)

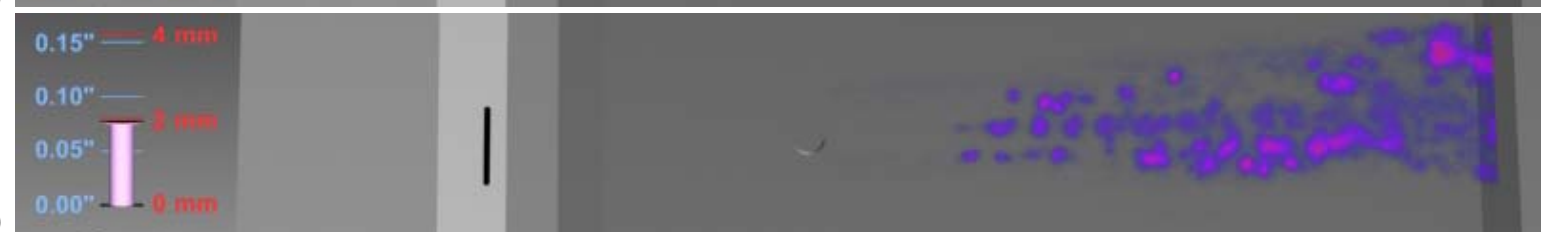

(h)

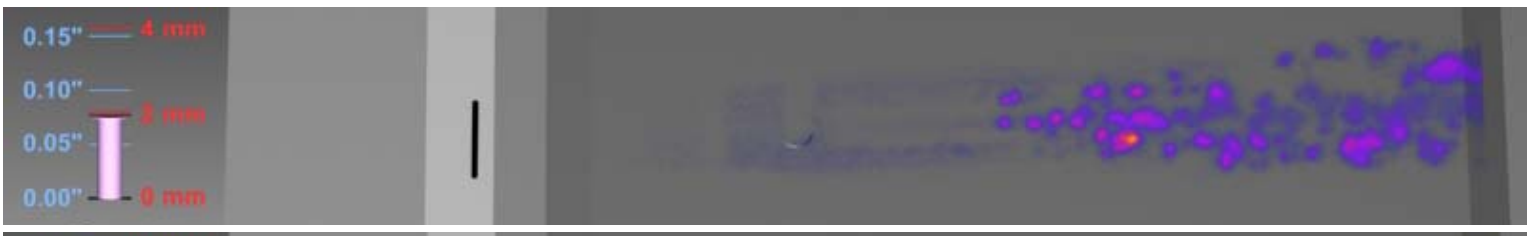

(i)

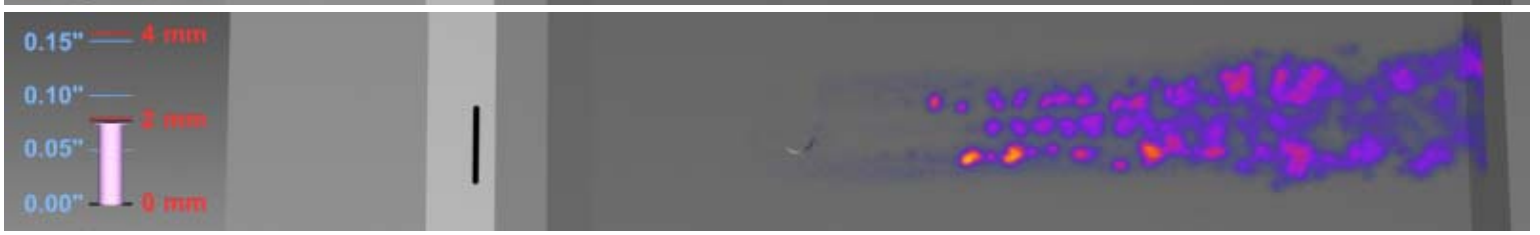

Figure A23: Test 443, Run 18, 1-mm tall by 4-mm wide cylinder, plate angle $=20^{\circ}, 11-\mathrm{mm}$ wide slot seeding, $\dot{m}=$ $300 \mathrm{sccm}, \mathrm{P}_{0}=9.32 \mathrm{MPa}$, sheet position $=2.0 \mathrm{~mm}$ above the plate, framing rate $=10 \mathrm{~Hz}$. 
(a)

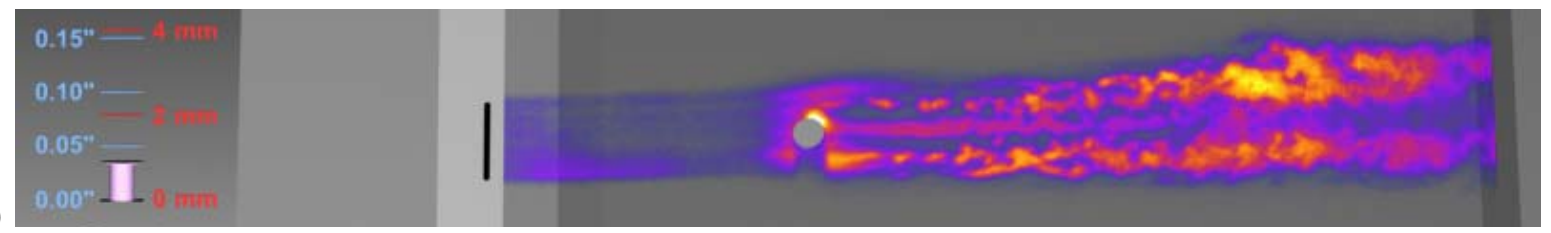

(b)

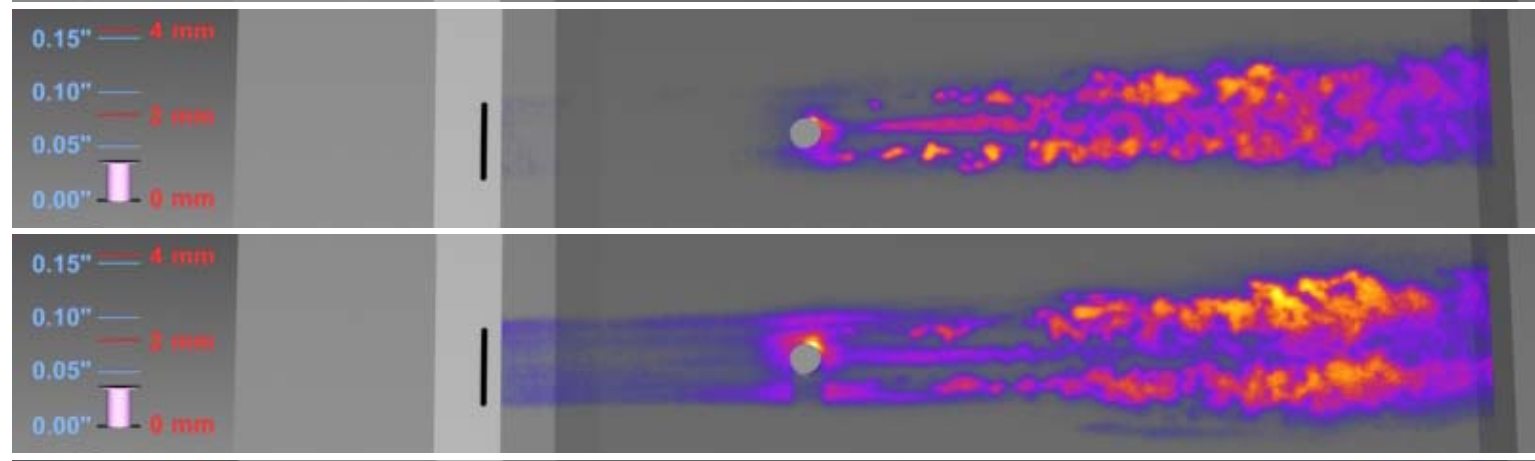

(c)

(d)

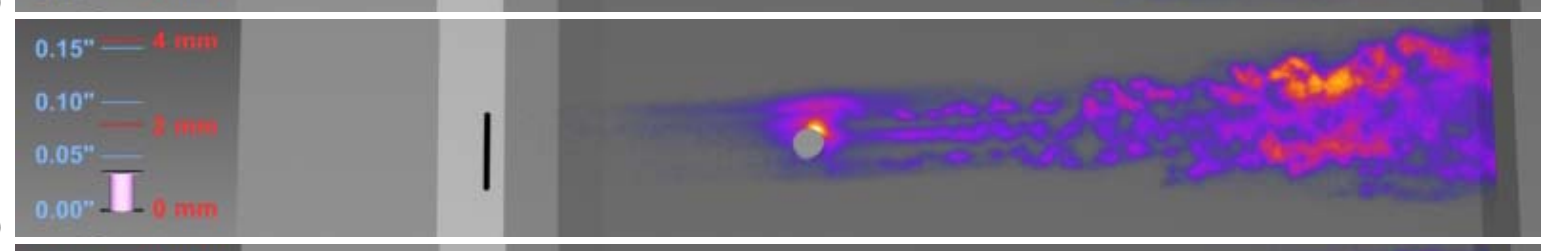

(e)

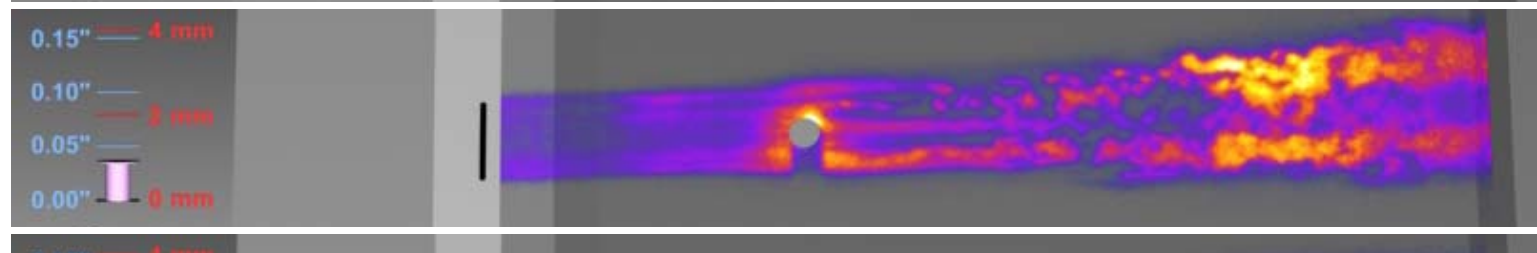

(f)
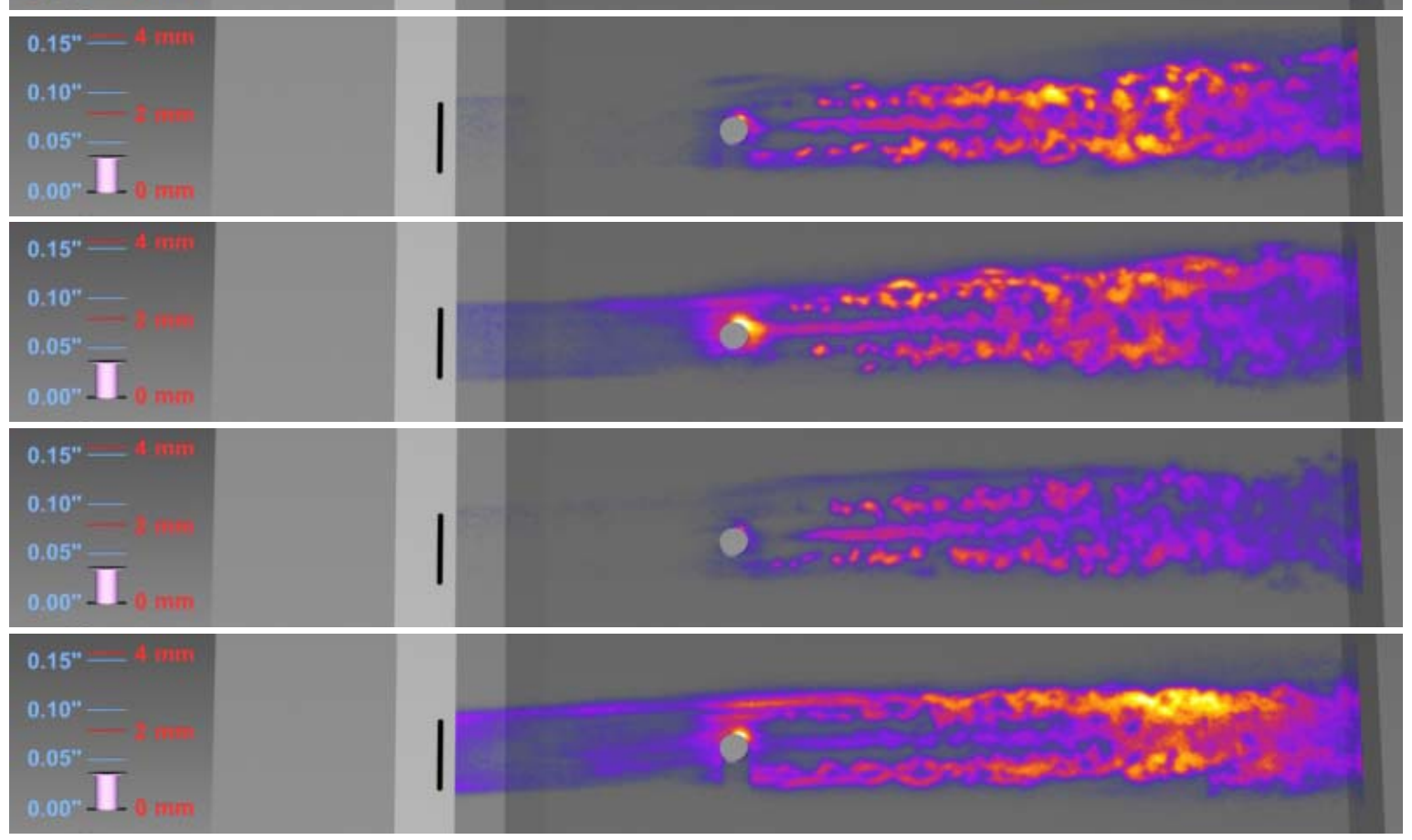

(i)

Figure A24: Test 443, Run 18, 1-mm tall by 4-mm wide cylinder, plate angle $=20^{\circ}, 11-\mathrm{mm}$ wide slot seeding, $\dot{m}=$ $300 \mathrm{sccm}, \mathrm{P}_{0}=9.32 \mathrm{MPa}$, sheet position $=0.9 \mathrm{~mm}$ above the plate, framing rate $=10 \mathrm{~Hz}$. 
(a)

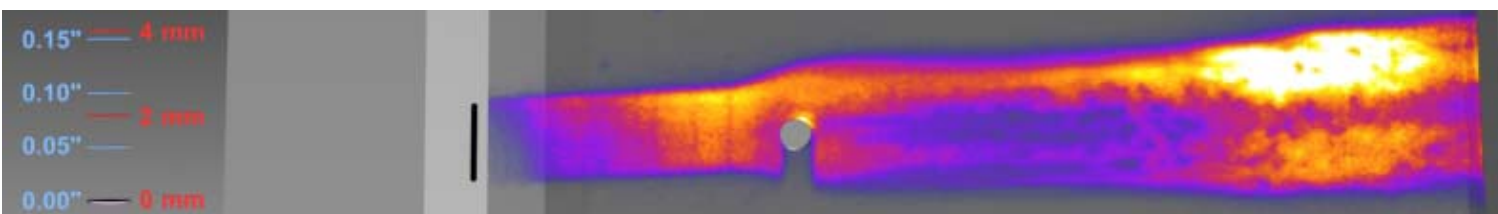

(b)

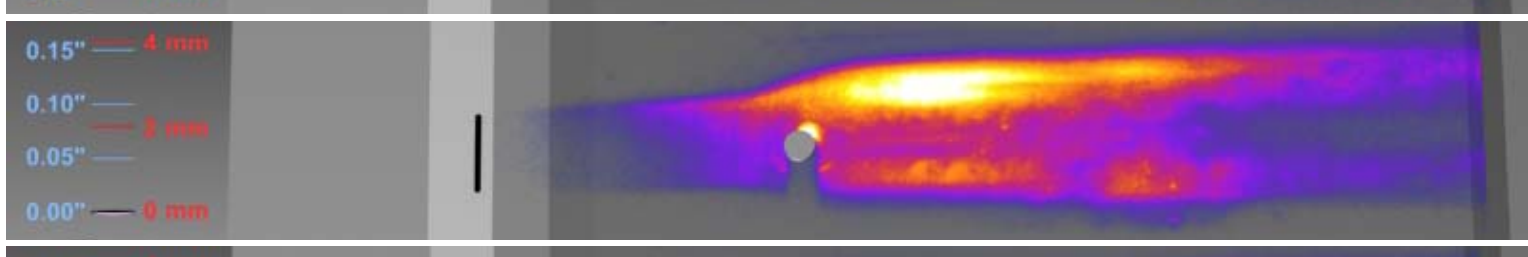

(c)

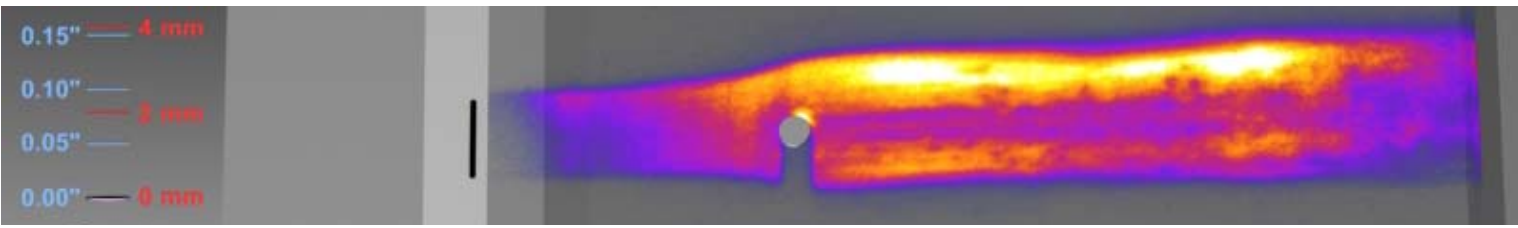

(d)

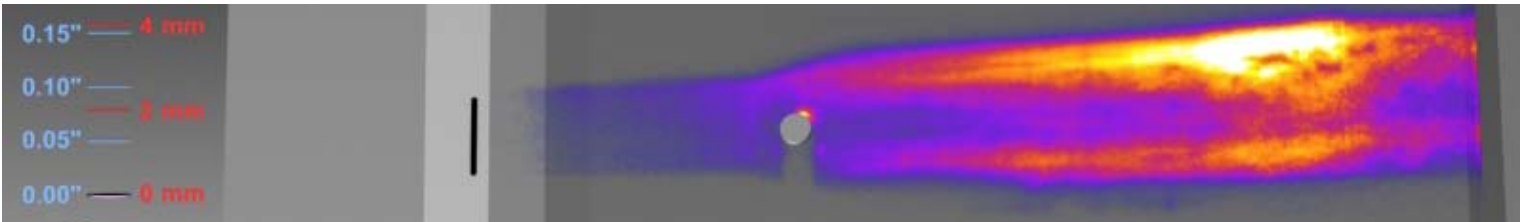

(e)

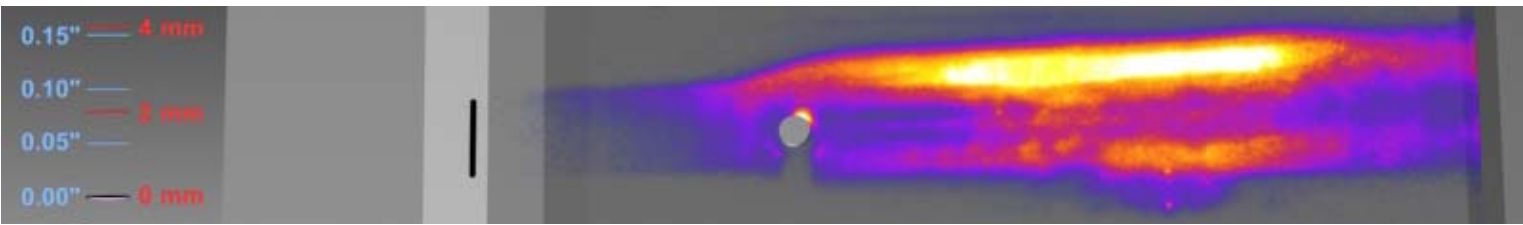

(f)

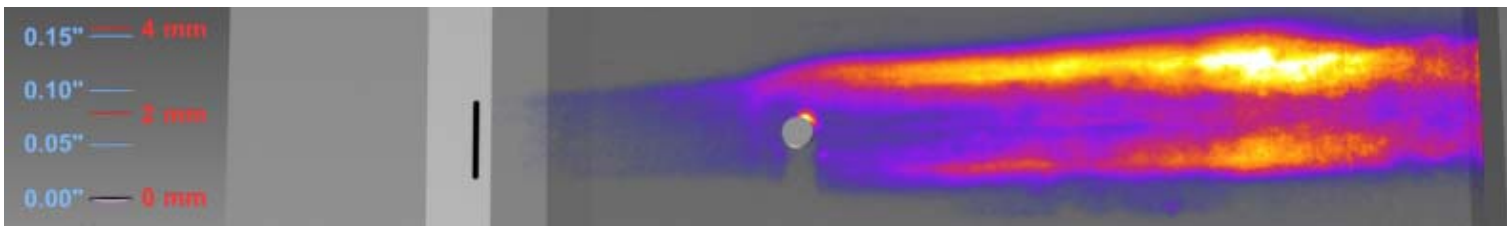

(g)

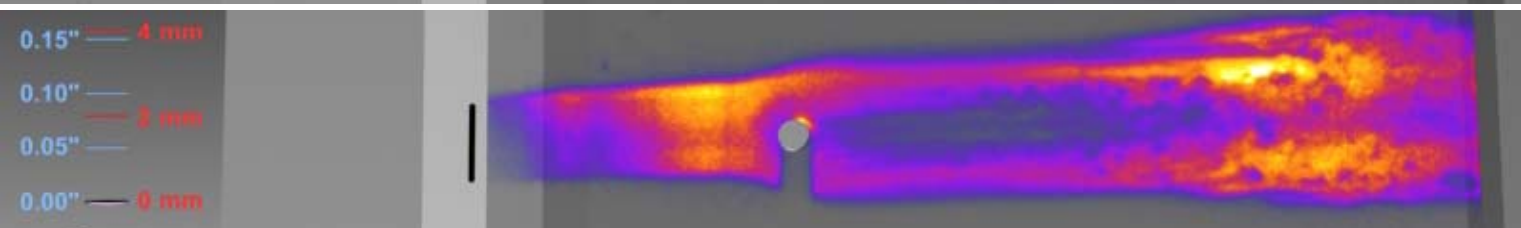

(h)

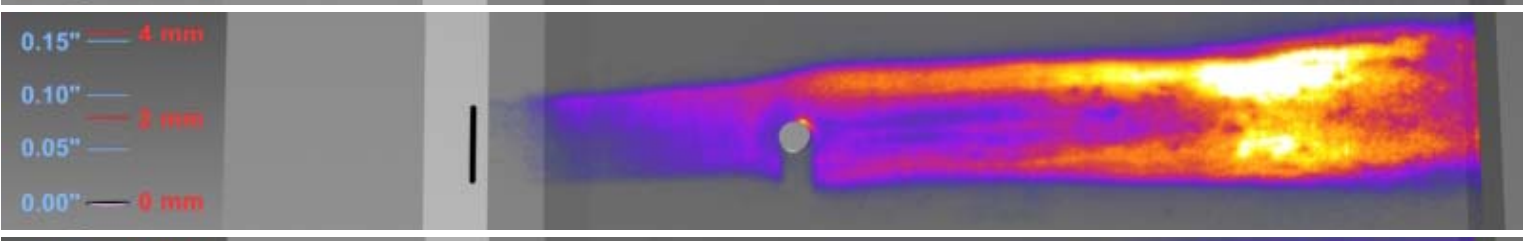

(i)

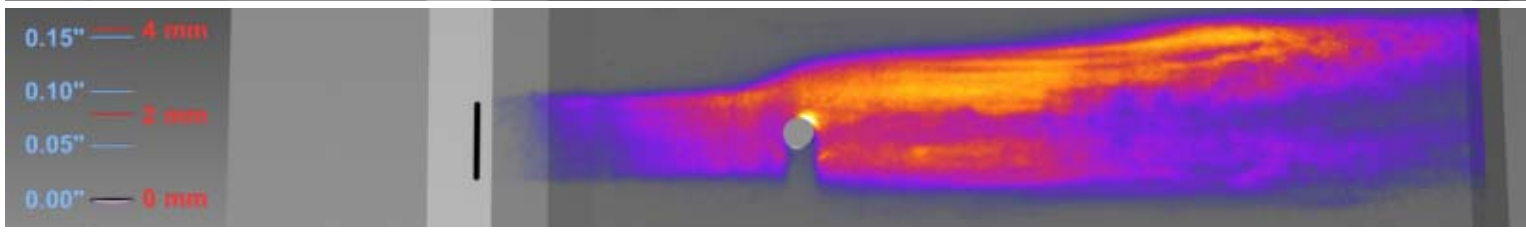

Figure A25: Test 443, Run 18, 1-mm tall by 4-mm wide cylinder, plate angle $=20^{\circ}, 11-\mathrm{mm}$ wide slot seeding, $\dot{\mathrm{m}}=$ $300 \mathrm{sccm}, \mathrm{P}_{0}=9.32 \mathrm{MPa}$, sheet position $=0.0 \mathrm{~mm}$ above the plate, framing rate $=10 \mathrm{~Hz}$. 
(a)

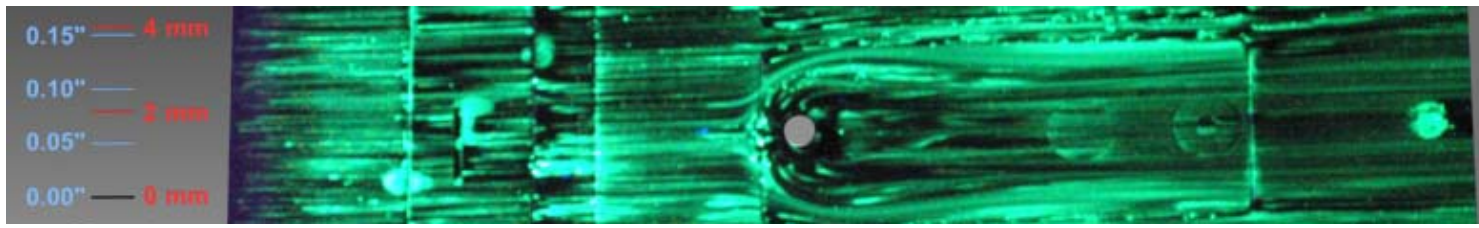

(b)

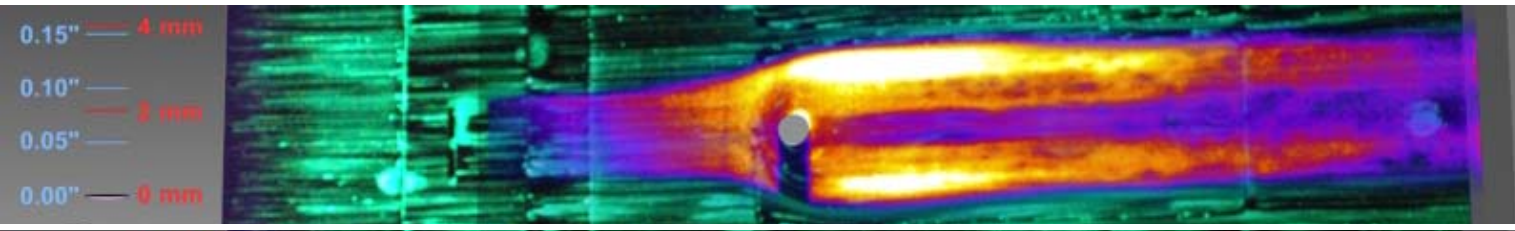

(c)

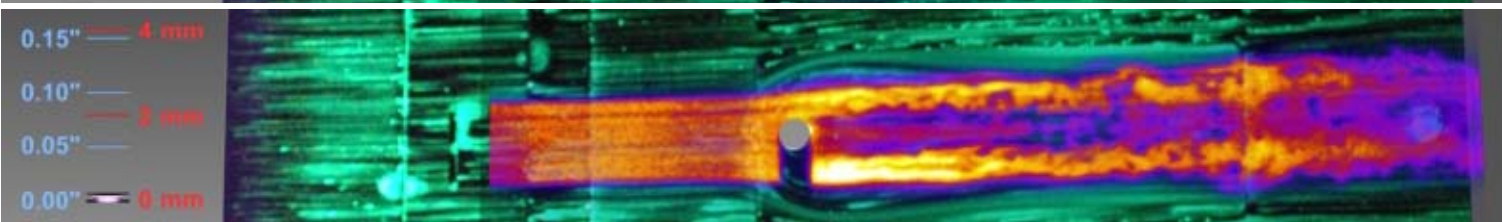

(d)

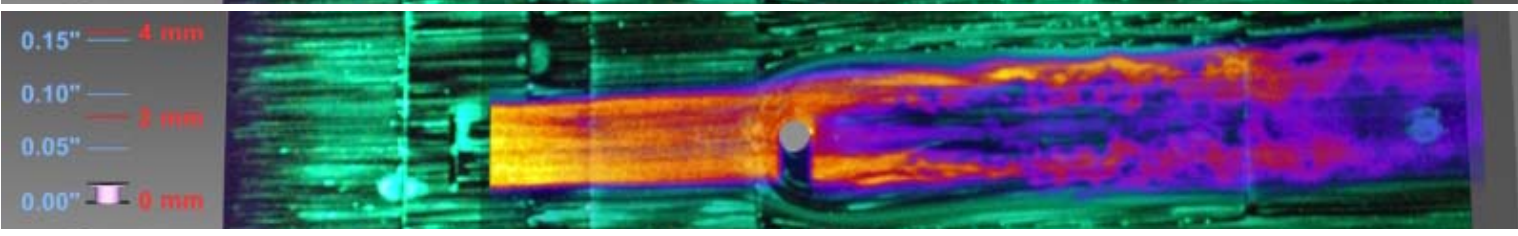

(e)

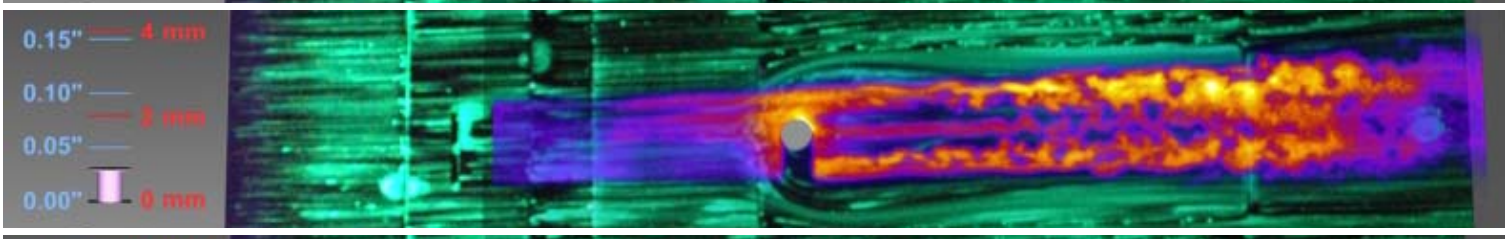

(f)

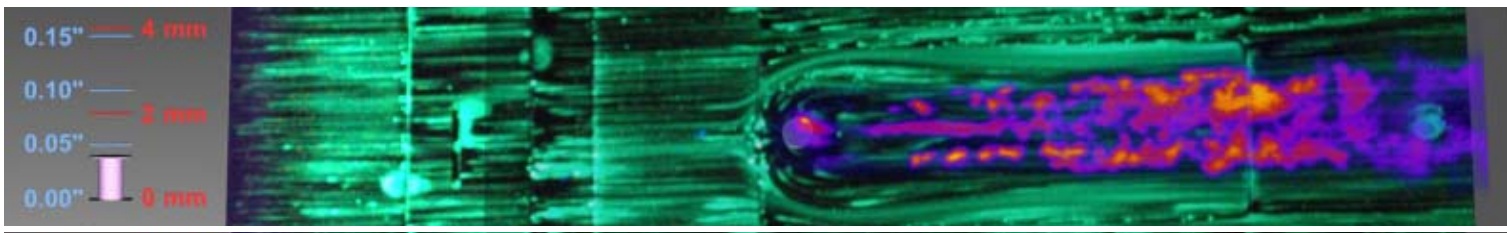

(g)

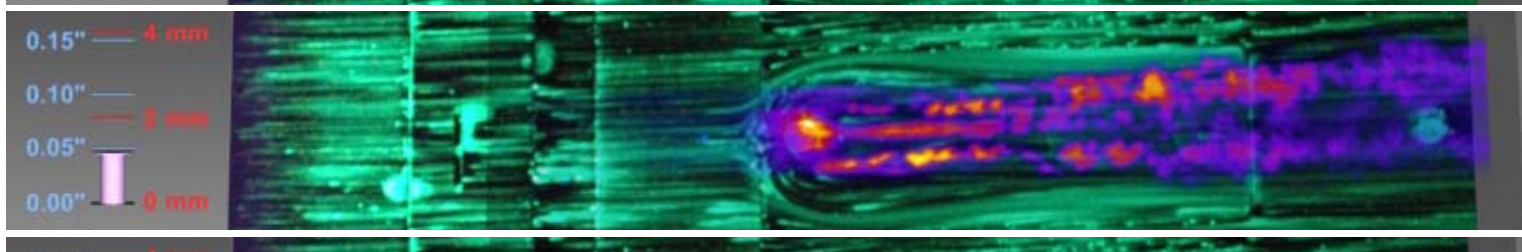

(h)

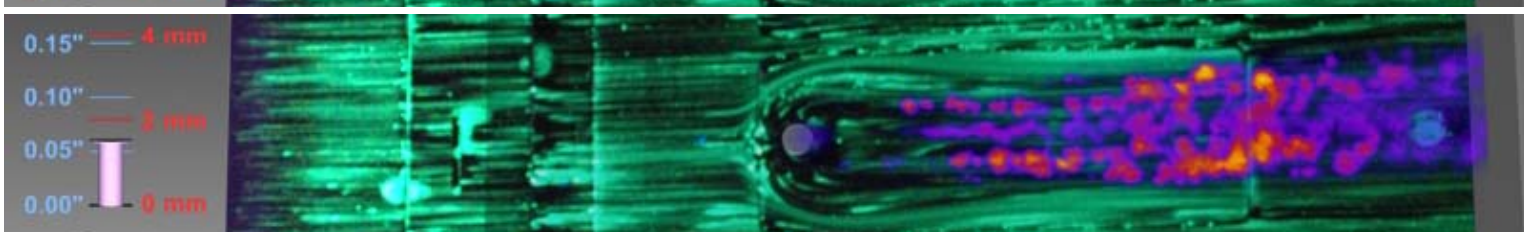

(i)

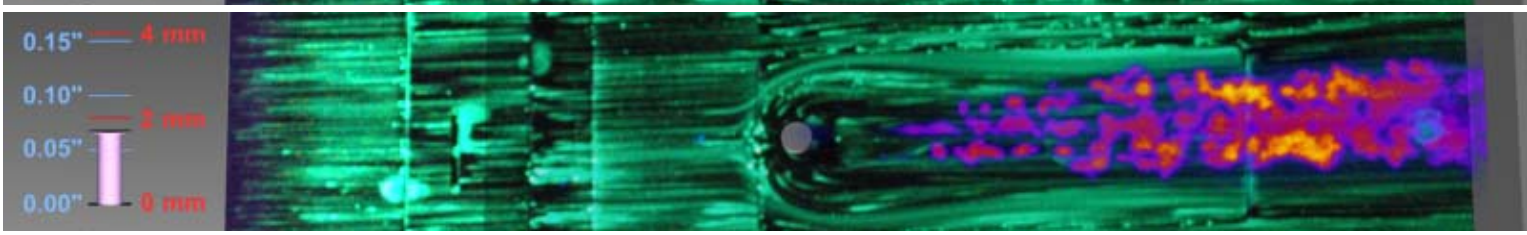

Figure A26: Test 443, Run 18, 1-mm tall by 4-mm wide cylinder with oil flow, plate angle $=20^{\circ}$, 11 -mm wide slot seeding, $\dot{m}=300 \mathrm{sccm}, \mathrm{P}_{0}=9.32 \mathrm{MPa}$, sheet position varies from 0.0 to $1.75 \mathrm{~mm}$ above the plate in $0.25 \mathrm{~mm}$ increments in (b)-(i), (a) shows oil flow only, framing rate $=10 \mathrm{~Hz}$. 
(a)

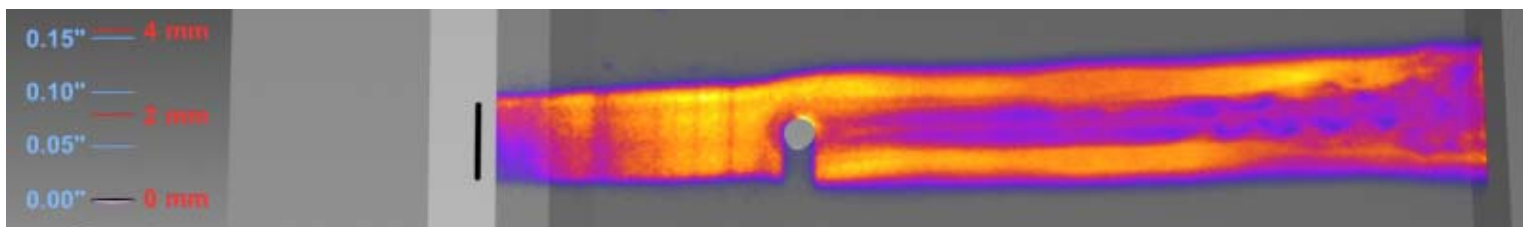

(b)

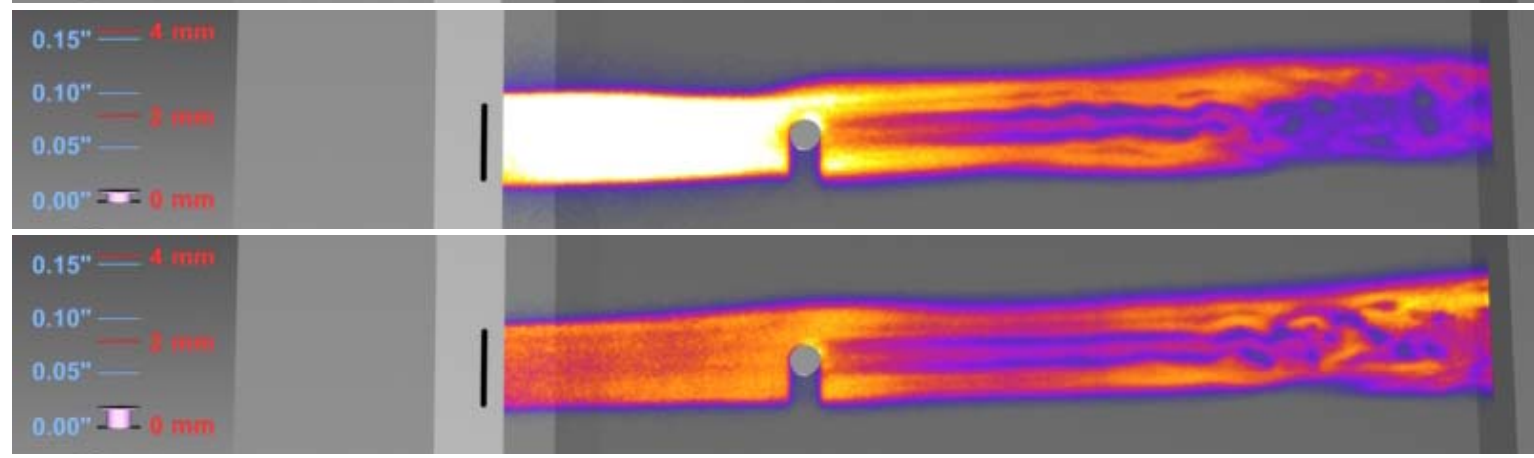

(c)

(d)

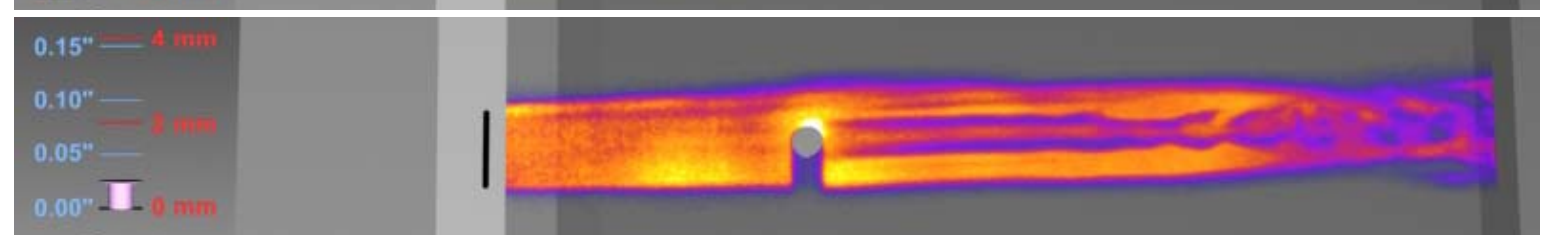

(e)

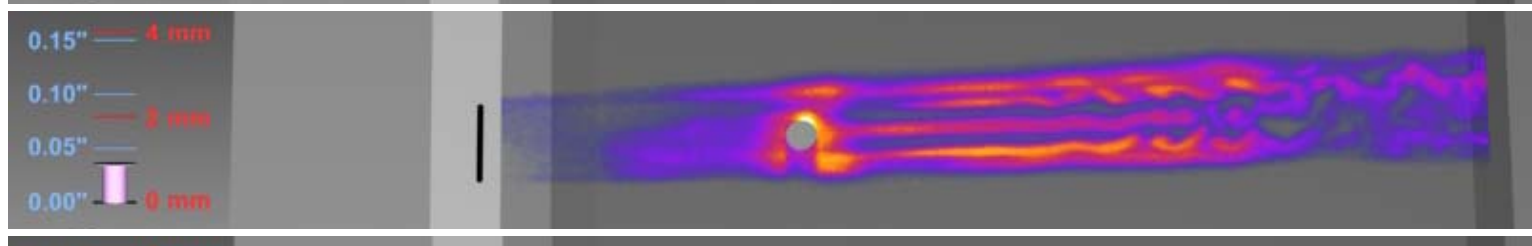

(f)

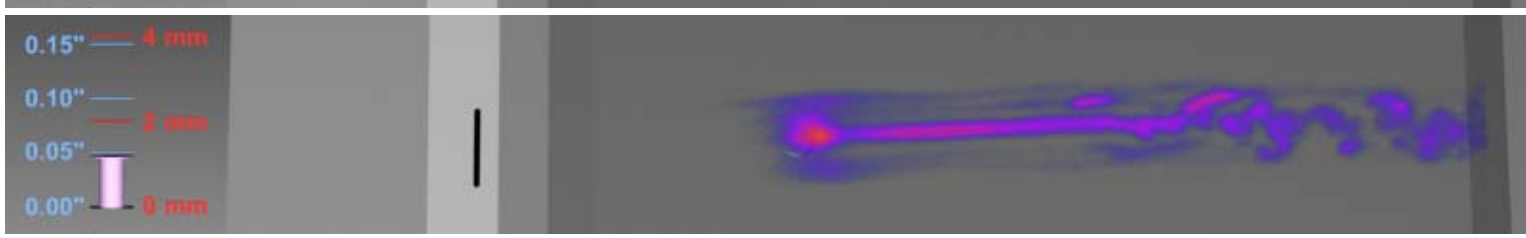

(g)

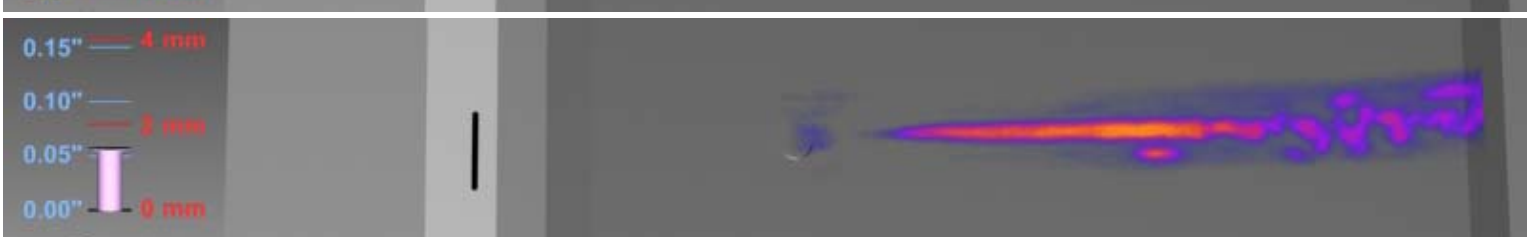

(h)

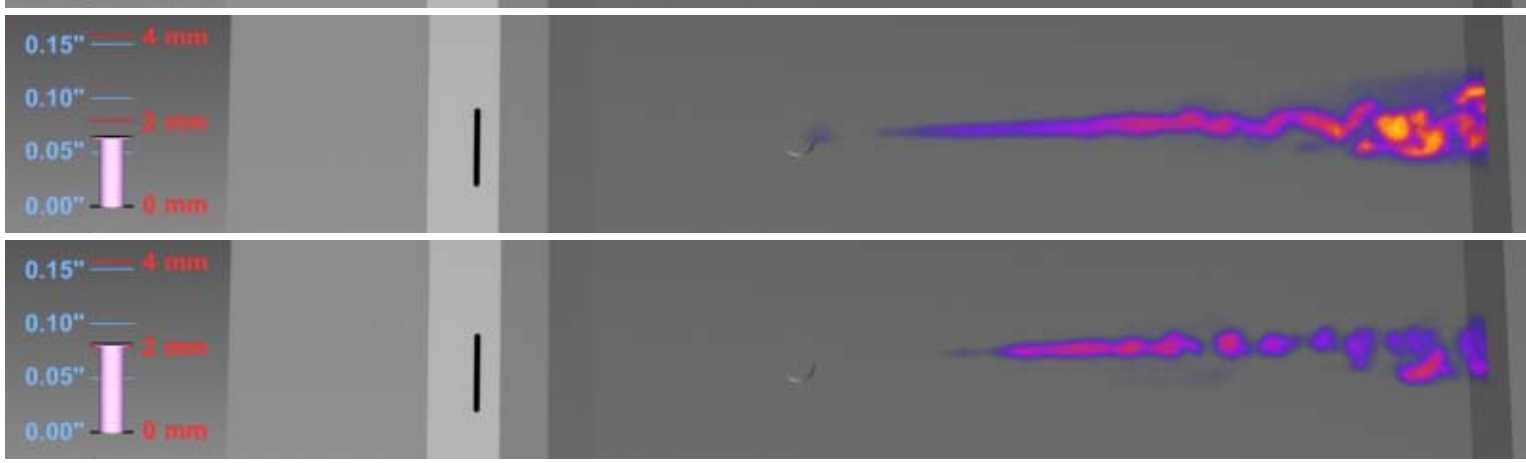

Figure A27: Test 443, Run 19, 1-mm tall by 4-mm wide cylinder, plate angle $=20^{\circ}, 11$-mm wide slot seeding, $\dot{m}=$ $300 \mathrm{sccm}, \mathrm{P}_{0}=4.97 \mathrm{MPa}$, sheet position varies from 0.0 to $2.0 \mathrm{~mm}$ above the plate in $0.25 \mathrm{~mm}$ increments in (a)-(i), framing rate $=10 \mathrm{~Hz}$. 
(a)

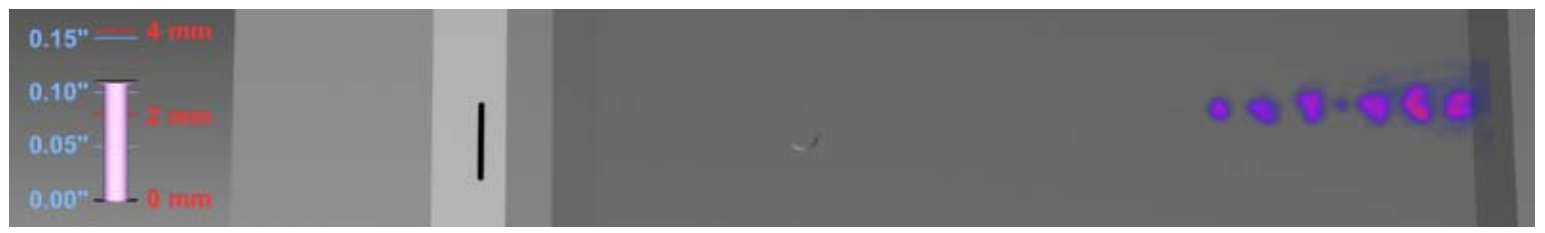

(b)

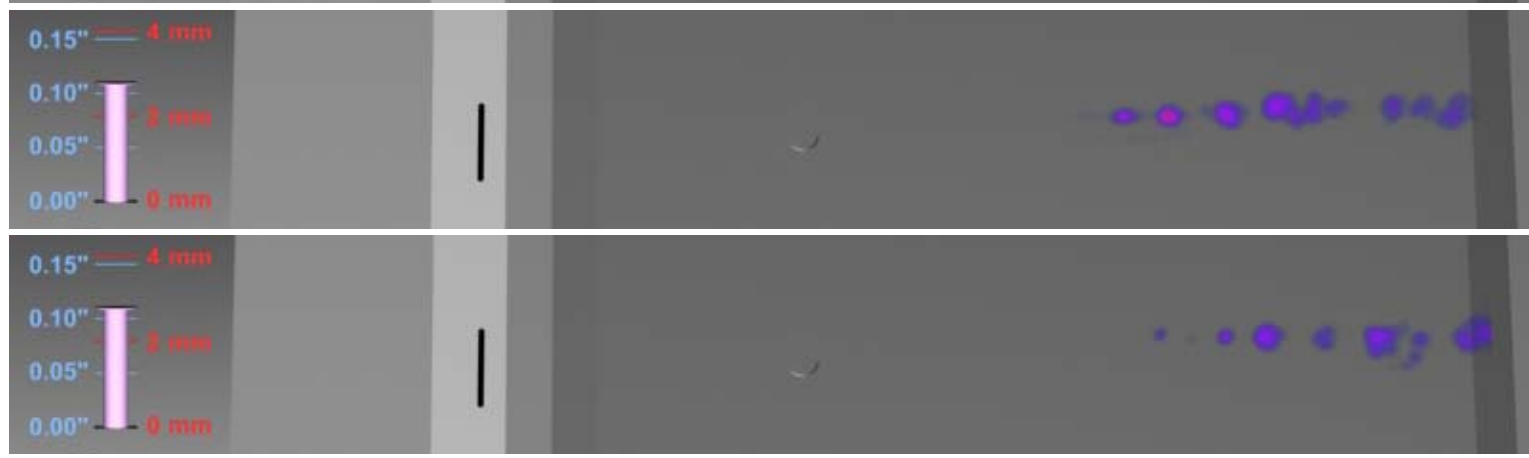

(c)

(d)

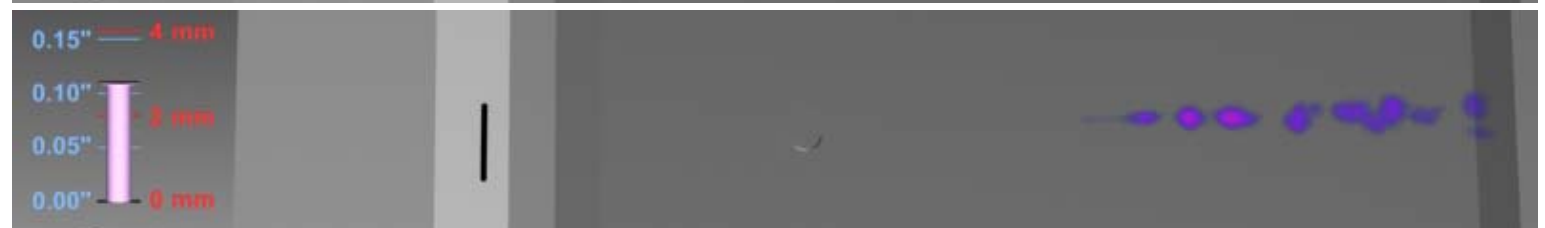

(e)

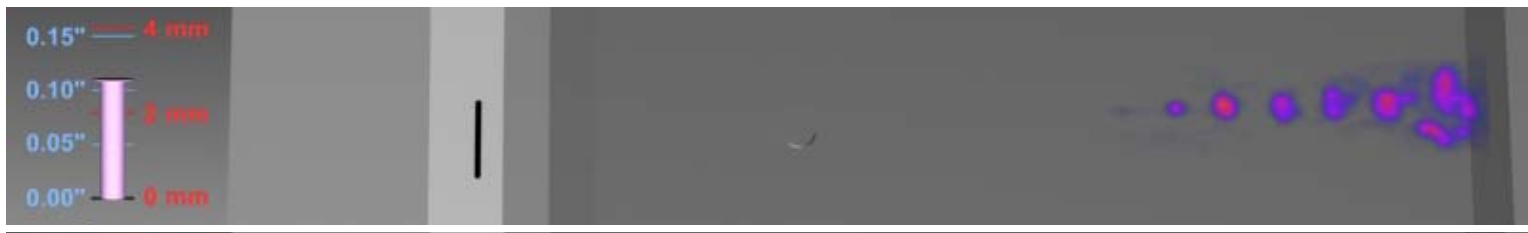

(f)

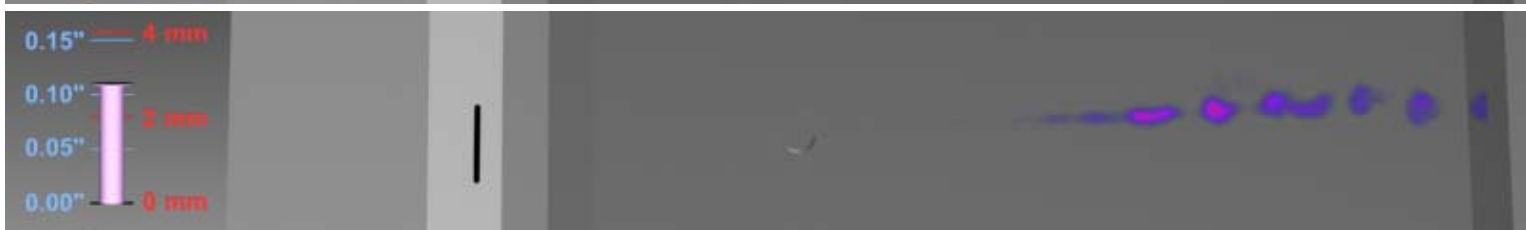

(g)
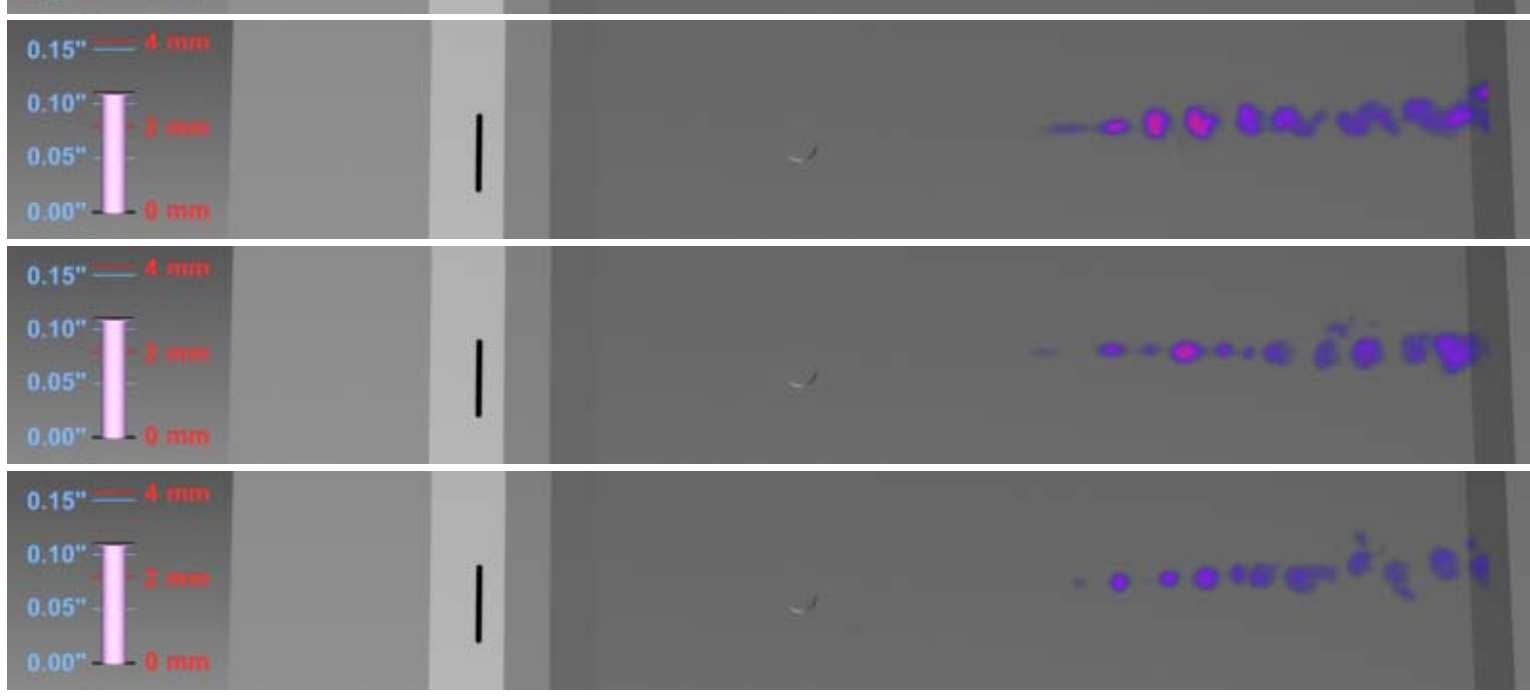

(i)

Figure A28: Test 443, Run 19, 1-mm tall by 4-mm wide cylinder, plate angle $=20^{\circ}, 11-\mathrm{mm}$ wide slot seeding, $\dot{m}=$ $300 \mathrm{sccm}, \mathrm{P}_{0}=4.97 \mathrm{MPa}$, sheet position $=2.8 \mathrm{~mm}$ above the plate, framing rate $=10 \mathrm{~Hz}$. 
(a)

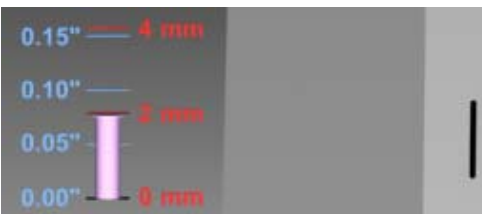

(b)

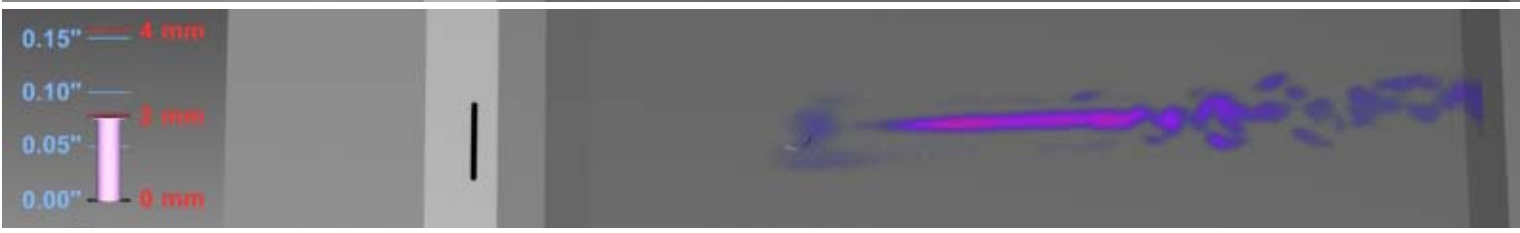

(c)

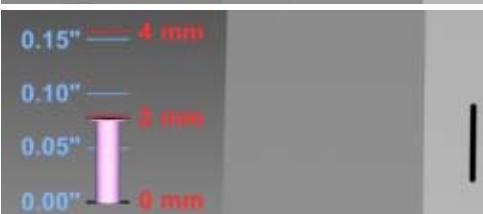

(d)

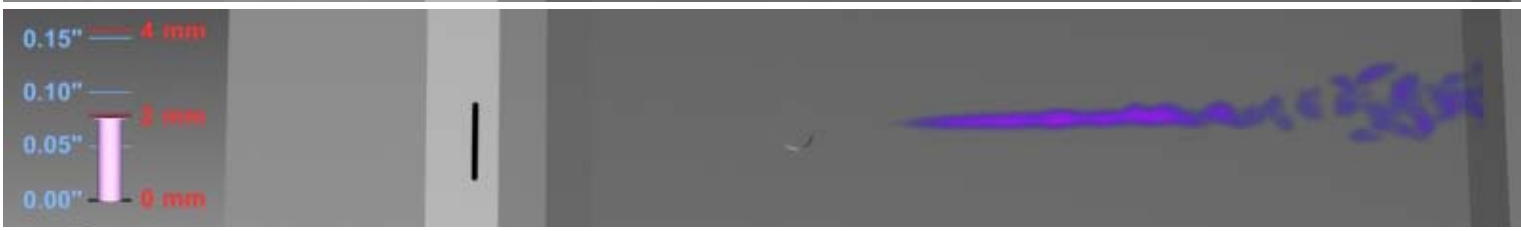

(e)

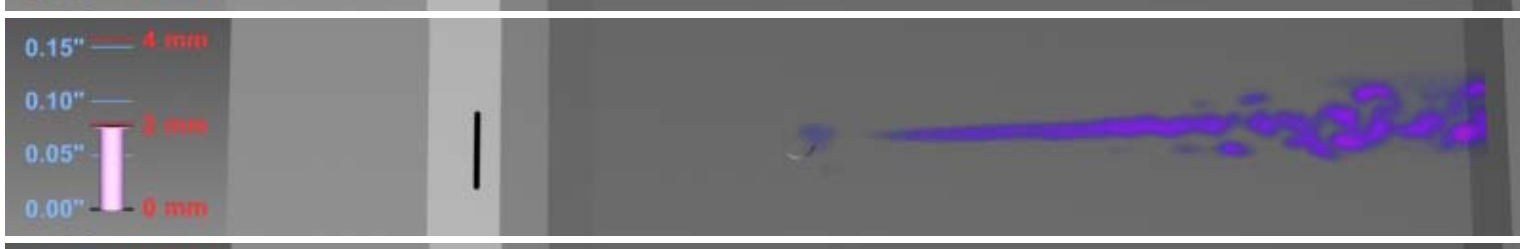

(f)

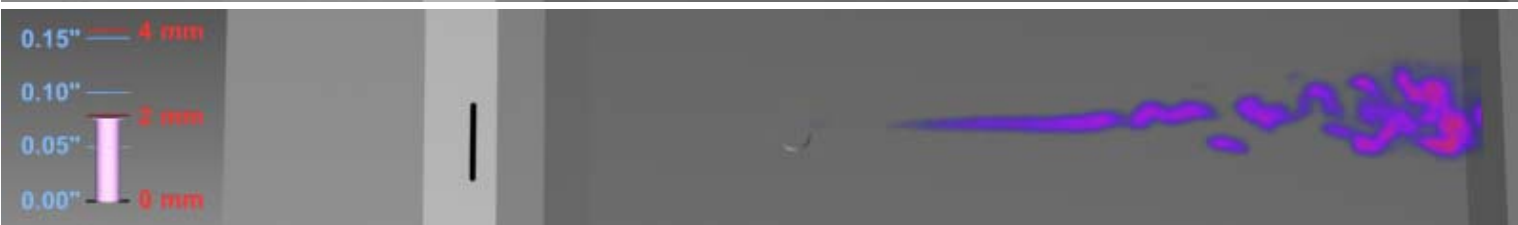

(g)

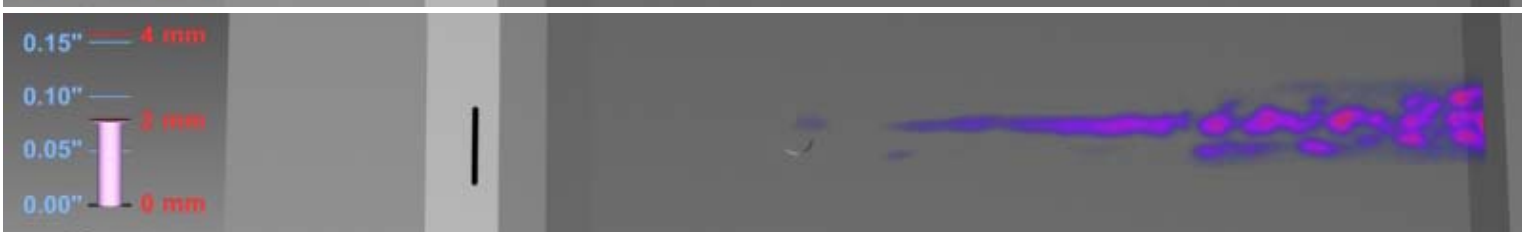

(h)

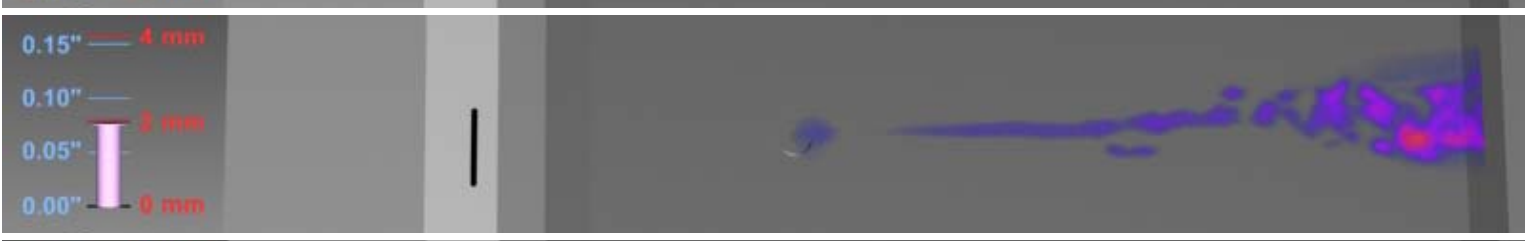

(i)

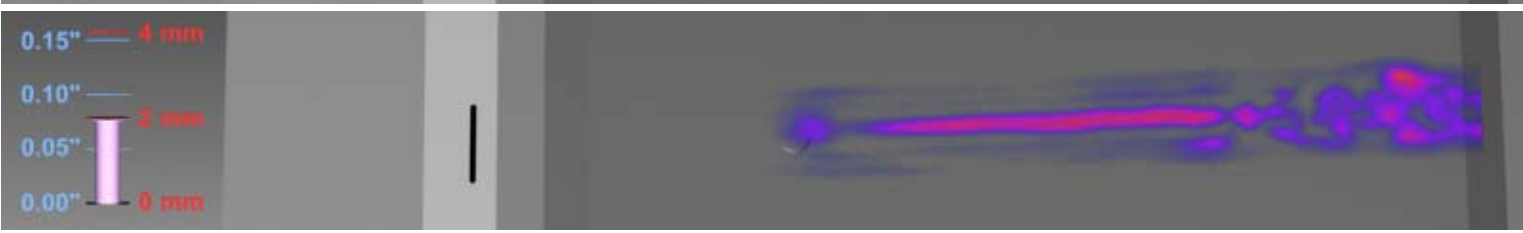

Figure A29: Test 443, Run 19, 1-mm tall by 4-mm wide cylinder, plate angle $=20^{\circ}, 11$-mm wide slot seeding, $\dot{m}=$ $300 \mathrm{sccm}, \mathrm{P}_{0}=4.97 \mathrm{MPa}$, sheet position $=2.0 \mathrm{~mm}$ above the plate, framing rate $=10 \mathrm{~Hz}$. 
(a)

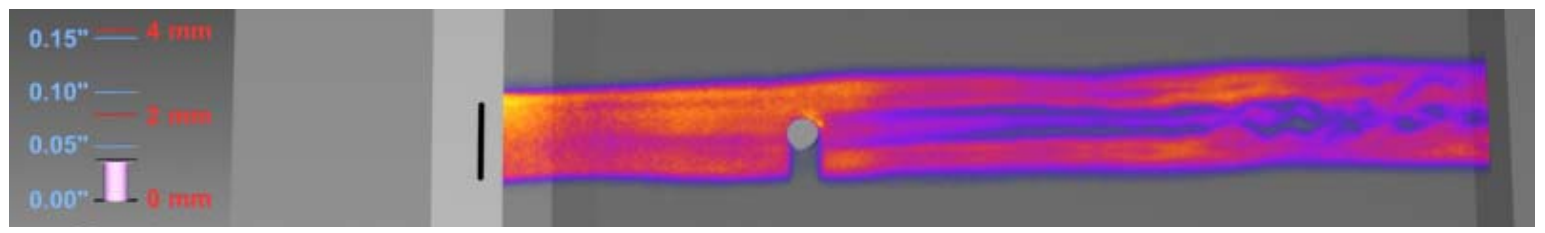

(b)

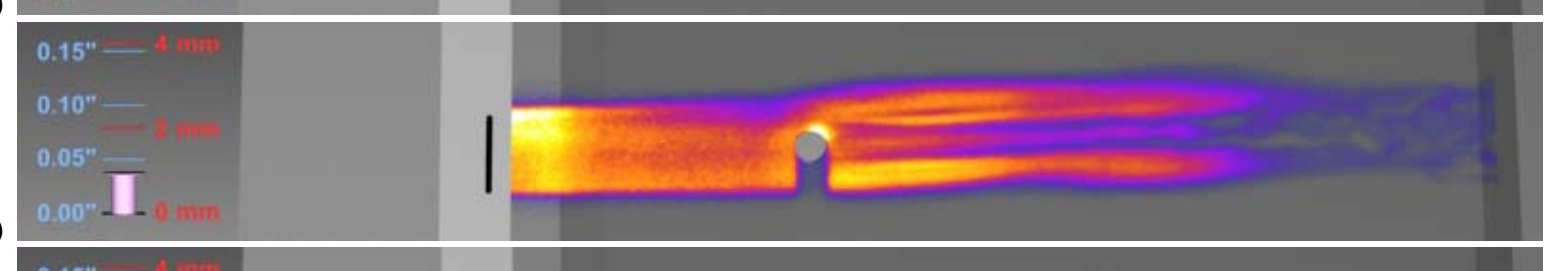

(c)

(d)
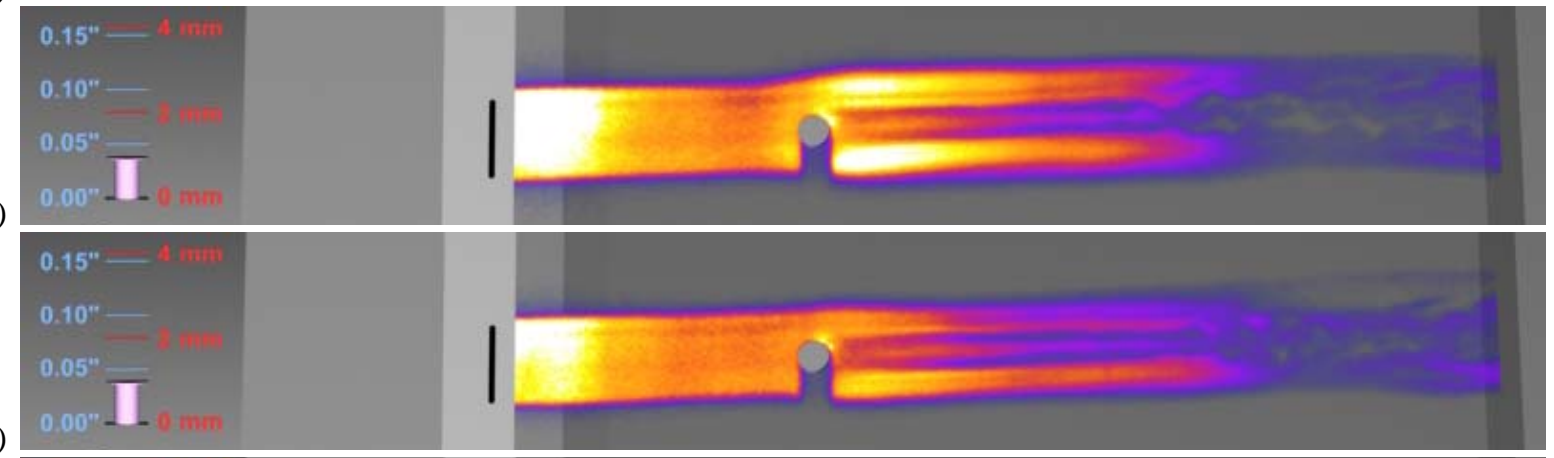

(e)

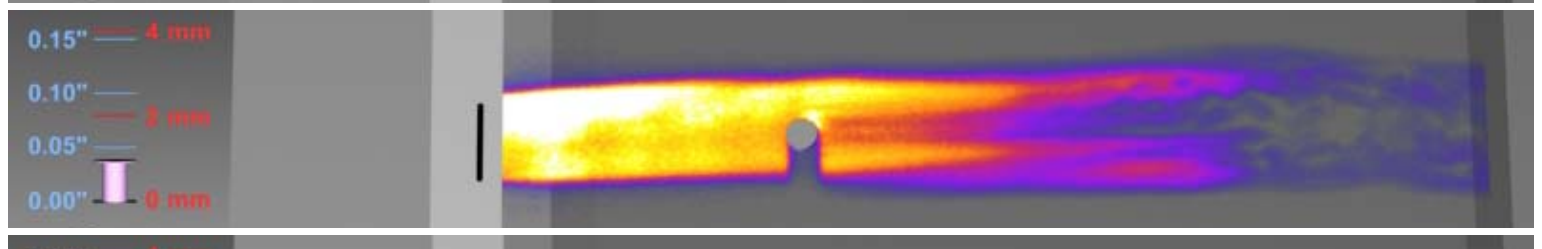

(f)

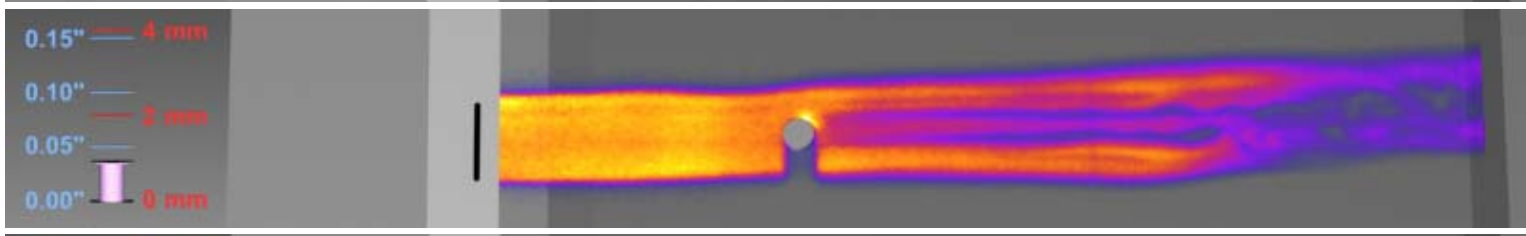

(g)

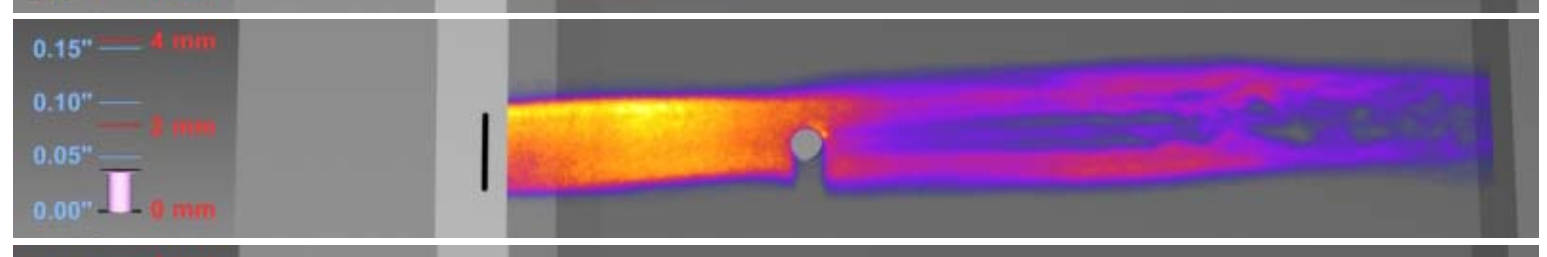

(h)

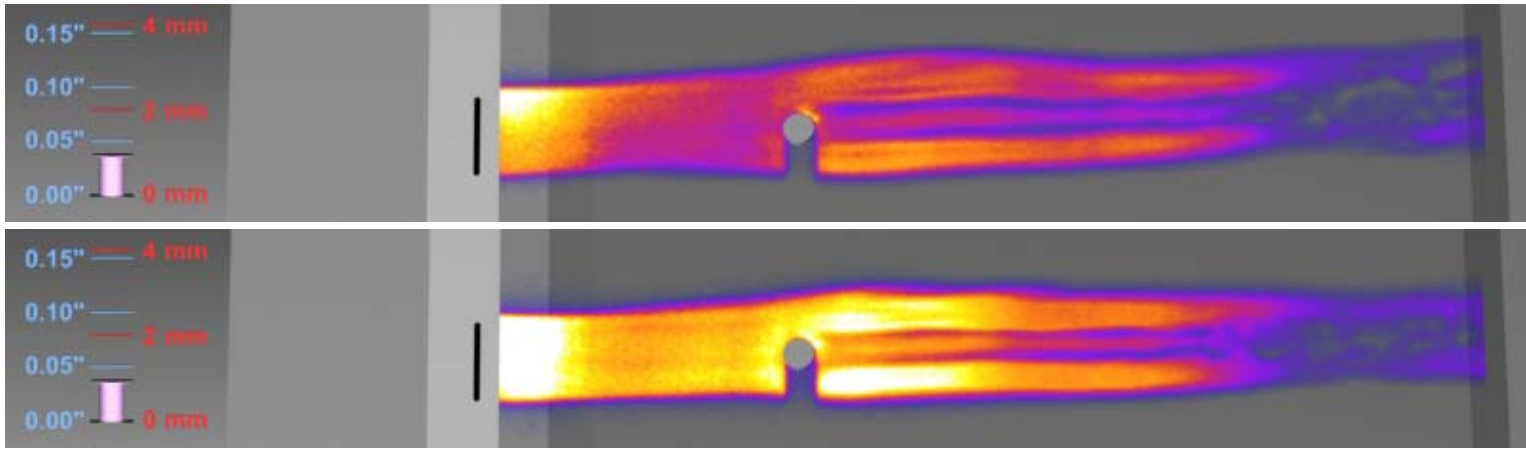

(i)

Figure A30: Test 443, Run 19, 1-mm tall by 4-mm wide cylinder, plate angle $=20^{\circ}, 11-\mathrm{mm}$ wide slot seeding, $\dot{m}=$ $300 \mathrm{sccm}, \mathrm{P}_{0}=4.97 \mathrm{MPa}$, sheet position $=1.0 \mathrm{~mm}$ above the plate, framing rate $=10 \mathrm{~Hz}$. 
(a)

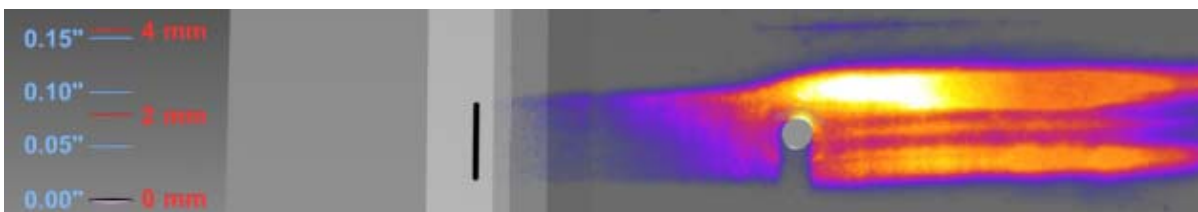

(b)

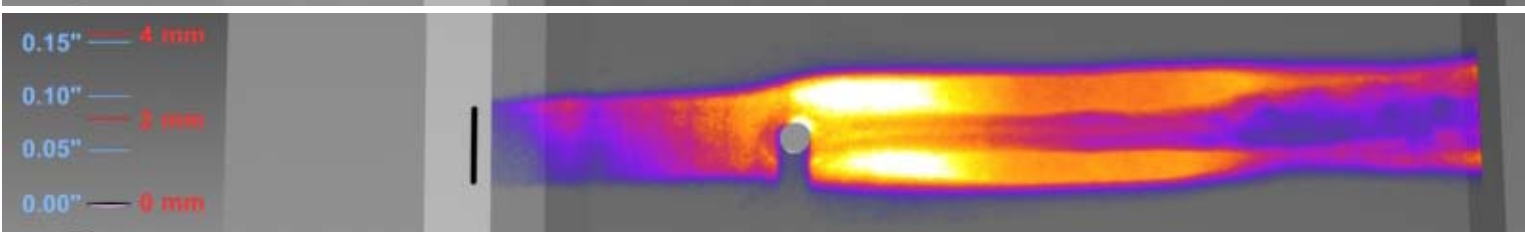

(c)

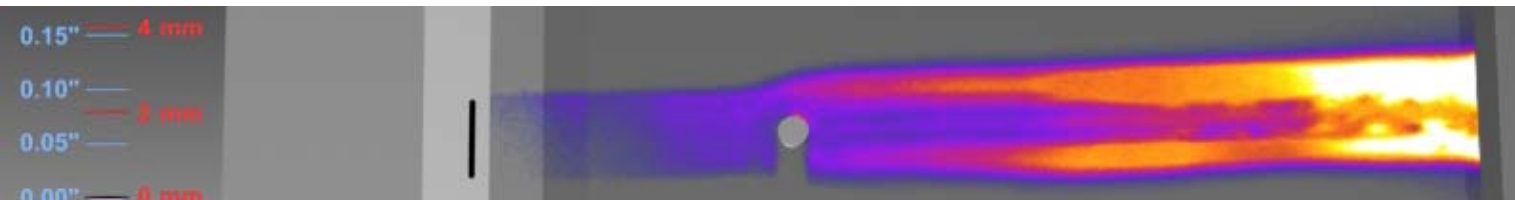

(d)

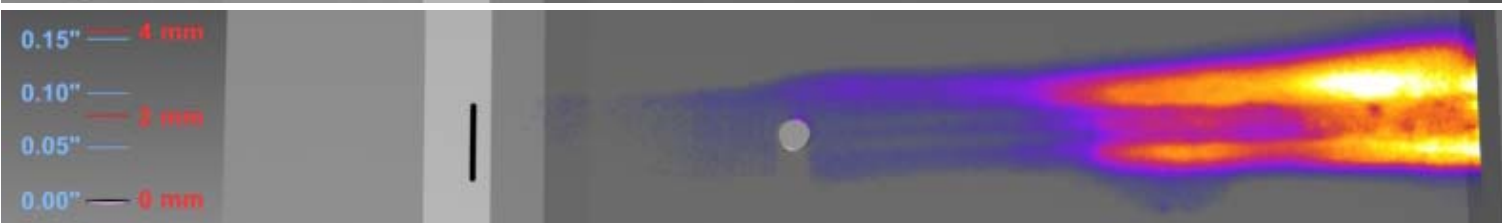

(e)

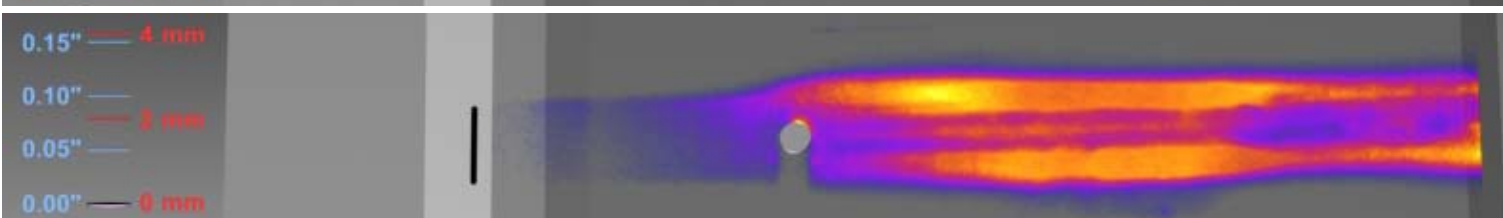

(f)

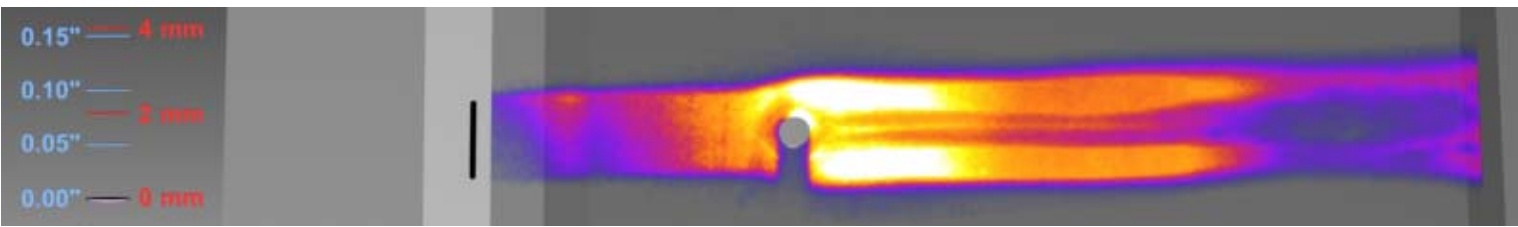

(g)

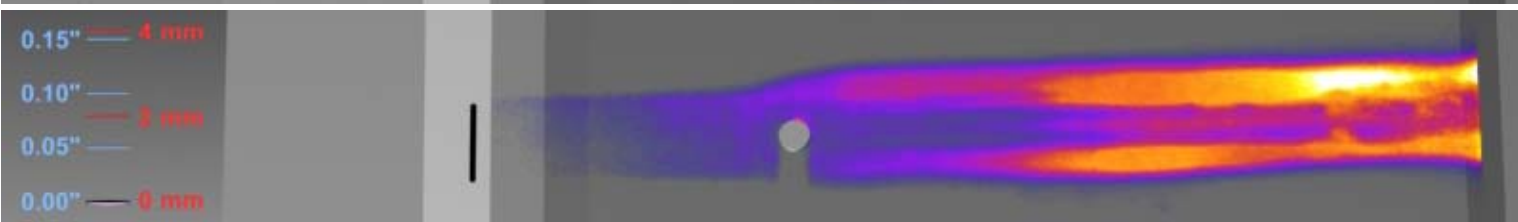

(h)

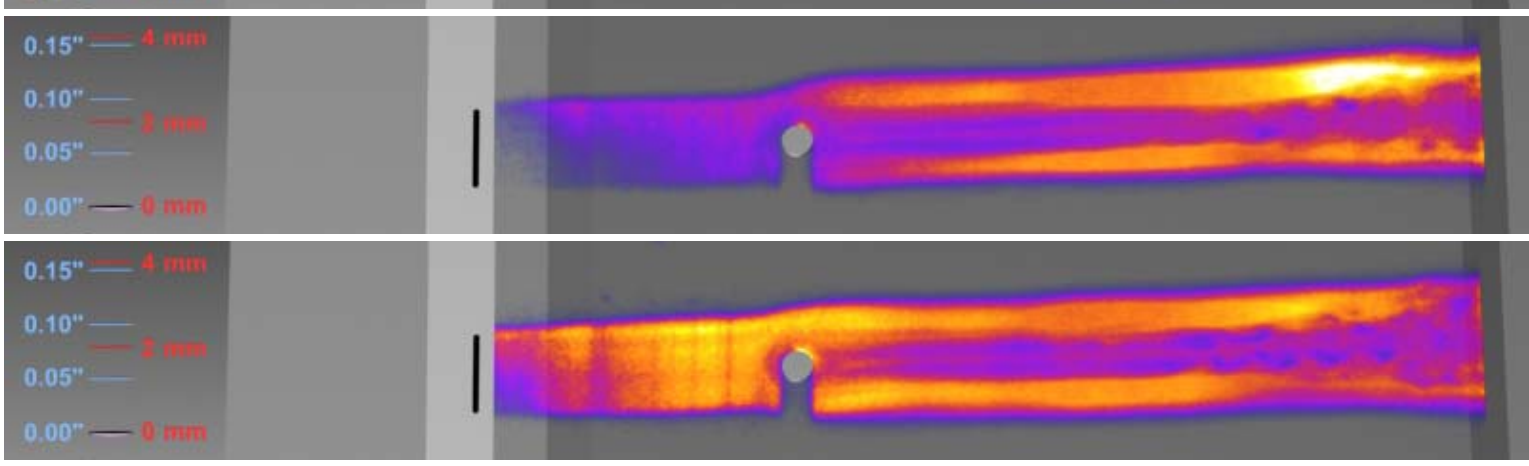

Figure A31: Test 443, Run 19, 1-mm tall by 4-mm wide cylinder, plate angle $=20^{\circ}, 11$-mm wide slot seeding, $\dot{m}=$ $300 \mathrm{sccm}, \mathrm{P}_{0}=4.97 \mathrm{MPa}$, sheet position $=0.0 \mathrm{~mm}$ above the plate, framing rate $=10 \mathrm{~Hz}$. 
(a)

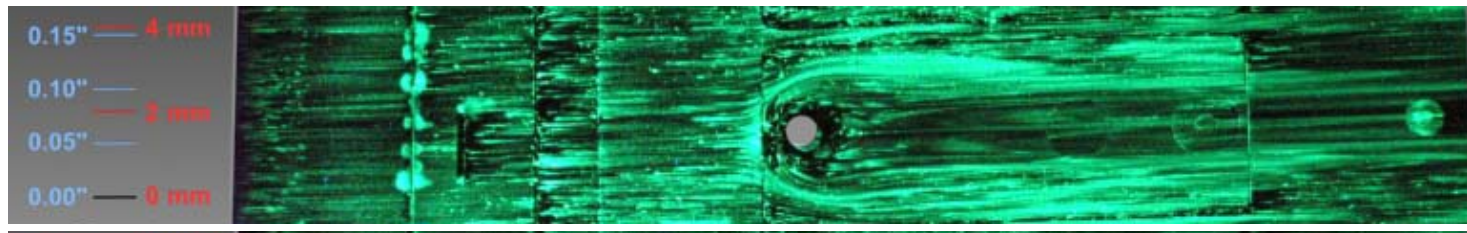

(b)

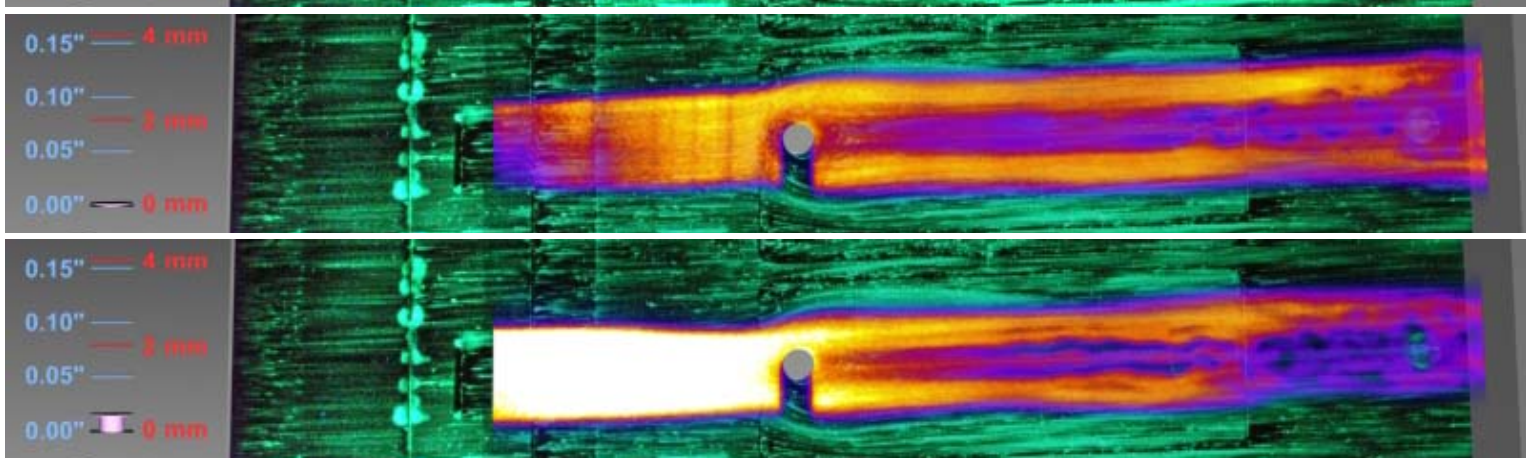

(c)

(d)

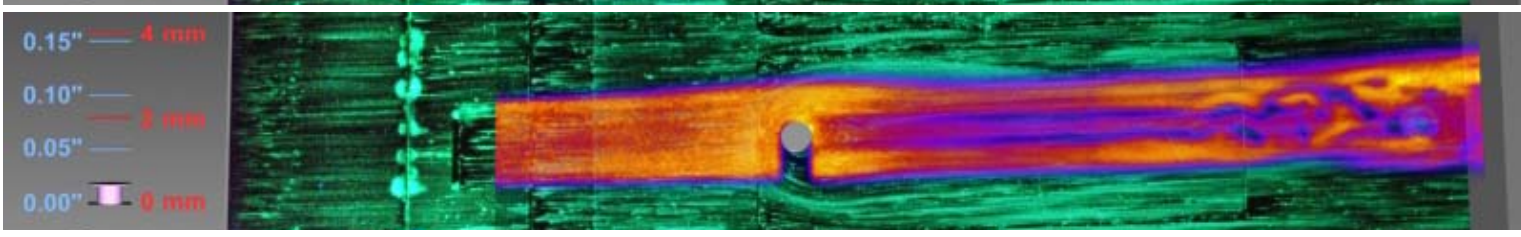

(e)

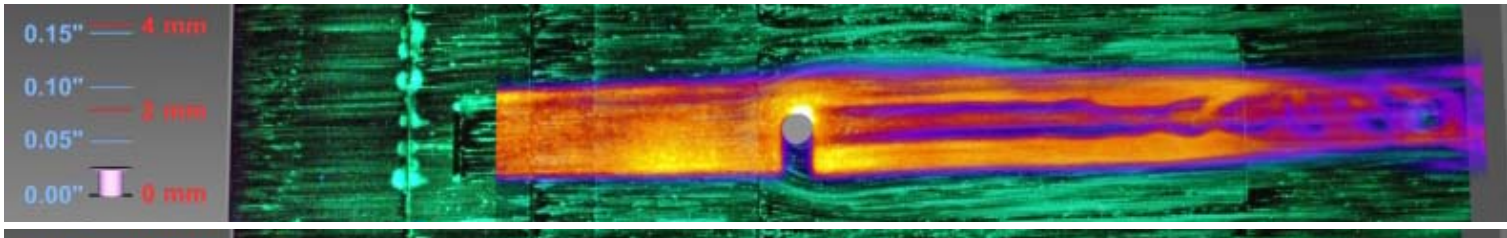

(f)

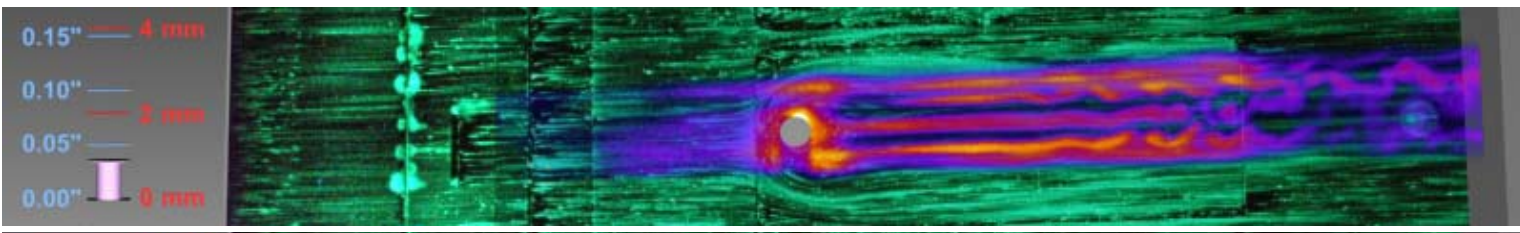

(g)

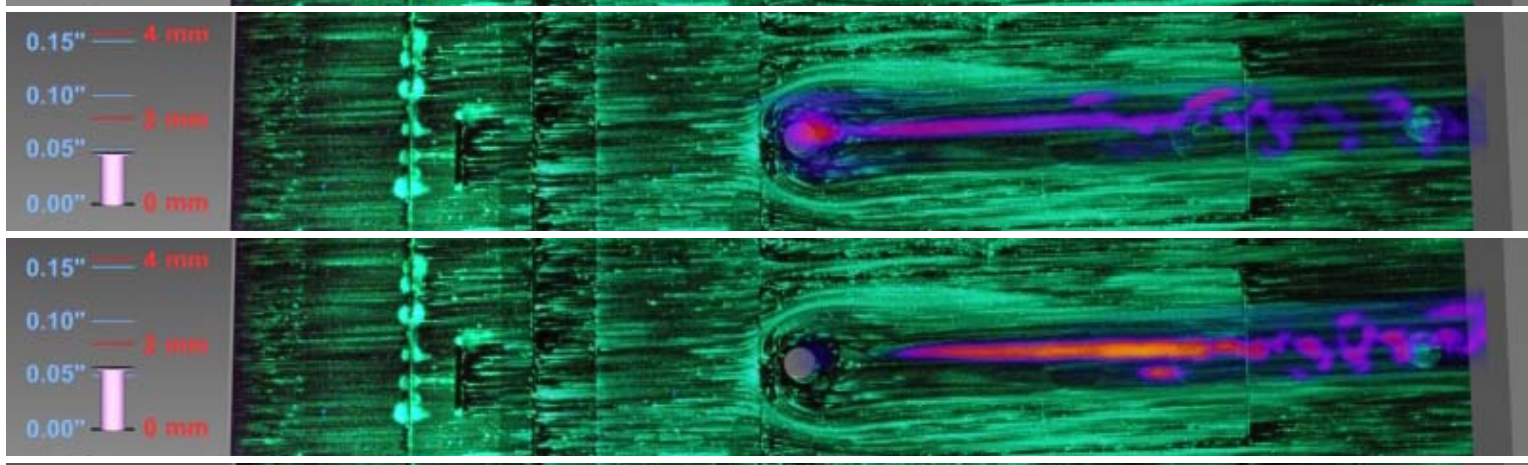

(i)

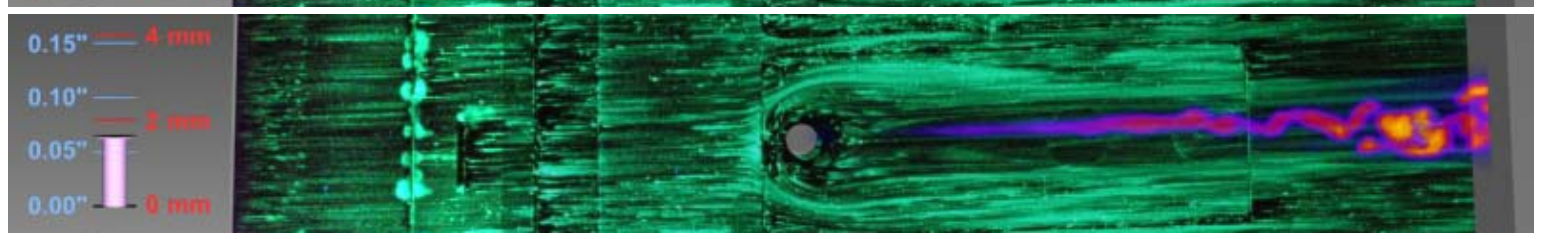

Figure A32: Test 443, Run 19, 1-mm tall by 4-mm wide cylinder, plate angle $=20^{\circ}, 11$-mm wide slot seeding, $\dot{m}=$ $300 \mathrm{sccm}, \mathrm{P}_{0}=4.97 \mathrm{MPa}$, sheet position varies from 0.0 to $1.75 \mathrm{~mm}$ above the plate in $0.25 \mathrm{~mm}$ increments in (b)(i), (a) shows oil flow only, framing rate $=10 \mathrm{~Hz}$. 
(a)

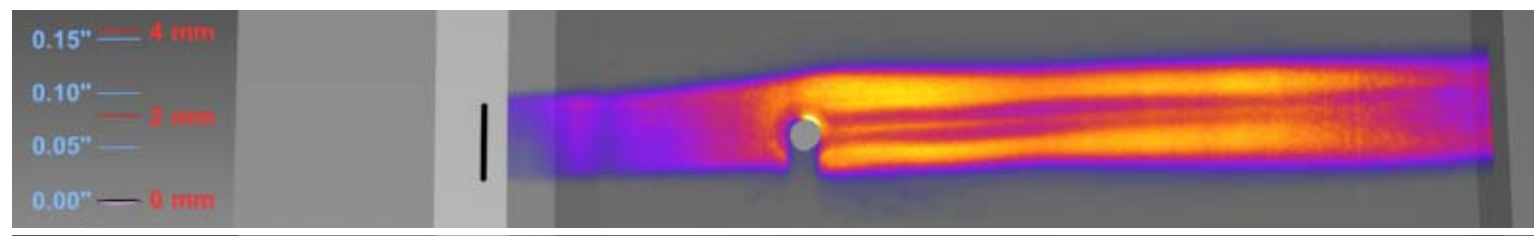

(b)

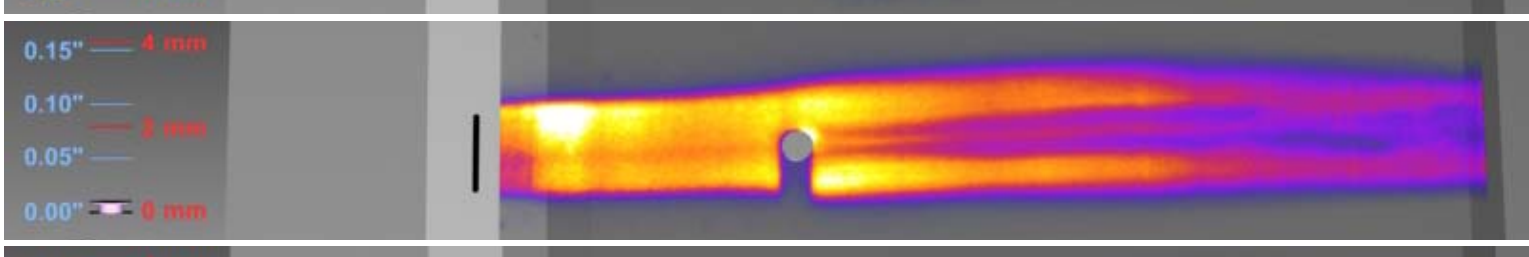

(c)

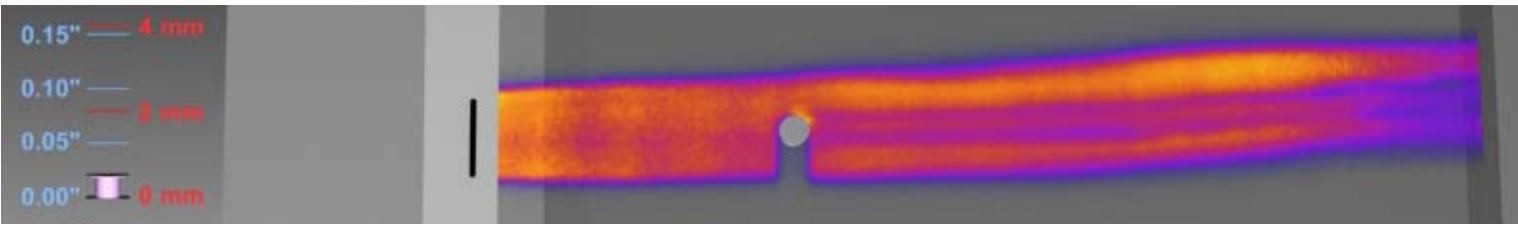

(d)

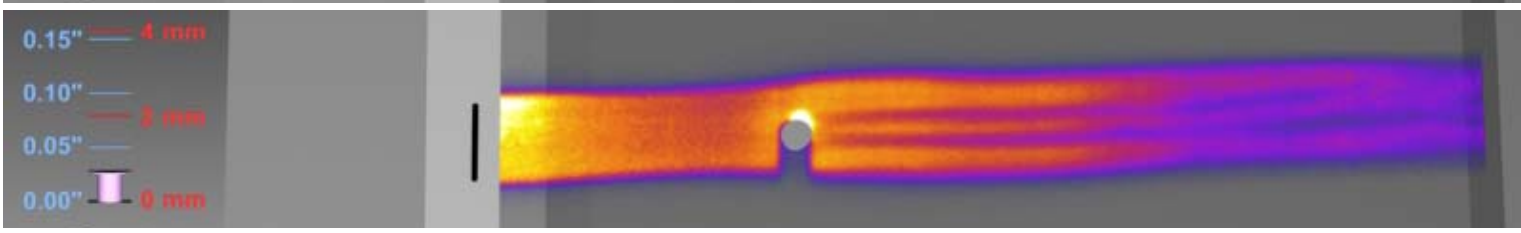

(e)

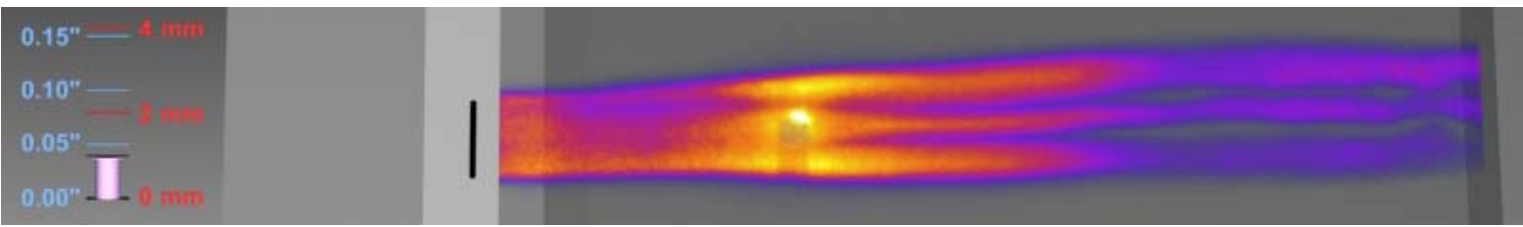

(f)

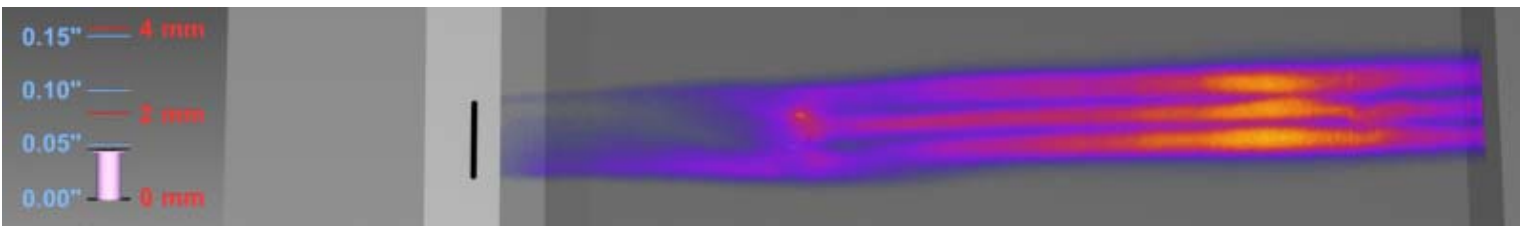

(g)

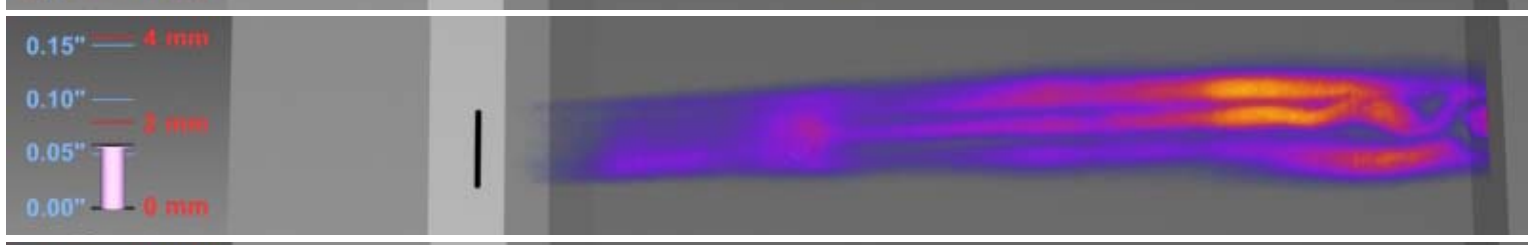

(h)

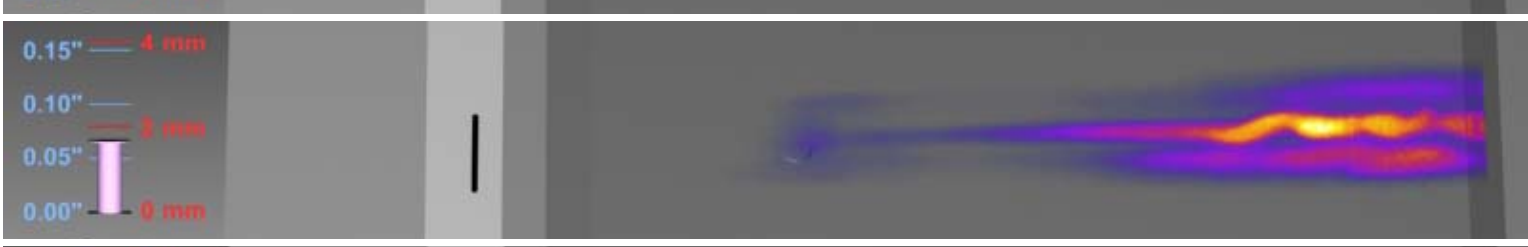

(i)

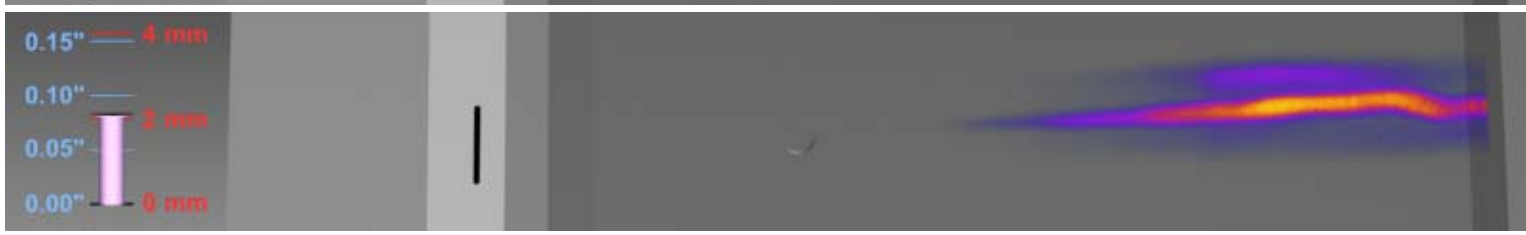

Figure A33: Test 443, Run 20, 1-mm tall by 4-mm wide cylinder, plate angle $=20^{\circ}$, 11-mm wide slot seeding, $\dot{m}=$ $300 \mathrm{sccm}, \mathrm{P}_{0}=2.41 \mathrm{MPa}$, sheet position varies from 0.0 to $2.0 \mathrm{~mm}$ above the plate in $0.25 \mathrm{~mm}$ increments in (a)-(i), framing rate $=10 \mathrm{~Hz}$. 
(a)

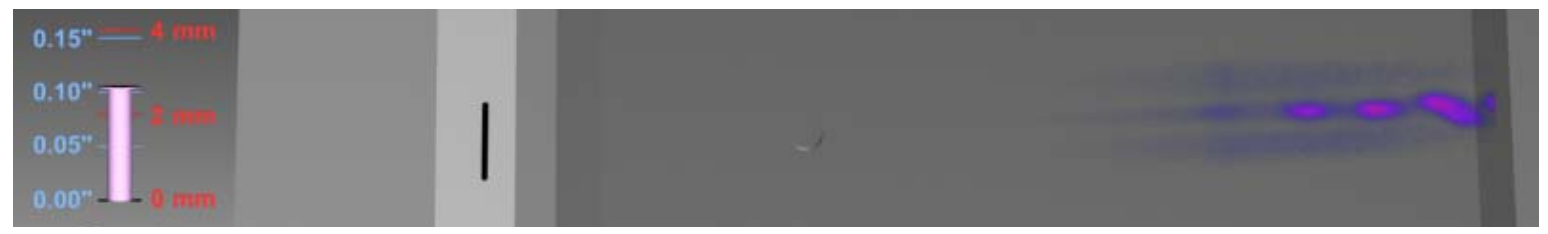

(b)

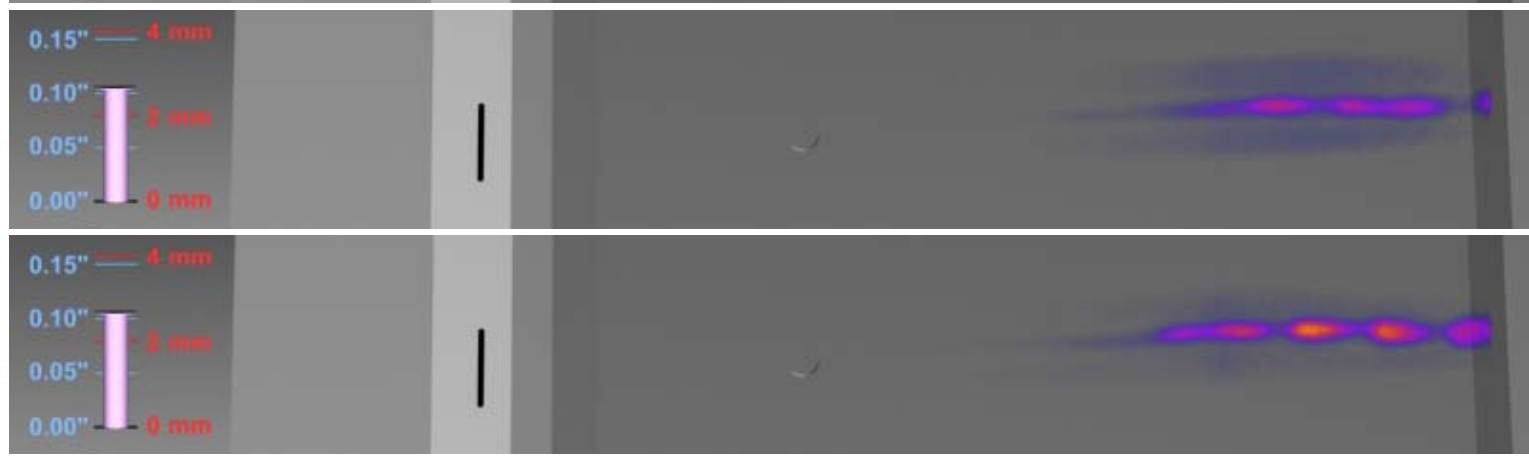

(c)

(d)

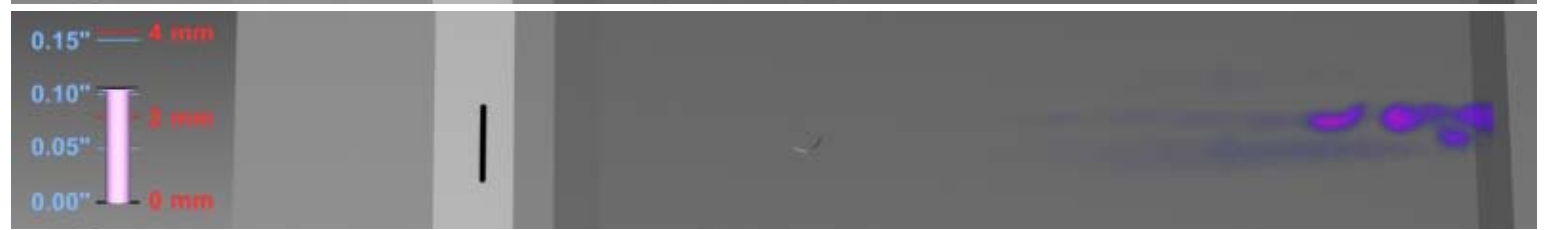

(e)

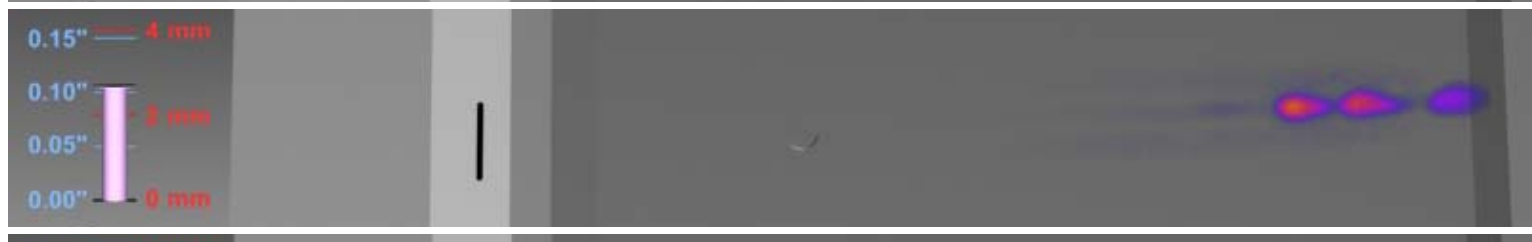

(f)

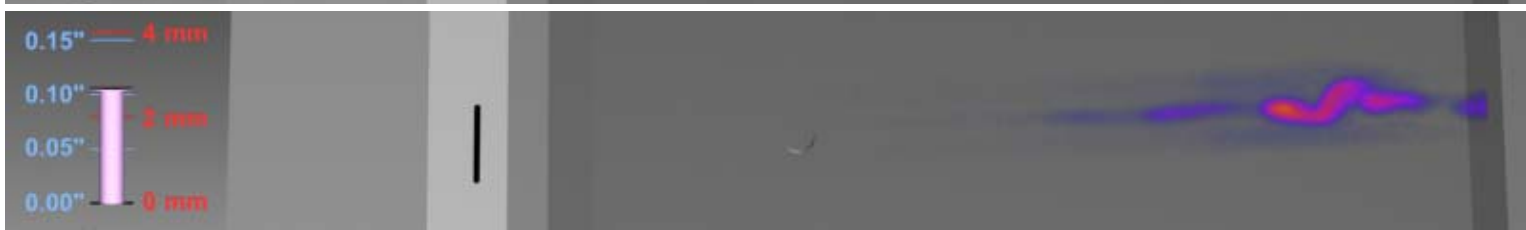

(g)

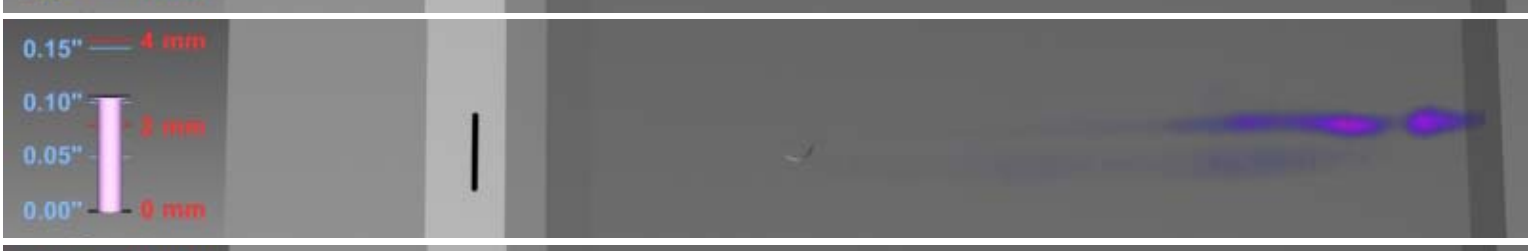

(h)

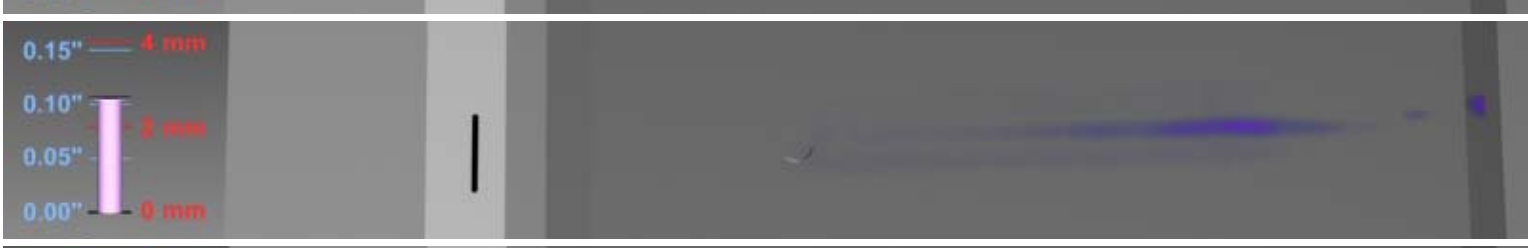

(i)

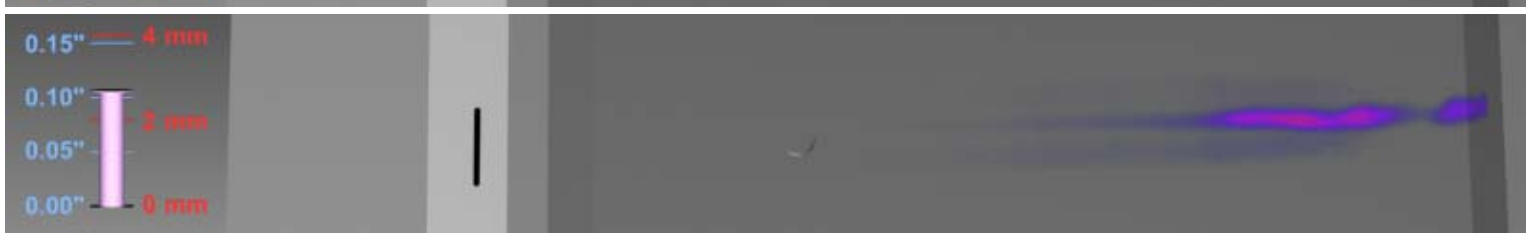

Figure A34: Test 443, Run 20, 1-mm tall by 4-mm wide cylinder, plate angle $=20^{\circ}, 11$-mm wide slot seeding, $\dot{m}=$ $300 \mathrm{sccm}, \mathrm{P}_{0}=2.41 \mathrm{MPa}$, sheet position $=2.7 \mathrm{~mm}$ above the plate, framing rate $=10 \mathrm{~Hz}$. 
(a)

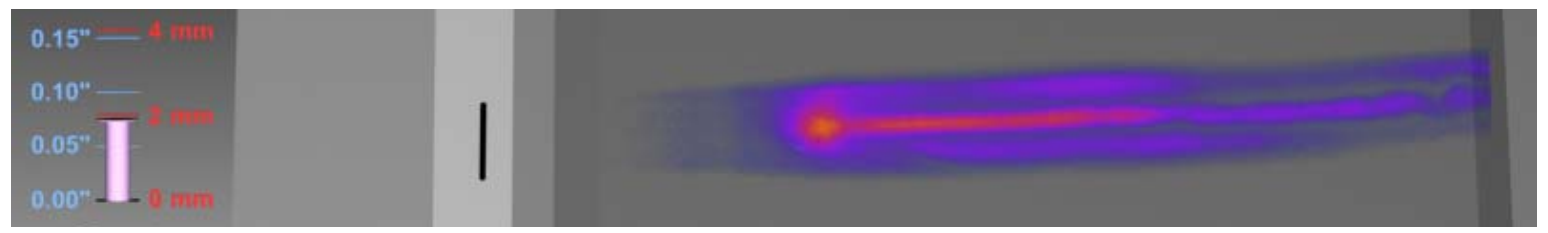

(b)

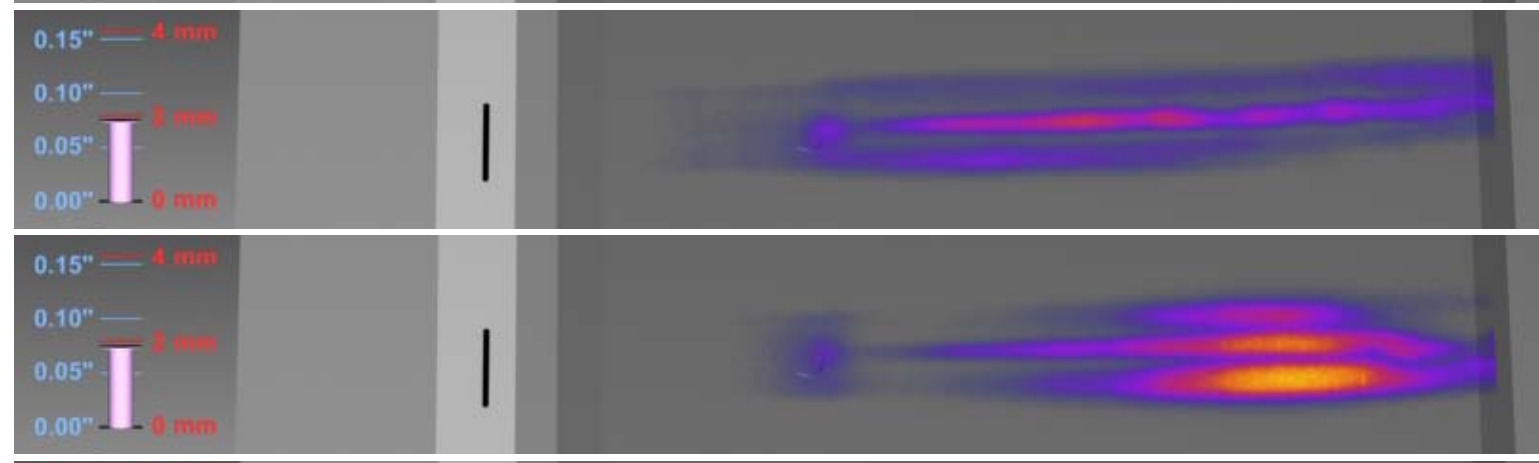

(d)

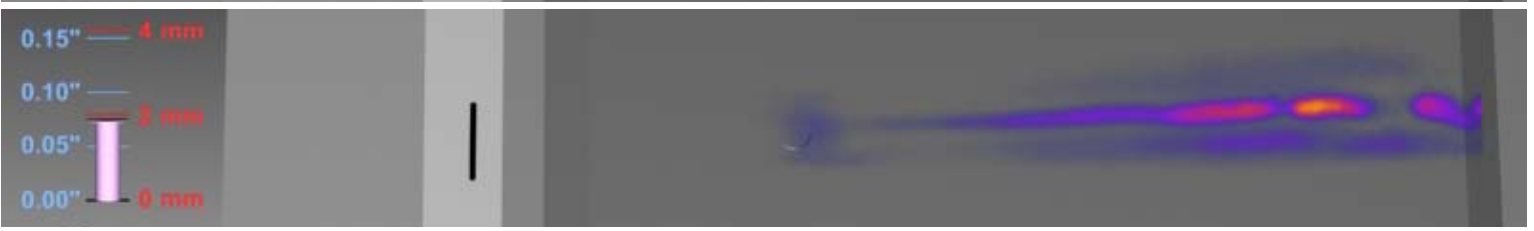

(e)

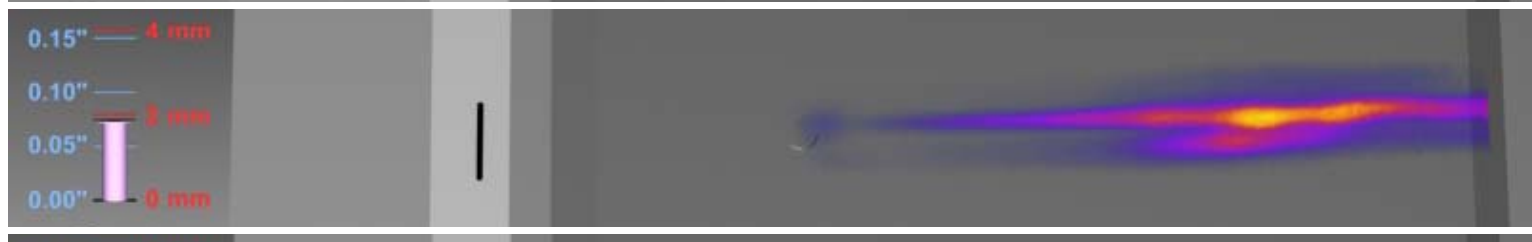

(f)

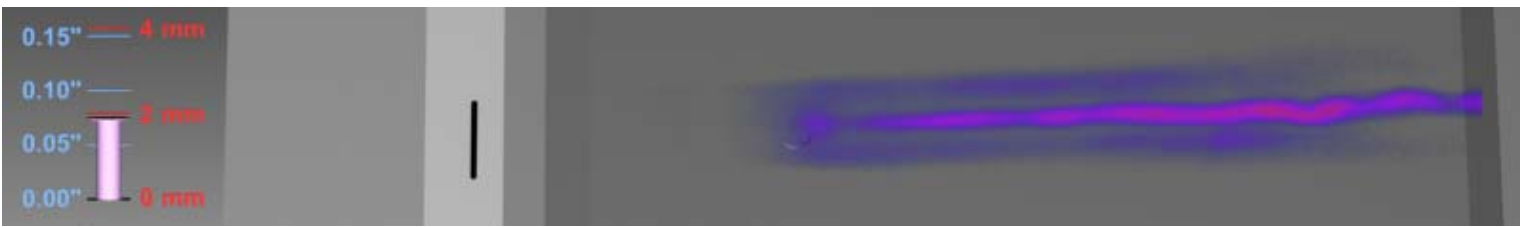

(g)

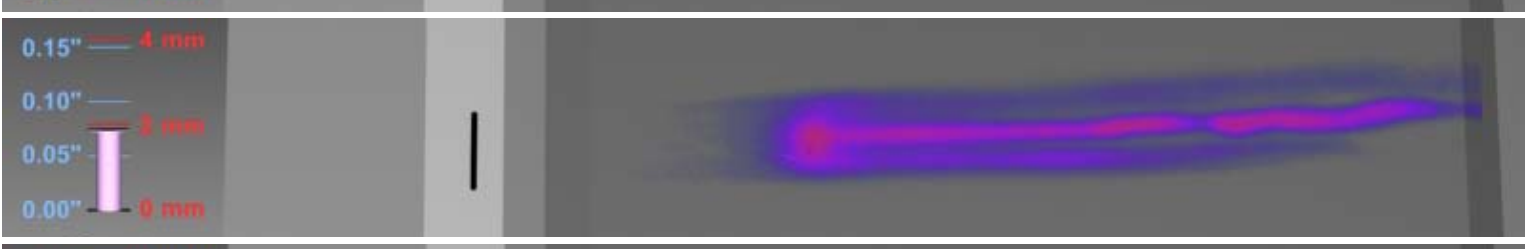

(h)

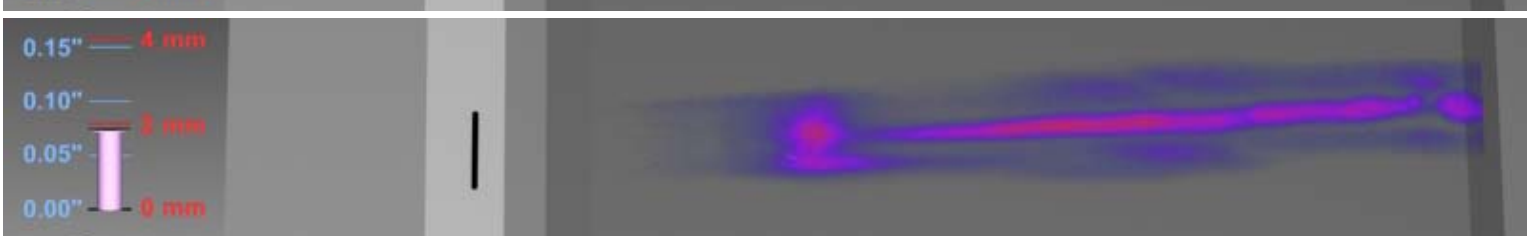

(i)

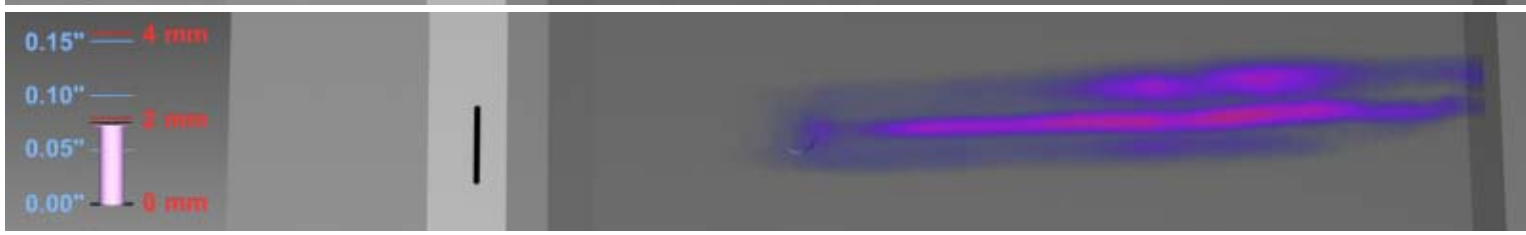

Figure A35: Test 443, Run 20, 1-mm tall by 4-mm wide cylinder, plate angle $=20^{\circ}, 11-\mathrm{mm}$ wide slot seeding, $\dot{m}=$ $300 \mathrm{sccm}, \mathrm{P}_{0}=2.41 \mathrm{MPa}$, sheet position $=1.9 \mathrm{~mm}$ above the plate, framing rate $=10 \mathrm{~Hz}$. 
(a)

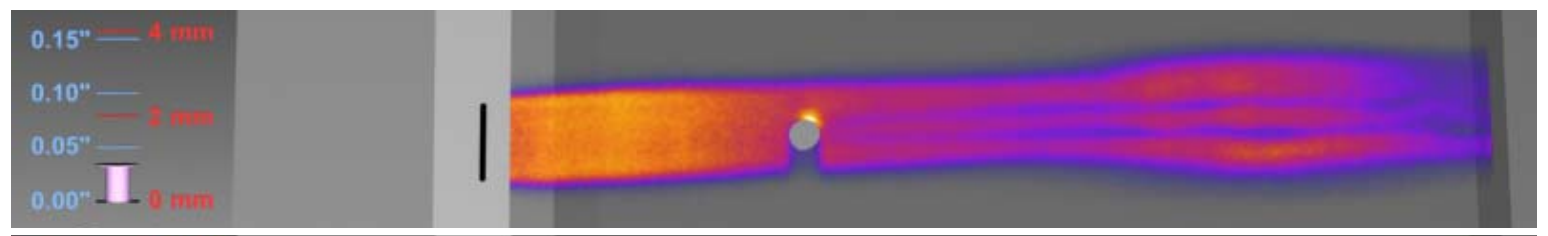

(b)

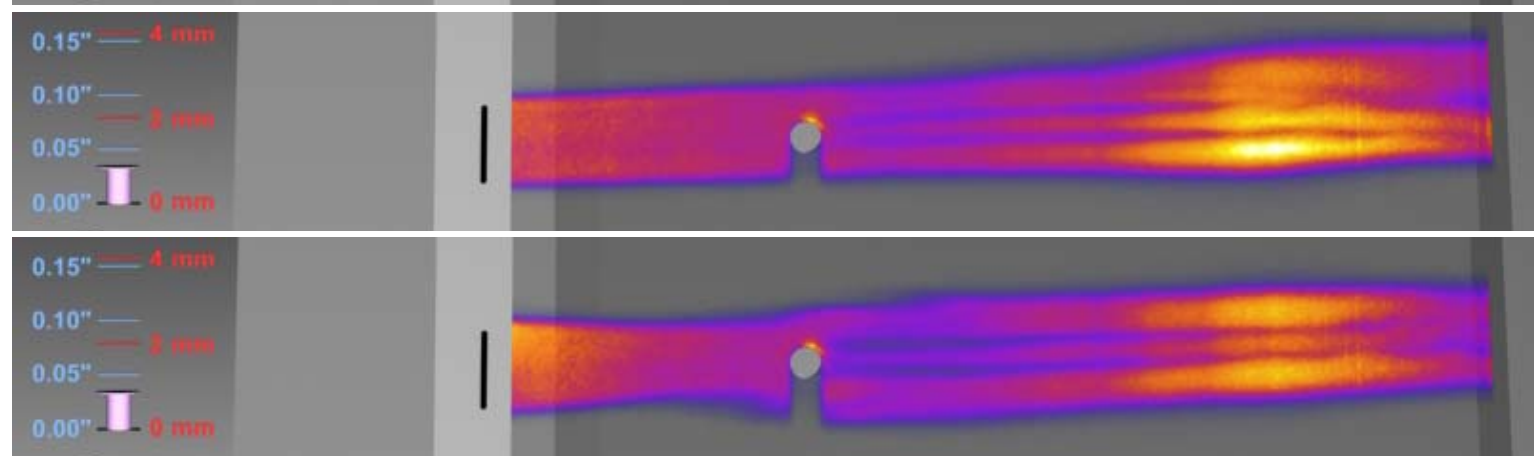

(c)

(d)

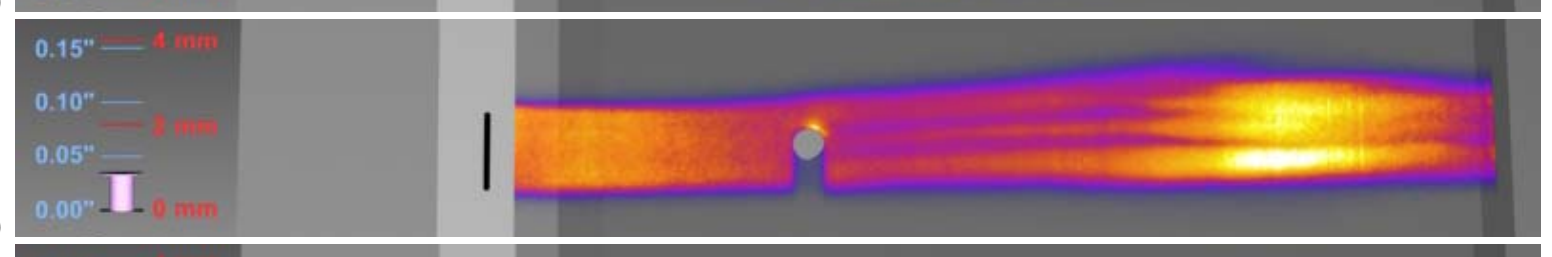

(e)

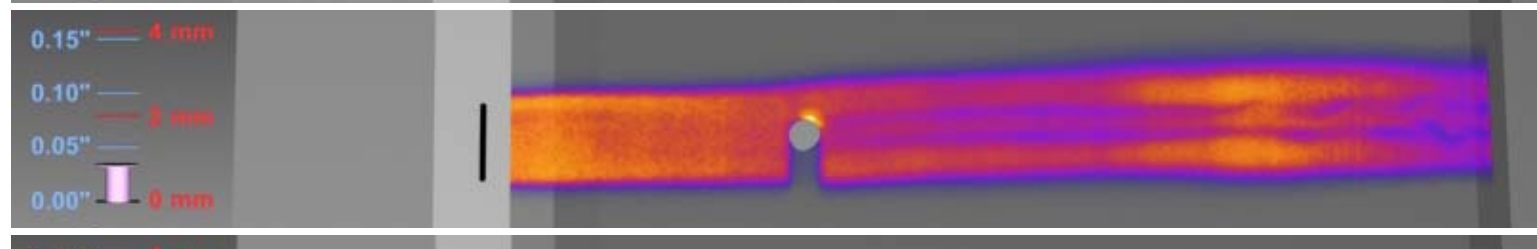

(f)

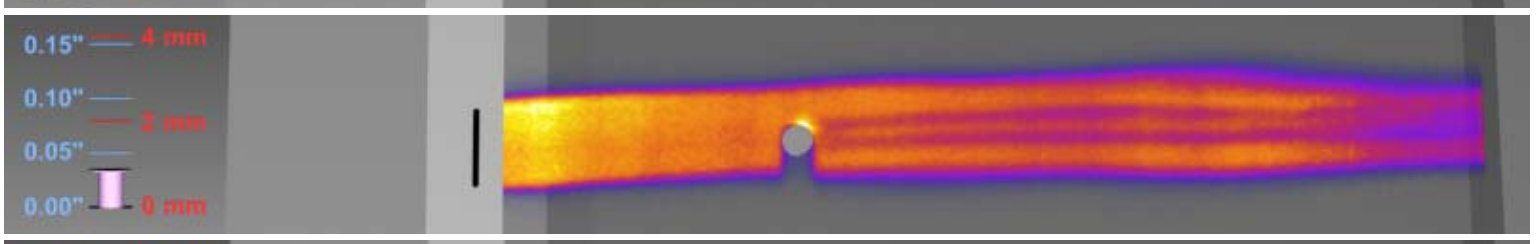

(g)

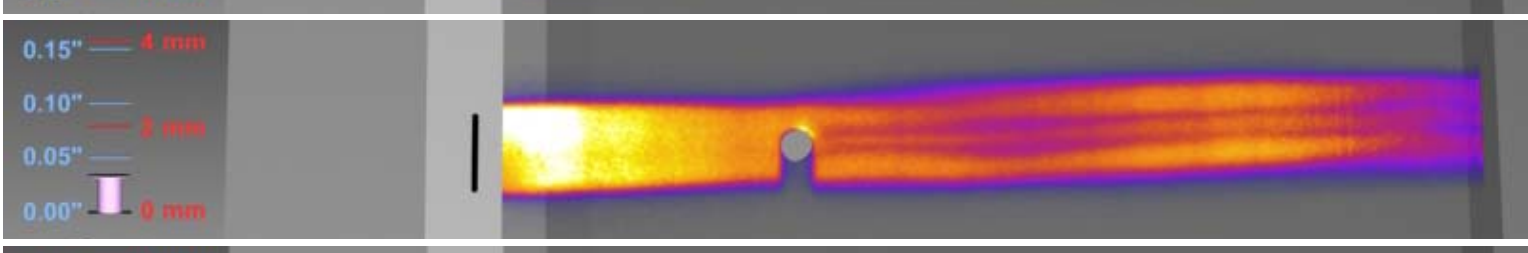

(h)

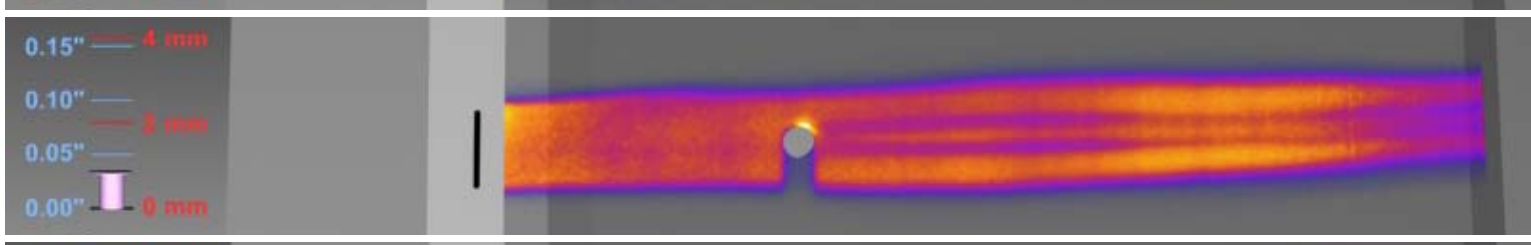

(i) A36: Test 443, Run 20, 1-mm tall by 4-mm wide cylinder, plate angle $=20^{\circ}, 11-\mathrm{mm}$ wide slot seeding, $\dot{m}=$ $300 \mathrm{sccm}, \mathrm{P}_{0}=2.41 \mathrm{MPa}$, sheet position $=0.9 \mathrm{~mm}$ above the plate, framing rate $=10 \mathrm{~Hz}$. 
(a)

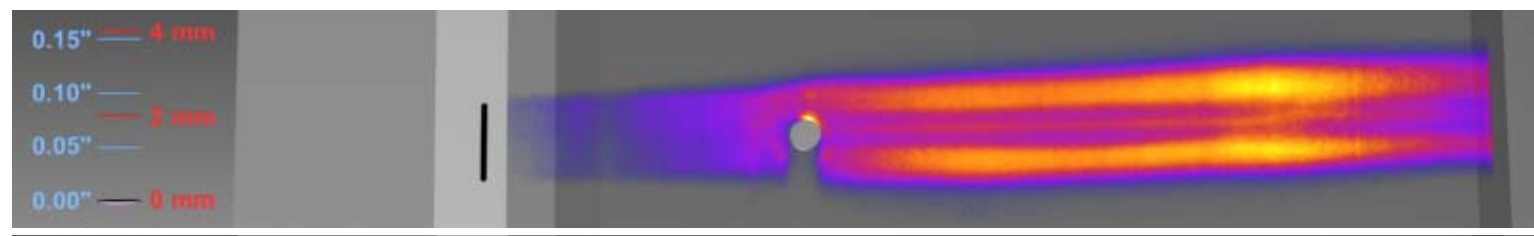

(b)

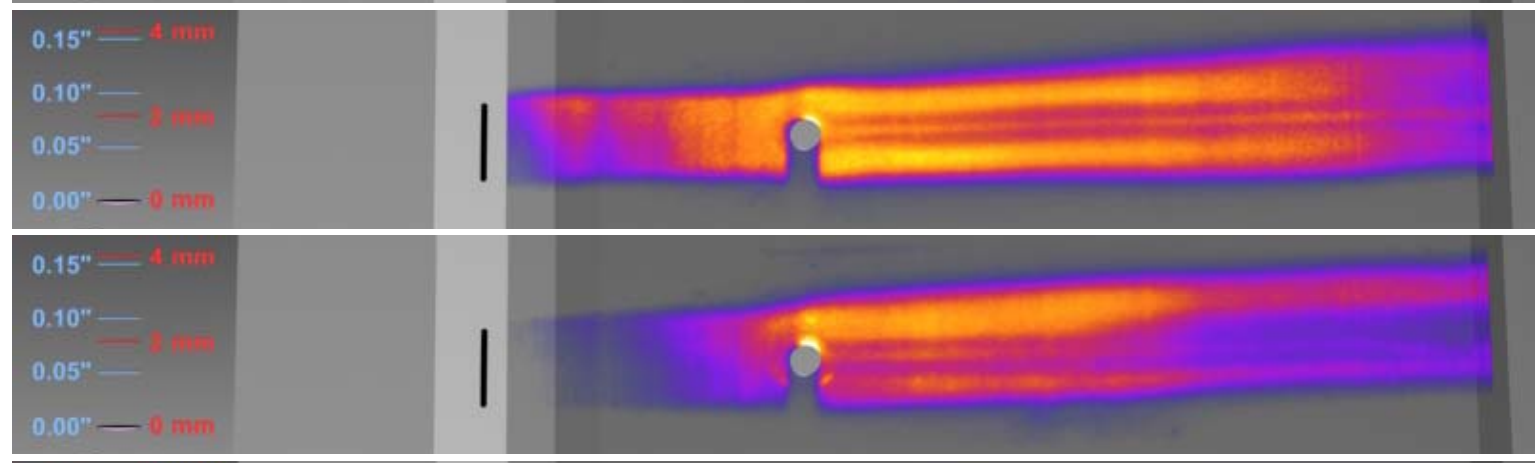

(c)

(d)

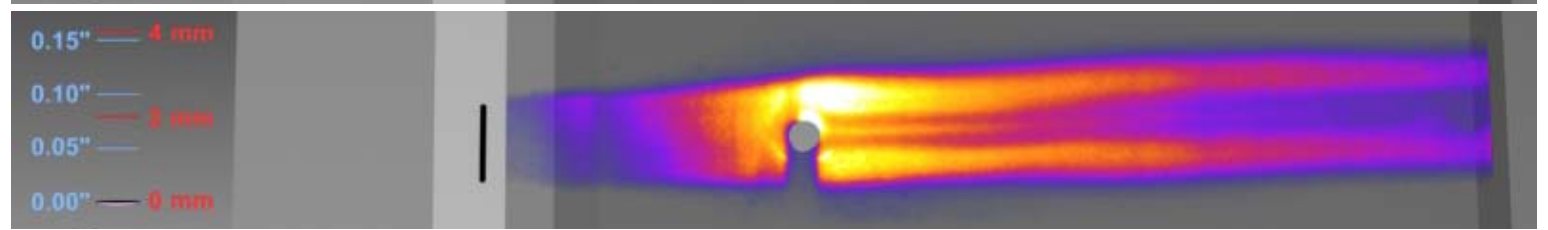

(e)

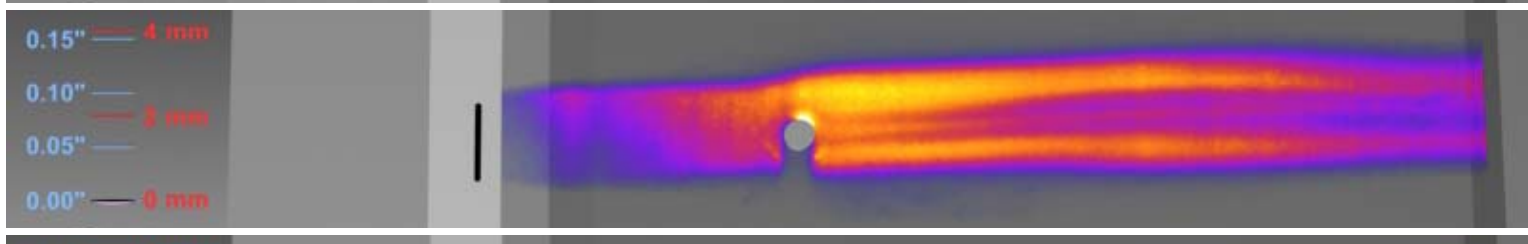

(f)
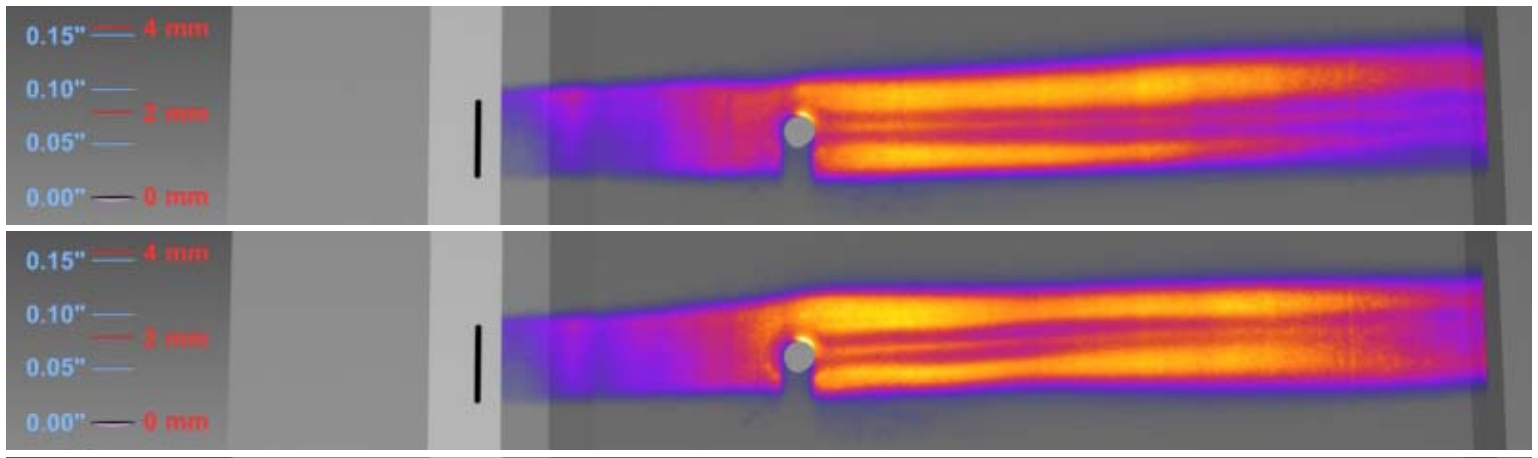

(g)

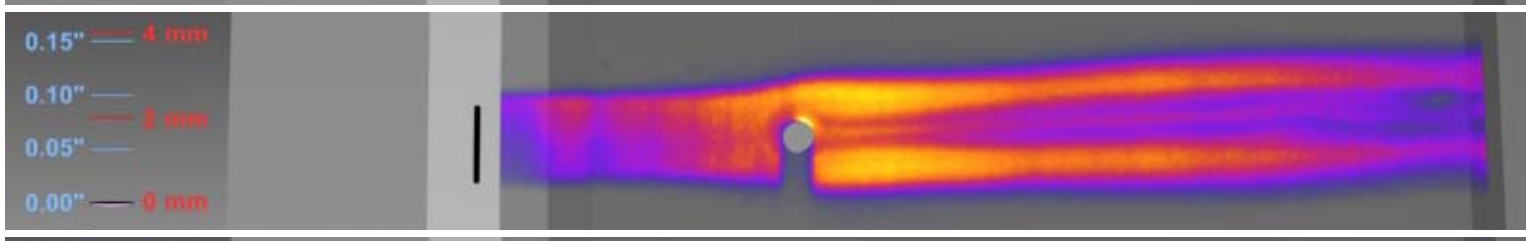

(i)

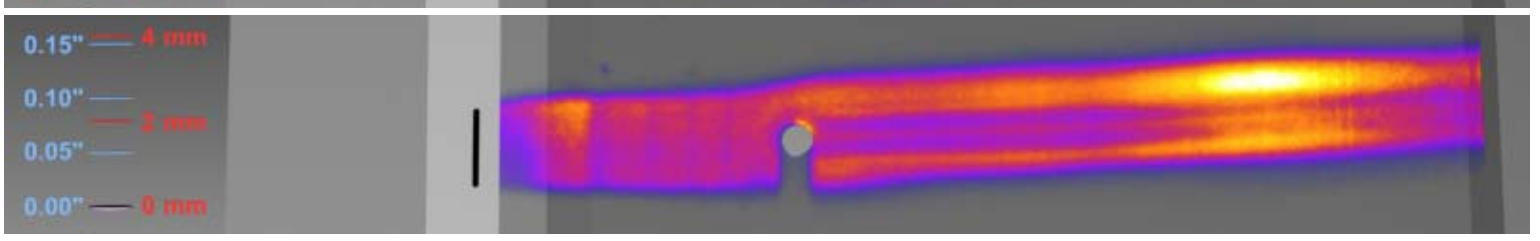

Figure A37: Test 443, Run 20, 1-mm tall by 4-mm wide cylinder, plate angle $=20^{\circ}, 11$-mm wide slot seeding, $\dot{m}=$ $300 \mathrm{sccm}, \mathrm{P}_{0}=2.41 \mathrm{MPa}$, sheet position $=0.0 \mathrm{~mm}$ above the plate, framing rate $=10 \mathrm{~Hz}$. 
(a)

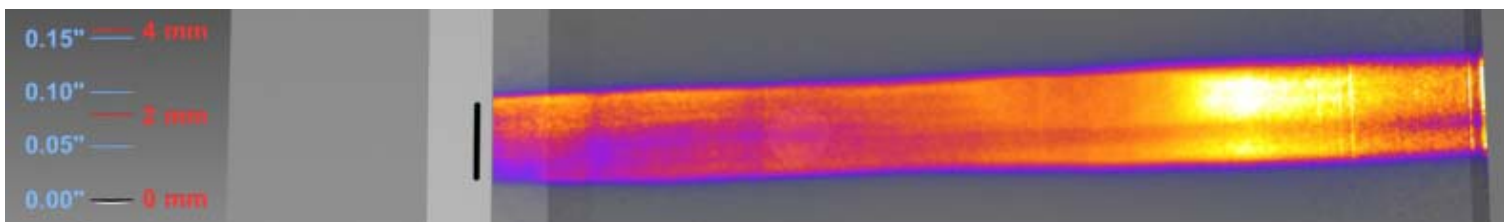

(b)

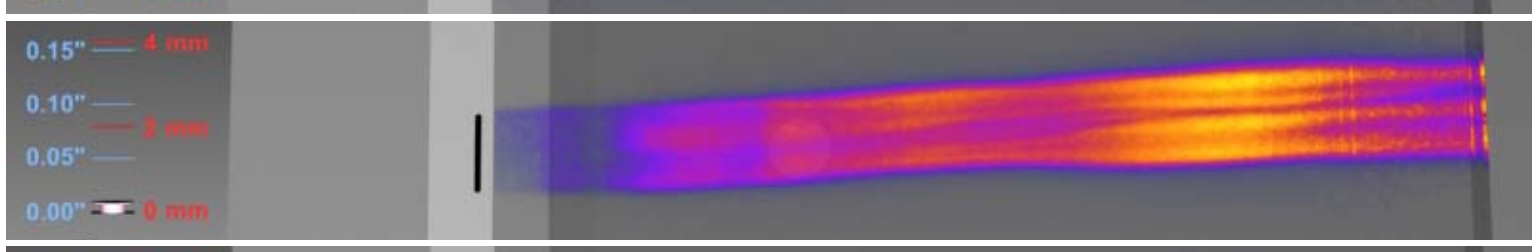

(c)

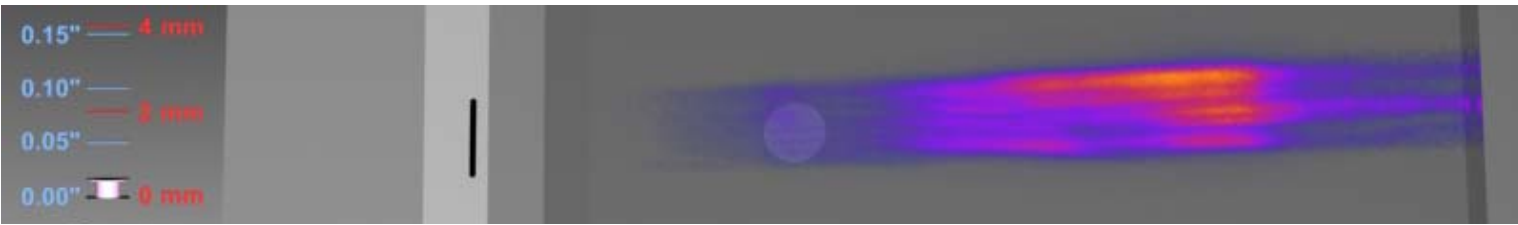

(d)

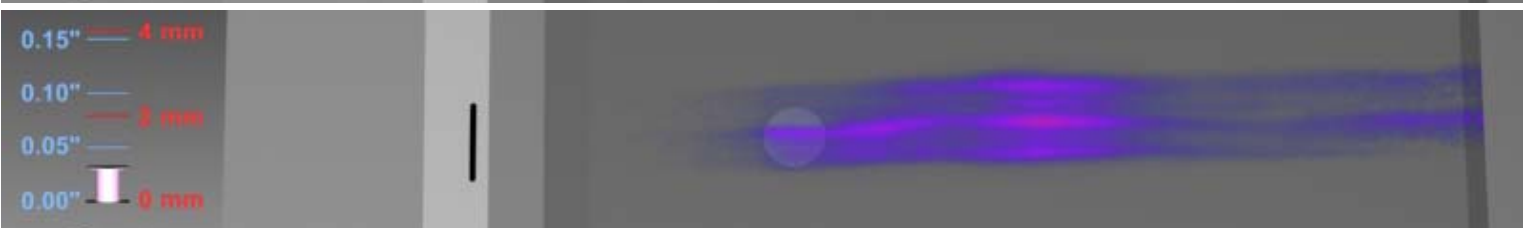

(e)

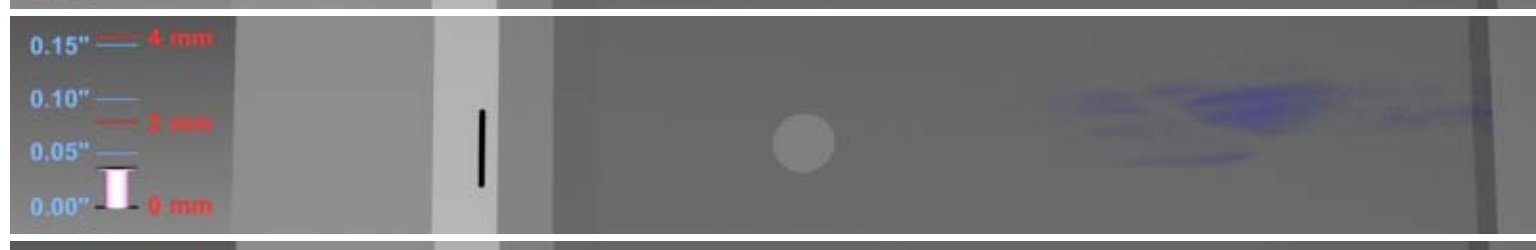

(f)

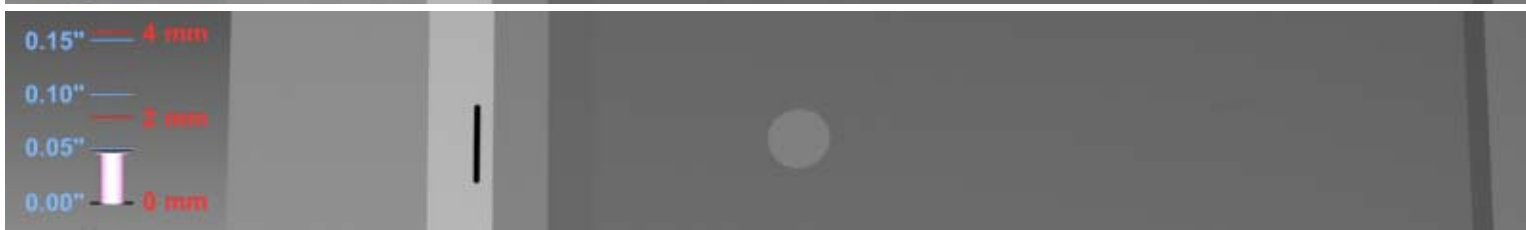

(g)

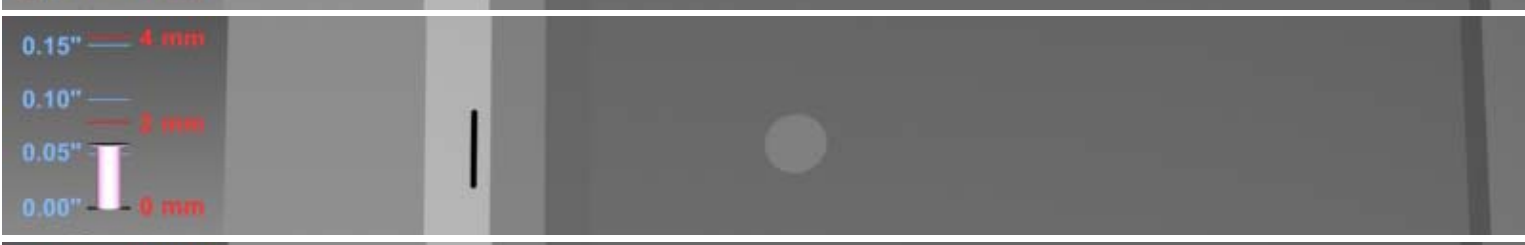

(h)
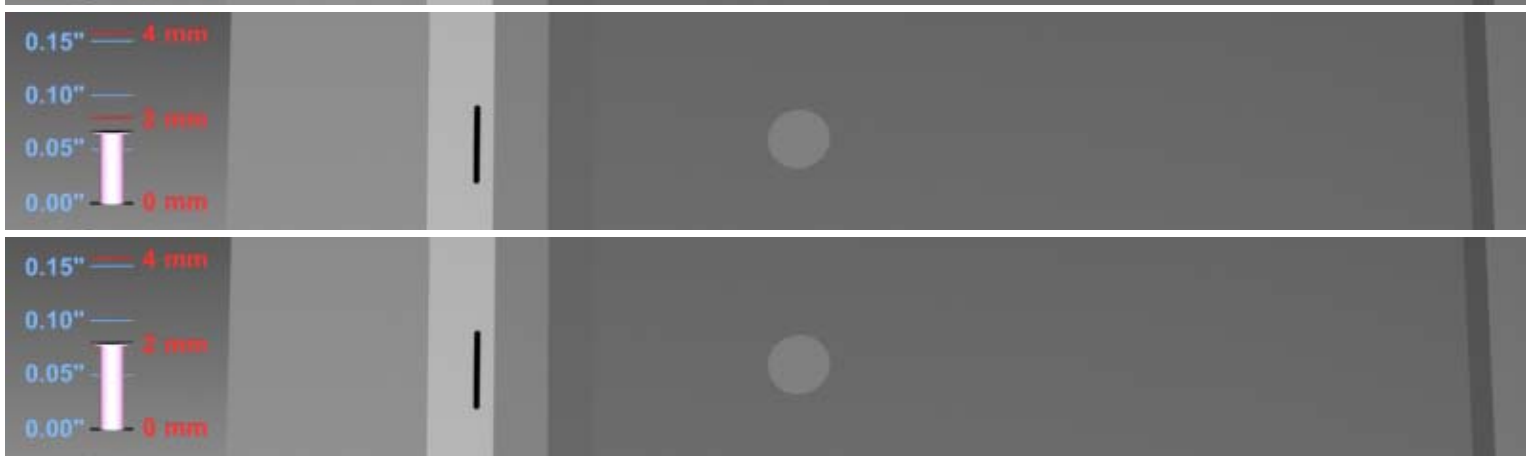

Figure A38: Test 443, Run 23, no trip, plate angle $=20^{\circ}$, 11 -mm wide slot seeding, $\dot{m}=300$ sccm, $\mathrm{P}_{0}=9.35 \mathrm{MPa}$, sheet position varies from 0.0 to $2.0 \mathrm{~mm}$ above the plate in $0.25 \mathrm{~mm}$ increments in (a)-(i), framing rate $=10 \mathrm{~Hz}$. 
(a)

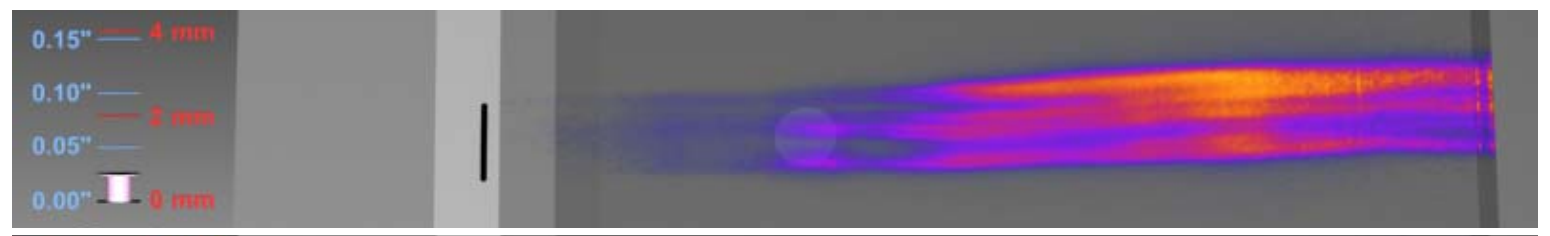

(b)

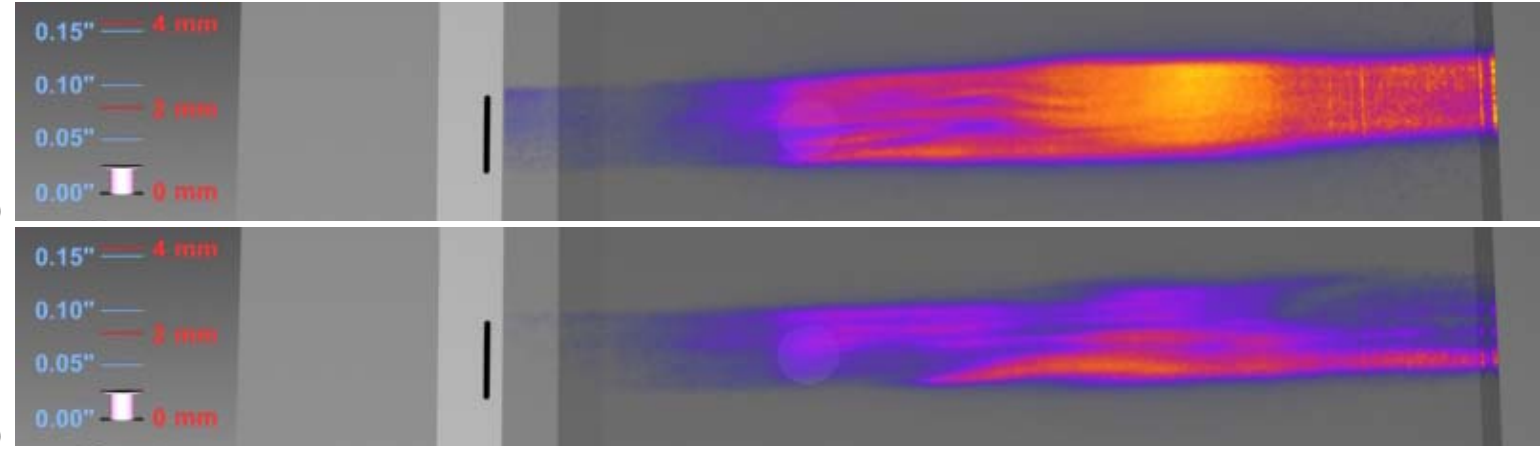

(d)

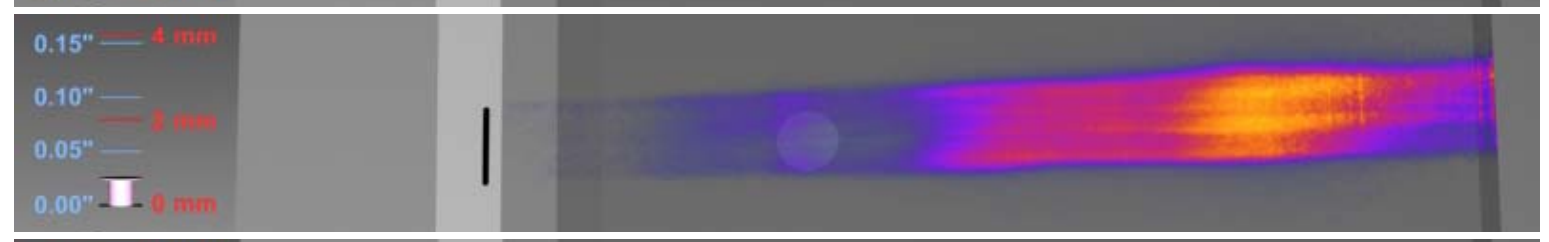

(e)

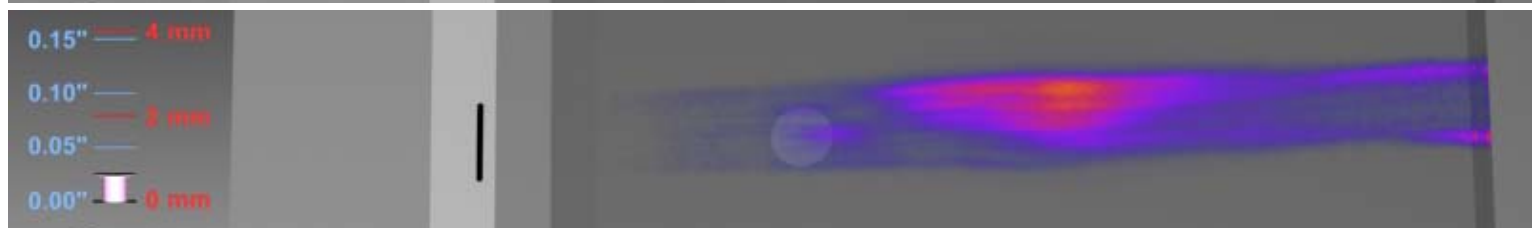

(f)

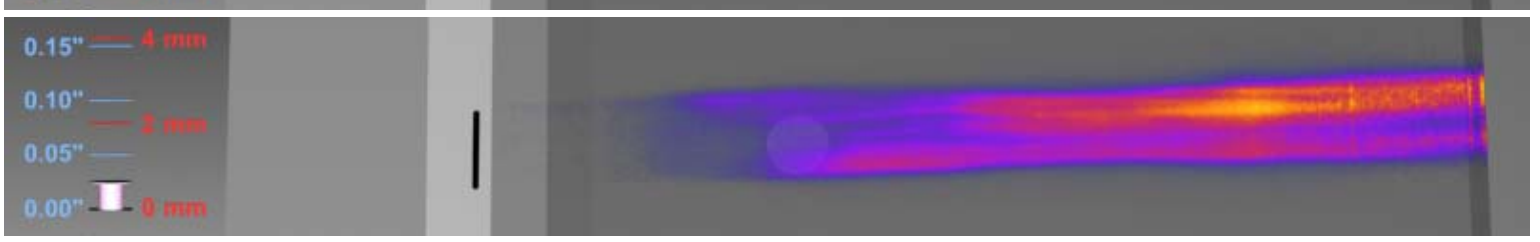

(g)
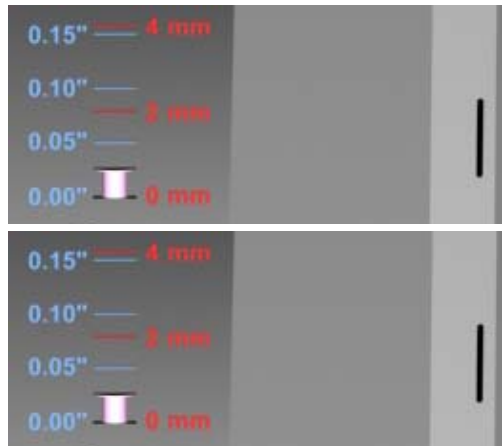

(h)

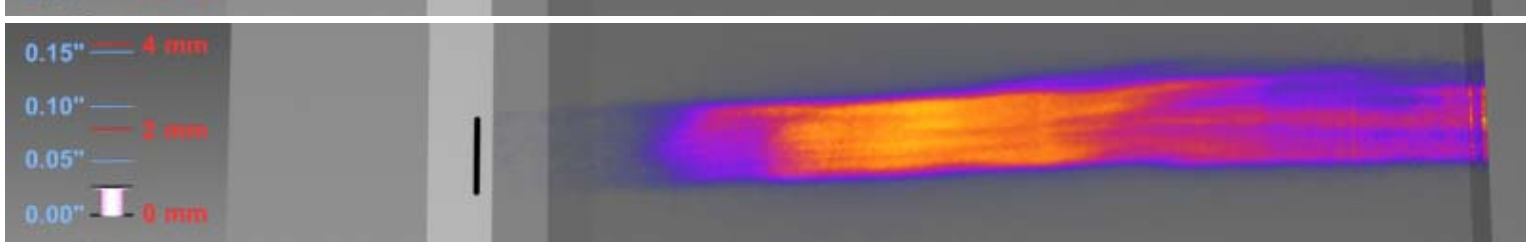

Figure A39: Test 443, Run 23, no trip, plate angle $=20^{\circ}$, 11 -mm wide slot seeding, $\dot{m}=300 \mathrm{sccm}, \mathrm{P}_{0}=9.35 \mathrm{MPa}$, sheet position $=0.7 \mathrm{~mm}$ above the plate, framing rate $=10 \mathrm{~Hz}$. 
(a)

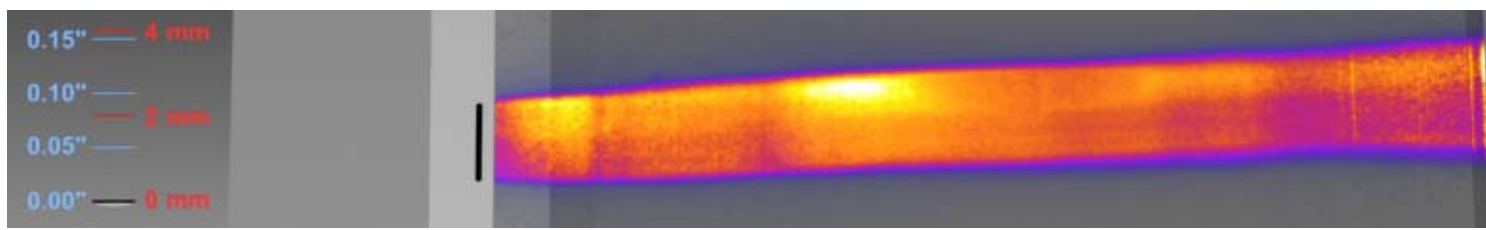

(b)

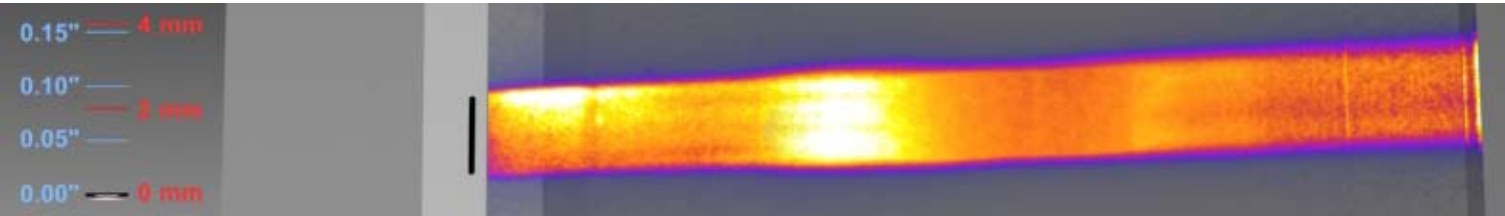

(c)

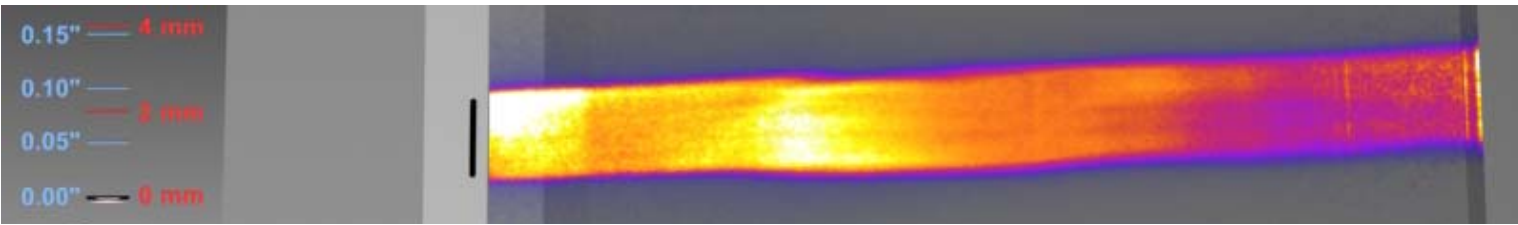

(d)

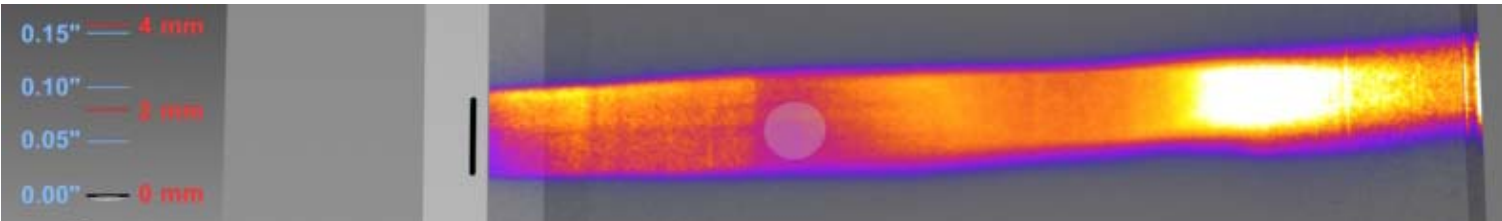

(e)

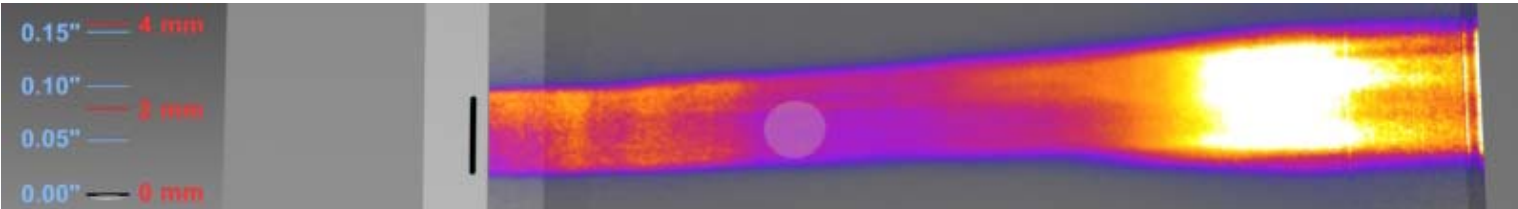

(f)

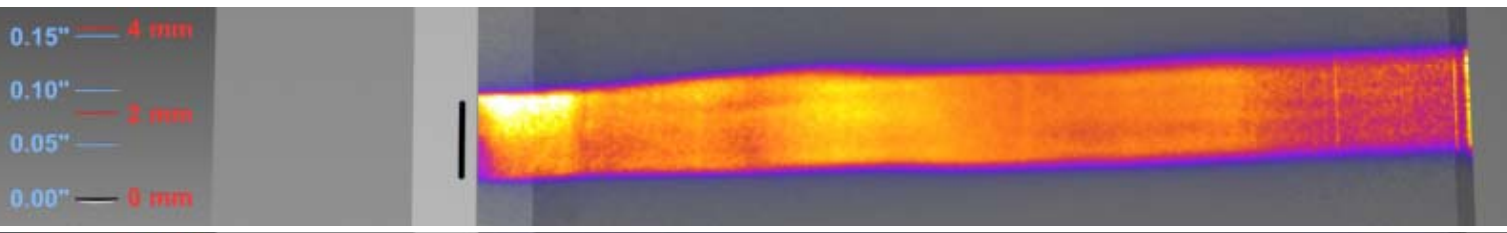

(g)

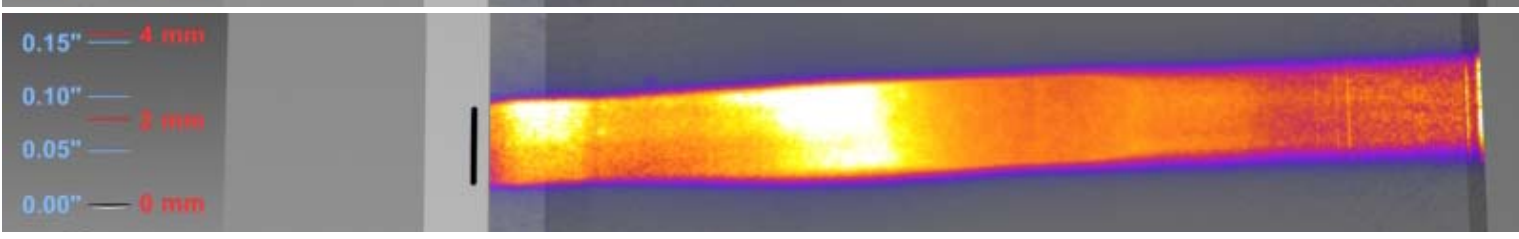

$0.15^{\prime \prime}-\left(1 x^{\prime}-1\right.$
$0.10^{\prime \prime}-$
$0.05^{\prime \prime}$
$0.00^{\prime \prime}-0$

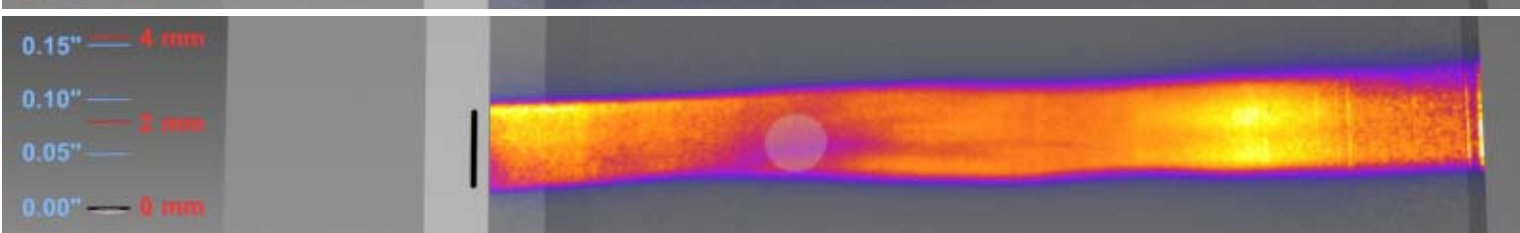

Figure A40: Test 443, Run 23, no trip, plate angle $=20^{\circ}$, 11 -mm wide slot seeding, $\dot{m}=300 \mathrm{sccm}, \mathrm{P}_{0}=9.35 \mathrm{MPa}$, sheet position $=0.0 \mathrm{~mm}$ above the plate, framing rate $=10 \mathrm{~Hz}$. 
(a)

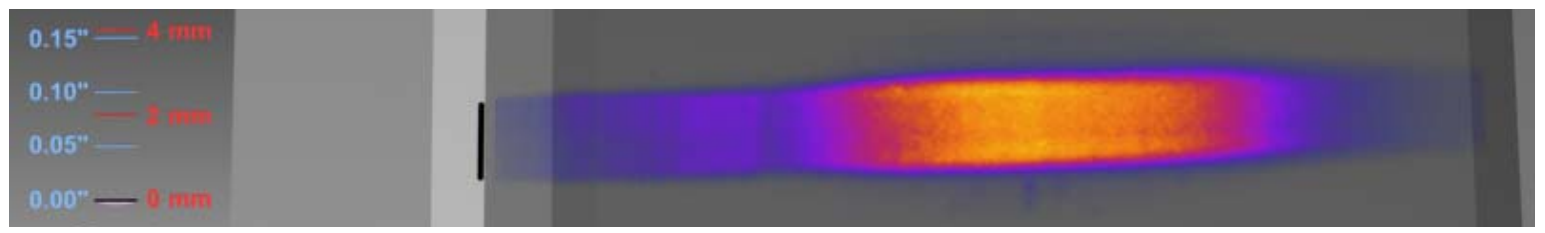

(b)

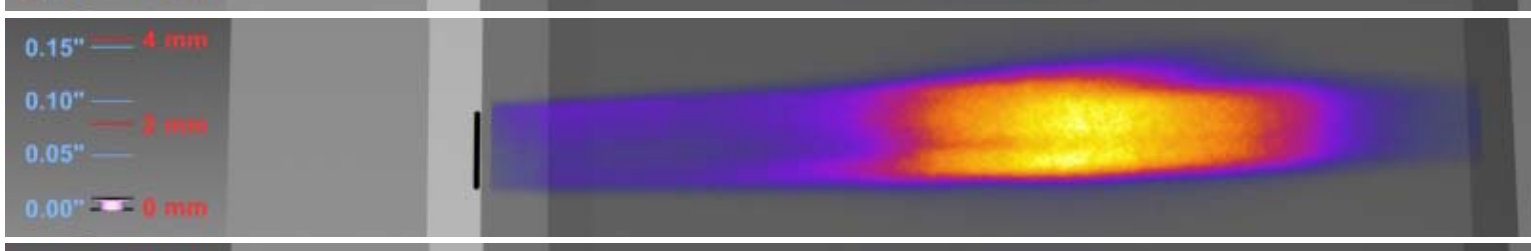

(c)

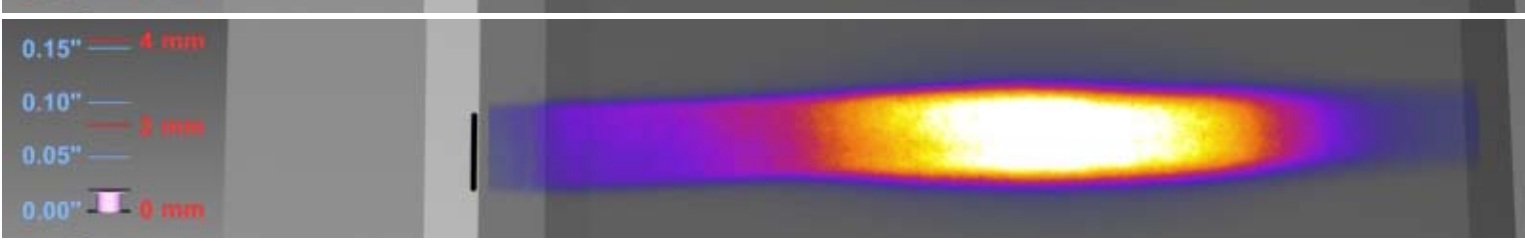

(d)

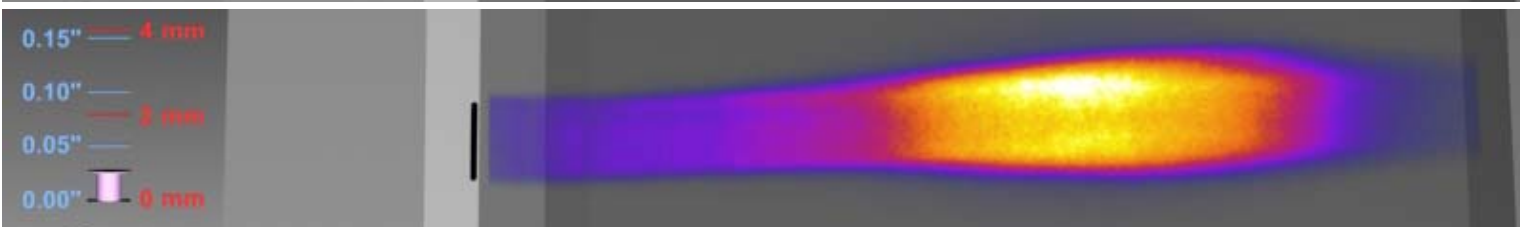

(e)

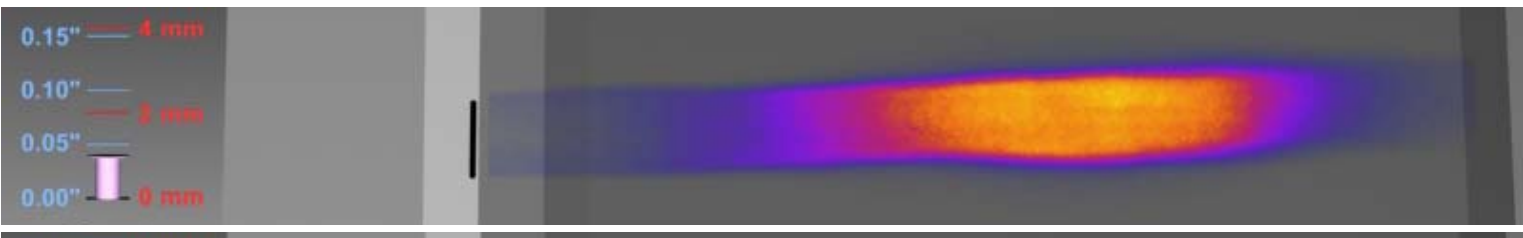

(f)

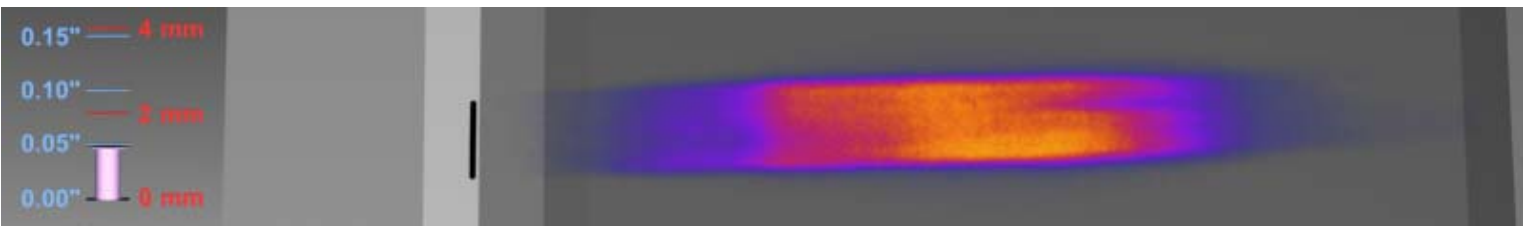

(g)

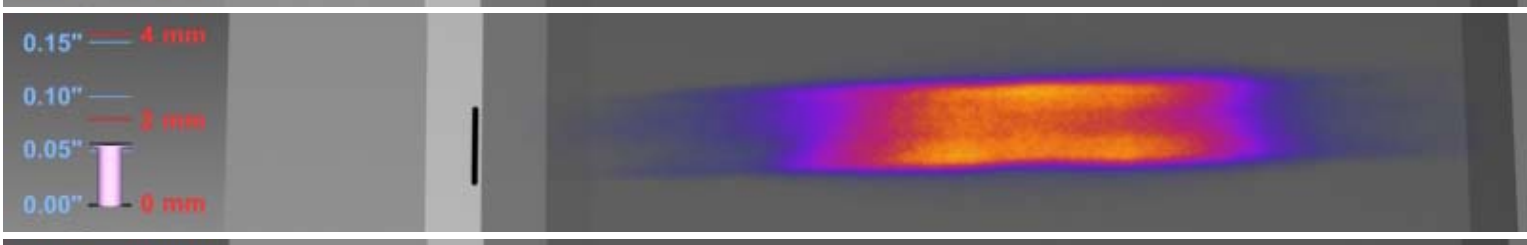

(h)
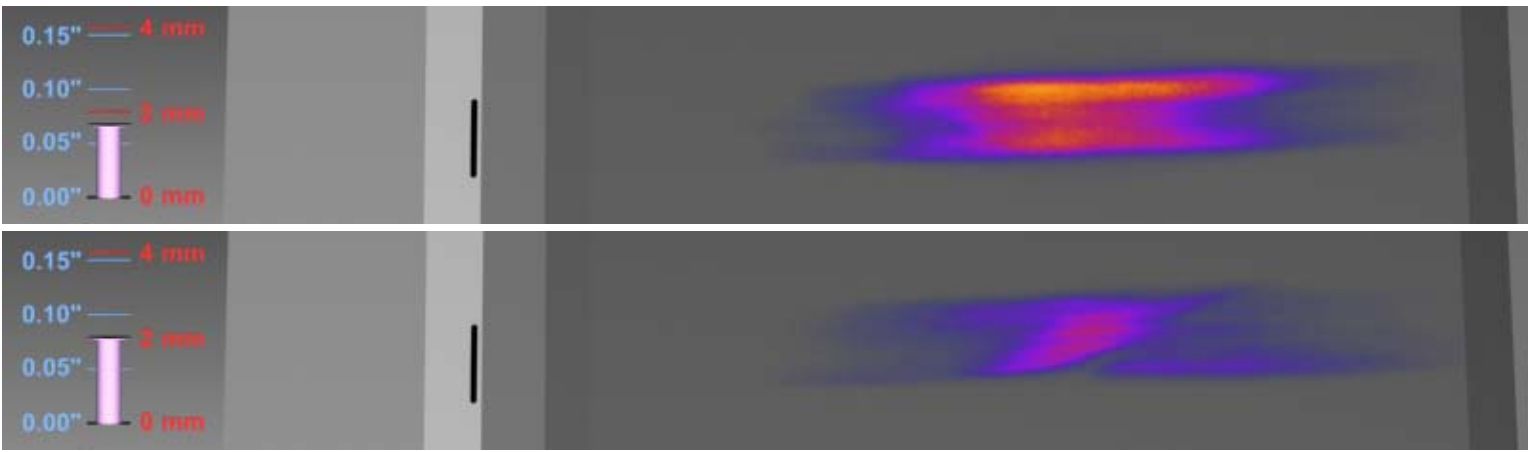
(i)

Figure A41: Test 443, Run 25, no trip, plate angle $=20^{\circ}$, 11 -mm wide slot seeding, $\dot{m}=300$ sccm, $\mathrm{P}_{0}=4.98 \mathrm{MPa}$, sheet position varies from 0.0 to $2.0 \mathrm{~mm}$ above the plate in $0.25 \mathrm{~mm}$ increments in (a)-(i), framing rate $=10 \mathrm{~Hz}$. 
(a)

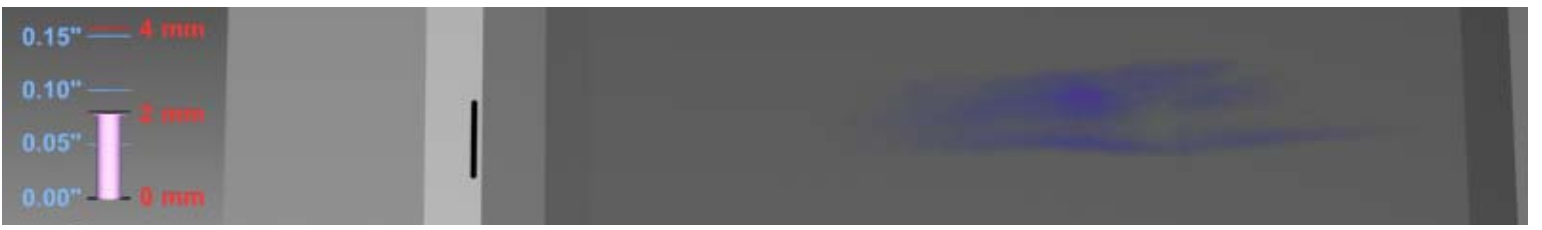

(b)

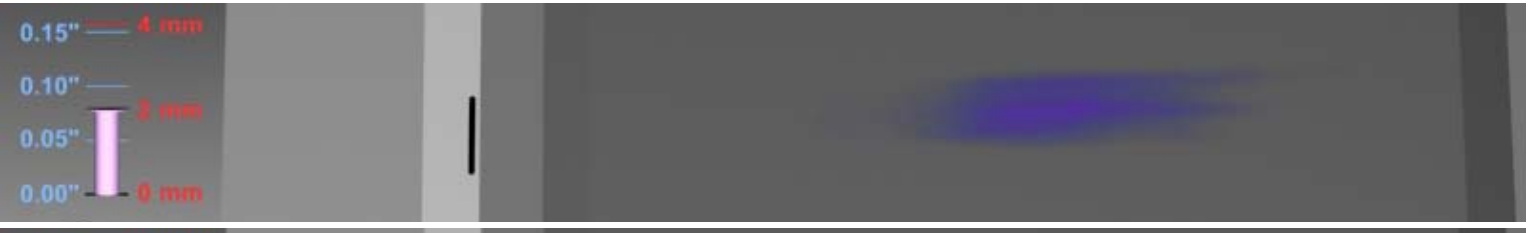

(c)

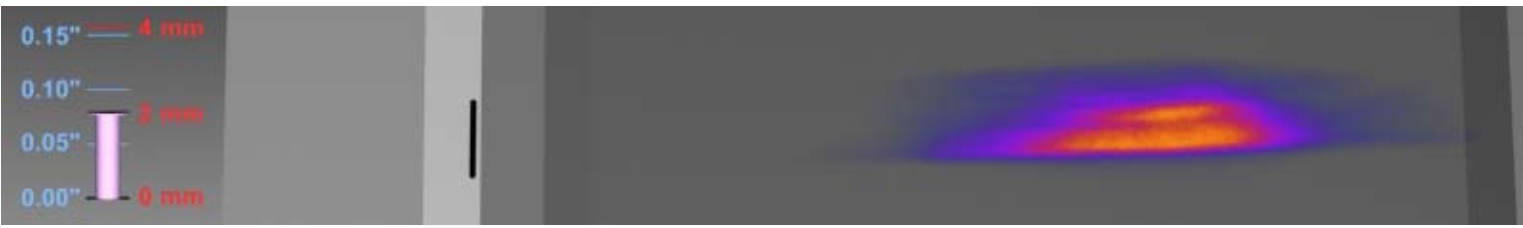

(d)

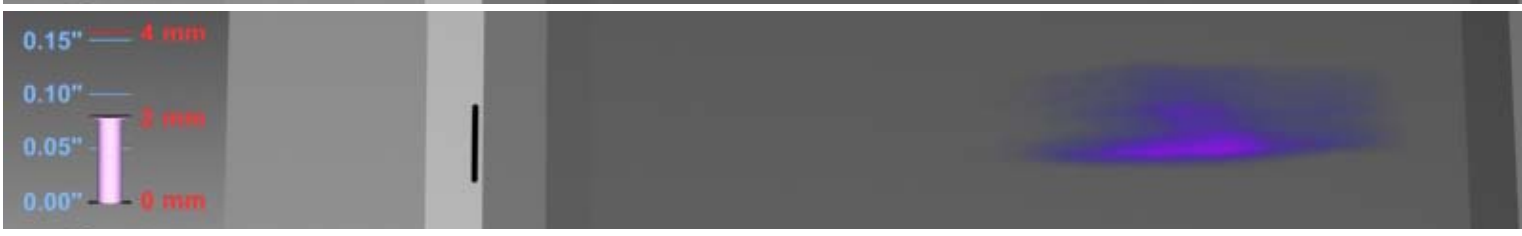

(e)

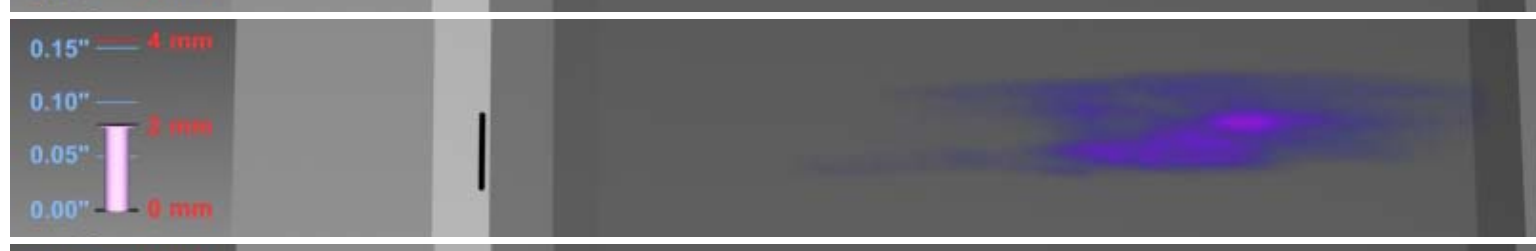

(f)
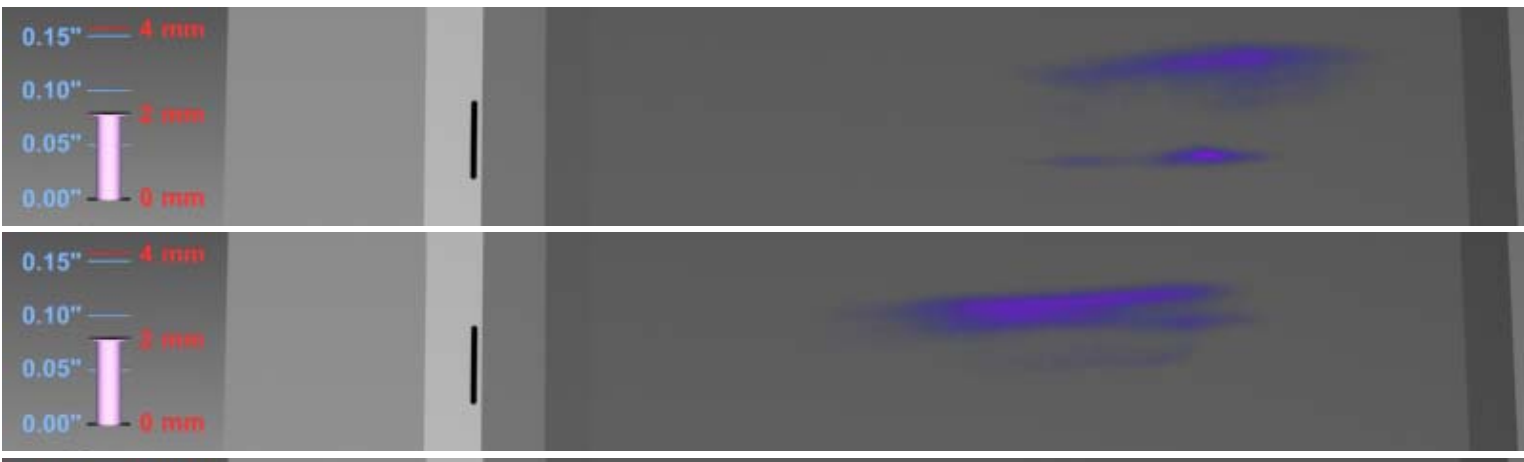

(g)

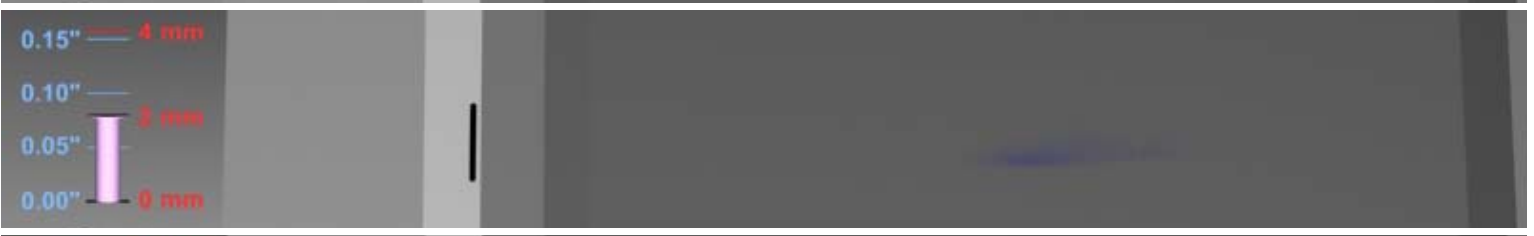

(h)

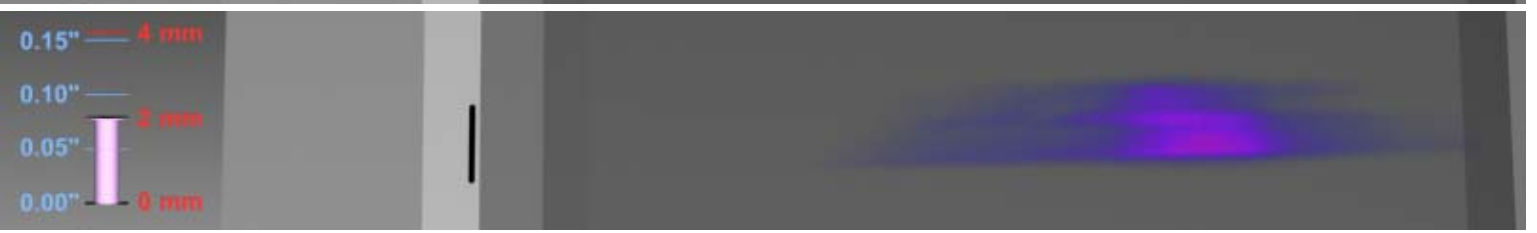

Figure A42: Test 443, Run 25, no trip, plate angle $=20^{\circ}$, 11-mm wide slot seeding, $\dot{m}=300 \mathrm{sccm}, \mathrm{P}_{0}=4.98 \mathrm{MPa}$, sheet position $=2.0 \mathrm{~mm}$ above the plate, framing rate $=10 \mathrm{~Hz}$. 
(a)

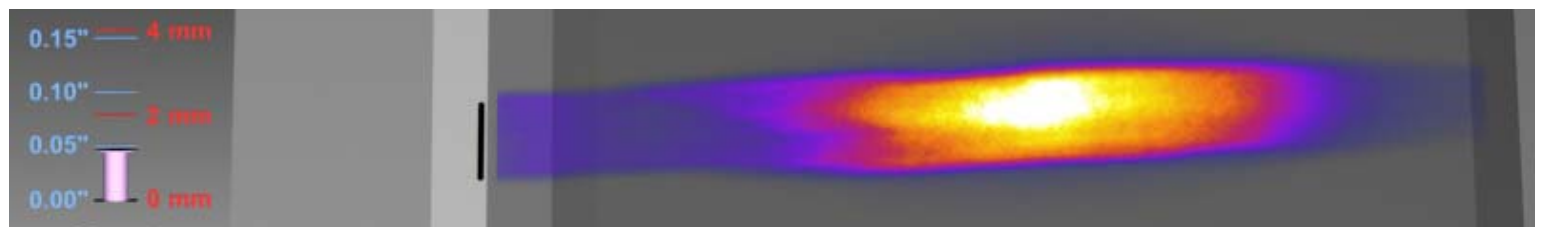

(b)

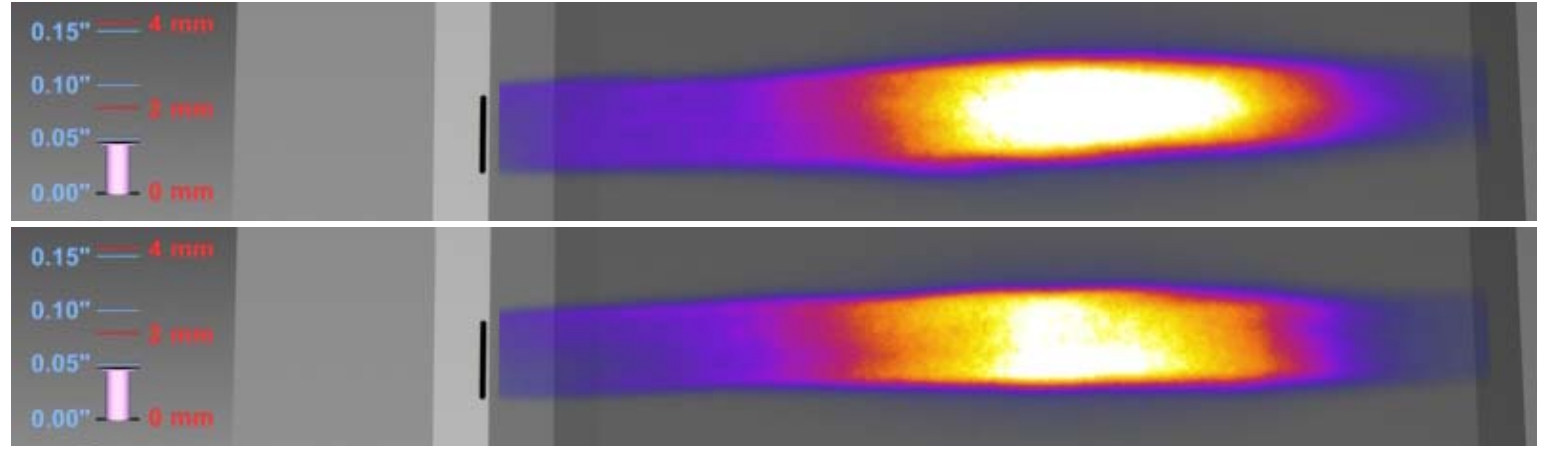

(c)

(d)

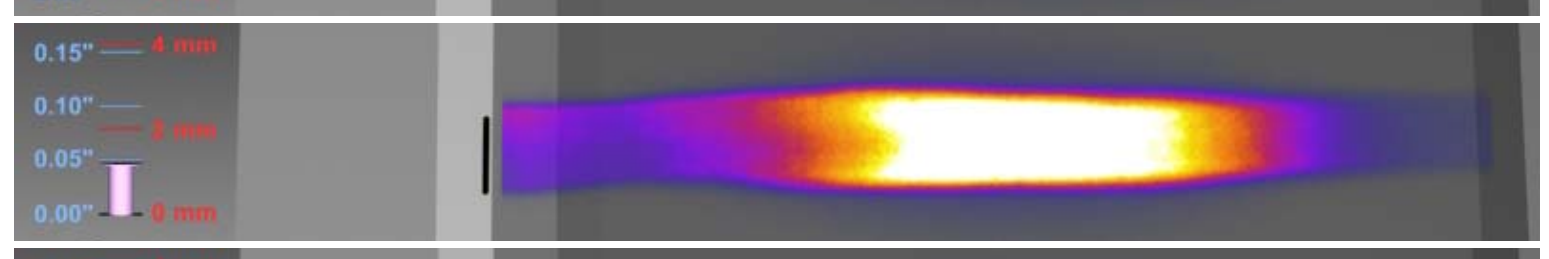

(e)

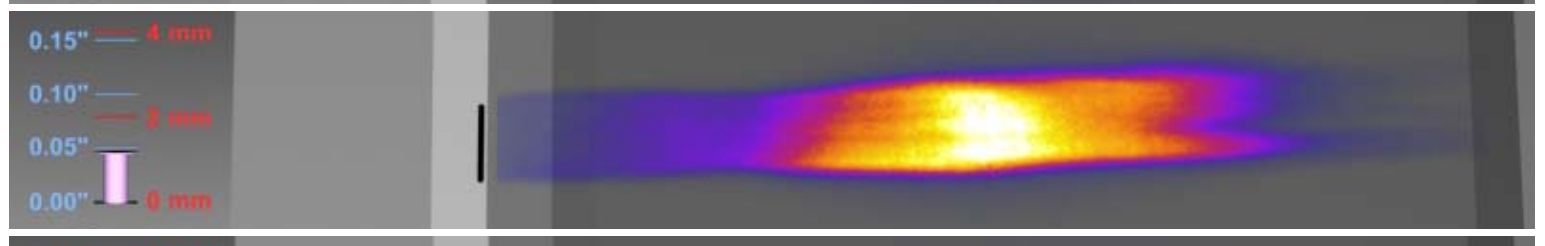

(f)
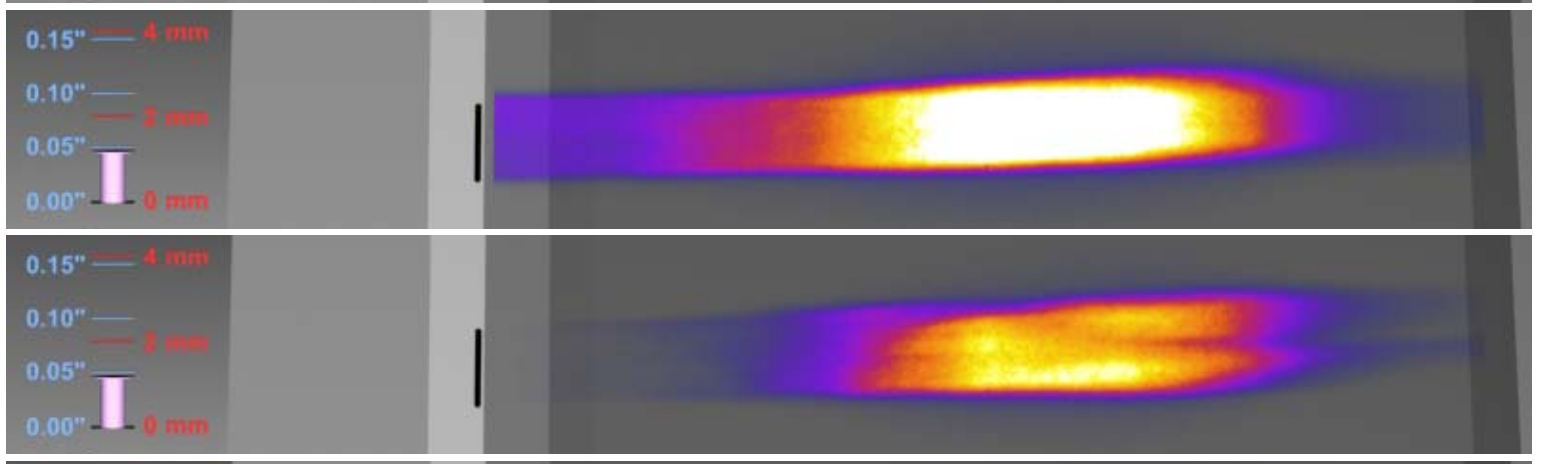

(g)

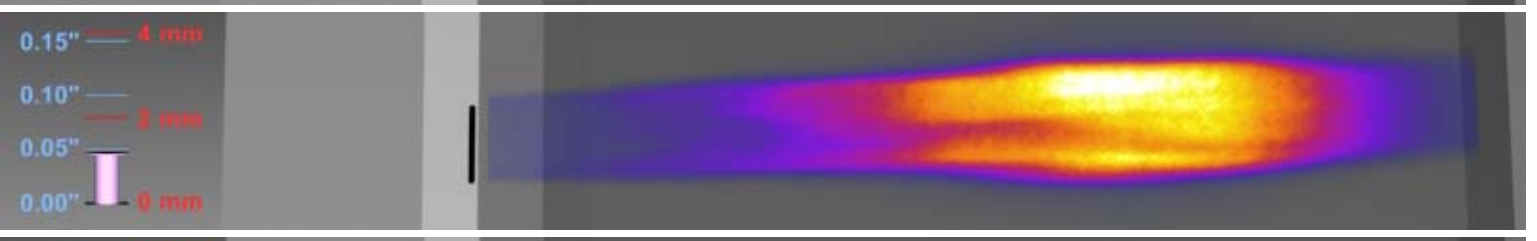

(h)

(i) A43: Test 443, Run 25, no trip, plate angle $=20^{\circ}$, 11-mm wide slot seeding, $\dot{m}=300 \mathrm{sccm}, \mathrm{P}_{0}=4.98 \mathrm{MPa}$, sheet position $=1.2 \mathrm{~mm}$ above the plate, framing rate $=10 \mathrm{~Hz}$. 
(a)

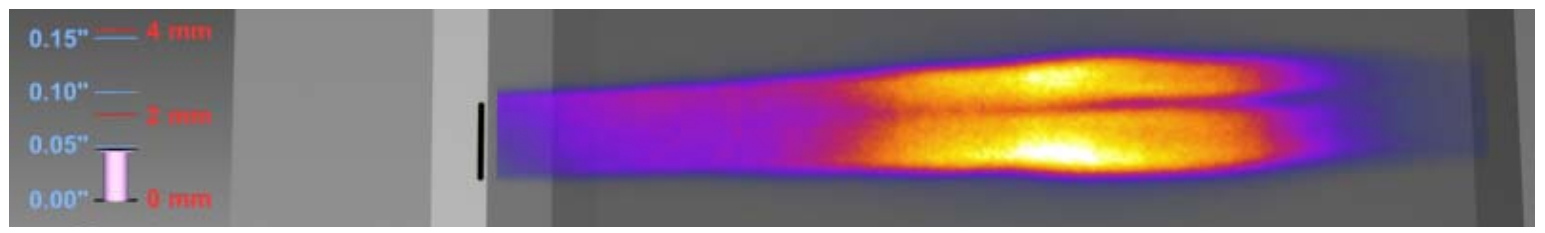

(b)

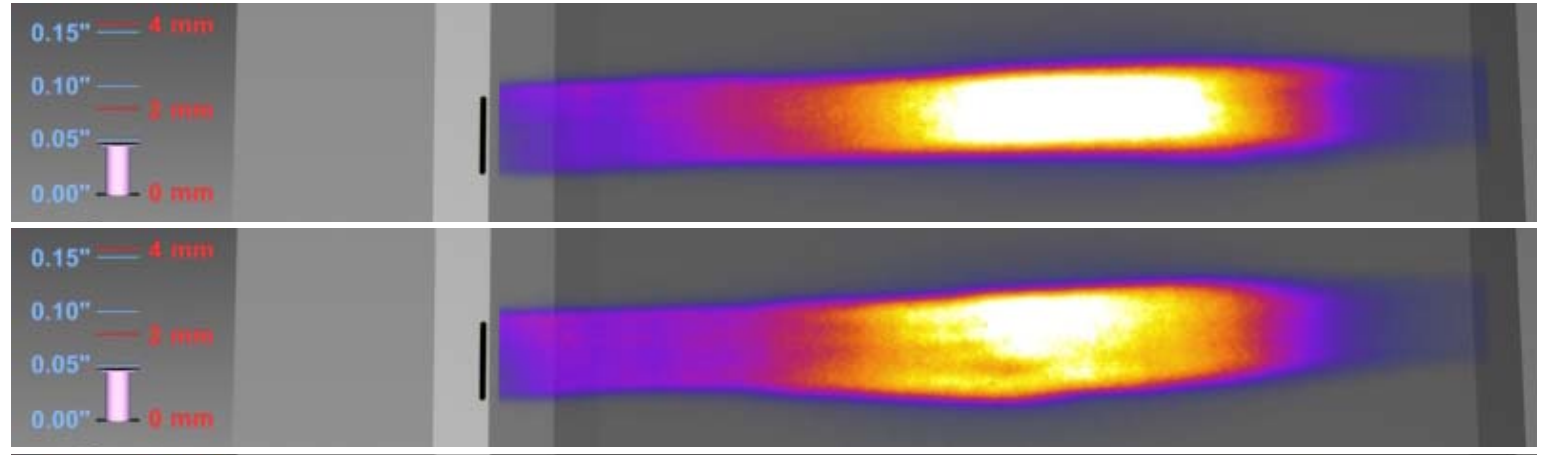

(c)

(d)

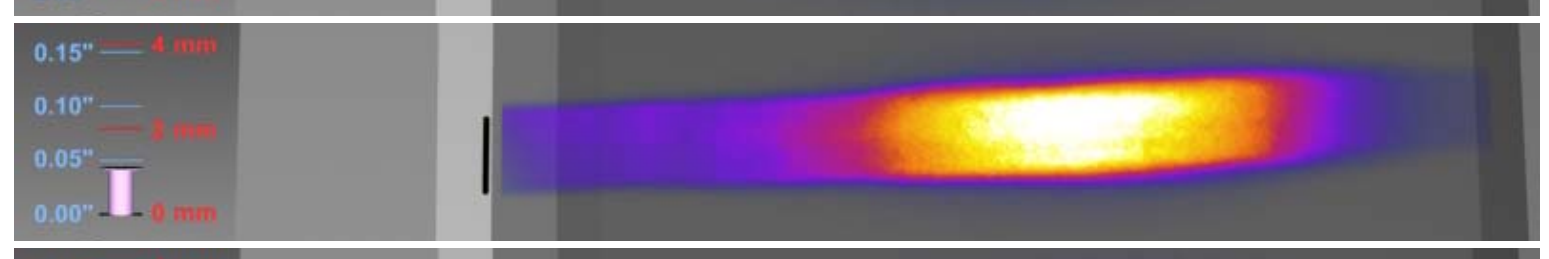

(e)

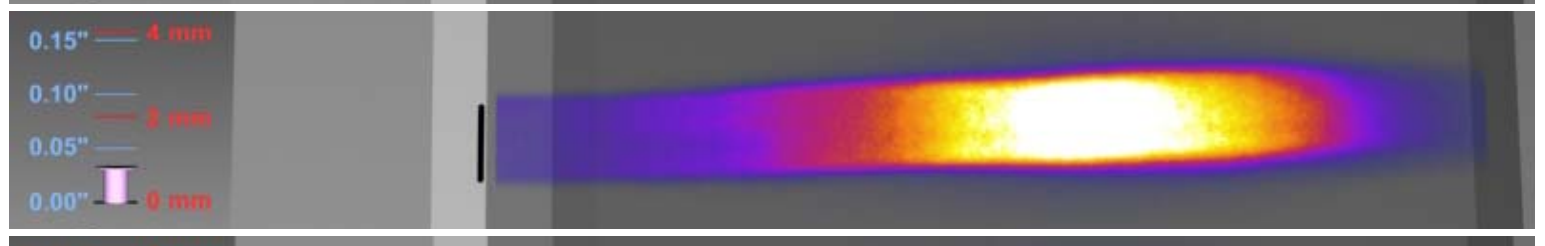

(f)

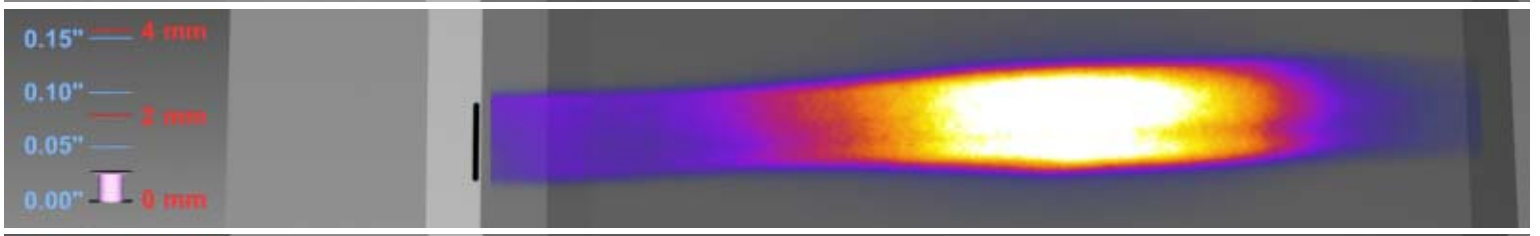

(g)

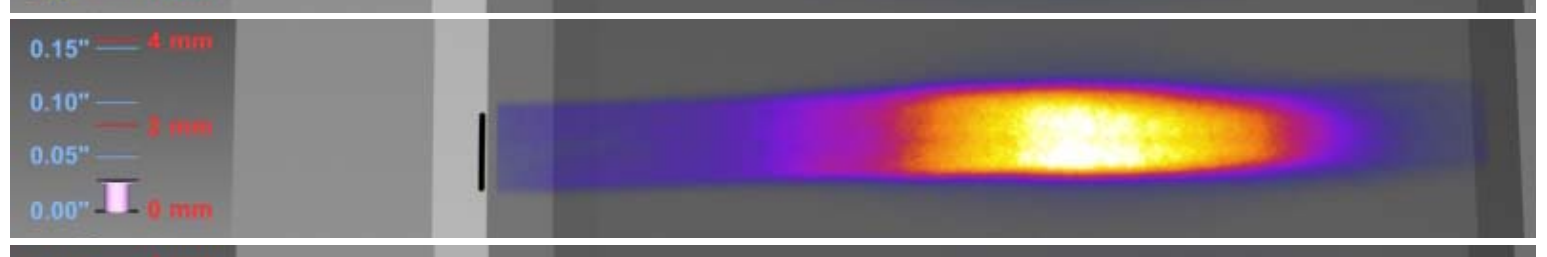

(h)
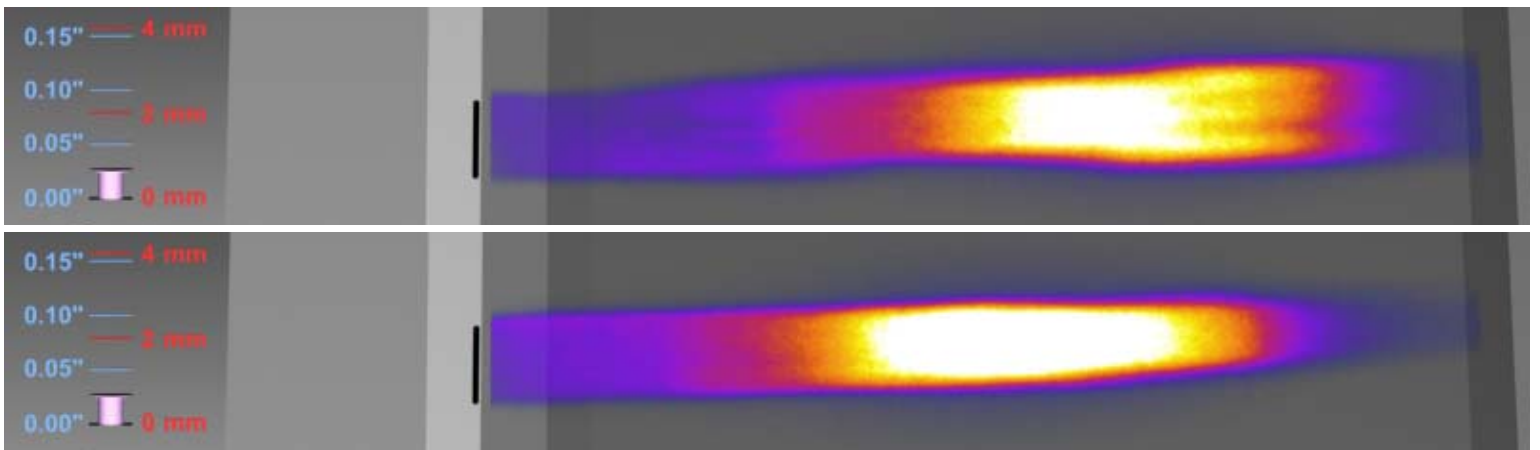

Figure A44: Test 443, Run 25, no trip, plate angle $=20^{\circ}$, 11 -mm wide slot seeding, $\dot{m}=300 \mathrm{sccm}, \mathrm{P}_{0}=4.98 \mathrm{MPa}$, sheet position $=0.7 \mathrm{~mm}$ above the plate, framing rate $=10 \mathrm{~Hz}$. 
(a)

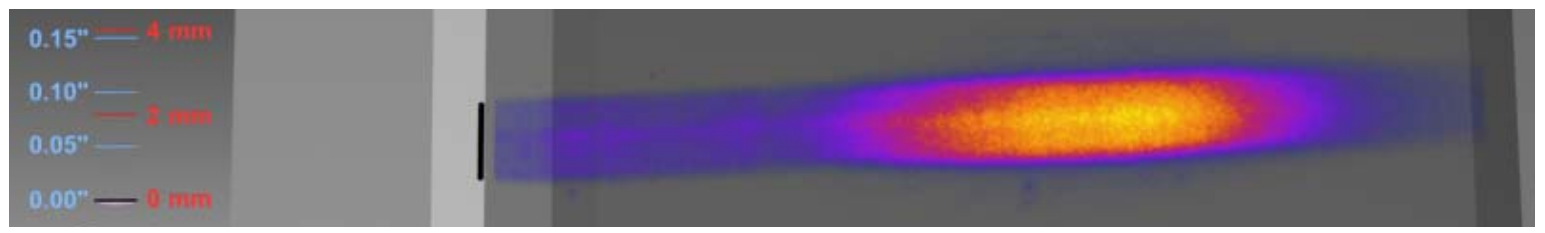

(b)

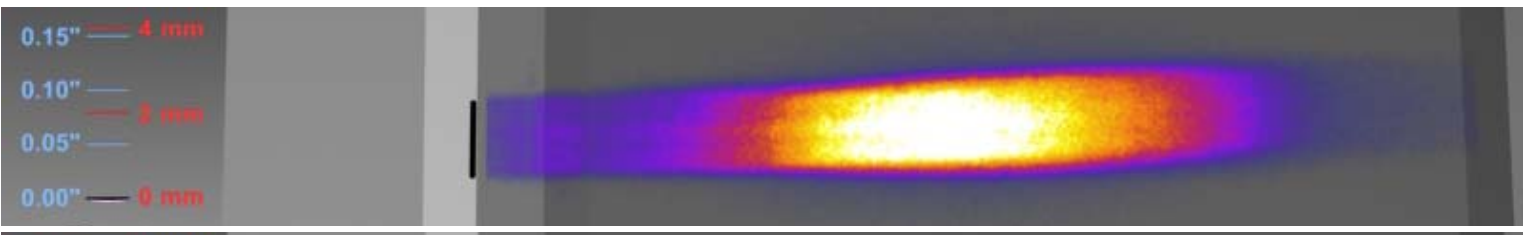

(c)

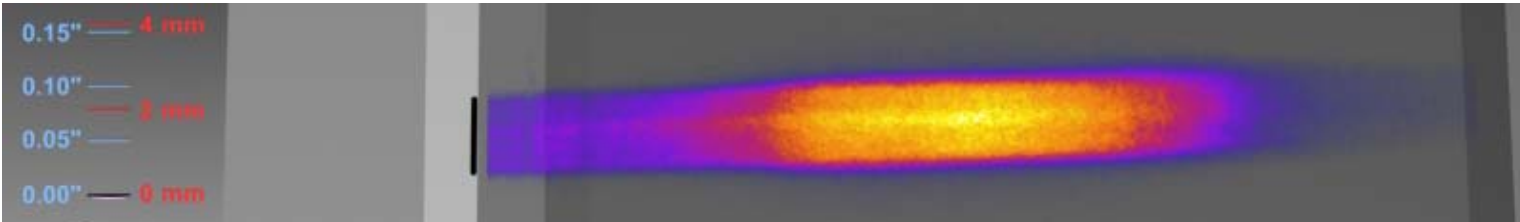

(d)

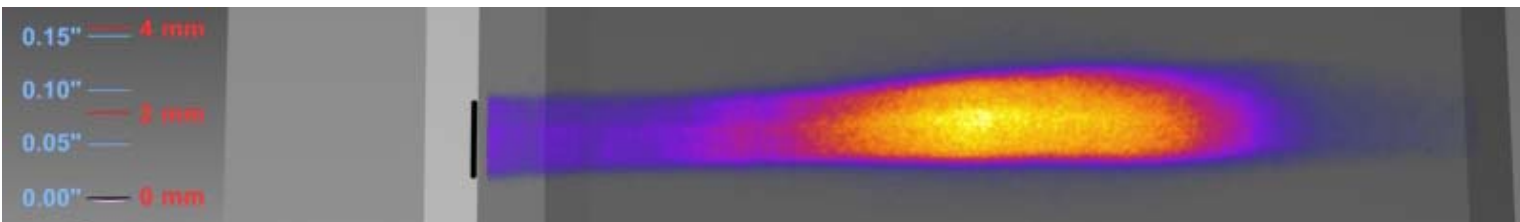

(e)

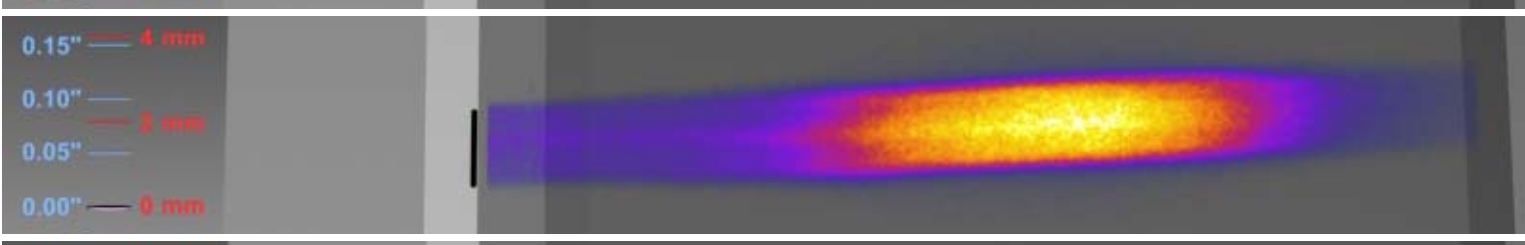

(f)
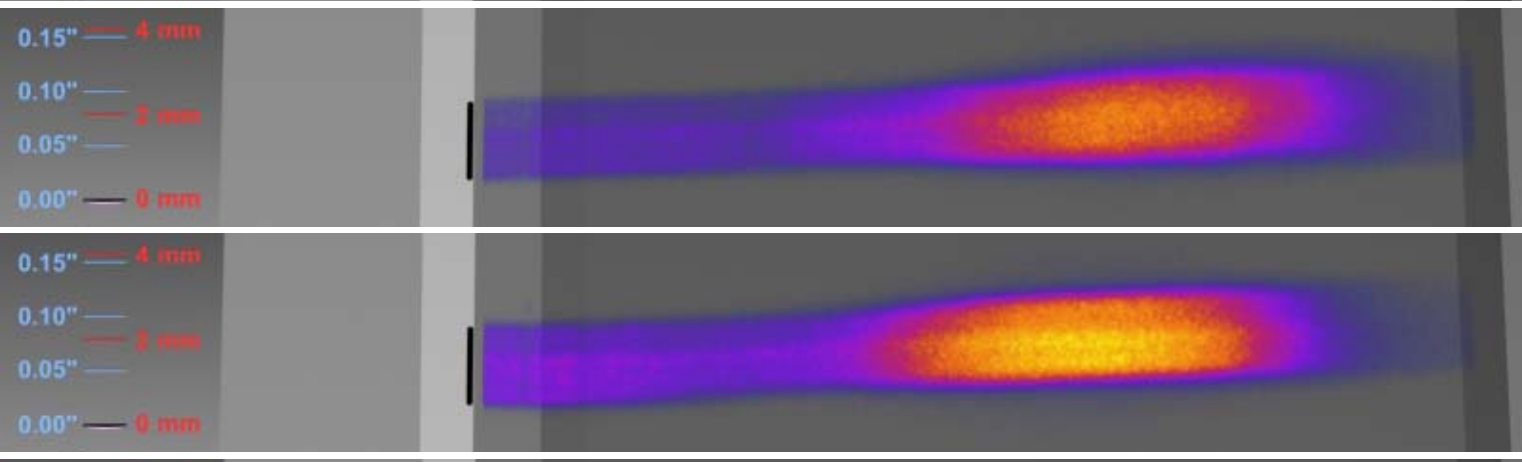

(g)

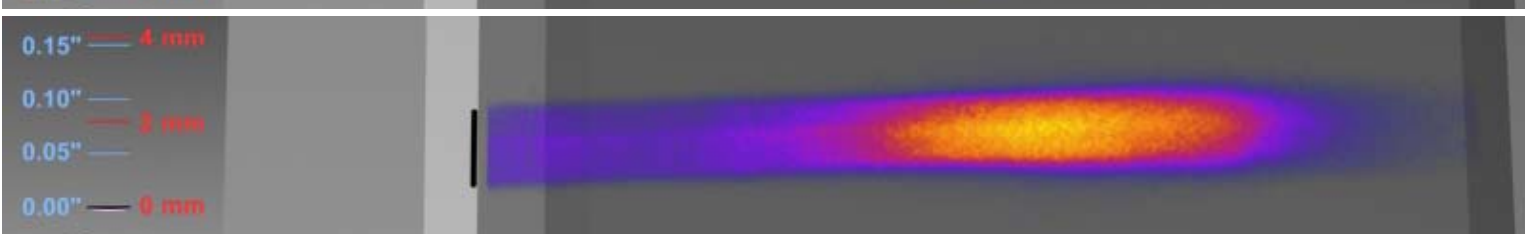

(h)

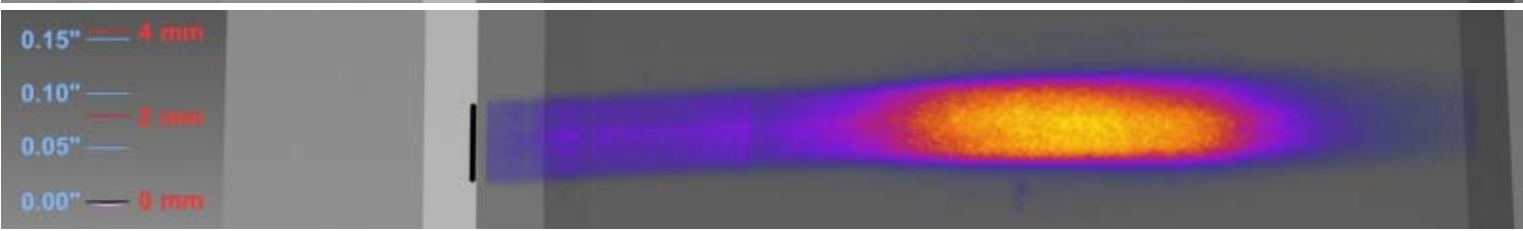
(i)

Figure A45: Test 443, Run 25, no trip, plate angle $=20^{\circ}$, 11 -mm wide slot seeding, $\dot{m}=300 \mathrm{sccm}, \mathrm{P}_{0}=4.98 \mathrm{MPa}$, sheet position $=0.0 \mathrm{~mm}$ above the plate, framing rate $=10 \mathrm{~Hz}$. 


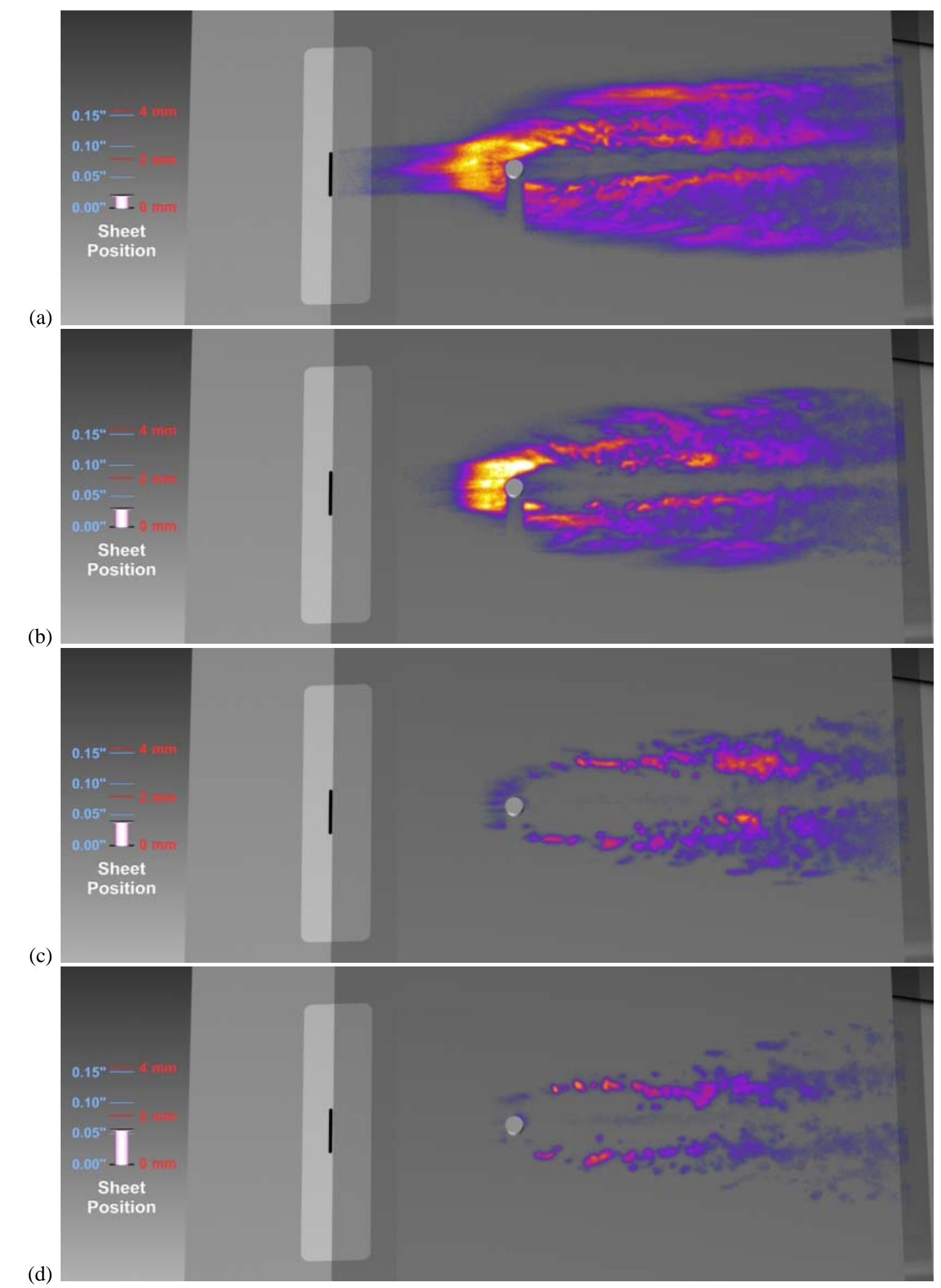

Figure A46: Test 443, Run 31, 2-mm tall by 4-mm wide cylinder, plate angle $=20^{\circ}$, 11-mm wide slot seeding, $\dot{m}=$ $300 \mathrm{sccm}, \mathrm{P}_{0}=9.33 \mathrm{MPa}$, sheet position $=0.6,0.8,1.0$ and $1.5 \mathrm{~mm}$ above the surface in (a)-(d) respectively, framing rate $=10 \mathrm{~Hz}$. 


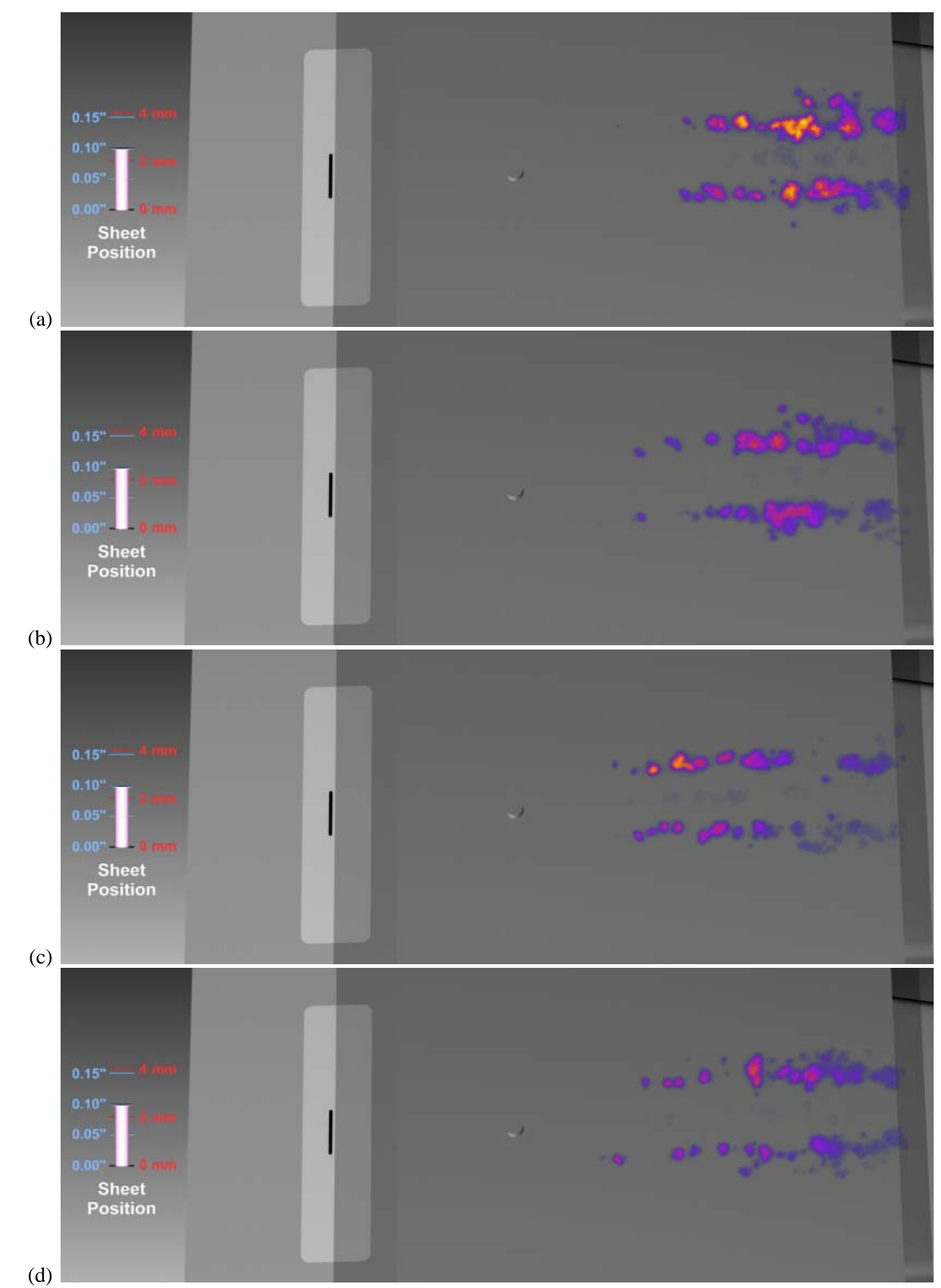

Figure A47: Test 443, Run 31, 2-mm tall by 4-mm wide cylinder, plate angle $=20^{\circ}, 11$-mm wide slot seeding, $\dot{m}=$ $300 \mathrm{sccm}, \mathrm{P}_{0}=9.33 \mathrm{MPa}$, sheet position $=2.6 \mathrm{~mm}$ above the plate, framing rate $=10 \mathrm{~Hz}$. 


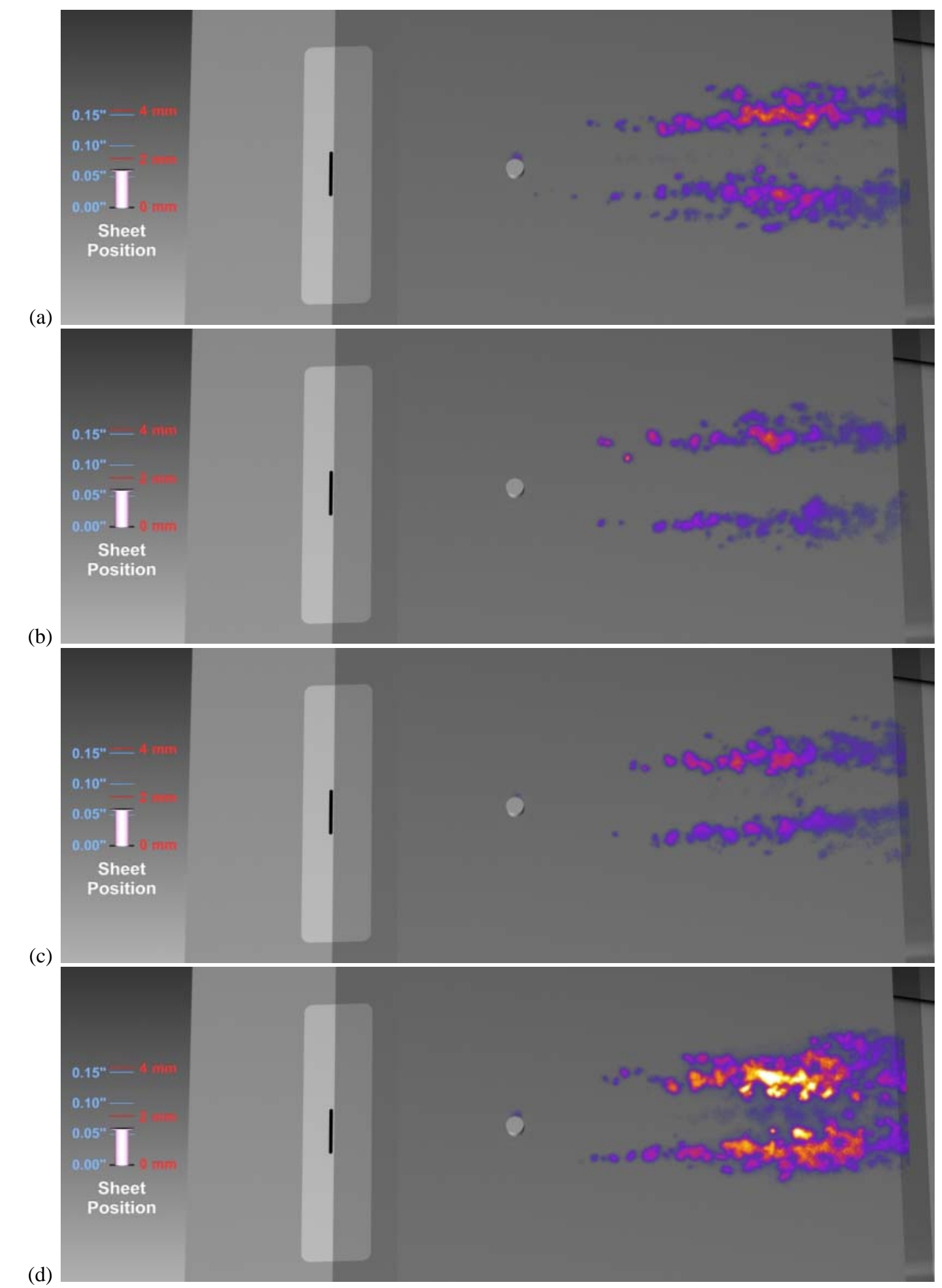

Figure A48: Test 443, Run 31, 2-mm tall by 4-mm wide cylinder, plate angle $=20^{\circ}$, 11-mm wide slot seeding, $\dot{m}=$ $300 \mathrm{sccm}, \mathrm{P}_{0}=9.33 \mathrm{MPa}$, sheet position $=1.6 \mathrm{~mm}$ above the plate, framing rate $=10 \mathrm{~Hz}$. 


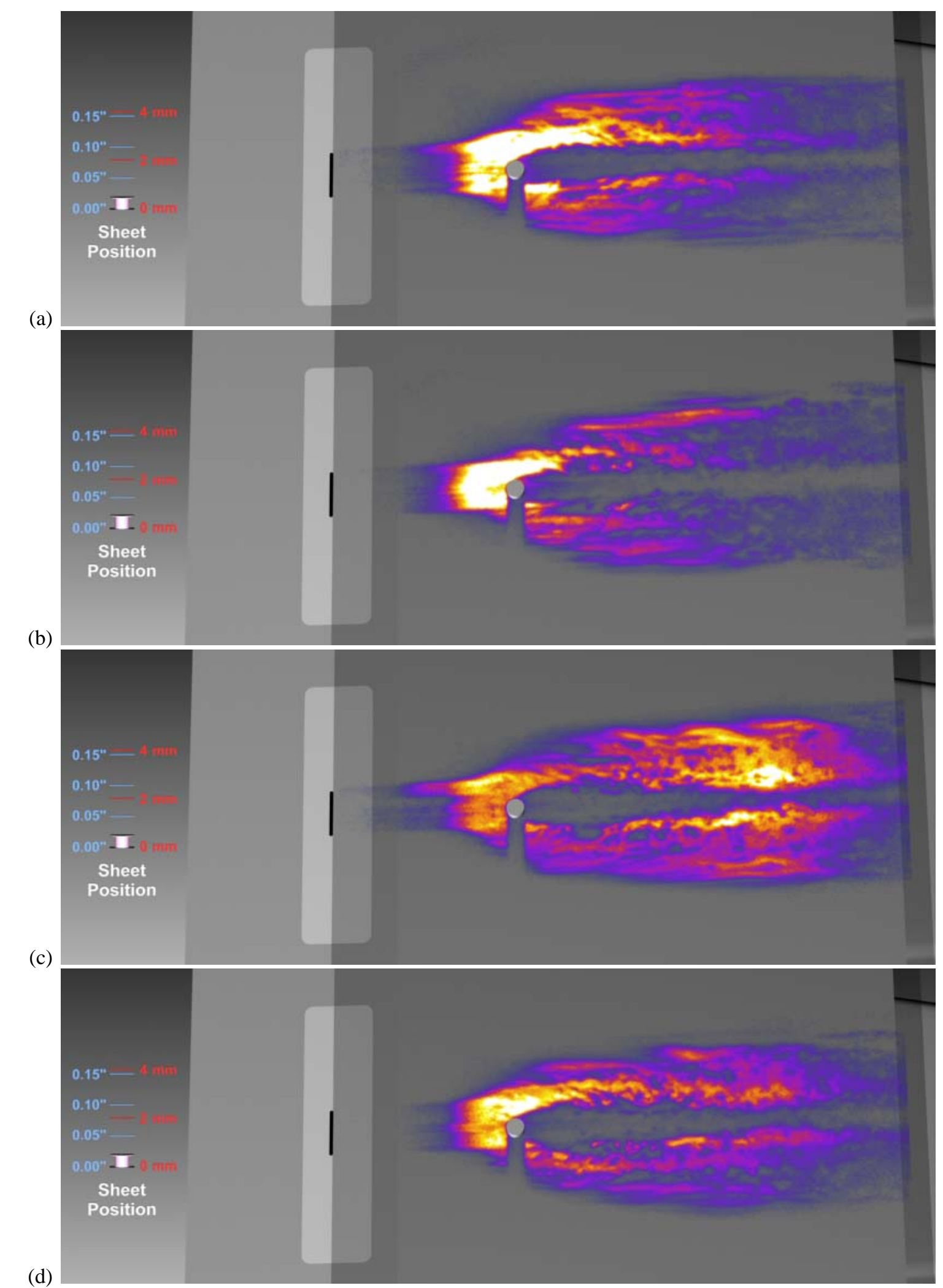

Figure A49: Test 443, Run 31, 2-mm tall by 4-mm wide cylinder, plate angle $=20^{\circ}$, 11-mm wide slot seeding, $\dot{m}=$ $300 \mathrm{sccm}, \mathrm{P}_{0}=9.33 \mathrm{MPa}$, sheet position $=0.5 \mathrm{~mm}$ above the plate, framing rate $=10 \mathrm{~Hz}$. 
(a)

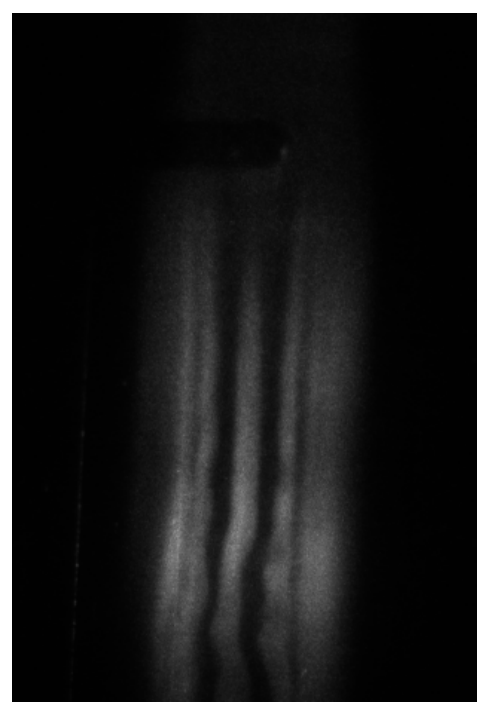

(b)
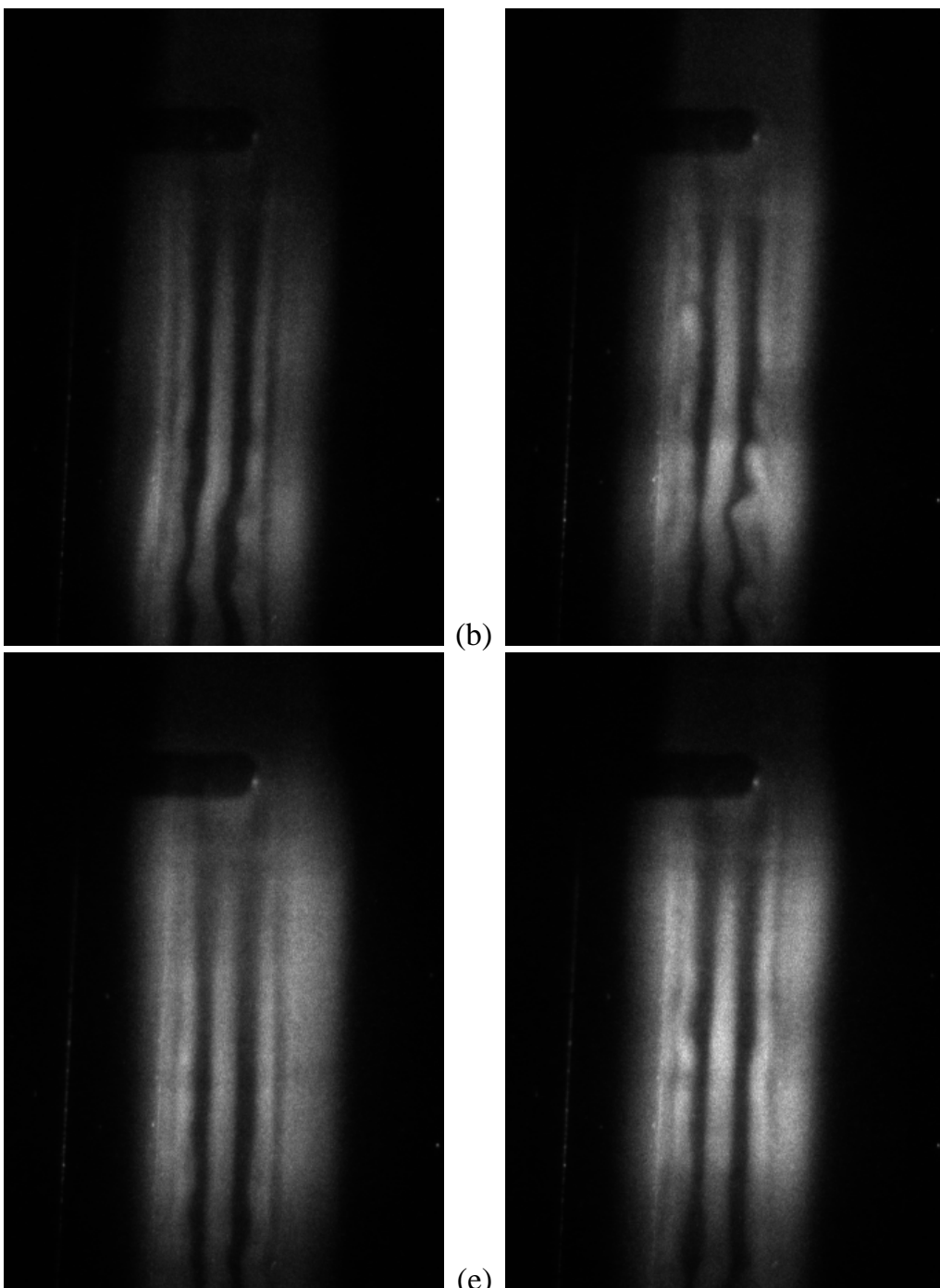

(d)

(e)

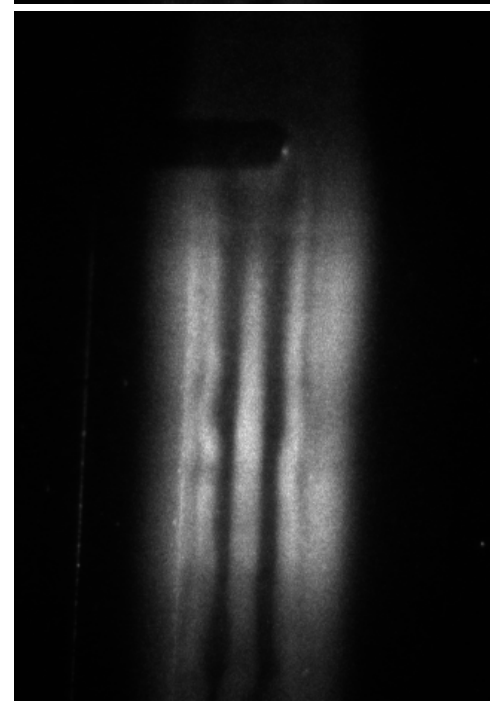

(c)

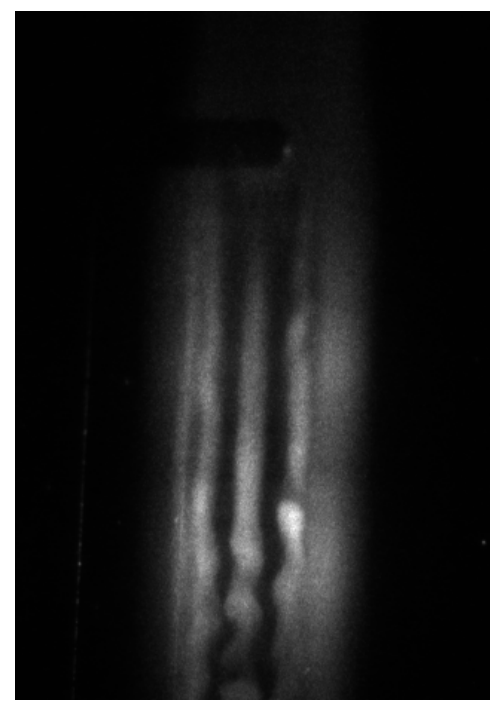

(f)

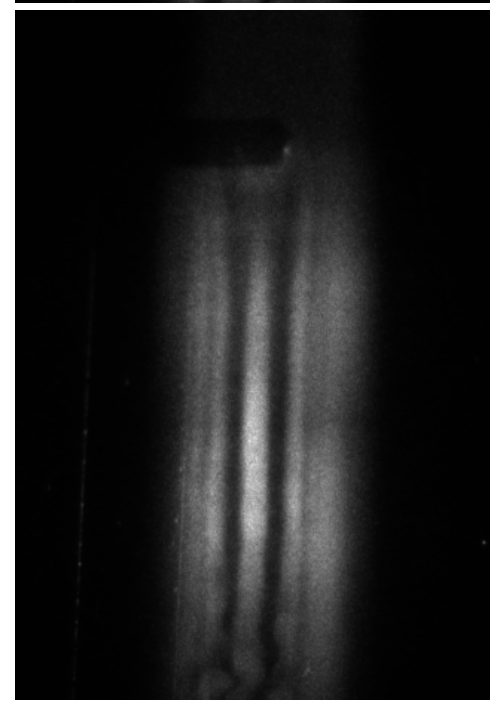

(g) (h)
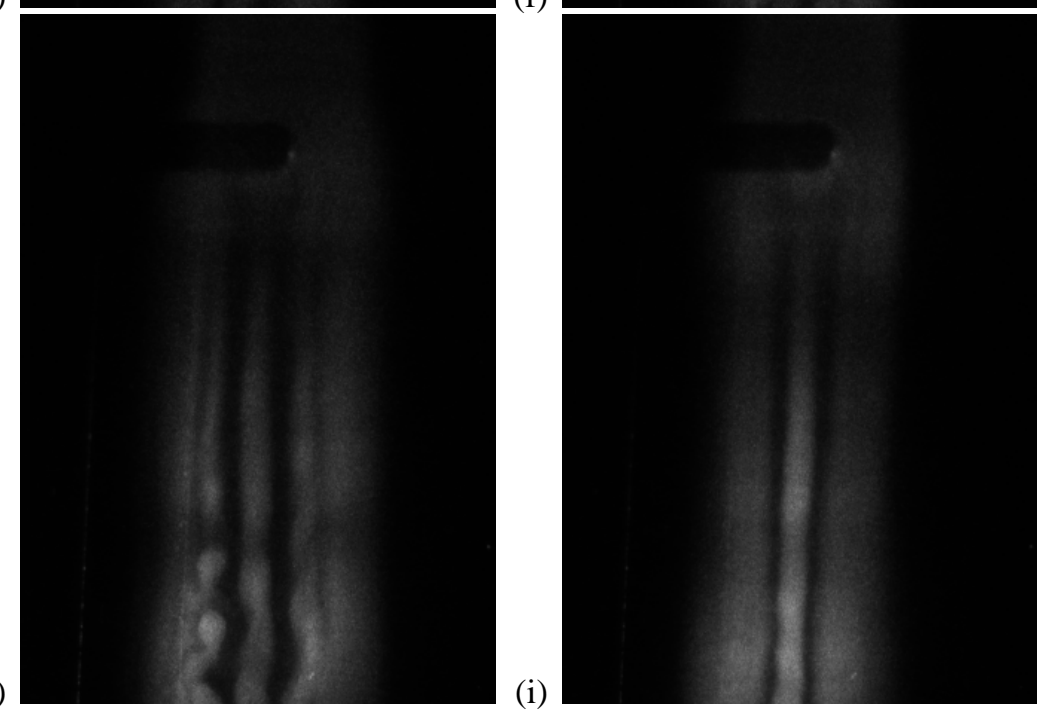

(i)

Figure A50: Test 443, Run 47, 1-mm tall by 4 -mm wide cylinder trip, plate angle $=20^{\circ}$, 11 -mm wide slot seeding, $\dot{m}=310 \mathrm{sccm}, \mathrm{P}_{0}=4.97 \mathrm{MPa}$, thick laser sheet PLIF visualization, framing rate $=10 \mathrm{~Hz}$. 
(a)

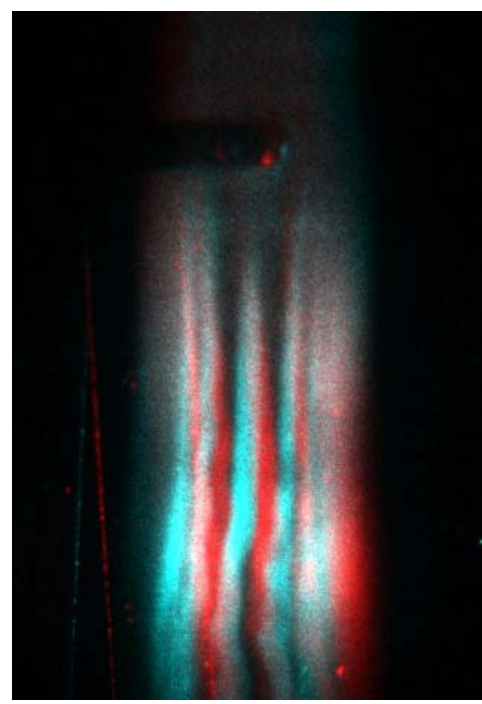

(b)
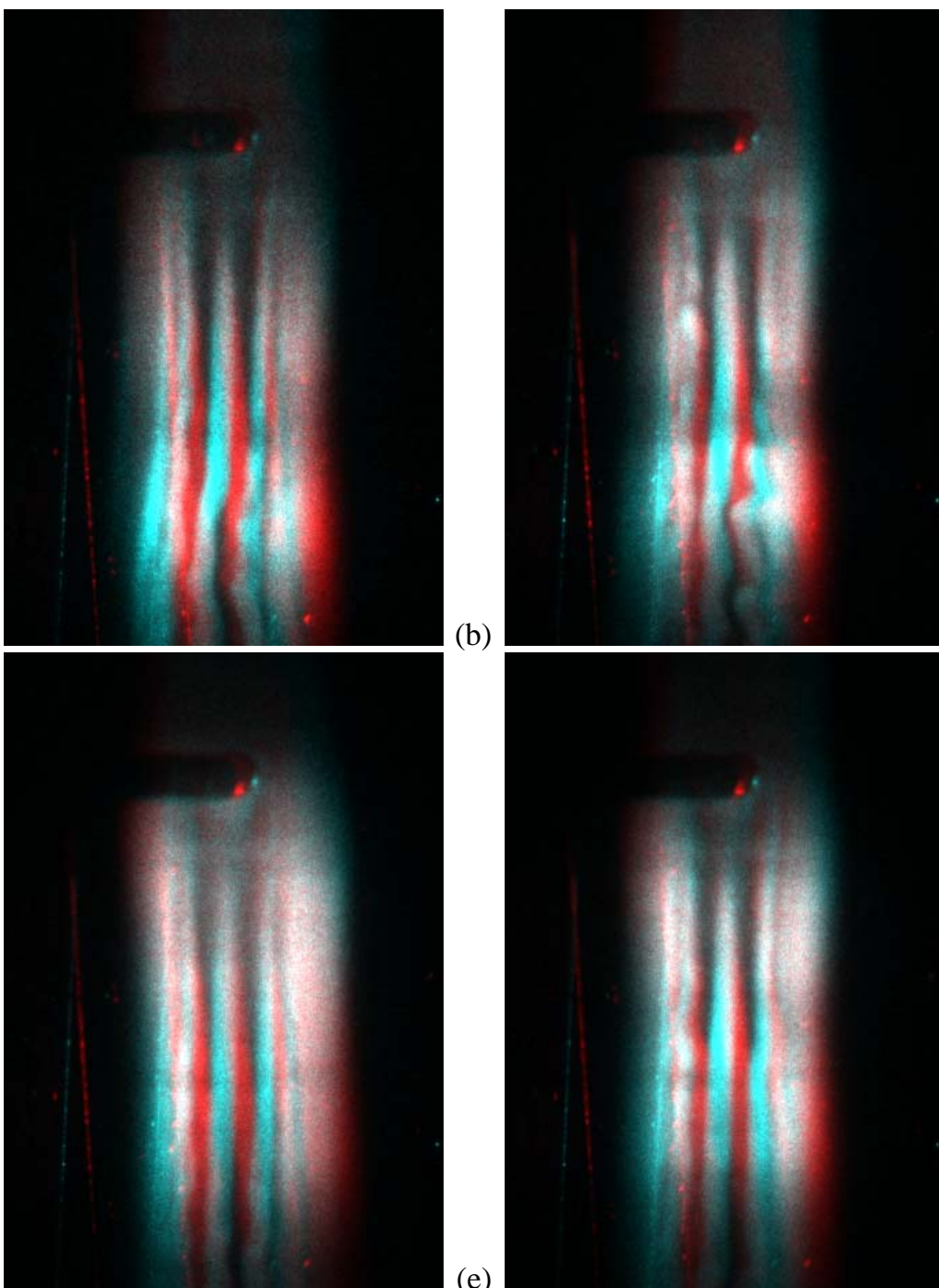

(d)

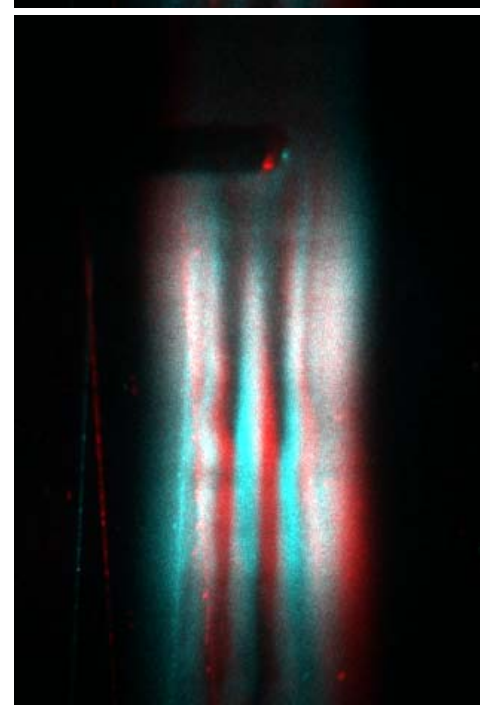

(c)

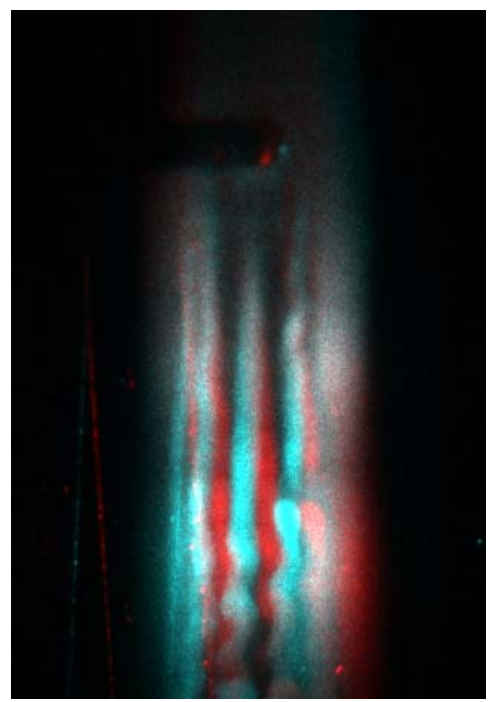

(f)
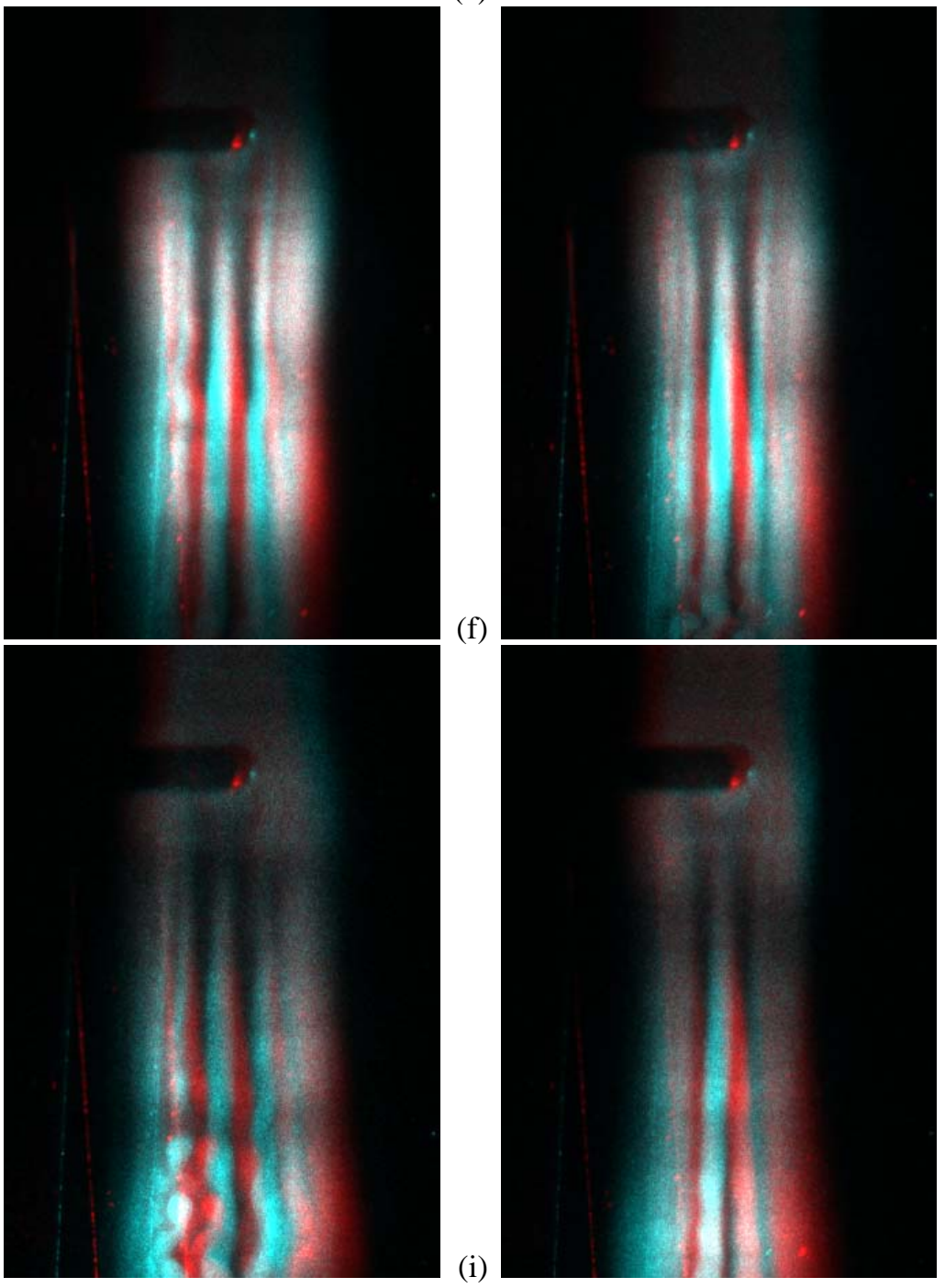

(g) (h) (i)

Figure A51: Test 443, Run 47, 1-mm tall by 4-mm wide cylinder trip, plate angle $=20^{\circ}$, 11-mm wide slot seeding, $\dot{m}=310 \mathrm{sccm}, \mathrm{P}_{0}=4.97 \mathrm{MPa}$, stereoscopic PLIF visualization, framing rate $=10 \mathrm{~Hz}$. 
(a)

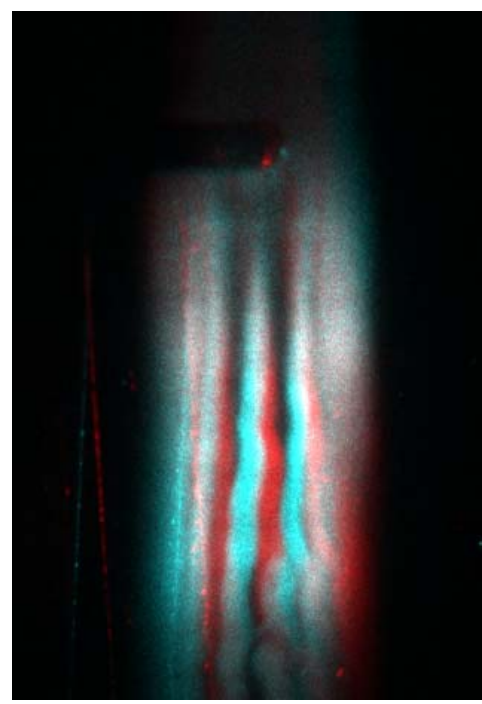

(b)

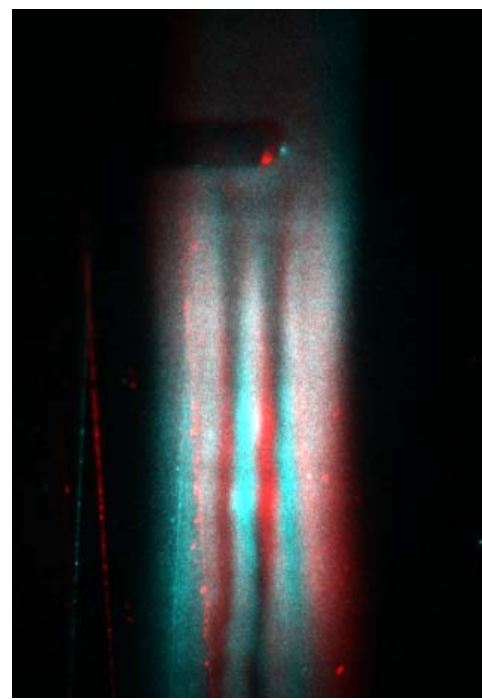

(c)

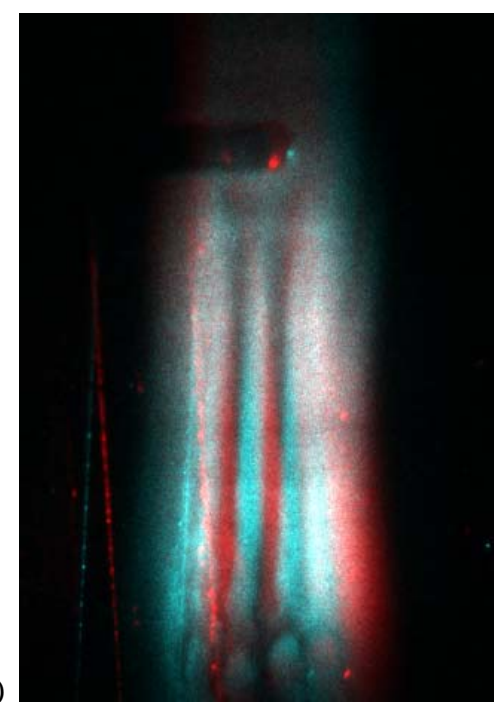

(e)
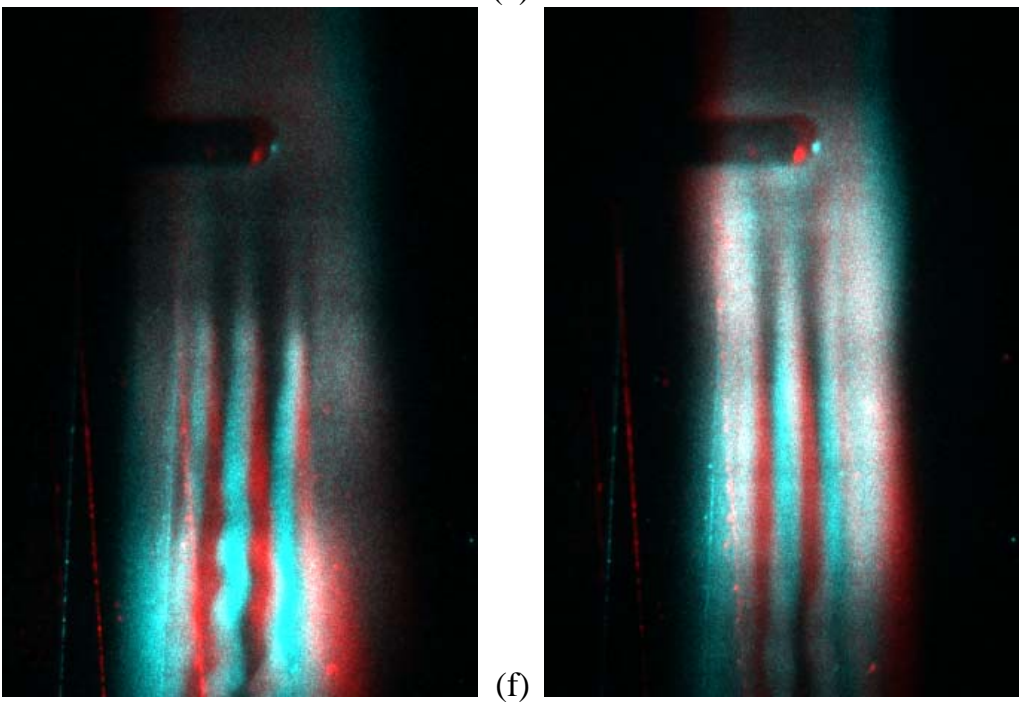

(d)
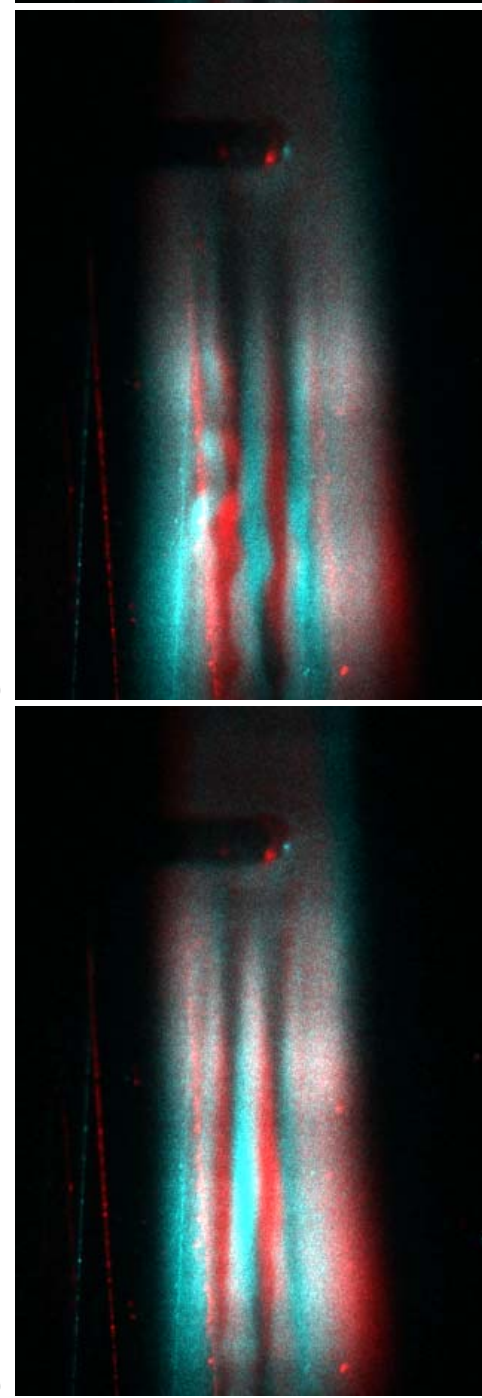

)
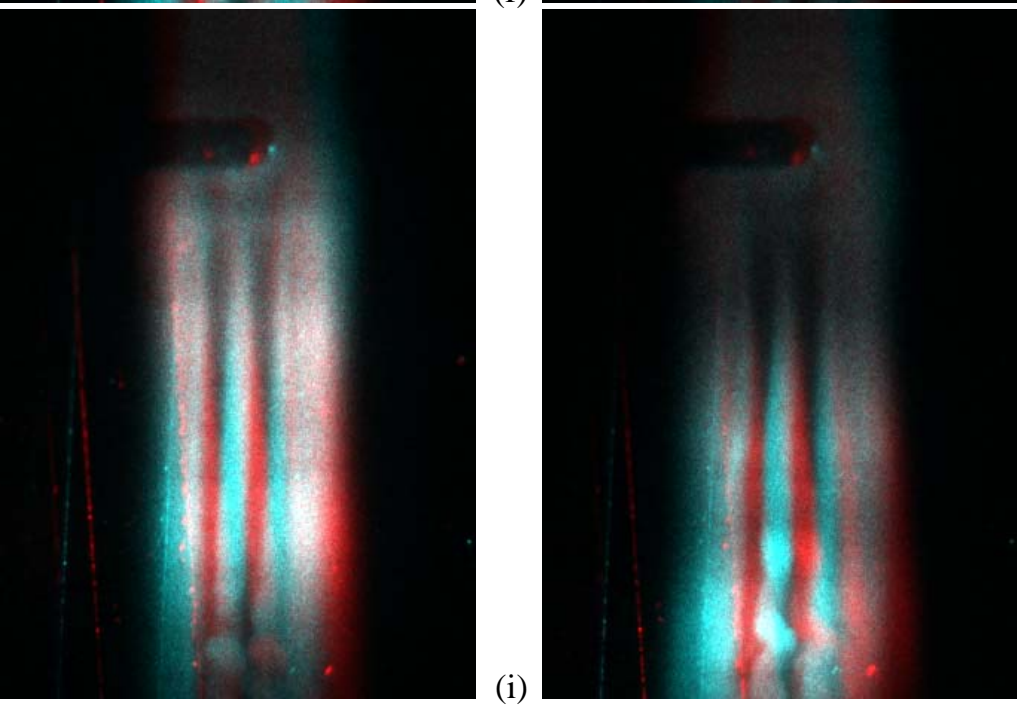

(i)

Figure A52: Test 443, Run 47, 1-mm tall by 4-mm wide cylinder trip, plate angle $=20^{\circ}$, 11-mm wide slot seeding, $m=310 \mathrm{sccm}, \mathrm{P}_{0}=4.97 \mathrm{MPa}$, stereoscopic PLIF visualization, framing rate $=10 \mathrm{~Hz}$. 
(a)

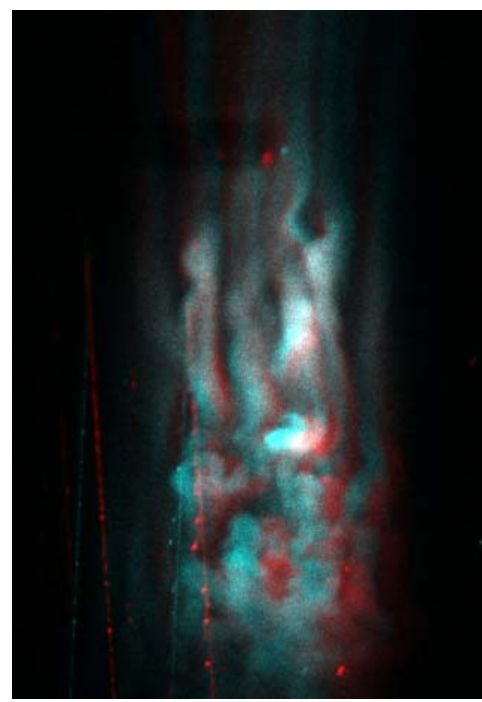

(b)

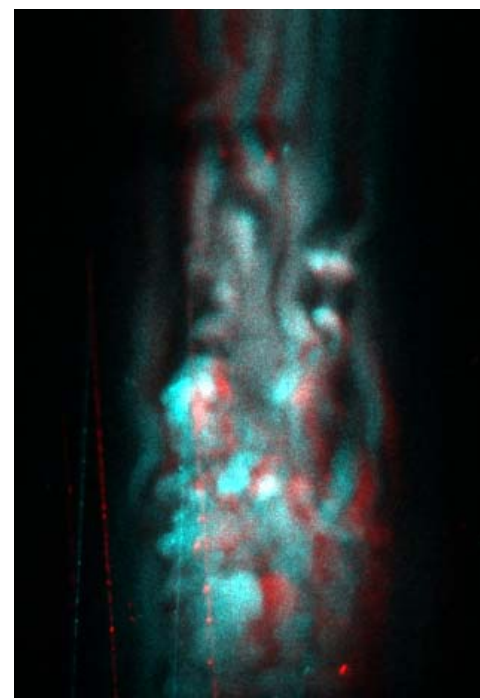

(c)

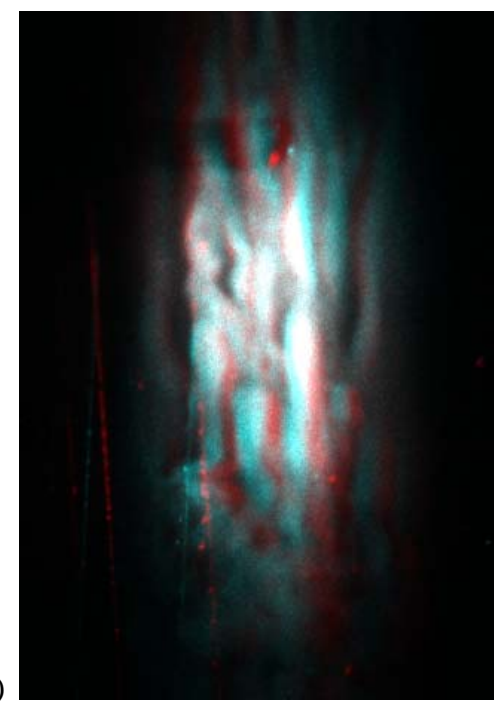

(d)

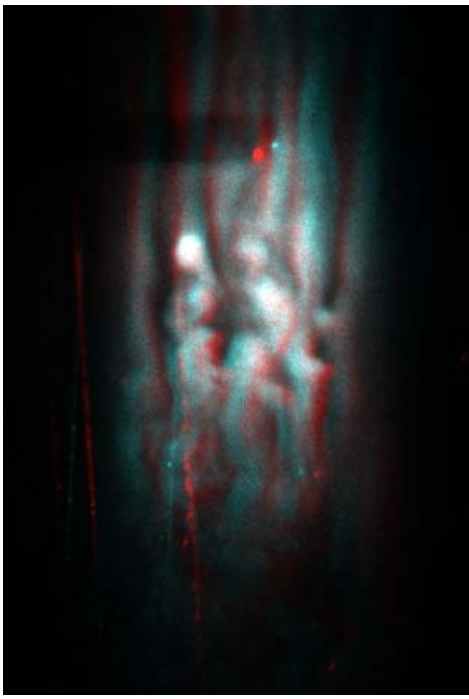

(e)

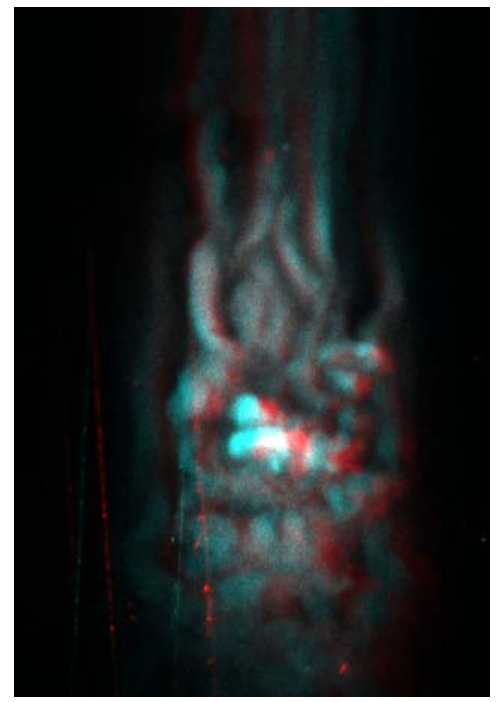

(f)
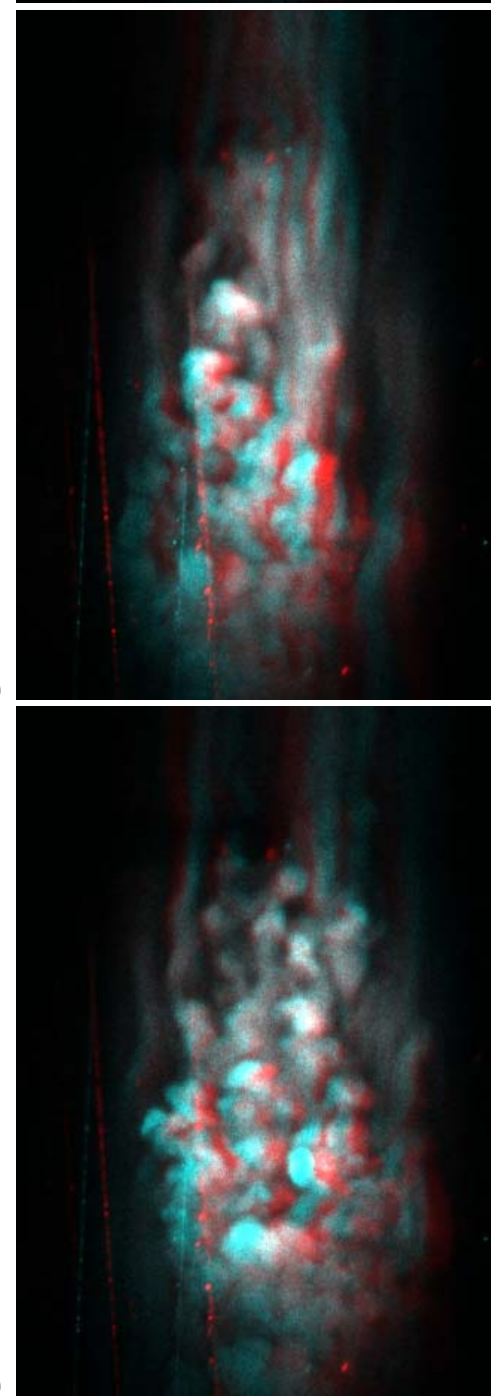

(h)

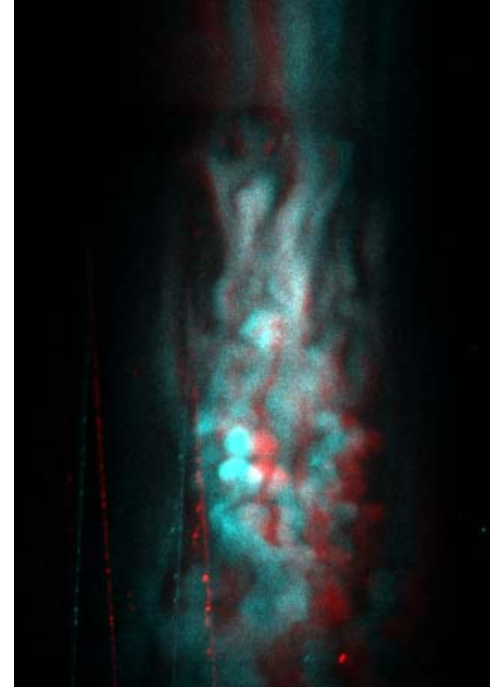

(i)

Figure A53: Test 443, Run 47, 1-mm tall by 4-mm wide cylinder trip, plate angle $=20^{\circ}, 11-\mathrm{mm}$ wide slot seeding, $\dot{m}=4300 \mathrm{sccm}, \mathrm{P}_{0}=4.97 \mathrm{MPa}$, stereoscopic PLIF visualization, framing rate $=10 \mathrm{~Hz}$. 
(a)

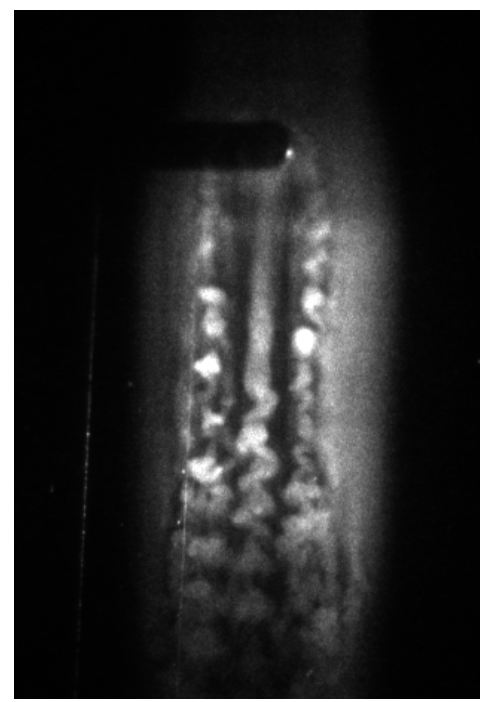

(b)

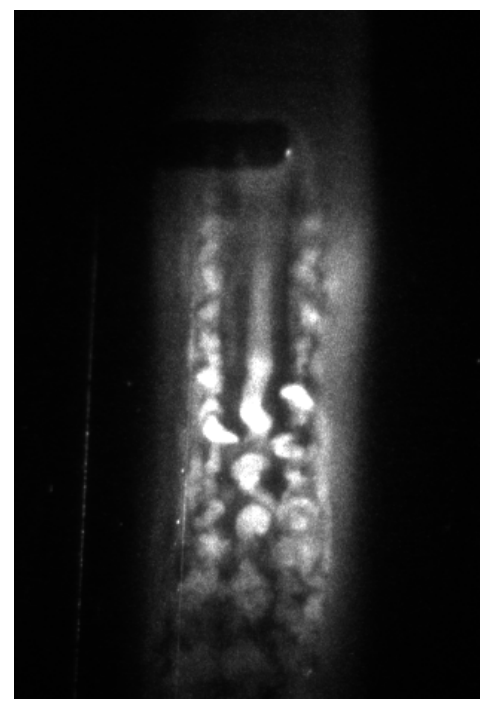

(c)

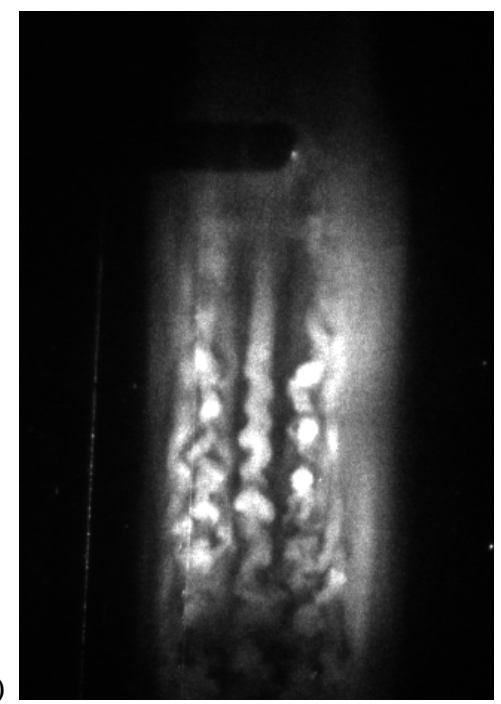

(d)

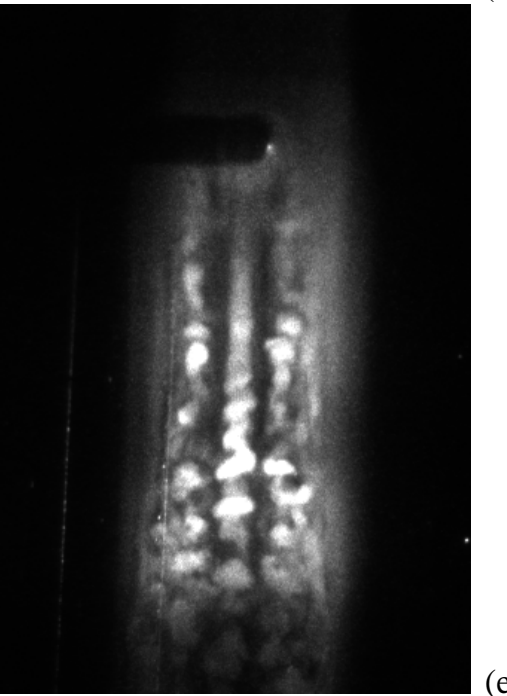

(e)
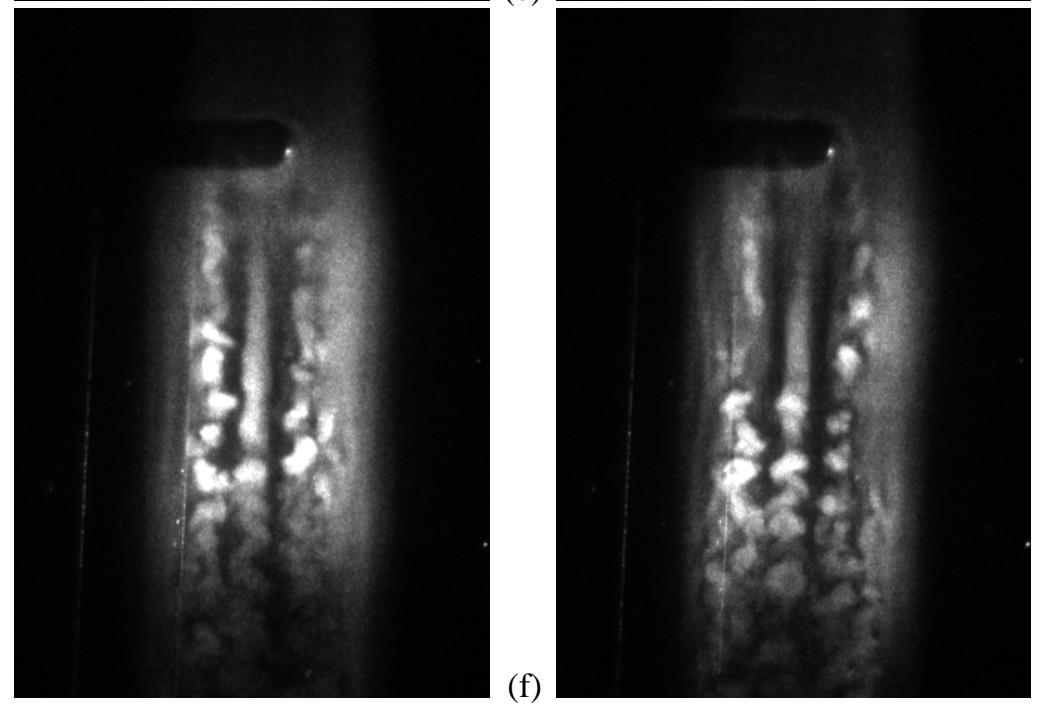

(f)
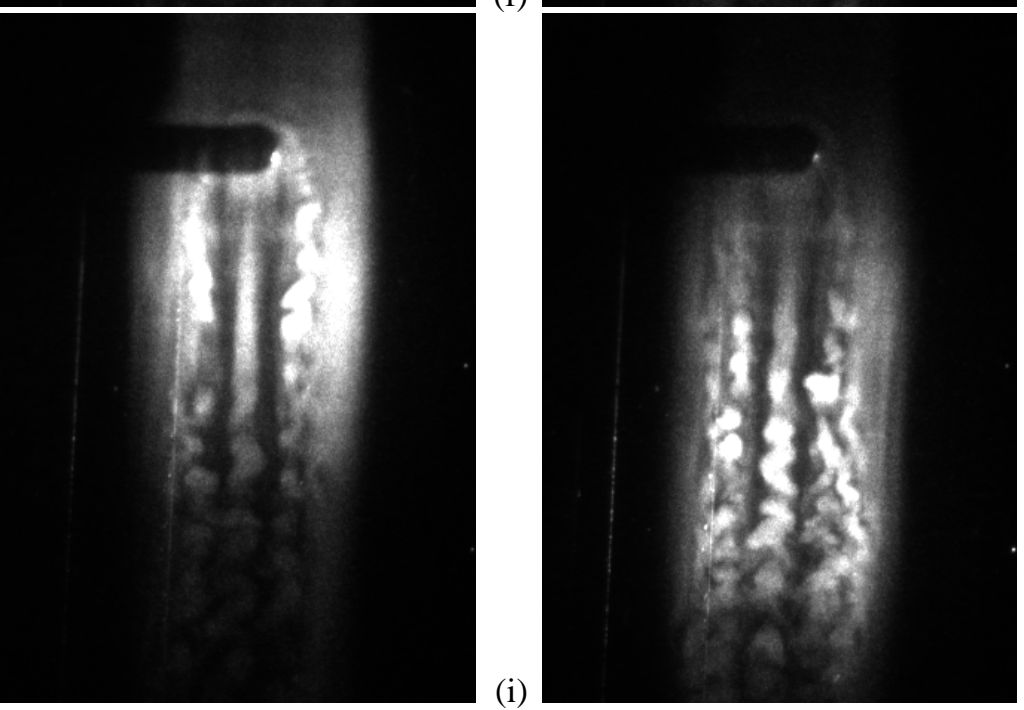

(i)

(g) (h)

Figure A54: Test 443, Run 48, 1-mm tall by 4-mm wide cylinder trip, plate angle $=20^{\circ}$, 11 -mm wide slot seeding, $\dot{m}=620 \mathrm{sccm}, \mathrm{P}_{0}=9.35 \mathrm{MPa}$, thick laser sheet PLIF visualization, framing rate $=10 \mathrm{~Hz}$. 
(a)

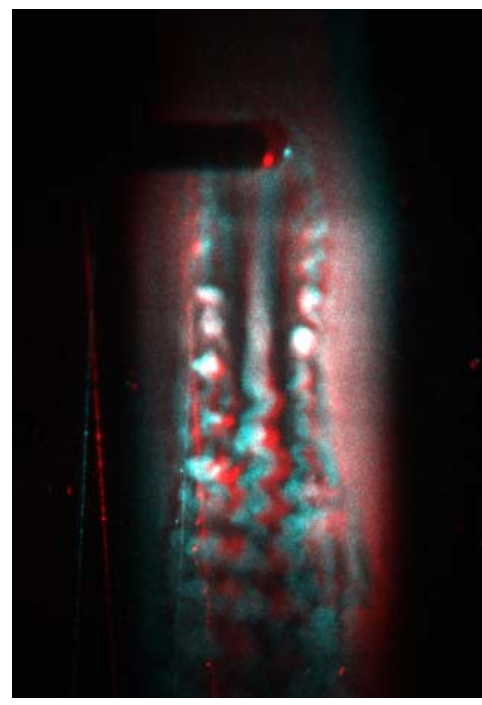

(b)

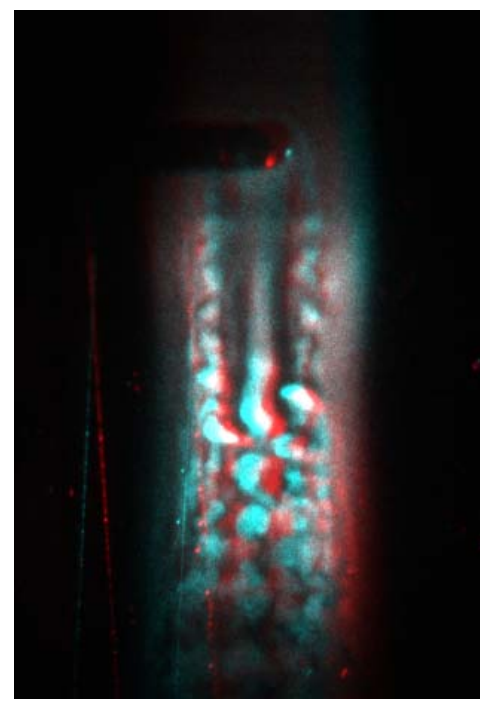

(c)

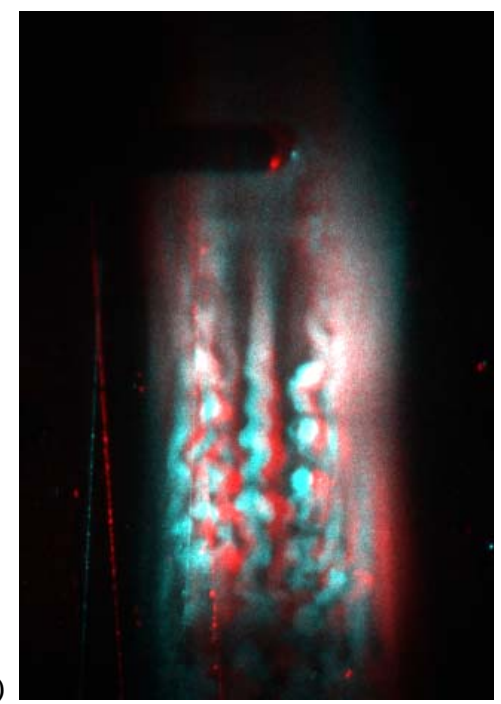

(d)

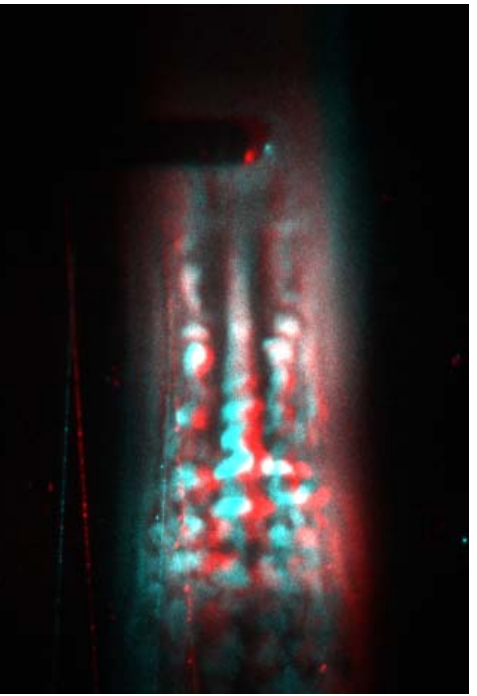

(e)
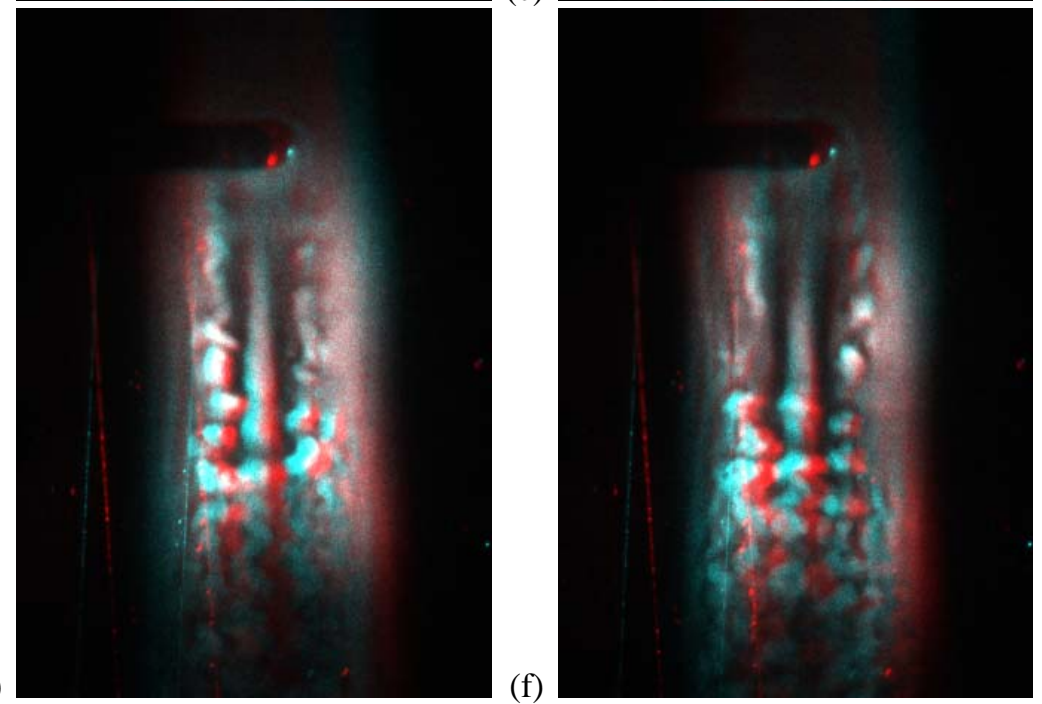

(f)
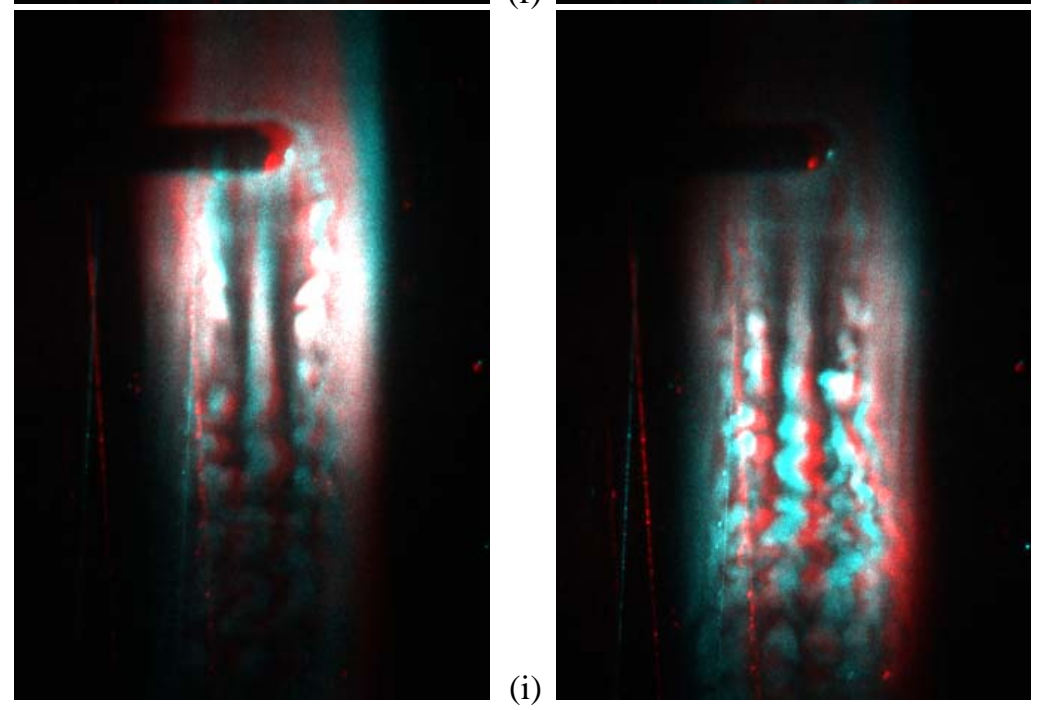

(i)

Figure A55: Test 443, Run 48, 1-mm tall by 4-mm wide cylinder trip, plate angle $=20^{\circ}, 11-\mathrm{mm}$ wide slot seeding, $\dot{m}=620 \mathrm{sccm}, \mathrm{P}_{0}=9.35 \mathrm{MPa}$, stereoscopic PLIF visualization, framing rate $=10 \mathrm{~Hz}$. 
(a)

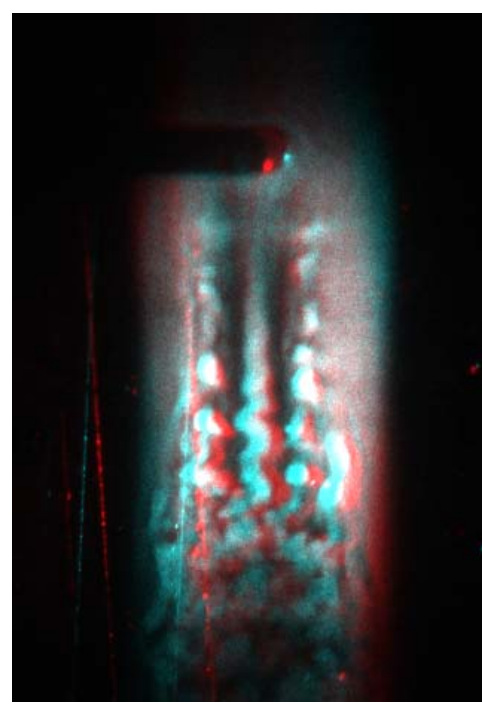

(b)

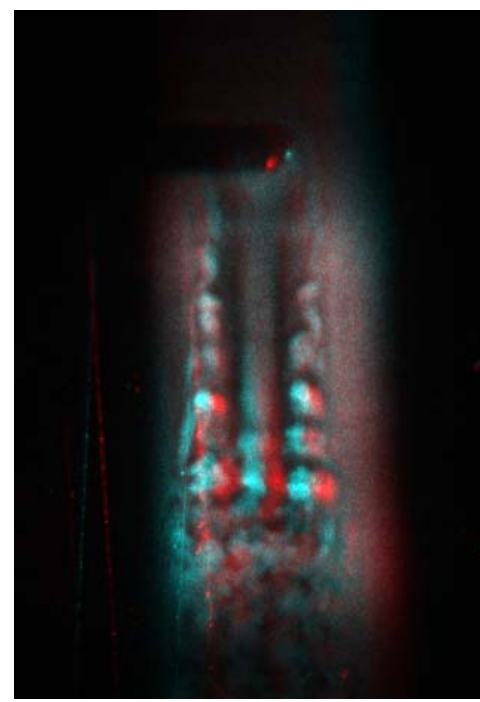

(c)

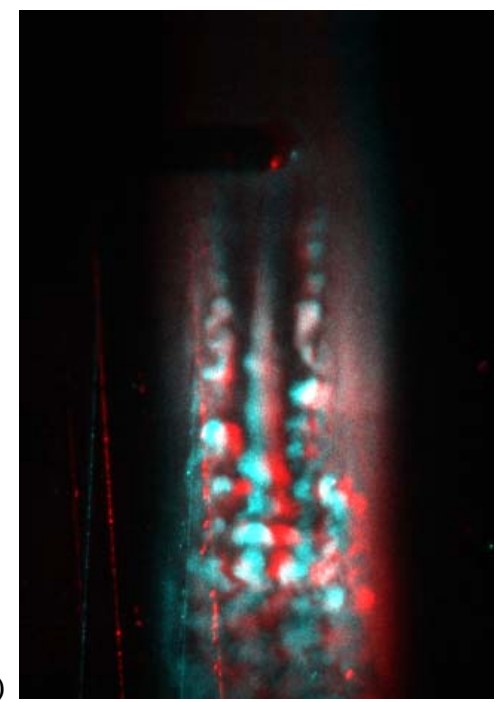

(d)

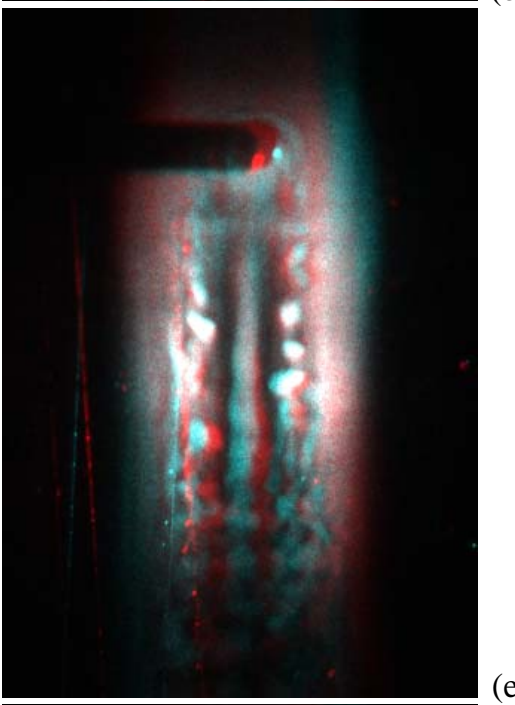

(e)
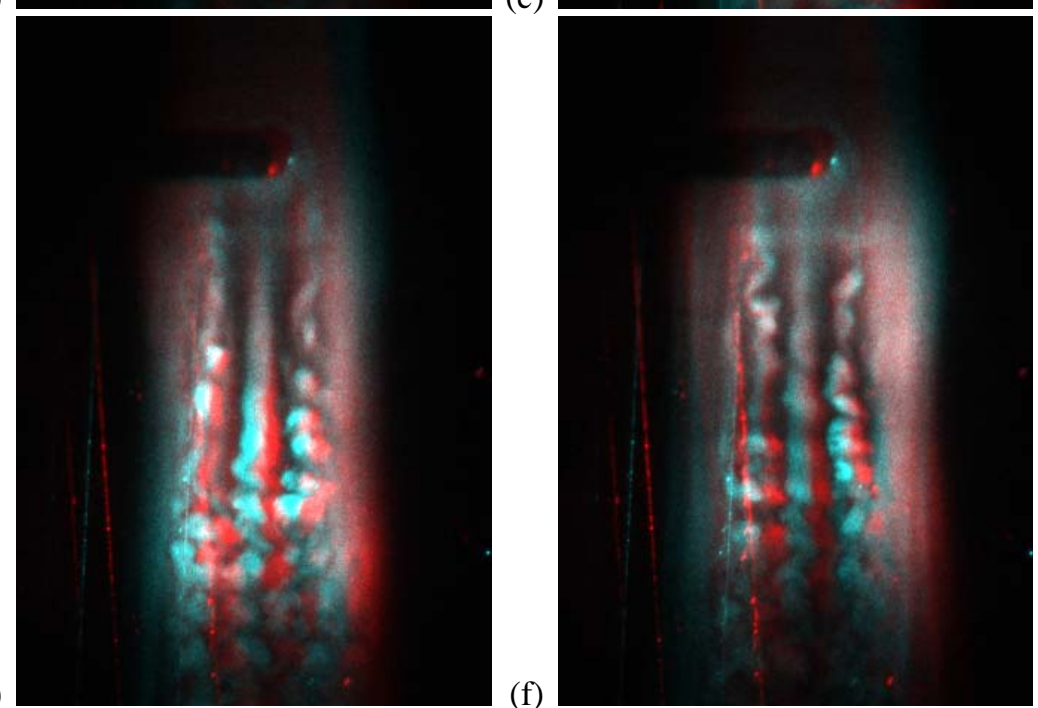

(f)
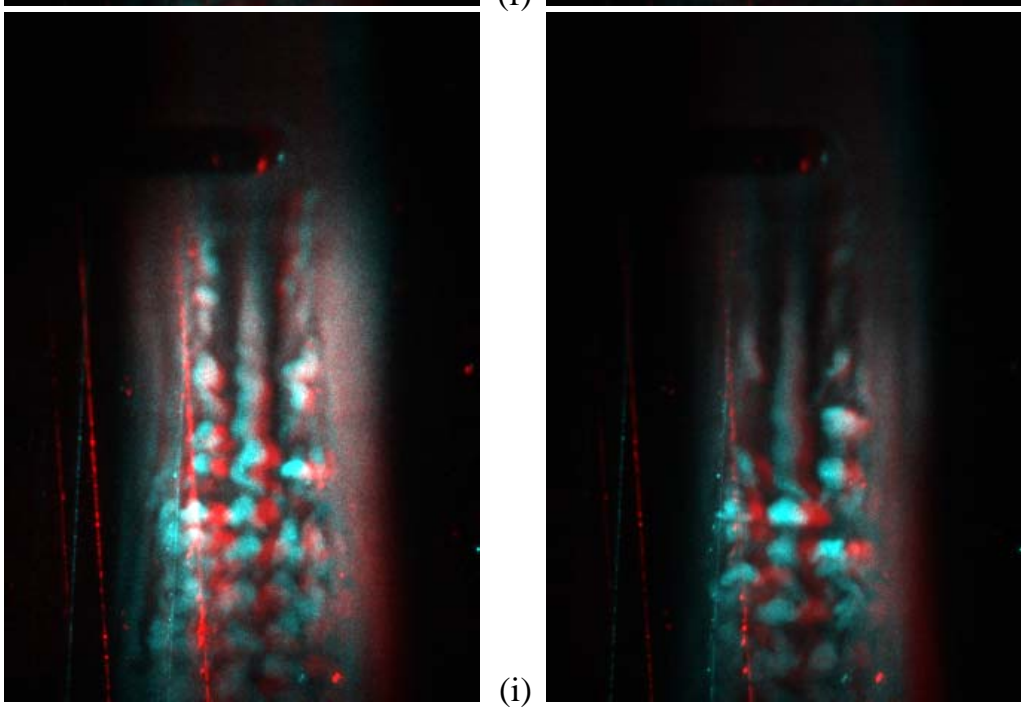

(i)

(g) (h)

Figure A56: Test 443, Run 48, 1-mm tall by 4-mm wide cylinder trip, plate angle $=20^{\circ}, 11$-mm wide slot seeding, $\dot{m}=620 \mathrm{sccm}, \mathrm{P}_{0}=9.35 \mathrm{MPa}$, stereoscopic PLIF visualization, framing rate $=10 \mathrm{~Hz}$. 
(a)

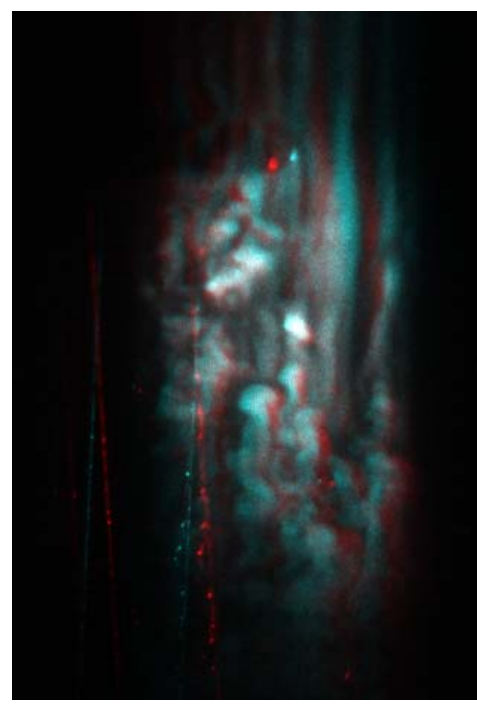

(b)
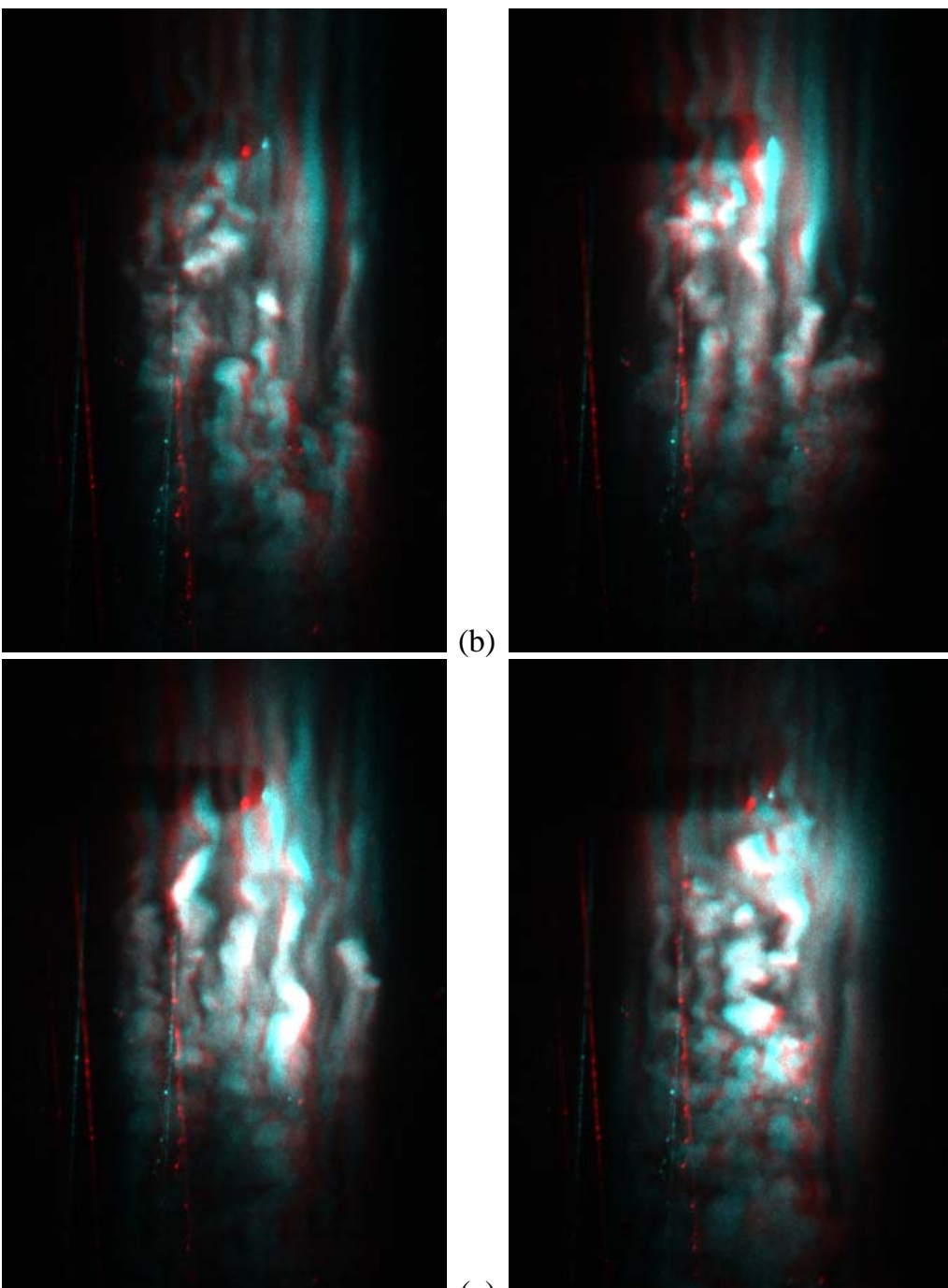

(d)

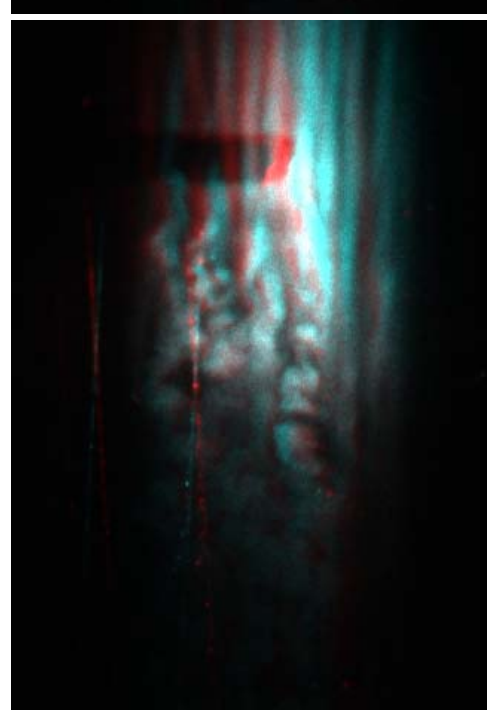

(e)

(h)

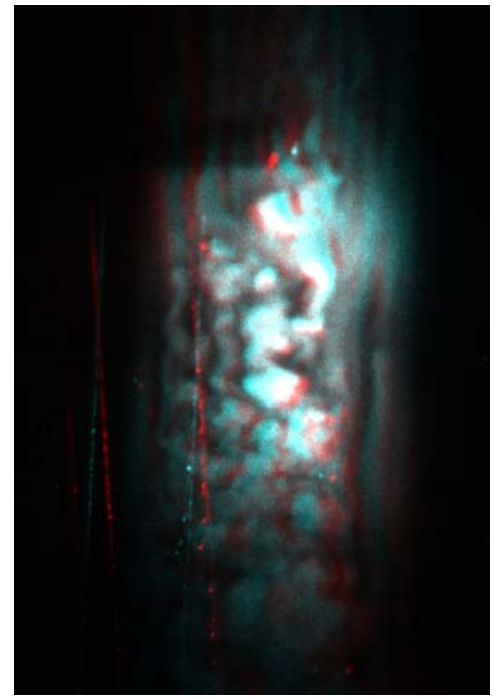

(c)

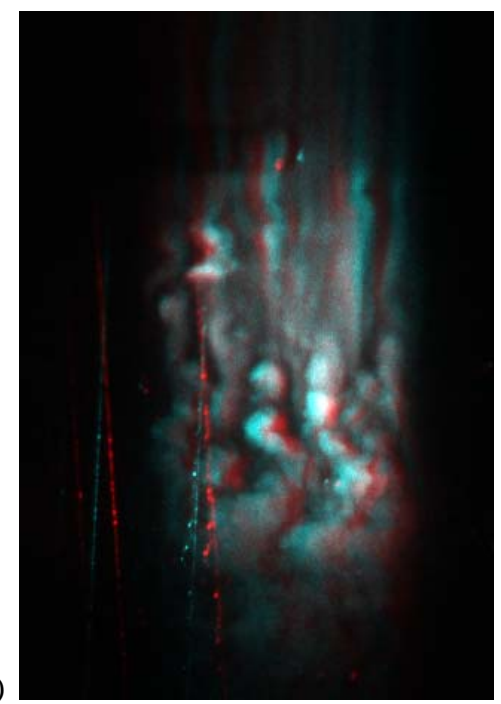

(f)
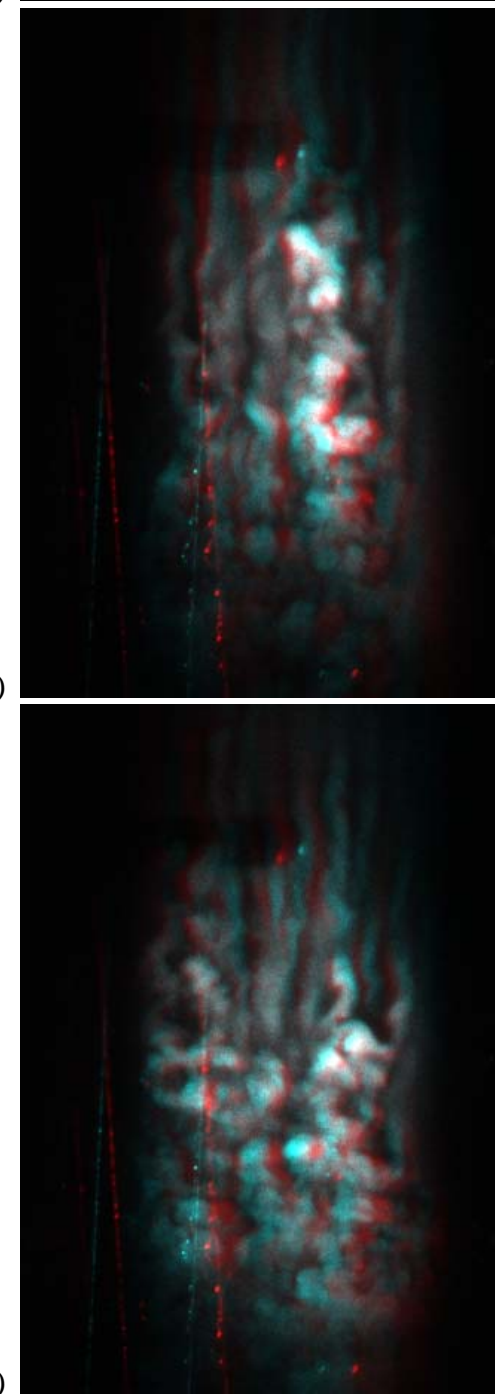

(i)

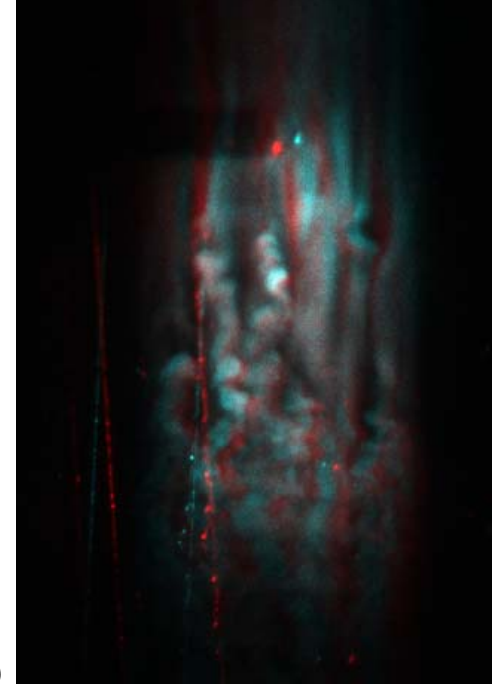

Figure A57: Test 443, Run 48, 1-mm tall by 4-mm wide cylinder trip, plate angle $=20^{\circ}, 11-\mathrm{mm}$ wide slot seeding, $\dot{m}=4200 \mathrm{sccm}, \mathrm{P}_{0}=9.35 \mathrm{MPa}$, stereoscopic PLIF visualization, framing rate $=10 \mathrm{~Hz}$. 
(a)

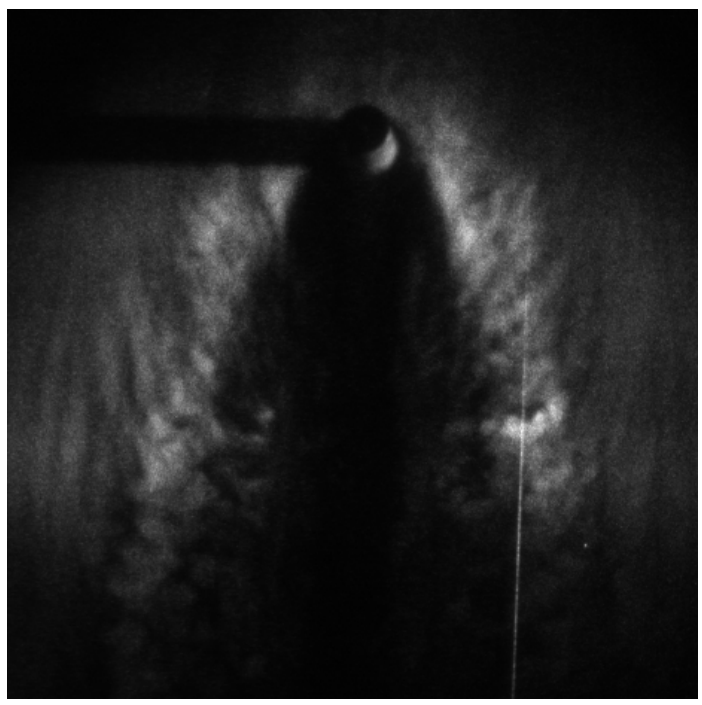

(c)

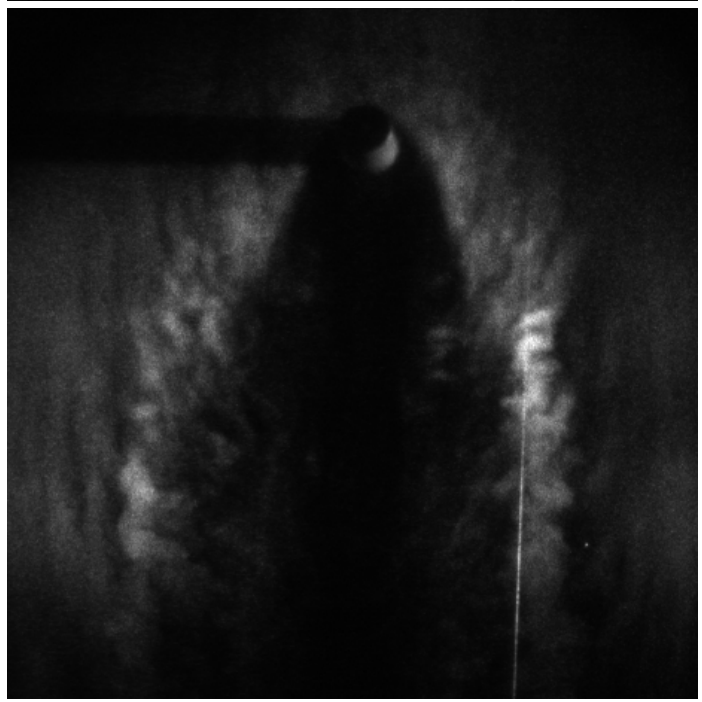

(e)

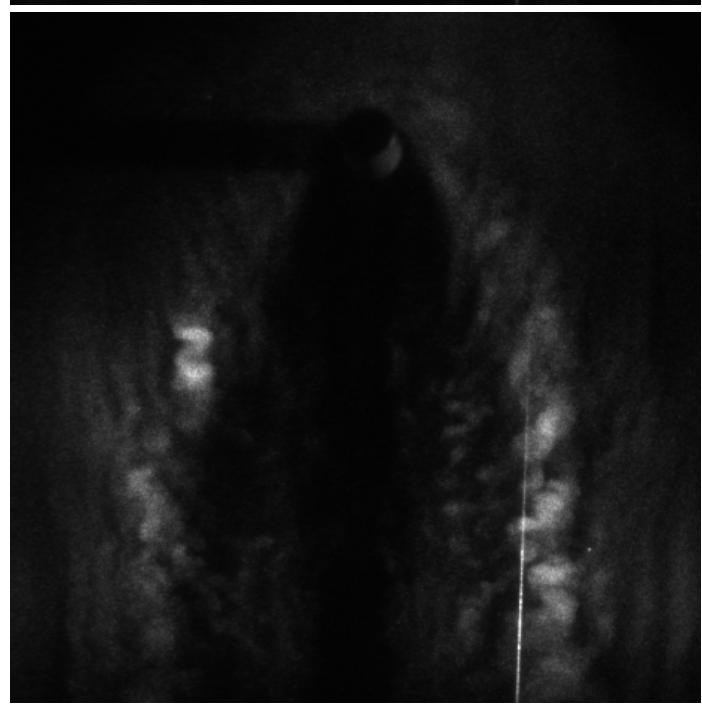

(b)

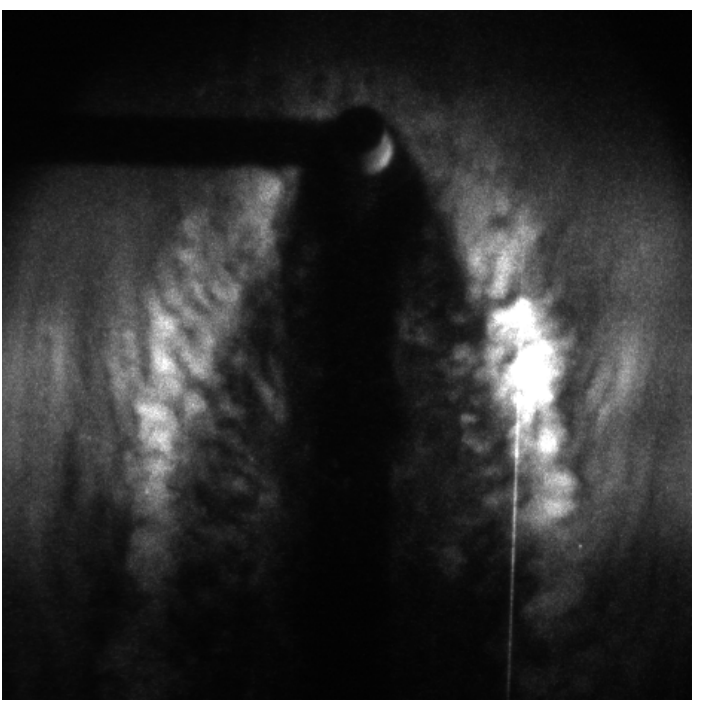

(d)
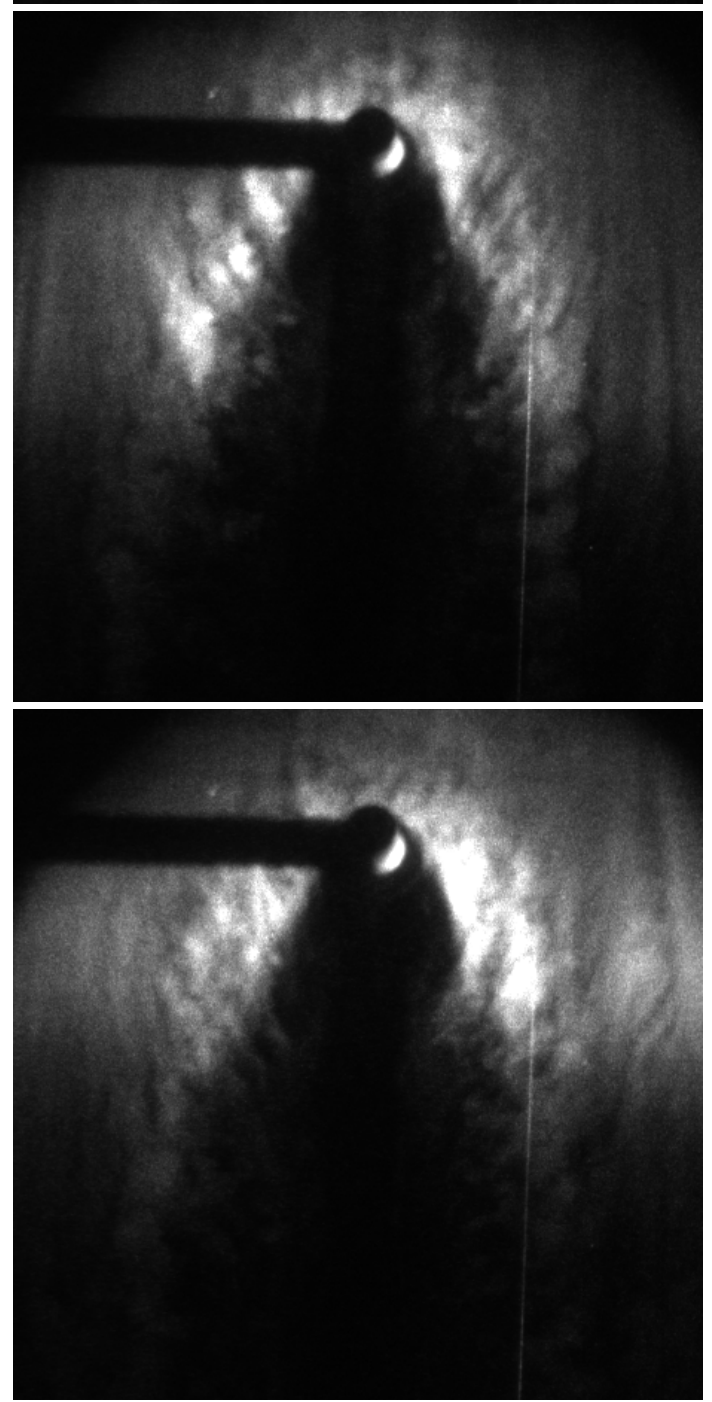

(f)

Figure A58: Test 443, Run 49, 4-mm tall by 4-mm wide cylinder trip, plate angle $=20^{\circ}$, 11-mm wide slot seeding, $\dot{m}=620$ sccm, $\mathrm{P}_{0}=9.35 \mathrm{MPa}$, thick laser sheet PLIF visualization, framing rate $=10 \mathrm{~Hz}$. 
(a)

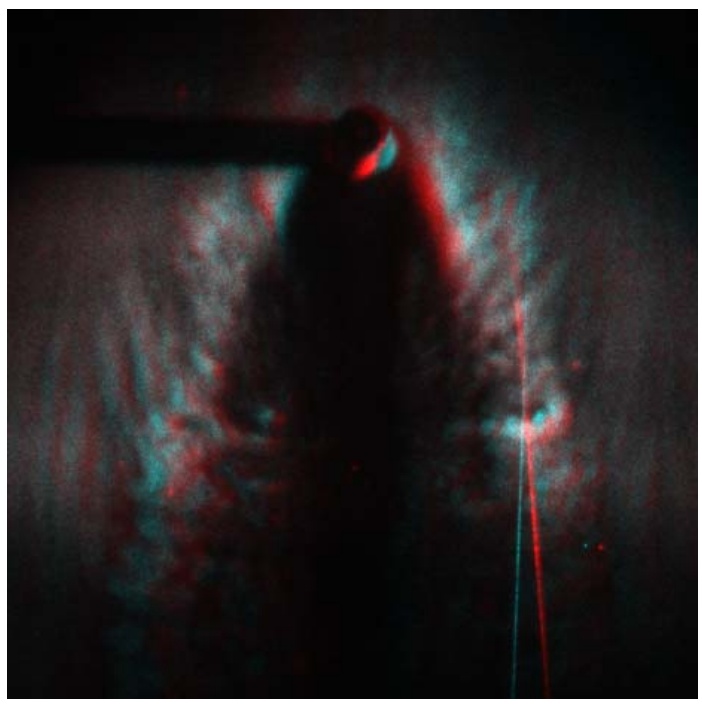

(c)

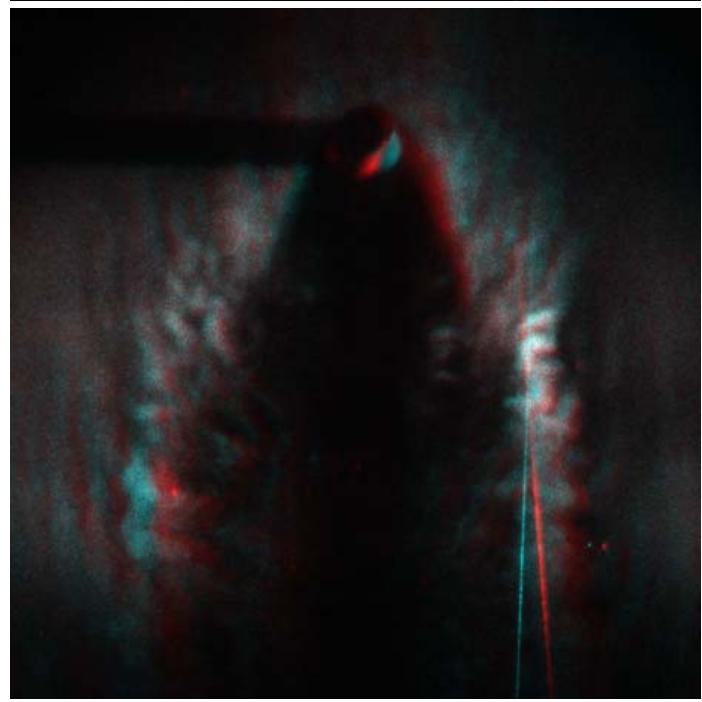

(e)

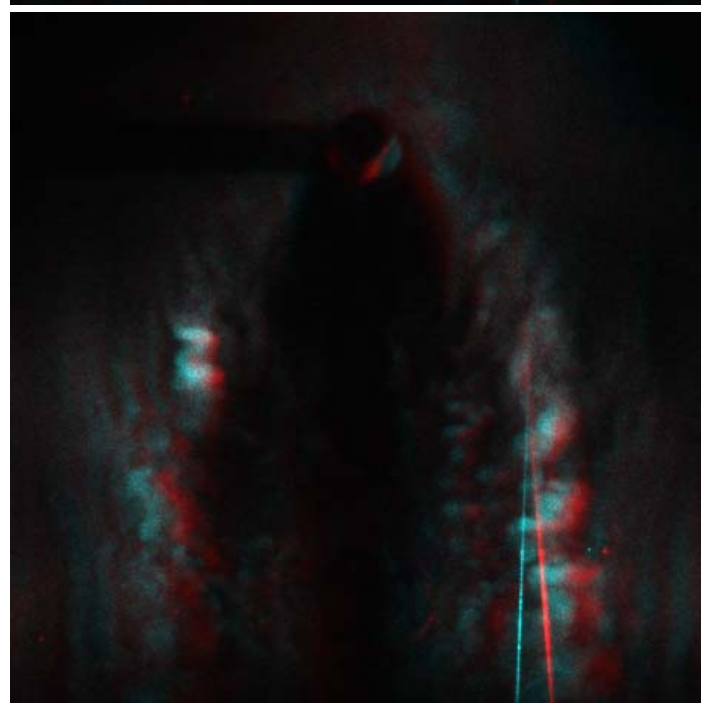

(b)

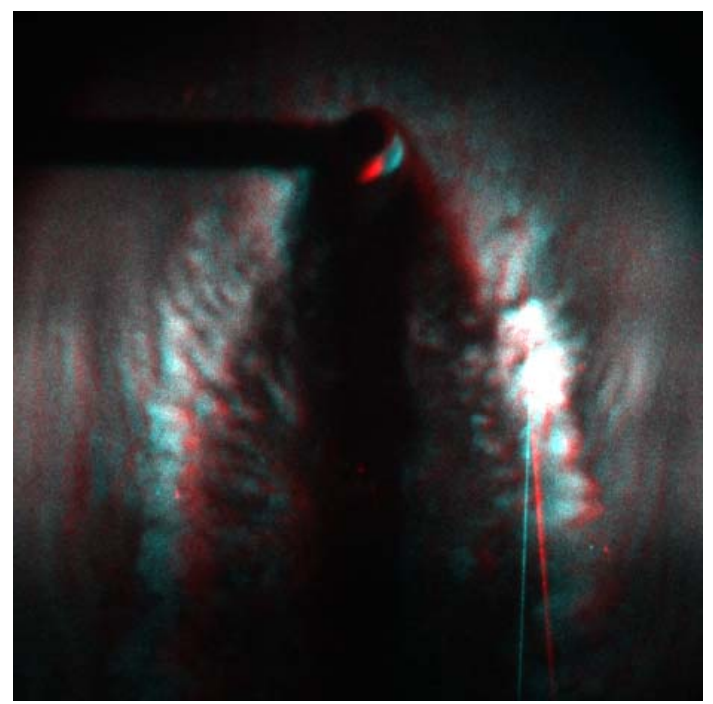

(d)
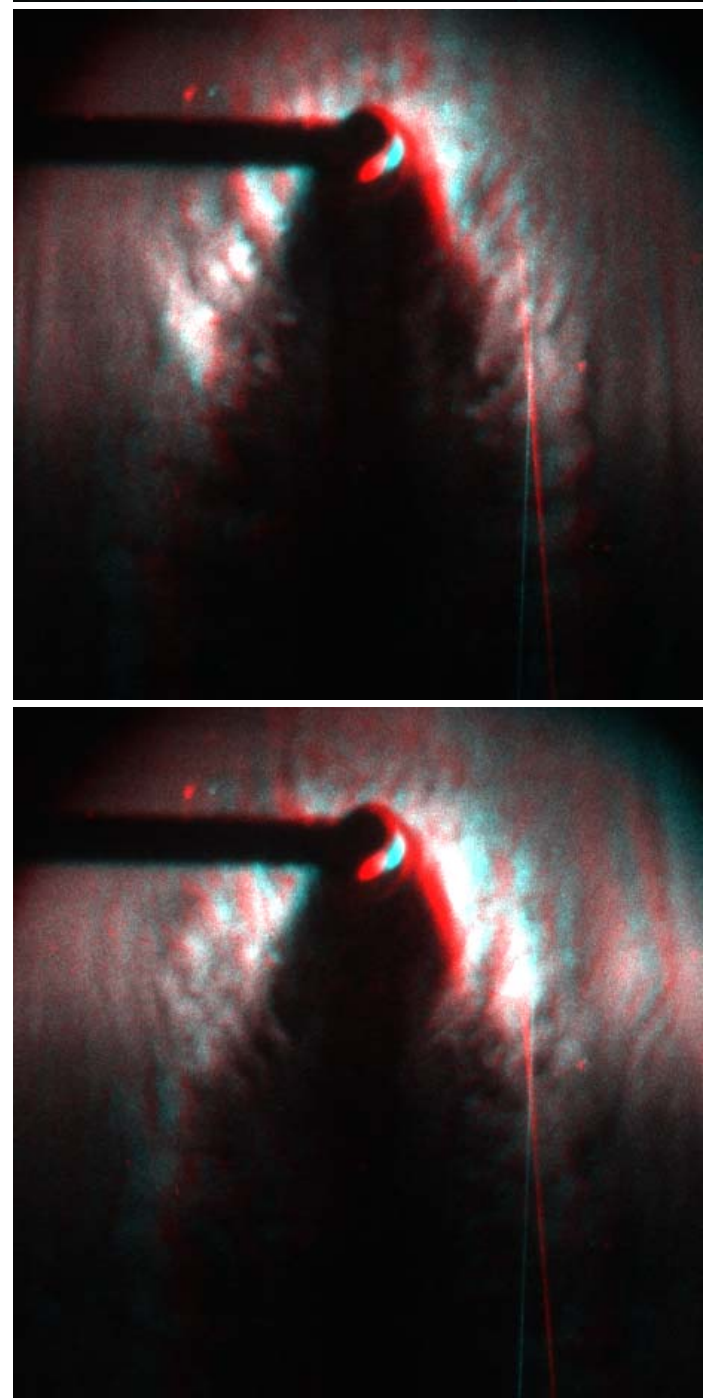

(f)

Figure A59: Test 443, Run 49, 4-mm tall by 4-mm wide cylinder trip, plate angle $=20^{\circ}$, 11-mm wide slot seeding, $\dot{m}=620 \mathrm{sccm}, \mathrm{P}_{0}=9.35 \mathrm{MPa}$, stereoscopic PLIF visualization, framing rate $=10 \mathrm{~Hz}$. 
(a)

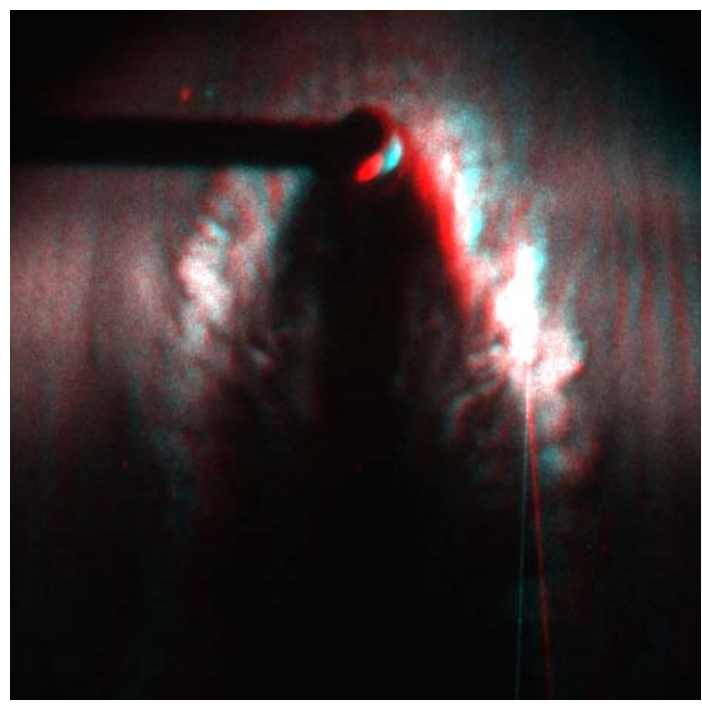

(c)

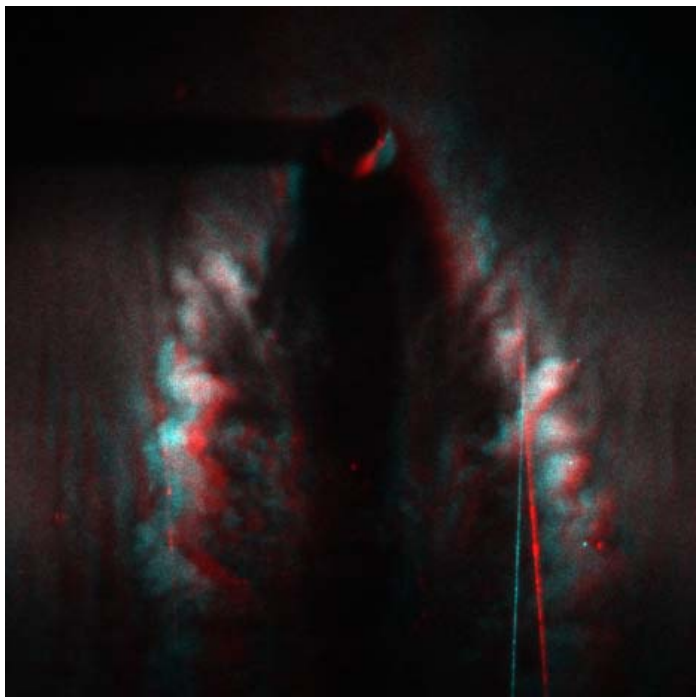

(e)

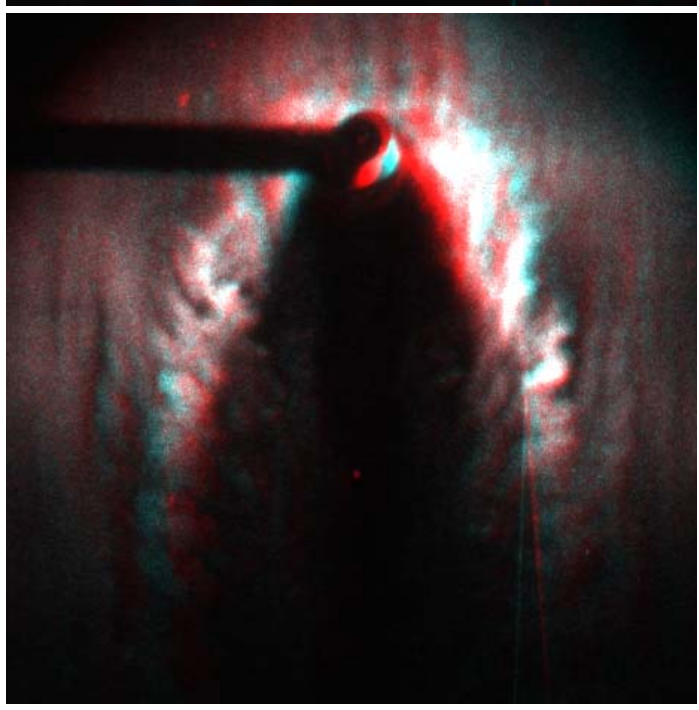

(b)

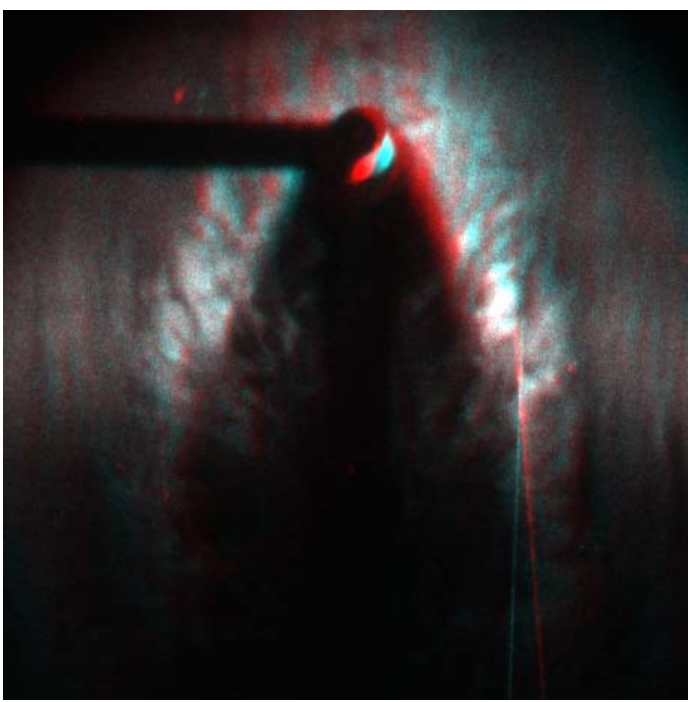

(d)
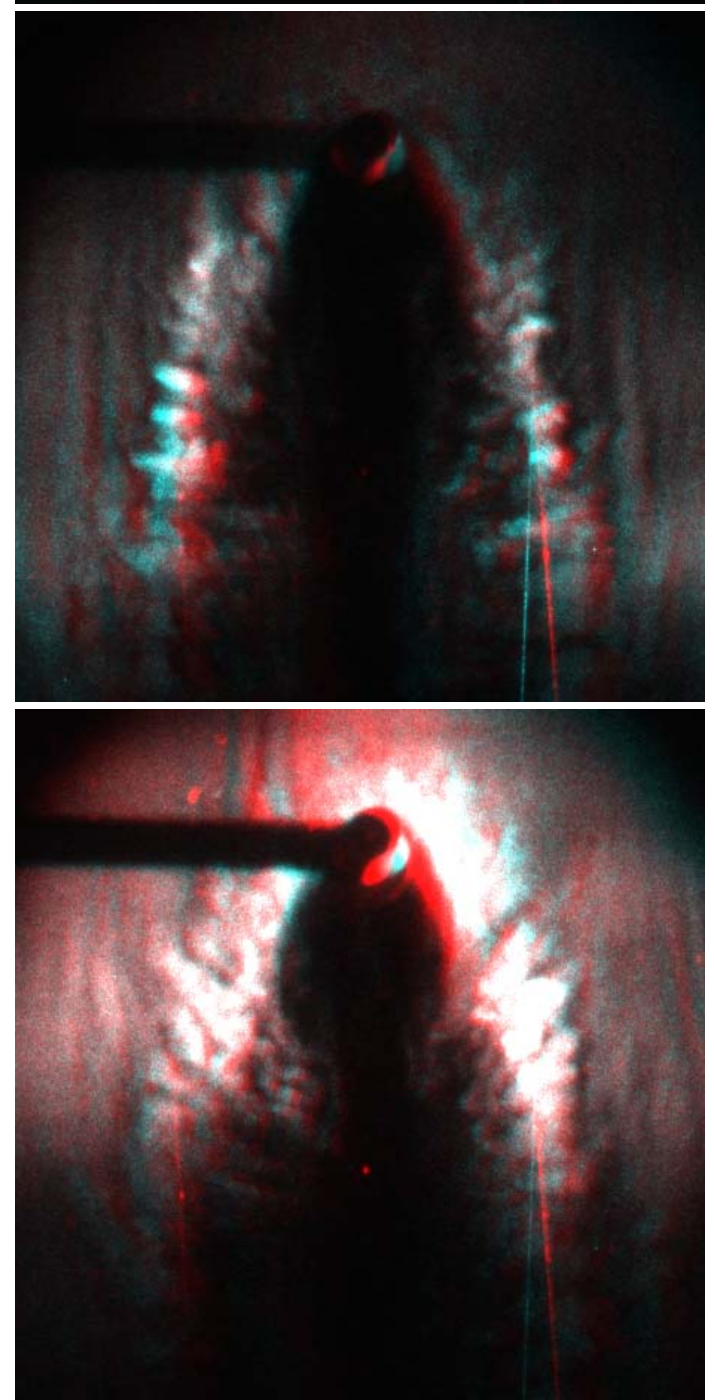

(f)

Figure A60: Test 443, Run 49, 4-mm tall by 4-mm wide cylinder trip, plate angle $=20^{\circ}, 11$-mm wide slot seeding, $\dot{m}=620 \mathrm{sccm}, \mathrm{P}_{0}=9.35 \mathrm{MPa}$, stereoscopic PLIF visualization, framing rate $=10 \mathrm{~Hz}$. 
(a)

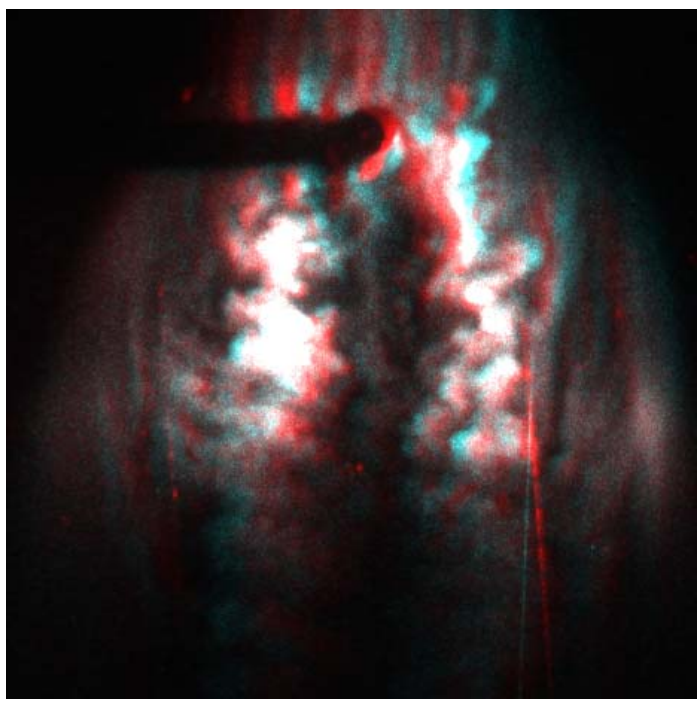

(c)

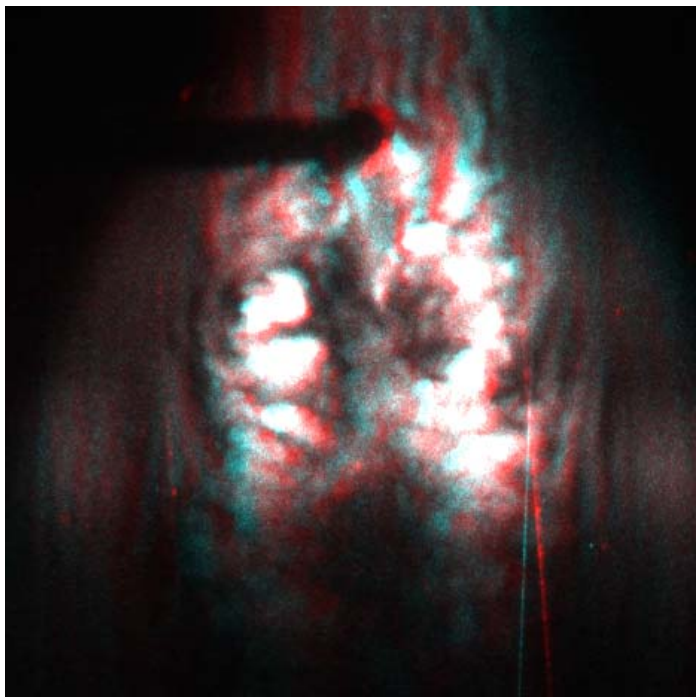

(e)

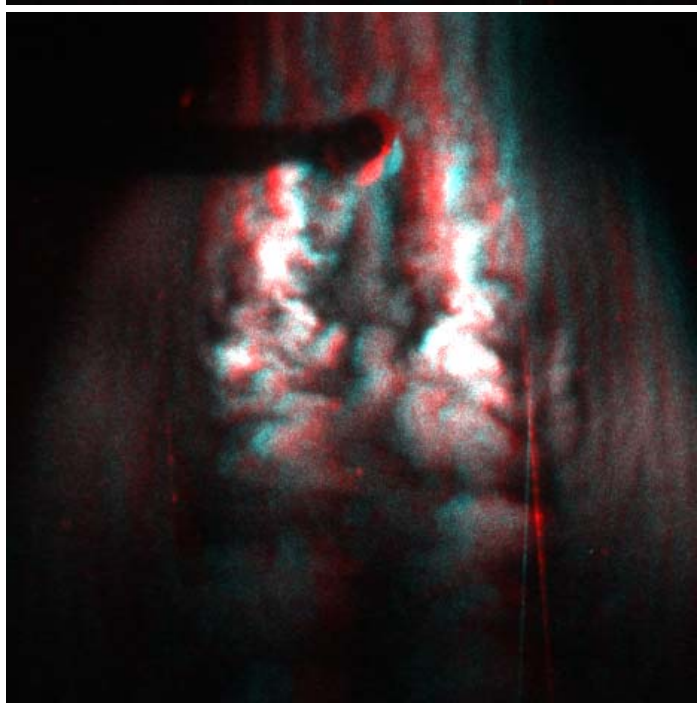

(b)

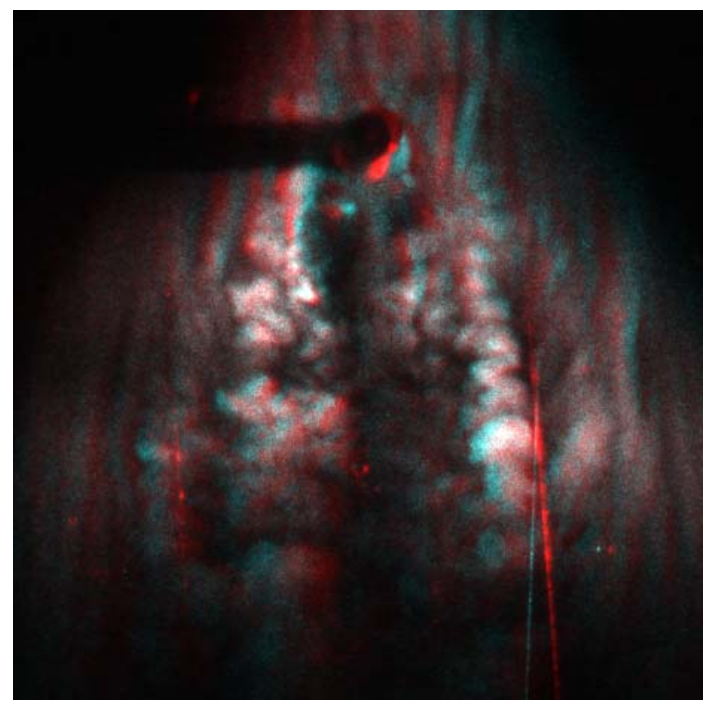

(d)
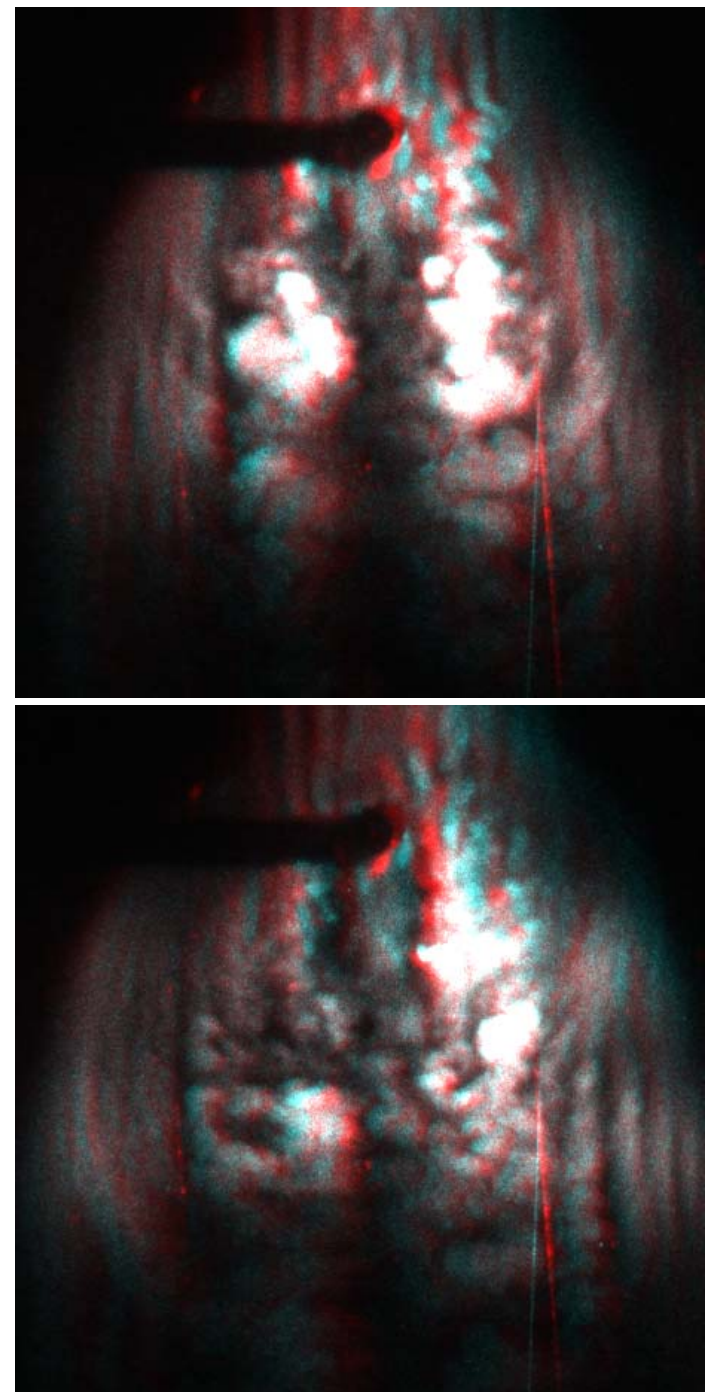

(f)

Figure A61: Test 443, Run 49, 4-mm tall by 4-mm wide cylinder trip, plate angle $=20^{\circ}, 11$-mm wide slot seeding, $\dot{m}=4400$ sccm, $\mathrm{P}_{0}=9.35 \mathrm{MPa}$, stereoscopic PLIF visualization, framing rate $=10 \mathrm{~Hz}$. 


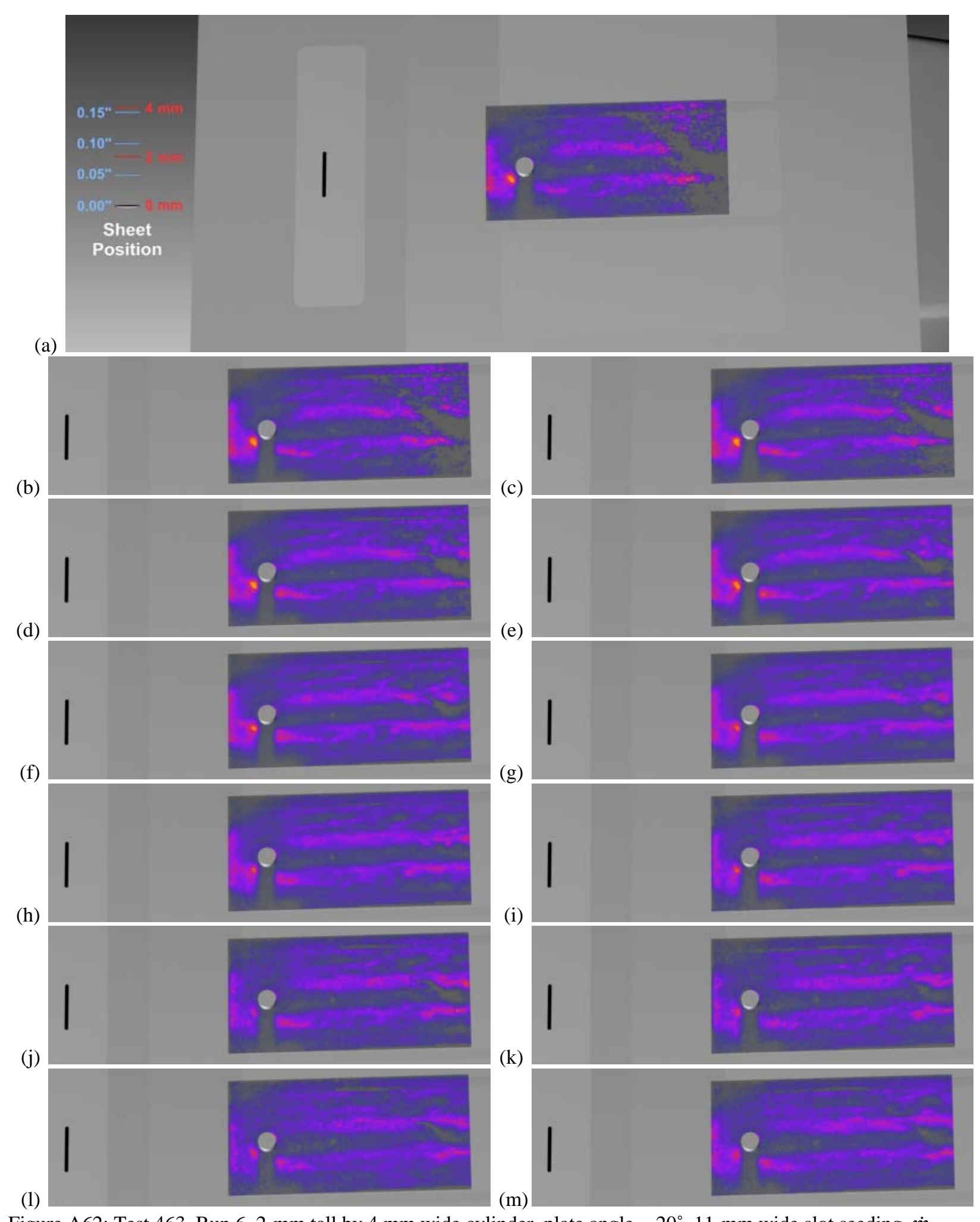

Figure A62: Test 463, Run 6, 2-mm tall by $4 \mathrm{~mm}$ wide cylinder, plate angle $=20^{\circ}$, 11-mm wide slot seeding, $\dot{m}=$ $300 \mathrm{sccm}, \mathrm{P}_{0}=4.97 \mathrm{MPa}$, sheet position $=0.1 \mathrm{~mm}$ above surface, framing rate $=500 \mathrm{kHz}$. 


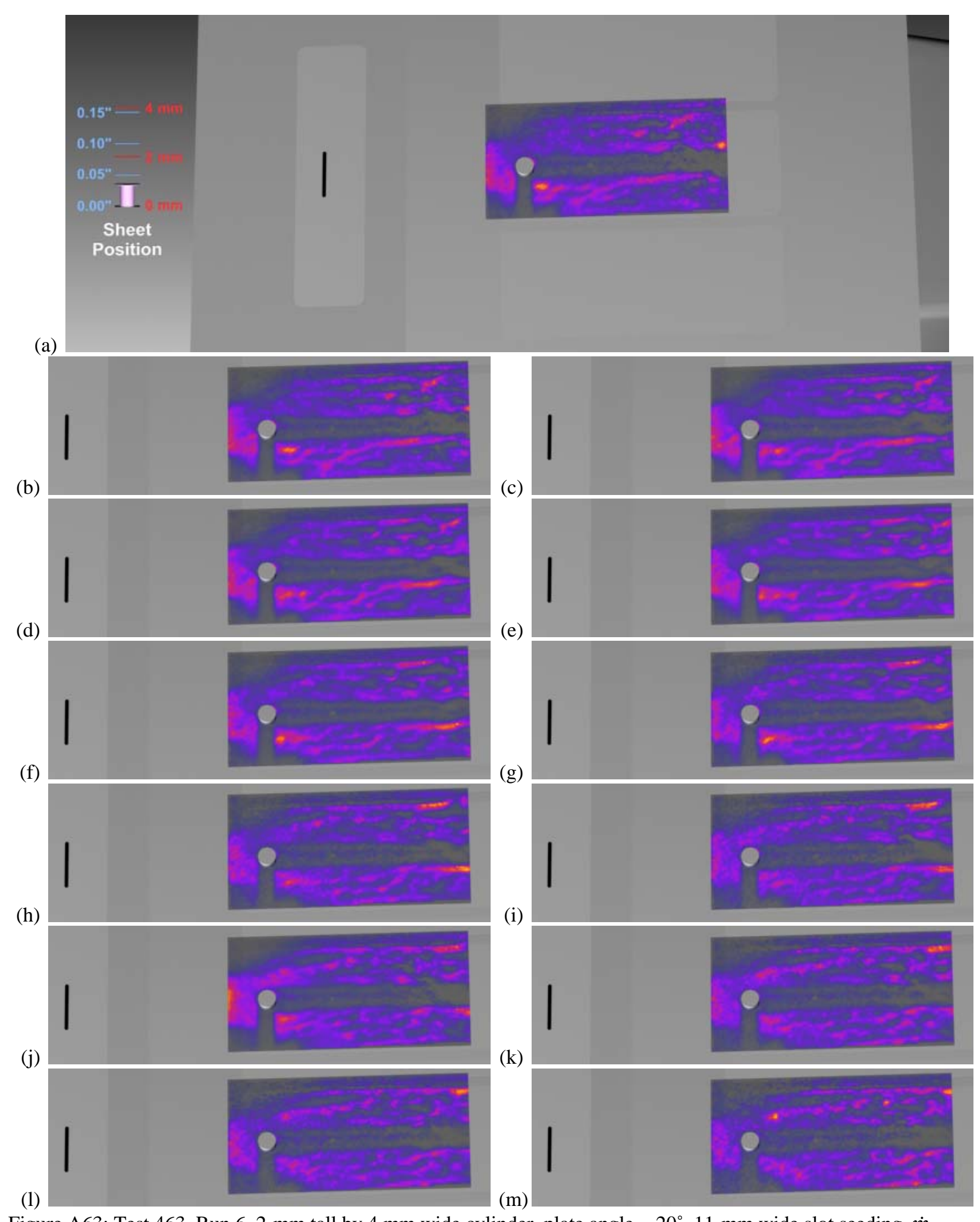

Figure A63: Test 463, Run 6, 2-mm tall by $4 \mathrm{~mm}$ wide cylinder, plate angle $=20^{\circ}$, 11-mm wide slot seeding, $\dot{m}=$ $300 \mathrm{sccm}, \mathrm{P}_{0}=4.97 \mathrm{MPa}$, sheet position $=0.9 \mathrm{~mm}$ above surface, framing rate $=500 \mathrm{kHz}$. 


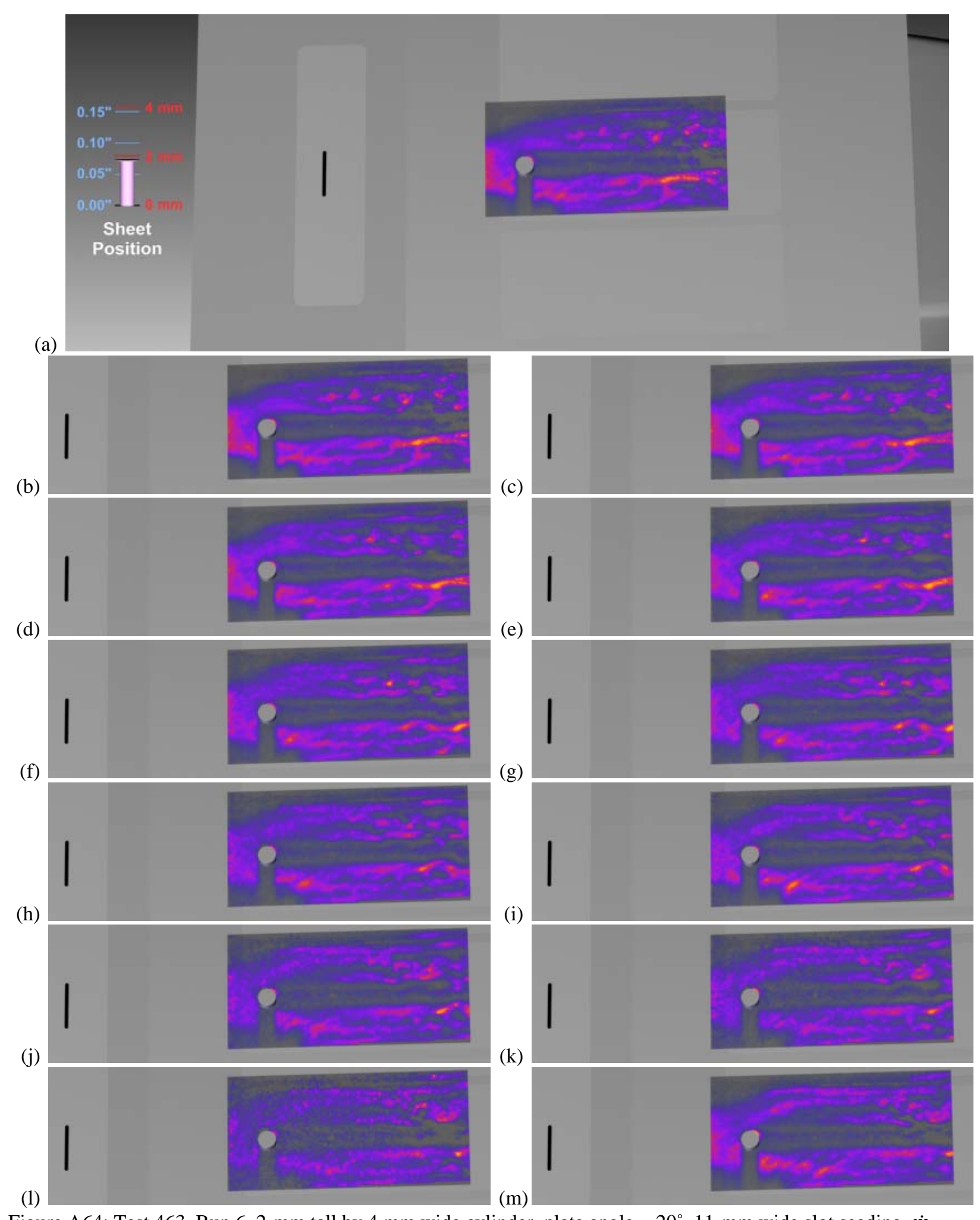

Figure A64: Test 463, Run 6, 2-mm tall by $4 \mathrm{~mm}$ wide cylinder, plate angle $=20^{\circ}$, 11-mm wide slot seeding, $\dot{m}=$ $300 \mathrm{sccm}, \mathrm{P}_{0}=4.97 \mathrm{MPa}$, sheet position $=1.9 \mathrm{~mm}$ above surface, framing rate $=500 \mathrm{kHz}$. 


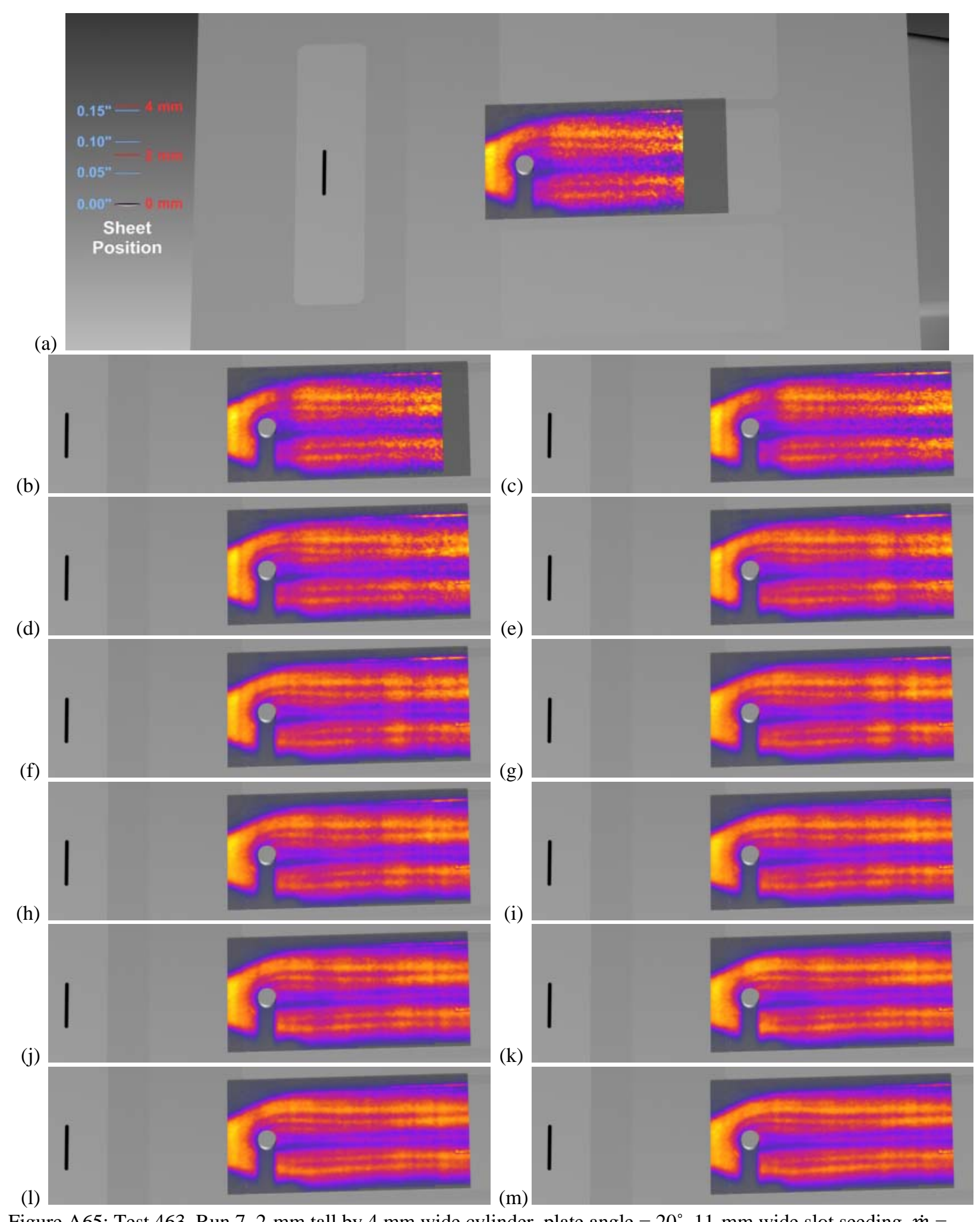

Figure A65: Test 463, Run 7, 2-mm tall by $4 \mathrm{~mm}$ wide cylinder, plate angle $=20^{\circ}$, 11-mm wide slot seeding, $\dot{m}=$ $300 \mathrm{sccm}, \mathrm{P}_{0}=2.4 \mathrm{MPa}$, sheet position $=0 \mathrm{~mm}$ above surface, framing rate $=500 \mathrm{kHz}$. 


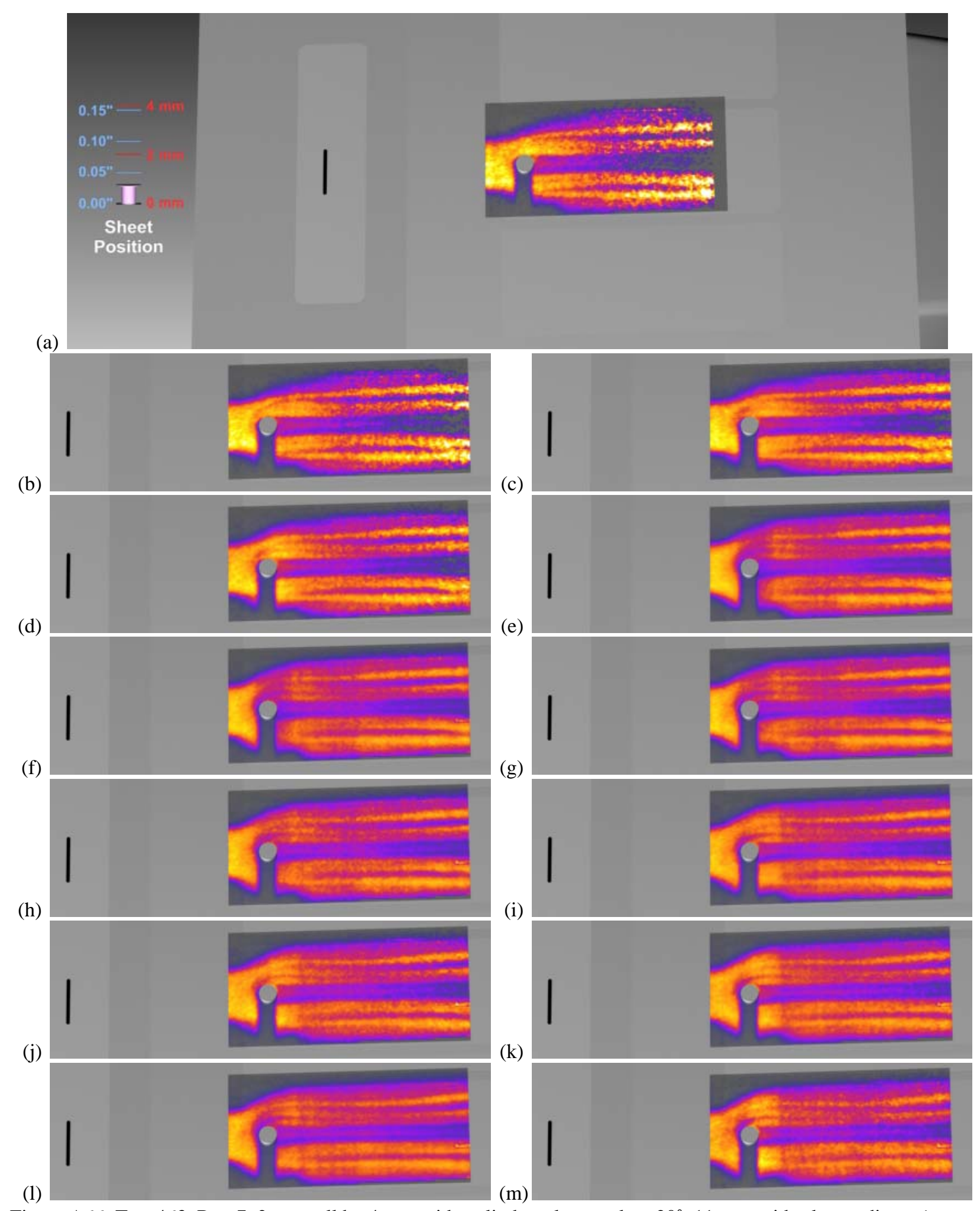

Figure A66: Test 463, Run 7, 2-mm tall by $4 \mathrm{~mm}$ wide cylinder, plate angle $=20^{\circ}, 11$-mm wide slot seeding, $\dot{m}=$ $300 \mathrm{sccm}, \mathrm{P}_{0}=2.4 \mathrm{MPa}$, sheet position $=0.8 \mathrm{~mm}$ above surface, framing rate $=500 \mathrm{kHz}$. 


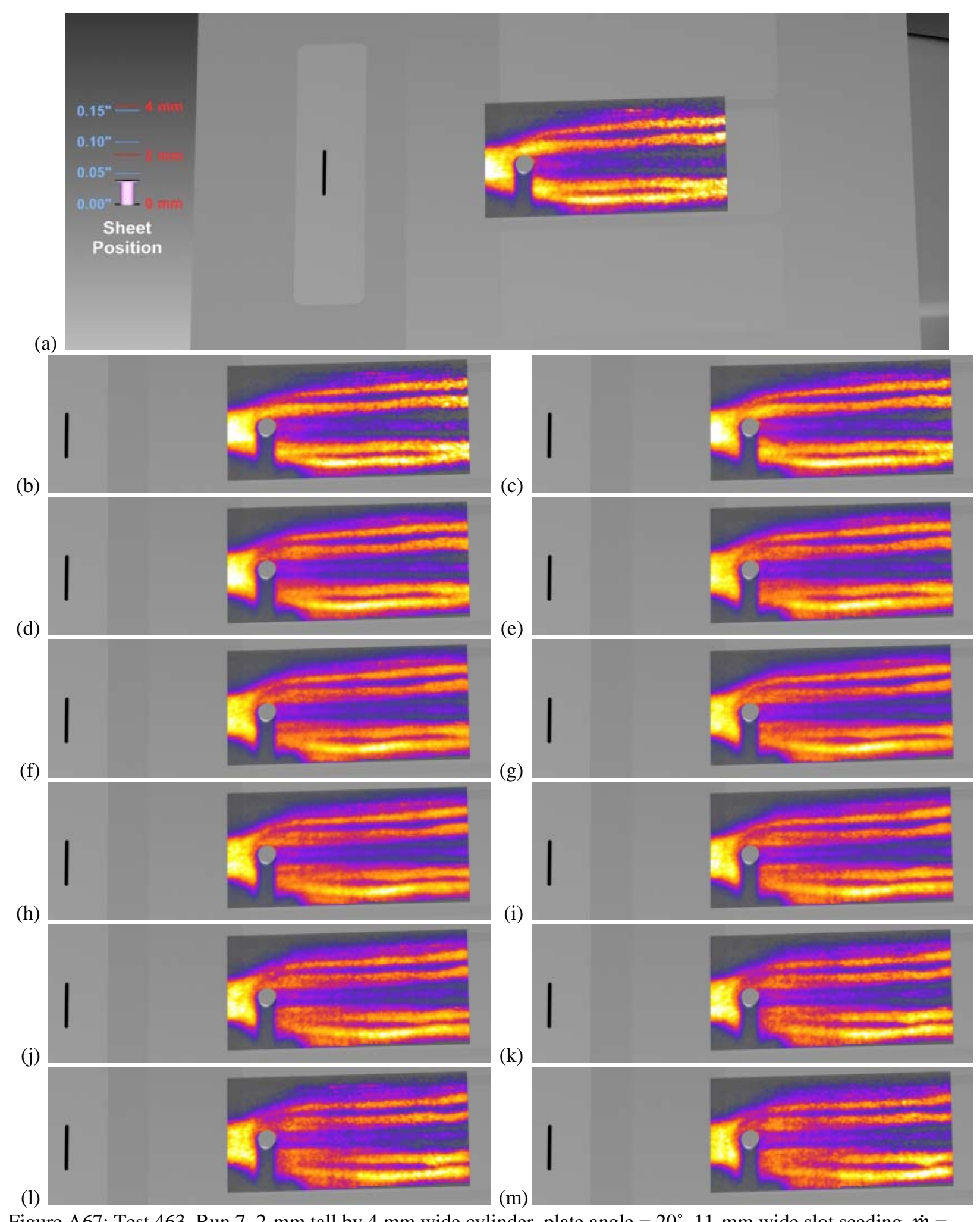

Figure A67: Test 463, Run 7, 2-mm tall by $4 \mathrm{~mm}$ wide cylinder, plate angle $=20^{\circ}$, 11-mm wide slot seeding, $\dot{m}=$ $300 \mathrm{sccm}, \mathrm{P}_{0}=2.4 \mathrm{MPa}$, sheet position $=1 \mathrm{~mm}$ above surface, framing rate $=500 \mathrm{kHz}$. 


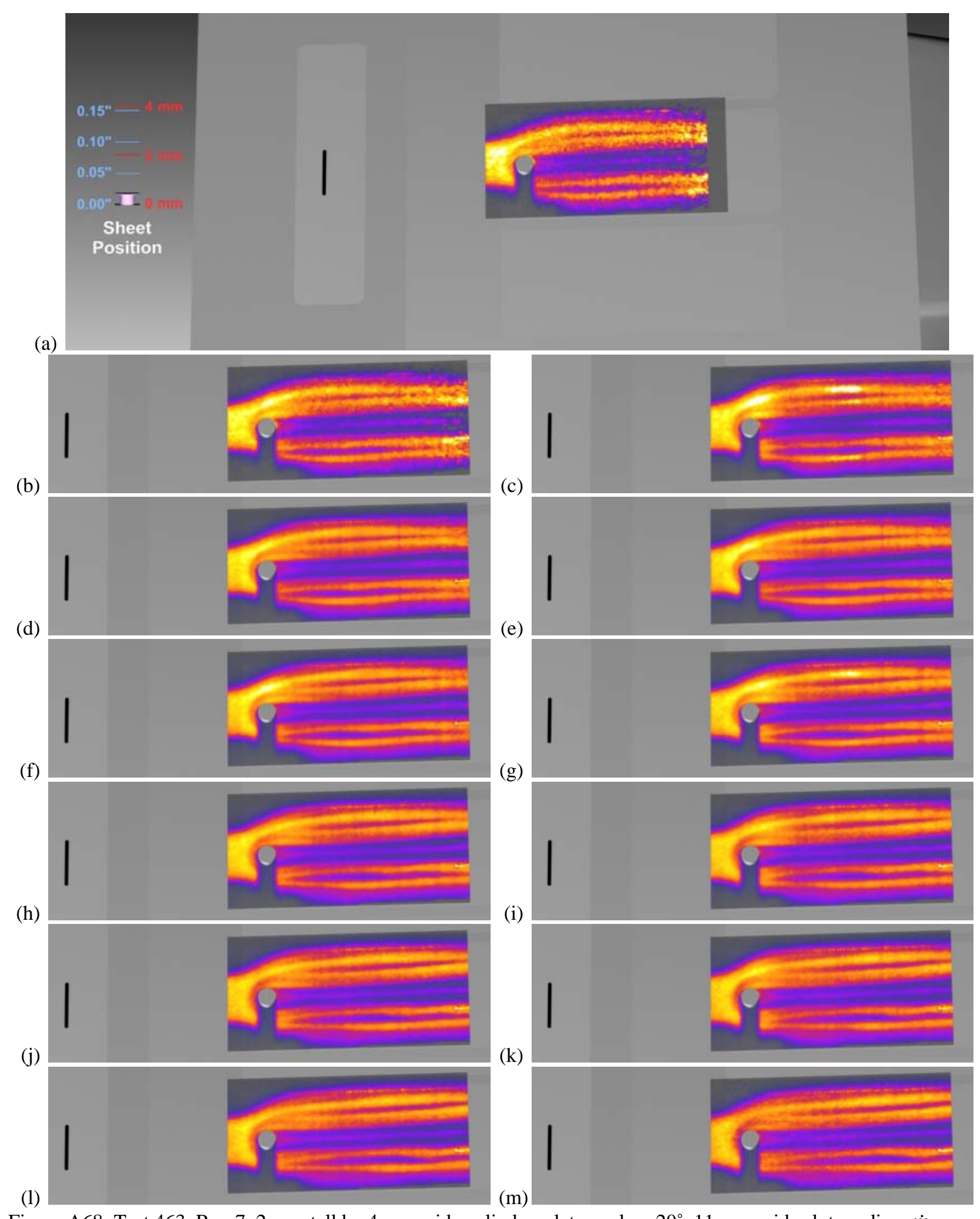

Figure A68: Test 463, Run 7, 2-mm tall by $4 \mathrm{~mm}$ wide cylinder, plate angle $=20^{\circ}$, 11-mm wide slot seeding, $\dot{m}=$ $300 \mathrm{sccm}, \mathrm{P}_{0}=2.4 \mathrm{MPa}$, sheet position $=0.5 \mathrm{~mm}$ above surface, framing rate $=500 \mathrm{kHz}$. 


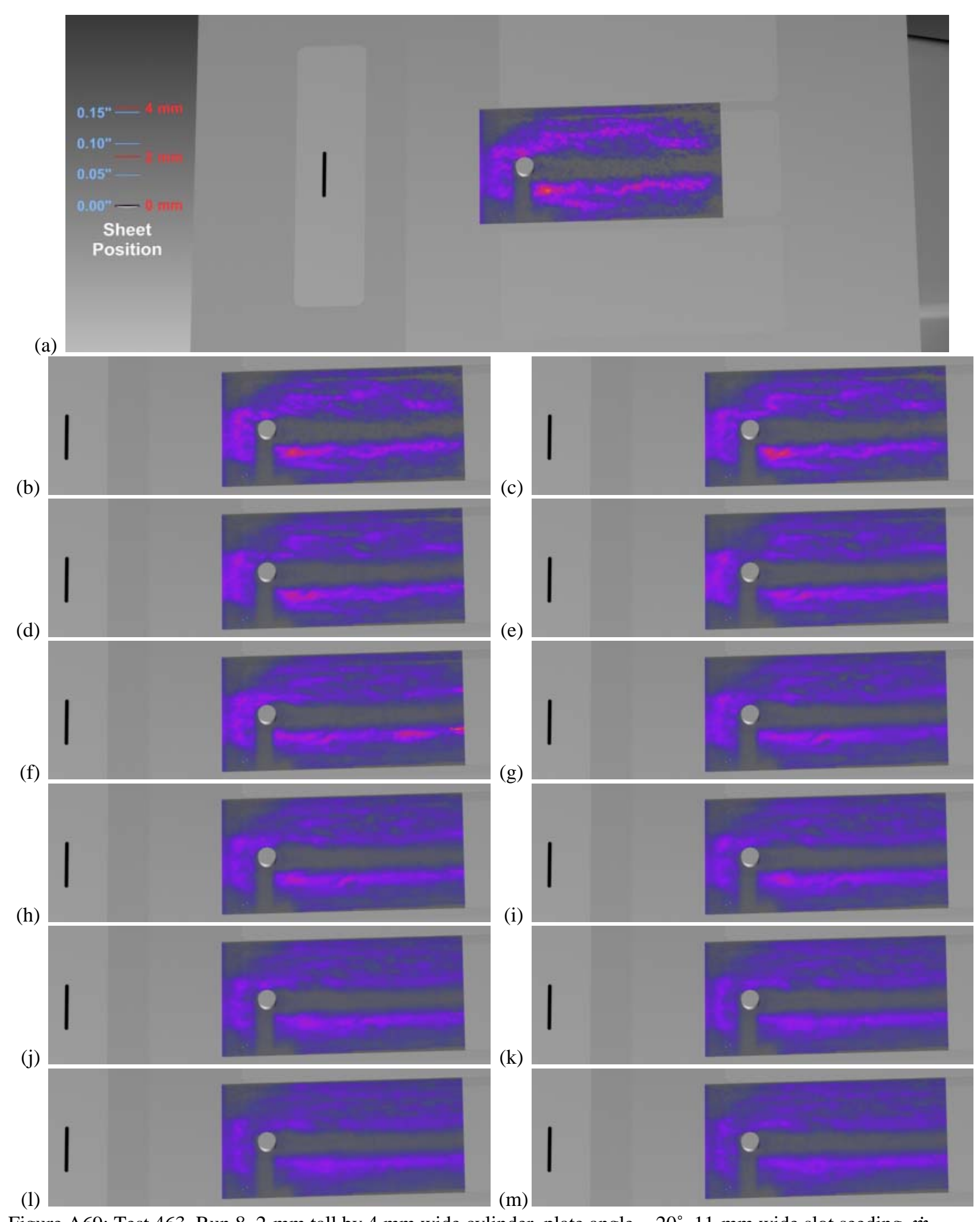

Figure A69: Test 463, Run 8, 2-mm tall by $4 \mathrm{~mm}$ wide cylinder, plate angle $=20^{\circ}$, 11-mm wide slot seeding, $\dot{m}=$ $300 \mathrm{sccm}, \mathrm{P}_{0}=9.34 \mathrm{MPa}$, sheet position $=0.1 \mathrm{~mm}$ above surface, framing rate $=500 \mathrm{kHz}$. 


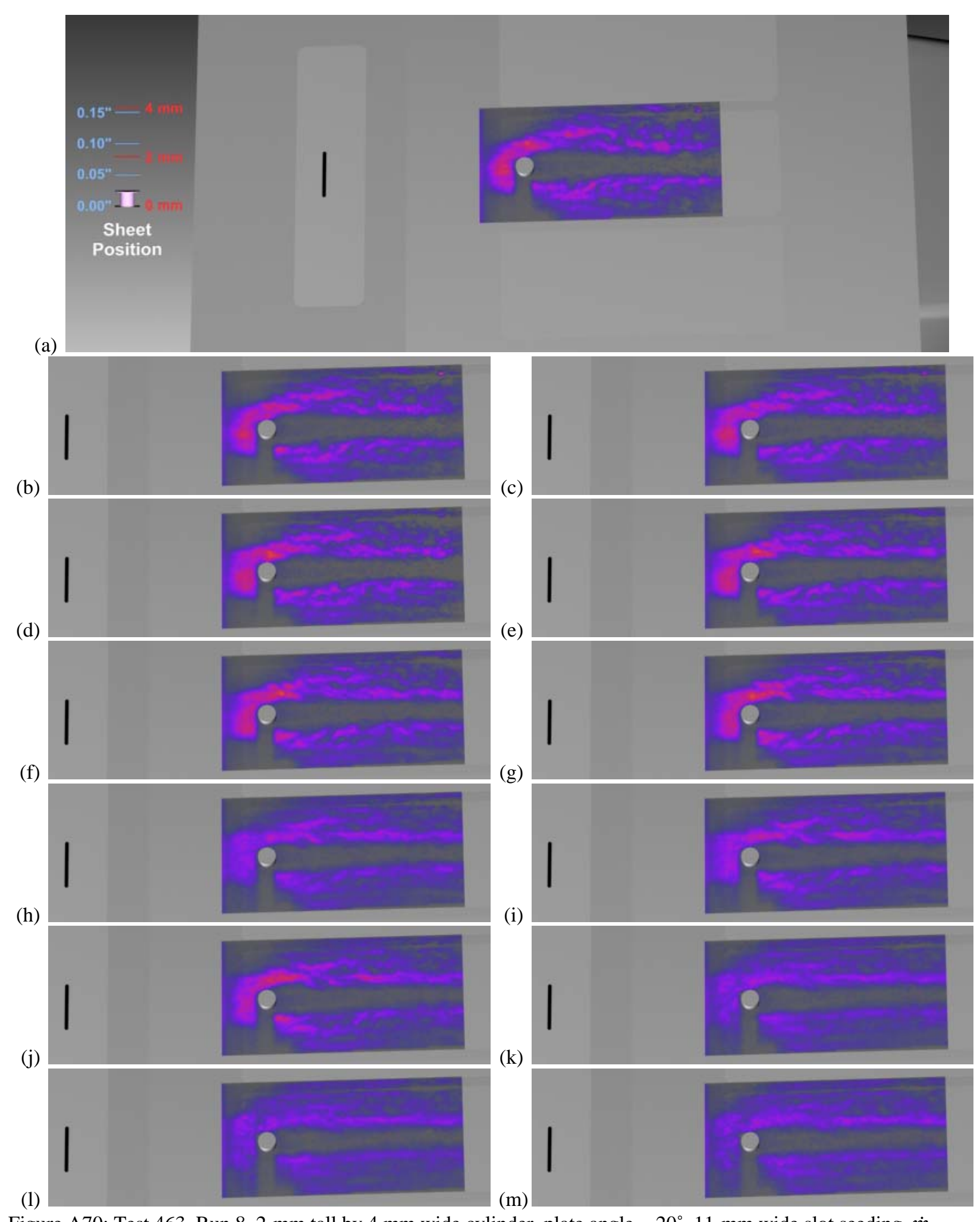

Figure A70: Test 463, Run 8, 2-mm tall by $4 \mathrm{~mm}$ wide cylinder, plate angle $=20^{\circ}$, 11-mm wide slot seeding, $\dot{m}=$ $300 \mathrm{sccm}, \mathrm{P}_{0}=9.34 \mathrm{MPa}$, sheet position $=0.6 \mathrm{~mm}$ above surface, framing rate $=500 \mathrm{kHz}$. 


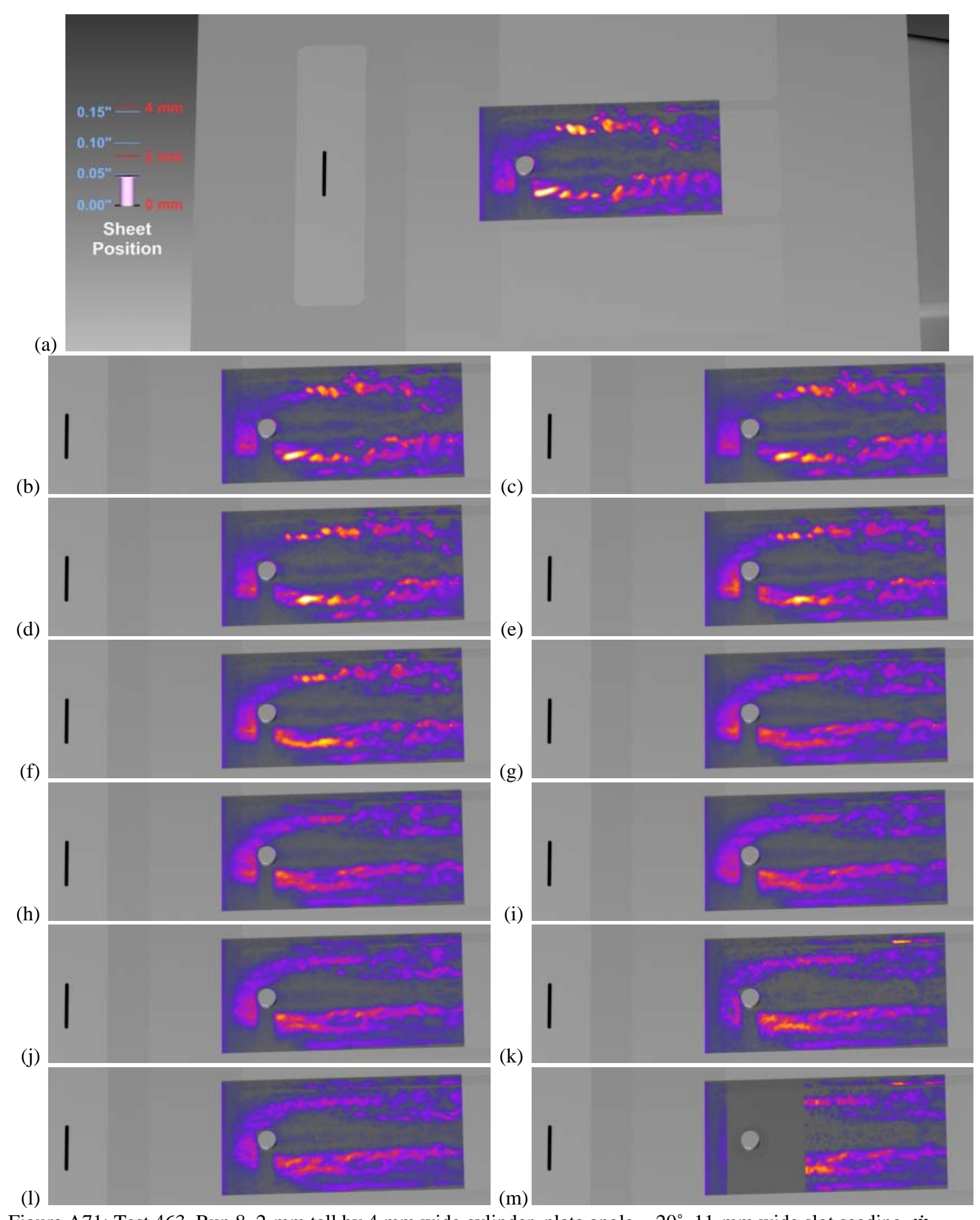

Figure A71: Test 463, Run 8, 2-mm tall by $4 \mathrm{~mm}$ wide cylinder, plate angle $=20^{\circ}$, 11-mm wide slot seeding, $\dot{m}=$ $300 \mathrm{sccm}, \mathrm{P}_{0}=9.34 \mathrm{MPa}$, sheet position $=1.2 \mathrm{~mm}$ above surface, framing rate $=500 \mathrm{kHz}$. 


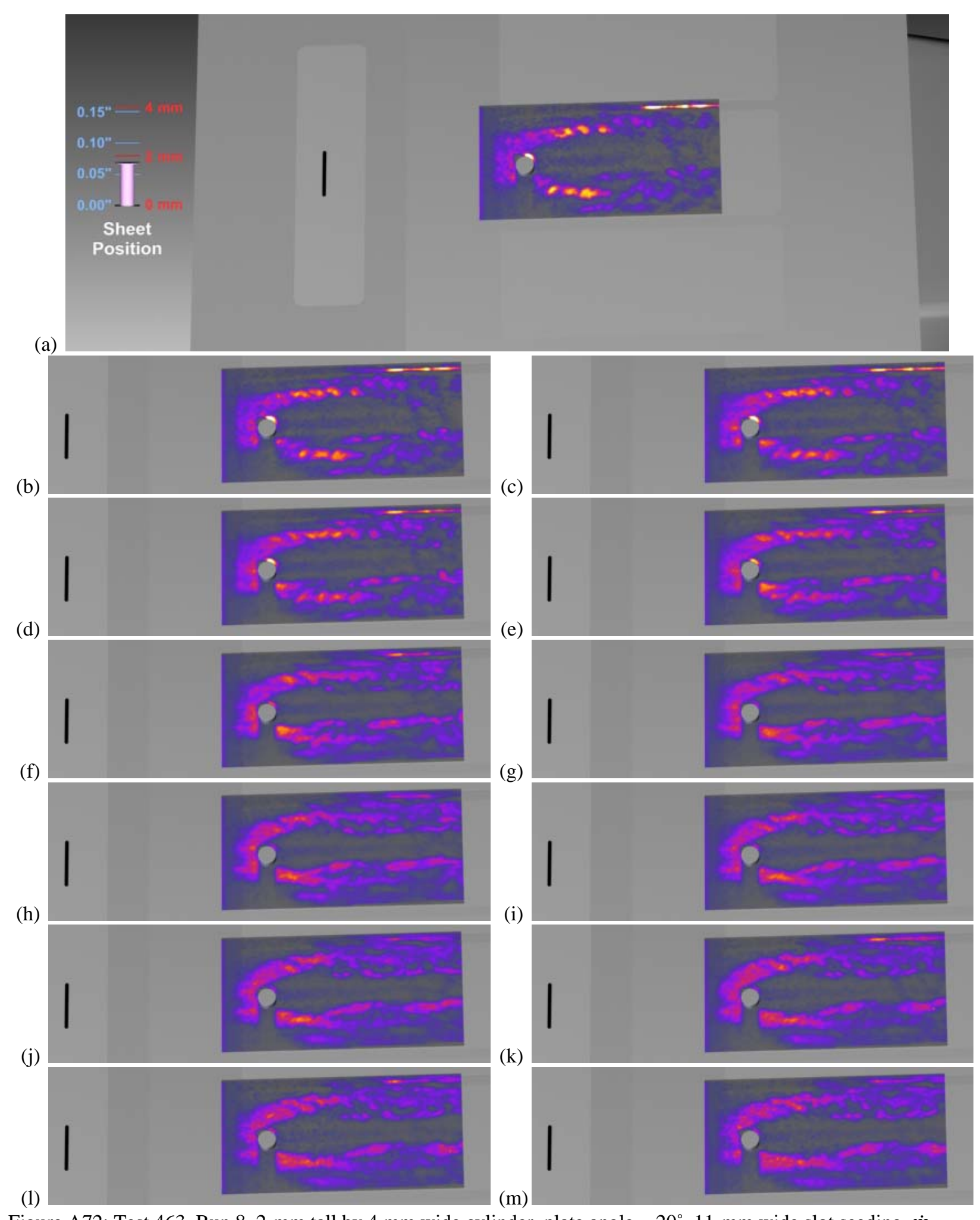

Figure A72: Test 463, Run 8, 2-mm tall by $4 \mathrm{~mm}$ wide cylinder, plate angle $=20^{\circ}$, 11-mm wide slot seeding, $\dot{m}=$ $300 \mathrm{sccm}, \mathrm{P}_{0}=9.34 \mathrm{MPa}$, sheet position $=1.8 \mathrm{~mm}$ above surface, framing rate $=500 \mathrm{kHz}$. 


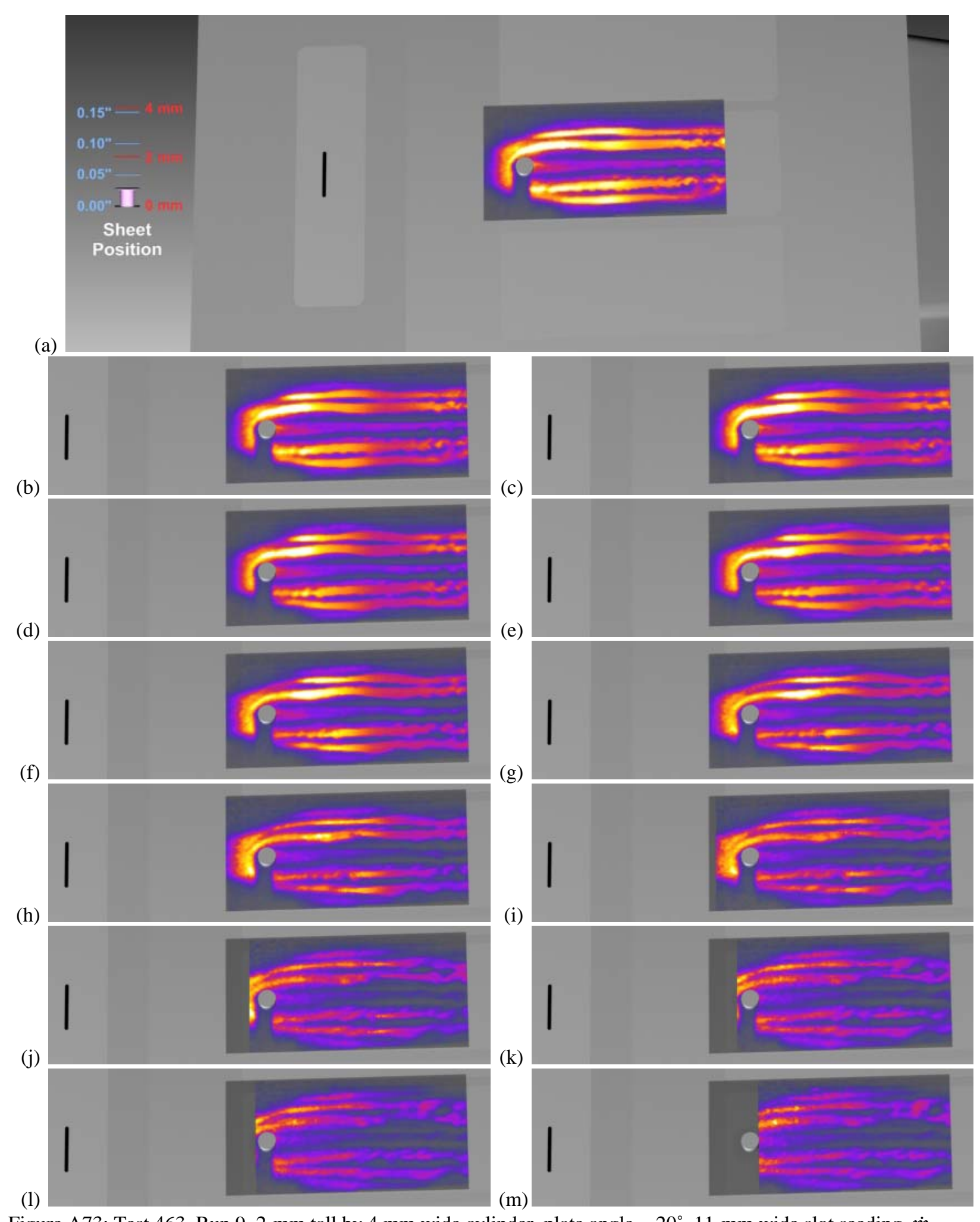

Figure A73: Test 463, Run 9, 2-mm tall by $4 \mathrm{~mm}$ wide cylinder, plate angle $=20^{\circ}, 11$-mm wide slot seeding, $\dot{m}=$ $300 \mathrm{sccm}, \mathrm{P}_{0}=3.45 \mathrm{MPa}$, sheet position $=0.8 \mathrm{~mm}$ above surface, framing rate $=500 \mathrm{kHz}$. 


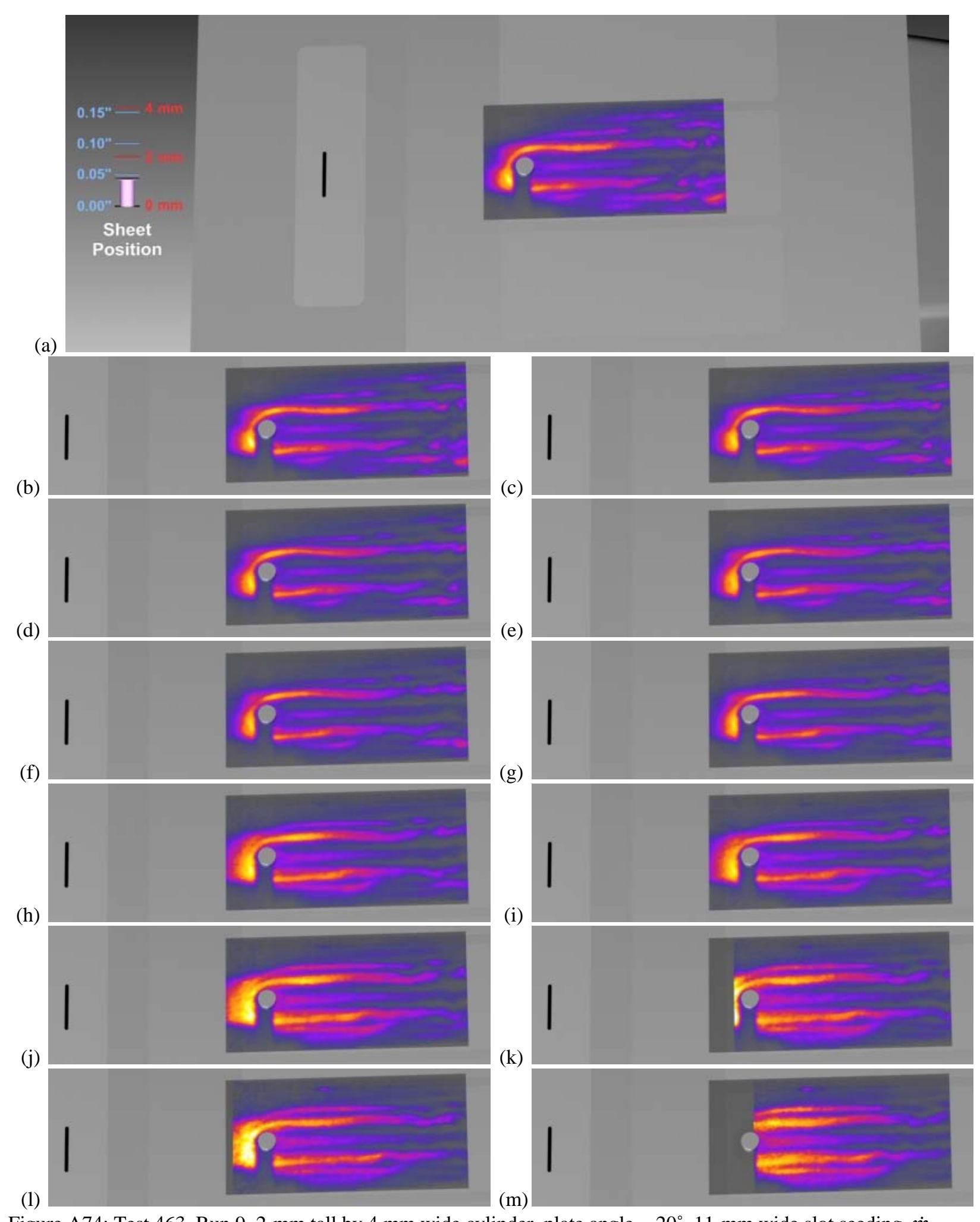

Figure A74: Test 463, Run 9, 2-mm tall by $4 \mathrm{~mm}$ wide cylinder, plate angle $=20^{\circ}$, 11-mm wide slot seeding, $\dot{m}=$ $300 \mathrm{sccm}, \mathrm{P}_{0}=3.45 \mathrm{MPa}$, sheet position $=1.2 \mathrm{~mm}$ above surface, framing rate $=500 \mathrm{kHz}$. 


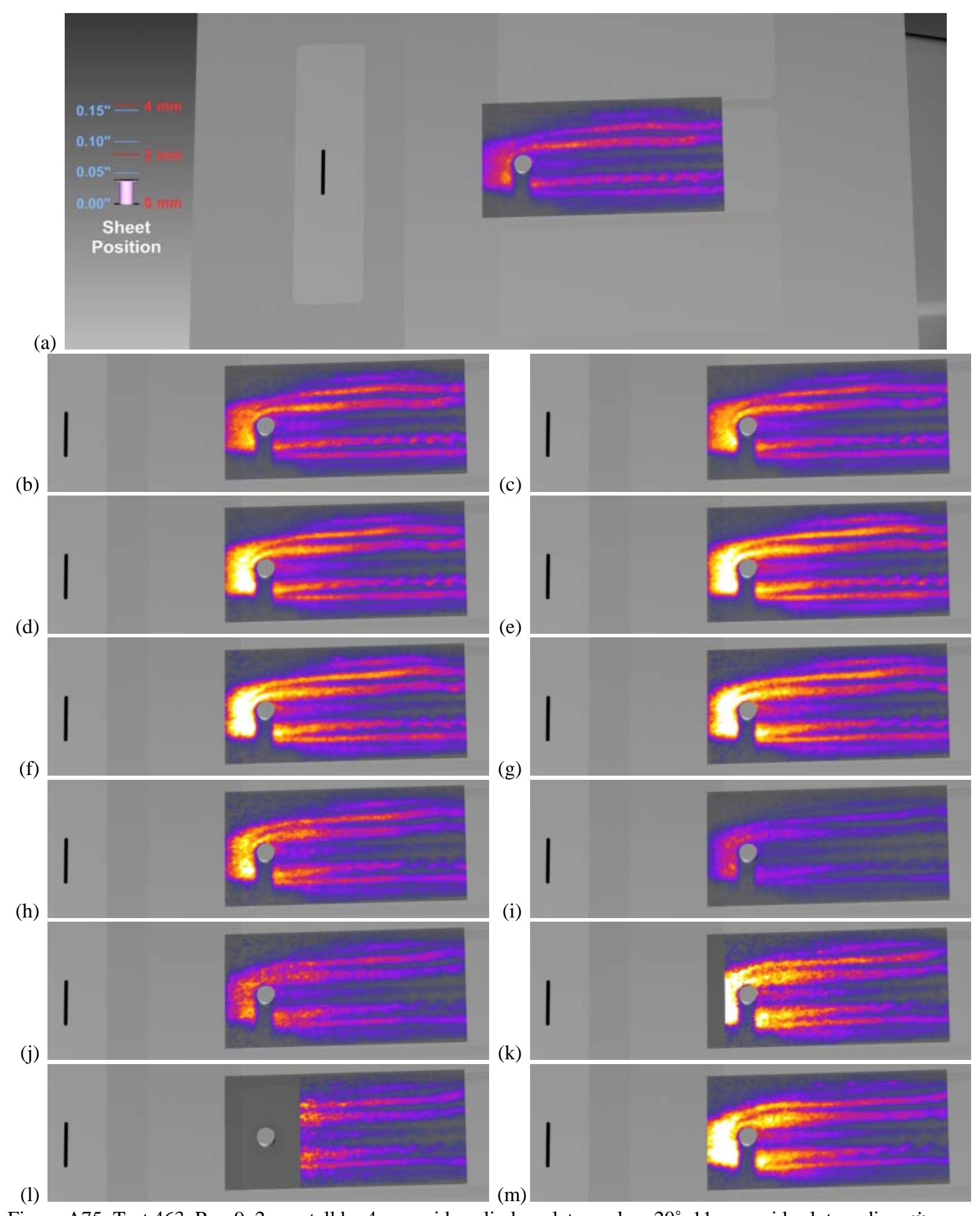

Figure A75: Test 463, Run 9, 2-mm tall by $4 \mathrm{~mm}$ wide cylinder, plate angle $=20^{\circ}$, 11-mm wide slot seeding, $\dot{m}=$ $300 \mathrm{sccm}, \mathrm{P}_{0}=3.45 \mathrm{MPa}$, sheet position $=1 \mathrm{~mm}$ above surface, framing rate $=500 \mathrm{kHz}$. 


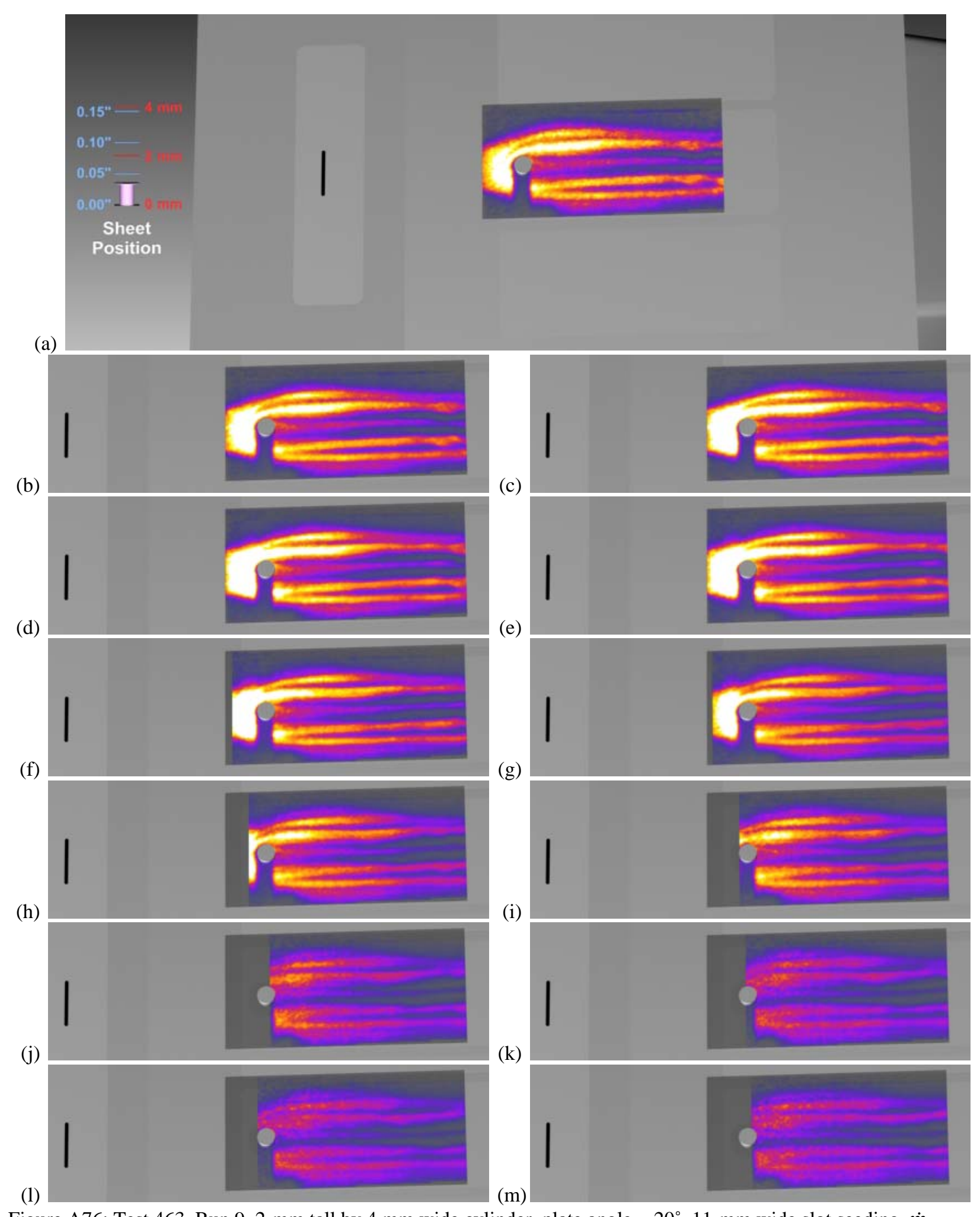

Figure A76: Test 463, Run 9, 2-mm tall by $4 \mathrm{~mm}$ wide cylinder, plate angle $=20^{\circ}$, 11-mm wide slot seeding, $\dot{m}=$ $300 \mathrm{sccm}, \mathrm{P}_{0}=3.45 \mathrm{MPa}$, sheet position $=0.9 \mathrm{~mm}$ above surface, framing rate $=500 \mathrm{kHz}$. 


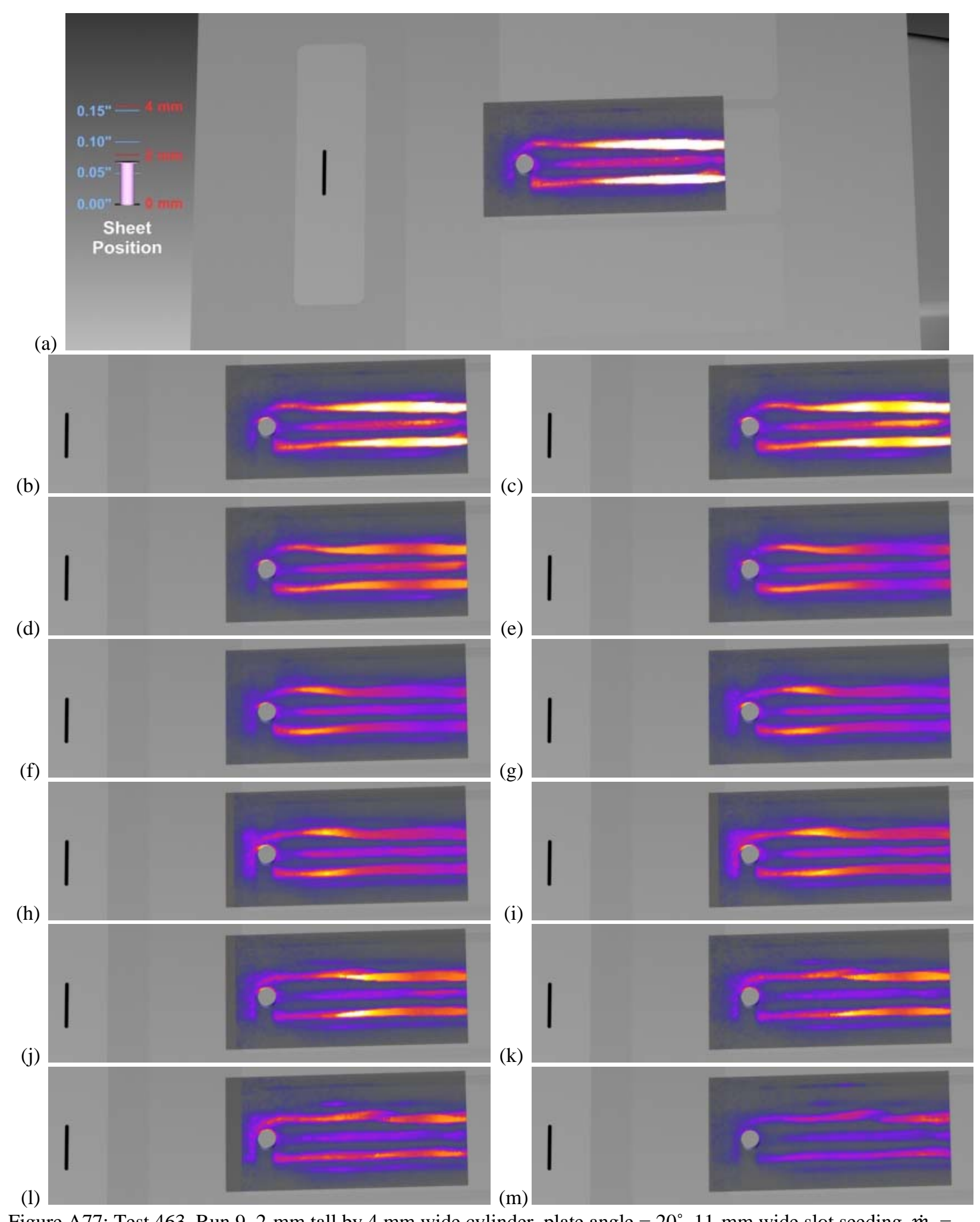

Figure A77: Test 463, Run 9, 2-mm tall by $4 \mathrm{~mm}$ wide cylinder, plate angle $=20^{\circ}$, 11-mm wide slot seeding, $\dot{m}=$ $300 \mathrm{sccm}, \mathrm{P}_{0}=3.45 \mathrm{MPa}$, sheet position $=1.8 \mathrm{~mm}$ above surface, framing rate $=500 \mathrm{kHz}$. 


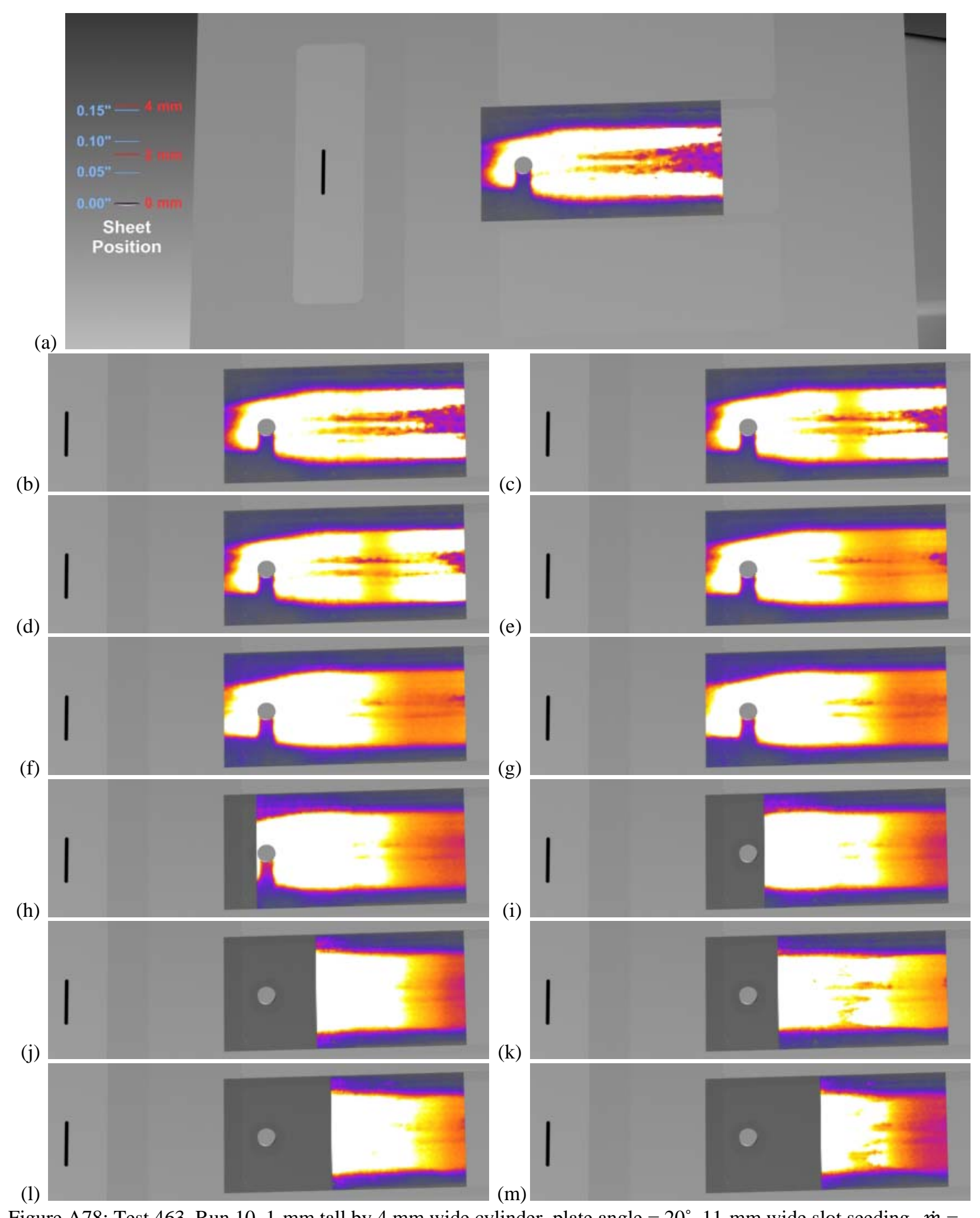

Figure A78: Test 463, Run 10, 1-mm tall by $4 \mathrm{~mm}$ wide cylinder, plate angle $=20^{\circ}, 11$-mm wide slot seeding, $\dot{m}=$ $300 \mathrm{sccm}, \mathrm{P}_{0}=6.91 \mathrm{MPa}$, sheet position $=0 \mathrm{~mm}$ above surface, framing rate $=500 \mathrm{kHz}$. 


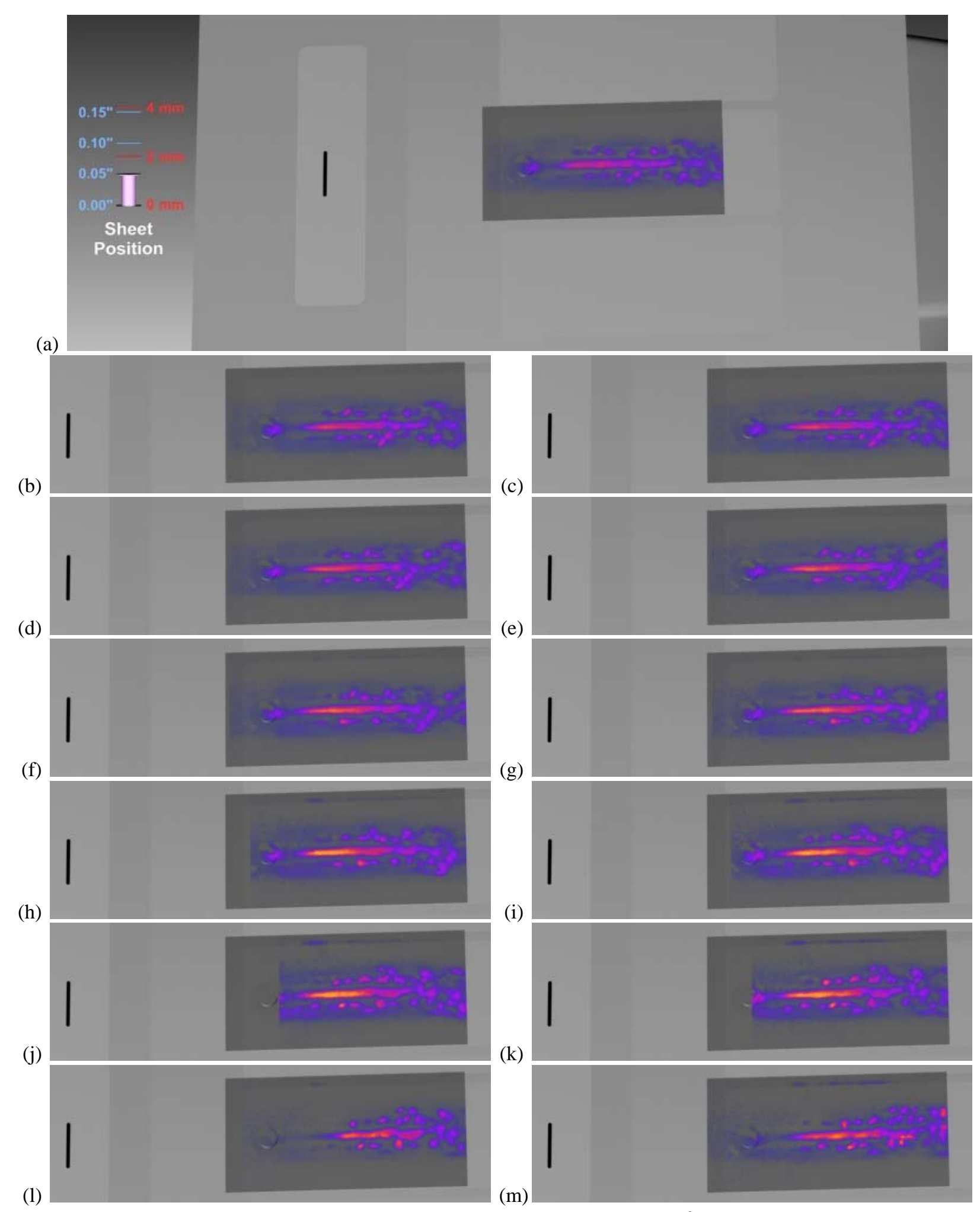

Figure A79: Test 463, Run 10, 1-mm tall by $4 \mathrm{~mm}$ wide cylinder, plate angle $=20^{\circ}, 11$-mm wide slot seeding, $\dot{m}=$ $300 \mathrm{sccm}, \mathrm{P}_{0}=6.91 \mathrm{MPa}$, sheet position $=1.3 \mathrm{~mm}$ above surface, framing rate $=500 \mathrm{kHz}$. 


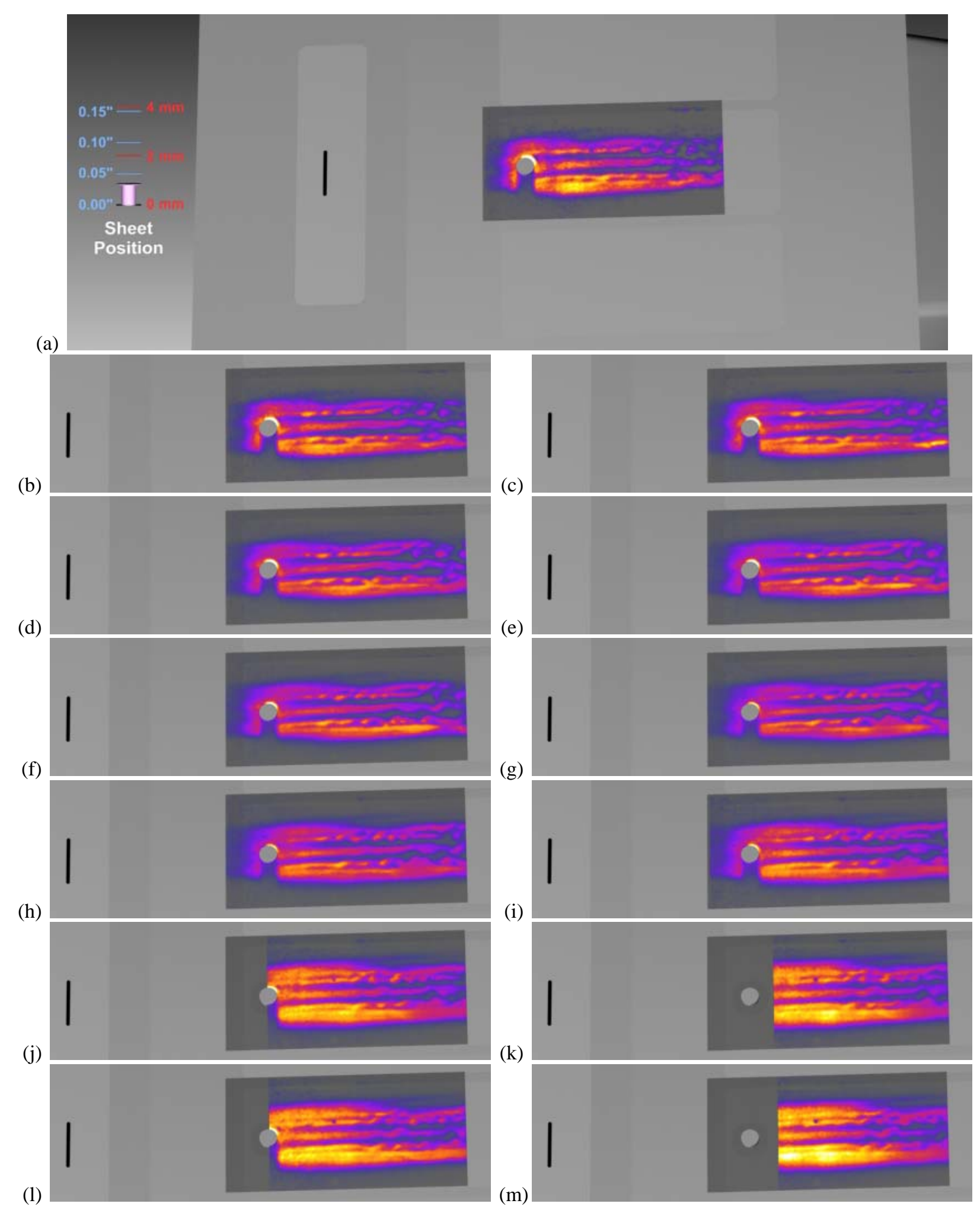

Figure A80: Test 463, Run 10, 1-mm tall by $4 \mathrm{~mm}$ wide cylinder, plate angle $=20^{\circ}, 11$-mm wide slot seeding, $\dot{m}=$ $300 \mathrm{sccm}, \mathrm{P}_{0}=6.91 \mathrm{MPa}$, sheet position $=0.9 \mathrm{~mm}$ above surface, framing rate $=500 \mathrm{kHz}$. 


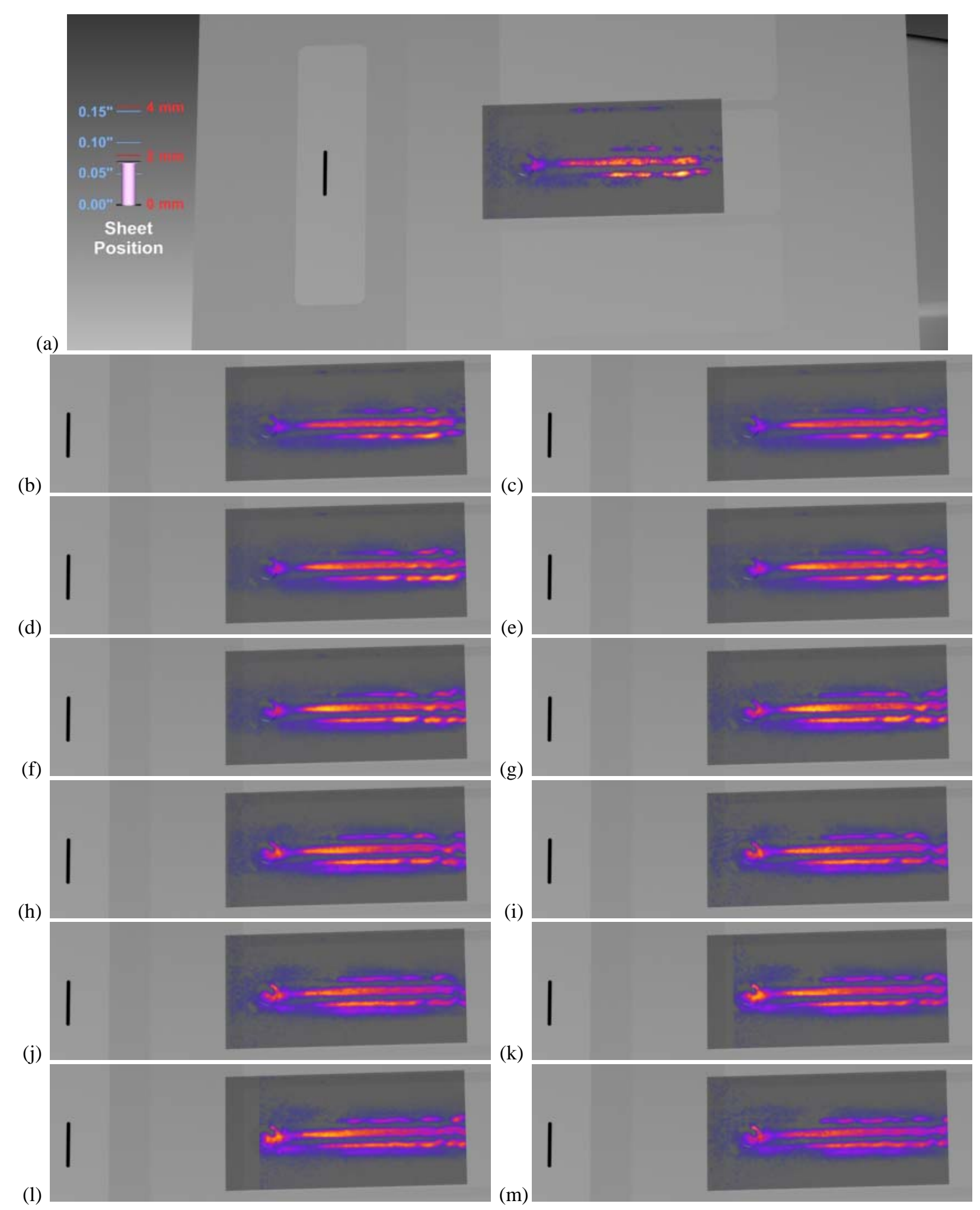

Figure A81: Test 463, Run 10, 1-mm tall by $4 \mathrm{~mm}$ wide cylinder, plate angle $=20^{\circ}, 11$-mm wide slot seeding, $\dot{m}=$ $300 \mathrm{sccm}, \mathrm{P}_{0}=6.91 \mathrm{MPa}$, sheet position $=1.8 \mathrm{~mm}$ above surface, framing rate $=500 \mathrm{kHz}$. 


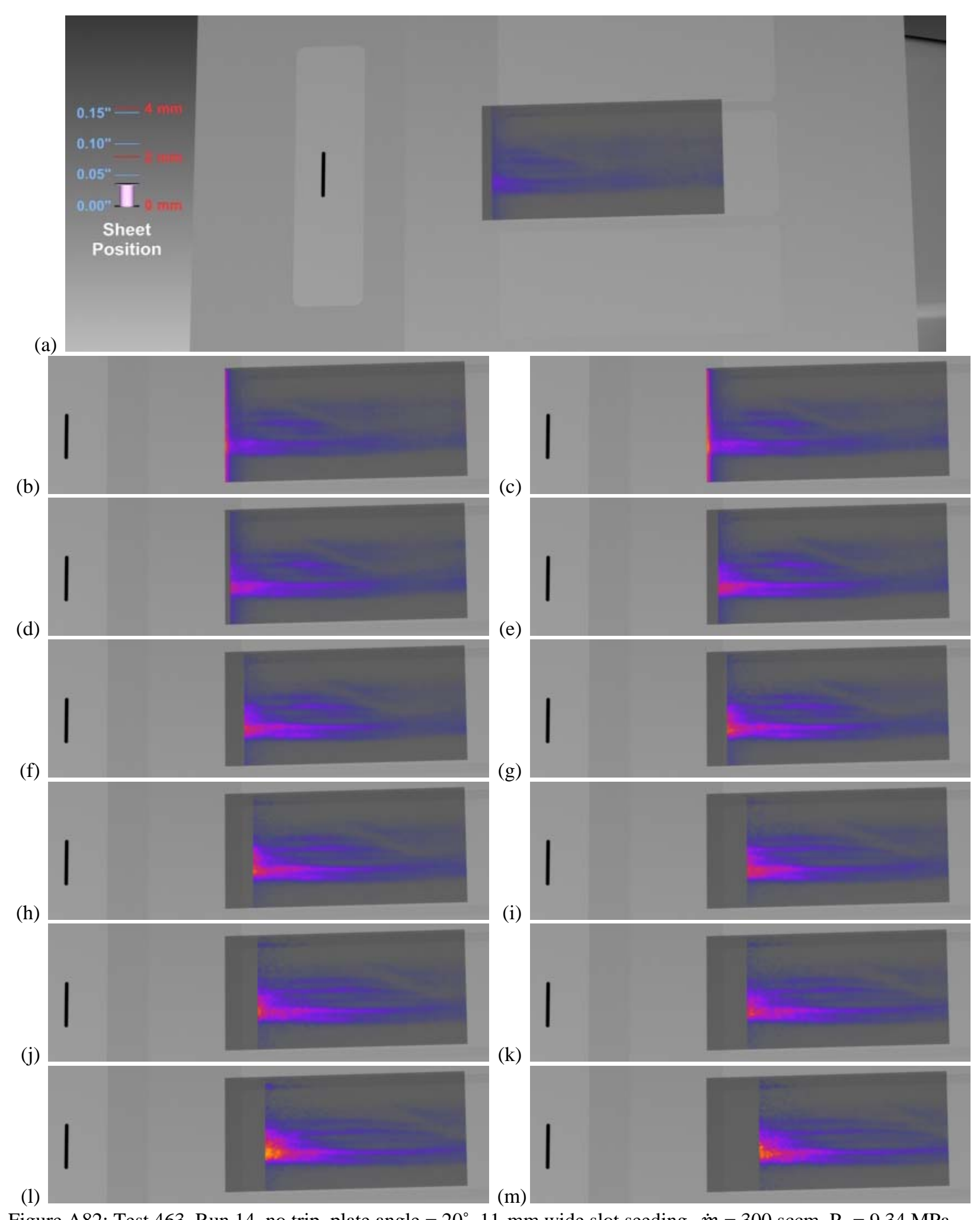

Figure A82: Test 463, Run 14, no trip, plate angle $=20^{\circ}$, 11-mm wide slot seeding, $\dot{m}=300$ sccm, $\mathrm{P}_{0}=9.34 \mathrm{MPa}$, sheet position $=0.9 \mathrm{~mm}$ above surface, framing rate $=500 \mathrm{kHz}$. 


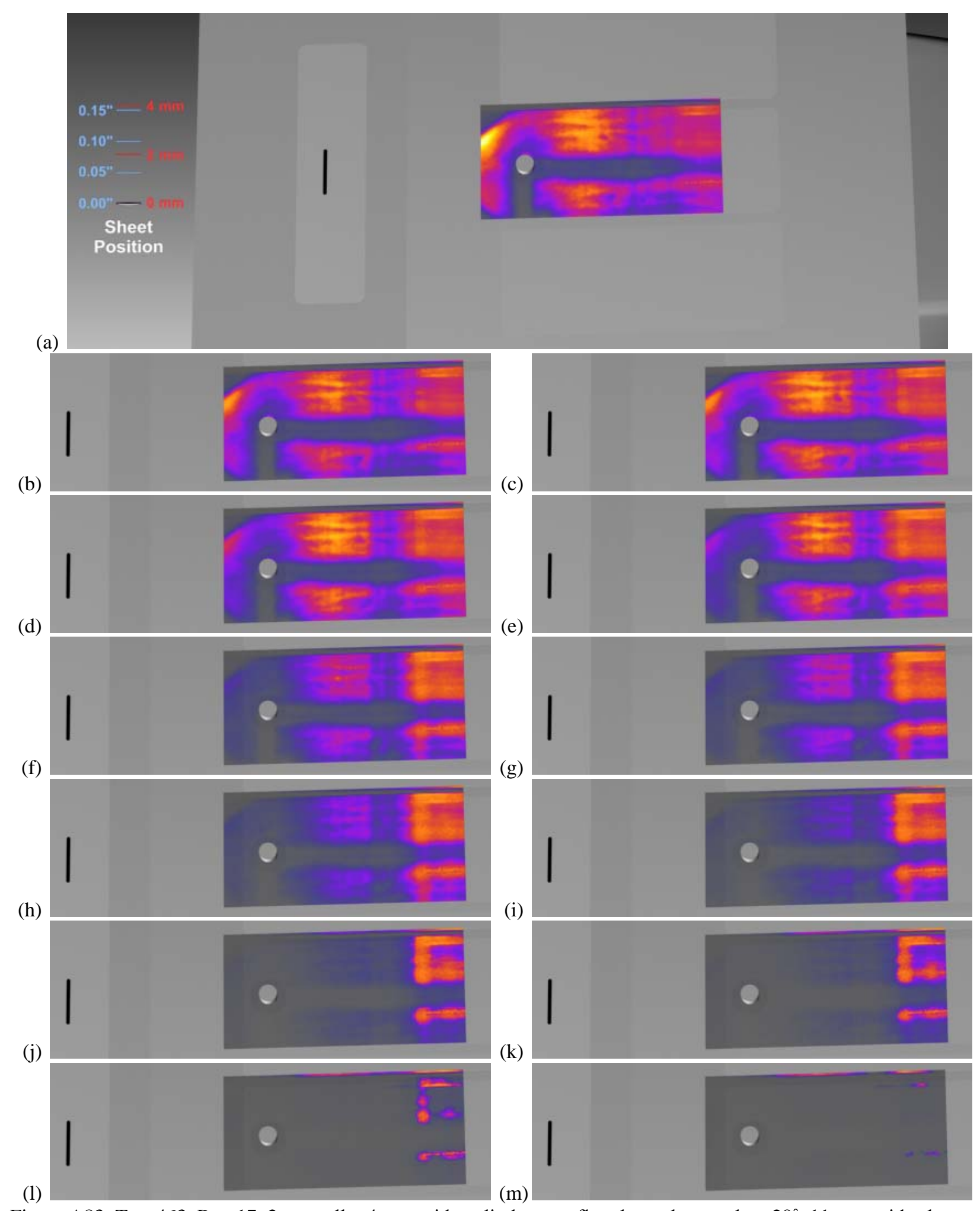

Figure A83: Test 463, Run 17, $2 \mathrm{~mm}$ tall $\mathrm{x} 4 \mathrm{~mm}$ wide cylinder on a flat plate, plate angle $=20^{\circ}$, 11-mm wide slot seeding, $\dot{m}=300 \mathrm{sccm}, \mathrm{P}_{0}=4.97 \mathrm{MPa}$, sheet position $=0.1 \mathrm{~mm}$ above surface, framing rate $=1 \mathrm{MHz}$. 


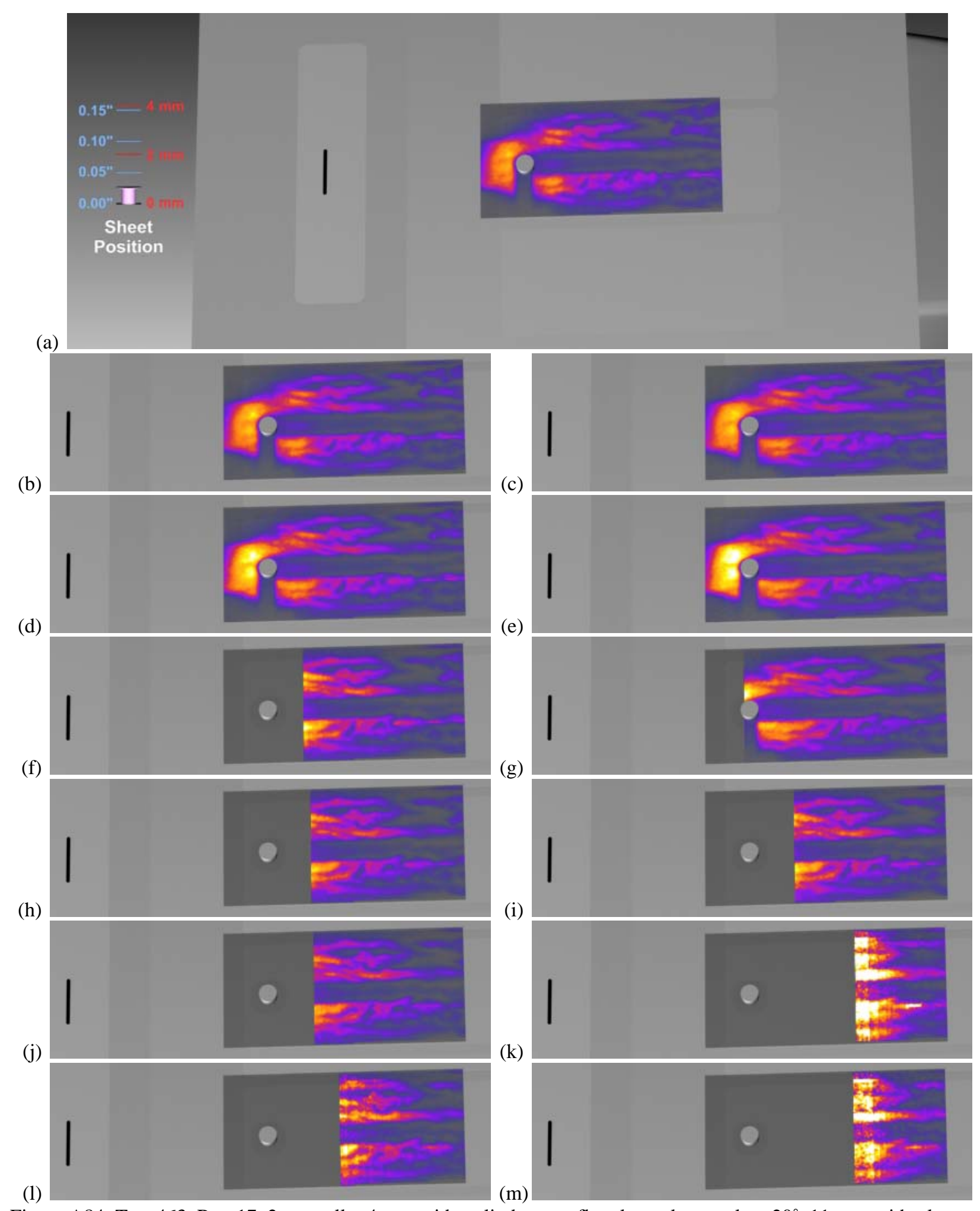

Figure A84: Test 463, Run 17, $2 \mathrm{~mm}$ tall $\mathrm{x} 4 \mathrm{~mm}$ wide cylinder on a flat plate, plate angle $=20^{\circ}, 11$-mm wide slot seeding, $\dot{m}=300 \mathrm{sccm}, \mathrm{P}_{0}=4.97 \mathrm{MPa}$, sheet position $=0.7 \mathrm{~mm}$ above surface, framing rate $=1 \mathrm{MHz}$. 


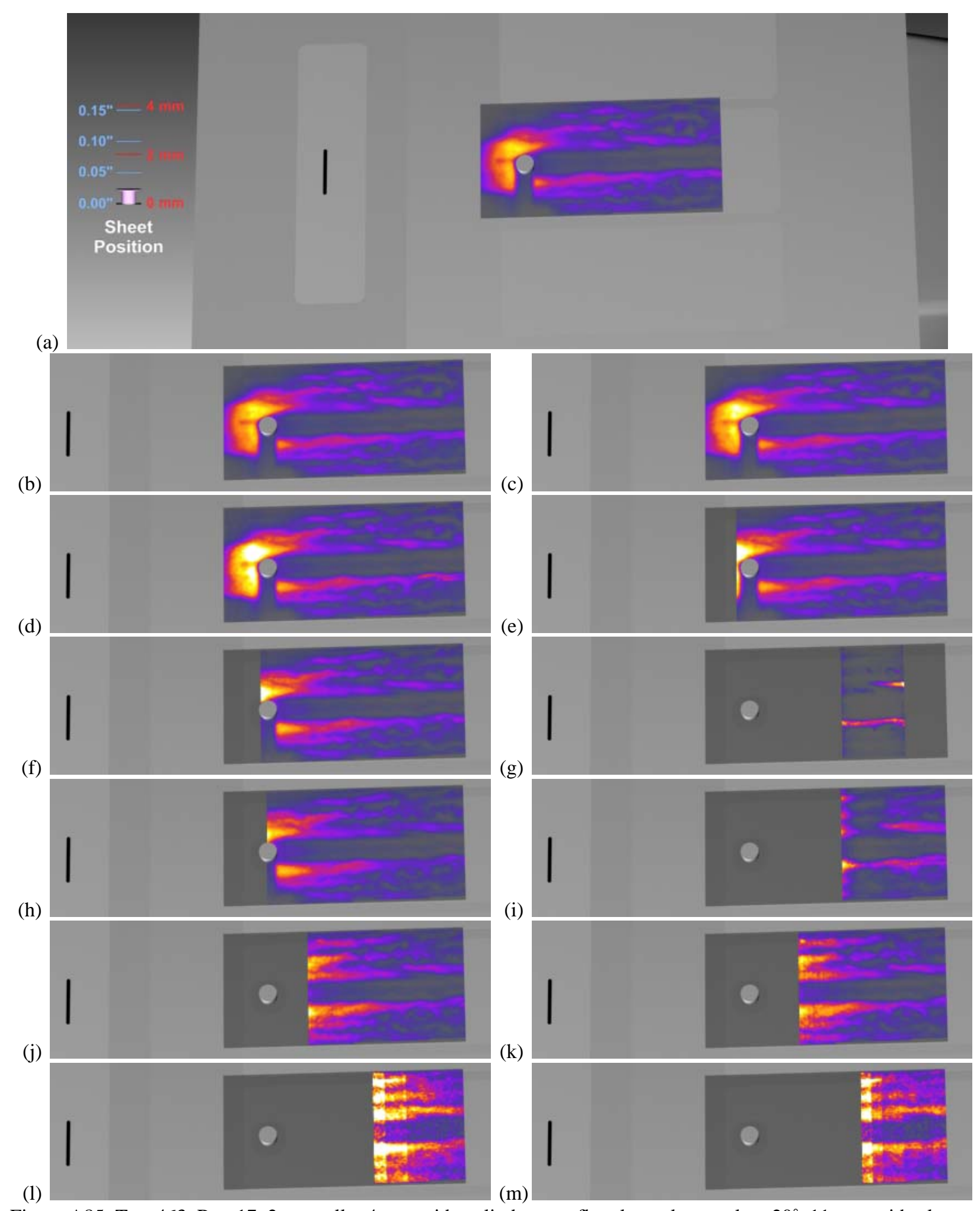

Figure A85: Test 463, Run 17, $2 \mathrm{~mm}$ tall $\mathrm{x} 4 \mathrm{~mm}$ wide cylinder on a flat plate, plate angle $=20^{\circ}$, 11-mm wide slot seeding, $\dot{m}=300 \mathrm{sccm}, \mathrm{P}_{0}=4.97 \mathrm{MPa}$, sheet position $=0.6 \mathrm{~mm}$ above surface, framing rate $=1 \mathrm{MHz}$. 


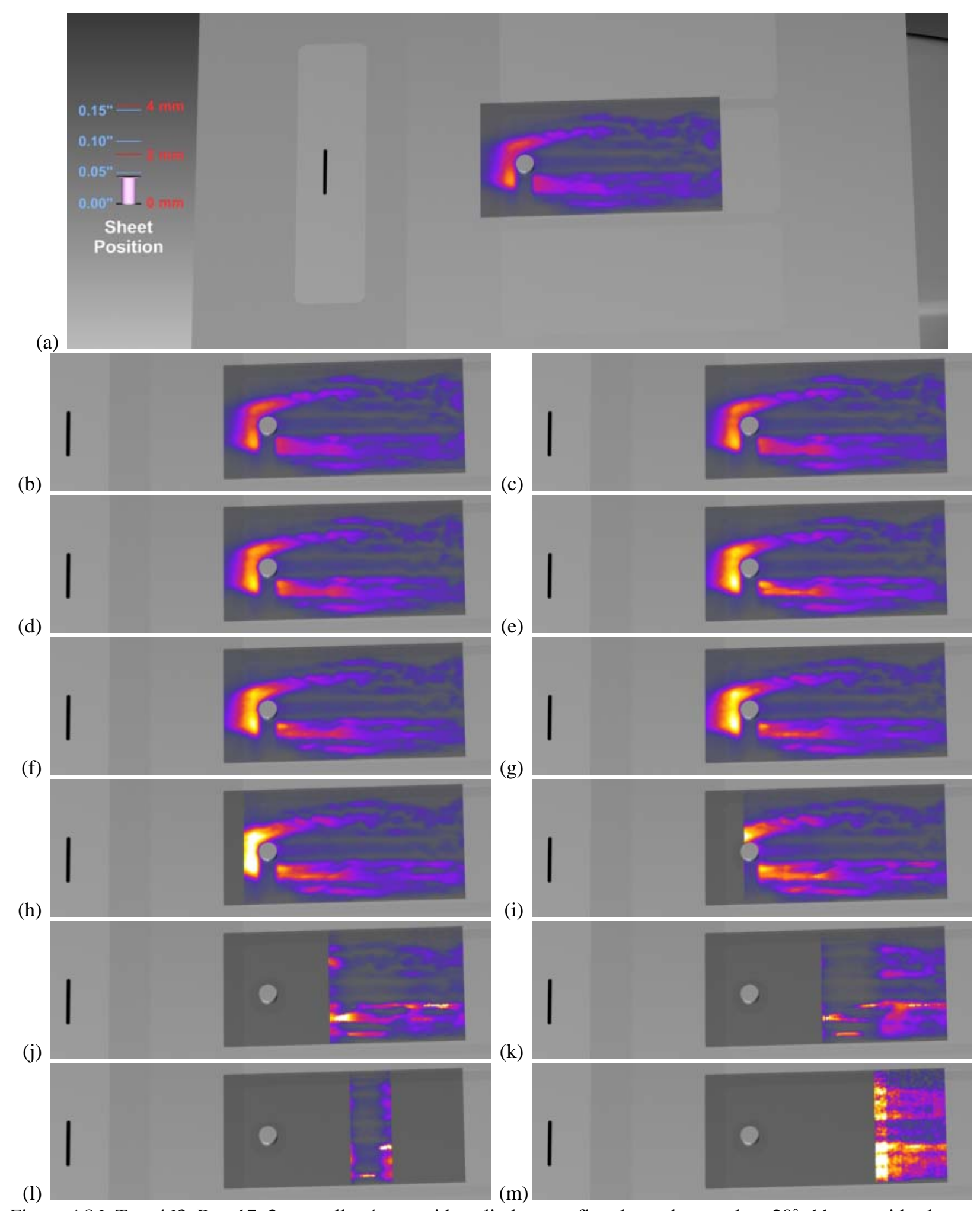

Figure A86: Test 463, Run 17, $2 \mathrm{~mm}$ tall $\mathrm{x} 4 \mathrm{~mm}$ wide cylinder on a flat plate, plate angle $=20^{\circ}$, 11-mm wide slot seeding, $\dot{m}=300 \mathrm{sccm}, \mathrm{P}_{0}=4.97 \mathrm{MPa}$, sheet position $=1.1 \mathrm{~mm}$ above surface, framing rate $=1 \mathrm{MHz}$. 


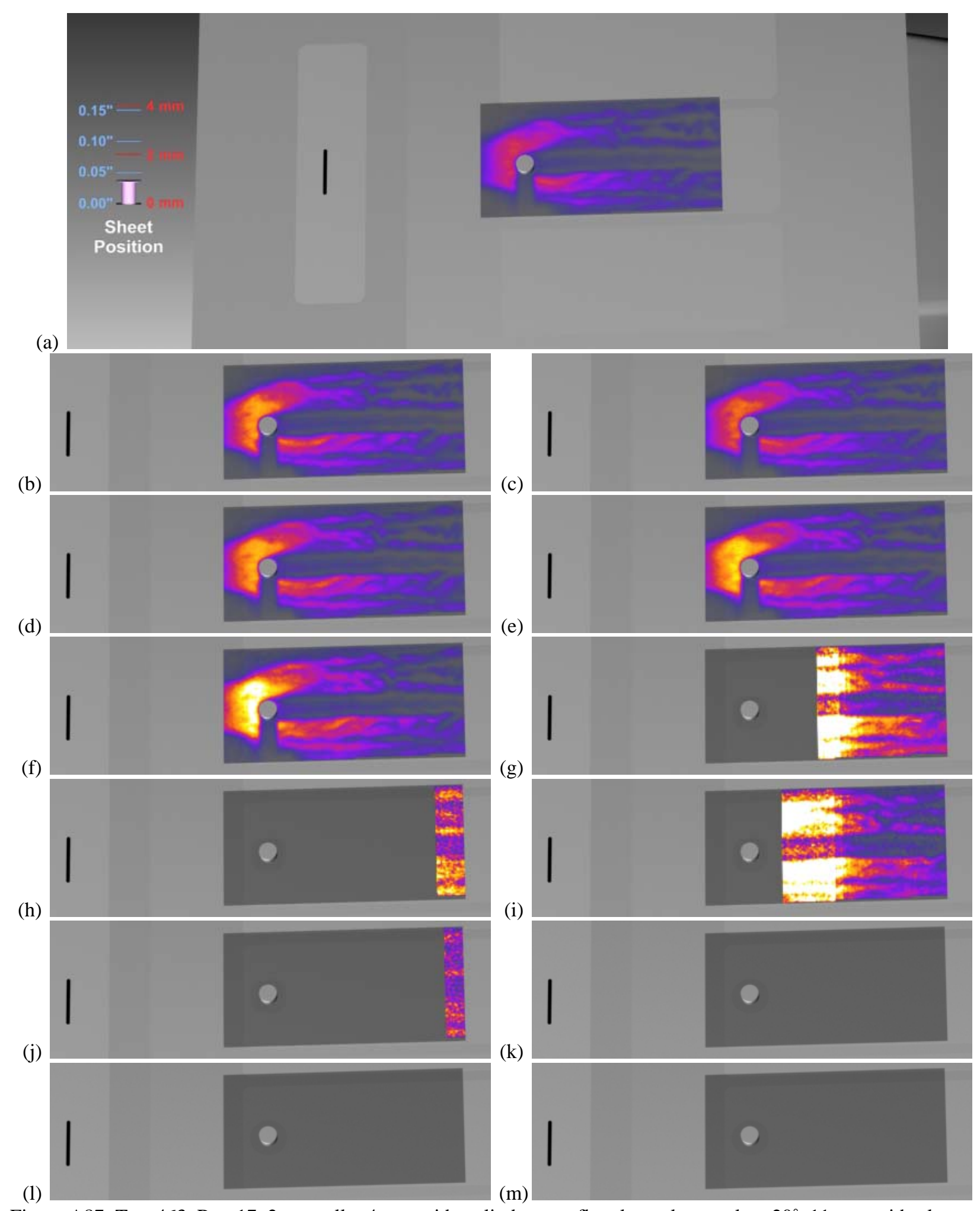

Figure A87: Test 463, Run 17, $2 \mathrm{~mm}$ tall $\mathrm{x} 4 \mathrm{~mm}$ wide cylinder on a flat plate, plate angle $=20^{\circ}$, 11-mm wide slot seeding, $\dot{m}=300 \mathrm{sccm}, \mathrm{P}_{0}=4.97 \mathrm{MPa}$, sheet position $=1.0 \mathrm{~mm}$ above surface, framing rate $=1 \mathrm{MHz}$. 


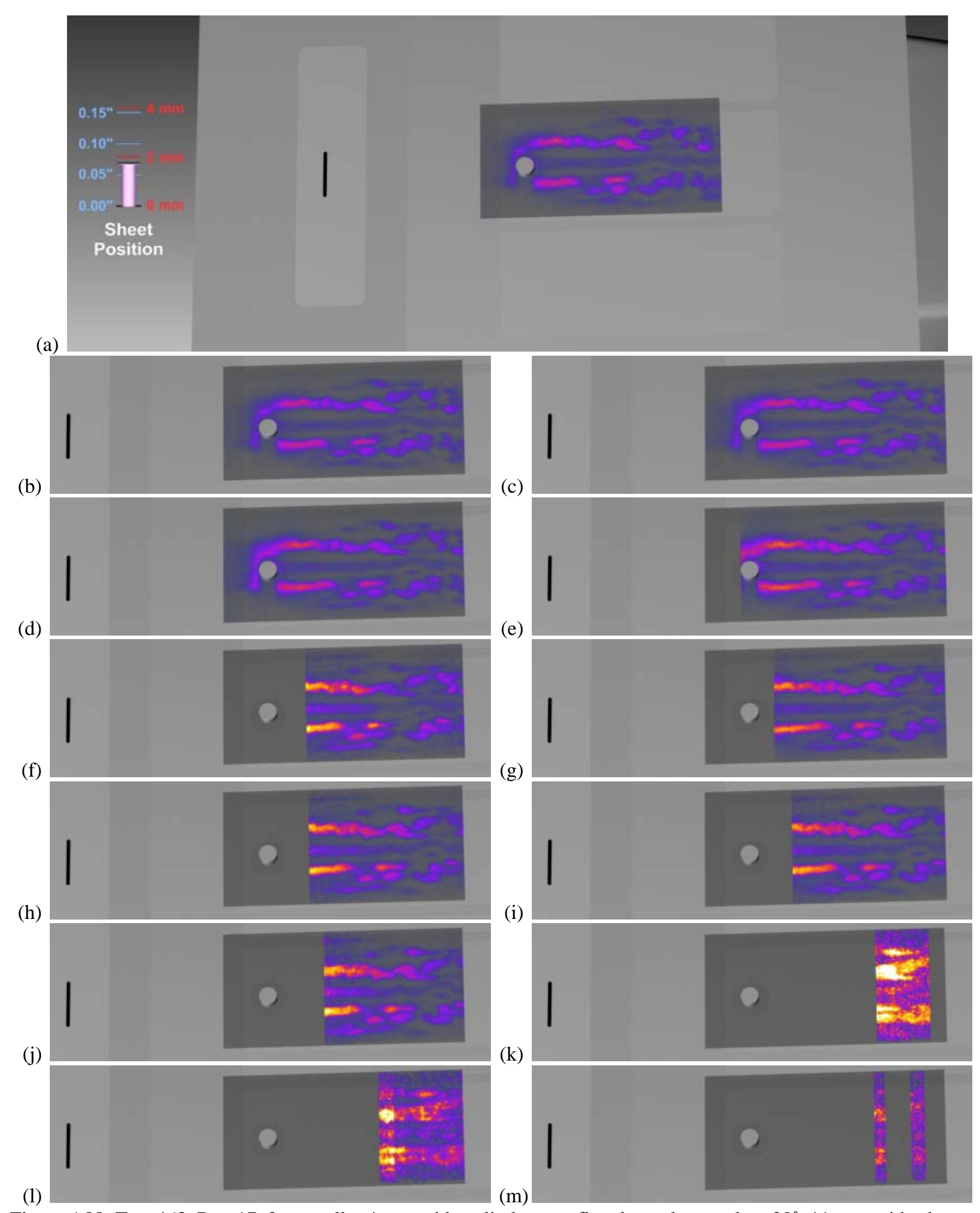

Figure A88: Test 463, Run 17, $2 \mathrm{~mm}$ tall $\mathrm{x} 4 \mathrm{~mm}$ wide cylinder on a flat plate, plate angle $=20^{\circ}$, 11-mm wide slot seeding, $\dot{m}=300 \mathrm{sccm}, \mathrm{P}_{0}=4.97 \mathrm{MPa}$, sheet position $=1.8 \mathrm{~mm}$ above surface, framing rate $=1 \mathrm{MHz}$. 


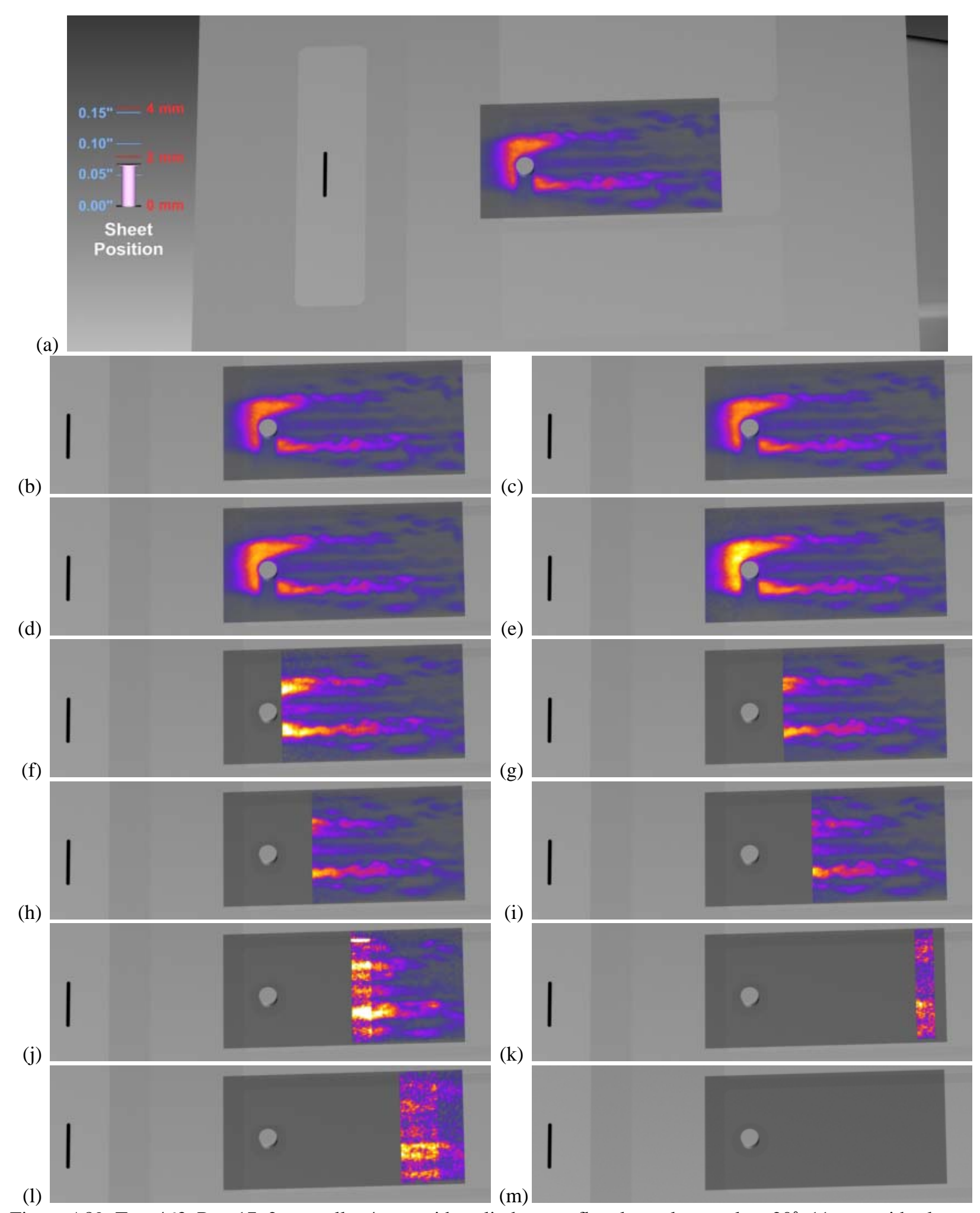

Figure A89: Test 463, Run 17, $2 \mathrm{~mm}$ tall $\mathrm{x} 4 \mathrm{~mm}$ wide cylinder on a flat plate, plate angle $=20^{\circ}$, 11-mm wide slot seeding, $\dot{m}=300 \mathrm{sccm}, \mathrm{P}_{0}=4.97 \mathrm{MPa}$, sheet position $=1.7 \mathrm{~mm}$ above surface, framing rate $=1 \mathrm{MHz}$. 


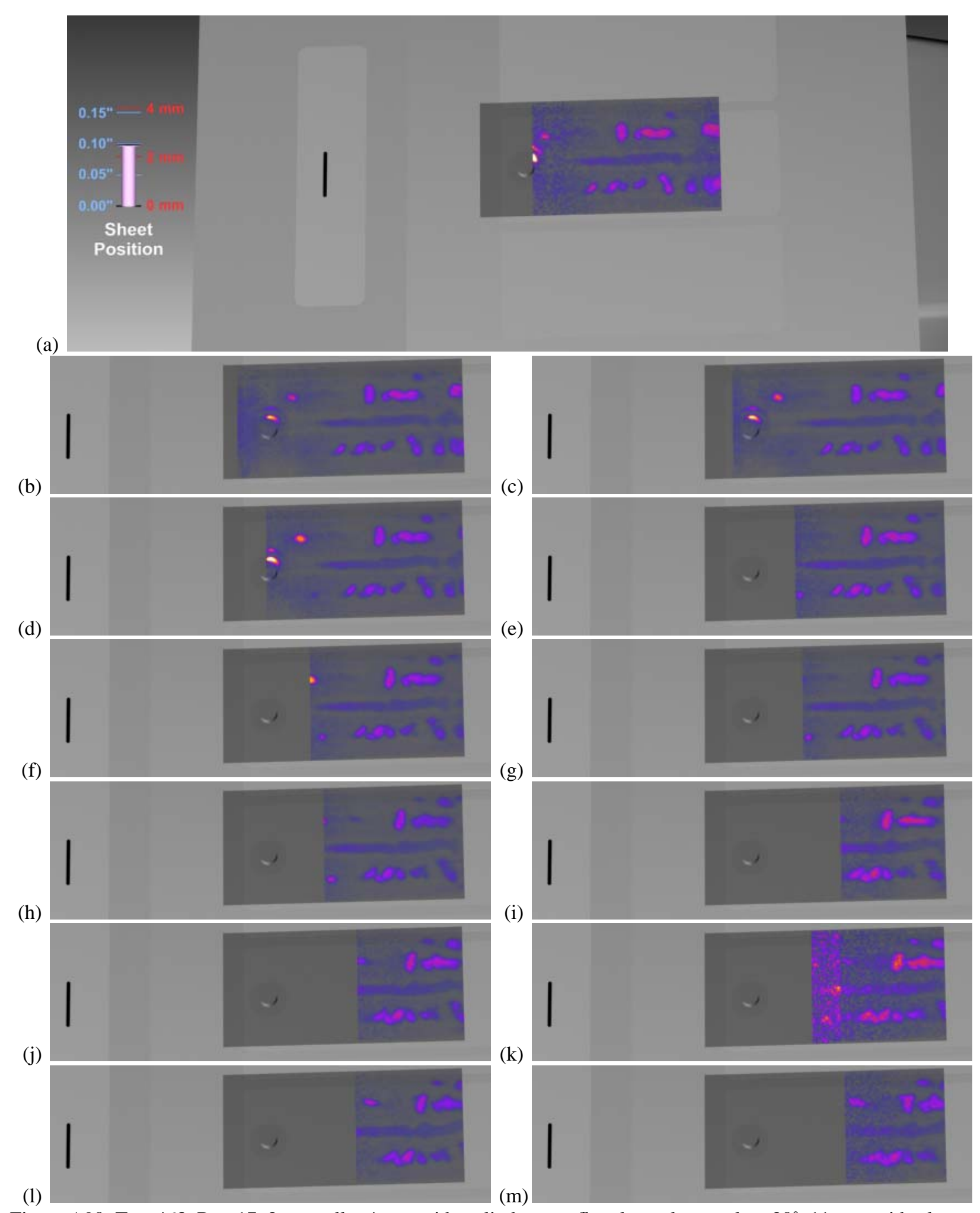

Figure A90: Test 463, Run 17, $2 \mathrm{~mm}$ tall $\mathrm{x} 4 \mathrm{~mm}$ wide cylinder on a flat plate, plate angle $=20^{\circ}, 11$-mm wide slot seeding, $\dot{m}=300 \mathrm{sccm}, \mathrm{P}_{0}=4.97 \mathrm{MPa}$, sheet position $=1.7 \mathrm{~mm}$ above surface, framing rate $=1 \mathrm{MHz}$. 